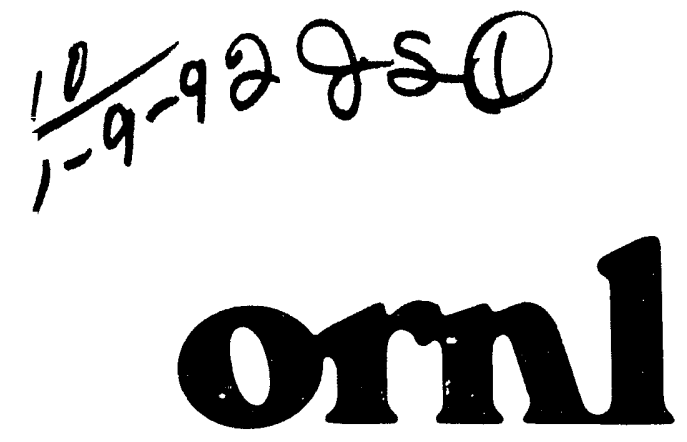

OAK RIDGE

NATIONAL

LABORATORY

MARTIN MARUETAA
ORNL/TM-11851

\title{
Technoeconomic Evaluation of the Extractive Fermentation of Butanol as a Guide to Research in This Area of Biotechnology
}

Robert M. Busche

MANAGED BY

MARTIN MARIETTA ENERGY SYSTEMS, INC.

FOR THE UNITED STATES

DEPARTMENT OF ENERGY 
This report has been reproduced directly from the best available copy.

Available to DOE and DOE contractors from the Office of Scientific and Technical information, P.O. Box 62, Oak Ridge, TN 37831; prices available from (615) 576-8401, FTS 626-8401.

Available to the public from the National Technical Information Service, U.S. Department of Commerce, 5285 Port Royal Rd., Springfield, VA 22161.

This report was prepared as an account of work sponsored by an agency of the United States Government. Neither the United States Government nor any agency thereof, nor any of their employees, makes any warranty, express or implied, or assumes any legal liability or responsibility for the accuracy, completeness, or usefulness of any information, apparatus, product, or process disclosed, or represents that its use would not infringe privately owned rights. Reference herein to any specific commercial product, process, or service by trade name, trademark, manufacturer, or otherwise, does not necessarily constitute or imply its endorsement, recommendation, or favoring by the United States Government or any agency thereof. The views and opinions of authors expressed herein do not necessarily state or reflect those of the United States Government or any agency thereot. 


\title{
TECHNOECONOMIC EVALUATION \\ OF THE EXTRACTIVE FERMENTATION OF BUTANOL AS A GUIDE TO RESEARCH IN THIS AREA OF BIOTECHNOLOGY
}

Robert M. Busche

Bio En-Gene-Er Associates, Inc.

Wilmington, Delaware 19803-2439

\author{
Prepared for the \\ Office of Conservation and Renewable Energy \\ (65 1014801 ) \\ U.S. Department of Energy \\ Washington, DC \\ and \\ National Corn Growers Association \\ St. Louis, Missouri
}

Date Published - Scptember 1991

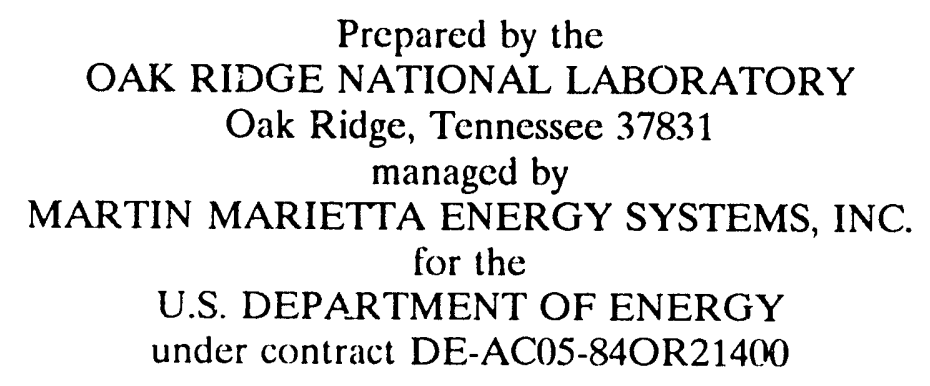


TABLE OF CONTENTS

EXECUTIVE SUMMARY $\ldots \ldots \ldots \ldots \ldots \ldots \ldots \ldots$

1. INTRODUCTION $\ldots \ldots \ldots \ldots \ldots \ldots \ldots \ldots \ldots \ldots \ldots$

2. MARKET POSITION $\ldots \ldots \ldots \ldots \ldots \ldots \ldots \ldots \ldots \ldots$

3. HISTORICAL DEVELOPMENT ............. 9

4. TECHNICAL BACKGROUND $\ldots \ldots \ldots \ldots \ldots \ldots \ldots \ldots \ldots$

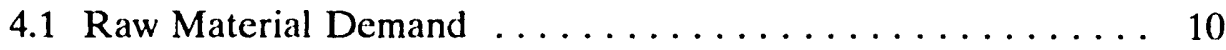

4.2 Product Inhibition $\ldots \ldots \ldots \ldots \ldots \ldots \ldots \ldots \ldots \ldots \ldots \ldots \ldots \ldots \ldots$

5. PROCESS DEVELOPMENT STRATEGY $\ldots \ldots \ldots \ldots \ldots \ldots 17$

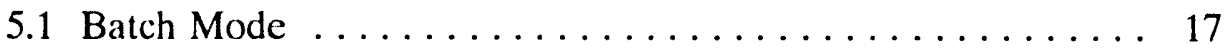

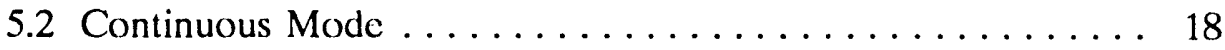

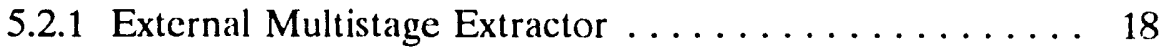

5.2.2 External Extraction with Single Recycle . . . . . . . 19

5.2.3 External Extraction with Double Recycle .......... 19

5.2 .4 In Situ Extraction ................... 20

6. EXTRACTIVE FERMENTATION PROCESS SCENARIOS $\ldots \quad .21$

6.1 Process Scenario for External Extraction . . . . . . . . . 21

6.2 Process Scenario for In Situ Extraction . . . . . . . . . 23

7. OPERATING CONDITIONS ............... 23

8. EXTRACTION PERFORMANCE ............. 26

9. PROJECTED ECONOMICS OF THE CONVENTIONAL WEIZMANN PROCESS $\ldots \ldots \ldots \ldots \ldots \ldots \ldots \ldots \ldots \ldots \ldots$

10. PROJECTED ECONOMICS OF THE EXTERNAL

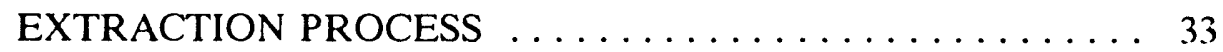

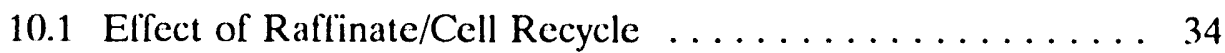

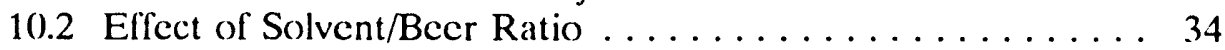

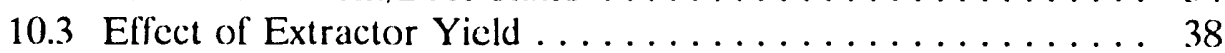

10.4 Effect of Product Concentration ............... 38

10.5 Effect of Sugar Price and Yicld . . . . . . . . . . . 44

10.6 Effect of Recovery of Raffinate Chemicals . . . . . . . 48 
11. PROJECTED ECONOMICS OF THE IN SITU EXTRACTION

PROCESS $\ldots \ldots \ldots \ldots \ldots \ldots \ldots \ldots \ldots \ldots \ldots \ldots \ldots \ldots$

11.1 Effect of Solvent/Feed Ratio .................. 51

11.2 Effect of Butanol Concentration ............... 51

11.3 Effect of Sugar Price $\ldots \ldots \ldots \ldots \ldots \ldots \ldots \ldots \ldots \ldots$

12. RECOMMENDATIONS $\ldots \ldots \ldots \ldots \ldots \ldots \ldots \ldots \ldots$

13. CONCLUSIONS $\ldots \ldots \ldots \ldots \ldots \ldots \ldots \ldots \ldots \ldots \ldots \ldots \ldots \ldots \ldots$

14. ACKNOWLEDGMENT $\ldots \ldots \ldots \ldots \ldots \ldots \ldots \ldots \ldots$

15. REFERENCES $\ldots \ldots \ldots \ldots \ldots \ldots \ldots \ldots \ldots \ldots \ldots \ldots \ldots$

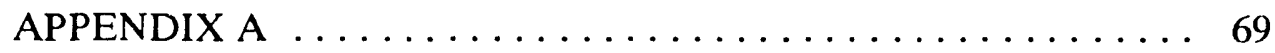

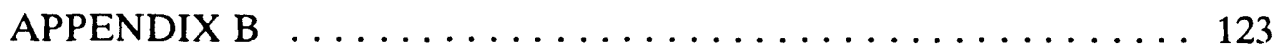

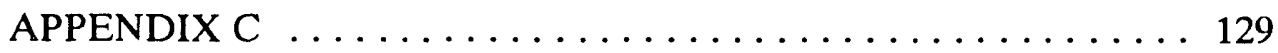

APPENDIX D $\ldots \ldots \ldots \ldots \ldots \ldots \ldots \ldots \ldots \ldots \ldots \ldots \ldots \ldots$

APPENDIX E $\ldots \ldots \ldots \ldots \ldots \ldots \ldots \ldots \ldots \ldots \ldots \ldots \ldots \ldots$

APPENDIX F $\ldots \ldots \ldots \ldots \ldots \ldots \ldots \ldots \ldots \ldots \ldots \ldots \ldots \ldots \ldots \ldots$

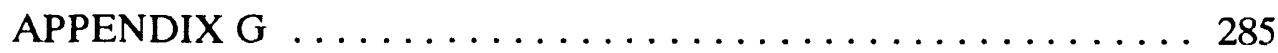




\section{EXECUTTVE SUMMARY}

From World War I until the early 1930s, butanol was produced from corn by the Weizmann ABE process. Thereafter, however, the fermentation process was replaced by the cheaper Oxo process for producing synthetic butanol from propylene. The U.S. annual production is now close to 1 billion lb.

Synthetic butanol currently sells for $\$ 0.38 / \mathrm{b}$ at a crude oil price of $\$ 17-\$ 20 / \mathrm{bbl}$. However, considering the increases expected again in crude oil prices in the 1990s, a doubling in the price for propylene-derived butanol is not inconceivable.

At the current state of the art, the $\mathrm{ABE}$ process would require butanol prices in excess of $\$ 1.20 / \mathrm{hb}$ to be competitive. Two major problems exist: (1) low product concentrations in the beer resulting from inhibition of the functioning of the organism byproduct, which lead to high process investment charges; and (2) poor yields related to large losses of substrate to carbon dioxide and by-products, coupled with high prices for corn syrup compared with presently depressed oil prices.

To circumvent the inhibition problem, two scenarios have been developed for a new extractive fermentation process to remove the product from the field of fermentation "as rapidly as it forms." Both models rely on continuous fermentation instead of batch operation to effect significant cost reductions.

In the external multistage extraction model, the fermenter is operated with a large recycle of cells to increase volumetric productivity while maintaining product concentration in the beer that is fed to the extractor close to the threshold of inhibition at $13 \mathrm{~g} / \mathrm{L}$. As a result, it appears that fermenter volume and investment could be dramatically reduced. Raffinate is also recycled to improve the recovery of valuable by-products. Selling prices below $\$ 0.43 / \mathrm{lb}$ look feasible.

In the in situ single-stage extraction model, the fermentation is operated with simultaneous extraction at high ratios of solvent to aqueous feed so that, in fact, the product is removed into the solvent phase as rapidly as it forms. It appears that effective concentrations in the fermenter could reach $-200 \mathrm{~g} / \mathrm{L}$ while maintaining product concentration at $13 \mathrm{~g} / \mathrm{L}$ in the aqueous phase containing the organism. Since total plant investment decreases in direct proportion to increases in concentration, major cost 
reductions could be expected. For this case, cell density and product cost would be about the same as in the external extraction case.

For either model, genetically engineering an organism that was less inhibited by product or substrate or that produced only butanol as the preferred product would lead to further cost reductions. If the goal case can be achieved, cost would be reduced to $\$ 0.35 / \mathrm{lb}$ butanol. However, the microbiological research program that would be required would have a lower probability of success than the engineering program required to develop the extraction processes based on the existing organism.

Certainly, these improvements would represent major breakthroughs in not only the Weizmann process, but, generically, in any fermentation process that suffers from product inhibition (which is most of them). Continuing research along these lines is highly recommended. Suggestions for fine-tuning the program are appended. 


\section{INTRODUCTION}

Since the Middle East oil crisis of 1973, many people in government, academia, and industry have been concerned about the strategic implications of a loss of a major source of crude oil for American industry. Accordingly, over the past decade a large number of research programs have been directed toward exploring the potential use of abundant renewable materials as basic feedstocks for fuels and chemicals. The Biocatalyst Project, administered by the Jet Propulsion Laboratory as part of the Energy Conversion and Utilization Technologies (ECUT) program of the Department of Energy, is now in the forefront of this effort.

This report represents the completion of a part of an overall project to cvaluate the technical and economic status of several newly conceptualized processes for producing butanol, acetone, ethanol, acetic acid, and aerobically produced specialty chemicals, which are candidates for research support. The objectives of the project are to identify strengths and weaknesses in the proposed processes and to assist in developing an ongoing research strategy along economically relevant lines.

The products to be studied presently comprise a collective U.S. market for 10.7 billion $\mathrm{lb}$ valued at $\$ 2.8$ billion. If their manufacturing processes were converted from petroleum feedstocks to corn, they would consume 556 million bushels.

Furthermore, if ethanol could be produced at a low enough price to serve as the precursor to ethylene and butadiene, it and its derivatives could account for 159 billion $\mathrm{lb}$, or $50 \%$ of the U.S. production of 316 billion lb of synthetic organic chemicals, presently valued at $\$ 113$ billion. ${ }^{1}$ This use would consume 3.4 billion bushels, or $\sim 45 \%$ of the corn crop. In addition, the use of butanol for diesel blends or in jet fuel blends to enhance the range of military aircraft could further increase its market.

At the present state of the antiquated art for producing these products from corn by fermentation, product costs do not appear competitive compared with prices for incumbent petroleum-based chemicals. Industrial interest in fermentation chemicals had revived in the 1970s as a result of the oil crisis. However, the subsequent softening in oil prices in the 1980s removed the competitive edge of renewable materials compared with 
fossil feedstocks and resulted in an almost complete loss of momentum in research in this area of biotechnology.

In general, fermentation processes have two major problems:

1. inherently poor yiclds resulting from the production of by-products, including high levels of carbon dioxide and hydrogen needed to maintain the electronic balance of the metabolism of the organism, coupled with the current relatively high cost of renewable sugars and starches compared with the presently depressed prices for petroleum; and

2. the inhibition of most organisms by their own products, which causes the fermentation to shut down after reaching only low, $\sim 1 \%$, product concentrations, as a result of which the recovery of product from dilute aqueous solution is accordingly expensive.

The problem resulting from the cost of raw materials will presumably rectify itself in a few years after increased demand for oil relative to Middle East production capacity and further Middle East turmoil again foree oil prices to rise. Conoco predicts that this will happen in the 1990s (Fig. 1), at which time it is expected that the United States will be importing half of its oil supply instead of the 7\% it imported at the time of the 1973 oil crisis. ${ }^{2}$

One possible solution to the inhibition problem would be to apply extraction or distillation as an integral part of the fermentation process so as to remove the product from the ficld of fermentation as rapidly as it forms. In the case of an external multistage extraction process, this is expected to permit a proportionate increase in cell density while maintaining product concentration in the fermenter near the threshold of inhibition. As a result, volumetric productivity increases and fermenter in astment and cost decrease. Alternatively, in the case of an in situ single-stage fermenter/extractor, this is expected to increase productivity and effective product concentration while maintaining low concentrations in the beer.

In the current technocconomic study of the butanol process, the state of the art for its fermentative manufacture was defined. From this, scenarios for an improved process were developed based on the expectations for adapting extractive fermentation to 


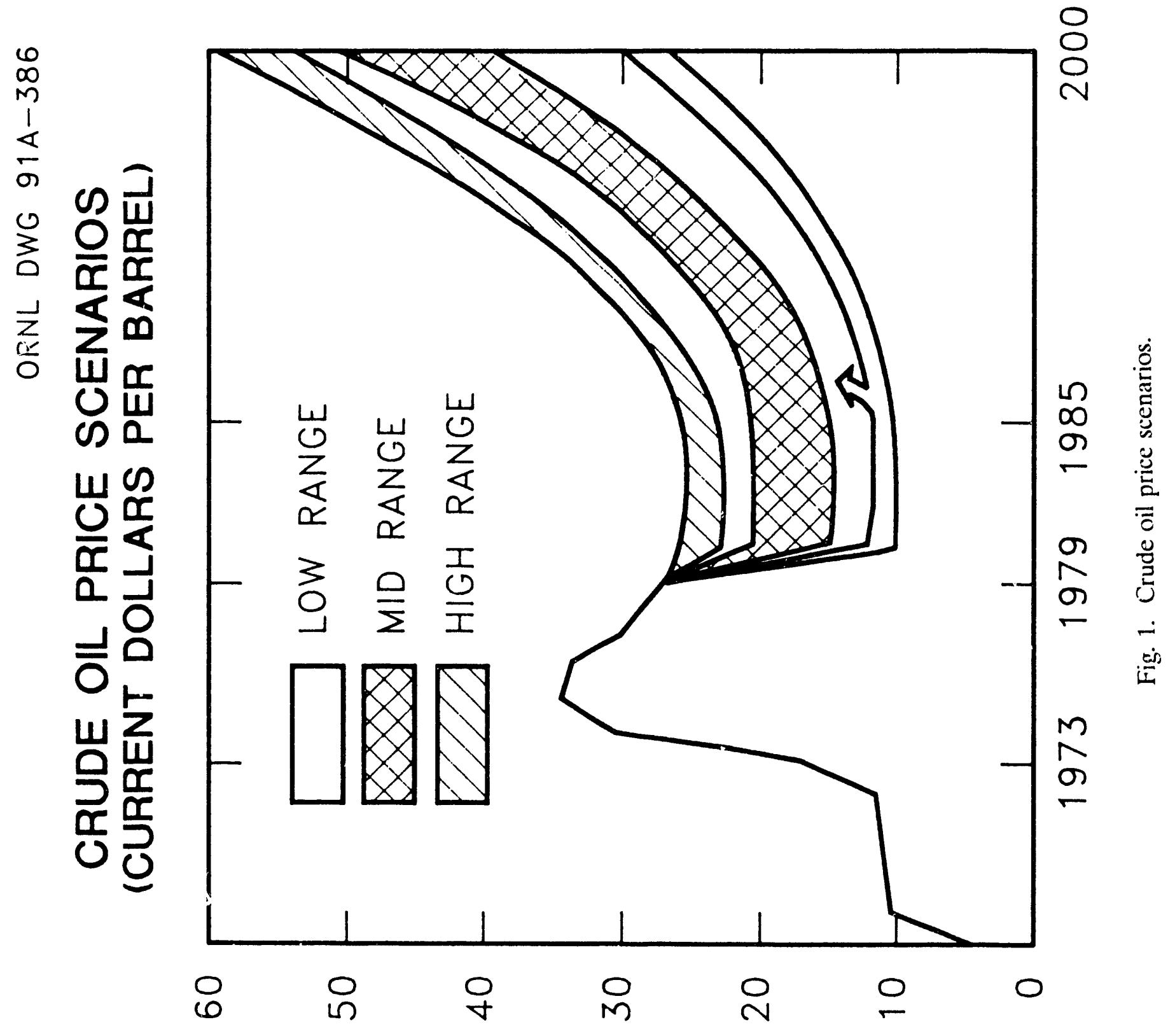


reach plausible cell densities and effective conentration levels. The economics of these scenarios were then developed. The sensitivity of the economics to attaining, exceeding, or falling short of goals for key operating parameters was also determined. It is hoped that the results will provide a strong perspective as to the relative merits for supporting research on any of the alternatives and the direction the research should be channeled so as to be economically relevant and improve the technocconomic position of the process.

\section{MARKET POSITION}

The United States leads among world producers of butanol (Table 1). ${ }^{3}$ Production has risen steadily at 5\% annually since 1964 to a production level of $\sim 918$ million lb of $n$-butanol plus 190 million $\mathrm{lb}$ of isobutanol. European production is less and stagnani; Japanese production has declined to where Japan is now a net importer.

Almost all butanol is made from propylene by the Oxo process. Consumption favors $n$-butanol in all market areas, but especially in U.S. markets. As a result, newer plants are based on a rhodium catalyst system that enhances the production of $n$-butanol, the preferred isomer.

Table 1. Butanol production $\left(10^{6} \mathrm{lb}\right)$

\begin{tabular}{lccc}
\hline Country & $n$-Butanol & Isobutanol & $n$ /iso \\
\hline United States & 842 & 143 & 5.9 \\
Europe & 300 & 220 & 1.4 \\
Japan & 43 & 36 & 2.6 \\
\hline
\end{tabular}

The major U.S. end uses for $n$-butanol in descending order are: butyl acrylates/methacrylates for latex paints, textile finishes, and floor polishes; glycol ethers as 
solvents for surface coatings and adhesives; butyl acetate as a rapid drying solvent for varnishes; dibutylphthalate and other plasticizers for a variety of adhesives, packaging films, and surface coatings; amino acids; butylamines for use in pesticides and rubber processing; and uses in fire-resistant hydraulic fluids. Demand is expected to increase by $3.2-3.5 \%$ annually to reach 920 million $\mathrm{lb}$ in 1988.

Isobutanol has similar uses but favors direct solvent use. It also finds use in lube oil additives. It is generally inferior to $n$-butanol in properties and finds use only if price is sufficiently discounted -- historically $15 \%$.

The use of $n$-butanol and isobutanol in blends with methanol as an octane enhancer for gasoline has been introduced but has faced environmental challenges and stiff competition from Oxinol tert-butanol blends and ethanol blends. The economics associated with butanol blends do not appear attractive when compared with methanol/ethanol blends. However, butanol might find better use as blends with diesel oil and with jet fuel for military aircraft.

Acetone, the primary by-product in butanol manufacture by fermentation, has actually a more important role than butanol in world markets. Demand in the United States over the decade of the 1980 s has been relatively flat, at $\sim 2.5$ billion lb. Most of this total has been for producing methacrylates or acrylic resins (Fig. 2). It must be noted that the acetone supply is derived from two sources: (1) as a by-product in the conversion of cumene to phenol, or (2) by direct synthesis from isopropanol. U.S producers are listed in Table 2 on the basis of their raw material. By-product acetone tracks the demand for adhesives for plywood for the building trades and is always sold off first at a discount under the cost of isopropylene-derived product. Accordingly, acetone derived from fermentation must either compete with the latter or be discounted as the former. Recent producers of sugar-derived acetone are listed in Table 3. Of these, only the South African plant is significant. 
ORNL DWG $91 \mathrm{~A}-384$

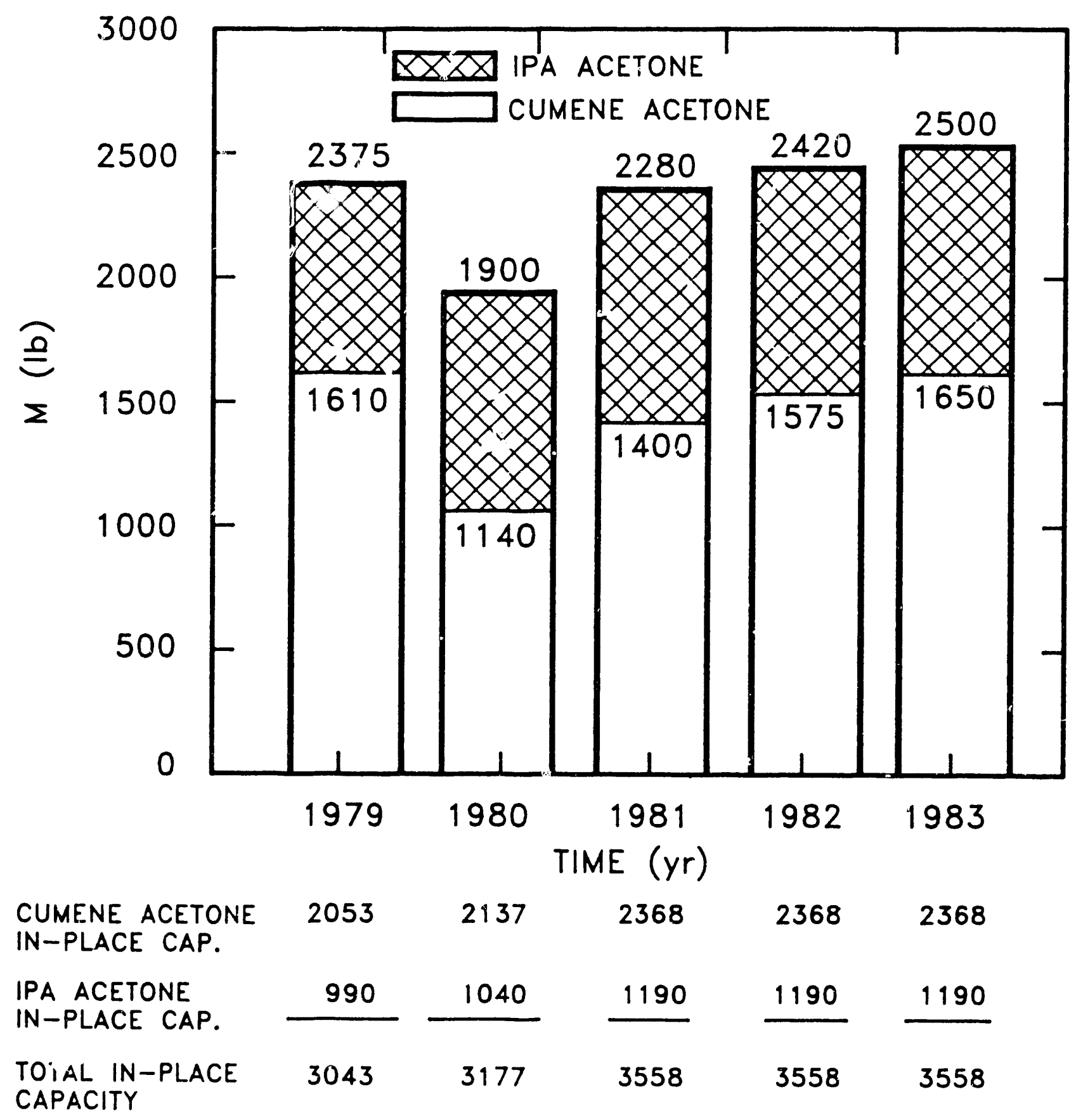

Fig. 2. U.S. Acetone Industry supply/demand. 
Table 2. U.S. acetone producers' "in-place" capacities

\begin{tabular}{|c|c|c|c|c|c|}
\hline \multirow[b]{2}{*}{ Producer } & \multicolumn{5}{|c|}{ Output $\left(10^{6} \mathrm{lb}\right)$} \\
\hline & 1980 & 1981 & 1982 & 1983 & 1984 \\
\hline \multicolumn{6}{|l|}{ Cumene-based } \\
\hline Allied & 360 & 360 & 360 & 360 & 360 \\
\hline Chevron & 33 & 33 & 33 & 33 & 33 \\
\hline Clark & 54 & 54 & 54 & 54 & 54 \\
\hline Dow & 284 & 284 & 284 & 284 & 284 \\
\hline General Electric & 30 & 240 & 240 & 240 & 240 \\
\hline Georgia Pacific & 177 & 198 & 198 & 198 & 198 \\
\hline Getty (Skelly) & 57 & 57 & 57 & 57 & 57 \\
\hline Monsanto & 300 & 300 & 300 & 300 & 300 \\
\hline Shell & 300 & 300 & 300 & 300 & 300 \\
\hline U.C.C. & 230 & 230 & 230 & 230 & 230 \\
\hline U.S. Steel & 312 & 312 & 312 & 312 & 312 \\
\hline Subtotal & 2137 & 2368 & 2368 & 2368 & 2368 \\
\hline Eastman & 80 & 80 & 80 & 80 & 80 \\
\hline Exxon & 140 & 140 & 140 & 140 & 140 \\
\hline Shell & 650 & 800 & 800 & 800 & 800 \\
\hline U.C.C. & 170 & 170 & 170 & 170 & 170 \\
\hline Subtotal & 1040 & 1190 & 1190 & 1190 & 1190 \\
\hline GRAND TOTAL & 3177 & 3558 & 3558 & 3558 & 3558 \\
\hline
\end{tabular}


Table 3. Producers of sugar-derived acetone

\begin{tabular}{ll}
\hline \multicolumn{1}{c}{ Producer } & \multicolumn{1}{c}{ Status } \\
\hline National Chemical Products Co. & Operating \\
Transvaal, Union South Africa & $12 \times 25 \mathrm{M}$ gal \\
Egyptian Sugar and Distillation Co. & Operating \\
El-Hawamdia, Giza, A.R.E. & \\
Soviet Union & Operating \\
& \\
Commercial Solvents Corp. & Torn Down \\
Terre Haute, IN & $50 \mathrm{M}$ gal \\
Chase Chemical & Torn Down \\
Puerto Rico & $2 \times 60 \mathrm{M}$ gal \\
& \\
Publicker Industries & Standby \\
Philadelphia, PA & $140 \mathrm{Million} \mathrm{PPY}$ \\
& $5 \times 2000 \mathrm{M}$ gal (EtOH) \\
& $10 \times 500 \mathrm{M}$ gal \\
& $9 \times 250 \mathrm{M}$ gal \\
& $12 \times 18 \mathrm{M}$ gal \\
& $6 \times 1 \mathrm{M}$ gal \\
\hline
\end{tabular}




\section{HISTORICAL DEVELOPMENT}

The original Weizmann process for fermenting starch-containing grains to butanol and acetone was developed under the stimulus of the World War I demand for acetone for manufacturing "cordite," a double-based smokeless powder used for British naval guns. The process made a successful transition to civilian products but was finally supplanted in the 1950 s by cheaper petrochemical processes. Interest in the fermentation process revived following the energy crisis of 1973 but was cooled again as a result of the softening in oil prices in the early 1980 s. $^{4}$

A few years prior to World War I, Weizmann had developed an organism, $C$. acetobutylicum, which successfully fermented starchy grains to produce a mixture of acetone, butanol, and ethanol (ABE). He applied for and received patents on the so-called ABE process. ${ }^{5,6}$ With the outbreak of World War I, a plant was built at Kings Lynn, England, in 1914 to produce acetone by fermentation, but operation was a failure until Weizmann was placed in charge and installed his process. Because of the shortage of corn in England, the process was transferred to Canada in August 1916, where it operated until November 1918. To supplement this output, a butanol-acetone piant was built in Terre Haute, Indiana, which operated from May to November 1918 under the auspices of the War Production Board. While the Terre Haute Plant operated, there was no use for butanol and it was stored.

However, shortly after the end of the war, the Dupont Company developed Duco nitrocellulose lacquers for use in automobile finishes. It was found that $n$-butyl acetate was the solvent of choice for this coating system. In order to supply the $n$-butanol required for making the acetate, the Terre Haute plant was reactivated in 1920 as a private venture by Commercial Solvents Corporation, which had acquired an exclusive license under the Weizmann U.S. patent. Commercial Solvents used 50,000)-gal fermenters to make the solvents. The plant is now owned by International Minerals and Chemical Corporation. It no longer operates the fermentation equipment but has maintained the historical cultures and has advised that the technology is available for licensing. 
When the patents expired in 1936, new ABE plants were built in Philadelphia (Publicker), Baltimore, Puerto Rico, and Japan. A flow sheet for the process as practiced by Publicker in the late 1930s and 1940s is shown in Fig. 3. The Publicker plant was based on the use of 5000-gal Hortonspheres through 500,000-gal tanks for the batch fermenter train. Operation of this plant was described in detail by Beesch. ${ }^{7}$ The plant was, since, shut down but was considered for reactivation in the late 1970s. The decision to proceed was deferred as a result of the oil glut of the 1980s.

At the present time, the only $\mathrm{ABE}$ plant operating in the free world is at Germiston in the Union of South Africa at National Chemical Products, Ltd., a division of Sentrachem Ltd. Started up in 1936, its operation was described by Spivey. ${ }^{8}$

\section{TECHNICAL BACKGROUND}

\subsection{RAW MATERIAL DEMAND}

Raw material economics has always been one of the most important parameters in the choice between fermentation processes for producing solvents. Until 1938, the ABE process operated solely on corn using $C$. acetobutylicum, with a solvents yield of $-26.5 \%$, based on dry corn and comprising roughly $60 \%$ butanol, $30 \%$ acetone, and $10 \%$ ethanol. ${ }^{911}$ After 1938, new organisms were developed which allowed the use of cheaper molasses.

Early in this study, it became apparent that the stoichiometry of the fermentation was very important to the economics. The metabolic pathway followed by $C$. acetobutylicum is outlined in Fig. 4. Electron balance must be maintained among the competing reactions. Yields to the various products are outlined in Table 4. Even though the organism operates near its biological maximum, carbon yields are poor because of the large losses to carbon dioxide. In general, the solvents are formed in the ratio $60 \%$ $n$-butanol, $30 \%$ acetone, and $10 \%$ ethanol, with the corresponding release of hydrogen and carbon dioxide. 


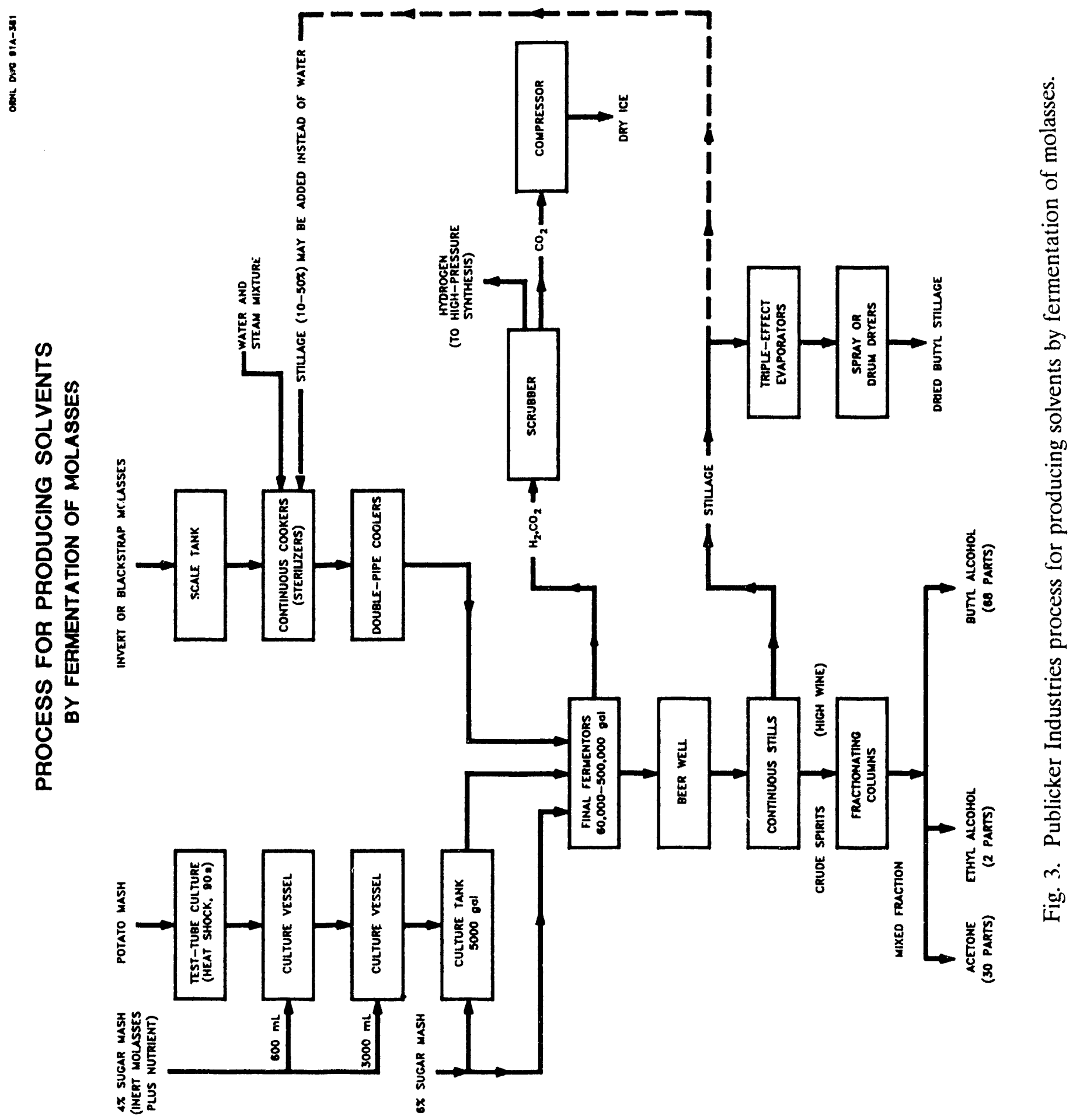




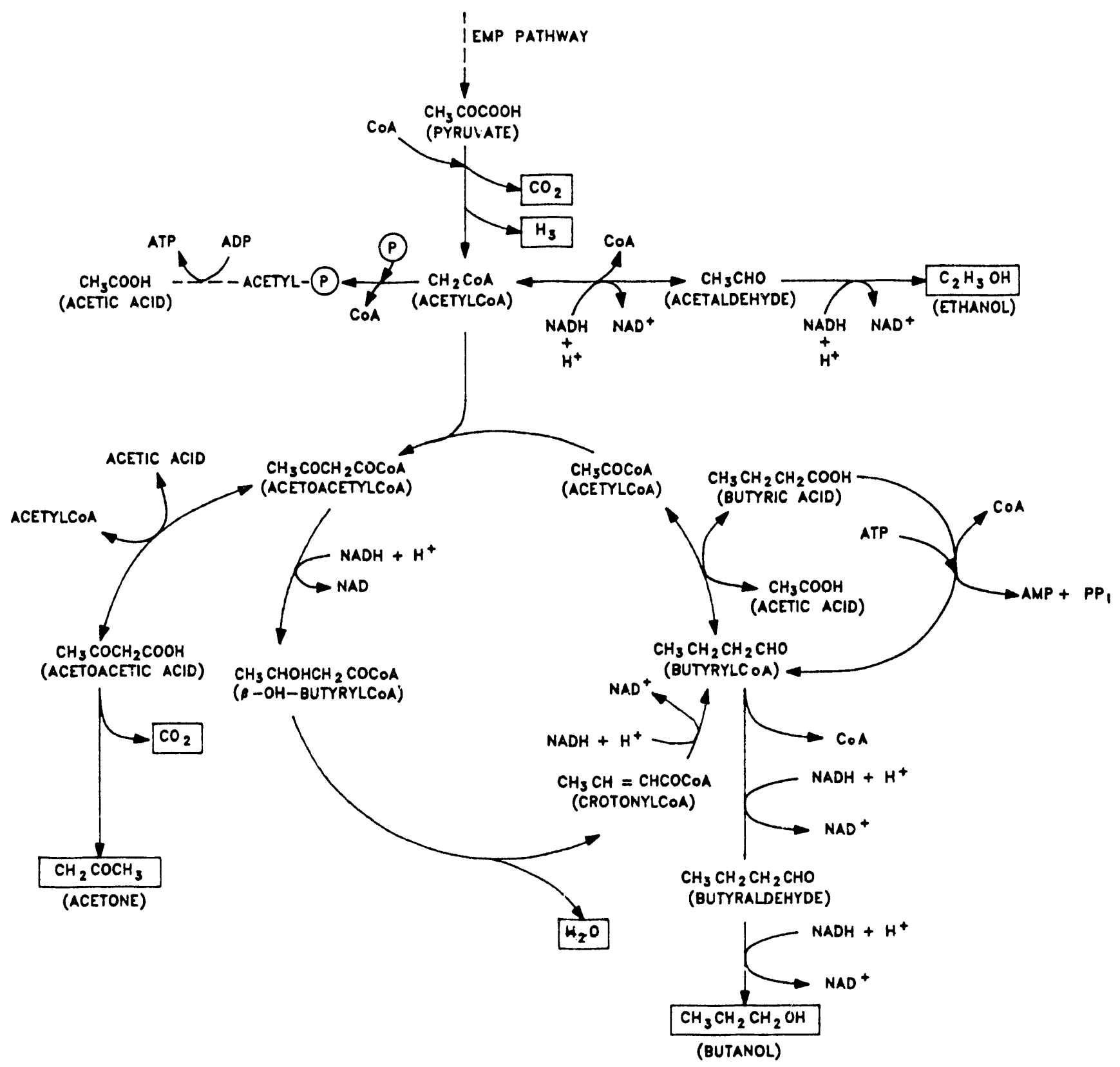

Fig. 4. Metabolic pathway for glucose utilization by clostridium bacteria. 
Table 4. Fermentation stoichiometry (per $100 \mathrm{~mol}$ butanol)

Basecase

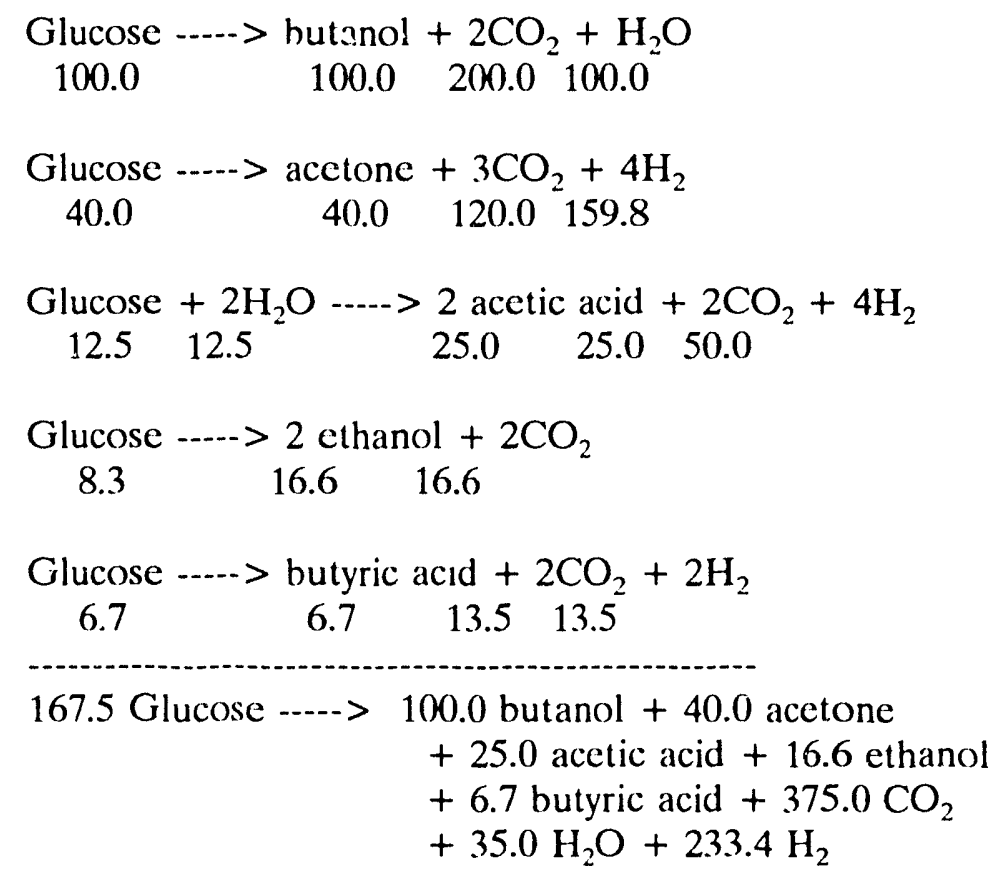

Goal case

100.0 glucose.$--->100.0$ butanol $+200.0 \mathrm{CO}_{2}+100.0 \mathrm{H}_{2} \mathrm{O}$ 
Alternatively, Weimer has postulated that an organism could be developed that would produce only butanol according to the "goal" case of Table 3. This could theoretically be accomplished, although with great difficulty, by blocking the acetyl CoA to acetaldehyde (to ethanol) pathway and the acetoacetyl CoA to acetone pathway. ${ }^{12}$ Small amounts of acetaldehyde might be added to the fermentation to inhibit the ethanol route, but since acetaldehyde is toxic in general, it might completely stop the fermentation.

\subsection{PRODUCT INHIBITION}

As with most fermentations, $C$. acetobutylicum is inhibited by its own substrate and products. Previous studies have shown that butanol is the most toxic of the products. ${ }^{13,14}$ The fermentation is totally inhibited by butanol concentrations of $10-15 \mathrm{~g} / \mathrm{L}$. ${ }^{4,15,16}$ Thus, in normal batch operation, productivity is limited to $\sim 0.25 \mathrm{~g} /(\mathrm{L} \cdot \mathrm{h})$, or $13 \mathrm{~g} / \mathrm{L}$ butanol after 40 batch hours plus $12 \mathrm{~h}$ of fermenter turnaround. Productivities in the range 0.16-0.58 $\mathrm{g} /(\mathrm{L} \cdot \mathrm{h})$ have been reported by various investigators. ${ }^{12-17}$ This experience has suggested to many that the fermentation rate and product concentration could be raised to commercial levels by removing the butanol from the fermentation medium as fast as it forms. This assumption forms the basis for the scenarios developed for economic evaluation in this study.

Various methods for removing inhibitory products have been proposed. These include adsorption by activated carbon, ${ }^{17-20}$ ion-exchange resins, ${ }^{21-24}$ or polymeric resins; ${ }^{25-28}$ extraction by aqueous solvents ${ }^{29-35}$ or organic solvents; ${ }^{36-42}$ or membrane separation. ${ }^{43-44}$ The use of organic solvents is the subject of the butanol study.

As a preamble to extraction studies, a number of laboratories have tested the toxicity of various solvents toward the organism..$^{37,39,40,45-50}$ In many cases, it was found that good solvents for the products were also toxic to the organism.

In the early 1980s, Leung of $\mathrm{MIT}^{45}$ studied at small scale the extraction of butanol with corn oil during fermentation to minimize inhibition.

Gill and Ratledge ${ }^{51}$ suggested that the toxicity of hydrocarbons toward specific organisms might be related to the aqueous solubility of these compounds. They showed that toxicity was reduced by adding a nontoxic compound such as hexadecane to the organic phase. 
Evans and Wang at Michigan ${ }^{52}$ studied extractive fermentation at a 10-mL-test-tube scale with the aim of optimizing distribution coefficient versus toxicity by using mixtures of toxic solvents having good distribution cocfficients with nontoxic hydrocarbons having low solubility in the aqueous fermentation rnedium. Oleyl alcohol was found to be a good solveni with low toxicity.

Japanese investigators also demonstrated the use of oleyl alcohol to extract butanol in batch experiments. ${ }^{39}$

Blanch and coworkers at the University of California at Berkeley ${ }^{53}$ also showed in limited small-scale tests using oleyl alcohol as a solvent that the effect of butanol inhibition could be reduced and volumetric productivity increased by removing butanol in either hatch $^{54}$ or fed-batch culture. ${ }^{55}$ The feasibility of using continuous processing in extractive fermentation was also demonstrated at bench scale in experiments in which the fermenter broth was continuously recycled to an external extraction column. ${ }^{56}$ In the fed-batch experiments using oleyl alcohol as a solvent, they were able to increase productivity to 1.5 $\mathrm{g} /(\mathrm{L} \cdot \mathrm{h})$. However, in these experiments, productivity can also be inhibited by the high concentrations of the sugar substrate required in batch culture to drive the fermentation to high butanol productivities. True continuous culture would circumvent this problem. Blanch's continuous runs were operated for over $55 \mathrm{~h}$ at double the productivity of batch or fed-batch culture.

In normal batch operation, productivity is limited to $\sim 0.25 \mathrm{~g} /(\mathrm{L} \cdot \mathrm{h})$, or $13 \mathrm{~g} / \mathrm{L}$ butanol after 40 batch h plus $12 \mathrm{~h}$ of fermenter turnaround. Productivities in the range of $0.16-0.58 \mathrm{~g} /(\mathrm{L} \cdot \mathrm{h})$ have been reported by various investigators. $33,39,41,42,56,57$

The extractive fermentation program at Batlelle Memorial Institute picked up where these studies leli olf. Since early 1986, Battelle had been developing its "multi-phase fluidized-bed (MPFB) bioreactor" concept with the intent to enhance fermentation by removing product as fast as it forms using an in situ solvent (oleyl alcohol, etc.) that is not soluble in the acpucous fermentation medium and nontoxic to the organism. A schematic drawing of the bioreactor concept is shown in Fig. 5. This design was used to represent the fermenters in this study. The production of butanol/acetone/ethanol by C. acetobutylicum was chosen as the demonstration example for the generic process. More recently, this program has been transferred to the Oak Ridge National Laboratory. If 


\section{MULTIPHASE FLUIDIZED BED BIOREACTOR \\ WITH CEL RECYCLE}

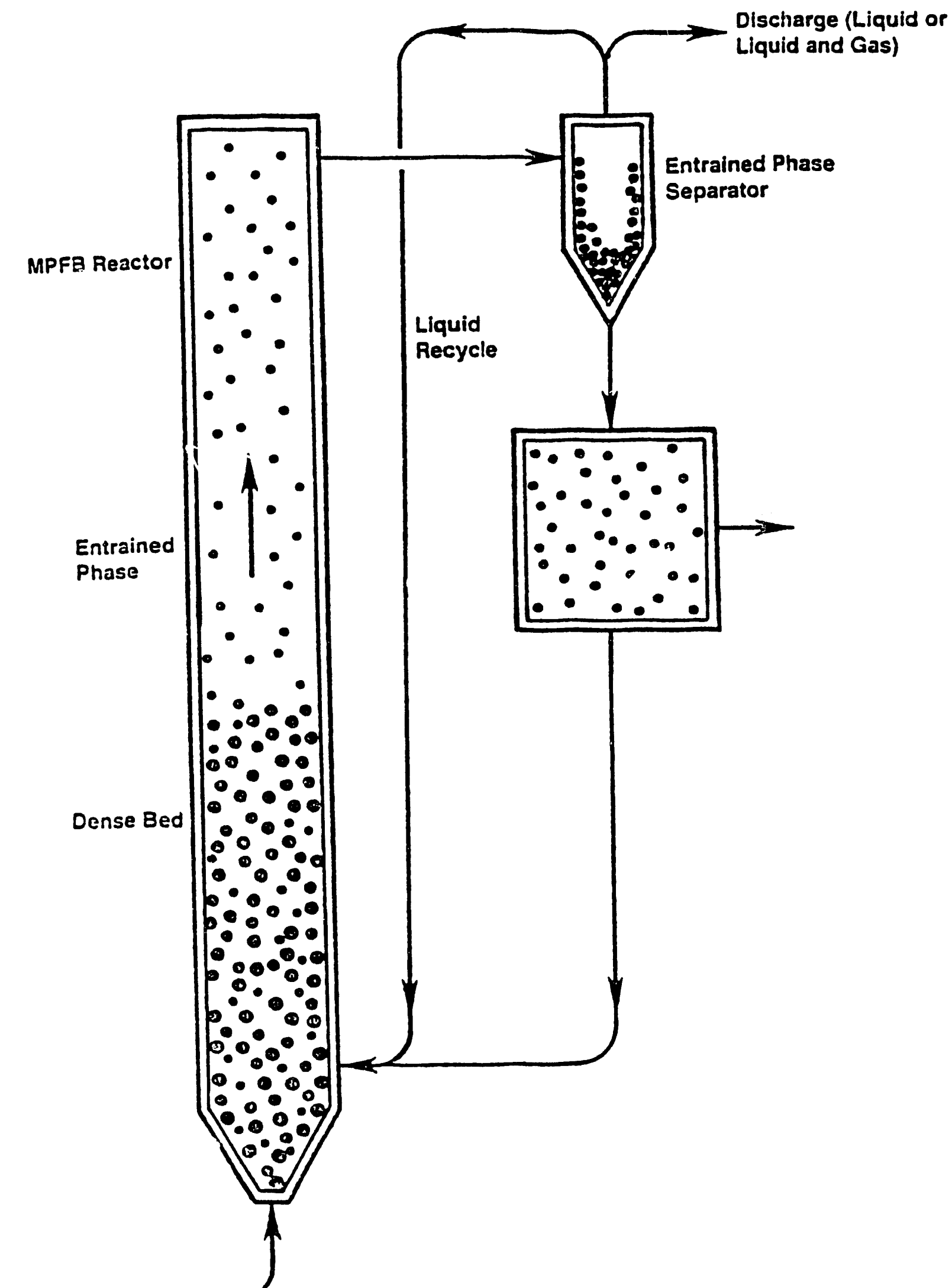

Feed (Liquid or

Fig. 5. Muli:phase Fluidized Bed (MPFB) Concept.

Liquid and Gas) 
successful with this system, the Oak Ridge program will be expanded to the acetic acid, ethanol, and other systerns.

The goals of the Battelle program are to (1) demonstrate the feasibility of the MPFB hioreactor for producing bulk chemicals in a continuous mode and (2) provide a generic design basis which can be scaled up beyond the current prepilot seale to the commercial production of butanol as weli as other major products.

The program to date has been aimed at developing a cell immobilization system; testing this in a fluidized-bed reactor; determining in separate tests the toxicity of the candidate solvents for butanol toward the immobilized organism: and in the course of all this, to design, build, and operate a process development unit (prepilot plant) to study operation. Progress has been reported in a number of papers. ${ }^{58-62}$

\section{PROCESS DEVELOPMENT STRATEGY}

\section{5.: BATCH MODE}

In the operation of the conventional batch fermenter on $C$. acetobutylicum, it is well known that as the product accumulates in the fermenter, a point is reached at which product inhibition of the functioning of the organism shuts down the fermentation. The amount of product produced is controlled by the volume of the fermenter at the maximum attainable product concentration -- usually a low amount.

In view of this limitation of the batch system, many researchers have evaluated the advantages of removing product from the batch fermenter "as fast as it forms" by various means in order to sustain the fermentation over a longer period. In such cases, additional substrate must be added in such a manner as to not adversely affect the fermentation by substrate inhibition. This condition usually results in the use of a fed-batch mode.

In effect, however, this approach actually represents a conversion of the batch mode to a continuous mode in which cells are prevented from leaving the fermenter. Thus, in any continuous system, the product is always being removed from the fermenter as fast as it forms. 


\subsection{CONTINUOUS MODE}

It appears then that the development of a continuous fermentation system is a fundamental requirement for improving the economic viability of the butanol process. As noted earlier, Blanch has operated a small research unit in a continuous mode, as has Scott of Oak Ridge National Laboratory. ${ }^{63}$ Scott incorporates lactic acid in an immobilized cell system to act as a mild hydrogen donor to scavenge oxygen so as to maintain a strict anaerobic environment and prevent cell deactivation. These results are encouraging, but it is imperative that continuous operation be demonstrated over an extended period at pilot scale.

This study was based on a continuous fermentation system in which product is removed from the broth either by an external, multistage extractor or by the in situ, singlestage extraction of product within the fermenter itself. The two approaches differ in that in the external case, the beer (at the maximum allowable, but low, product concentration) is the feed to a multistage extraction; whereas in the in situ case, the same beer is the raffinate leaving a single-stage extraction. In the latter case, the "effective concentration" in the fermenter (the"feed" to the in situ extraction) can be 10-15 times higher than in the aqueous beer phase, although the single-stage extraction is not as efficient as the multistage approach. Work is under way now to develop a model based on an in situ multistage extractor/fermenter design.

\subsubsection{External Multistage Extractor}

This study was based on a continuous fermentation system in which cells are maintained in the fermenter at a desired, maximum level. Thus, assuming that specific productivity [g product/g cell h] remains constant at constant (but maximum allowable) product concentration, the higher the cell density the greater the volumetric productivity [g product/L.h], the shorter the fermentation time, and, hence, the smaller the fermenter size and investment required for a desired design capacity; or, for an existing fermentation plant, the greater the throughput and production level.

This result can be accomplished either by immobilizing the cells to prevent their loss from the fermenter or by filtering the cells from the beer and recycling them. The 
immobilization approach would be preferred; it would avoid passing cells through a filter and, possibly, an extractor with possible deactivation of cells by me hanical attrition or exposure to solvent and with possible plugging of the trays of an extractor.

If for some reason the cells cannot be immobilized, then, two alternative recycle schemes can be considered: (1) a single recycle in which the cells are carried with the beer through an extractor and recycled with raffinate to the fermenter, or (2) a double recycle in which the cells are separated from the beer before the extractor and recycled, while cell-free raffinate from the extractor is recycled separately so as to assist in recovering by-products.

The economic consequences for any of the above options would be the same except for certain limiting situations.

\subsubsection{External Extraction With Single Recycle}

The single-recycle mode was used as the basis for this study as a "worse" case. It has the drawback that recycled cell density and recycled raffinate cannot be controlled scparately. Thus, for this model, a point is reached at a recycle ratio of 15:1 beyond which recycle supplies all the need for makcup water in the fresh feed. To go higher would require that the $45 \%$ syrup feed to the fermenter be evaporated to maintain the water balance. This would greatly increase the cost of substrate and must be avoided.

This limitation may be moot, however, since at a 15:1 recycle, cell density has been increased from $1.4 \mathrm{~g} / \mathrm{L}$ at zero recycle to $22.3 \mathrm{~g} / \mathrm{L}$, dilution rate from $0.03 \mathrm{~h}^{-1}$ to $0.48 \mathrm{~h}^{-1}$, and volumetric productivity from 0.4 to $6.3 \mathrm{~g} / \mathrm{L} \cdot \mathrm{h}$. As a result, total plant investment has been reduced from $\$ 228$ million to $\$ 41$ million for a 160 -million-pound-per-year production level and the cost-plus-return selling price from $\$ 1.08 / \mathrm{lb}$ butanol to $\$ 0.43 / \mathrm{hb}$. Although cell density could presumably be increased above $22 \mathrm{~g} / \mathrm{L}$ without adverse effect, further increases would lead to marginal improvements in cost.

\subsubsection{External Extraction with Double Recycle}

In the double-recycle case, cell density could reach higher levels than are possible with the water balance limitations of the single-recycle case. Likewise, cells would have 
less exposure to solvent with concomitant possible toxic side effects. Process control would have to be more sophisticated, but this is not an insurmountable problem.

In either recycle case, the recycle of raffinate is mainly desirable to reduce product losses to the aqueous purge. It also reduces the flu' $d$ load on the first raffinate still, thereby reducing the cost of this large still and its attendant steam consumption and investment. Raffinate recycle is also ostensibly desirable to (1) enhance the extraction of less extractable by-products such as acetone and ethanol; (2) conserve process water; and (3) recycle cells in the single-recycle case. None of the latter arguments are critical to the process. For the basis of this study, the recovery of low-boiling chemicals by distillation of the raffinate is definitely economically justified. This prevents the loss at any recycle ratio of valuable byproducts that are not recovered by extraction. Conservation of process water is of minor importance compared with other cost elements. As noted, cell recycle can be accomplished by separate recycle or immobilization.

\subsubsection{In Situ Extraction}

In the in situ case, cells are either recycled, immobilized, or allowed to grow in a first-stage fermenier to the level required to produce the desired amount of product in a second stage, such that the raffinate (beer) leaving the fermenter does not exceed the threshold level of inhibition of product concentration. The effective concentration í.e., amount produced divided by the aqueous flow) would be many times this since the bulk of the product leaves in tr. $\approx$ solvent extract. The multiplier would approximately equal the solvent/feed ratio times the distribution coefficient ( $\sim 12 \mathrm{X}$ for the basis used).

In summary then, in external extraction, cell density is increased to reduce fermentation time and fermenter volume at the maximum allowable product concentration in the beer for a desired production level. In situ extraction cell density is increased to increase effective product concentration at the maximum allowable product concentration in the aqueous beer and constant fermertation time for the desired production level. 


\section{EXTRACTIVE FERMENTATION PP.OCESS SCENARIOS}

\subsection{PROCESS SCENARIO FOR EXTERNAL EXTRACTION}

A flow sheet for the process model of external extraction is shown in Fig. 6. Beer leaving the fermenter train is passed to a multis age extractor (or first to a filter in the double-recycle model), whereupon substantially all of the product is removed by a selective solvent. Some of the by-product acetone and ethanol are removed also, as are the higherboiling acids: acetic and butyric. Actually, as a worse case it was assumed, rather than known, that the acids would be, indeed, pioduced and that the pH of extraction would be low enough for the acids to exist as acids rather than as salts. In salt form, they could not be extracted and would stay with the raffinate.

The aqueous raffinate, containing cells in the single-recycle case, leaving the extractor is recycled to the desired, highest possible level to the fermenter. The balance is filtered to remove residual eells. The filtrate is sent to the raffinate still train to recover by-product aceione and ethanol. Residual salts either fed to or produced in the fermenters are purged as the tails from the first raffinate still.

Butanol, by-product acetone and ethanol, and solvent contained in the extract are separated in a low-boilers still train. Butanol and acetone are recovered in pure form in this train, whereas crude ethanol is sent to the raffinate train for purification. Solvent is separated from lower-boiling wastes in the high-boilers still and recycled to the extractor.

One potential problem with the use of a high-boiling solvent must be noted. If the solubility of solvent in water is high enough, the loss of solvent to the aqueous waste leaving the process as the tails from the first raffinate still might be unaceptablr. A solubility of $20 \mathrm{ppm}$ was assumed in this siudy, while Blanch assumed $100 \mathrm{ppm}$ in his study. Either would be satisfactory. 


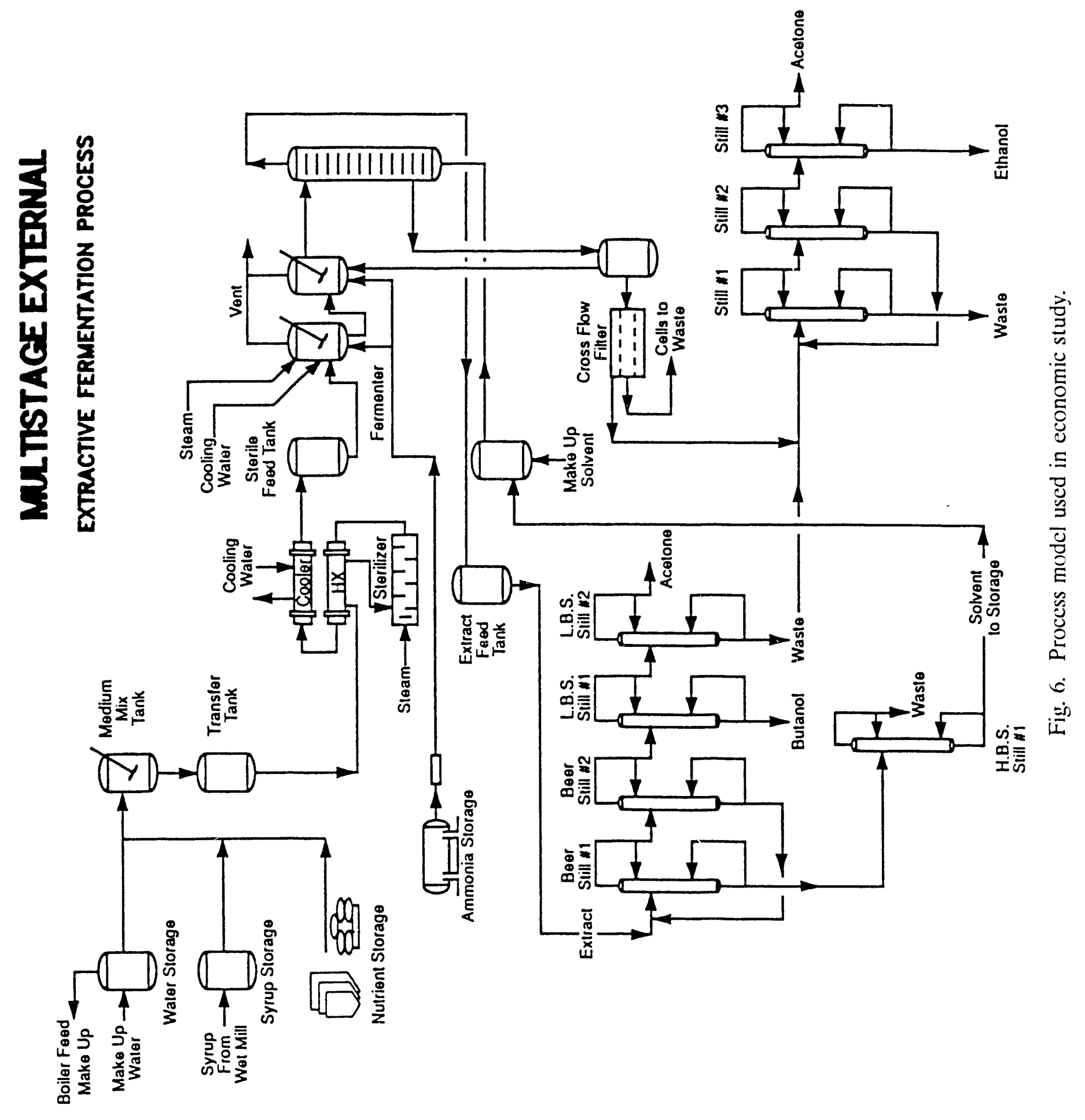




\subsection{PROCESS SCENARIO FOR IN SITU EXTRACTION}

The flow shect for the in situ case is substantially the same as for the external extractor case except that extraction is carried out in the fermenter and no additional extraction vessel is required. Also, aqueous raffinate containing cell debris and unextracted product and by-products is sent to waste disposal. The evaluation of this case did not include the recovery of ehemicals in the raffinate. This would probably be justified; in which case the raflinate still train used for the cxternal extractor case would be added. The process is shown in Fig. 7.

\section{OPERATING CONDITIONS}

It was assumed that the plant would be sited in the Midwest adjoining a wet corn mill, with dilute $45 \%$ syrup supplied over the fence by pipeline. Capacity was sized to a 18()-million-pound-per-ycar butanol plant having a midpoint of construction in 1984 and operating in 1988 at a 160-million-pound-per-year production rate. Technical and financial data for the cases evaluated are provided in the appendixes.

Basic data for the external extraction process are outlined in Table 5 according to whether they are independent or dependent variables. Key equipment dimensions are shown to provide a feeling for scale of the operation. The basecase data for fermentation without eell recyele were obtained from the literature sourees discussed earlier. Distribution coefficients were determined by Battelle. ${ }^{62}$ A comparison with the basis used by Blanch is shown in Table 6.

The main differences among cases were whether or not ralfinate with eells was recycled and whether raffinate chemicals were recovered. As can be noted from the selling prices required to provide an adequate return on investment, the recycle of eells 


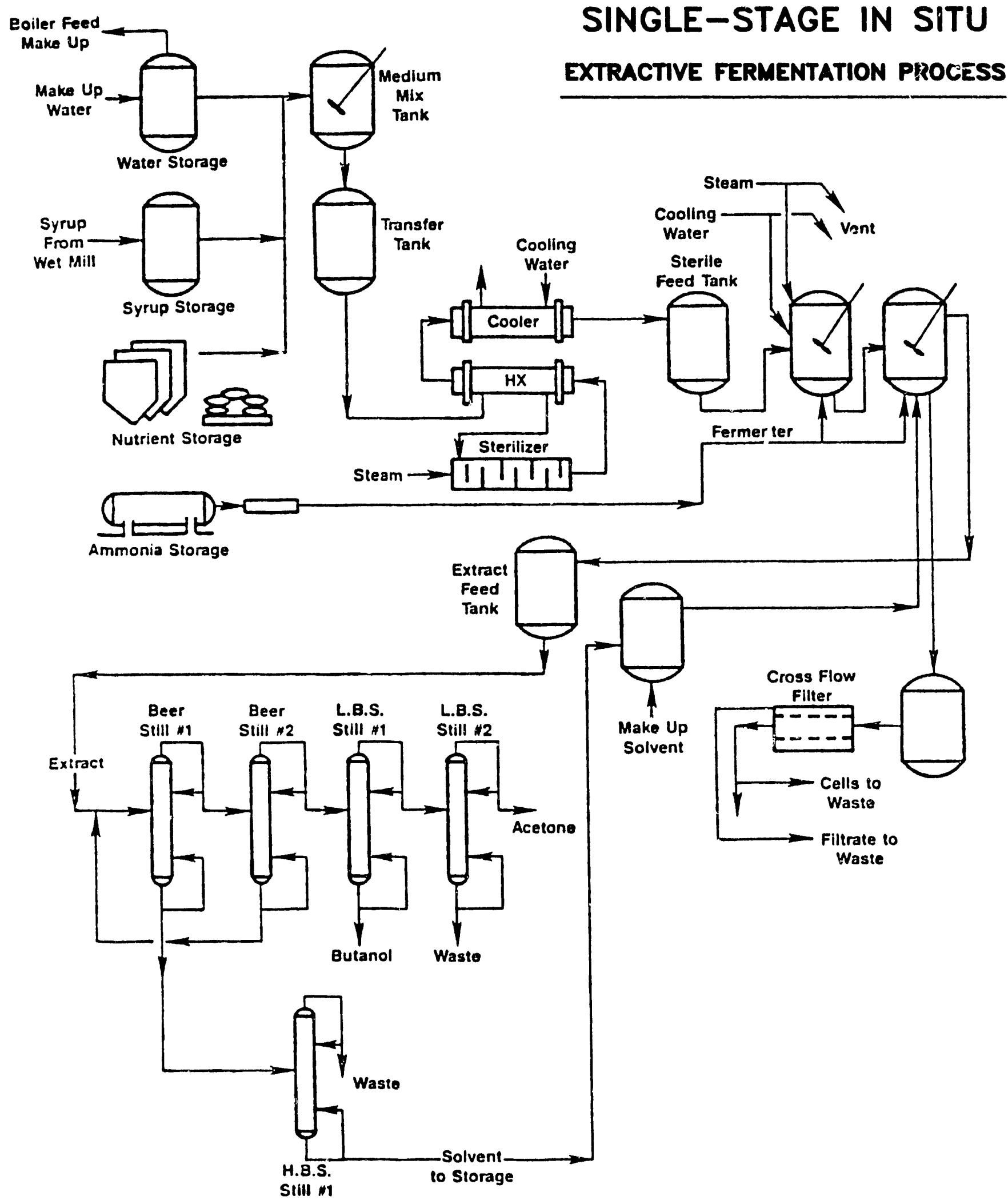

NO RECOVERY OF RAFFIMATE CHEMICALS

Fig. 7. Process model used in economic study. 


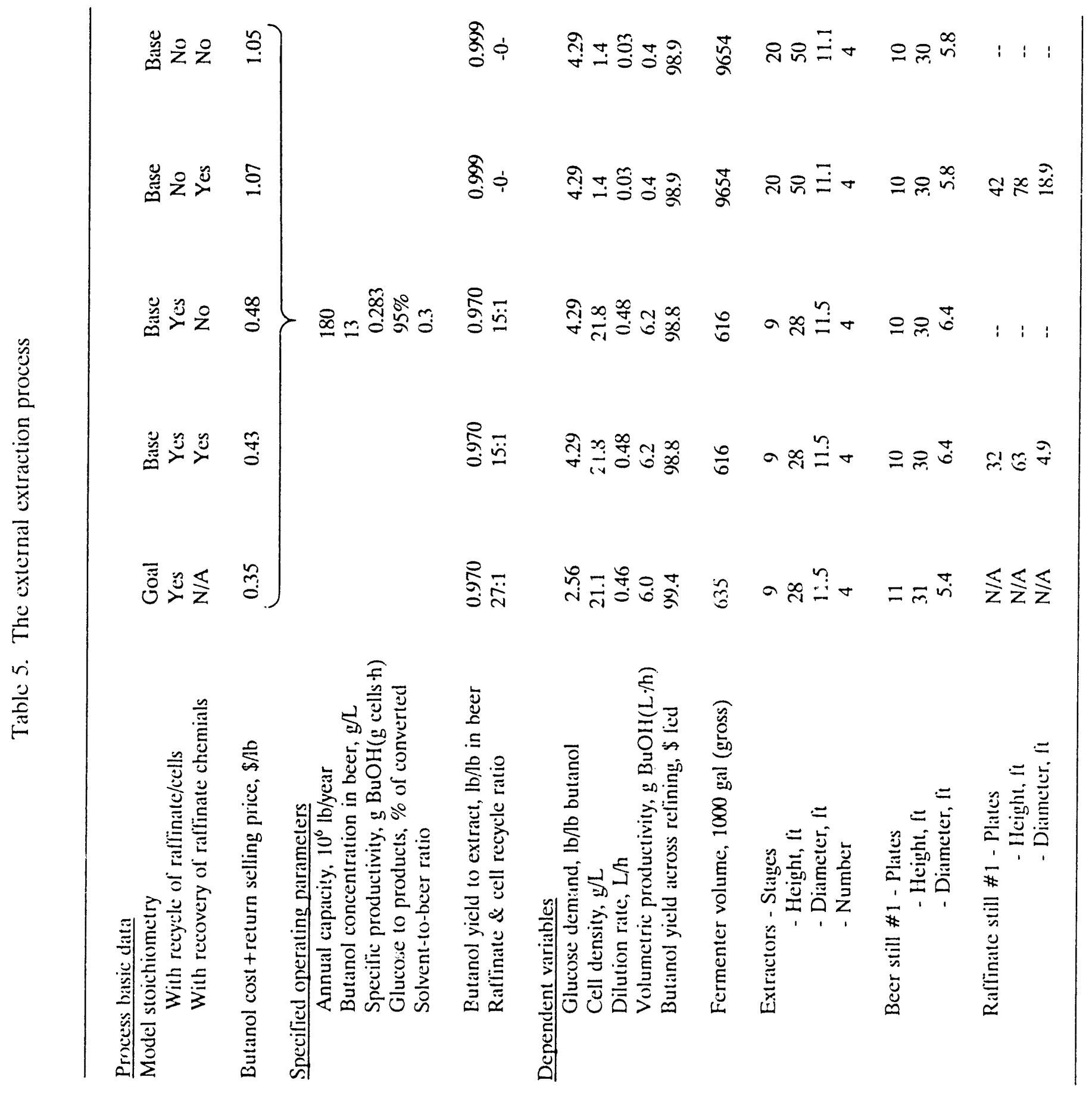


Table 6. Blanch's basis comparison

\begin{tabular}{lll}
\hline & This study & Blanch's study \\
& & \\
\hline Product concentration, $\mathrm{g} / \mathrm{L}$ & 13.0 & 13.7 \\
Fermentation time, $\mathrm{h}$ & 33 & 30 \\
Cell density, $\mathrm{g} / \mathrm{L}$ & 1.4 & 3.0 \\
Specific productivity, $\mathrm{g} / \mathrm{g} \cdot \mathrm{h}$ & 0.28 & -- \\
Volumetric productivity, $\mathrm{g} / \mathrm{L} \cdot \mathrm{h}$ & 0.39 & $0.46-(0.89$ \\
Distribution coellicient & & \\
Butanol & 4.3 & 2.6 \\
Acctone & 0.5 & 0.3 \\
Ethanol & 0.2 & 0.1 \\
Acctic acid & 0.2 & n.a. \\
Butyric acid & 0.2 & n.a. \\
& & \\
\hline
\end{tabular}

to the highest operable density is of utmost importance. Recovery of rafinate chemicals is also justifical.

Data used for the in situ extraction case are the same as the above except that no recycle or rallinate recovery was involved.

\section{EXTRACTION PERFORMANCE}

In continuous, multistage, countercurrent extraction, a trade-off must be made between the required number of stages, which determines the cost of the extractor, and 
the solvent-to-aqueous feed ratio, which determines the cost of recovering product from the solvent extract. The number of stages required for the separation approaches infinity as the product of the distribution coefficient times the solvent/feed ratio approaches unity. As a practical matter, this product should be rontrolled fairly close to unity for a proper balance. This effect can be seen from the data of Appendix $G$, which define the number of stages required for any given solvent/feed ratio and desired yield of product to extract.

For the external extractor basecase, a solvent/feed ratio of 0.3 at a distribution cocfficient of $4.3(0.3 \times 4.3=1.29)$ called for 9 stages at a yicld of $97 \%$. As will be discussed later, this combination appeared to provide the lowest cost. The yiclds of acetone (15\%) and ethanol $(6 \%)$ are low because of their low distribution coeflicients and low solvent/feed ratio.

For the in situ extraction case, the fermenter acts as a single stage of extraction. Consequently, the efficiency is lower than for the multistage case, but much higher effective concentrations of product in the fermenter can be realized.

Yiclds of product to extract for single-stage extraction for various distribution coefficients and solvent/beer ratios are shown in Table 7 and Fig. 8. At a distribution coeflicient of 4.3 , a solvent/beer ratio of 3.5 would lead to a distribution of $93.8 \%$ of the butanol solute in the fermenter to the extract. At its lower distribution coeflicient, the yield of acetone to extract would be $\sim 60 \%$, and the yield of ethanol, $40 \%$.

The improvement possible in effective concentration is shown in Table 8 and Fig. $S$, ill which "initial" concentration represents the amount of product produced relative to the aqueous flow. Thus, at a solvent/feed ratio of 3.5 , the effective concentration of product in the fermenter can be increased to close to $200 \mathrm{~g} / \mathrm{L}$ while maintaining the actual concentration in the aqueous raflinate (beer) at $13 \mathrm{~g} / \mathrm{L}$, the threshold of inhibition.

It should be noted that although oleyl alcohol was used as a model solvent in this study, as well as in many others reported in the literature, it could not be used in commercial practice since it boils too high to allow a practical separation of product from the extract. It was assumed in this study that the oleyl alcohol was either in a carrier of nonyl alcohol or that a new solvent was identified that had the solvent characteristics of olcyl alcohol and the distillation characteristics of nonyl alcohol. 


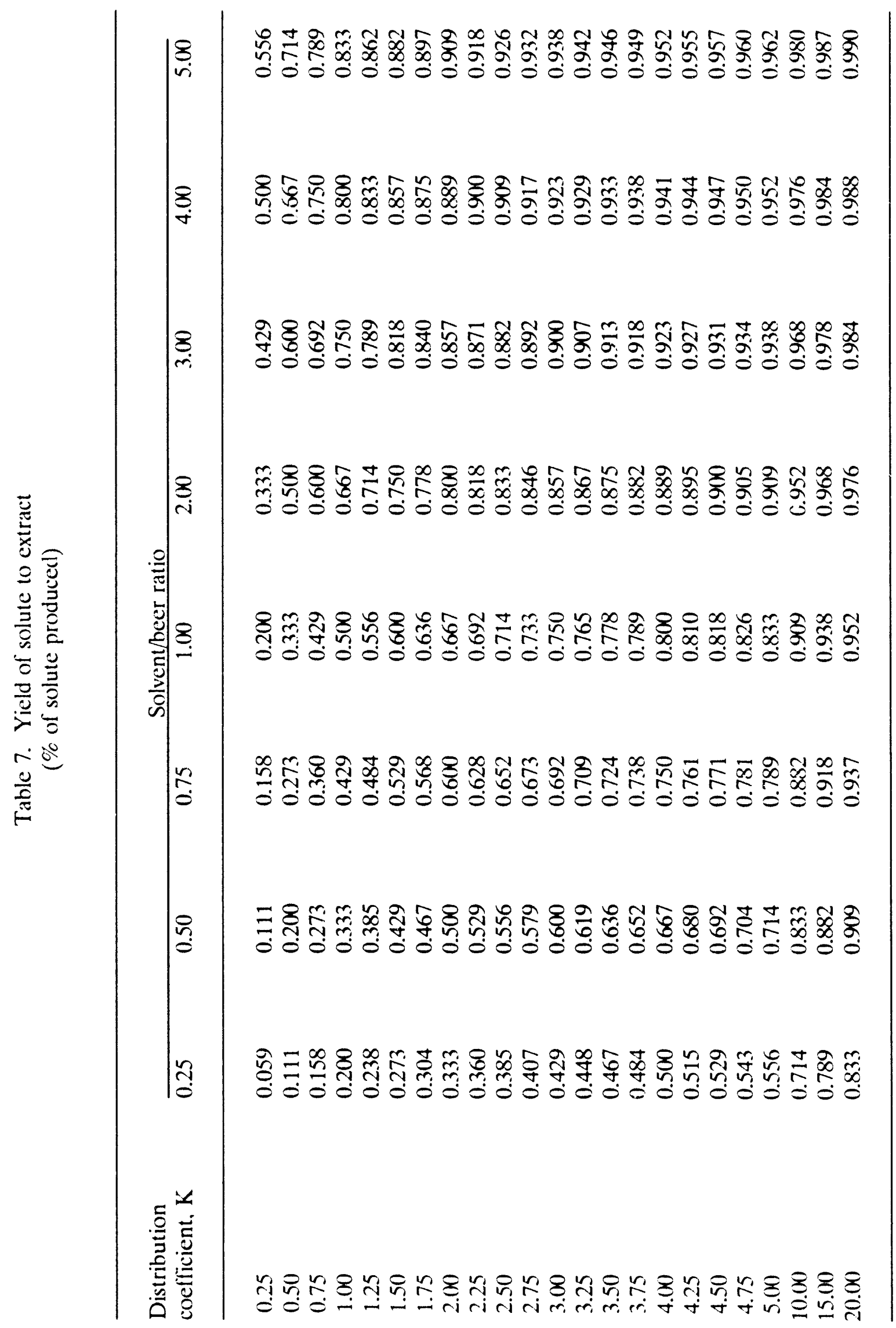




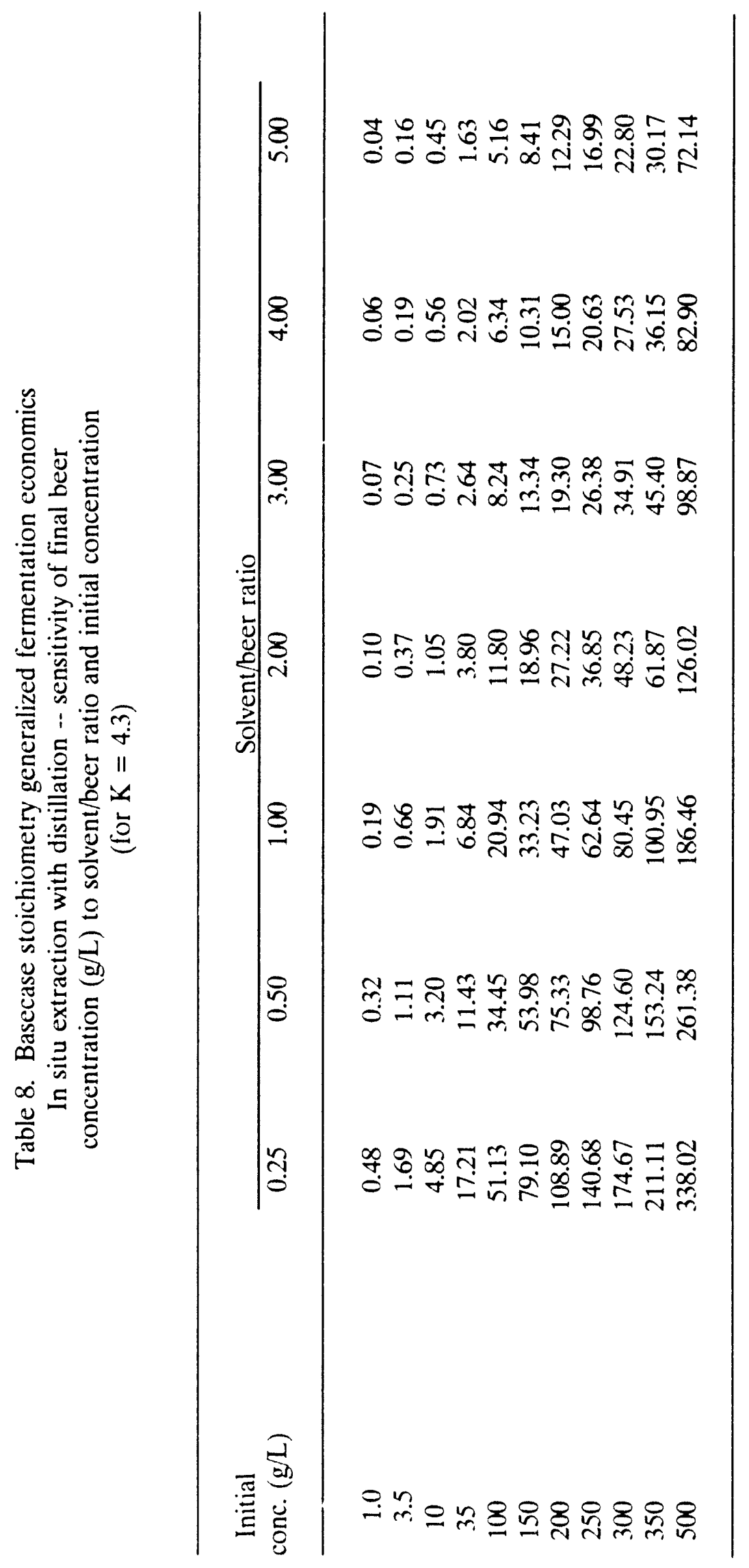




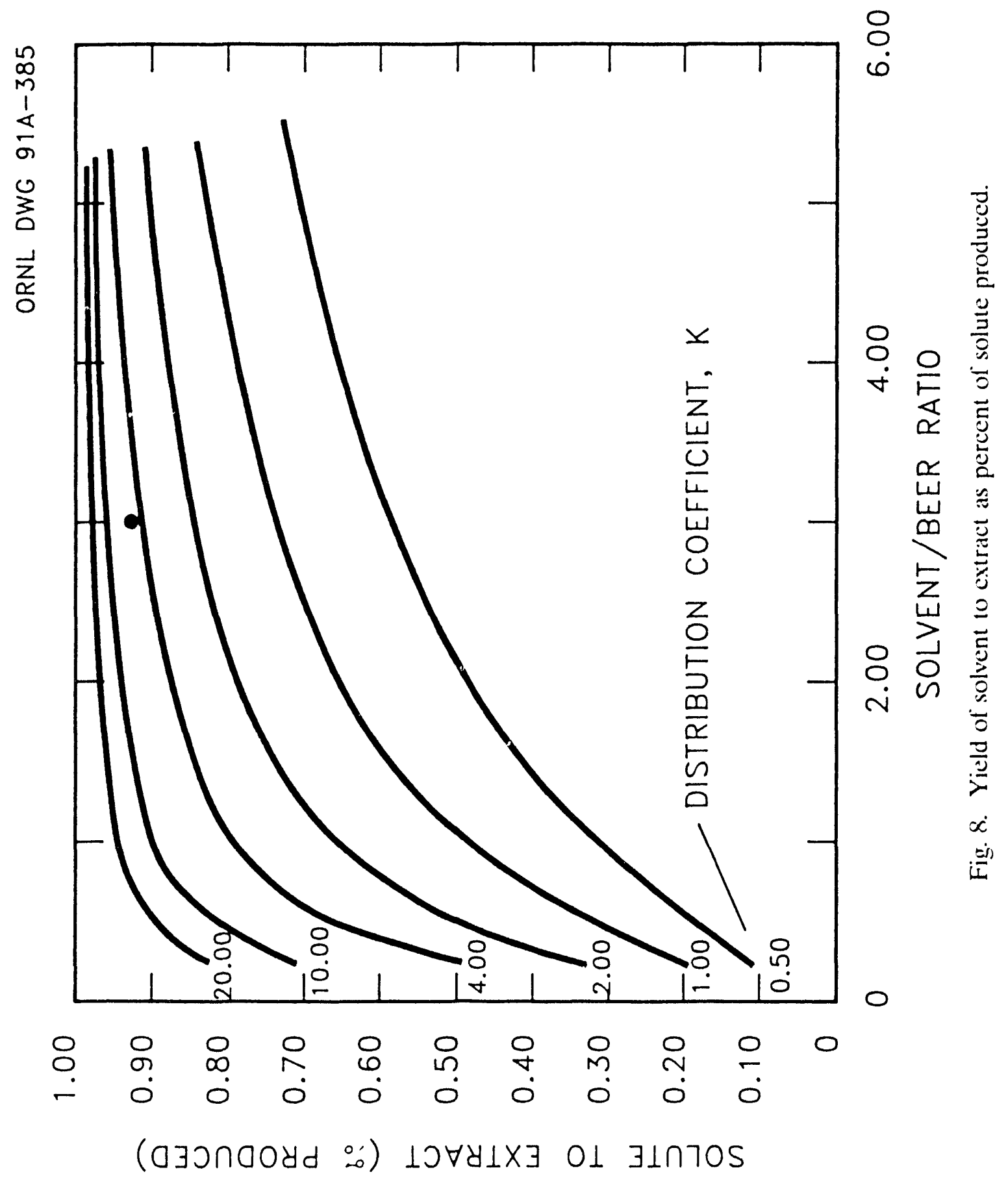




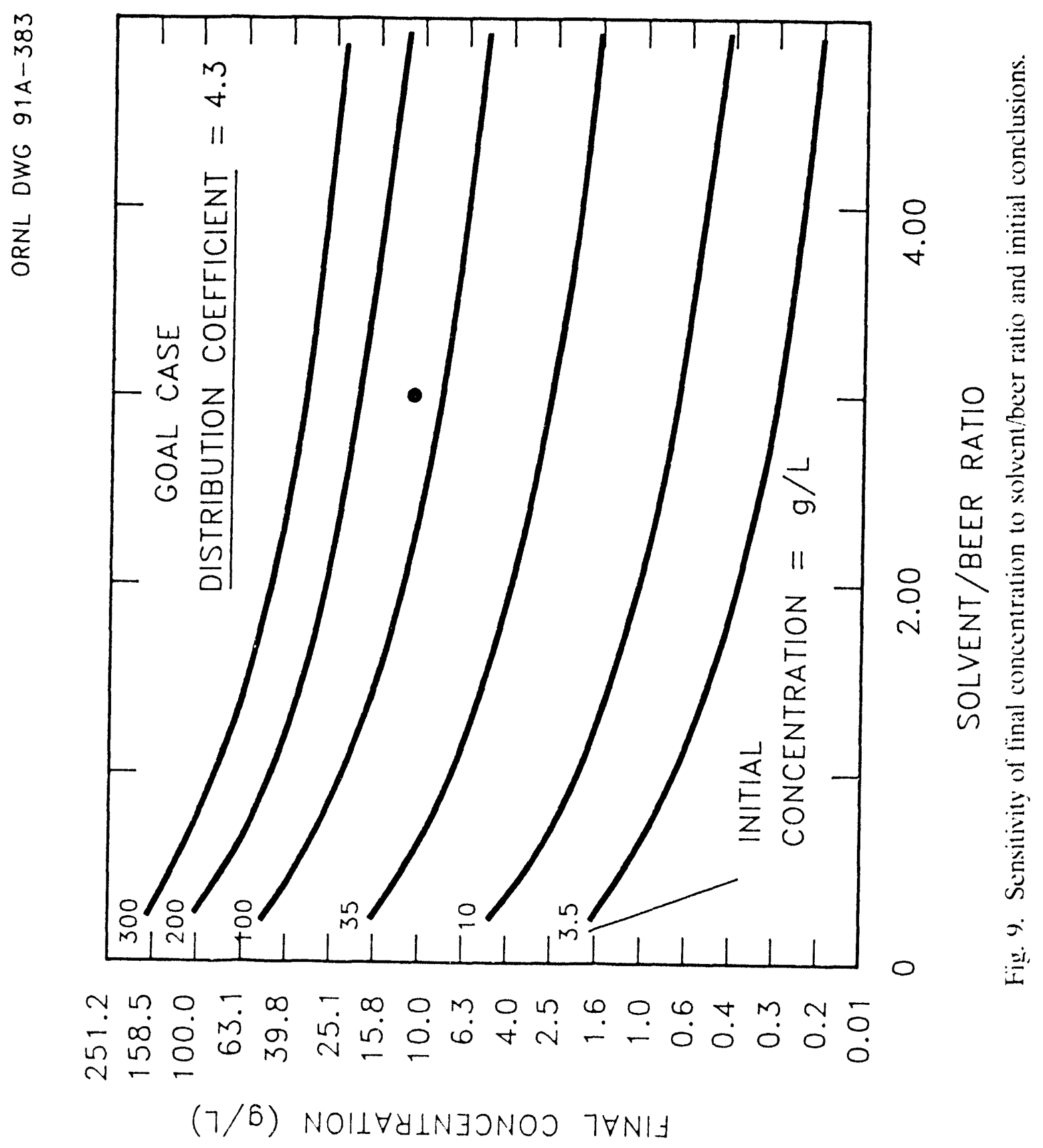




\section{PROJECTED ECONOMICS OF THE CONVENTIONAL WEIZMANN PROCESS}

Butanol currently sells for $\$ 0.38 / \mathrm{b}$ at a crude oil price of $\$ 15-\$ 20 / \mathrm{bbl}$. This price has been fairly stable since 1982. However, considering the increases expected again in crude oil prices in the 1990s, a doubling in the price for oil-based butanol is not inconceivable.

Lenz and Moreira analyzed the economics of the conventional Weizmann process utilizing a molasses substrate and found that the process would have to operate at a loss because of the combined elfect of raw material costs and dilute concentrations. ${ }^{\text {.t }}$

The current study also concluded that the Weizmann process would operate at a loss in the present economic environment. Butanol from a 170)-million-pound-per-year plant based on the conventional process and operating at 150 million lb/year in 1987 would cost $-\$ 1.19,1 \mathrm{~b}$ for the lermentation operation alone (including raw matterials cost but excluding recovery and purificalion costs). ${ }^{\text {s. }}$ This cost includes a 30$)^{\prime}$ e pretax return on investment.

The investment at the midpoint of construction of 1983 is compriscel of $\$ 230$ million in direct process equipment: $\$ 3.3$ million in allocated power, services, and general lacilities: and $\$ 30$ million in working capitat. Of the dircet process investment. \$2(1) million alone is lor lermenters (16 million gal) and their ancillaries priced installed at $\$ 12.50$ gross gal. A contingency factor of $30 \%$ was applied to the investment. Werking capital is high because of the high value of the product inventery and accounts receriable.

This estimale assumes a butanol concentration of $1.3 \mathrm{~g} \mathrm{~L}$ at a batch time of $40 \mathrm{~h}$ and a turnaround of $12 \mathrm{~h}$. Of the glueose converted, $5 \% \mathrm{c}$ is used for ecell growth and $95 \%$ for products according to the stovichiometry of Table 4. The product cost sheet is summarized in Table 9. 
Table 9. Product cost sheet of the Weizmann Process

\begin{tabular}{|c|c|}
\hline Factor & $\operatorname{cost}(\$)$ \\
\hline Raw materials & 0.30 \\
\hline Utilitics & 0.14 \\
\hline Labor-related & 0.10 .3 \\
\hline Depreciation & 0.14 \\
\hline Other cappital-rolated & 01.115 \\
\hline Const of manuficture & 0.50 \\
\hline SE, RSD, administration & 0.10 \\
\hline Cist of silk's & 0.72 \\
\hline Pretax curnings & 0.58 \\
\hline By-produce cordis & $(0.11)$ \\
\hline (ist-plus-return price & 1.19 \\
\hline
\end{tabular}

Wang compared the conomics of extractive batch fermenter designs with conventional hateh designs and concluded that the extraction design appleared to be considerathly more prolitible than the conventional design." Blanch made a similar conclusion with rexpect to a led-batch extractive design.".

10. PROJIECIIED IECONOMICS OF TIII IEXTIERNAL. IEXTRACIION PROCIESS

Fxternal multistane atration combined with raftinate/eell recyele and recovery of chemicats in the ratlinate appears to have a dramatic eflect on reducing product costs. 
For the first time in over 30 years, it now seems that the fermentation process is within striking distance of competing with synthetic butanol at $\$ 0.38 / \mathrm{b}$. The economics of the live cases develoned for the external extraction process are summarized in Table 10. These cases are also elaborated upon in Appendixes A through E. The mandatory prices are based on attaining a pretax return on an investment of $30 \%$.

With a $15: 1$ recycle and recovery of raffinate chemicals, the cost-plus-return price appears to be reduced to $\$(0.43 / \mathrm{b}$. With a double recycle and higher iecycle ratio, further reductions appear possible, although these may be marginal.

The development of organisms that are less inhibited by product and/or nave a selective stoichiometry favoring the production of butanol appears to reduce cost below the present price for synthetic butanol.

These conclusions will be discussed further in the following sections.

\subsection{EFFECT OF RAFFINATE/CELL RECYCLE}

Cost is primarily sensitive to the recycle of cells. As shown in Table 11, cost decreases with increases in recycle ratio over the entire range studied. This effect mainly results from a concomitant reduction in fermenter and total plant investment. For this model, recyele ratios over 15:1 would require evaporation of the syrup -- an undesirable requirement. However, this could be avoided by using a double recycle if cell concentrations above $22 \mathrm{~g} / \mathrm{L}$ could be tolerated in the fermenter. Total llow through the fermenter remains constant irrespective of recycle ratio for a fixed, limiting product concentration and annual production rate. Cell density increases as cell recycle increases; thus, fermentation time and fermenter investment can be decreased correspondingly to balance production rate at a constant specific productivity.

\subsection{EFFECT OF SOLVENT/BEER RATIO}

The solvent-to-beer ratio should be minimized for optimum costs. The cost tradeoff of operating parameters is shown in Table 12. For the conditions of this study, the best ratio appears to be 0.3 . For a distribution coefticient of 4.3 , the plates required in the extractor approach inlinity at a ratio of 0.23 , but drop sharply to reasonable levels 


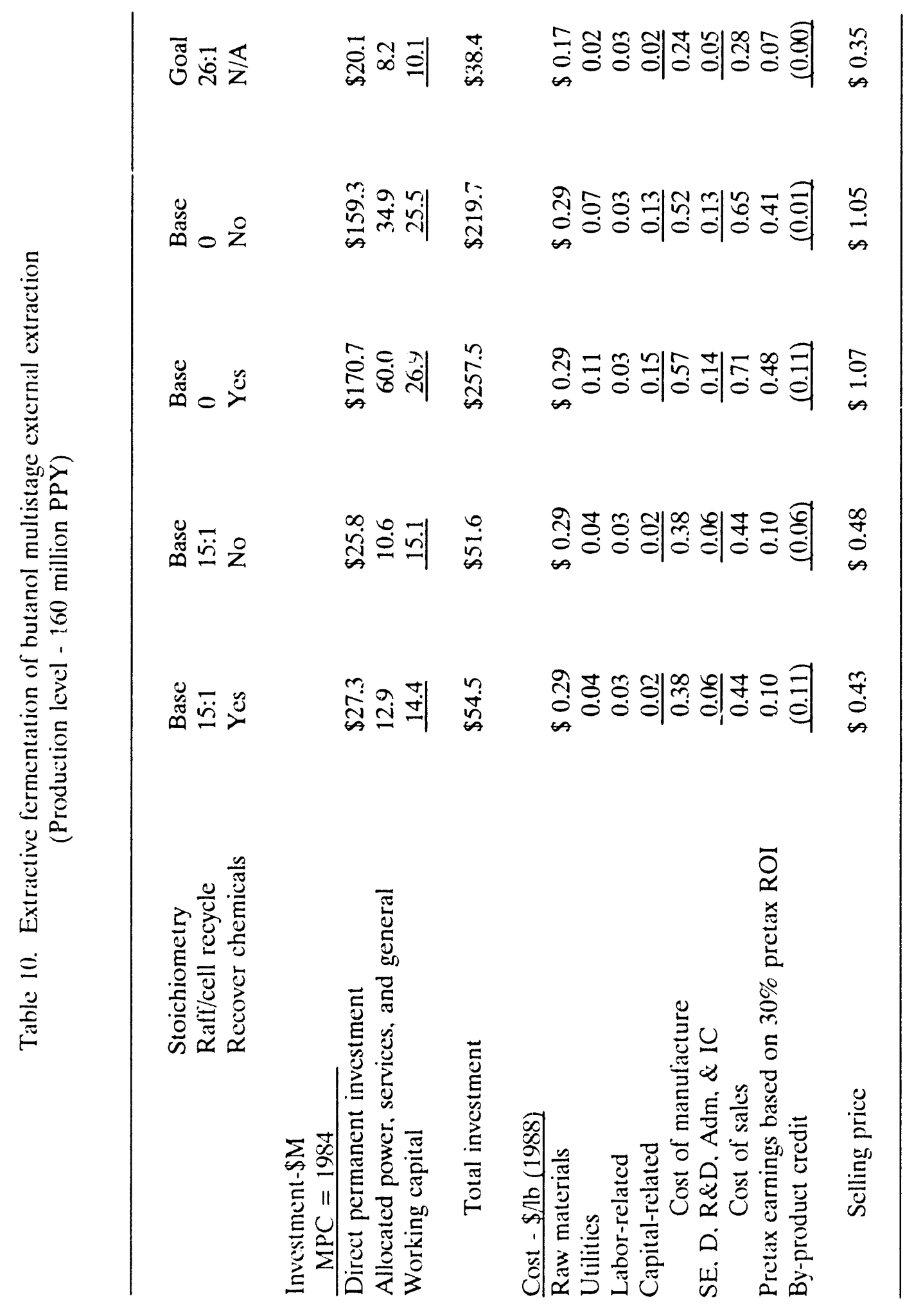




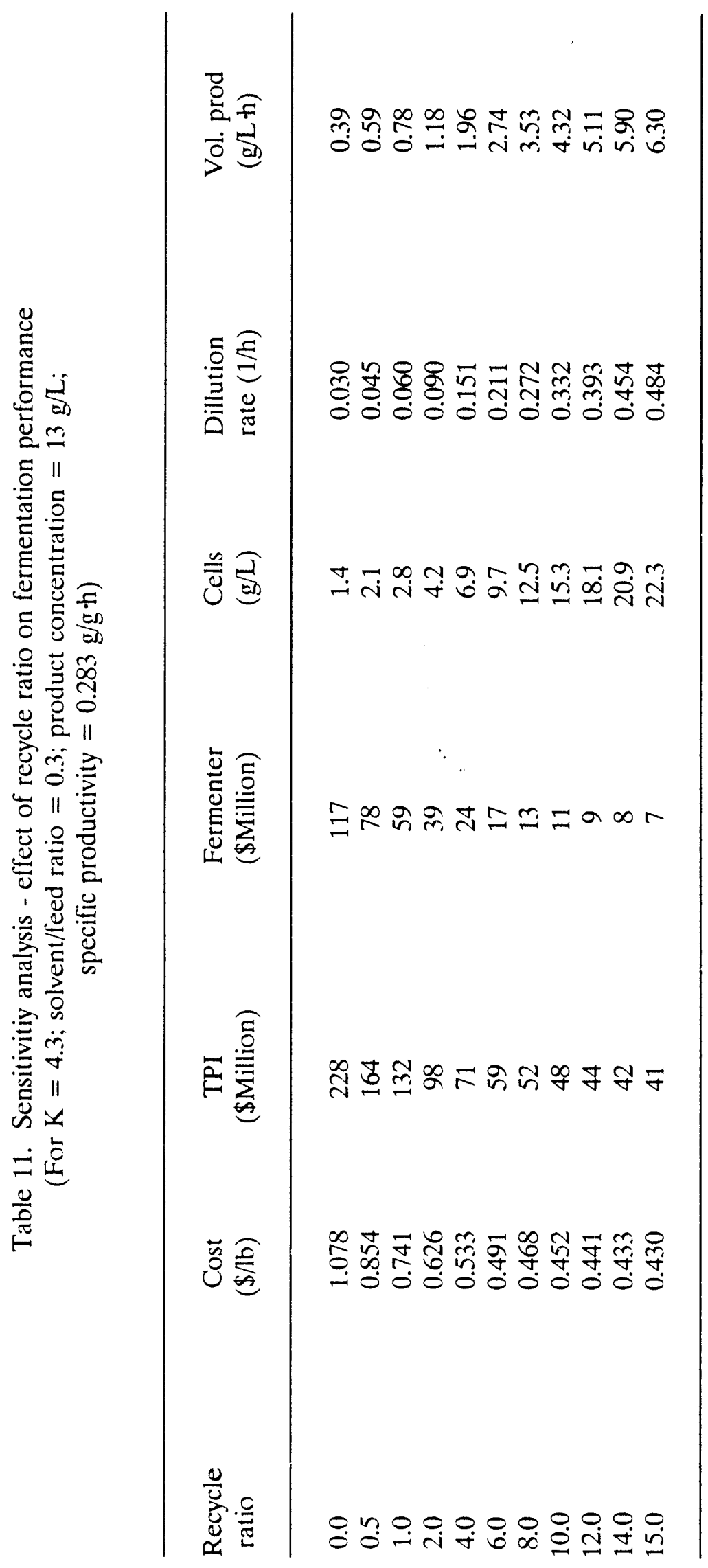




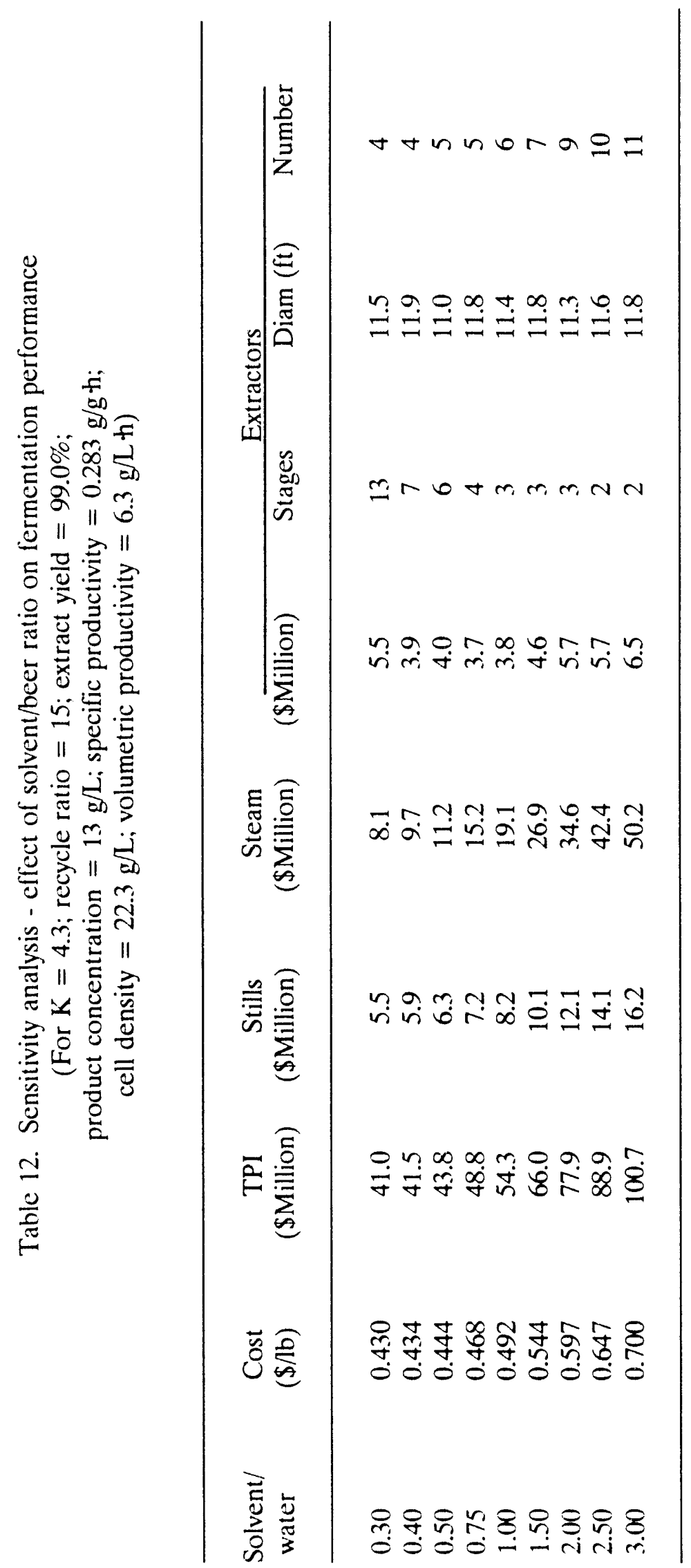


at slightly higher ratios. As the solvent-to-beer ratio increases, the number of extractor stages that are required decreases but throughput and, hence, extractor diameter and/or number increase. These opposing effects modulate extractor investment. However, both distillation and steam investment increase in order to process the higher volume of thuid downstream.

\subsection{EFFECT OF EXTRACTOR YIELD}

A similar trade-off occurs in the choice of yield of product to extract. As yield increases, so does the number of stages required to effect the separation, in opposition to the improvement in process efliciency. As shown in Table 13 and Fig. 10, a cost optimum in reached at a yield of $\sim 97 \%$. Above this, cost rises sharply. If cells are not recycled, the optimum would occur at a higher yicld.

\subsection{EFFECT OF PRODUCT CONCENTRATION}

The basecases of this study were developed using a $13-\mathrm{g} / \mathrm{L}$ product coneentration -- supposedly close to the aceptable limit of feedback inhibition. Lower and higher concentrations were also explored. Higher concentrations would require developing an organism that is less sensitive to product inhibition. This appears to be a worthwhile research goal. The results are summarized in Table 14 and Fig. 11. Note that dilution rate is synonymous with raflinate/recycle ratio.

At any product concentration, it is clearly desirable to operate at as high a recycle ratio (dilution rate) as possible. At $13 \mathrm{~g} / \mathrm{L}$ and a $15: 1$ ratio, cell density would reach 22 $\mathrm{g} / \mathrm{L}$. This level appears operable, but densities required for concentrations above, say, 50 $\mathrm{g} / \mathrm{L}$ (even if attainable without inhibition) may produce unaceeptable viscositics and adverse effects on the organism in the lermenter. As a practical matter, it does not appear that increasing concentration much above $25 \mathrm{~g} / \mathrm{L}$ will have a significant effect on reducing further the cost of the product.

It can also be noted that at a $15: 1$ ratio $\left(0.48 \mathrm{~h}^{-1}\right.$ dilution rate) the cost curve is close to the "infinite " dilution rate curve, at which point further decreases in lermenter volume have an insignilicant cllect on cost and investment. 
Table 13. Sensitivity of product cost to yield to extract and solvent/heer ratio

\begin{tabular}{|c|c|c|c|c|}
\hline \multirow[b]{2}{*}{ S/B Ratio: } & \multicolumn{2}{|c|}{ 15:1 Raffinate recycle } & \multicolumn{2}{|c|}{ No recycle } \\
\hline & 0.3 & 0.4 & 0.3 & $\overline{0.4}$ \\
\hline \multicolumn{5}{|c|}{ Yicld (lb/lb fed) } \\
\hline 0.85 & $\$ 0.440$ & $\$ 0.450$ & $\$ 1.236$ & $\$ 1.250$ \\
\hline 0.90 & 0.434 & 0.443 & 1.172 & 1.183 \\
\hline 0.93 & 0.430 & 0.440 & 1.137 & 1.148 \\
\hline 0.95 & 0.429 & 0.438 & 1.116 & 1.126 \\
\hline 0.96 & 0.428 & 0.437 & 1.105 & 1.115 \\
\hline 0.97 & 0.428 & 0.435 & 1.095 & 1.104 \\
\hline 0.98 & 0.428 & 0.434 & 1.085 & 1.094 \\
\hline 0.99 & 0.430 & 0.434 & 1.078 & 1.085 \\
\hline 0.999 & 0.436 & 0.438 & 1.075 & 1.080 \\
\hline 0.9999 & $-\cdots$ & 0.442 & -- & 1.083 \\
\hline
\end{tabular}


Table 14. Sensitivity of fermentation performance

to product concentration

(With recovery of raffinate chemicals)

\begin{tabular}{|c|c|c|c|c|c|c|}
\hline $\begin{array}{l}\text { Product } \\
\text { conc. (g/L) }\end{array}$ & $\begin{array}{c}\text { BuOH cost } \\
(\$ / \mathrm{lb})\end{array}$ & $\begin{array}{c}\text { TPI } \\
(\$ / \mathrm{lb})\end{array}$ & $\begin{array}{l}\text { Ferm inv } \\
\$ / l b\end{array}$ & $\begin{array}{l}\text { Ferm inv } \\
\text { TPPI }\end{array}$ & $\begin{array}{l}\text { Cells } \\
(\mathrm{g} / \mathrm{L})\end{array}$ & $\begin{array}{c}\text { Dil rate } \\
(1 / \mathrm{h})\end{array}$ \\
\hline \multicolumn{7}{|c|}{ For no ralfinate/eell recycle } \\
\hline 3 & 3.51 & 5.18 & 2.91 & $56 \%$ & 0.3 & 0.0 .3 \\
\hline 6 & 1.94 & 2.66 & 1.46 & 55 & 0.6 & 0.03 \\
\hline 13 & 1.10 & 1.30 & 0.67 & 52 & 1.4 & 0.03 \\
\hline 18 & 0.89 & 0.97 & 0.49 & 50 & 1.9 & 0.03 \\
\hline 25 & 0.74 & 0.73 & 0.35 & 48 & 2.7 & 0.03 \\
\hline 50 & 0.55 & 0.41 & 0.17 & 42 & 5.4 & 0.03 \\
\hline 100 & 0.45 & 0.25 & 0.09 & 35 & 10.7 & 0.03 \\
\hline
\end{tabular}

For $0.5: 1$ raffinate/eell recycle

3

6

13

18

25

50

100

\section{3}

6

1.3

18

25

50

10()

3
6
13
18
25
50
100
2.53

1.45

0.87

0.72

0.62

0.48

0.41

1.56

0.96

0.63

0.55

0.49

0.42

0.38

1.07

0.71

0.51

0.46

0.43

0.38

0.36
3.60

1.87

0.93

0.70

0.53

0.31

0.20
1.94

0.97

0.45

0.32

0. 23

0.12

0.06
54

52

48

46

44

37

30
0.5

1.0

2.1

2.9

4.0

8.0

15.9
0.05

0.05

0.05

0.05

0.05

0.05

0.05

For 2:1 raflinate/ecll recycle

$\begin{array}{lllll}2.02 & 0.97 & 48 & 0.9 & 0.09 \\ 1.07 & 0.49 & 45 & 1.9 & 0.09 \\ 0.55 & 0.22 & 41 & 4.1 & 0.09 \\ 0.42 & 0.16 & 38 & 5.7 & 0.09 \\ 0.33 & 0.12 & 35 & 7.9 & 0.09 \\ 0.21 & 0.06 & 28 & 15.8 & 0.09 \\ 0.14 & 0.03 & 21 & 31.8 & 0.09\end{array}$

For 5:1 raffinate/cell recycle

$\begin{array}{lllll}1.22 & 0.49 & 40 & 1.9 & 0.18 \\ 0.66 & 0.24 & 37 & 3.8 & 0.18 \\ 0.36 & 0.11 & 32 & 8.2 & 0.18 \\ 0.28 & 0.08 & 29 & 11.3 & 0.18 \\ 0.22 & 0.06 & 26 & 15.8 & 0.18 \\ 0.15 & 0.03 & 20 & 31.7 & 0.18 \\ 0.11 & 0.02 & 14 & 64.0 & 0.18\end{array}$


Table 14. (continued)

\begin{tabular}{|c|c|c|c|c|c|c|}
\hline \multicolumn{7}{|c|}{ For $10: 1$ ralfinate/cell recycle } \\
\hline 3 & 0.84 & 0.85 & 0.26 & 31 & 3.4 & 0.32 \\
\hline 6 & 0.59 & 0.47 & 0.13 & 28 & 6.9 & 0.33 \\
\hline 13 & 0.45 & 0.26 & 0.06 & 24 & 15.0 & 0.33 \\
\hline 18 & 0.42 & 0.21 & 0.04 & 21 & 20.8 & 0.33 \\
\hline 25 & 0.40 & 0.17 & 0.03 & 19 & 29.0 & 0.33 \\
\hline 50 & 0.37 & 0.12 & 0.02 & 13 & 58.6 & 0.33 \\
\hline 100 & 0.36 & 0.09 & 0.009 & 9 & 119.6 & 0.34 \\
\hline \multicolumn{7}{|c|}{ For $15: 1$ raflinate/cell recycle } \\
\hline 3 & 0.75 & 0.70 & 0.18 & 26 & 5.0 & 0.47 \\
\hline 6 & 0.54 & 0.39 & 0.09 & 23 & 10.0 & 0.47 \\
\hline 13 & 0.43 & 0.22 & 0.04 & 19 & 21.8 & 0.48 \\
\hline 18 & 0.40 & 0.18 & 0.0 .3 & 17 & 30.3 & 0.48 \\
\hline 25 & 0.38 & 0.15 & 0.02 & 15 & 42.3 & 0.48 \\
\hline 50 & 0.36 & 0.11 & 0.01 & 10 & 86.0 & 0.49 \\
\hline 100 & 0.36 & 0.09 & 0.006 & 7 & 177.7 & 0.50 \\
\hline
\end{tabular}




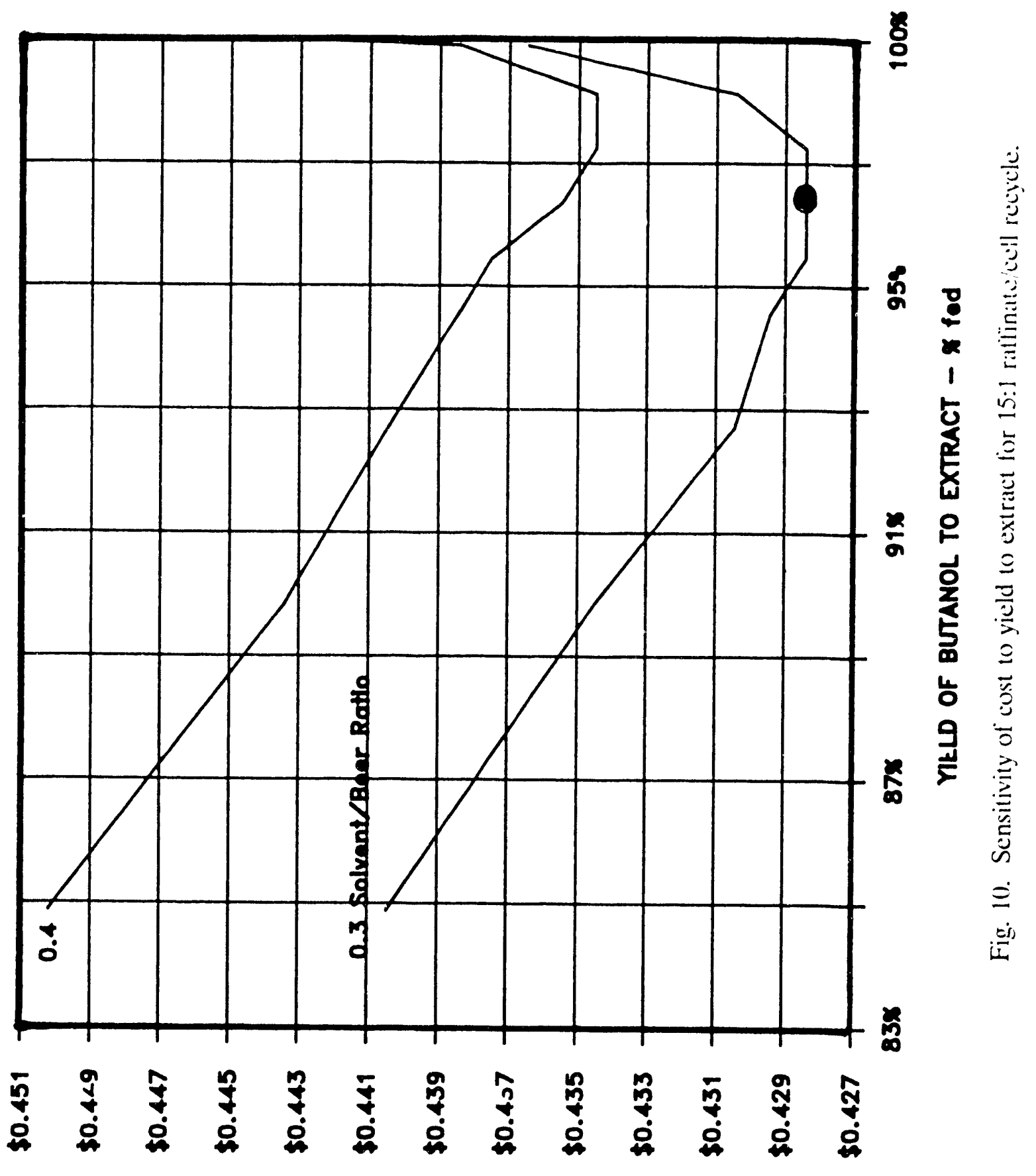

$91 / \$-3018 d$ NynL3y-snาd-1500 


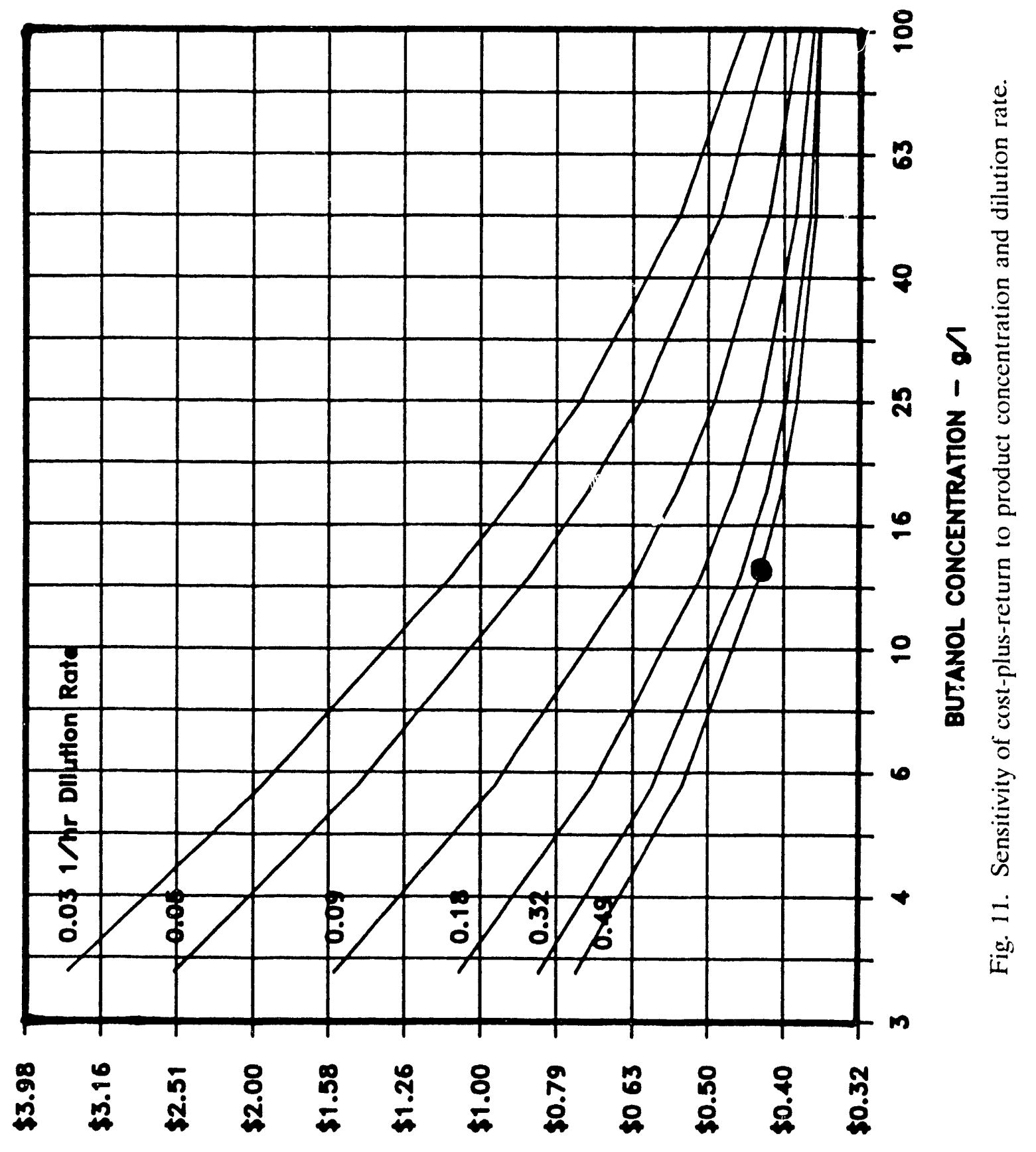

91/\$ - 3018d NynL3y-snTd-1500 


\subsection{EFFECT OF SUGAR PRICE ANI) YILILD}

The cost of the sugar substrate is a very important element of cost, particularly if the enginecring improvements discussed in the previous section can be realized. For Basecalse $A$, the cost of substrate amounts to $6.5 \%$ of the $\$ 10.43 / 1 \mathrm{~h}$ selling price and $7.4{ }^{\circ}$; of the cost of manulacture. This cost combines the effects of sugar price and yield to product.

If an organism can be developed to provide the stoichiometry indicated as the goal case of Table 4. the cost-plus-return selling price could be recluced from $\$(1) .43 \mathrm{th}$ to $\$ 0.35 /$ th (i.c., below the current price for synthetic butanol). This case is summariced in Tahle 10 and Appendix E:

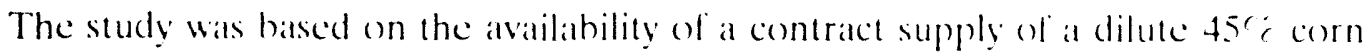
syrup from an adjoining wet mill at a transter price of $\$ 0.065$. Ih expuivalent glucose. Unfortunately, such price information is considered proprictary hy wet millers and is not fortheoming. However, a recent analysis of the lecdstock costs for fermentation cothanol plants"s showed that over the period 1QS1 - 20S6 at an average price for \#2 sellow corn ex Chicage of $\$ 2.86$ hushel the average price for corn net of by-produce credits was

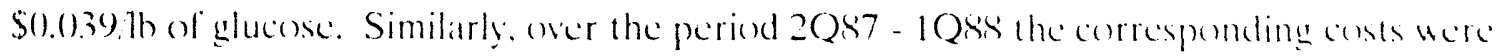
$\$ 1.79$ hushel and $\$(1.10199$ th of sugar.

A corn price of \$2.60 bushel appears to correspond to a sugar cose of $-\$ 90.181 \mathrm{lb}$ of starch aceording to the correlation of puhlished data shown in l"ig. 12.

Finally, the following estimate was made by the author ats an approximalle, it mot qualified. evaluation of substrate costs. The basis was for an carly gest plant processing

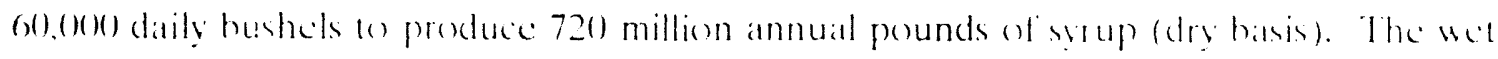
mill yield was andumed to he 31 .6 th of starch hushel. Insestment in the wet mill wis estimated to he $\$ 40$ million (Tahle 15 ).

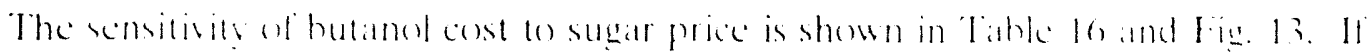

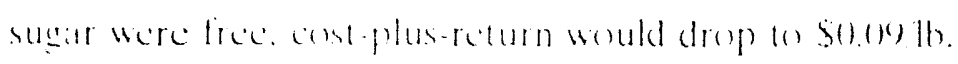

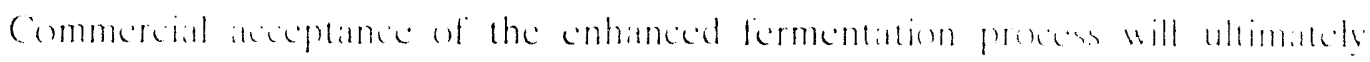

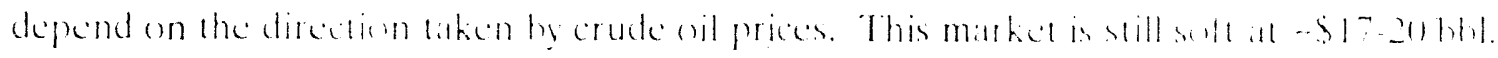




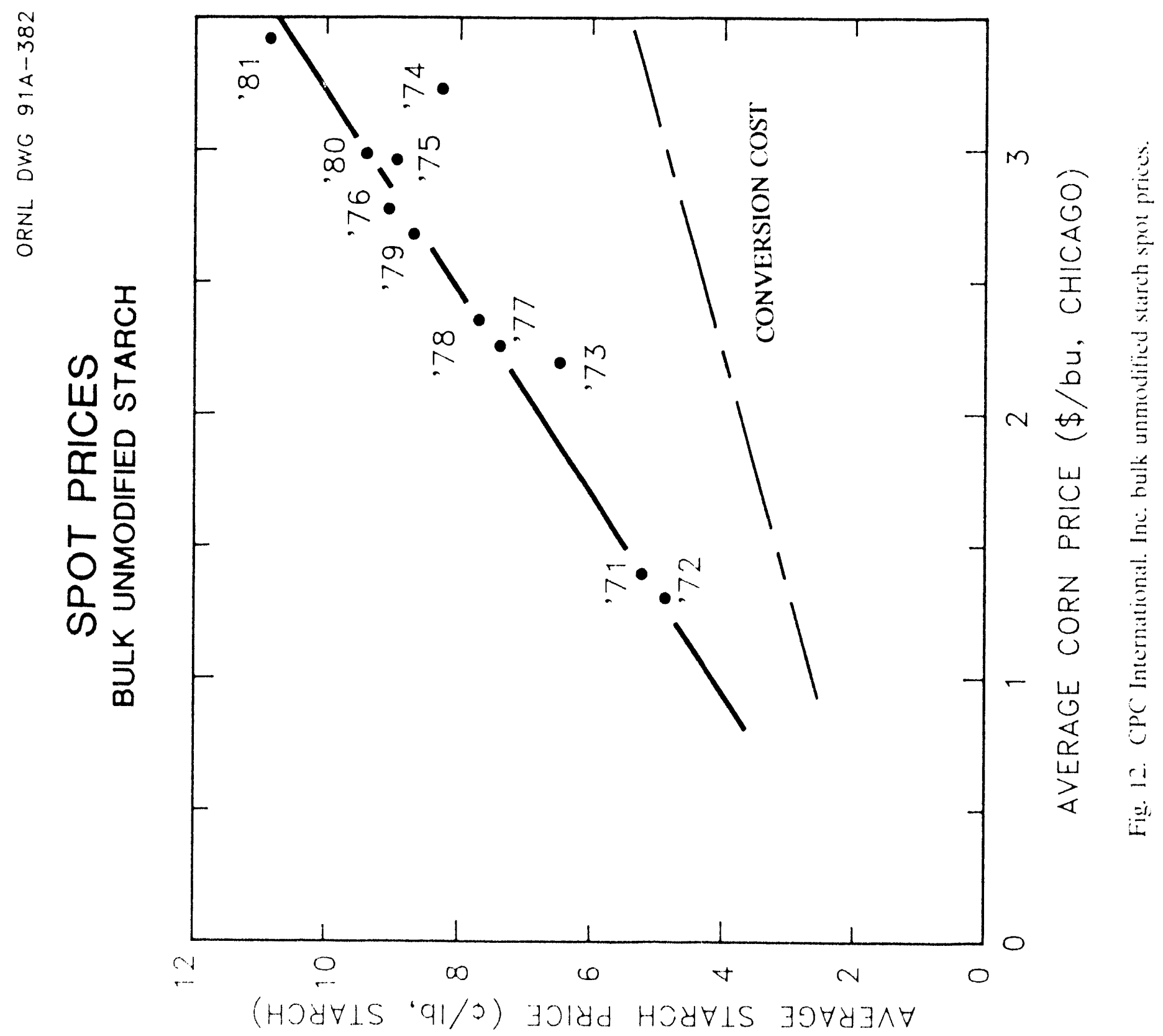




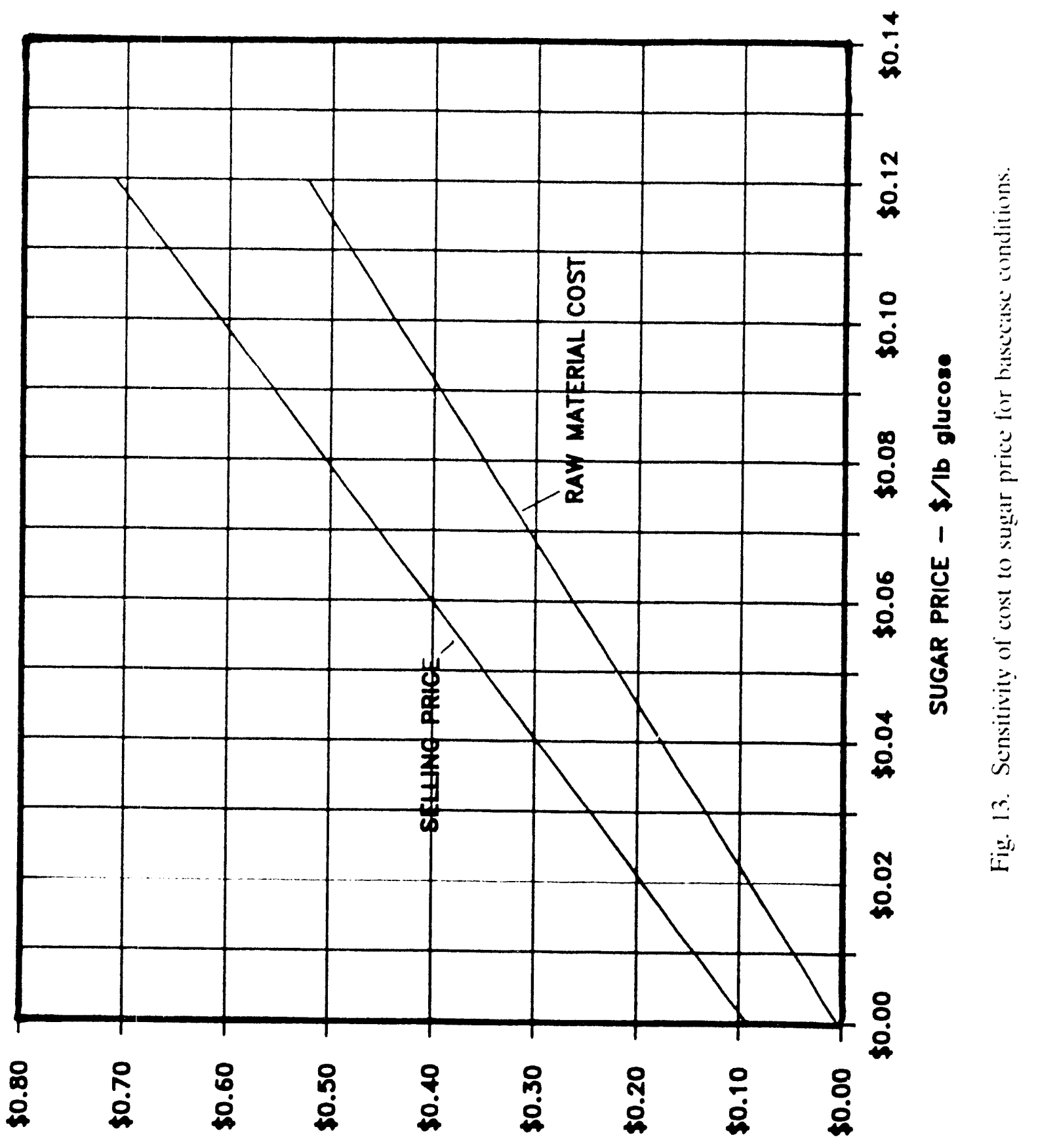

$91 / \$-1500$ 
Table 15. Approximate cealuation of substrate costs

\begin{tabular}{|c|c|}
\hline & $\$ / \mathrm{lb}$ glucose \\
\hline Corn (a $\$ 2.60)$ bushel & $\$ 0.074$ \\
\hline \multicolumn{2}{|l|}{ Coproduct credits } \\
\hline Corn oil $1.7 \mathrm{lb} / \mathrm{bu}(\mathrm{a} \$ 0.26 / \mathrm{lb}$ & $(0.013)$ \\
\hline onc, Giluten meial $2.2 \mathrm{lb} / \mathrm{hu}$ (1t) $\$ 26.5 / \mathrm{ton}$ & $(0.0() S)$ \\
\hline Gluten liced $11.5 \mathrm{lb} / \mathrm{bu}(a \mathrm{a} \$ 125 / \mathrm{ton}$ & $(0.02(1))$ \\
\hline Total credits & $(0.041)$ \\
\hline Net corn & 0.03 .3 \\
\hline Encymes & 0.006 \\
\hline Labor & 0.007 \\
\hline Utilitics & 0.008 \\
\hline Maintenance, taxes, and insurance & $0 .(10) 4$ \\
\hline Depreciation & $0.00(14$ \\
\hline Net cost of manufacture & 0.062 \\
\hline Selling, administrative, \& research & $0 .(0) 2$ \\
\hline Earnings before taxes & 0.017 \\
\hline Sclling price & $\$() .081$ \\
\hline \multicolumn{2}{|l|}{ Savings for contract $45 \%$ syrup } \\
\hline Stcam $1.15 \mathrm{lb} / \mathrm{lb}$ & $(0.006)$ \\
\hline Labor & $(0 .(0) 1)$ \\
\hline Maintenance and depreciation & $(0 .(0) 1)$ \\
\hline S, A, \& R & $(0 .(0) 1)$ \\
\hline Earnings & $(0 .()(0) 7)$ \\
\hline Adjusted contract price & $\$(1.065$ \\
\hline
\end{tabular}


Table 16. Sensitivity analysis

Ellect of sugar price on product cost

\begin{tabular}{lccc}
\hline $\begin{array}{l}\text { Sugar } \\
\text { price } \\
(\$ / 1 \mathrm{~b})\end{array}$ & $\begin{array}{c}\text { Cost of } \\
\text { raw material } \\
(\$ / \mathrm{lb})\end{array}$ & $\begin{array}{c}\text { Cost of } \\
\mathrm{mfg} . \\
(\$ / 1 \mathrm{~b})\end{array}$ & $\begin{array}{c}\text { Cost-plus- } \\
\text { return } \\
(\$ / 1 \mathrm{~b})\end{array}$ \\
\hline 0.00 & 0.004 & 0.099 & 0.092 \\
0.02 & 0.090 & 0.186 & 0.196 \\
0.04 & 0.177 & 0.273 & 0.299 \\
0.06 & 0.264 & 0.360 & 0.402 \\
0.08 & 0.351 & 0.447 & 0.506 \\
0.10 & 0.438 & 0.534 & 0.609 \\
0.12 & 0.525 & 0.621 & 0.713 \\
\hline
\end{tabular}

However, James MeNabb of Conoco ${ }^{2}$ has pointed out that OPEC is presently operating at only $60 \%$ of eapacity. By the early 1990 s, production is expected to reach $80 \%$; market power will shift back from the buyer to the seller, with a corollary increase in oil prices. As a result, he forecasts that although oil prices will remain in the low $\$ 2$ )s until 1990, they will reach the mid $\$ 30$ ) by 1995 and $\$ 50$ per barrel by the year 2000). Thus, a doubling of the $\$(0.38$ price for butanol over the next decade is not out of the question.

\subsection{EFFECT OF RECOVERY OF RAFFINATE CHEMICALS}

Since hutanol is the primary product of the ABE system, the process model was designed to extract substantially all of it with a reasc nable number of extraction stages. However, since the major by-products acetone and ethanol have lower distribution coeflicients, a considerable portion of that which is produced remains in the aqueous rallinate. For this system, it appears that the recovery of these by-products from the rallinate purge is economically justified provided that raffinate and eells are recyeled at the level studied. Indeed, the return on the additional investment required amounts to $220 \%$. The cases with and without recovery compare as follows (Table 17). 
Table 17. Eflect of recovery of raffinate chemicals

\begin{tabular}{lccc}
\hline Expense, \$/lb & No recovery & Recovery & Difference \\
\hline Cost of sales & $\$ 0.444$ & $\$(0.439$ & $(\$ 0.0(15)$ \\
Acetone credit & $(0.062)$ & $(0.084)$ & $(0.022)$ \\
Ethanol credit & $\frac{--}{0.382}$ & $\frac{(0.028)}{0.327}$ & $\frac{(0.028)}{(0.055)}$ \\
Investment, \$/lb & & & \\
New stills &.- & 0.009 & 0.009 \\
By-product storage & $0.0(04$ & 0.008 & 0.004 \\
Steam & $\underline{0.035}$ & $\underline{0.045}$ & $\underline{0.010}$ \\
& 0.039 & 0.062 & 0.023 \\
Total plt. invest & 0.203 & 0.228 & $0.025^{*}$ \\
\hline
\end{tabular}

* \$4.5 million for 180 MM PPY plant

Return on additional investnient $=\$(0.055 / \$ 0.025=220 \%$

\section{PROJECTED ECONOMICS OF THE IN SITU EXTRACTION PROCESS}

The cost-plus-return for butanol as manufactured by the in situ extraction model would amount to $\$ 0.49 / \mathrm{hb}$ at the same $22-\mathrm{g} / \mathrm{L}$ cell density as for the external extraction case, for which the cost was $\$ 0.48 / \mathrm{lb}$ without recovery of raffinate chemicals. Both models would benefit further by recovery of the by-products, but that scheme was not included in the in situ model. As with the external extractor case, the minimum eost of the in situ case depends on the extent to which eell density can be increased. In the case of the in situ basecase, increasing the solvent to feed ratio at a fixed dilution rate is the main operating approach. A basecase ratio of 3.5 at a $0.03 \mathrm{~h}^{-1}$ dilution rate was used here. A summary of the economic picture is provided in Table 18 and Appendix F. 
Table 18. Single-stage in situ extraction fermentation of butanol

(No recovery of raflinate chemicals)

Basecase stoichiometry

Summary

Production level

163 Million PPY

Investment, \$Million

$\mathrm{MPC}=1984$

Direct permanent investment

21.6

Allocated power, services, and general

Working capital

Total investment

$\frac{15.8}{47.0}$

\section{Cost, \$/b (1988)}

Raw materials

0.30

Utilities

0.05

Labor-related

0.03

Capital-related

0.02

Cost of manufacture

0.40

SE, D, R\&D, Adm, and IC

Cost of sales

$\frac{0.06}{0.46}$

0.09

Pretax earnings based on: $30 \%$ pretax ROI

$(0.06)$

Selling price

0.49

Financial Criteria

Net ROI 3rd year (assumed)

$16 \%$

Investors rate of return (20 operating years)

$17 \%$

Years to break even - Annual cash 1987

- Cumulative cash 1991

- Cum. disc. cash (NPV) 1995

Net present value \$Million (20 years (a) $12 \%$ ) $\$ 16.4$ 


\subsection{EFFECT OF SOLVENT/FEED RATIO}

Cost is reduced by raising the solvent/feed ratio to the highest level possible. As noted in Table 19 , cost might be reduced to $\$ 0.39 / \mathrm{lb}$ at a $20: 1$ ratio if a cell density of 120 $\mathrm{g} / \mathrm{L}$ were operable, but that seems doubtful. A large portion of the saving is related to the reduction in fermenter volume and investment that results from the trade-off with cell density at constant specific productivity. At high solvent/feed ratios, fermenter investment becomes insignificant compared with total plant investment, and in effect an "infinite" ratio is reached.

Distribution coefficient is an important adjunct to solvent/feed ratio. This effect is shown in Table 20 and Fig. 14. If a coefficient of 4.3 cannot be attained in commercial operation, the reduction in performance can be compensated for to some extent by raising the ratio.

\subsection{EFFECT OF BUTANOL CONCENTRATION}

For the in situ evaluation, butanol concentration was held constant at $13 \mathrm{~g} / \mathrm{L}$, the threshold of inhibition for the existing organism. However, cost performance could be improved if product inhibition could be reduced. The effect of increasing the allowable concentration in conjunction with changes in solvent/feed ratio is shown in Table 21 and Fig. 15 .

Similarly, cost could be reduced by developing a more active organism with a higher specific productivity that could operate at a dilution rate higher than the $0.03 \mathrm{~h}^{-1}$ used in the in situ basecase. The combined effect of improvements in both dilution rate and product concentration is shown in Table 22 and Fig. 16. Data for an "infinite" dilution rate are included. That curve represents the limit to which the fermenter performance can be pushed. 


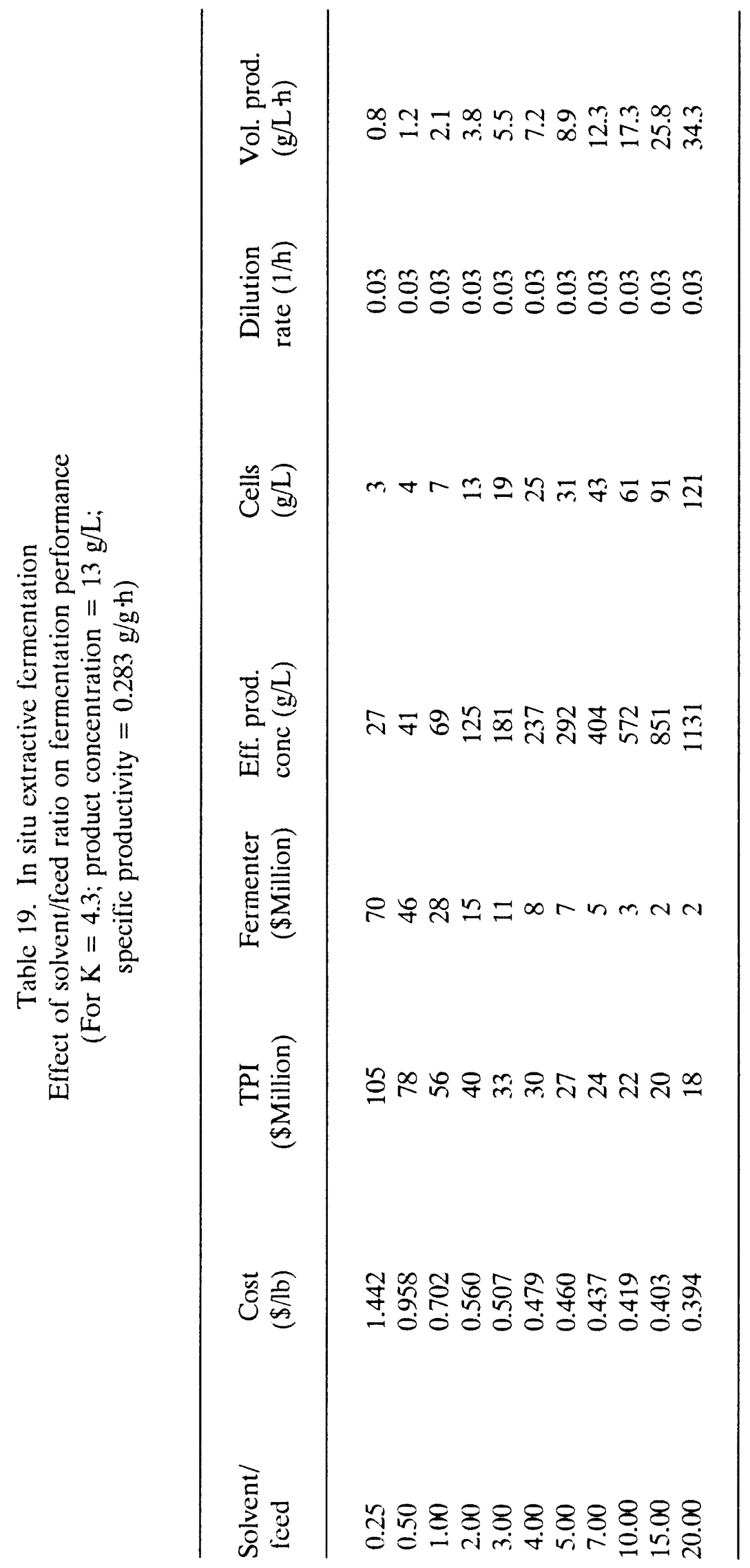




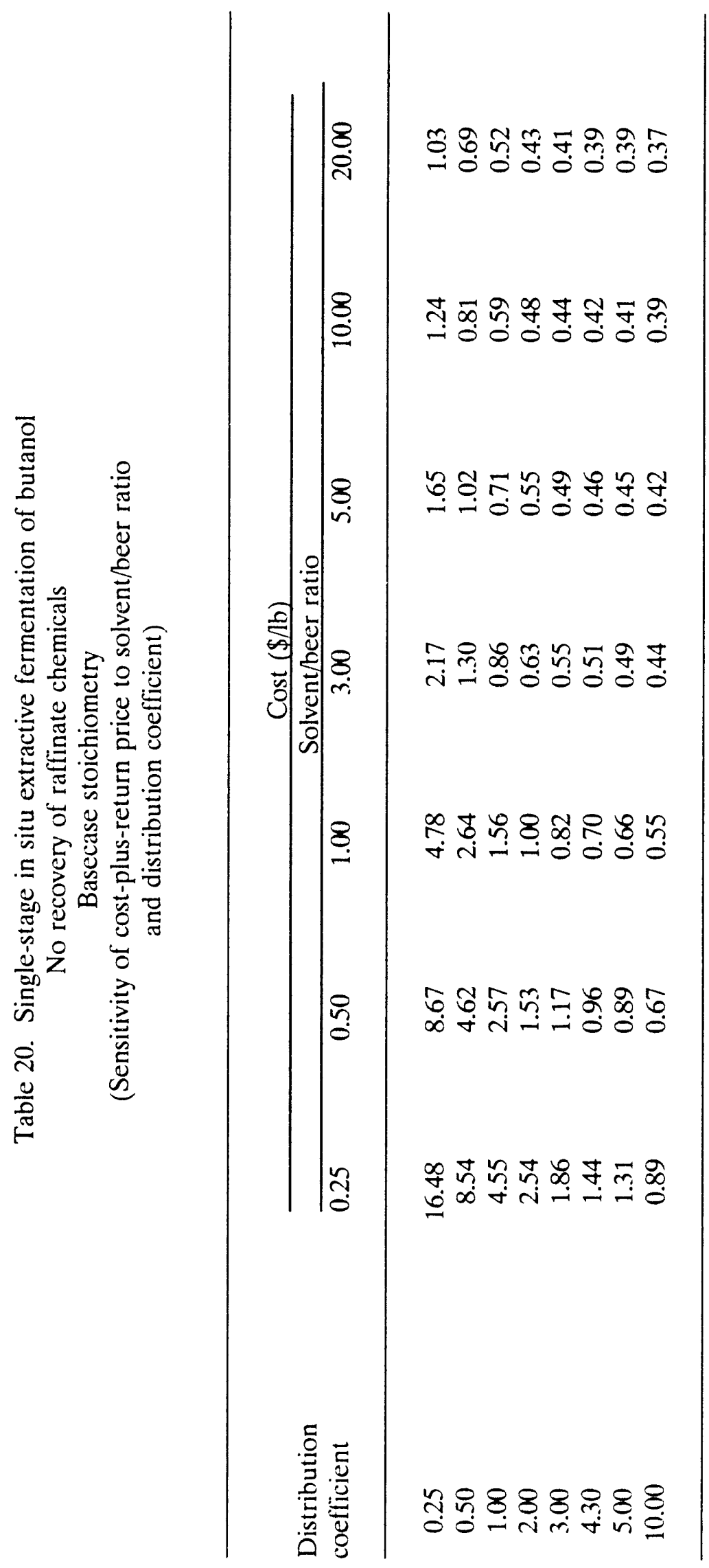




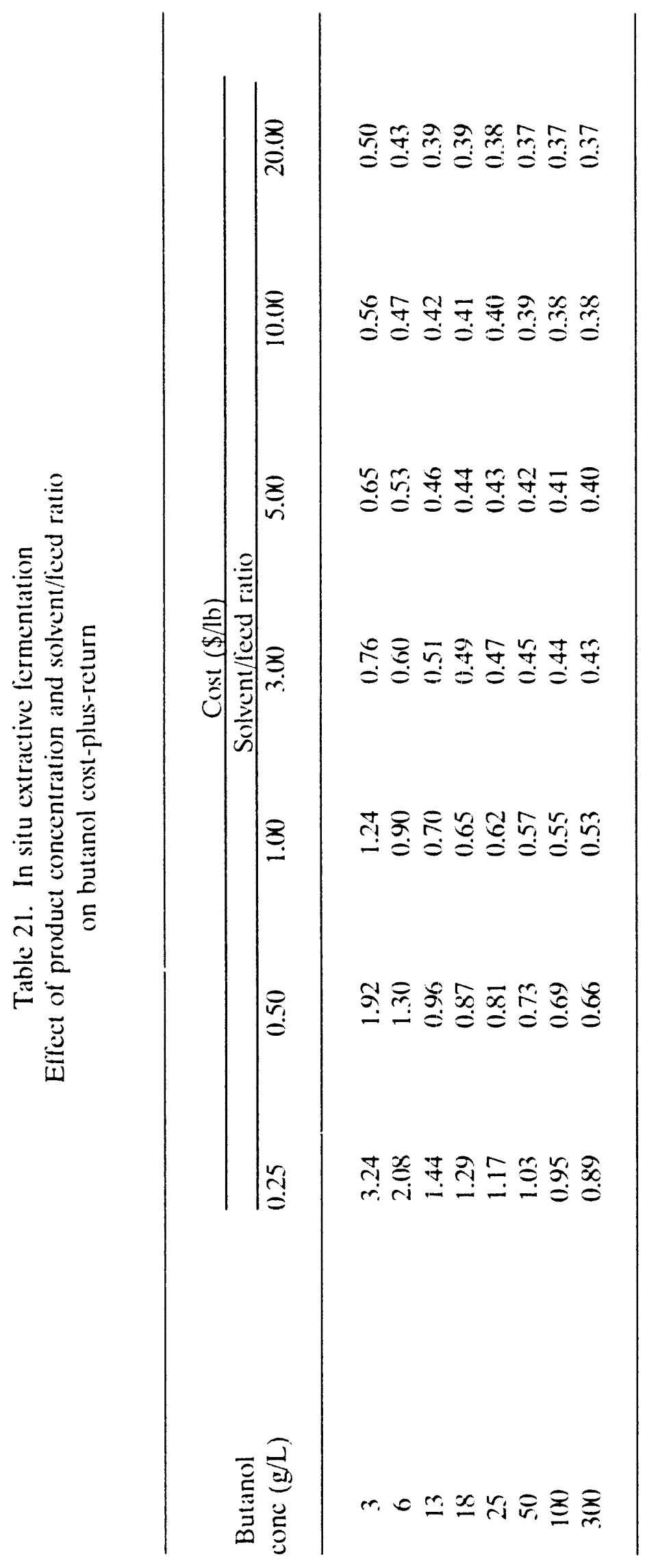


Table 22. Single-stage in situ extractive fermentation of butanol

No recovery of raflinate chemicals

Basecase stoichiometry

(Sensitivity of cost-plus-return price)

\begin{tabular}{cccccc}
\hline \multirow{2}{*}{$\begin{array}{c}\text { Raflinate } \\
\text { concentration } \\
(\mathrm{g} / \mathrm{L})\end{array}$} & 0.01 & 0.03 & 0.10 & 0.30 & Cost $(\$ / \mathrm{lb})$ \\
\cline { 2 - 6 } & & & & & \\
\cline { 2 - 6 } & & & \multicolumn{5}{c}{ Dilution rate $(1 / \mathrm{h})$} \\
& & & & & \\
1 & 2.25 & 1.40 & 1.10 & 1.02 & 0.97 \\
3 & 1.05 & 0.76 & 0.66 & 0.63 & 0.62 \\
10 & 0.62 & 0.53 & 0.50 & 0.49 & 0.49 \\
30 & 0.49 & 0.46 & 0.45 & 0.45 & 0.45 \\
100 & 0.44 & 0.44 & 0.43 & 0.43 & 0.43 \\
300 & 0.43 & 0.43 & 0.43 & 0.43 & 0.43 \\
& & & & & \\
\hline
\end{tabular}




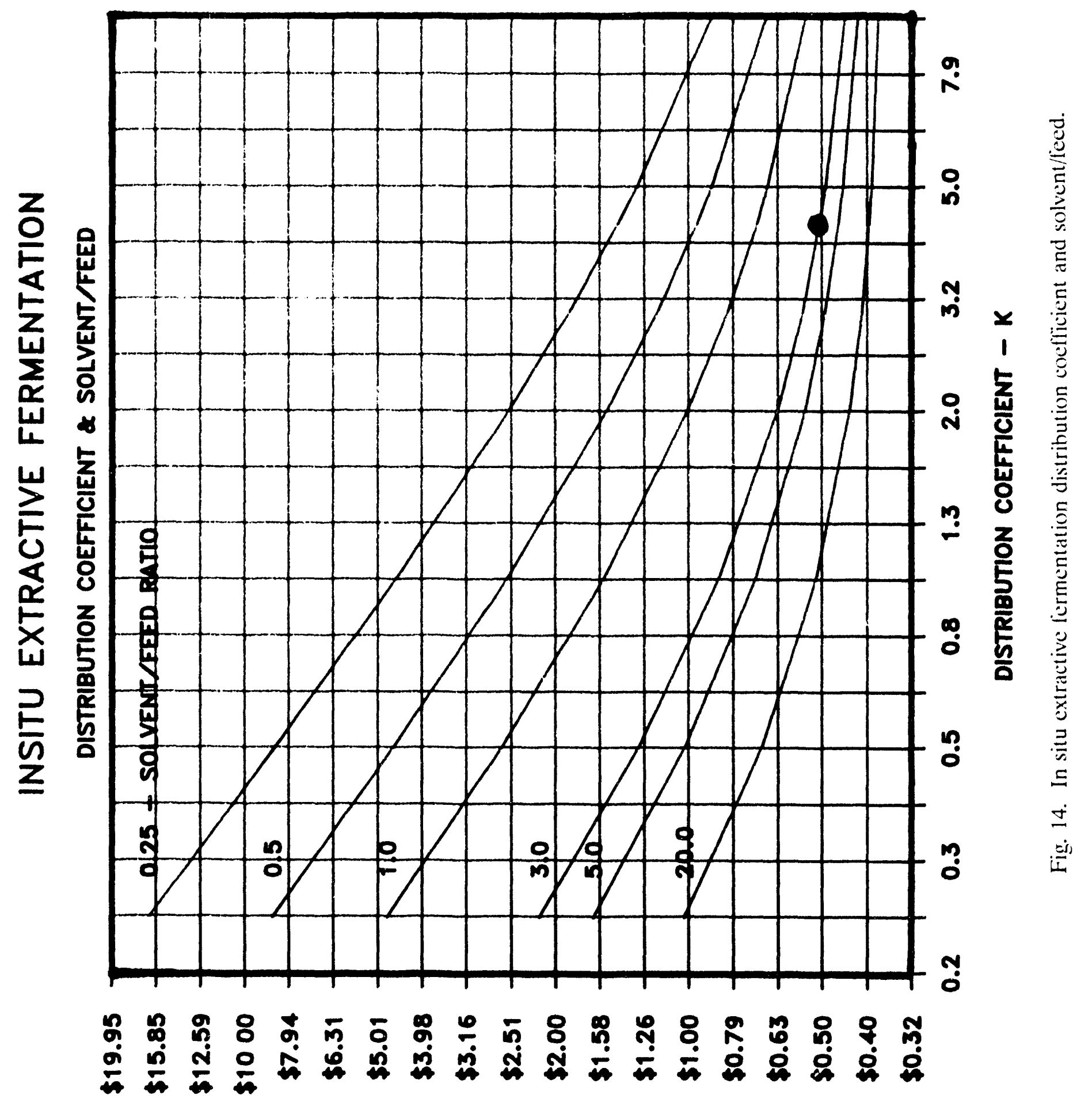

q1/\$ - 3018d NYnL3y-snาd-1500 


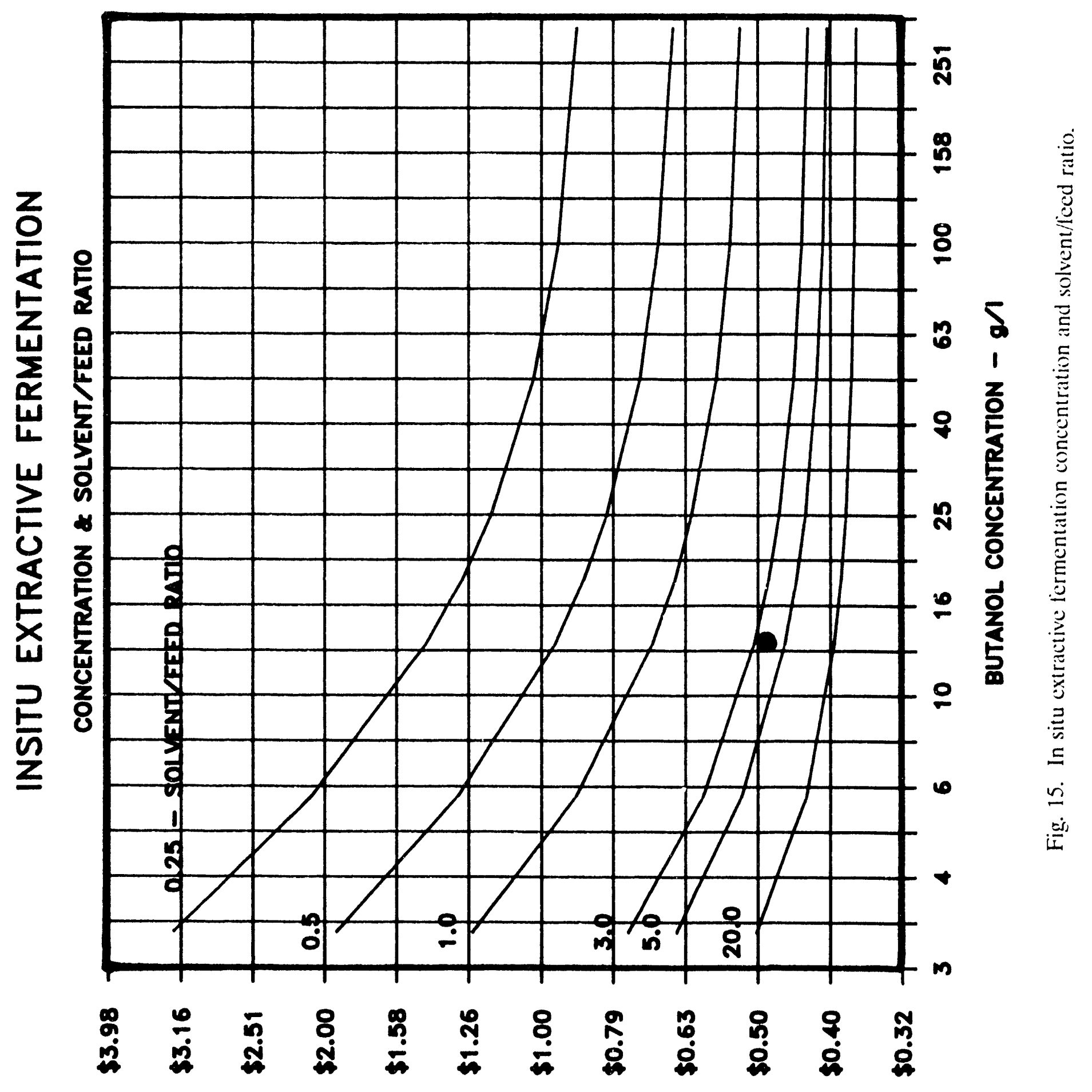

वi/\$ - 3oldd Nanızม-snาd-1500 


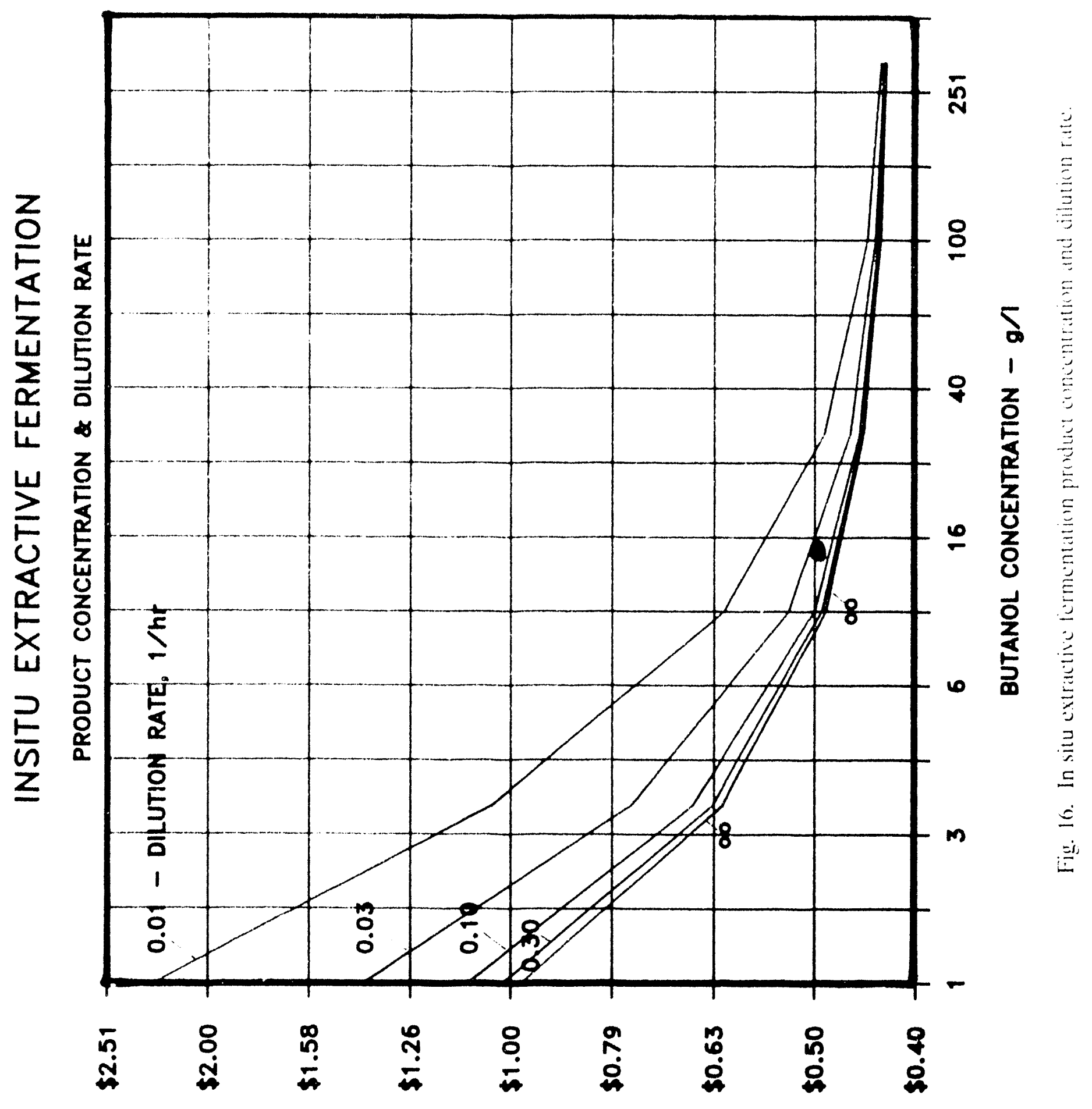

$91 / \$$ - Jolyd Nyn13y-Snרd-1500 


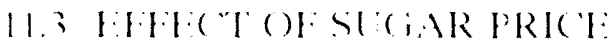

As might be eypected, cost is highly sensitive to sugar price. This cflect is shown in Tathe 23 and Fig. 17. it will be very important to the viability of either exiraction mode 16) provide a cost-eflective supply of syrup to the eommercial venture.

\section{RICOMMENDATIONS}

It appeared from the results of the study that either the external or in situ extraction processes would be equally effective in reducing the cost of producing butanol to commercially aceeptable kevels. However, the external process would appear to be more casy to develop and operate on a commercial scale. Accordingly, the following recommendations are made as a guide to further research in this area:

1. Demonstrate continuous operation of the lermentation process on a rack or pilot scalc over an extended run time, say, $1000 \mathrm{~h}$.

2. Immobilize the cells and/or add a crossllow filter or similar separation device so as to retain the cells in the fermenter or recycle them to the desired maximum eell density while increasing flow through the lermenter to hold product concentration at its optimum level relative to feclback inhibition.

3. In a separate system, test the continuous countercurrent extraction of butanol, acelone, and ethanol by oleyl alcohol or an improved solvent using a synthetic beer that mimics the expected impurity and salt levels of the fermentation beer (or use the actual heer if such is available at that time).

4. Concurrenlly, in a separate small-scale laboratory study, seck a solvent having belter distillation characteristics than oleyl alcohol with at least the same 
Table 23. Single-stage in situ extractive fermentation of butanol

No recovery of raffinate chemicals

Basecase stoichiometry (Elfect of sugar price on product cost)

\begin{tabular}{lccc}
\hline $\begin{array}{l}\text { Sugar } \\
\text { price } \\
(\$ / / b)\end{array}$ & $\begin{array}{c}\text { Cost of } \\
\text { raw mat. } \\
(\$ / / \mathrm{b})\end{array}$ & $\begin{array}{c}\text { Cost of } \\
\text { mfg. } \\
(\$ / \mathrm{h})\end{array}$ & $\begin{array}{c}\text { Cost plus } \\
\text { retuin } \\
(\$ / 1 \mathrm{~b})\end{array}$ \\
\hline 0.0() & $0.00)$ & 0.10 & \\
0.04 & 0.19 & 0.29 & 0.15 \\
0.06 & 0.28 & 0.38 & 0.37 \\
0.08 & 0.38 & 0.48 & 0.48 \\
0.10 & 0.47 & 0.57 & 0.59 \\
0.12 & 0.56 & 0.66 & 0.70 \\
\hline
\end{tabular}




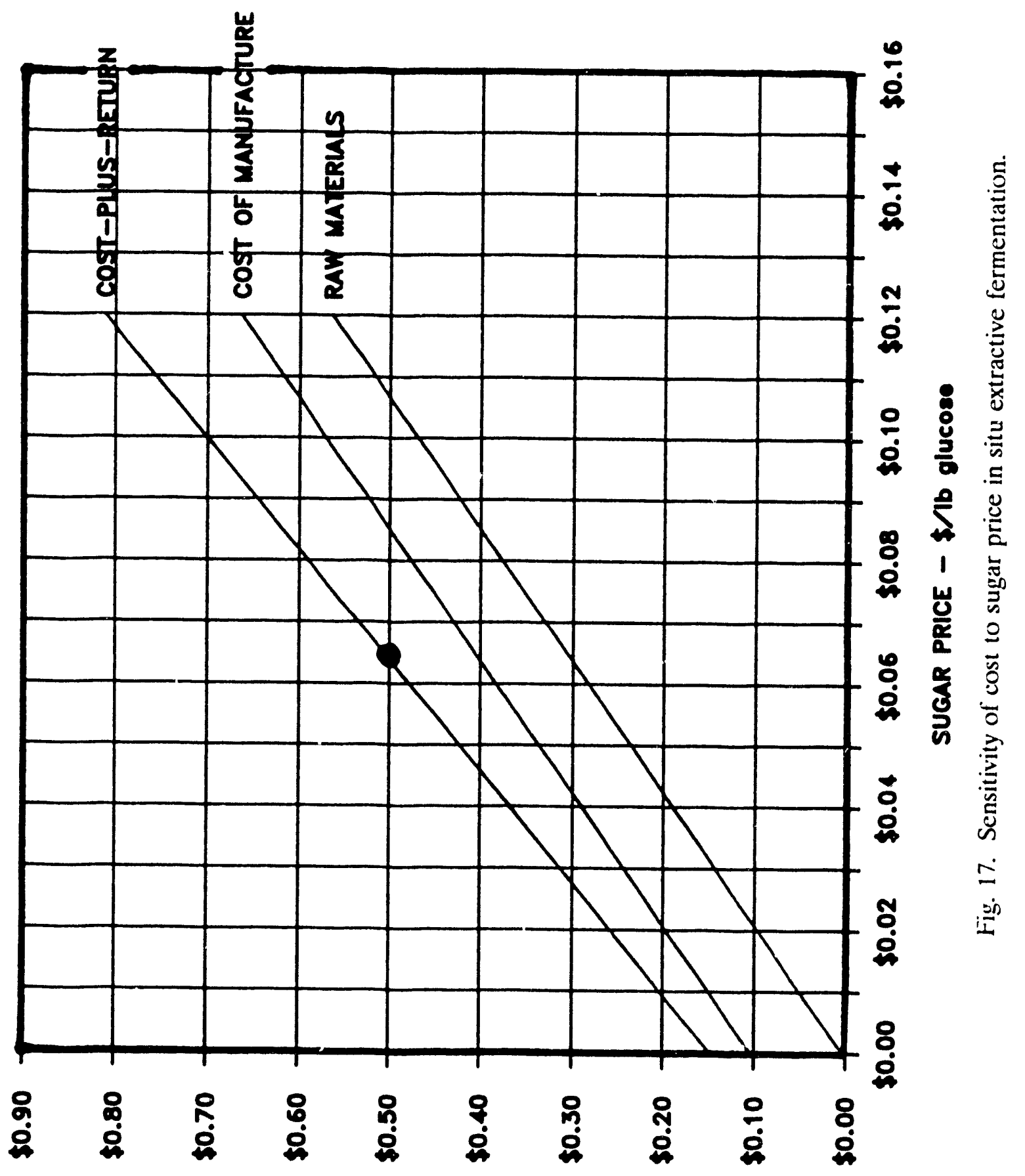

$91 / \$-1500$ 
distribution coefficient and low toxicity. A solvent boiling below water might be considered. Although this would require a different process scenario than the one used in this study for a high-boiling solvent, it might offer advantages over the high-boiling recovery process. In the selection of a new solvent, attention should be paid to the following potential problems: (1) separation of high-boiling acids, if any are present and at a sufficiently low $\mathrm{pH}$ to be extractable, might be difficult; (2) if the solvbility of water in the solvent is much higher than the $20 \mathrm{ppm}$ that was assumed, problems with azcotropes in the recovery still train involving acetone, butanol, and ethanol could arise; and (3) if the solubility of solvent in water is much higher than the $20 \mathrm{ppm}$ that was assumed, additional investment would be required to recover and/or dispose of solvent in the waste raffinate.

5. Integrate the fermentation unit with the extiction unit and demonstrate operability with.out cell retention or recycle, then with cell 'suildup, and finally with raffinate recycle to assess possible adverse effects from the buildup of unextracted products or toxins.

6. Consider the concurrent development of a genetically revised organism that produces only butanol as its solvent slate and/or has an enhanced specilic productivity.

7. Continue the comparative evaluation of the economics of both processes as new information is obtained from the research program. In the event a clear preference for the in situ process emerges, adapt the experimental program to the direct feed of solvent to the fermenter and demonstrate the in situ precess.

\section{CONCLUSIONS}

Based on the economic analyses, it appears that the extractive fermentation system could substantially reduce the cost of butanol and other similar high-boiling fermentation products that are now produced at low product concentrations as a result of product inhibition. Such an economic breakthrough cannot be realized until the 
system has been fully demonstrated in a continuous process over an extended period at pilot scale, optimized according to the findings of this study, and scaled up for the specific fermentation process of interest.

However, there appears to $\mathrm{b} \approx$ no inherent design limitation in effecting the engineering improvements required in the process operation.

Such may not be the case in attempting to develop an organism with an improved stoichiometry and/or specific productivity. The goal is sufficiently important, however, to warrant the laboratory effort.

Certainly, these improvements would represent major breakth roughs in not only the butanol process, but, generically, in any fermentation process that suffers from product inhibition (which is most of them), particularly where the product boils higher than water.

\section{ACKNOWLEDGMENT}

This work was jointly sponsored by the Jet Propulsion Laboratory (JPL) of the California Institute of Technology and the National Corn Growers Association (NCGA). It was conducted under contract No. 958353 with the Laboratory as part of its project on biocatalysis. The JPL project is a part of the Energy Conversion Utilization Technologies (ECUT) program of the U.S. Department of Energy. The author wishes to thank Dr. M. N. Dastoor of JPL and Mr. D. W. Ragsdale of NCGA for their inputs and support of the contracted study.

\section{REFERENCES}

1. R. M. Busche, The Biomass Alternative - A Report On The Potential of Renewable Resources as Feedstocks for U.S. Organic Chemicals, Bio En-Gene-Er Associates, Inc., Wilmington, DE, April 10, 1987. 
2. J. E. McNabb, World Energy Outlook Through 2000, Conoco, Inc., Wilmington, DE, September 1986.

3. R. M. Busche, Butanol - Industrial Market Volume and Potential, Bio En-Gene-Er Associates, Inc., Wilmington, DE, May 6, 1986.

4. M. T. Walton and J. L. Martin, "Production of Butanol-Acetone by Fermentation," Chapt. 6 in Microbial Technology, 2nd ed., Vol 1, H. J. Peppler and D. Perlman, eds., Academic Press, New York, 1979.

5. C. Weizmann, Brit. Patent 4845, 1915.

6. C. Weizmann, U.S. Patent 1,315,585, 1919.

7. S. C. Beesch, Ind. Eng. Chem., 44, 1677 (1952).

8. M. J. Spivey, Process Biochem., p. 2 (Nov. 1978).

9. C. L. Gabriel, Ind. Eng. Chem., 20, 1063 (1928).

10. C. L. Gabriel and F. M. Crawford, Ind. Eng. Chem., 22, 1163 (1930).

11. W. N. McCutchan and R. J. Hickey, pp. 347-52 in Industrial Fermentations, Vol I, L. A. Underkofler and R. J. Hickey, eds., Chem. Publ. Co., New York, 1954.

12. P. J. Weimer, Central Research \& Development Department, E.I. Dupont de Nemours \& Co. Inc., Wilmington, DE, private communication, May 1987.

13. R. Ryden, pp. $125-48$ in Biochemical Engineering, R. Steel, ed., Heywood, London, 1958.

14. A. R. Moreira, D. C. Ulmer, and J. C. Linden, Biotechnol. Bioeng. Symp., 11, $567-79$ (1981).

15. J. J. H. Hastings, pp. 31-44 in Economic Microbiology, Vol II, A. H. Rose, ed., Academic Press, New York, 1978.

16. J. C.-Y. Leung and D. I. C. Wang, pp. 348-52 in Proc. 2nd World Congress of Chemical Engineering and World Chemical Exposition, Montreal, Canada, Oct. 4-9, 1981, C. W. Robinson, ed.

17. G. F. Andrews and S. Elkeechen, Chem. Eng. Comm., 29, 139 (1984).

18. R. E. Heady and J. R. Frankiewicz, U.S. Patent 4,520,104, 1985.

19. H. Y. Wang, F. M. Robinson, and S. S. Lee, Biotechnol. Bioeng. Symp., 11, 555 (1981). 
20. S. S. Lee and H. Y. Wang, Biotechnol. Bioeng. Symp., 11, 221, (1982).

21. R. G. Denkewalter and J. Gillin, German Patent 1,062,891, 1959.

22. A. Kitai, H. Tone, T. Ishikura, and A. Ozaki, J. Ferment. Technol., 46, 442 (1968).

23. H. Tone, A. Kitai, and A. Ozaki, Biotechnol. Bioeng., 10, 689 (1968).

24. S. K. Tangu and T. K. Ghose, Process Biochem., p. 24 (Aug/Sept 1981).

25. P. J. Evans and H. J. Wang, Appl. Environ. Microbiol., 47, 1323 (1984).

26. M. Larsson, O. Holst, and B. Mattiasson, pp. 313-16 in Third European Congress on Biotechnology, Vol. II, Muenchen, FRG, Sept. 10-14, 1984.

27. H. Y. Wang, L. A. Kominek, and J. L. Jost, Adv. Biotechnol., 1, 601-7 (1981).

28. H. Y. Wang, Ann. N.Y. Acad. Sci., 413, 313-21 (1983).

29. R. Hernandez-Mena, J. A. Ribaud, and A. E. Humphrey, pp. 21-5 in Sixth International Fermentation Symposium, London, Ontario, July 1980.

30. M. Piziss and G.-G. Heden, Biotechnol. Bioeng., 7, 355 (1965).

31. I. Kuhn, Biotechnol. Bioeng., 22, 2393 (1980).

32. B. Hahn-Hagerdal, B. Mattiassen, and P.-A. Albertsson, Biotechnol. Lett. 3, 53 (1981).

33. B. Mattiasson, M. Suominen, E. Andersson, L. Haggstrom, P.-A. Albertsson, and B. Hahn-Hagerdal, Enzyme Eng., 6, 153-55 (1982).

34. B. Mattiasson and B. Hahn-Hagerdal, pp. 131-34 in Immobilized Cells and Organelles, Vol. I, B. Mattiasson, ed., CRC Press, Boca Raton, FL, 1983.

35. M. Larsson and B. Mattiasson, Ann. N. Y. Acad. Sci., 434, 144-47 (1984).

36. R. K. Finn, J. Ferment. Technol., 44, 144-47 (1984).

37. M. Minier and G. Goma, Biotechnol. Bioeng., 24, 1565 (1982).

38. J. Ribaud, M.S. Thesis, Univ. Pennsylvania, 1980.

39. S. Ishii, M. Taya, and T. Kobayashi, J. Chem. Eng. Jpn., 18, 125 (1985).

40. M. Taya, S. Ishii, and T. Kobayashi, J. Ferment. Technol., 63, 181 (1985). 
41. S. R. Roffler, H. W. Blanch, and C. R. Wilke, Bioproc. Eng. 2 (1), 1, (1987).

42. S. R. Roffler, H. W. Blanch, and C. R. Wilke, Bioproc. Eng. (in press).

43. M. Matsumura and H. Markl, Biotechnol. Bioeng., 28, 5341986.

44. R. W. Traxler, E. M. Wood, J. Mayer, and M. P. Wilson, Dev. Ind. Microbiol., 26,519 (1985).

45. J. C.-Y. Leung, "The Production of Acetone and Butanol by Clostridium acetobutylicum Using Free and Immobilized Cells," Ph.D. Thesis, Massachusetts Institute of Technology, Cambridge, MA, 1982.

46. R. K. Finn, J. Ferment. Technol., 44, 305-10 (1966).

47. M. J. Playne and B. R. Smith, Biotechnol. Bioeng., 25, 1251-65 (1983).

48. W. L. Griffith, A. L. Compere, and J. M. Googin, Dev. Ind. Microbiol., 24, $347-52$ (1983).

49. W. L. Griffith, A. L. Compere, and J. . 1. Googin, Dev. Ind. Microbiol., 24, 795-800 (1983).

50. Y. J. Jeon and Y. Y. Lee, "Membrane Assisted Extractive Butanol Fermentation", presented at 8th Symposium on Biotechnology for Fuels and Chemicals, Gatlinburg, TN (1986).

51. C. O. Gill and Ratledge, J. Gen. Microbiol., 72, 165-72 (1972).

52. P. J. Evans and H. Y. Wang, "Response of Clostridium acetobutylicum to the Presence of Mixed Extractants," Dept. of Chemical Engineering, Univ. of Michigan, 1985.

53. S. Roftler, H. W. Blanch, and C. R. Wilke, Biotechnol. Prog., 3, 131 (1987).

54. B. L. Maiorella, H. W. Blanch, and C. R. Wilke, Process Biochem., 18,(4), 5 (1983).

55. B. L. Maiorella, H. W. Blanch, and C. R. Wilke, Biotechnol. Bioeng., 226, 1003 (1984).

56. S. R. Roftler, H. W. Blanch, and C. R. Wilke, Biotechnol. Bioeng., in press.

57. B. M. Ennis and L. S. Maddox, Biotechnol. Lett., 7, 601 (1985).

58. B. Allen et al., "Quarterly Technical Progress Report for Second Quarter 1986 on Multi-phase Fluidized Bed -- An Advanced Bioreactor Concept," Contract 
No. 957241 to California Institute of Technology, Jet Propulsion Laboratory, Battelle Memorial Institute, July 14, 1986.

59. B. Allen et al., "Fiscal Year Progress Report on Multi-phase Fluidized Bed -An Advanced Bioreactor Concept," Contract No. 957241 to California Institute of Technology, Jet Propulsion Laboratory, Battelle Memorial Institute, Sept. 12, 1986.

60. B. Allen et al., "Quarterly Technical Progress Report for Third Quarter 1986 on Multi-phase Fluidized Bed -- An Advanced Bioreactor Concept," Contract No. 957241 to California Institute of Technology, Jet Propulsion Laboratory, Battelle Memorial Institute, Oct. 14, 1986.

61. B. Allen et al., "Quarterly Technical Progress Report for Fourth Quarter 1986 on Multi-phase Fluidized Bed -- An Advanced Bioreactor Concept," Contract No. 957241 to California Institute of Technology, Jet Propulsion Laboratory, Battelle Memorial Institute, Jan. 14, 1987.

62. B. Allen et al., "Final Technical Progress Report on Multi-phase Fluidized Bed -- An Advanced Bioreactor Concept," Contract No. 957241 to California Institute of Technology, Jet Propulsion Laboratory, Battelle Memorial Institute, Dec. 14, 1987.

63. F. Godia, H. I. Adler, B. H. Davison, and C. D. Scott, "Use of Immobilized Microbial Membrane Fragments to Reduce Oxygen Content and Enhance Acetone-Butanol Fermentation," Oak Ridge National Laboratory, (1989).

64. T. G. Lenz and A. R. Moreira, Ind. Eng. Chem. Prod. Res. Dev., 19, 478 (1980).

65. R. M. Busche, "FERMENT" Economic Analysis Software Program, Bio En-Gene-Er Associates, Inc., Wilmington, DE, 1987.

66. P. J. Evans and H. J. Wang, "A Comparative Design and Economic Analysis of an Acetone-Butanol Fermentation Facility With and Without In situ Solvent Extraction," Dept. of Chem. Engr., Univ. of Michigan, 1986.

67. S. Roffler, H. W. Blanch and C. R. Wilke, Biotechnol. Prog., 3,(3), 131-40 (1987).

68. S. M. Lewis and W. M. Grimes, Economic Time Series Analyses of the Alcohol Industry, Enzyme Technology Corp, Ashland, OH, 1988. 
APPENDIX A. BASECASE STOICHIOMETRY

WITH 15:1 RAFFINATE/CELLS RECYCLE

WITH RECOVERY OF RAFFINATE CHEMICALS 
MULTISTAGE EXTRACTIUE FERMENTATION OF BUTANOL WITH RECOUERY OF RAFFINATE

BASECASE STOICHIOMETRY

SUMMARY

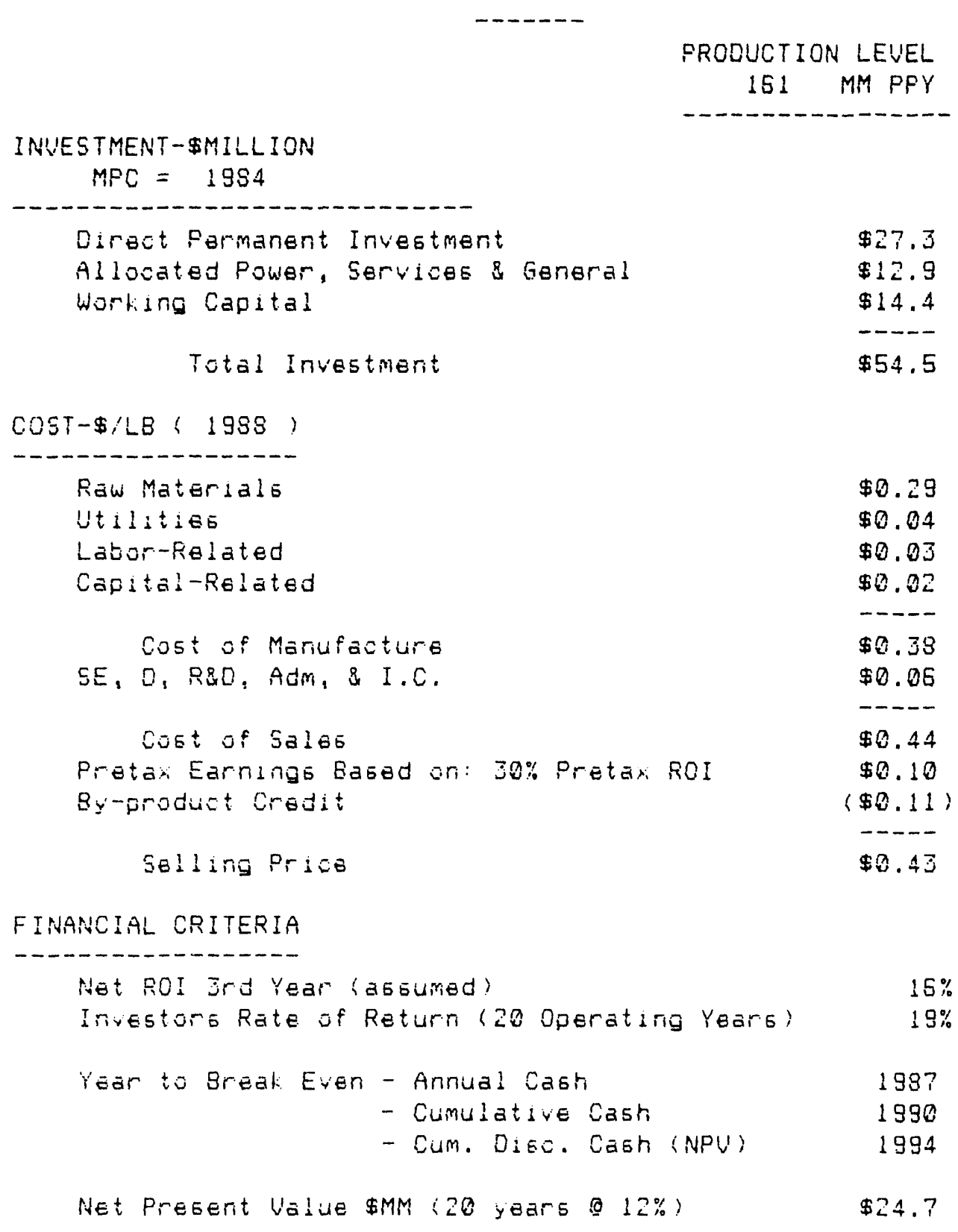


MULTISTAGE EXTRACTIUE FERMENTATION OF BUTANOL WITH RECOUERY OF RAFFINATE

BASECASE STOICHIOMETRY

INUESTMENT

CONDITIONS

-...........

-.-.-.-.-.

Sited in Iowa on the Mississipgi River adjacent to a corni wet mill and a utility puwer hube for over-the-fence supply of syrup and power.

\begin{tabular}{|c|c|c|c|c|}
\hline & $\begin{array}{l}\text { UNITS } \\
\text { UND }\end{array}$ & & $\begin{array}{l}\text { THIS } \\
\text { OASE } \\
-\end{array}$ & \\
\hline CAPACITY O 3000 HRS & $M M P P Y$ & & 179.2 & \\
\hline MID-POINT OF CONSTRUCTION & YEAR & & 1934 & \\
\hline CONSTRUCTION COST INOEX & $1980=100$ & & 128 & \\
\hline INUESTMENT CONTINGENCY & $\%$ INSTALLEI & & $30 \%$ & \\
\hline FERMENTER UNIT INUESTMENT & $\begin{array}{l}\text { \$GR.GAL. - } \\
\$: G R \text {. GAL. - }\end{array}$ & $\begin{array}{l}\text { OWTH } \\
\text { OD.N }\end{array}$ & $\begin{array}{l}\$ 12.90 \\
\$ 10.94\end{array}$ & \\
\hline $\begin{array}{l}\text { *40\% REEUMmERIEJ for re } \\
\text { OIRECT PERMANENT INUESTMENT }\end{array}$ & Procesgea & & & \\
\hline$\ldots \ldots \ldots \ldots \ldots \ldots \ldots \ldots \ldots \ldots$ & SCALE & & THI & IS CASE \\
\hline & FACTOR & & \$MM & FIANN.LE. \\
\hline & $-\cdots-\cdots$ & & $\cdots-\cdots$ & $-\cdots \cdots-$ \\
\hline FERMENTATION SECTION & & & & \\
\hline Receiving, Prep o Sterilization & 0.50 & & $\$ 3.92$ & $\$ 2.022$ \\
\hline Air Compression \& Aeration & 0.50 & & 0.00 & 0.000 \\
\hline Fermantation & $0.89-1.00$ & & 7.58 & 0.042 \\
\hline Entraction & & & 4.38 & 0.024 \\
\hline Producticell Sefaration & 0.75 & & 0.99 & 0.005 \\
\hline Fermeritation Sub-total & & & $\$ 15.89$ & $\$ 0.094$ \\
\hline OTSTILLATION SEOTION & STILLS & $4 x \cdot 5$ & & \\
\hline Seer Stil1 \#1 & $\$ 0.31$ & $\$ 1.03$ & $\$ 1.34$ & $\$ 0.007$ \\
\hline Bear Stili \#2 & 0.20 & 0.24 & 0.43 & 0.002 \\
\hline Low-8oilers Stili \#1 & 0.20 & 0.14 & 0.34 & 0.002 \\
\hline Low-Boilers Stil1 \#2 & 3. 35 & 0.11 & 0.47 & 0.003 \\
\hline Low-8011605 Eti11 $\# 3$ & 0.00 & 0.20 & 0.20 & 2.600 \\
\hline High-8011er Stid1\#1 & 0.77 & 0.31 & 1.08 & 0.006 \\
\hline Raffirate Etili:\#1 & 0.48 & 8.51 & 0.99 & 0.006 \\
\hline Raffirite Still \#2 & Q. $2 S$ & 0.14 & 0.42 & 0.002 \\
\hline Roffiriate Still \#J & 9.32 & 0.10 & 0.42 & 0.002 \\
\hline Distillation Suttotal & & & $\$ 5.49$ & $\$ 0.03$ \\
\hline STORAGE SECTIOA & & & & \\
\hline Storage - Butariol & & & $\$ 3.45$ & $\$ 0.019$ \\
\hline Storige - Acetorie & & & $\$ 1.05$ & 0.005 \\
\hline Storage - Ethanol & & & $\$ 0.35$ & 0.002 \\
\hline & & & & $\tan x$ \\
\hline Storage subtital & & & $\$ 4.8 ?$ & $\$ 0.03$ \\
\hline TOTAL OIRECT PLANT & & & $\$ 27.25$ & 50.152 \\
\hline
\end{tabular}


MULTISTAGE EXTRACTIUE FERMENTATION OF BUTANOL WITH RECOUERY OF RAFFINATE BASECASE STOICHIOMETRY

INVESTMENT

\section{- - - - - - - -}

ALLOCATEO PERMANENT INUESTMENT

\begin{tabular}{|c|c|c|c|c|c|}
\hline & APII! & UNIT & & & \\
\hline UNITS & BASECASE & THIS CASE & UNITS & \$MM & \$IANN.LB. \\
\hline$-\cdots-\cdots$ & $-\cdots-\cdots$ & $-\cdots---$ & $\ldots-\cdots$ & -....- & $\cdots-\cdots$ \\
\hline ELECTRICITY & $\$ 183$ & $\$ 183$ & 1,965 & $\$ 0.35$ & $\$ 0.002$ \\
\hline STEAM & $\$ 45$ & $\$ 45$ & 182,481 & 8.21 & 0.046 \\
\hline COOLING WATER & $\$ 52$ & $\$ 52$ & 7,045 & 0.37 & 0.002 \\
\hline PROCESS WATER & $\$ 313$ & $\$ 313$ & 31 & 0.01 & .000 \\
\hline WASTE DISPOSAL & $\$ 3$ & $\$ 3$ & 116,431 & 0.32 & 0.002 \\
\hline GEN'L \& SERUICES & $10 \%$ & $10 \%$ & $\$ 36.5$ & 3.55 & 0.020 \\
\hline TOTAL ALLOCATED PLAN & & & & $\$ 12.92$ & $\$ 0.072$ \\
\hline TOTAL PERMANENT INUESTMEN & & & & $\$ 40.18$ & $\$ 0.224$ \\
\hline WORKING CAPITAL & & & & & \\
\hline & & DAYS & DAYS & & \\
\hline & BASIS & BASECASE & THIS CASE & $\$ M M$ & $\$ / A N N . L B$. \\
\hline & $\cdots-\cdots$ & --- & $-\cdots--$ & ----- & $-\cdots--$ \\
\hline RAW MAT'L INUENTORY & \$RAW MATL & 2 & 2 & $\$ 0.29$ & $\$ 0.002$ \\
\hline SEMI-FINISHED PROOUCT & $\$(R+M) / 2$ & 5 & 5 & 0.74 & 0.004 \\
\hline FINISHED PRODUCT & $\$ C O M$ & 30 & 30 & 5.05 & 0.023 \\
\hline CASH & $\$(\operatorname{Cos}-0)$ & E & 6 & 1.12 & 0.006 \\
\hline ACCOUNTS RCD. -TRADE & $\$ S P$ & 30 & 30 & 5.58 & 0.032 \\
\hline ACCOUNTS RCO. -MISC. & $\%$ COM & $0.9 \%$ & $0.9 \%$ & 0.55 & 0.003 \\
\hline DEFERRED CHARGES & $\%$ COM & $1.5 \%$ & $1.5 \%$ & 0.92 & 0.005 \\
\hline TOTAL WORKING CAPITAL & & & & $\$ 14.37$ & $\$ 0.050$ \\
\hline
\end{tabular}


MULTISTAGE EXTRACTIUE FERMENTATION OF BUTANOL WITH RECOUERY OF RAFFINATE

BASEOASE STOICHIOMETR:

\begin{tabular}{|c|c|c|c|}
\hline \multirow[b]{2}{*}{ FRTCES Z COST FACTORE } & \multicolumn{3}{|c|}{ INFLATION } \\
\hline & SASECASE & FACTOR & THIS DASE \\
\hline$\cdots \cdots$ & n......... & $\ldots \ldots$ & $\cdots \cdots-\cdots$ \\
\hline GFERotirig Year & 1988 & 1988 & 1983 \\
\hline \multicolumn{4}{|l|}{ Pon Materióls } \\
\hline - Biogugar Syruf & $\$ 0.055$ it. & 1.00 & \$0.DES It. A.5. \\
\hline -Arthy Amorio & $\$ 0.048 / 15$. & 1.80 & $\$ 0.045: 15$. \\
\hline 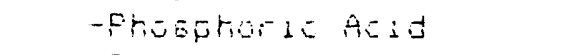 & $\$ 0.155 / 16$ & 1.80 & \$0.155it. \\
\hline -Putásiumi Crigride & $\$ 0.055,15$ & 1.00 & $\$ 0.053 ; 15$. \\
\hline -Mingr Mutrienta & $\$ 0.451 ;$ it. & 1.00 & $\$ 0.451 ; 1 t$. \\
\hline \multicolumn{4}{|l|}{ Utilities } \\
\hline -Electricity & $\$ 0.040, Y \mathrm{VWH}$ & 1.00 & $\$ 0.040: K W H$ \\
\hline -5team & $\$ 2.20 \therefore$ M L. & 1.00 & $\$ 2.20 ， M I 5$. \\
\hline - Coolding water & $\$ 0.34$ /M gal. & 1.00 & $\$ 0.04: M g a 1$. \\
\hline -Prodess Wetier & $\$ 0.50$, M gal. & 1.00 & $\$ 0.50 \therefore$ जäl. \\
\hline -Biodegradation & $\$ 0.04$ /1t. 0.6. & 1.00 & $\$ 0.04: 15.0 .5$. \\
\hline -Laritfill & $\$ 0.05$ ，化. d.5. & 1.00 & $\$ 0.05$ :1t. 4.5. \\
\hline \multicolumn{4}{|l|}{ Labor-Reláted } \\
\hline -Dir, Op. Wages u Een. & $\$ 25.40$ iman-rir. & 1.80 & \$2E.40 inuri-rir. \\
\hline -Dir. Salaries \& Berefi & $13 \%$ DOWBE & -- & $13 \%$ Dows \\
\hline -Of. Supplieg \& Service & $5 \%$ DOWLE & -- & $5 \%$ \% 0048 \\
\hline -6POH or Operationio & $25 \%$ nOW5\&8 & -- & $23 \% 00 W 5 \% B$ \\
\hline - Control Lat & $\$ 19.22$ mian-hr. & 1.00 & $\$ 19.22$ manthir. \\
\hline -Tech. Assist, to Mfig. & $\$ 22.0 E$ iman-rir. & 1.00 & $\$ 22.05$ iman-hr. \\
\hline \multicolumn{4}{|l|}{ Gápital-Related } \\
\hline -Mant. Wages \& Ban. & $1.7 \% \mathrm{DPI}$ & -- & $1.7 \% \mathrm{OPI}$ \\
\hline Malnt. Sálaries \& Ben. & 25\% MWLS & $\ldots$ & $25 \%$ MWR \\
\hline -Mairit. Mat'l \& Service & $40 \%$ MWRB & - & $40 \%$ MWRS \\
\hline -Maint. Overhead & $4 \%$ MWSB & -- & $4 \%$ MWLS \\
\hline -EPOH on Mainteriarice & $23 \%$ Muss B & -- & $23: M W 5 \& B$ \\
\hline -Tàes \& Irsurarize & $0 . \overline{3} \% D P I$ & -- & $0.3 \% \mathrm{OPI}$ \\
\hline -Depreciation - DPI & $3 \%$ OFI & -- & $3 \%$ DPI \\
\hline -DEpireaiation - APSBG & $6 \% A P S B G$ & -- & $E \because A P S \& G$ \\
\hline \multicolumn{4}{|l|}{ Cost of Manufacture } \\
\hline - EEllirg Empense & $3 \%$ Sales & -- & $3 \%$ Sales \\
\hline -Distribution & $\$ 0.01 ; 15$. & -- & $\$ 0.01$ iti. \\
\hline -Research \& Development & $4.5 \%$ Salas & - & $5 \%$ Sale \\
\hline -Adrinistrative ExfEnse & $2 \%$ SalE & -- & $2 \% 3 \pi 1 \leq 5$ \\
\hline -Iricantive Compenoation & E\% PTE & - & $E \% H[E$ \\
\hline \multicolumn{4}{|l|}{ Cost of Saler } \\
\hline -Pretan Earninga & $30 \%$ TIFR & -- & $30 \%$ TIFR \\
\hline -Credit: Acetorie & $\$ 0.27,15$. & 1.00 & $\$ 0.27 \div 16$. \\
\hline - Credit: Ethoñu & $\$ 2.23$; it. & 1.00 & \$0.29:15. \\
\hline -Product Selling Price & $\$ 0.00 ， 16$. & 1.00 & $\$ 0.00 \div 16$. \\
\hline
\end{tabular}




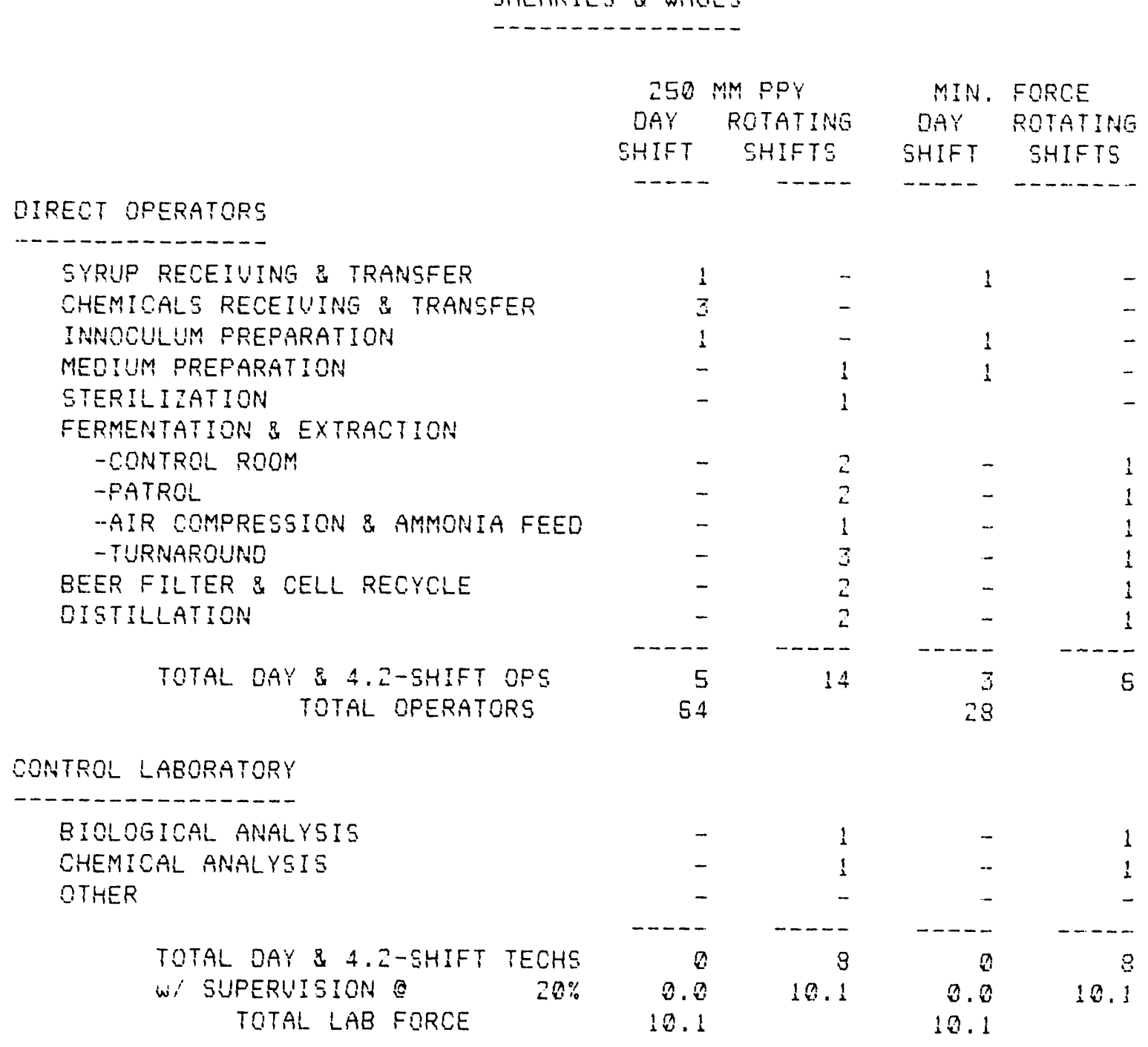

TECHNICAL ASSISTANCE TO MANUFACTURING

\begin{tabular}{|c|c|}
\hline PROCESS ENGINEERS & 1 \\
\hline WAGES, SALARIES \& BENEFITS SCHEDULE- & 1988 \\
\hline 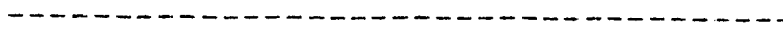 & ---- \\
\hline OPERATING WAGES - \$IHOUR & $\$ 20.14$ \\
\hline TECHNICIANS - ANNUAL \$ & $\$ 30,500$ \\
\hline PROCESS ENGINEERS - ANNUAL $\$$ & $\$ 35,000$ \\
\hline PENSION - AS \% OF COMPENSATION & 3. $1 \%$ \\
\hline FICA & $5.8 \%$ \\
\hline UNEMPLOYMENT COMPENSATION & $0.6 \%$ \\
\hline GROUP LIFE INGURANCE & $0.7 \%$ \\
\hline MEDICAL INSURANCE & $3.5 \%$ \\
\hline DENTAL INSURANCE & $0.8 \%$ \\
\hline SAUINGS PLAN & $2.5 \%$ \\
\hline V'ACATI GN & $7.4 \%$ \\
\hline ILLNESS & $1.4 \%$ \\
\hline ABSENCE WITH PERMISSION & Q. $2 \%$ \\
\hline TOTAL BENEFITS & $31.1 \%$ \\
\hline
\end{tabular}


HULTISTAGE EXTRAOTIUE FERMENTATION OF BUTANOL WITH SECOUERY OF RAFFINATE

BASECASE STOICHTOMETRY

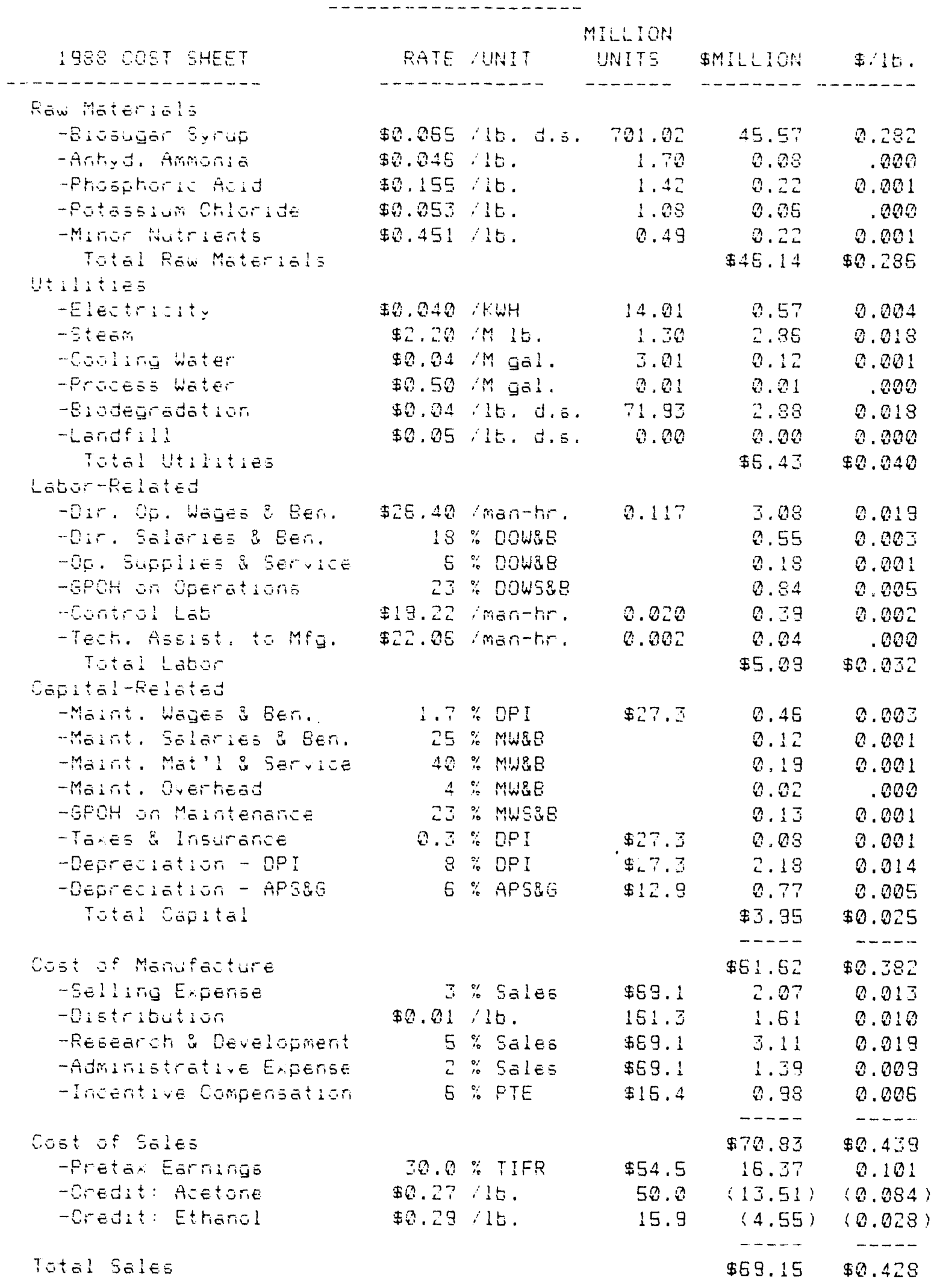


MULTISTAGE EXTRACTIUE FERMENTATION OF BUTANOL WITH RECOUERY OF RAFFINATE BASECASE STOICHIOMETRY

CASH FLOW IMILLION DOLLARS; YEAR;

Seeriário:

1. Investment oplit evenly over three soristrustion years.

2. Plant operates at $50 \%$ of full 50 ale the first year.

3 . " $" 75 \% "$ " " thie becond year.

4. " " $100 \% "$ " " the third year.

5. " $100 \% "$ " "thereafter.

5. Five year degreciation rate; rialf-year convertion;

(20, 32, 19.2, 11.5, 11.5, 5.8\%). Ta, 34\% federal, 3\% state.

7. Note that the cash flow analysis has meariarig only if the

product can te sold at the frice indicated on the ast sheet.

\begin{tabular}{|c|c|c|c|c|c|c|c|}
\hline \multirow[b]{2}{*}{ YEAR } & \multicolumn{2}{|c|}{ INUESTMENT } & \multirow{2}{*}{ DEP } & \multirow{2}{*}{ COST EX 0} & \multirow{2}{*}{ S.ALES } & NET EARN & \multirow{2}{*}{ ANN CASH } \\
\hline & PI & $W C$ & & & & $-\cdots$ & \\
\hline-3 & --- & $-\cdots$ & & & & & \\
\hline $198 \overline{3}$ & $\$ 9.09$ & & & & & & $(\$ 9.09)$ \\
\hline 334 & $\$ 3.89$ & & & & & & $1 \$ 9.09$ \\
\hline 985 & $\$ 9.09$ & $\$ 14.37$ & & & & & $(\$ 23.45)$ \\
\hline 1385 & & & $\$ 5.45$ & $\$ 35.99$ & $\$ 43.60$ & $\$ 8.74$ & $(\$ 0.18)$ \\
\hline 1987 & & & $\$ 8.72$ & $\$ 53.70$ & $\$ E 5.40$ & $\$ 1.39$ & $\$ 10.50$ \\
\hline 1388 & & & $\$ 5.23$ & $\$ 67.83$ & $\$ 37.21$ & $\$ 8.33$ & $\$ 14.11$ \\
\hline 1989 & & & $\$ 3.14$ & $\$ 57.83$ & $\$ 87.21$ & $\$ 10.20$ & $\$ 1 \Xi . j 4$ \\
\hline 1990 & & & $\$ 3.14$ & $\$ 57.38$ & \$37.21 & $\$ 10.20$ & $\$ 13.34$ \\
\hline 1991 & & & $\$ 1.58$ & $\$ 57.83$ & $\$ 97.21$ & $\$ 11.13$ & $\$ 12.76$ \\
\hline 1992 & & & & $\$ E ? .38$ & $\$ 87.21$ & $\$ 12.18$ & $\$ 12.18$ \\
\hline 1993 & & & & $\$ 67.88$ & $\$ 87.21$ & $\$ 12.18$ & $\$ 12.18$ \\
\hline 1994 & & & & $\$ 67.88$ & $\$ 37.21$ & $\$ 12.18$ & $\$ 12.18$ \\
\hline 935 & & & & $\$ 67.88$ & $\$ 37.21$ & $\$ 12.18$ & $\$ 12.18$ \\
\hline 1995 & & & & \$5?.88 & $\$ 87.21$ & $\$ 12.18$ & $\$ 12.18$ \\
\hline 1997 & & & & $\$ 67.33$ & $\$ 87.21$ & $\$ 12.18$ & $\$ 12.18$ \\
\hline 8 & & & & $\$ E 7.88$ & $\$ 87.21$ & $\$ 12.18$ & $\$ 12.18$ \\
\hline 1933 & & & & $\$ 57.83$ & $\$ 37.21$ & $\$ 12.18$ & $\$ 12.18$ \\
\hline 2000 & & & & $\$ 57.83$ & $\$ 87.21$ & $\$ 12.13$ & $\$ 12.18$ \\
\hline 2001 & & & & $\$ 57.38$ & $\$ 87.21$ & $\$ 12.18$ & $\$ 12.13$ \\
\hline 2082 & & & & $\$ 57.88$ & $\$ 37.21$ & $\$ 12.13$ & $\$ 12.18$ \\
\hline 103 & & & & $\$ 57.88$ & $\$ 37.21$ & $\$ 12.13$ & $\$ 12.18$ \\
\hline 204 & & & & \$E? . BS & $\$ 87.21$ & $\$ 12.18$ & $\$ 12.18$ \\
\hline 05 & & $(\$ 14.57)$ & & $\$ 57.83$ & $\$ 87.21$ & $\$ 12.18$ & $\$ 26.55$ \\
\hline
\end{tabular}

NET RETURN ON INUESTMENT-JRO DPERATING YEAR $=15.6 \%$ 
MULTISTAEE EXTRACTIVE FERMENTATION OF BUTANOL WITH RECOUERY OF RAFFINATE BASECASE STOICHIOMETPY

CAEH FLOW (MILLION DOLLARS,YEAR,

Soerigrio:

1. Irvestrient afilit everly over the soristruction years.

2. Flant operateb at $50 \%$ of full soale the first year.

ב. " " "

4 4. " $100 \%$ " "the third year.

5. " $" 100 \% "$ " thereafter.

E. Five year depregiation rate: half-year comverition;

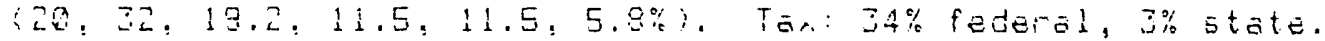

i. Wote that the cash flow anolysis has riearing orily if the

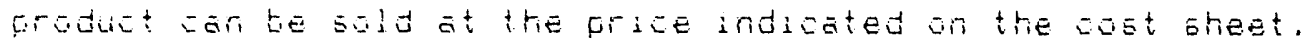

OUM CAEH NOU O $12 \%$ \%I只

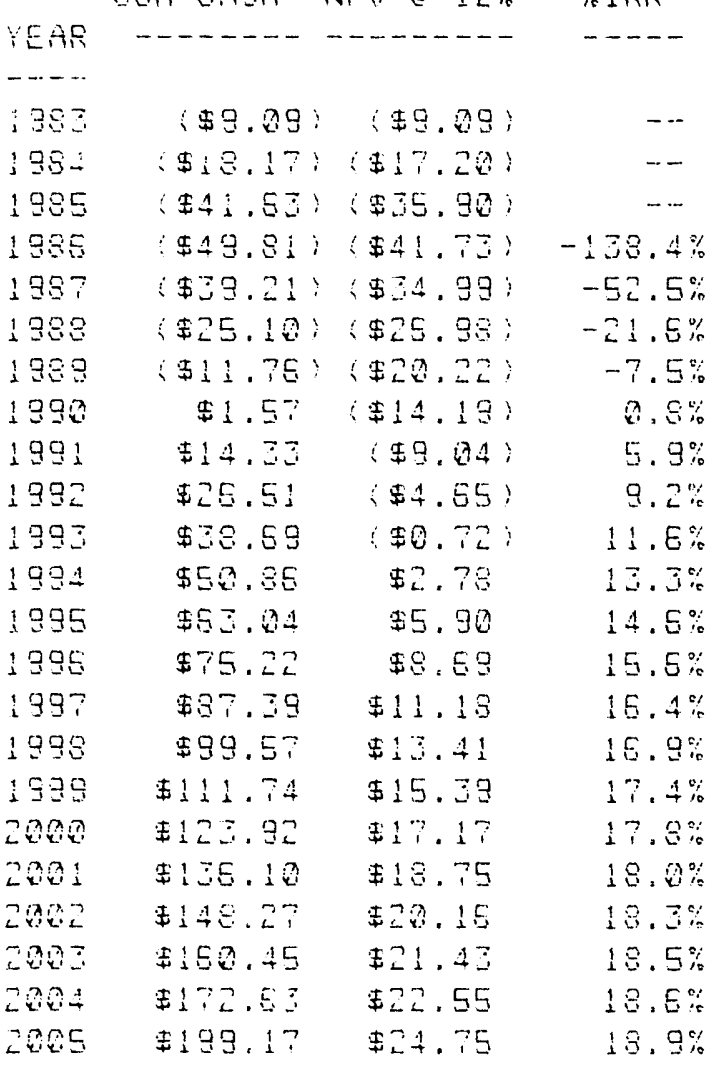


MULTISTAGE EXTRACTIUE FERMENTATION OF BUTANOL

WITH RECOUERY OF RAFFINATE

BASECASE STOICHIOMETRY

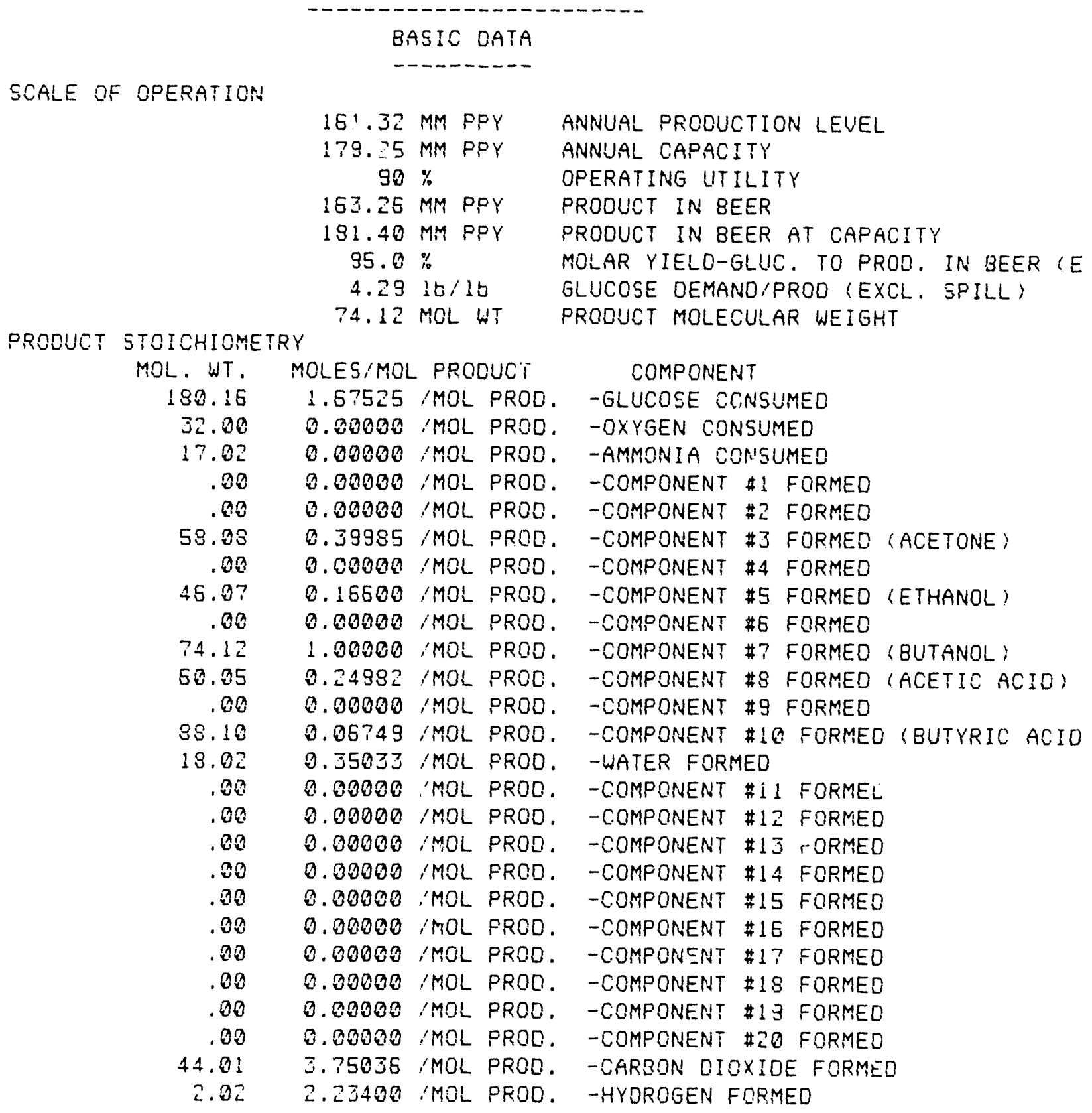

NUTRIENTS IN FERMENTER FEED

$3 \%$

$30.9 \mathrm{mg} / \mathrm{g}$ cells

El.5 mg'g cells

2?. 9 Mg'G CElL MINOR NUTRIENTS 
MULTISTAGE EXTRACTIUE FERMENTATION OF BUTANOL WITH RECOUERY OF RAFFINATE

BASECASE STOICHIOMETRY

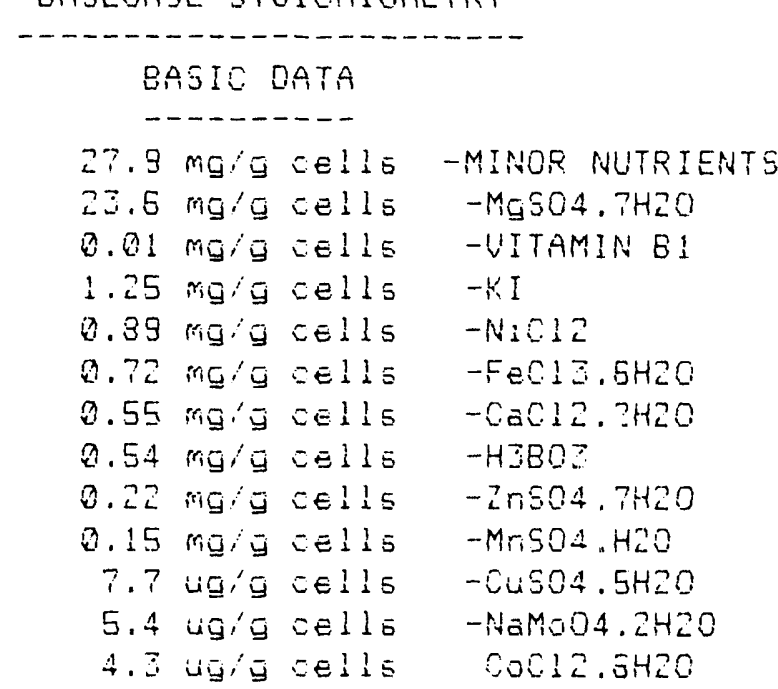

FERMENTATION

TYPE

STAGES

CONLITIONS

STAGE: GROWTH PRODUCTION

$$
\begin{aligned}
& \text { Q :A OR 1: -ANAEROBIC :O; OR AEROBIC :1; }
\end{aligned}
$$

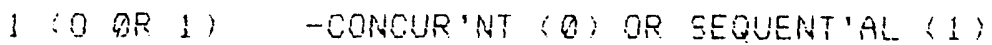

E. 5 S

0

$\therefore \therefore .5$

0.230

$$
0.00
$$

0.00

$-.95$

.11

5

$-$

5

-

2E4.13
6.5

$$
139.1
$$

21.8 9i1

B. 4751 ihr

0.28j gig*tir

E. 13 gil*tir

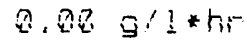

a $m ! 1 * 5 r$

(n) $m, \cdots M$

a. 1 5!

$5 \mathrm{C}$

19 táligriol

ż. IL Btuitrogal

\section{- TEMPEFATURE}

$-F H$

- PRODUCT CONCENTRATION IN BEER

- CELL DENGITY (CHO ONLY)

-DILUTION RATE

-PRODUCT FRODURTIUITY

* Spesifis Productivity

* Volunetrie Productivity - CELL PRONUCTIUITY S CHO ONLY;

-OXYGEN TRANSFERRED

-OXYGEM FER I GXYGEN STOICH. REMANO

- GLLCOSE SPILL

- COOLING WATER TEMPERATURE

-HEAT EVOLVEO-PRODUCT FORMATION

- HEHT REMOVEO BY COOLING COILS

- ACTIVE VOLLIME REOUIRED

- HEADSPACE

-GROSS VOLUME anol. 15\% 5Fares and

-GROSS SIZE

- MUMEER OF UNITS

PFODUET SEPARATION

$$
\begin{aligned}
& 4805 i 1
\end{aligned}
$$

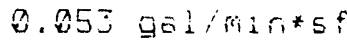

$$
\begin{aligned}
& 4,257 \text { 54 } f
\end{aligned}
$$

-CELL CONC. (CHO) EX FILTER

-FILTER THROLGHFUT

-FILTER SIZE

PRODUET RECQUERY E PURTFICATIUN 
MULTISTAGE EXTRACTIVE FERMENTATION OF BUTANOL

WITH REZOUERY OF RAFFINATE

BASECASE STOICHIOMETRY

BASIC DATA

MATERIALS OF CONETRUOTION

FERMENTERS \& EXTRACTERS

STILLS

HEAT EXCHANGERS

STORAGE TANKS

FOR WHICH:

$\begin{array}{cc}\text { CHOICES } & \text { SELECTION } \\ 1,3 & 1 \\ 1,2,3,4,5 & 1 \\ 1,3,5,5 & 1 \\ 1,3 & 1\end{array}$

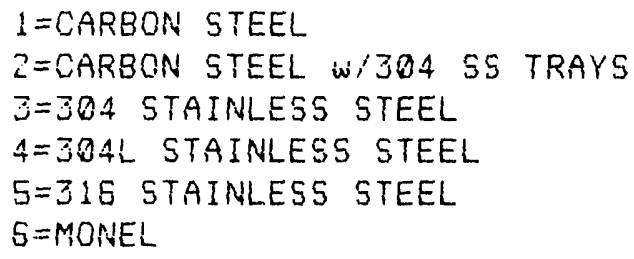

RETURN ON INUESTMENT

To Calculate Sellimg Price Required to Provide a Fixed Return, Enter the Desired Return on Irivestment: $O R$

To Calculate the ROI Pasulting from a Fixed Market Price, Eriter Marlet Price for: 1989 (Year) ilt.

Enter an Investment Contimgency to Represent the Risl: Level of the Basid Data $30 \%$

UENTURE TIMING

Midpoint of Construction (i.e. 13XX) 1994

Operating Year (i.e. $19 x \times$ ) 1338

\section{EXTRACTION}

Solvent

0 leyl Alcohol $\mathrm{CH} 3(\mathrm{CH}) 7 \mathrm{CH}=\mathrm{CH}(\mathrm{CH}) 7 \mathrm{CH} 2 \mathrm{OH}$

Solvent Molecular Weight $2 E 8.5$

Solvent Ratio, r
Mutual Solutilities

0. 30 1b/lb water in feed

l'ater in Solvent, w

solvent in Watei, 5

$(1+5))(1+w)$

0.000020 ltilt 601 vent

Garima

$0.00002016 / 1$ t water

1.00000

$-0.70000$

Water in Solvent Feed, m 0.000000 lb.llt solvent

Solvent in Aqueous feed 0.000019 lb/lt water in feed

Raffiriate Recycle

15.0 lb raffinata waterilt water

Solvent/water Balances

Extract - Solvent

- Water

in fermenter feed

Raffinate - Water

- Solvert

Solute Distritution

Phi K Extract Raffinate

0.30000 ibilb water in feed

0.00001 1b/lb water in feed

0.99999 1bilb water in feed

0.00002 ltill water in feed 
MULTISTAGE EXTRACTIUE FERMENTATION OF EUTANOL WITH RECOUERY OF RAFFINATE BASECASE STOIOHIOMETPY

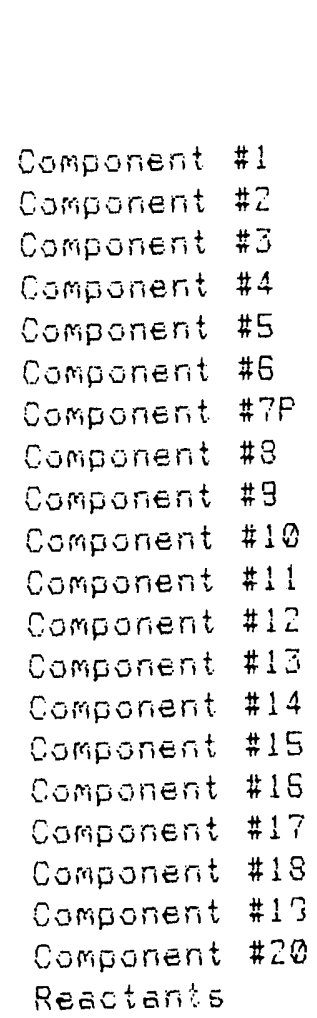

BASIC DATA

D.OEZ

a. 052

0.199

Q. 062

Q. 115

Q. 082

8. 972

๑. 116

๑. 052

Q. 115

Q. 1252

จ. 362

ด. 062

0. 862

จ. Q62

0. 082

D. 062

D. 062

D. $\triangle 5 E$

ถ. 062

0.062

0.00
0.00
0.50
0.00
0.20
0.00
4.30
0.20
0.00
0.20
0.00
0.00
0.00
0.00
0.00
0.00
0.00
0.00
0.00
0.00
0.00

1

\begin{tabular}{|c|c|c|c|c|}
\hline 6. 808 & 1.000 & 战优 & in & beer \\
\hline 8.000 & 1.000 & 地/佔 & in & beer \\
\hline 0.146 & 0.854 & lt: 15 & $1 \pi$ & beer \\
\hline 0.000 & 1.000 & lbilt & $1 n$ & beer \\
\hline 0.058 & 0.342 & lbit & $1 n$ & beer \\
\hline 0.000 & 1.000 & 战/1 & $i n$ & teer \\
\hline 0.9700 & 0.030 & 彭优 & $i n$ & beer \\
\hline 0.058 & 0.942 & 1tilt & in & eer \\
\hline 0.000 & 1.000 & $1 b i 1 b$ & $i n$ & eer \\
\hline 0.058 & 0.942 & $15 ; 1 b$ & in & eer \\
\hline 0.000 & 1.000 & 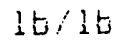 & in & Eer \\
\hline 0.000 & 1.000 & 1tito & in & eer \\
\hline 0.000 & 1.000 & 比：战 & $i \pi$ & LEer \\
\hline 0.000 & 1.000 & 比，化 & in & jeer \\
\hline 0.000 & 1.000 & lt: 15 & $i n$ & eEt \\
\hline 0.000 & 1.900 & 1t: lo & in & tueEr \\
\hline 0.8000 & 1.000 & 比优 & in & SEE \\
\hline 0.000 & 1.000 & 1b/1b & $i n$ & Leer \\
\hline 0.000 & 1.000 & $15: 1 t$ & $i n$ & bieer \\
\hline 0.000 & 1.000 & $15 / 15$ & $\ln$ & beer \\
\hline 0.000 & 1.082 & $1 t / 1 t$ & in & toeer \\
\hline
\end{tabular}

2

0.9951

0. 8457

5000 $10 E$

11.6

9

Co Areailextractor, sa ft

Number of Extractors

Eritractor Diameter, ft

Theoretical Stages

Extractor Height, ft

Exitractors Cost - \$1000 $3096 \mathrm{MPC}$

-All Carbori Steel

-Ali 304 Stainless Steel

23

- Bare Equipment

$\$ 1,053.3$

$\$ 1,430,6$ 


\begin{tabular}{|c|c|c|c|c|c|}
\hline tage to stage & elá & $\begin{array}{c}0515: 101 \\
0.03000\end{array}$ & 0.27395 & 0.47036 & 0.51942 \\
\hline Stage & Feed & 1 & 2 & 3 & 4 \\
\hline Extract & & & & & \\
\hline -Sulvent & & 0.30000 & 0.30000 & 0.30000 & 0.30000 \\
\hline -water & & 0.00001 & 0.00001 & 0.00001 & 0.00001 \\
\hline -Total & & 0.30000 & 0.30001 & 0.30001 & 0.30001 \\
\hline Róffinate & & & & & \\
\hline -Solvent & 0.00002 & 0.00002 & 0.00002 & 0.00002 & 0.00002 \\
\hline -water & 1.00000 & 1.00000 & 1.00000 & 1.00000 & 1.00000 \\
\hline -Total & 1.00002 & 1.00002 & 1.00002 & 1.00002 & 1.00002 \\
\hline oduet parti & 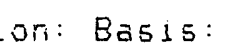 & 比化 & te inf $f$ & & \\
\hline Based on $f_{1 x}$ & ed yield = & $97.00 \%$ & & & \\
\hline Solvent/Feed & 只atio， $r=$ & 0.30 & & & \\
\hline 4.30 & istages= & 9 & & & \\
\hline Exitract & & 0.97000 & 0.72194 & 0.52964 & 2. 33058 \\
\hline Raffinate & 1.00000 & 0.75134 & 0.55964 & 0.41053 & 0.23502 \\
\hline
\end{tabular}




\begin{tabular}{|c|c|c|c|c|c|c|c|}
\hline $\begin{array}{r}0.7490 \\
5\end{array}$ & $\begin{array}{r}0.245 E \\
5\end{array}$ & $\begin{array}{r}x .93400 \\
?\end{array}$ & $\begin{array}{r}0.34785 \\
6\end{array}$ & $\begin{array}{r}9.9895 E \\
9\end{array}$ & $\begin{array}{r}102: 90 \\
10\end{array}$ & $\begin{array}{r}2.4598 \\
11\end{array}$ & $\begin{array}{r}1.05542 \\
12\end{array}$ \\
\hline & 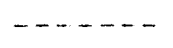 & $\ldots \ldots$ & . & - & & & \\
\hline$\therefore 30300$ & 0.30030 & 2.30303 & 0.50003 & 3.30006 & 0.30000 & D. 30030 & 0.50000 \\
\hline 3.20021 & 2.30001 & 0.02301 & 0.30301 & 0.03001 & 0.30001 & 0.00301 & 0.00001 \\
\hline 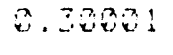 & Q & 0.53021 & 3. 30381 & 0.30001 & B. 20302 & 0.50001 & 0.50001 \\
\hline$B=$ & ‥ & ㄱ. 39020 & ‥2002 & 3.03022 & 3. 20032 & 0.83002 & . \\
\hline 30000 & 1. 33030 & 1.02020 & $\therefore .00003$ & 1.00000 & 1.20000 & 1.00000 & 1.36 \\
\hline $1.3 \times 23$ & $\therefore .0002=$ & $\therefore .30002$ & 1.39002 & $\therefore .00002$ & 1.08022 & 1.00302 & $\therefore$ \\
\hline
\end{tabular}

$\begin{array}{lllllll}0.25502 & 0.17544 & 0.10500 & 0.05217 & 0.01044 & -0.02130 & -2.04698 * * * * * * \\ 0.23544 & 0.13500 & 0.05217 & 0.04044 & 0.00510 & -0.01538 & -0.03542 * * * * * *\end{array}$




\begin{tabular}{|c|c|c|c|c|c|c|c|}
\hline $\begin{array}{r}.08149 \\
13 \\
\end{array}$ & $\begin{array}{r}.09317 \\
14\end{array}$ & $\begin{array}{r}1.10222 \\
15\end{array}$ & $\begin{array}{r}1.10924 \\
16\end{array}$ & $\begin{array}{r}1.11468 \\
17\end{array}$ & $\begin{array}{r}1.11890 \\
18\end{array}$ & $\begin{array}{r}1.12217 \\
19\end{array}$ & 11 \\
\hline $\begin{array}{l}0.30000 \\
0.00001 \\
0.30001\end{array}$ & $\begin{array}{l}0.30000 \\
0.00001 \\
0.30001\end{array}$ & $\begin{array}{l}0.30000 \\
0.00001 \\
0.30001\end{array}$ & $\begin{array}{l}0.30000 \\
0.00001 \\
0.30001\end{array}$ & & & & $\begin{array}{l}0.30000 \\
0.00001 \\
0.30001\end{array}$ \\
\hline $\begin{array}{l}0.00002 \\
1.00000 \\
1.00002\end{array}$ & $\begin{array}{l}0.00002 \\
1.00000 \\
1.00002\end{array}$ & $\begin{array}{l}2.00 \\
1.00 \\
1.00\end{array}$ & $\begin{array}{l}0.00002 \\
1.00000 \\
1.00002\end{array}$ & $\begin{array}{l}0.00002 \\
1.00000 \\
1.00002\end{array}$ & $\begin{array}{l}0.00002 \\
1.00000 \\
1.00002\end{array}$ & $\begin{array}{l}0.00002 \\
1.00000 \\
1.00002\end{array}$ & $\begin{array}{l}0.00002 \\
0.99999 \\
1.00001\end{array}$ \\
\hline
\end{tabular}

$-0.03149-0.03317-0.10222-0.10924$

$-0.11458-0.11830-0.12217-0.12471$

$-0.05317-0.07222-0.07924-0.08468$

$-0.08390-0.09217-0.09471-0.09657$ 
SUL垃

2. 30002

‥ .00000000

Q. 302020

$-0.12557$ 


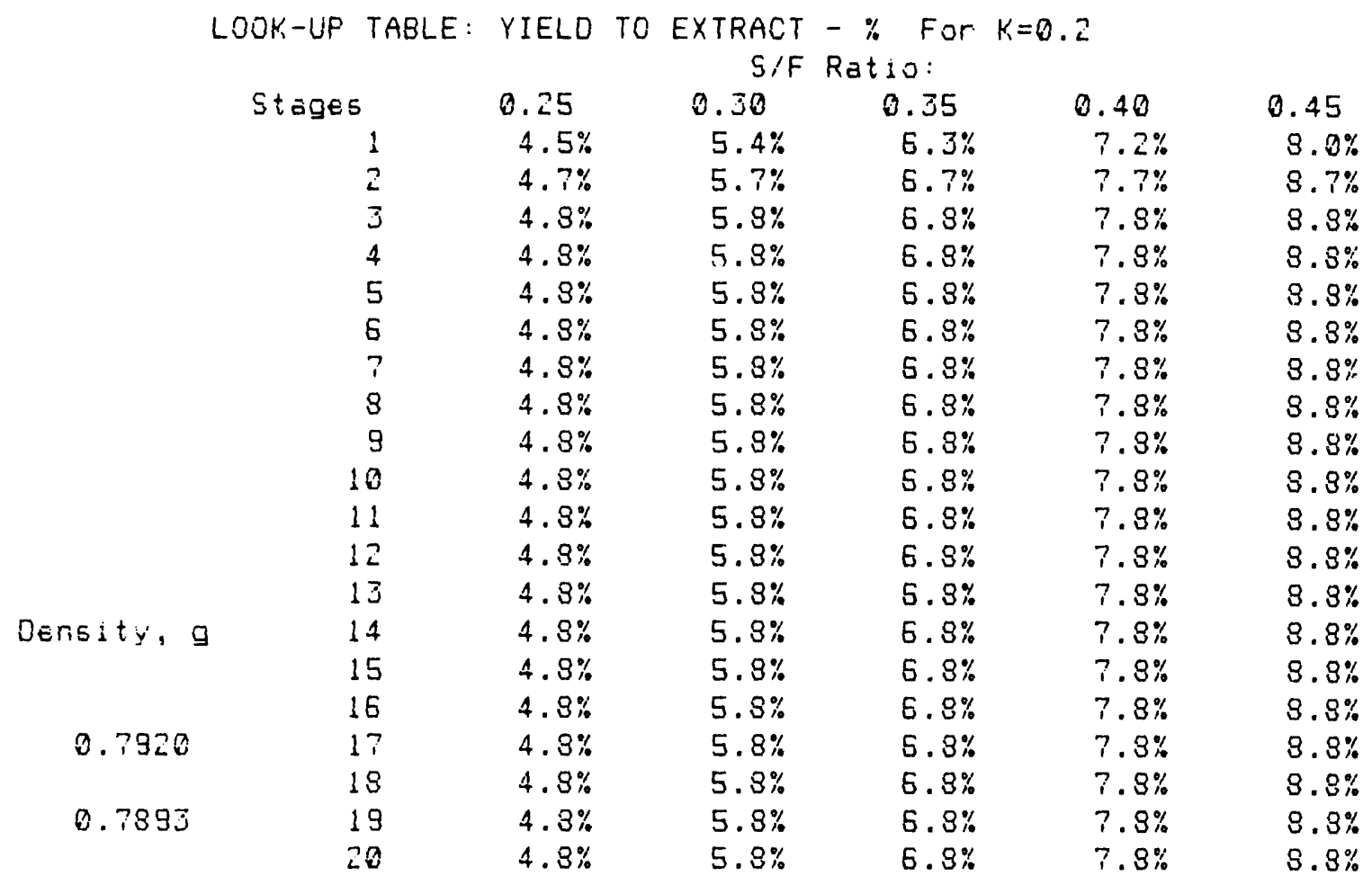




\begin{tabular}{|c|c|c|c|c|c|c|c|}
\hline D. 50 & 1.00 & 1.50 & 2.00 & 2.50 & 3.00 & 3.50 & 4.00 \\
\hline $8.9 \%$ & $15.0 \%$ & $22.0 \%$ & $2.8 .3 \%$ & $33.1 \%$ & $37.4 \%$ & $41.1 \%$ & $47.3 \%$ \\
\hline $9.7 \%$ & $19.1 \%$ & $27.0 \%$ & $55.8 \%$ & $A 2.7 \%$ & $48.8 \%$ & $54.2 \%$ & $58.9 \%$ \\
\hline $9.8 \%$ & $13.5 \%$ & $=7.0 \%$ & $38.3 \%$ & $45.5 \%$ & $53.9 \%$ & $50.4 \%$ & $55.1 \%$ \\
\hline $9.8 \%$ & $13.8 \%$ & $23.5 \%$ & $39.2 \%$ & $48.2 \%$ & $5 E .5 \%$ & $53.8 \%$ & TA. $1 \%$ \\
\hline $9.8 \%$ & $17.8 \%$ & $29.8 \%$ & $39.6 \%$ & $49.1 \%$ & $57.9 \%$ & $55.9 \%$ & 그.8\% \\
\hline $3.8 \%$ & $13.8 \%$ & $29.8 \%$ & $39.7 \%$ & $49.5 \%$ & $58.8 \%$ & $57.2 \%$ & $74.5 \%$ \\
\hline $9.3 \%$ & $19.8 \%$ & $29.3 \%$ & $39.8 \%$ & $49.7 \%$ & $59.2 \%$ & $58.1 \%$ & $75.0 \%$ \\
\hline $9.8 \%$ & $19.8 \%$ & $29.9 \%$ & $39.9 \%$ & $49.3 \%$ & $59.5 \%$ & $68.5 \%$ & 75. $8 \%$ \\
\hline $9.8 \%$ & $13.3 \%$ & $29.9 \%$ & $33.9 \%$ & $43.8 \%$ & $59.6 \%$ & $59.1 \%$ & $77.5 \%$ \\
\hline $3.8 \%$ & $19.8 \%$ & $29.3 \%$ & $39.9 \%$ & $43.8 \%$ & $59.7 \%$ & EB.J\% & $78.0 \%$ \\
\hline $3.3 \%$ & $19.8 \%$ & $29.9 \%$ & $39.9 \%$ & $49.8 \%$ & $59.8 \%$ & $63.5 \%$ & $78.4 \%$ \\
\hline $9.8 \%$ & $13.8 \%$ & $23.9 \%$ & $33.9 \%$ & $49.8 \%$ & $59.9 \%$ & $53.5 \%$ & $73.7 \%$ \\
\hline $3.8 \%$ & $19.8 \%$ & $29.9 \%$ & $33.9 \%$ & $49.3 \%$ & $59.9 \%$ & E9.7\% & $79.0 \%$ \\
\hline $9.3 \%$ & $19.8 \%$ & $29.9 \%$ & $33.9 \%$ & $43.8 \%$ & $59.9 \%$ & $59.3 \%$ & $73.2 \%$ \\
\hline $3.8 \%$ & $19.8 \%$ & $29.9 \%$ & $39.9 \%$ & $49.3 \%$ & $59.9 \%$ & E $3.8 \%$ & $79.3 \%$ \\
\hline $9.8 \%$ & $19.8 \%$ & $29.9 \%$ & $39.9 \%$ & $49.8 \%$ & $53.9 \%$ & $59.9 \%$ & $73.4 \%$ \\
\hline $9.8 \%$ & $19.8 \%$ & $23.9 \%$ & $39.9 \%$ & $49.8 \%$ & $59.9 \%$ & $59.9 \%$ & $79.5 \%$ \\
\hline $3.8 \%$ & $19.8 \%$ & $29.9 \%$ & $39.9 \%$ & $49.8 \%$ & $53.9 \%$ & $59.8 \%$ & $73.5 \%$ \\
\hline $9.3 \%$ & $19.8 \%$ & $23.9 \%$ & $39.9 \%$ & $49.8 \%$ & $59.9 \%$ & $69.9 \%$ & $79.7 \%$ \\
\hline $9.8 \%$ & $13.8 \%$ & $29.9 \%$ & $33.9 \%$ & $49.8 \%$ & $59.9 \%$ & $53.9 \%$ & Т. $3 \%$ \\
\hline
\end{tabular}




$\begin{array}{ll}4.50 & 5.00 \\ 47.3 \% & 49.9 \% \\ 53.1 \% & 55.5 \% \\ 70.8 \% & 74.9 \% \\ 75.5 \% & 79.9 \% \\ 78.5 \% & 83.3 \% \\ 80.7 \% & 85.6 \% \\ 82.4 \% & 87.4 \% \\ 83.5 \% & 88.9 \% \\ 84.5 \% & 89.9 \% \\ 85.3 \% & 90.8 \% \\ 85.0 \% & 91.5 \% \\ 86.5 \% & 92.2 \% \\ 87.0 \% & 92.8 \% \\ 87.3 \% & 93.3 \% \\ 87.7 \% & 93.7 \% \\ 87.9 \% & 34.1 \% \\ 88.2 \% & 34.4 \% \\ 88.4 \% & 94.7 \% \\ 83.5 \% & 94.9 \% \\ 88.5 \% & 95.1 \%\end{array}$




\begin{tabular}{|c|c|c|c|c|c|c|c|}
\hline & IE & $\begin{array}{l}\text { TO EVTKACT } \\
\text { SIF RATLO }\end{array}$ & & & & & \\
\hline Stăす̄ & a. 25 & 0.30 & 6.35 & 0.40 & 2.45 & 0.50 & 1.00 \\
\hline 1 & $10.0 \%$ & $12.5 \%$ & $14.6 \%$ & $15.3 \%$ & $18.0 \%$ & $19.8 \%$ & $\dot{Z} \dot{Z}$. \\
\hline 2 & $11.2 \%$ & $14.0 \%$ & $15.2 \%$ & $13.0 \%$ & $21.0 \%$ & $24.0 \%$ & $42.8 \%$ \\
\hline$\dot{3}$ & $12.0 \%$ & $14.5 \%$ & $27.6 \%$ & $19.3 \%$ & $22.0 \%$ & $25.0 \%$ & $43.3 \%$ \\
\hline 4 & $12.0 \%$ & $21.5 \%$ & $17.1 \%$ & $20.0 \%$ & $23.0 \%$ & $25.0 \%$ & $43.0 \%$ \\
\hline 5 & $12.0 \%$ & $14.5 \%$ & $17.1 \%$ & $20.0 \%$ & $23.02 \%$ & $25.0 \%$ & $48.5 \%$ \\
\hline 5 & $12.0 \%$ & $14.5 \%$ & $17.1 \%$ & $20.0 \%$ & $23.2 \%$ & $25.0 \%$ & $49.0 \%$ \\
\hline 7 & $12.8 \%$ & $14.6 \%$ & $17.1 \%$ & $20.0 \%$ & $23.8 \%$ & $25.6 \%$ & $49.5 \%$ \\
\hline 3 & $12.0 \%$ & $14.5 \%$ & $17.1 \%$ & $20.0 \%$ & $23.0 \%$ & $25.0 \%$ & $49.6 \%$ \\
\hline 3 & $12.0 \%$ & $14.5 \%$ & $17.1 \%$ & $20.0 \%$ & $23.0 \%$ & $25.0 \%$ & $49.7 \%$ \\
\hline 10 & $12.0 \%$ & $14.6 \%$ & $17.1 \%$ & $20.0 \%$ & $23.0 \%$ & $25.0 \%$ & $49.7 \%$ \\
\hline 11 & $12.0 \%$ & $14.6 \%$ & $17.1 \%$ & $20.8 \%$ & $23.0 \%$ & $25.2 \%$ & $49.7 \%$ \\
\hline 12 & $12.0 \%$ & $14.6 \%$ & $17.1 \%$ & $20.0 \%$ & $23.8 \%$ & $25.0 \%$ & $49.7 \%$ \\
\hline 13 & $12.0 \%$ & $14.5 \%$ & $17.1 \%$ & $20.0 \%$ & $25.0 \%$ & $25.0 \%$ & $49.7 \%$ \\
\hline 14 & $12.0 \%$ & $14.5 \%$ & $17.1 \%$ & $20.0 \%$ & $23.0 \%$ & $25.8 \%$ & $49.7 \%$ \\
\hline 15 & $12.0 \%$ & $14.5 \%$ & $17.1 \%$ & $20.0 \%$ & $23.0 \%$ & $25.0 \%$ & $49.7 \%$ \\
\hline 15 & $12.0 \%$ & $14.6 \%$ & $17.1 \%$ & $20.0 \%$ & $23.0 \%$ & $25.0 \%$ & $49.7 \%$ \\
\hline 17 & $12.0 \%$ & $14 . E \%$ & $17.1 \%$ & $20.8 \%$ & $23.0 \%$ & $25.0 \%$ & $49.7 \%$ \\
\hline 13 & $12.0 \%$ & $14.5 \%$ & $17.1 \%$ & $20.0 \%$ & $23.0 \%$ & $25.0 \%$ & $49.7 \%$ \\
\hline 19 & $12.0 \%$ & $14.5 \%$ & $17.1 \%$ & $20.0 \%$ & $23.0 \%$ & $25.8 \%$ & $49.7 \%$ \\
\hline 20 & $12.0 \%$ & $14.5 \%$ & $17.1 \%$ & $20.0 \%$ & $23.0 \%$ & $25.0 \%$ & $49.7 \%$ \\
\hline
\end{tabular}




\begin{tabular}{|c|c|c|c|c|c|c|c|}
\hline 1.50 & 2.00 & 2.50 & 3.00 & 3.50 & 4.00 & 4.50 & 5.80 \\
\hline $42.0 \%$ & $49.0 \%$ & $55.3 \%$ & $59.5 \%$ & $52.0 \%$ & $56.0 \%$ & $59.0 \%$ & $71.2 \%$ \\
\hline $56.0 \%$ & $55.0 \%$ & $73.0 \%$ & $73.0 \%$ & $32.0 \%$ & $85.0 \%$ & $87.0 \%$ & $83.8 \%$ \\
\hline $55.0 \%$ & $74.9 \%$ & $32.0 \%$ & $37.0 \%$ & $91.8 \%$ & $95.0 \%$ & $94.0 \%$ & $96.0 \%$ \\
\hline $57.0 \%$ & $73.0 \%$ & $87.5 \%$ & $92.3 \%$ & $35.1 \%$ & $97.0 \%$ & $37.7 \%$ & $98.4 \%$ \\
\hline E9. & $83.0 \%$ & $91.0 \%$ & $35.0 \%$ & $37.0 \%$ & $93.3 \%$ & $99.1 \%$ & $39.3 \%$ \\
\hline $71.0 \%$ & $35.0 \%$ & $93.2 \%$ & $35.0 \%$ & $98.4 \%$ & $39.2 \%$ & $99.5 \%$ & $99.7 \%$ \\
\hline $71.5 \%$ & $37.0 \%$ & $34.9 \%$ & $97.0 \%$ & $99.0 \%$ & $33.5 \%$ & $99.8 \%$ & $39.9 \%$ \\
\hline $72.5 \%$ & $38.0 \%$ & $95.0 \%$ & $98.0 \%$ & $99.5 \%$ & $39.8 \%$ & $39.9 \%$ & $100.0 \%$ \\
\hline $73.0 \%$ & $89.0 \%$ & 고. $5 \%$ & $99.1 \%$ & $99.7 \%$ & $39.9 \%$ & $100.0 \%$ & $100.0 \%$ \\
\hline $73.5 \%$ & $30.0 \%$ & $37.0 \%$ & $99.5 \%$ & $39.8 \%$ & $100.0 \%$ & $100.0 \%$ & 100.0 \\
\hline $74.0 \%$ & $91.0 \%$ & $33.0 \%$ & $99.5 \%$ & $99.9 \%$ & $100.0 \%$ & $100.0 \%$ & 100.0 \\
\hline $74.2 \%$ & $32.0 \%$ & $33.5 \%$ & $39.7 \%$ & 3马. $9 \%$ & $100.0 \%$ & $100.0 \%$ & 100.0 \\
\hline $74.3 \%$ & $92.5 \%$ & $38.7 \%$ & $99.8 \%$ & $100.0 \%$ & $100.0 \%$ & $100.0 \%$ & 100.0 \\
\hline $74.5 \%$ & $9 j .0 \%$ & $39.8 \%$ & $39.9 \%$ & $100.0 \%$ & $100.0 \%$ & $100.0 \%$ & 100.0 \\
\hline $74.6 \%$ & $33.5 \%$ & $99.2 \%$ & $93.9 \%$ & $100.0 \%$ & $100.0 \%$ & $100.0 \%$ & 100.0 \\
\hline $74.6 \%$ & $34.0 \%$ & $99.4 \%$ & $39.9 \%$ & $100.0 \%$ & $100.0 \%$ & $100.0 \%$ & 100.0 \\
\hline $74.7 \%$ & $34.2 \%$ & $99.5 \%$ & $99.9 \%$ & $100.0 \%$ & $100.0 \%$ & $100.0 \%$ & 100.0 \\
\hline $74.7 \%$ & $94.5 \%$ & 99.5\% & $99.9 \%$ & $100.0 \%$ & $100.0 \%$ & $100.0 \%$ & 100.0 \\
\hline $74.7 \%$ & $95.0 \%$ & $99.7 \%$ & $39.3 \%$ & $100.0 \%$ & $100.0 \%$ & $100.0 \%$ & 100.0 \\
\hline $74.8 \%$ & $35.0 \%$ & $39.8 \%$ & $99.9 \%$ & $100.0 \%$ & $100.0 \%$ & $100.0 \%$ & 100.0 \\
\hline
\end{tabular}




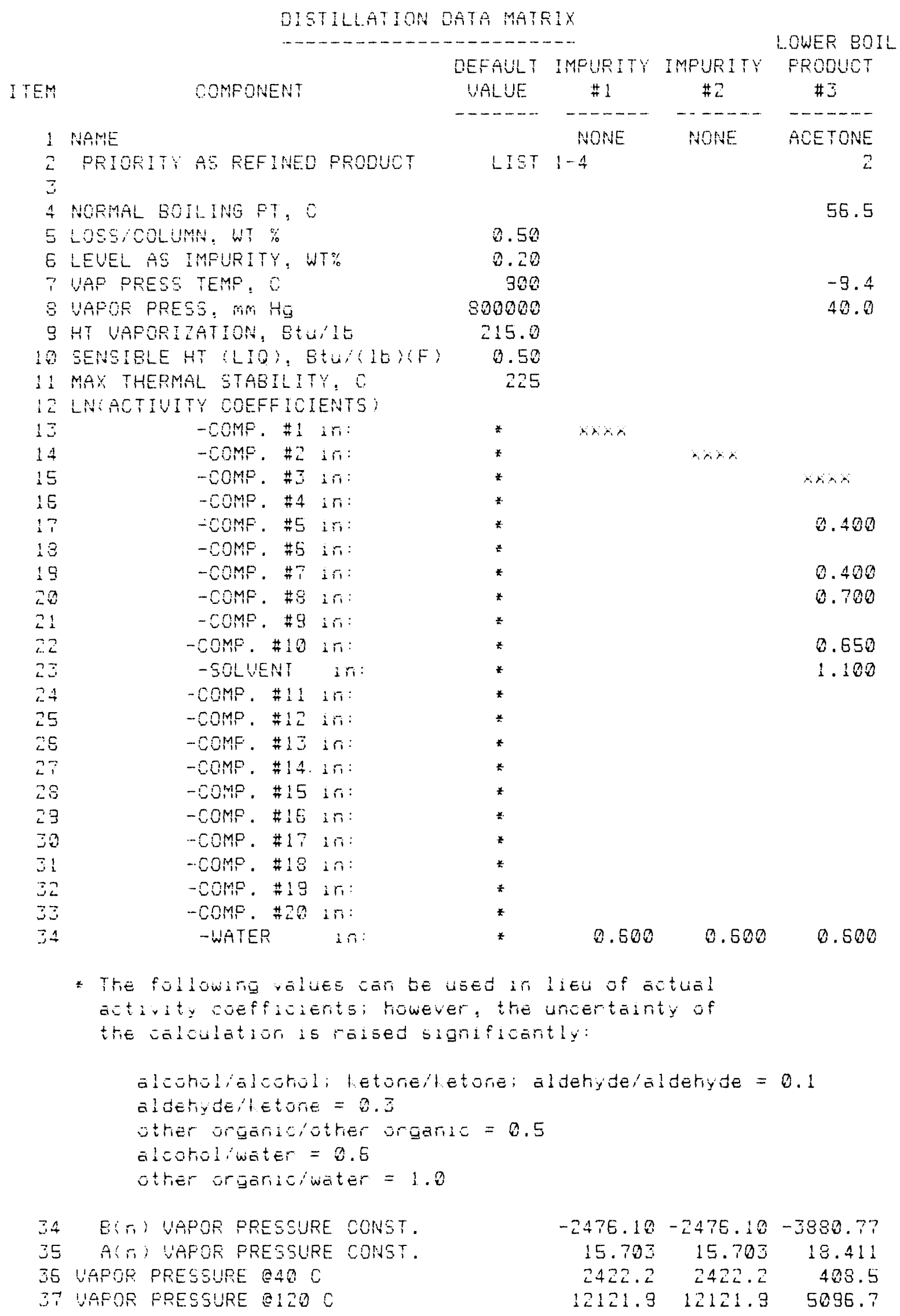




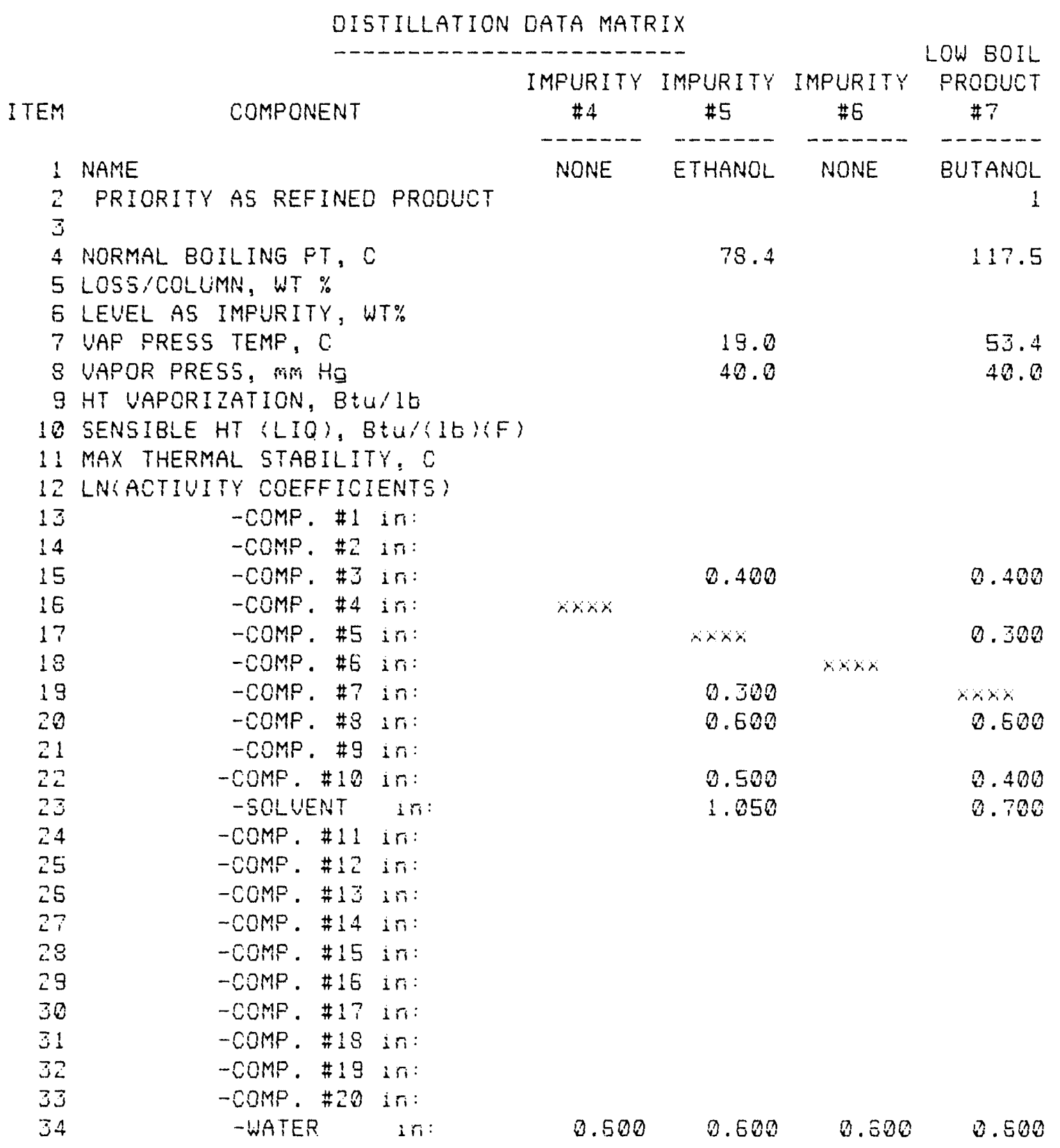

* Trie following values can be used ir lieu of actuál adtivity coefficients; however, the uncertalnty of the oaloulation is raised sigrificantly:

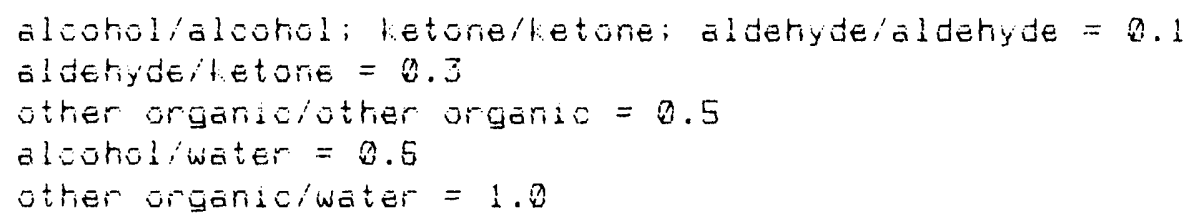




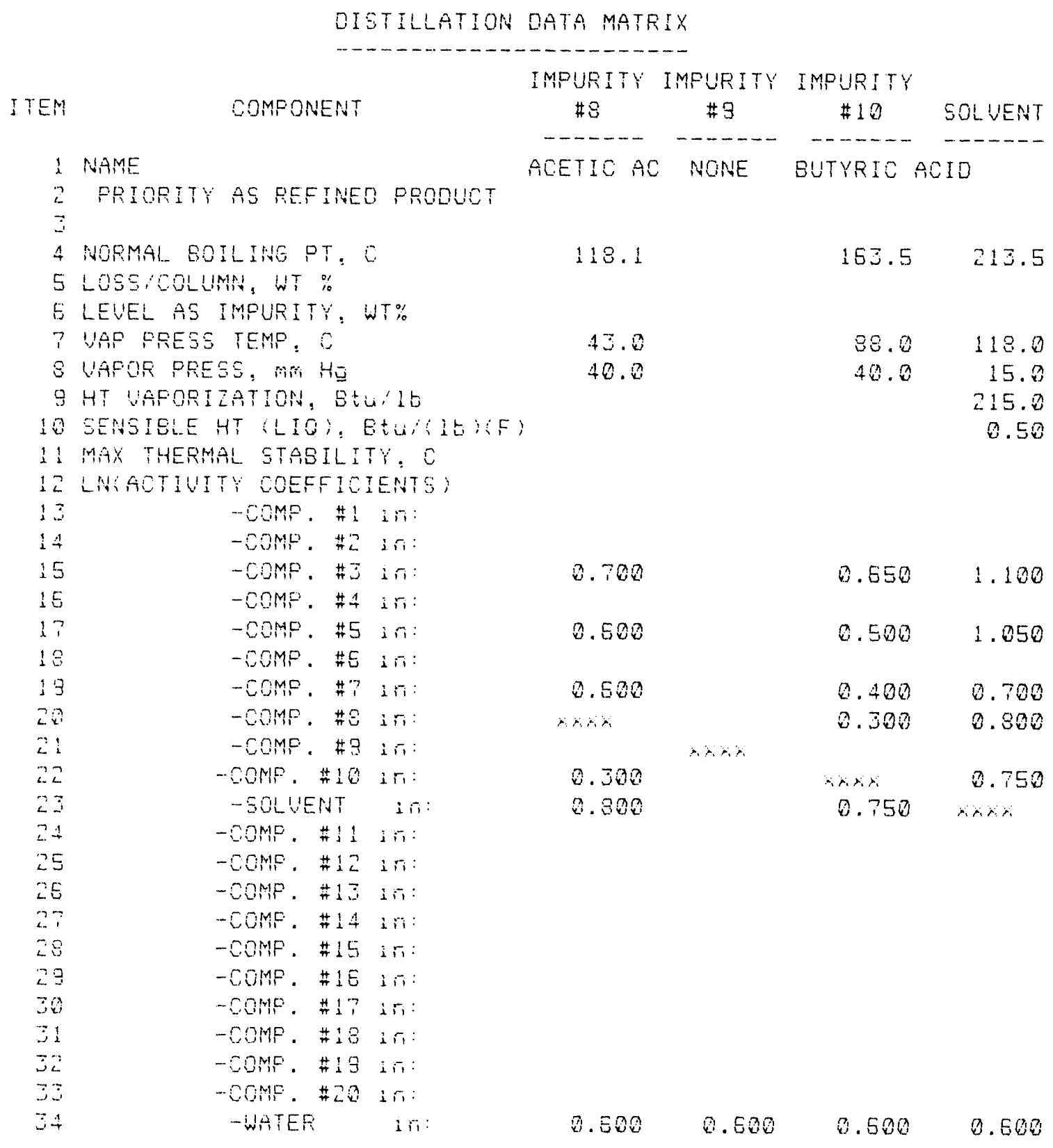

* The followirg values zari toe used ir lieu of actuel

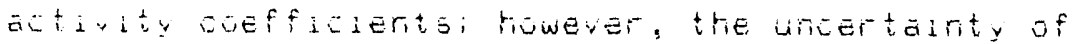

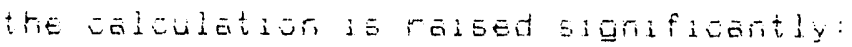

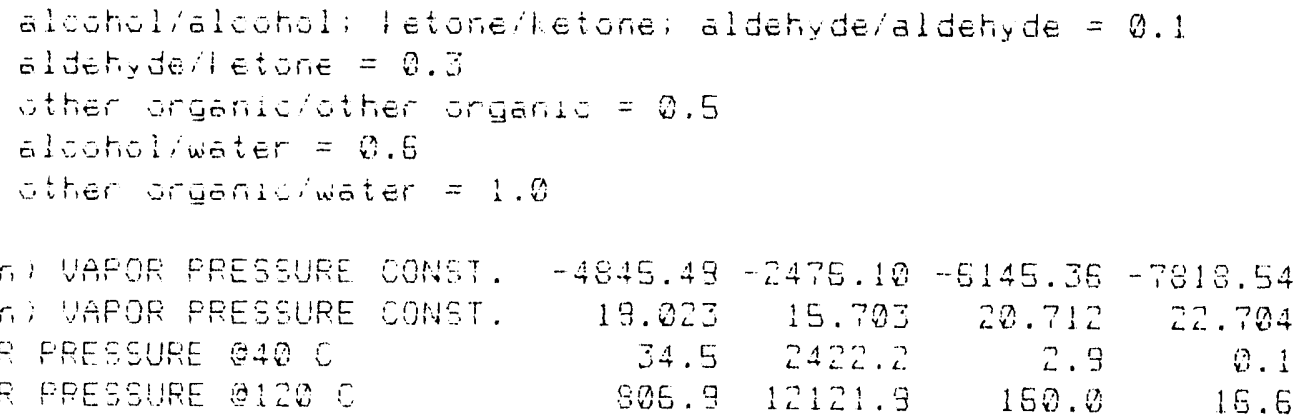


DISTILLATION DATA MATRIX

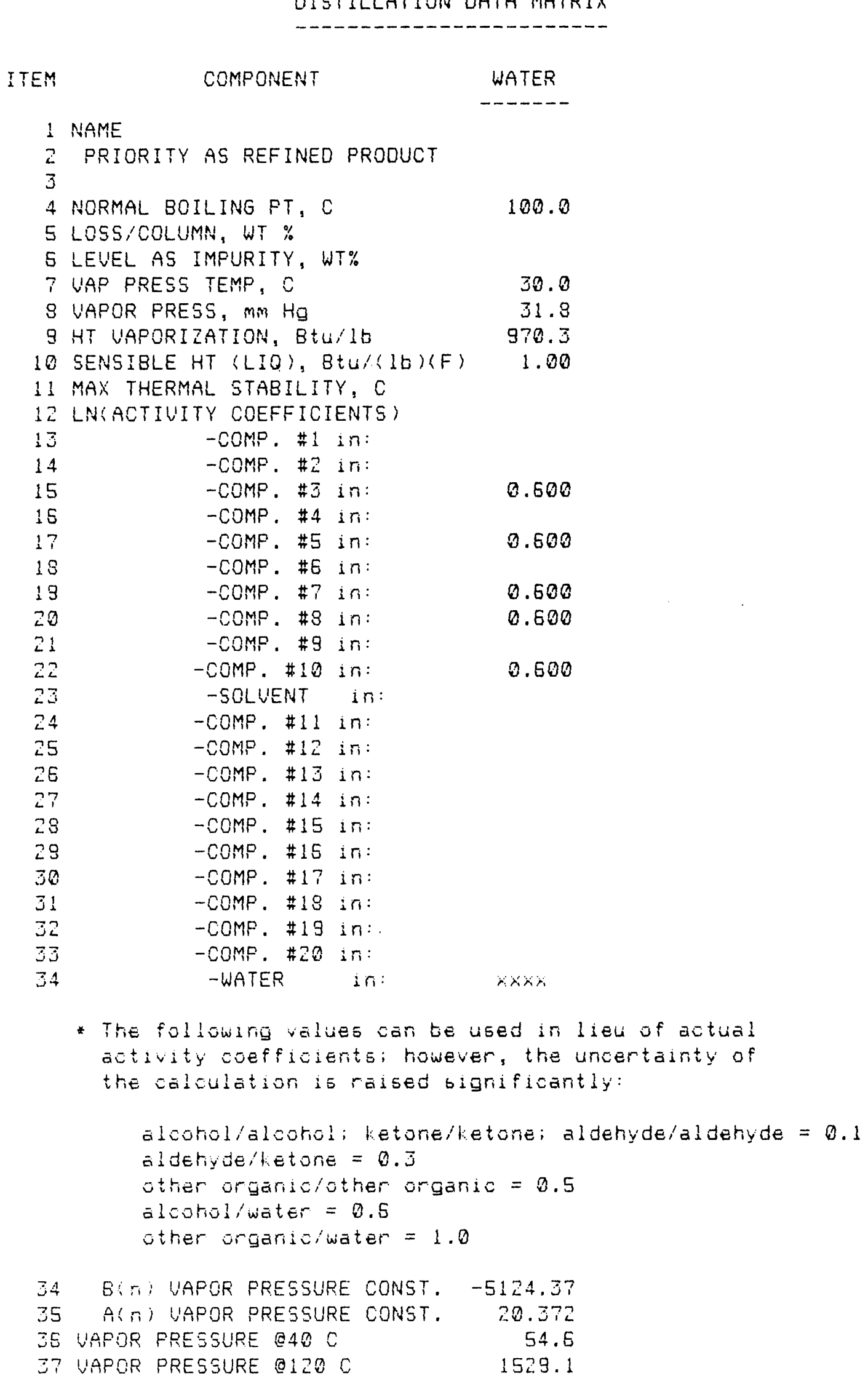




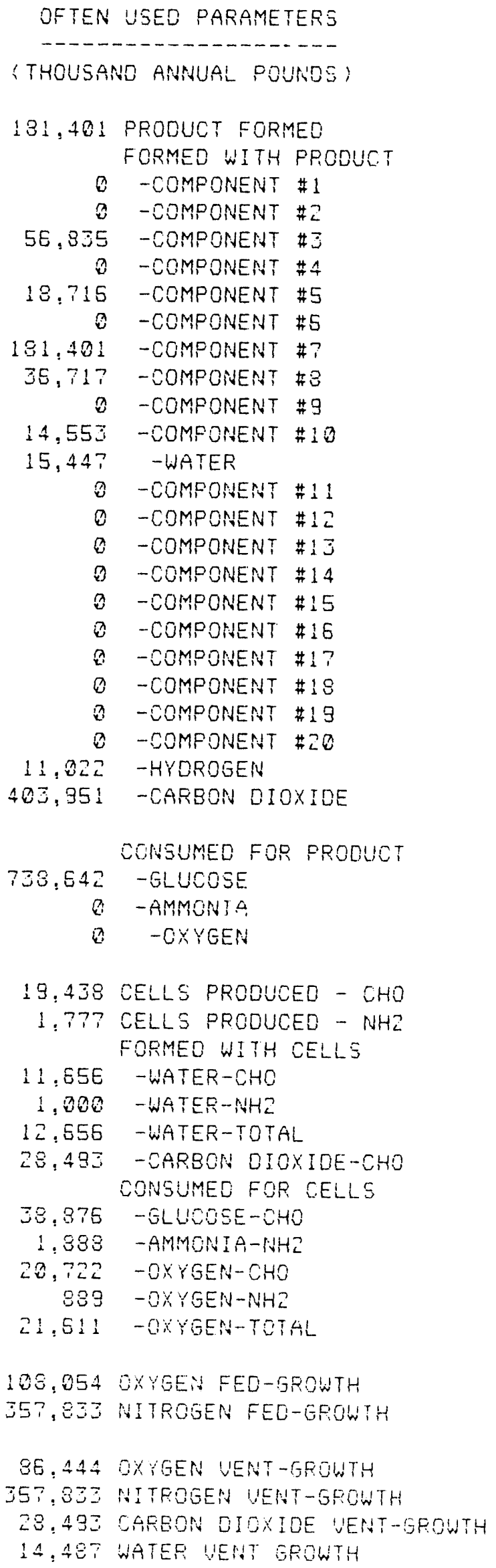




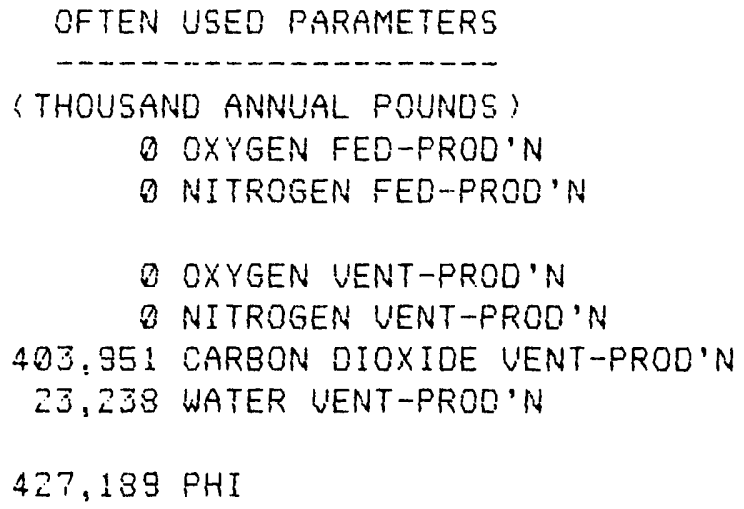


MULTISTAGE EXTRACTIUE FERMENTATION OF BUTANOL WITH RECOUERY OF RAFFINATE

BASECASE STOICHIOMETRY

\begin{tabular}{lr} 
WATER BALANCE \\
WATER IN: \\
\hline MAKE UP WATER & \\
BIOSUGAR SYRUP & 120,779 \\
STERILIZER STEAM & 778,913 \\
FORMED WITH CELLS & 50,804 \\
FORMED WITH PRODUCT & 12,855 \\
& 15,447 \\
TOTAL IN & $-\cdots,-13$ \\
\hline
\end{tabular}

WATER OUT:

AOUEOUS WASTE CONDENSATE MAKEUF TO P.H FERMENTER VENTS

PURGED WITH CELLS MOISTURE IN PRODUCTS

841,353

50,304

37,725

48,595

121

TOTAL OUT

988,500 
MULTISTAGE EXTRACTIUE FERMENTATION OF EUTANOL WITH RECOUERY OF RAFFINATE

BASECASE STOICHIOMETRY

MATERIAL BALANCE FLOWEULET

THOUGAND ANNUAL POUNDS (33O DAYS) O CAFACITY

\begin{tabular}{|c|c|c|c|c|c|c|}
\hline & STREAM & $\begin{array}{c}1 \\
\text { CORN } \\
\text { SYRUP }\end{array}$ & $\begin{array}{c}2 \\
\text { ANHYO } \\
\text { AMMONIS }\end{array}$ & $\begin{array}{c}3 \\
\text { NUTRIENTS }\end{array}$ & $\begin{array}{c}4 \\
\text { MIX } \\
\text { MATER }\end{array}$ & $\begin{array}{c}5 \\
\text { MIXED } \\
\text { MEDIUM }\end{array}$ \\
\hline$p$ & $\ldots \ldots \ldots$ & $-\cdots--$ & $\ldots-\cdots$ & -- & - n- - - & $\ldots-\cdots$ \\
\hline$R$ & CELLS -CHO & 2 & 0 & 0 & 0 & $\theta$ \\
\hline 0 & $-N+142$ & 0 & $\theta$ & 0 & 0 & . \\
\hline 0 & -MINERALS & 0 & 0 & 8 & 0 & a) \\
\hline U & -TOTAL & 0 & 0 & 0 & 0 & 0 \\
\hline $\mathrm{C}$ & COMPONENT \#1 & 0 & 0 & 0 & 0 & 0 \\
\hline$T$ & COMPONENT \#Z & 0 & $D$ & a & 0 & $a$ \\
\hline$\# 2$. & COMPONENT \#Z & 0 & 0 & 0 & 0 & 0 \\
\hline & COMPONENT \#4 & 0 & 6 & 8 & 0 & 2 \\
\hline & COMPONENT \#5 & 0 & 0 & 3 & 0 & 0 \\
\hline & COMPONENT \#E & 0 & - & 0 & 3 & $\theta$ \\
\hline \#1 & COMPONENT \#? & 0 & 0 & 0 & 8 & 0 \\
\hline & COMPONENT \#B & 0 & 0 & 0 & 0 & 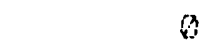 \\
\hline & COMPONENT \#G & 0 & 0 & 0 & 0 & 0 \\
\hline & COMFONENT \#10 & 3 & n & 0 & 0 & 0 \\
\hline & COMFONENT \#11 & 0 & 0 & 0 & 0 & 0 \\
\hline & COMPONENT \#12 & 0 & 0 & 0 & 0 & 0 \\
\hline & COMPONENT \#13 & 0 & 0 & 0 & 8 & 0 \\
\hline$\#$ & COMPONENT \#14 & 0 & 0 & 3 & 8 & Q \\
\hline & COMEONENT \#15 & 0 & 0 & 0 & 0 & 0 \\
\hline & COMPONENT \#15 & 0 & 0 & 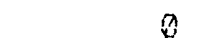 & 0 & 0 \\
\hline & COMPONENT \#17 & 0 & 0 & $D$ & 0 & 0 \\
\hline$\#$ & COMPONENT \#18 & $n$ & 0 & $\theta$ & $\theta$ & 8 \\
\hline & COMPONENT \#13 & 0 & 8 & 8 & 3 & 2 \\
\hline & COMPONENT \#20 & 0 & 0 & $\theta$ & 0 & $\theta$ \\
\hline & GLUCOSE & 778.913 & $\theta$ & 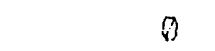 & 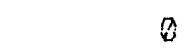 & 778.913 \\
\hline & AMMONIA & 0 & 1,838 & 8 & 0 & 0 \\
\hline & FHOSPHORIC ACID & 0 & $\theta$ & 1,573 & 3 & 1.573 \\
\hline & POTASSIUM CHLORIDE & 0 & 0 & 1,197 & 0 & 1,197 \\
\hline & MINOR NUTRIENTS & 0 & 0 & 543 & 0 & 543 \\
\hline & WATER & 778,913 & 0 & 0 & 59,975 & 833,883 \\
\hline & SOLVENT & 0 & 0 & 0 & 0 & 0 \\
\hline & CAREON DIOXIDE & 0 & D & $\theta$ & 0 & 0 \\
\hline & OXYGEN & 0 & 0 & 0 & 0 & 0 \\
\hline & NITROGEN & 0 & 0 & 0 & 0 & $\mathrm{a}$ \\
\hline & HYOROGEN & 0 & 0 & $\theta$ & 0 & 0 \\
\hline & $\cdots \cdots$ & $\ldots \ldots$ & $\cdots-\cdots-$ & $-\cdots-\cdots$ & $-\cdots-\cdots$ & $\cdots \cdots-\cdots$ \\
\hline & $\begin{array}{l}\text { GRANO TOTAL } \\
\text { CHECY ON TOTAL }\end{array}$ & 1.557 .325 & 1,388 & 5,313 & 59,975 & $1: 521: 114$ \\
\hline & TEMFERATURE： $C$ & 20 & 20 & 20 & 20 & 20 \\
\hline & PRESSURE, PSIA & 14.7 & 14.7 & 14.7 & 20.8 & 25.6 \\
\hline & STATE & SOL'N & LIOUID & SOLIOS & LIQUID & $50 \mathrm{~L} N$ \\
\hline
\end{tabular}


MULISTRGF EXTRAOTIUE FEFMENTATION OE EUTANOL WITH FECOUERY OF RAFFINATE

BAEECASE STOICHIOMETEY

MATERIAL EALANCE FLOWSHEET

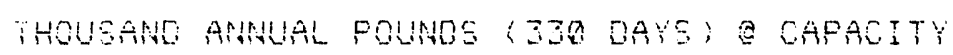


MULTISTAGE EXTRACTIUE FERMENTATION OF BUTANOL WITH RECOUERY OF RAFFINATE

BASECASE STOICHIOMETPY

MATERIAL BALANCE FLOWSHEET

THOUSAND ANNUAL POUNOS (3ZO GAYS) E CAFACITY

\begin{tabular}{|c|c|c|c|c|c|c|}
\hline & & $\begin{array}{c}11 \\
\text { COMEINEO } \\
\text { FEED }\end{array}$ & $\begin{array}{c}12 \\
\text { BEER } \\
\# 1\end{array}$ & $\begin{array}{c}13 \\
\text { BEER } \\
\# ?\end{array}$ & $\begin{array}{c}14 \\
\text { UENT } \\
\text { GROUTH }\end{array}$ & $\begin{array}{c}15 \\
\text { UENT } \\
\text { PROD'N }\end{array}$ \\
\hline$P$ & - & $\ldots \ldots-$ & ---1 & 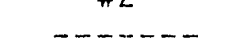 & $-0-1$ & - \\
\hline$R$ & CELLS - CHO & 0 & 13,433 & 313,588 & 0 & 0 \\
\hline 0 & $-\mathrm{NHZ}$ & 0 & 1,777 & 28,571 & 0 & 0 \\
\hline D & -MINERALS & 0 & 543 & 3,763 & 0 & 0 \\
\hline U & -TOTAL & 0 & 21,758 & 351,021 & 0 & 0 \\
\hline$\Sigma$ & COMPONENT \#1 & 0 & 0 & $\theta$ & 8 & 8 \\
\hline$T$ & COMPONENT \#Z & $\theta$ & . & 0 & 0 & $\theta$ \\
\hline$\# 2$ & COMFONENT \#3 & 0 & 0 & 285.998 & 0 & 0 \\
\hline & COMPONENT \#4 & 0 & a & 0 & 0 & 0 \\
\hline & COMPONENT \#5 & 3 & 0 & 150,561 & 2 & 0 \\
\hline & COMPONENT \#S & 0 & 0 & 0 & 0 & 0 \\
\hline$\# 1$ & COMPONENT \#? & 0 & 0 & 135,554 & $\theta$ & 0 \\
\hline & COMPONENT \#B & D & 0 & 315,184 & 0 & 0 \\
\hline & COMPCNENT \#马 & 0 & 0 & 0 & 0 & 0 \\
\hline & COMPONENT \#10 & 0 & 0 & 124,924 & 0 & 0 \\
\hline & COMPONENT \#11 & 0 & 0 & 0 & 8 & 0 \\
\hline & COMPONENT \#12 & $\theta$ & 0 & 0 & $\theta$ & 0 \\
\hline & COMPONENT \#13 & 0 & 0 & 8 & 0 & 0 \\
\hline$\#$ & COMPONENT \#14 & 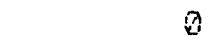 & 0 & 0 & 0 & 8 \\
\hline & COMPONENT \#15 & $\theta$ & 0 & 0 & 8 & 0 \\
\hline & COMPONENT \#15 & 0 & $\theta$ & i & 0 & 0 \\
\hline & COMPONENT \#IT & 8 & 0 & Q & 0 & 0 \\
\hline$\#$ & COMPONENT \#18 & 0 & 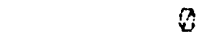 & 0 & 0 & 0 \\
\hline & COMPONENT \#13 & 0 & 0 & 0 & 0 & 0 \\
\hline & COMPONENT \#ZW & 0 & 0 & 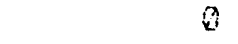 & 0 & 0 \\
\hline & GLUCOSE & 778,313 & 740,037 & 22.512 & 0 & 0 \\
\hline & AMMONIA & 1,388 & 0 & 0 & 8 & 0 \\
\hline & FHOSPHORIC ACID & 1,573 & 1,573 & 25,363 & $\theta$ & 0 \\
\hline & POTASSILM CHLORIDE & 1,197 & 1,137 & 19,317 & 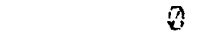 & $n$ \\
\hline & MINOR AUTRIENTS & 543 & 0 & 0 & 0 & $\theta$ \\
\hline & WATER & 899,592 & 837,851 & $14,357,985$ & 14,487 & 23,238 \\
\hline & SOLVENT & 0 & 0 & 2ES & 0 & 8 \\
\hline & CARBON DIOYIDE & 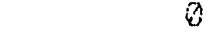 & 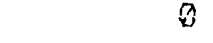 & 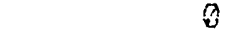 & 28,493 & 403,951 \\
\hline & OXYGEN & 108,054 & 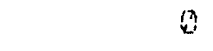 & 0 & 86.744 & 0 \\
\hline & MITPOEEN & 357,833 & 0 & $\theta$ & 357.835 & 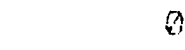 \\
\hline & HYOROGEN & 8 & 0 & 0 & 8 & 11.022 \\
\hline & $\cdots-\cdots$ & $\cdots-\cdots$ & $-\cdots-\cdots$ & $\cdots \cdots-\cdots$ & $\cdots \cdots$ & $\cdots \cdots-$ \\
\hline & $\begin{array}{l}\text { GRAND TOTAL } \\
\text { CHECK ON TOTAL }\end{array}$ & $2,149,094$ & $1,5 E 2,42 E$ & $15,849,935$ & 487.257 & 438,212 \\
\hline & TEMFERATURE : C & 33 & 33 & 33 & $3 j$ & 33 \\
\hline & PRESSURE， PSIA & - & 44.7 & 44.7 & 14.7 & 14.7 \\
\hline & STATE & - & SLURRY & GLURRY & GAS & 6.95 \\
\hline
\end{tabular}




\section{MULTISTAGE EXTRACTIUE FERMENTATION OF BUTANOL WITH RECOUERY OF RAFFINATE BASECASE STOICHIOMETRY}

MATERIAL BALANLE FLOWGHEET

THOUSAND ANHUAL POUNDS 330 DAYS: O CAPACITY

\begin{tabular}{|c|c|c|c|c|c|c|}
\hline & STREAM & $\begin{array}{l}\text { COMBINED } \\
\text { VENT }\end{array}$ & $\begin{array}{l}\text { SOLULNT } \\
\text { FEED }\end{array}$ & RAFFINATE & RECYCLE & $\begin{array}{l}\text { AQUEOUS } \\
\text { PURGE }\end{array}$ \\
\hline$P$ & $\cdots$ & $\cdots \cdots-\cdots$ & $\cdots \cdots-\cdots$ & $\cdots-\cdots--\cdots$ & $\cdots \cdots-\cdots$ & $\ldots \ldots$ \\
\hline 只 & CELLS - CHO & 0 & 0 & 313,588 & 294,150 & 19,438 \\
\hline 0 & $-N . H Z$ & 8 & 0 & 23,571 & 25,894 & 1.777 \\
\hline a & -MINERALS & 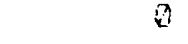 & $\theta$ & 8,763 & 8,220 & 543 \\
\hline 4 & -TOTAL & 0 & 8 & 351,021 & 329,253 & 21,758 \\
\hline c & COMFONENT \#1 & 0 & 0 & 0 & 0 & 0 \\
\hline$T$ & COMPONENT \#2 & 8 & a & 0 & 0 & D \\
\hline$\# 2$ & COMPONENT \#Z & 8 & 0 & 244,413 & 229,263 & 15,150 \\
\hline & COMEONENT \#4 & 0 & 0 & 0 & 0 & 0 \\
\hline & COMPONENT \#5 & 8 & 0 & 151,325 & 141,945 & 3,380 \\
\hline & COMPONENAT \#E & $\theta$ & 0 & 0 & 0 & 8 \\
\hline$\# 1$ & COMPONENT \#7 & 0 & 0 & 5,600 & 5,253 & 347 \\
\hline & COMPONENT \#Z & 0 & 0 & 295,863 & 278.457 & 13,402 \\
\hline & COMPONENT \#Q & 0 & $\theta$ & 8 & 0 & 0 \\
\hline & COMPONENT \#10 & 8 & D. & 117,654 & 110,371 & 7.293 \\
\hline & COMPONENT \#12 & 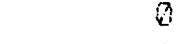 & 8 & 6 & 0 & 0 \\
\hline & COMPOHENT \#12 & 0 & 0 & 0 & 0 & 0 \\
\hline & COMPONENT \#1Z & 0 & 0 & 0 & 0 & 0 \\
\hline \# & COMPONENT \#14 & $\theta$ & 0 & 0 & 0 & $\theta$ \\
\hline & COMPONENT \#15 & 8 & 0 & 8 & D & 0 \\
\hline & COMPOHENT \#IL & a & 0 & $\theta$ & 0 & B \\
\hline & COMPONENT \#17 & 0 & 0 & (2) & 0 & 0 \\
\hline$\#$ & COMEONENT \#19 & 3 & 8 & 0 & D & 0 \\
\hline & COMPONENT \#19 & 8 & 8 & 8 & 0 & 0 \\
\hline & COMEONENT \#2O & 0 & 8 & 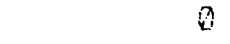 & 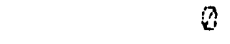 & Q) \\
\hline & GLUOOSE & 0 & 0 & 22,512 & 21,115 & $1, \Xi 95$ \\
\hline & AMMONIA & 0 & 0 & 0 & iv & 0 \\
\hline & FHOSFHORIO AOIO & 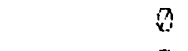 & 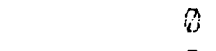 & 25.363 & 23,737 & 1,573 \\
\hline & FOTASEIUM CHLORIDE & 0 & 0 & 19.317 & 18.120 & 1.197 \\
\hline & MINOR NUTRIENTS & 0 & $\theta$ & 0 & 0 & 0 \\
\hline & WATER & 37.725 & 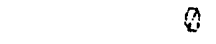 & $14,357,300$ & 13.467 .915 & 889,364 \\
\hline & GOLUENT & a & $4,307,395$ & 287 & 269 & 18 \\
\hline & CABEOH DIOXIDE & $4.22: 444$ & 0 & $\tilde{v}$ & $\theta$ & a \\
\hline & OXYGEN & $85: 44.4$ & Q & $\theta$ & 8 & $\theta$ \\
\hline & NITROGEN & 357.933 & 0 & 8 & D & 0 \\
\hline & HYOROGEN & 11.022 & 0 & 0 & 0 & 0 \\
\hline & $\begin{array}{l}\text { GRANO TOTAL } \\
\text { CHEO ON TOTAL }\end{array}$ & 325.469 & $4,307,395$ & $15,592,277$ & 14.625 .780 & 965,497 \\
\hline & TEMPEFATISE, C & 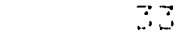 & 20 & & & 33 \\
\hline & FRESSURE, PSIA & $1+.7$ & 15 & & & 15 \\
\hline & STATE & GAE & LIOUIO & SLURRY & SLURRY & SLUERY \\
\hline
\end{tabular}


MULTISTAGE EXTRACTIUE FERMENTATION OF BUTANOL WITH RECOUERY OF RAFFINATE

BASECASE STOICHIOMETRY

MATERIAL BALANCE FLOWGHEET

THOUSAND ANNUAL POUNOS ( 330 DAYS) O CAFACITY

\begin{tabular}{|c|c|c|c|c|c|c|}
\hline P & CELLS - CHO & 19,438 & 0 & 0 & 0 & 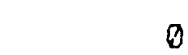 \\
\hline 0 & $-N H Z$ & 1,777 & 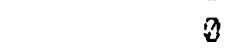 & 2 & 0 & \\
\hline $\mathrm{D}$ & -MINERALS & 543 & 0 & 0 & 0 & \\
\hline u & -TOTAL & 21,758 & 0 & 0 & 0 & \\
\hline $\mathrm{C}$ & COMFONENT \#1 & 0 & 0 & 0 & 0 & \\
\hline$T$ & COMPONENT \#Z & 0 & 0 & 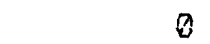 & 0 & \\
\hline$\# 2$ & COMPONENT $\# 3$ & 327 & 14,323 & 41,684 & 41,684 & 41,684 \\
\hline & COMPONENT \#A & 0 & 0 & 0 & 0 & 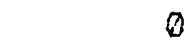 \\
\hline & COMFONENT \#5 & 512 & 8,863 & 9,335 & $9,3,36$ & 9,336 \\
\hline & COMPONENT \#E & 0 & $n$ & 0 & (i) & 0 \\
\hline$\# 1$ & COMPONENT $\# 7$ & 19 & 323 & 181,054 & 139,069 & 198,164 \\
\hline & COMPONENT \#8 & 1,005 & 17,397 & 18,315 & 13,351 & 395 \\
\hline & COMPONENT \#G & 0 & 0 & 0 & 0 & \\
\hline & COMFONENT \#1Q & 398 & 5,395 & 7,259 & 7,295 & 396 \\
\hline & COMPONENT \#11 & 0 & 0 & 0 & 0 & \\
\hline & COMPONENT \#12 & 0 & 0 & 0 & 0 & \\
\hline & COMPONENT \#13 & 0 & 0 & 0 & 0 & \\
\hline$\#$ & COMPONENT \#14 & 0 & 0 & 0 & 0 & \\
\hline & COMPONENT \#15 & 0 & 0 & 0 & 0 & \\
\hline & COMPONENT \#16 & $D$ & 0 & 0 & 0 & \\
\hline & COMPONENT \#17 & 0 & 0 & 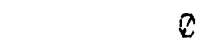 & 0 & \\
\hline$\#$ & COMPONENT \#18 & $\theta$ & 0 & 0 & 0 & \\
\hline & COMPONENT \#19 & 8 & 0 & 0 & 0 & \\
\hline & COMPONENT \#2R & 0 & 8 & 0 & $\theta$ & \\
\hline & GLUCOSE & 76 & 1,319 & $\theta$ & 0 & \\
\hline & AMMONIA & 0 & $c$ & 0 & 0 & \\
\hline & PHOSFHORIC ACID & 86 & 1,487 & 0 & 0 & \\
\hline & POTASSIUM CHLOEIOF & 65 & 1,132 & 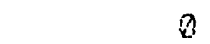 & 0 & \\
\hline & MINOR NUTRIENTS & 0 & 0 & 0 & Q & \\
\hline & WATER & 48,595 & 841,389 & 85 & 85 & 85 \\
\hline & SOLVENT & 1 & 17 & $4,307,378$ & $4,505,132$ & 193,154 \\
\hline & CARBON DIOXIDE & 0 & 0 & 0 & 0 & \\
\hline & OXYGEN & 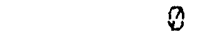 & 舟 & 0 & 0 & \\
\hline & NITROGEN & 0 & 0 & 0 & 0 & \\
\hline & HYOROGEN & 0 & 0 & 0 & 0 & \\
\hline & $\cdots$ & $-\cdots-\cdots$ & $-\cdots-\cdots$ & $\cdots \cdots$ & $\cdots \cdots$ & ---- \\
\hline & $\begin{array}{l}\text { GRAND TOTAL } \\
\text { CHECK ON TOTAL }\end{array}$ & 73,343 & 893,154 & $4,555,113$ & $4,781,004$ & 448,22 \\
\hline & TEMFERATURE， C & 33 & 33 & 33 & -- & 9 \\
\hline & FRESSURE, PSIA & 14.7 & 14.7 & 14.7 & -- & 11.5 \\
\hline & STATE & SLURRY & SOL'N & SOL'N & SOL'N & SOL \\
\hline
\end{tabular}


MULTISTAGE EXTRACTIUE FERMENTATION OF EUTANOL

WITH RECOULEY OF RAFFINATE

SASECASE STOICHIOMETRY

MATERIAL BALANCE FLOWSHEET

THOUSAND ANNUAL POUNDS (JID DAYS) E CAPACITY

\begin{tabular}{|c|c|c|c|c|c|c|}
\hline & STREAM & $\begin{array}{l}25 \\
\text { SEER \#1 } \\
\text { TAILS }\end{array}$ & $\begin{array}{c}27 \\
\text { BELE \#2 } \\
\text { MAKE }\end{array}$ & 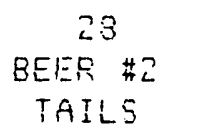 & $\begin{array}{c}29 \\
\text { LES \#1 } \\
\text { MAKE }\end{array}$ & $\begin{array}{c}30 \\
\text { LBS \#1 } \\
\text { TAILS }\end{array}$ \\
\hline$P$ & $\ldots$ & $--\cdots--$ & $\ldots \ldots$ & $\ldots \ldots$ & $\ldots \ldots$ & $\ldots-\ldots$ \\
\hline R & CELLS - CHO & 0 & $\theta$ & 8 & $\theta$ & 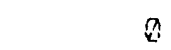 \\
\hline 0 & $-N 42$ & i & 0 & 0 & 2. & 0 \\
\hline D & -MINERALS & $\theta$ & 0 & Q & 6 & 0 \\
\hline U: & -TOTAL & 0 & 9 & 0 & $\theta$ & 9 \\
\hline$c$ & COMFONENT \#1 & 0 & 0 & $\theta$ & 0 & a \\
\hline$T$ & COMPONENT \#Z & 3 & (2) & 0 & $\theta$ & $\theta$ \\
\hline$\# 2$ & COMPONENT \#Z & 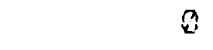 & 41,584 & $\theta$ & 41,325 & 350 \\
\hline & COMPOHENT \#4 & 0 & 8 & 0 & 0 & 0 \\
\hline & COMFONENT $\# 5$ & 0 & $3,3 J E$ & 0 & 8,978 & 358 \\
\hline & COMPONENT \#E & 0 & 0 & 8 & 3 & 3 \\
\hline$\# !$ & COMFONENT \#? & 905 & 130.149 & 18,315 & 901 & 179,248 \\
\hline & COMPONENT \#S & $17: 355$ & 560 & 35 & 8 & 350 \\
\hline & COMFONENT \#马 & 0 & 0 & 0 & : & 8 \\
\hline & COMPONENT \#10 & $E, 898$ & 360 & 35 & (2) & 360 \\
\hline & COMFONENT \#11 & 2 & 8 & 0 & 8 & 0 \\
\hline & COMPONENT \#12 & 0 & 8 & $\theta$ & 0 & $\theta$ \\
\hline & COMPONENT IIE & $\pi$ & 0 & 0 & 0 & $n$ \\
\hline$\#$ & COMPONENT $\# 14$ & $\pi$ & 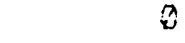 & 8 & ? & a) \\
\hline & COMFONENT \#15 & 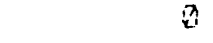 & 8 & 3 & () & 0 \\
\hline & COMPONENT \#15 & 8 & $\theta$ & 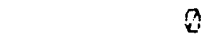 & $\theta$ & (6) \\
\hline & COMFONENT $\# 17$ & 0 & 0 & 8 & 0 & 0 \\
\hline$\#$ & COMPONENT \#13 & 2 & 6 & 8 & 0 & 0 \\
\hline & COMFOHENT $\# 13$ & 0 & 0 & 6 & 8 & a \\
\hline & COMPONENT \#ZO & 2 & $\theta$ & 0 & $\theta$ & 0 \\
\hline & GLUCOSE & 0 & 0 & b & 0 & 8 \\
\hline & AMMONIA & 0 & 0 & 0 & $a$ & 0 \\
\hline & PHOSFHORIC ACID & 3 & 0 & 3 & 8 & $\theta$ \\
\hline & FOTASEIUH CHLORIDE & 0 & 0 & Q & $\dot{u}$ & $\theta$ \\
\hline & MINOF NUTEIERTS & 2 & (3) & $\theta$ & 8 & 8 \\
\hline & WATER & 0 & SE & 8 & 0 & 85 \\
\hline & SOLUETT & 4.507 .019 & 360 & 197.80 .4 & 0 & $3 E 0$ \\
\hline & OARBON DIOXIDE & 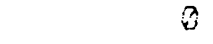 & 0 & 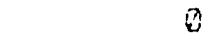 & 0 & 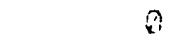 \\
\hline & OYYGEN & 0 & 8 & $\theta$ & 8 & 0 \\
\hline & MTTROGEN & 3 & 0 & 3 & 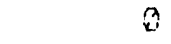 & 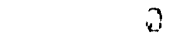 \\
\hline & HODOOGEN & 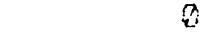 & 0 & $\theta$ & 8 & 0 \\
\hline & $\ldots$ & $\cdots$ & -0 & $\cdots-\cdots$ & - & 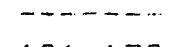 \\
\hline & $\begin{array}{l}\text { GEANE TOTAL } \\
\text { CHECL ON TOTAL }\end{array}$ & $4: \Xi \Xi 2,777$ & 근. 355 & 215,890 & 51,204 & 131,132 \\
\hline & TEMPEFATURE, 6 & 228 & 154 & 220 & $12 \Xi$ & 175 \\
\hline & FFESSURE, PSIA & 12.3 & 89.j & 96.2 & 98.3 & 100.5 \\
\hline & STATE & $50 \mathrm{~h}$ in & SOL in & $50 \mathrm{~L} \cdot \mathrm{H}$ & $30 L N$ & $501 \%$ \\
\hline
\end{tabular}


MULTISTAGE EXTRACTIUE FERMENTATION OF BUTANOL WITH RECOUERY OF RAFFINATE

BASECASE STOICHIOMETRY

MATERIAL BALANCE FLOWSHEET

THOUSAND ANNUAL POUNDS 330 DAYS) CO CAPACITY

\begin{tabular}{|c|c|c|c|c|c|c|}
\hline & STREAM & $\begin{array}{c}31 \\
\text { LBS \#2 } \\
\text { MAKE }\end{array}$ & $\begin{array}{c}32 \\
\text { LBS \#2 } \\
\text { TAILS }\end{array}$ & $\begin{array}{l}35 \\
\text { LBS \#3 } \\
\text { MAKE }\end{array}$ & $\begin{array}{l}34 \\
\text { LBS \#3 } \\
\text { TAILS }\end{array}$ & $\begin{array}{c}35 \\
\text { HES \#1 } \\
\text { MAKE }\end{array}$ \\
\hline $\mathrm{P}$ & $\ldots \ldots \ldots$ & $\ldots-\ldots$ & $\ldots \ldots$ & $-\cdots-\cdots$ & $\cdots \cdots-\cdots$ & $\ldots-\ldots$ \\
\hline 只 & CELLS -CHO & 8 & 0 & 0 & 0 & 0 \\
\hline 0 & $-\mathrm{NHZ}_{2}$ & 0 & 0 & (3) & $\theta$ & 0 \\
\hline $\mathrm{D}$ & -MINERALS & 0 & 0 & 0 & 8 & 8 \\
\hline u & -TOTAL & 0 & 0 & 0 & 0 & e \\
\hline c & COMPONENT \#1 & 0 & 8 & 0 & 0 & 8 \\
\hline$T$ & COMPONENT \#2 & 0 & 0 & 0 & 0 & c \\
\hline$\# 2$ & COMPONENT \#3 & 41,119 & 207 & 0 & 0 & $e$ \\
\hline & COMPONENT \#4 & 0 & 8 & 8 & 0 & B \\
\hline & COMPONENT \#5 & 32 & 8.395 & 0 & 0 & 8 \\
\hline & COMFONENT \#S & 0 & 0 & 0 & 4 & $b$ \\
\hline \#1 & COMPONENT \#7 & 8 & 301 & 0 & 0 & 905 \\
\hline & COMPONENT \#8 & 0 & 0 & a & $\theta$ & 17.865 \\
\hline & COMPONENT \#ق & 0 & 0 & $\theta$ & 0 & 0 \\
\hline & COMPONENT \#10 & 8 & 0 & 0 & 0 & 6,365 \\
\hline & COMPONENT \#11 & $\theta$ & 0 & a & 0 & 0 \\
\hline & COMPONENT \#12 & 0 & ( & (1) & 0 & 0 \\
\hline & COMPONENT \#1Z & 0 & 0 & 0 & 0 & 0 \\
\hline$\#$ & COMPONENT \#14 & 0 & 0 & Q & 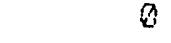 & 0 \\
\hline & COMPONENT \#15 & 0 & $\theta$ & 0 & 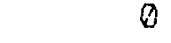 & 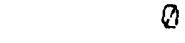 \\
\hline & COMPONENT \#1E & $\theta$ & 0 & 0 & 0 & 2 \\
\hline & COMPONENT \#17 & $\theta$ & 0 & a & 0 & $\theta$ \\
\hline$\#$ & COMPONENT \#18 & ( & 0 & 0 & 0 & $\theta$ \\
\hline & COMFONENT \#13 & 0 & 0 & 0 & 0 & 0 \\
\hline & COMPONENT \#20 & 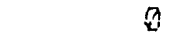 & 0 & 0 & 0 & $\boldsymbol{v}$ \\
\hline & GLUCOSE & 0 & 0 & 0 & (b) $-1-1-1-1$ & 8 \\
\hline & AMMONIA & 0 & 0 & 0 & 0 & 8 \\
\hline & PHOSPHORIC ACID & 0 & 0 & 0 & 0 & 8 \\
\hline & POTASSIUM CHLORIDE & 8 & 0 & 0 & 0 & 8 \\
\hline & MINOR NUTRIENTS & $\theta$ & 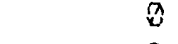 & 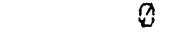 & 0 & 8 \\
\hline & WATER & 0 & 0 & 0 & $\theta$ & 0 \\
\hline & SOLVENT & 0 & 0 & 0 & 0 & 14 \\
\hline & CARBON DIOXIDE & 0 & 0 & 0 & a & 0 \\
\hline & OXYGEN & 0 & 0 & 6 & 0 & 0 \\
\hline & NITROGEN & 0 & 0 & 0 & 8 & 0 \\
\hline & HYUROGEN & 0 & 0 & 0 & 0 & $b$ \\
\hline & 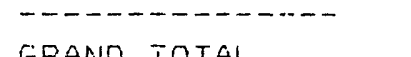 & 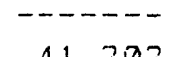 & & - & & \\
\hline & $\begin{array}{l}\text { GRANC TOTAL } \\
\text { CHECK ON TOTAL }\end{array}$ & 41,202 & 10,003 & 0 & 0 & 643 \\
\hline & TEMPERATURE, C & 100 & 120 & $\langle 273\rangle$ & 120 & 122 \\
\hline & PRESSURE, PSIA & 53.4 & 65.5 & .0 & .0 & 14.2 \\
\hline & STATE & SOL'N & SOL'N & SOL'N & SOL'N & SOL'N \\
\hline
\end{tabular}


MULTISTAGE EXTRACTIUE FERMENTATION OF BUTANOL WITH RECOUERY OF RAFFINATE BASECASE STOICHIOMETRY

MATERIAL BALANCE FLOWSHEET

THOUSAND ANNUAL POUNDS (3ZOD DAYS? CAPACITY

\begin{tabular}{|c|c|c|c|c|c|c|}
\hline & STREAM & $\begin{array}{c}36 \\
\text { HBS \#1 } \\
\text { TAILS }\end{array}$ & $\begin{array}{c}37 \\
\text { BYPRODUCT } \\
\text { FEED }\end{array}$ & $\begin{array}{l}38 \\
\text { RAFF \#1 } \\
\text { FEED }\end{array}$ & $\begin{array}{c}39 \\
\text { RAFF \#1 } \\
\text { MAKE }\end{array}$ & $\begin{array}{l}40 \\
\text { RAFF } 1 \\
\text { TAILS }\end{array}$ \\
\hline$P$ & $\ldots+\ldots+\ldots$ & $\ldots \ldots$ & $-\ldots-\cdots$ & $\ldots$ & $\ldots \ldots$ & …... \\
\hline$R$ & CELLS - CHO & 0 & 0 & $D$ & 0 & 0 \\
\hline 0 & $-N H 2$ & 0 & 8 & 0 & 8 & 6 \\
\hline 0 & -MINERALS & 0 & 0 & 0 & 0 & 0 \\
\hline $\mathrm{U}$ & -TOTAL & 0 & 0 & 0 & 0 & 0 \\
\hline$c$ & COMPONENT \#1 & 0 & 0 & 0 & $\theta$ & 0 \\
\hline$T$ & COMPONENT \#Z & 0 & 0 & 6 & 0 & $\theta$ \\
\hline$\# 2$ & COMPONENT \#3 & 0 & 14,530 & 14,530 & 14,530 & 10 \\
\hline & COMPONENT \#4 & 0 & 0 & 0 & 0 & 0 \\
\hline & COMPONENT \#5 & 0 & 17.765 & 19,530 & 19,442 & 39 \\
\hline & COMPONENT \#S & 0 & a & 0 & 0 & $\theta$ \\
\hline$\# 1$ & COMPONENT \#7 & 0 & 1,229 & 1,232 & 39 & 1,194 \\
\hline & COMPONENT \#B & 90 & 17,397 & 17,397 & 0 & $17: 397$ \\
\hline & COMFONENT \# 9 & 0 & 0 & 0 & 6 & 0 \\
\hline & COMPONENT \#10 & 34 & 6,895 & 6.895 & 0 & 5,835 \\
\hline & COMPONENT \#11 & 0 & 0 & 0 & 0 & 0 \\
\hline & COMPONENT \#12 & 0 & 0 & 0 & 0 & 0 \\
\hline & COMPONENT \#13 & 0 & 0 & 8 & 0 & 0 \\
\hline \# & COMPONENT \#14 & 0 & 0 & 0 & 0 & 0 \\
\hline & COMPONENT \#15 & 0 & 0 & $\theta$ & 0 & (1) \\
\hline & COMPONENT \#16 & 0 & 0 & 0 & 0 & 0 \\
\hline & COMPONENT \#17 & 0 & 0 & 0 & 0 & 0 \\
\hline$\#$ & COMPONENT \#18 & 0 & 0 & 0 & 0 & 0 \\
\hline & COMFONENT \#19 & 0 & $\theta$ & 0 & 0 & 0 \\
\hline & COMPONENT \#2O & 0 & (2) & 0 & 0 & 0 \\
\hline & GLUCOSE & 0 & 1,319 & 1,319 & 0 & 1,319 \\
\hline & AMMONIA & 0 & 0 & 0 & 0 & 0 \\
\hline & PHOSPHORIC ACID & 0 & 1,487 & 1,437 & $\theta$ & $1: 487$ \\
\hline & POTASSIUM CHLORIDE & 0 & 1,132 & 1,132 & 0 & 1.132 \\
\hline & MINOR NUTRIENTS & 0 & $\theta$ & 0 & Q & $\theta$ \\
\hline & WATER & 8 & 341,389 & 860,795 & 19,442 & $841: 353$ \\
\hline & SOLUENT & $4.307,004$ & 17 & 17 & 0 & 17 \\
\hline & CARBON DIOXIDE & 0 & b & 0 & 0 & $\theta$ \\
\hline & OXYGEN & 0 & 0 & 0 & 0 & 0 \\
\hline & NITROGEN & 6 & 0 & 6 & 0 & 8 \\
\hline & HYDROGEN & 0 & 0 & 0 & 0 & 0 \\
\hline & $\ldots \ldots$ & $\ldots \ldots$ & $\ldots \ldots$ & $\ldots \ldots$ & 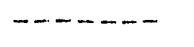 & $\ldots$ \\
\hline & $\begin{array}{l}\text { GRAND TOTAL } \\
\text { CHECK ON TOTAL }\end{array}$ & $4,307,128$ & $903: 157$ & 924,334 & 53,452 & 870,882 \\
\hline & TEMPERATURE, C & 220 & 34 & 35 & 35 & 105 \\
\hline & PRESSURE, PSIA & 17.7 & 14.7 & - & 15.1 & 17.6 \\
\hline & STATE & SOL'N & SOL'N & SOL'N & SOL'N & SOL'N \\
\hline
\end{tabular}


MULTISTAGE EXTRACTIUE FERMENTATION OF EUTANOL. WITH RECOUERY OF RAFFINATE

BASECASE STOICHIOMETRY

MATERIAL BALANCE FLOWSHEET

THOUSAND ANNUAL POUNDS 330 DAYS Q CAPACITY

\begin{tabular}{|c|c|c|c|c|c|c|c|}
\hline & STREAM & $\begin{array}{l}\text { RAFF \#2 } \\
\text { MAKE }\end{array}$ & $\begin{array}{l}\text { PAFE \#2 } \\
\text { TAILS }\end{array}$ & $\begin{array}{l}\text { RAFF \#3 } \\
\text { MAKE }\end{array}$ & $\begin{array}{l}\text { RAFF \#苛 } \\
\text { TAILS }\end{array}$ & $\begin{array}{l}\text { PEF INED } \\
\text { BUTANOL }\end{array}$ & $\begin{array}{l}\text { REFINED } \\
\text { ACETONE }\end{array}$ \\
\hline$p$ & $\cdots \cdots-\cdots \cdots-\cdots$ & $\cdots \cdots-$ & $-\cdots \cdots-$ & $\ldots \ldots-\cdots$ & $\cdots \cdots-\cdots$ & $-\cdots-\cdots$ & $-\cdots \cdots$ \\
\hline$R$ & CELLS - CHO & 8 & 0 & 0 & $\theta$ & $Q$ & 0 \\
\hline 0 & $-N H Z$ & 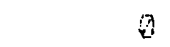 & 0 & 0 & $\theta$ & 0 & 8 \\
\hline 0 & -MINERALS & 0 & 0 & 0 & 0 & 0 & $\Delta$ \\
\hline 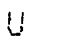 & -TOTAL & 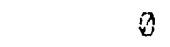 & $\theta$ & 0 & 8 & $\theta$ & 0 \\
\hline$C$ & COMPONENT \#1 & 3 & 8 & 8 & $\theta$ & 0 & 0 \\
\hline$T$ & COMPONENT \#2 & 8 & 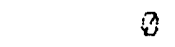 & A & 8 & 0 & 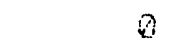 \\
\hline$\# 2$ & COMFONENT \#3 & 14,530 & 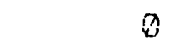 & 14.457 & 73 & 358 & 55,575 \\
\hline & COMPONENT \#4 & 0 & 0 & 0 & 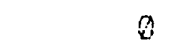 & $\theta$ & 0 \\
\hline & COMPONENT \#5 & 17.674 & 1.767 & 29 & 17.645 & 358 & 111 \\
\hline & COMPONENT \#E & 8 & $\theta$ & 0 & 0 & 0 & $\theta$ \\
\hline$\# 1$ & COMPONENT $\# 7$ & 35 & 4 & $\theta$ & 35 & 179,248 & $\theta$ \\
\hline & COMPONENT \#B & $\theta$ & 0 & (3) & 8 & 360 & 6) \\
\hline & COMPONENT \#G & 8 & 8 & $\theta$ & 8 & 0 & 0 \\
\hline & COMPONENT \#10 & $\hat{z}$ & 0 & 9 & $\theta$ & 350 & 3 \\
\hline & COMPONENT \#11 & $\theta$ & 8 & 0 & $\theta$ & 8 & 0 \\
\hline & COMPONENT \#12 & 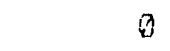 & 8 & 0 & 2 & $\hat{\theta}$ & 3 \\
\hline & COMPONENT \#13 & $\theta$ & 0 & 0 & 0 & $\theta$ & Q \\
\hline$\#$ & COMPONENT \#14 & 8 & 3 & 8 & $\theta$ & 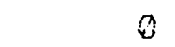 & 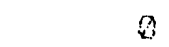 \\
\hline & COMPONENT \#15 & 0 & 0 & 0 & 8 & 8 & 0 \\
\hline & COMPOAENT \#1E & 8 & $\theta$ & 8 & $\theta$ & 3 & 0 \\
\hline & COMPONENT \#17 & 8 & $Q$ & $\theta$ & $\theta$ & 0 & 0 \\
\hline \# & COMPONENT \#18 & 8 & 8 & 0 & 0 & $\theta$ & 8 \\
\hline & COMPONENT \#19 & 8 & 8 & 0 & 0 & 8 & 6 \\
\hline & COMPONENT \#2O & $\therefore$ & 0 & 0 & 0 & 9 & 0 \\
\hline & GLUCOSE & 8 & 8 & $\theta$ & $\theta$ & 0 & (8) \\
\hline & AMMONIA & (2) & 0 & 0 & 8 & 8 & 0 \\
\hline & PHOSPHORIC ACID & 8 & 0 & $\theta$ & 0 & 0 & 0 \\
\hline & POTASSIUM CHLORIDE & 9 & 3 & 6 & $\theta$ & $\theta$ & 0 \\
\hline & MINOR NUTRIENTS & 0 & 8 & $\theta$ & 0 & Q & $a$ \\
\hline & WATER & 35 & 19,405 & 0 & 35 & $B E$ & 0 \\
\hline & SOLUENT & 0 & 0 & 0 & 0 & 350 & 0 \\
\hline & CARBON DIOXIDE & 8 & 8 & $\theta$ & 8 & 0 & in \\
\hline & OXYGEN & $\theta$ & 0 & 0 & $\theta$ & 0 & 8 \\
\hline & NITROGEN & 8 & 9 & 0 & 8 & 0 & 0 \\
\hline & HYOROGEN & 8 & 8 & 0 & 0 & 0 & 8 \\
\hline & $-\cdots--\cdots---\cdots$ & $\cdots-\cdots$ & $--\cdots--$ & $\cdots \cdots-$ & $\ldots \ldots$ & $-\cdots-\cdots$ & $\ldots \ldots \ldots$ \\
\hline & $\begin{array}{l}\text { GRAND TOTAL } \\
\text { CHECL ON TOTAL }\end{array}$ & 32,274 & 21,177 & 14.485 & 17.789 & 181,152 & $\begin{array}{r}55,587 \\
8\end{array}$ \\
\hline & TEMPERATURE, $C$ & 54 & 100 & 82 & 105 & 175 & 100 \\
\hline & , RESSURE, PSIA & 12.3 & 15.4 & 34.2 & 40.6 & 101 & 58 \\
\hline & STATE & SOL'N & SOL'N & SOL'N & SOL'N & $\theta$ & 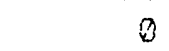 \\
\hline
\end{tabular}


MULTISTAGE EXTRACTIUE FERMENTATION OF BUTANOL WITH RECOUERY OF RAFFINATE

BASECASE STOICHIOMETRY

MATEFIAL BALANCE FLOWSHEET

THOUSAND ANAUAL POUNOS S BOE DAYS; E CAPACITY

\begin{tabular}{|c|c|c|c|c|c|c|}
\hline & GTREAM & $\begin{array}{l}47 \\
\text { REFINED } \\
\text { ETHANOL }\end{array}$ & $\begin{array}{c}48 \\
\text { ORGANIC } \\
\text { WASTE }\end{array}$ & $\begin{array}{c}49 \\
\text { MAKE-UP } \\
\text { SOLUENT }\end{array}$ & $\begin{array}{l}50 \\
\text { MAKE-LIP } \\
\text { WATER }\end{array}$ & $\begin{array}{c}51 \\
\text { STERILE } \\
\text { STEAM }\end{array}$ \\
\hline$P$ & stherim - & $\ldots \ldots$ & $\ldots \ldots$ & _....... & $\ldots$ & - \\
\hline$F$ & CELLS -CHO & 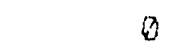 & 8 & 0 & 0 & 0 \\
\hline 0 & $-H+12$ & 0 & 0 & 0 & 0 & 0 \\
\hline $\mathrm{L}$ & -MINERALS & 0 & 0 & 0 & 0 & 0 \\
\hline ! & -TOTAL & 0 & 0 & 0 & 0 & () \\
\hline $\mathrm{C}$ & COMPONENT \#1 & 8 & 0 & 0 & 0 & 8 \\
\hline$T$ & COMPONENT \#Z & $a$ & 0 & 0 & 0 & 0 \\
\hline$\#=$ & COMPONENT \#3 & 73 & $(0)$ & 0 & 0 & 0 \\
\hline & COMPONENT \#4 & D & 0 & 2 & 0 & 0 \\
\hline & COMPONENT \#5 & 17,645 & 89 & 0 & 0 & Q \\
\hline & COMPONENT \#E & 0 & 0 & 0 & (2) & 0 \\
\hline$\# 1$ & COMFONENT \# 7 & 35 & 2,839 & 0 & 0 & 0 \\
\hline & COMPONENT \#B & 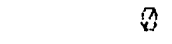 & 35,252 & 0 & 0 & 0 \\
\hline & COMFONENT \#9 & 0 & 0 & 0 & 0 & $\theta$ \\
\hline & COMPONENT \#10 & $D$ & 13,750 & 0 & a & 0 \\
\hline & COMPONENT \#11 & 0 & 0 & 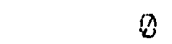 & 0 & 0 \\
\hline & OOMPONENT \#12 & 0 & 0 & 0 & 0 & 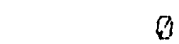 \\
\hline & COMPONENT \#13 & 0 & 0 & 0 & 0 & 0 \\
\hline \# & COMPONENT \#14 & 0 & $\theta$ & 0 & 0 & 0 \\
\hline & COMPONENT \#15 & 0 & 0 & 0 & 0 & 0 \\
\hline & COMPONENT \#1E & 0 & 0 & $\theta$ & 0 & 6) \\
\hline & COMPONENT \#17 & 0 & 0 & 0 & 0 & 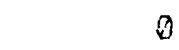 \\
\hline \# & COMEONENT \#18 & 0 & 0 & 0 & 0 & Q \\
\hline & COMFONENT \#19 & 0 & 0 & 0 & 0 & 6 \\
\hline & COMPONENT & 3 & 0 & 0 & 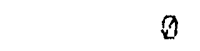 & \\
\hline & GLUCOSE & 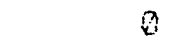 & 1,319 & 0 & 0 & 0 \\
\hline & AMMONIA & 0 & 0 & 0 & 0 & 0 \\
\hline & PHOSFHORIO ACID & 0 & 1,487 & $n$ & 0 & \\
\hline & POTASSIUM CHLORIDE & 3 & 1,132 & 0 & 0 & 0 \\
\hline & MINOR NUTRIENTS & a & 0 & 0 & 0 & 0 \\
\hline & WATER & 35 & 341,353 & 0 & 120,779 & 60,804 \\
\hline & SOLUENT & 0 & 31 & 374 & 0 & 0 \\
\hline & CAREON DIOXIDE & 0 & 0 & 3 & 0 & $\theta$ \\
\hline & OXYGEN & $\theta$ & 0 & $a$ & 0 & 0 \\
\hline & NITROGEN & 0 & 0 & 0 & $\theta$ & 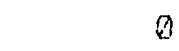 \\
\hline & HYOROGEN & 0 & 8 & 0 & 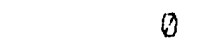 & $\theta$ \\
\hline & 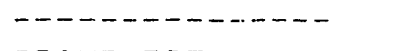 & $\cdots \cdots-$ & $-\cdots-\cdots$ & - & $\cdots \cdots$ & $\cdots$ \\
\hline & $\begin{array}{l}\text { GRAND TOTAL } \\
\text { CHECK ON TOTAL }\end{array}$ & 17.789 & 895,531 & $\begin{array}{l}374 \\
374\end{array}$ & 120,779 & 60,804 \\
\hline & TEMPERATURE, C & 105 & 40 & 20 & 20 & 141 \\
\hline & PRESSURE, PSIR, & 40.6 & 14.7 & 14.7 & 14.7 & 64.7 \\
\hline & STATE & $20 L, n$ & SOL' $N$ & LIOUID & LIOUIO & GAS \\
\hline
\end{tabular}


MULTISTAGE EXTRACTIUE FERMENTATION OF BUTANOL WITH RECOVERY OF RAFFINATE

BASECASE STOICHIOMETRY

MATERIAL BALANCE FLOWSHEET

THOUSAND ANNUAL POUNDS (330 DAYS) G CAFACITY

\begin{tabular}{|c|c|c|c|c|c|c|}
\hline & & $\begin{array}{c}52 \\
\text { FERM TP }\end{array}$ & $\begin{array}{c}53 \\
\text { DISTILL. } \\
\text { STSAM }\end{array}$ & $\begin{array}{l}54 \\
\text { COND. MU } \\
0.110\end{array}$ & $\begin{array}{c}55 \\
\text { MEDIUM }\end{array}$ & $\begin{array}{l}56 \\
\text { FERM' TR }\end{array}$ \\
\hline D & STREAM & STEAM & STEAM & P'WR H'SE & COOL WTR & COOL WTR \\
\hline$R$ & CELLS - CHO & 0 & 0 & 0 & 0 & 0 \\
\hline 0 & $-N H Z$ & 0 & 0 & $a$ & 0 & 0 \\
\hline 0 & -MINERALS & 0 & 0 & $a$ & 0 & 0 \\
\hline U & -TOTAL & 0 & 2 & 0 & 0 & 0 \\
\hline $\mathrm{C}$ & COMFONENT $\# 1$ & 0 & 0 & 0 & 0 & 0 \\
\hline$T$ & COMPONENT \#Z & 0 & 0 & 0 & $\theta$ & 0 \\
\hline$\# 2$ & COMFONENT \#3 & 0 & 0 & 0 & 0 & 0 \\
\hline & COMPONENT \#4 & $\theta$ & 0 & $\theta$ & 8 & 0 \\
\hline & COMPONENT \#5 & 0 & 0 & $\theta$ & $a$ & 0 \\
\hline & COMPONENT \#S & 8 & 0 & 0 & 0 & (i) \\
\hline$\# 1$ & COMPONENT \#7 & 0 & 0 & $\theta$ & 0 & 0 \\
\hline & COMPONENT \#B & b & 0 & 0 & 0 & (a) $-1-1-1-1$ \\
\hline & COMPONENT \#G & 0 & 0 & 0 & 8 & 0 \\
\hline & COMPONENT \#10 & 0 & 0 & 0 & 是 & 0 \\
\hline & COMPONENT \#11 & 0 & 0 & 0 & 0 & 0 \\
\hline & COMPONENT \#12 & 0 & 8 & a & 0 & 0 \\
\hline & COMPONENT \#13 & 0 & 0 & 0 & 0 & 0 \\
\hline \# & COMPONEEMT \#14 & $\theta$ & 0 & 0 & 0 & 0 \\
\hline & COMPONENT \#15 & 0 & 0 & B & 0 & 0 \\
\hline & COMPONENT \#16 & s & 0 & 3 & 0 & 0 \\
\hline & COMPONENT \#17 & 8 & 0 & 0 & $\theta$ & 0 \\
\hline$\#$ & COMPONENT \#13 & $\theta$ & 0 & $\theta$ & 0 & 0 \\
\hline & COMPONENT \#19 & $\theta$ & 0 & 8 & 0 & 0 \\
\hline & COMPONENT \#ZO & $\theta$ & 0 & D & 0 & 0 \\
\hline & GLUCOSE & 0 & 0 & 8 & 8 & $\theta$ \\
\hline & AMMONIA & a & 0 & 0 & 0 & 0 \\
\hline & PHOSPHORIC ACIO & $\theta$ & 0 & 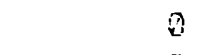 & 8 & 0 \\
\hline & POTASSIUM CHLORIDE & 0 & 8 & 0 & 8 & 0 \\
\hline & MINOR NUTRIENTS & 0 & 8 & $n$ & 6 & $\theta$ \\
\hline & WATER & 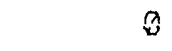 & $1,384,445$ & 50,804 & 784,895 & 12.750 .550 \\
\hline & SOLVENT & $\theta$ & 0 & 0 & () & 8 \\
\hline & CARBON DIOKIOE & 0 & 0 & 0 & 0 & 0 \\
\hline & OYYGEN & $\theta$ & 0 & 0 & $a$ & 0 \\
\hline & NITROGEN & $\theta$ & 0 & $\theta$ & 0 & 0 \\
\hline & HYOROGEN & $D$ & 0 & 0 & a & 0 \\
\hline & - & $\cdots \cdots$ & $\cdots \cdots$ & $\ldots \ldots$ & $\ldots \ldots$ & $\ldots \ldots$ \\
\hline & $\begin{array}{l}\text { GRAND TOTAL } \\
\text { OHECL ON TOTAL }\end{array}$ & 0 & 1.384 .445 & 50,804 & 784.895 & $12,760.550$ \\
\hline & TEMPERATURE, $\quad 0$ & 141 & 185 & 110 & 5 & 5 \\
\hline & PRESSURE, PSIA & 54.7 & 164.7 & 20.8 & 14.7 & 14.7 \\
\hline & STATE & 645 & 6,45 & LIOUIO & L. IOUID & EIOUID \\
\hline
\end{tabular}


MULTISTAGE EXTRACTIUE FERMENTATION OF BUTANOL WITH RECOUERY OF RAFFINATE BASECASE STOICHIOMETPY

MATERIAL BALANCE FLOWSHEET

THOUSAND ANNUAL POUNDS (33R DAYS) O CAFACITY

\begin{tabular}{|c|c|c|}
\hline & GTREA & $\begin{array}{c}\text { 57 } \\
\text { OISTILL. } \\
\text { COOL WTP }\end{array}$ \\
\hline $\mathrm{F}$ & 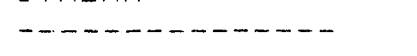 & $\ldots \ldots$ \\
\hline 只 & CELLE - CHO & 0 \\
\hline 0 & $-N N_{L}$ & Q \\
\hline$D$ & MINERALS & 8 \\
\hline U & -TOTAL & 0 \\
\hline$C$ & COMPONENT \#1 & 0 \\
\hline $\mathrm{T}$ & COMPONENT \#2 & i) \\
\hline$\# 2$ & COMPONENT \#S & ? \\
\hline & COMEONENT \#A & 8 \\
\hline & COMPONENT $\# 5$ & 0 \\
\hline & COMPONENT \#E & 8 \\
\hline+1 & COMPONENT \#? & 0 \\
\hline & COMFONENT \#3 & 0 \\
\hline & COMPONENT \#G & 0 \\
\hline & GOMPONENT \#1O & 8 \\
\hline & COMPONENT \#11 & D \\
\hline & COMFONENT \#12 & 0 \\
\hline & COMPONENT \#13 & 9 \\
\hline$\#$ & COMPONENT \#14 & 8 \\
\hline & COMPONENT \#15 & 0 \\
\hline & COMPONENT \#15 & 0 \\
\hline & COMPONEAT \#17 & 8 \\
\hline$\#$ & COMPONENT \#1S & 8 \\
\hline & COMPONENT \#19 & 9 \\
\hline & COMPONENT \#2B & 0 \\
\hline & GLUSOSE & 0 \\
\hline & AMHONIA & 0 \\
\hline & FHOSPHORIO ACIO & 0 \\
\hline & POTASSIUM CHLORIDE & 0 \\
\hline & MINOR MUTPIENTS & 8 \\
\hline & WATER & 14.345 .709 \\
\hline & SOLLIENT & 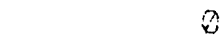 \\
\hline & CAPEON GIOXIOE & 8 \\
\hline & OXYGEN & a \\
\hline & NITROGEN & $a$ \\
\hline & HYOROGEN & Q \\
\hline & $\cdots \cdots---\cdots-\cdots$ & $\cdots-\cdots$ \\
\hline & $\begin{array}{l}\text { GFAHE TOTAL } \\
\text { CHECH ON TOTAL }\end{array}$ & $14,340,709$ \\
\hline & TEMPERATLIRE : $\mathrm{C}$ & 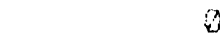 \\
\hline & $\begin{array}{l}\text { PRESSULE, PSIA } \\
\text { STATE }\end{array}$ & $\begin{array}{r}14.7 \\
\operatorname{LOSIO}\end{array}$ \\
\hline
\end{tabular}


M LS. MOLES,YEAR

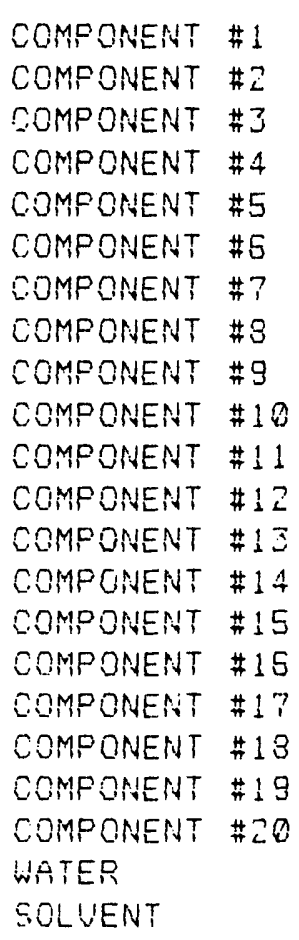

DISTILLATION CALCULATIONS

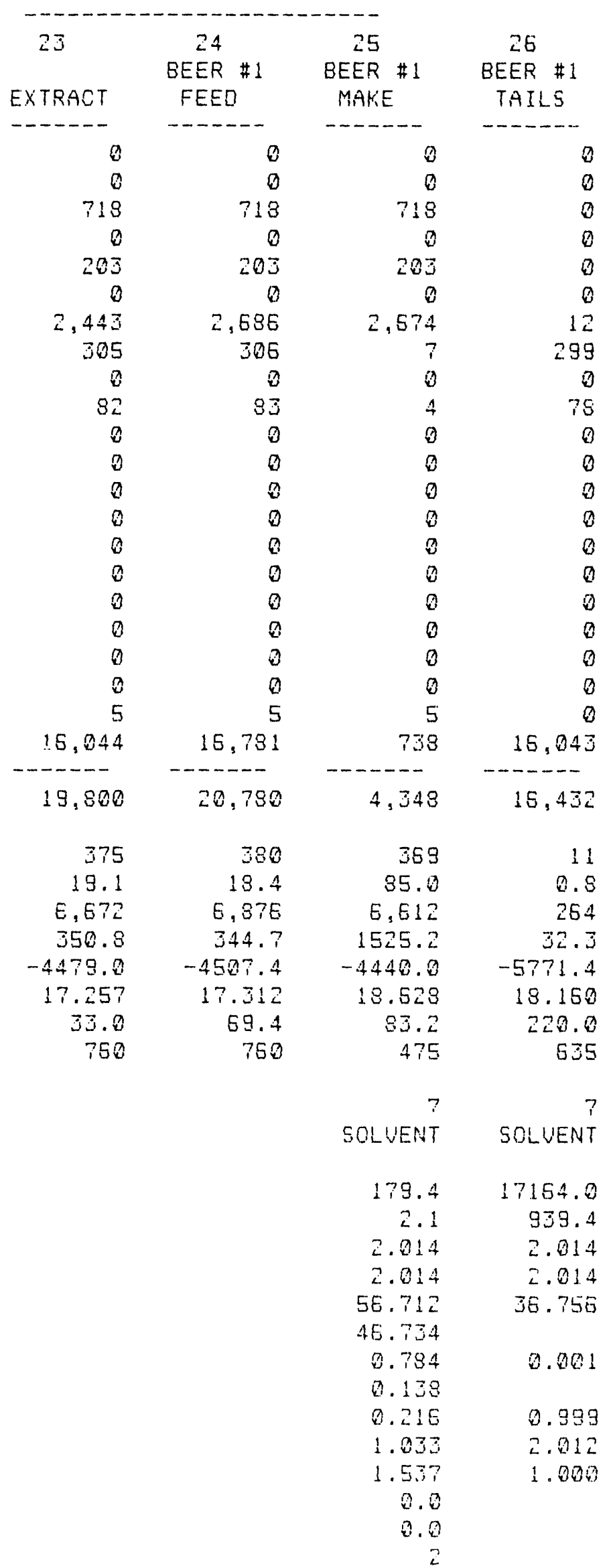


M LB. MOLES, YEAR

ACTUAL PLATES

FRESSURE MM HG (REUISED:

TEMPERATURE $O$ (REUISED)

AUERAGE MOLECULAR WEIGHT

GAS DENSITY - LBICF

CROSS SECTIONAL AREA - SO FT

COLUMN HEIGHT - FT

COLUMN DIAMETER

Y 1 (MPPY)

HV : HEAT VAPORIZ. -BEUILE;

CN (HEAT CAPACITY - Btu'LE'F)

HEAT LOAD - MM BTUIHr

CONDENSER COOLING WATER - GPM ( 15 C dT)

CALANDRIA STEAM - MPFH (150 PSIG)

COLUMN COST - \$1000 3035 MPC - BARE EOUIPMENT

- ALL CARBON STEEL

- C.S W/JO4 S.S. TRAYS

- ALL 304 STAINLESS STEEL

- ALL $304 L$ STAINLESS STEEL

- ALL 315 STAINLESS STEEL

CONDENSER OR CALANDRIA SURFACE - SO FT

COND. OR CALAND. COST - 10003086 MPC - BARE EOUIPMENT

- CARBON STEEL

- 304 STAINLESS STEEL

- JIE STAINLESS STEEL

-MONEL

SUBTOTAL

SUBTOTAL

SUBTOTAL

MINIMUM REFLUX RATIO

CT SUETOTAL \#

Cr SUETOTAL \#2

CI SUETOTAL \#Z

Cn CHECK

HV GURTOTAL \#1

H. SUETOTAL \#2

H. SUETOTAL \#ت

H. CHECK

MIN. FLATESENORMAL,

COL.COST-CIS NORMAL

COL. COST-SIS NORMAL

MIN.REFLUX: NORMAL:

O.S. AREG NORMAL)

HEAT LOAD N NORMAL:

CON,CAL COSTINORMAL)
DISTILLATION CALCULATIONS

\begin{tabular}{|c|c|c|c|}
\hline 23 & 24 & 25 & 25 \\
\hline & BEER \#1 & BEER \# 1 & BEER \#1 \\
\hline EXTRACT & FEED & MAKE & TAILS \\
\hline$\cdots \cdots-$ & $\ldots \ldots$ & $\ldots \ldots$ & $\ldots \ldots$ \\
\hline & & 10 & \\
\hline & & 595 & \\
\hline & & 90 & \\
\hline & & 103.09 & 253.67 \\
\hline & & 0.1532 & 0.3397 \\
\hline & & 31.8 & \\
\hline & & 29.3 & \\
\hline & & 5.4 & \\
\hline & & 133,154 & \\
\hline & & 215.1 & 215.0 \\
\hline & & 0.500 & 0.500 \\
\hline & & 13.394 & 94.957 \\
\hline$C d T$; & & 993 & \\
\hline & & & 110.50 \\
\hline
\end{tabular}

$\$ 56.4$

$\$ 73.6$

$\$ 107.2$.

$\$ 117.9$

$\$ 144.7$

1,556

$\$ 57.4 \$ 164.4$

$\$ 80.4$ \$230.2

F8E. 1 \$245.5

$\$ 111.9$ \$2 Q 2.6

$2,166,389$

2., 153.503

2.153,503

(0.4)

$124.532 \quad 453$

$396 \quad 12.427$

$39,168 \quad 2,153.503$

$0.500 \quad 0.500$

$53.575 \quad 195$

$170 \quad 5,344$

$42.589 \quad 926.209$

$215.1 \quad 215.0$ 
M LB. MOLEE;YEAR

COMPONENT $\# 1$
COMFONENT $\# 2$
COMPONENT $\# 3$
COMFONENT $\# 4$
COMPONENT $\# 5$
COMPONENT $\# 5$
COMPONENT $\# 7$
COMPONENT $\# 3$
COMPONENT $\# 3$
COMPONENT $\# 10$
COMPONENT $\# 11$
COMPONENT $\# 12$
COMPONENT $\# 12$
COMEONENT $\# 14$
COMPONENT $\# 15$
COMPONENT $\# 16$
COMPONENT $\# 17$
COMPONENT $\# 18$
COMPONENT $\# 19$
COMPONENT $\# 20$
WATER
SOLUENT

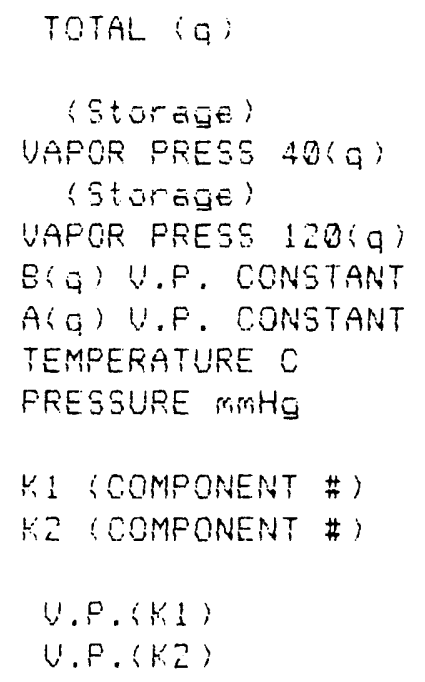

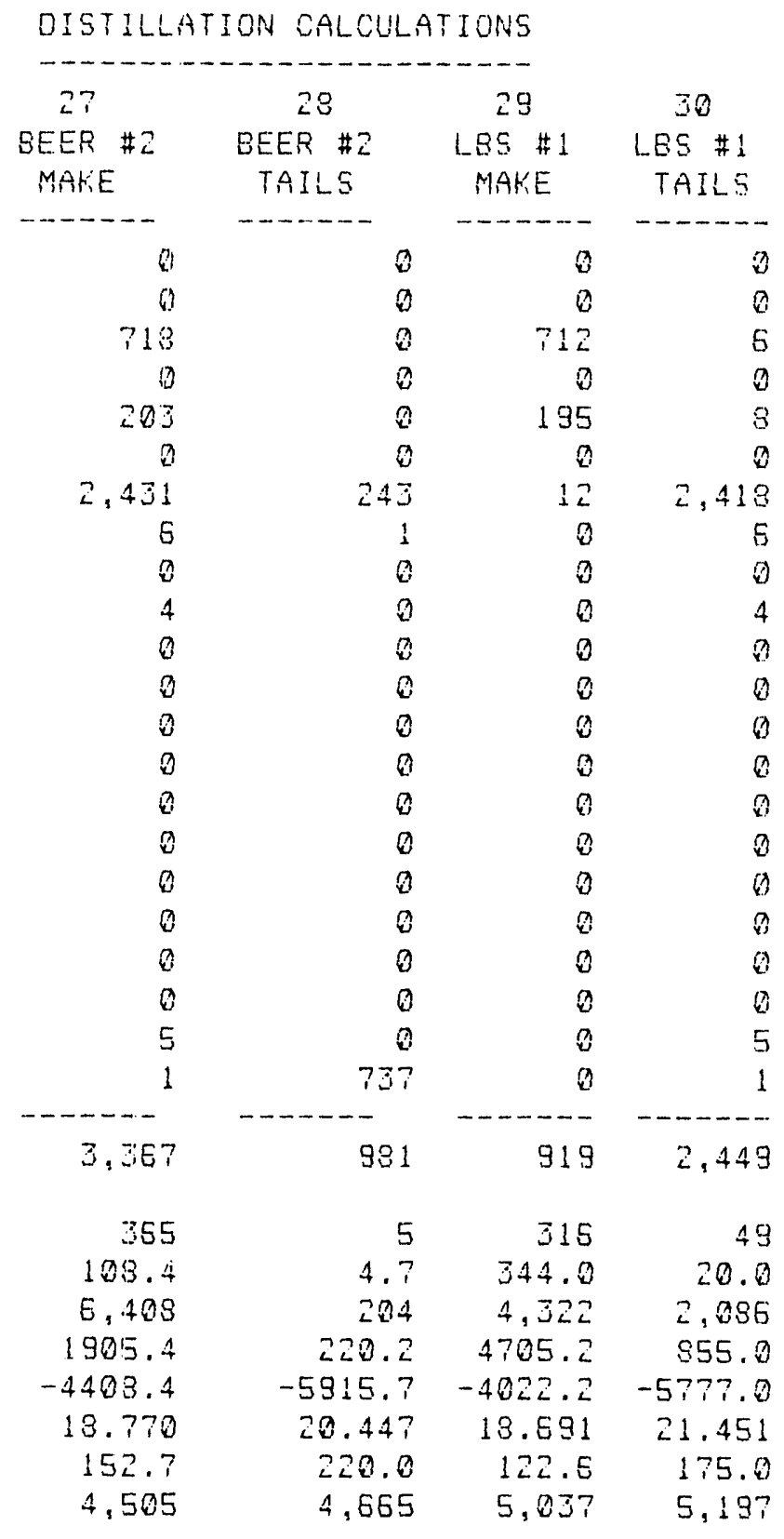

$\begin{array}{rrrr}7 & 7 & 6,5,4.3 & 6,5.4 .3 \\ \text { SOLVENT } & \text { SOLVENT } & 7 & 7 \\ 2621.7 & 17164.0 & 3833.5 & 17223.0 \\ 76.4 & 939.4 & 923.2 & 5206.5 \\ 2.014 & 2.014 & 1.350 & 1.350 \\ 2.014 & 2.014 & 1.350 & 1.350 \\ 17.053 & 2 E .000 & 3.187 & 4.458 \\ 21.527 & & 3.322 & \\ 0.999 & 0.243 & 0.941 & 0.003 \\ 0.794 & & 0.077 & \\ 0.001 & 0.752 & 0.059 & 0.997 \\ 1.000 & 1.485 & 1.001 & 1.347 \\ 2.012 & 1.044 & 1.304 & 1.000 \\ 0.0 & & 0.5 & \\ 0.0 & & 0.7 & \\ 3 & & 5 & \end{array}$


M LB. MOLESYYEAR

ACTUAL PLATES

PRESSURE MM HG (REUISED)

TEMPERATURE O (REUISED)

AUERAGE MOLECULAR WEIGHT

GAS DENSITY - LB/CF

CROSS SECTIONAL AREA - SO FT

COLUMN HEIGHT - FT

COLUMN DIAMETER

K1 (MPPY)

H. LHEAT VIAPORIZ.-BTUILE:

CI (HEAT CAPACITY - BtUILEIF)

HEAT LOAD - MM B BW/HR

CONDENSER COOLING WATER - GPM 1

CALANDRIA STEAM - MPFH : $15 \%$ PSIG;

COLUMN COET - \$1000 3035 MPO - BARE

- ALL CARBON STEEL

- C.5 W/304 S.S. TRAYS

- ALL 304 STAINLESS STEEL

- ALL 3O4L STAINLESS STEEL

- ALL 315 STAINLESS STEEL

CONDENGER OR CALANDRIA SURFACE -

COND. OR CALAND. COST - \$1000 303

-CARBON STEEL

-304 STAINLESS STEEL

- 315 STAINLESS STEEL

-MONEL

\section{SUBTORAL \\ SUBTOTAL \\ SUBTOTAL \\ MINIMUM REFLUX RATIO \\ OI SUETOTAL \# \\ Cri SUBTOTAL \#2 \\ CI SUBTOTAL \#ت \\ Cri CHECK}

HV SUETOTAL \#

HV SUETOTAL \#2

H. SUSTOTAL \#3

HV CHECK

MIN. PLATESINORMAL;

COL.COST-CIS NORMAL

COL.COST-S/S NORMAL

MIN.REFLUX SNORMAL:

C.S. AREA (NORMAL:

HEAT LOAO NORMAL:

CON,CAL CUST!NORMAL,

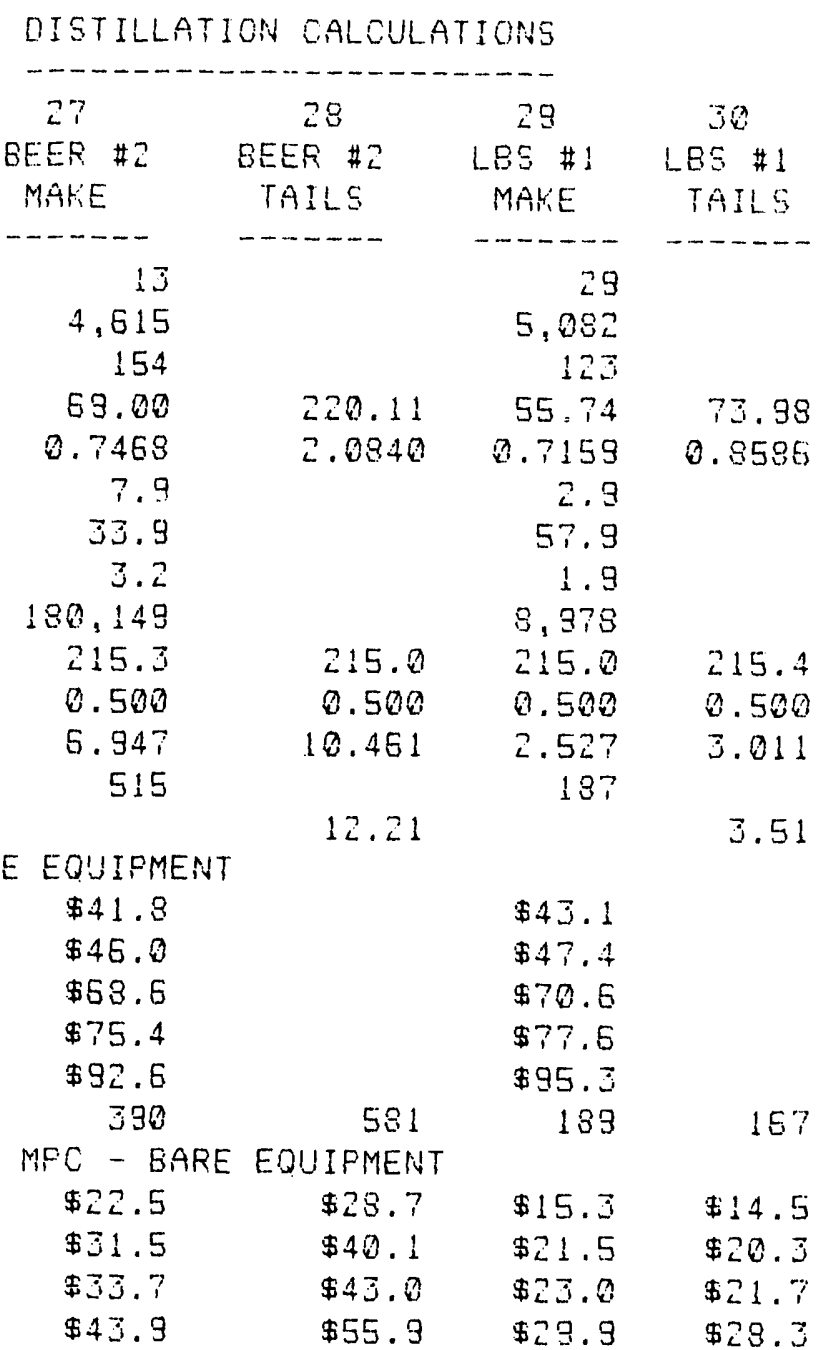

90,251

2.65

265

(0.8)

115,585

350

266

0.500

49,701

155

161

215.3

107,945

93,902

38,902

0.5

9,007

36

38,302

0.500

3.873

15

42,528

215.3
25,602

39,983

360

255

$0.500 \quad 0.500$

$11,003 \quad 38,593$

155

161

215.4

43

?1

3

15
3.1 
MLB. MOLESIYEAR

\begin{tabular}{|c|c|}
\hline COMFONENT & $\stackrel{+1}{* 1}$ \\
\hline COMPONENT & $\# \overrightarrow{2}$ \\
\hline COMEONENT & $\# \bar{j}$ \\
\hline COMPONENT & $\# 4$ \\
\hline COMPONENT & $\$ 5$ \\
\hline COMPONENT & $\# E$ \\
\hline COMPONENT & $\# ?$ \\
\hline COMPONENT & $\# 8$ \\
\hline COMPONENT & $\# 9$ \\
\hline COMPONENT & $\# 10$ \\
\hline COMPONENT & $\# \begin{array}{ll}1 & 1 \\
2 & 1\end{array}$ \\
\hline COMFONENT & $\# 12$ \\
\hline COMPONENT & $\# 13$ \\
\hline COMPONENT & $\# 14$ \\
\hline COMPONENT & $\# 15$ \\
\hline COMPONENT & $\# 16$ \\
\hline COMPONENT & $\# 17$ \\
\hline CGMPONENT & $\# 18$ \\
\hline COMPONENT & $\# 19$ \\
\hline $\begin{array}{l}\text { COMPONENT } \\
\text { WATES } \\
\text { SOL UENT }\end{array}$ & \pm 20 \\
\hline
\end{tabular}

TOTAL :G;

(Sturgige)

VAPOR PRESS 4B:

(Sturage?

VAPOR PRESS $120($ G)

B(G) U.P. CONSTANT

A:G; U.P. CONETANT

TEMPERATURE C

PRESSURE MIHG

ㄴ. (OOMPONENT \#)

KL (COMPONENT \#)

$$
\text { U.P. (K.: }
$$$$
\text { U.P. (L2: }
$$

GAMMA-L1 IN $\because 2$

GAMMA-KI IN KI

ALPHA

AUG COLUMN ALPHA

MOL FRACT. YL (MARE OR TAILS)

MOL FRACT. K1 (FEEO)

MOL FRACT, WL (MAKE OR TAILS)

ADJ. GAMMA-KI IN KI

AOS. GAMMA-KIZ IN $K 1$

MINIMUM REFLUX RATIO (ADUUSTED)

ACTUAL REFLUY RATIO

MINIMUM PLATES

\begin{tabular}{|c|c|c|c|}
\hline 31 & 32 & 33 & 34 \\
\hline$\angle 85 \# 2$ & L.85 \#2. & LSS \#3 & L.PS \#3 \\
\hline MAKE & TAILS & MALE & TAILE \\
\hline$\cdots-$ & $\cdots-\cdots-\cdots$ & $\cdots \cdots$ & $\cdots \cdots$ \\
\hline 8 & 0 & 8 & $\theta$ \\
\hline 0 & 8 & $\theta$ & $\theta$ \\
\hline 708 & 4 & 8 & 0 \\
\hline$\theta$ & 0 & 8 & 6 \\
\hline 工 & 193 & $\theta$ & 0 \\
\hline 0 & $\theta$ & 0 & 8 \\
\hline Q) & 12 & $\theta$ & ? \\
\hline 0 & 0 & 0 & 8 \\
\hline 0 & 0 & 8 & (3) \\
\hline 0 & 0 & 0 & 0 \\
\hline 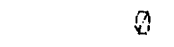 & 8 & 0 & 0 \\
\hline 8 & 8 & 0 & $\theta$ \\
\hline 3 & 0 & 0 & 0 \\
\hline$\theta$ & 0 & 0 & 0 \\
\hline 8 & 0 & 0 & 8 \\
\hline 8 & 0 & 0 & 0 \\
\hline 0 & (3) & 8 & 8 \\
\hline 0 & 8 & 0 & 8 \\
\hline 0 & 8 & 8 & $\hat{x}$ \\
\hline 8 & 8 & 0 & 0 \\
\hline$\theta$ & D & 0 & 8 \\
\hline 0 & 0 & 0 & 8 \\
\hline----- & ------ & $-----\cdots$ & $\cdots \cdots$ \\
\hline 710 & 209 & 0 & 0 \\
\hline 289 & 27 & $\theta$ & 8 \\
\hline 497.8 & 127.1 & .9 & .0 \\
\hline 3,515 & 707 & 6 & 0 \\
\hline 5392.7 & 3333.2 & .8 & .0 \\
\hline-3882.2 & -5048.5 & 8.0 & 0.0 \\
\hline 13.414 & 28.974 & -4.505 & -4.645 \\
\hline 102.7 & 120.0 & ERR & 120.0 \\
\hline 3.228 & 3,388 & $(150)$ & 8 \\
\hline 3 & 3 & 2,1 & $2: 1$ \\
\hline $4,5,5$ & $4,5,5$ & 3 & $\dot{U}$ \\
\hline 3231.3 & $5 \% 95.7$ & LKR & 1.0 \\
\hline 1935.6 & 3517.3 & ERR & 5936.7 \\
\hline 1.492 & 1.432 & 1.000 & 1.000 \\
\hline 1.437 & 1.492 & 1.000 & 1.000 \\
\hline 1.121 & 2. 1 131 & ERR & ERP \\
\hline 1.525 & & EFR & \\
\hline 0.397 & 6. 18 & ERR & ERP \\
\hline 0.735 & & 0.080 & \\
\hline 0.003 & 0.382 & ERR & ERR \\
\hline 1.000 & 1.471 & CRR & ERR \\
\hline 1.489 & $1.00 \%$ & ERP & ERR \\
\hline 0.0 & & 0.0 & \\
\hline 0.0 & & 0.0 & \\
\hline 21 & & 6 & \\
\hline
\end{tabular}


M LB. MOLESYYCAR

ACTUAL FLATES

FRESSUPE WT HE LPEUISEO :

TEMPERATURE O OREUISEL:

AVEFAGE MOLECULAR WESGHT

GAS DEHSTTY - LEIGF

CROSE SEOTINAL AEEA - SO FT

ELUMIV HEIGHT - FT

ERIIMN EITAMETER

I L $2 M P F$;

H. SHEAT VAPOPIZ. -EtUILE:

G IHEAT CAPACITY - EtLILEIF:

HEAT LOAD - MM DUMUH

GONENSEF COMLNG WATEP - GPM : 1

CALANDFIA STEAM - MFPH 150 PSIG;

COLIMH COST - 1000 JOSE MES - BARE

- FLL EAFEON STEEL

- 0.5 wIDO 5.S. TRAYS

- AH GX STAIHLESE BTEEL

- ALL jRAL STAINLESS BTEEL

- ALL IIG STAINLESS gTEEL

EOMOENSEF OF CALARDPIA G!RFACE -

GNO OR GALANE OOST - 18003086

- CAPQON ETEEL

ZZT STAINLESS STEEL

- JIG STAINLESE STEEL

- MOIIEL

EUETGTHL

SUSTOTAL

SIETETAL

MINIM!JM REFLUX RATIO

CA GUETOTHL \#2

CR SUETOTAL \#2

Or SLETOTAL \#ت

C. CHECH

H. SUETOTAL \#1

H. EUTOIAL \#2

H. BUETOTAL \#

H. SHEEH

MIN, PLATESENGFMAL:

GOL . OOST-EIS MORMAL

COL. COET-S:S MOPMAL

MIN, REFLUK! NOPMAL

O. . APEASHORMAL ;

HEAT LOALINOFMAL

COHAOAL COST:AOFMAL:
DISTILLATION CALCULATIONS

\begin{tabular}{|c|c|c|c|}
\hline 31 & 32 & $\Xi 5$ & 34 \\
\hline LES $\# \vec{L}$ & LES \#2 & LES \#3 & $\angle B S \# 5$ \\
\hline MALEE & TAILS & MAKE & TAILS \\
\hline$\cdots-\cdots$ & $\cdots \cdots \cdots$ & $\ldots-\cdots$ & $\cdots \cdots-\cdots$ \\
\hline 92 & & 0 & \\
\hline 5.819 & & 0 & \\
\hline 100 & & $27 \div$ & \\
\hline 58.05 & 47.91 & ERR & ER⿱口八 \\
\hline 3. 4698 & D. 4153 & ERR & ERR \\
\hline 1.3 & & 8.0 & \\
\hline 153.5 & & 0.6 & \\
\hline 1.5 & & 0.0 & \\
\hline $4 i, 113$ & & $\theta$ & \\
\hline 215.8 & 215.0 & ERR & ERR \\
\hline 0.530 & 0.506 & ERP & ERR \\
\hline 1.250 & 1.227 & 8.80 & 0.000 \\
\hline 31 & & 3 & \\
\hline & 1.45 & & 0.00 \\
\hline
\end{tabular}

$\$ 78.1$

\$.5.9

$\$ 8.9$

$\$ 3.0$

$\$ 125.5$

$\$ 0.0$

\$1 38.1

$\$ 153.5$

122

$12 L$

玉1工,

58

क.

$\$ 0.0$

680

0

\$17.7

$\$ 10.2$

\$2.

$\$ 2.0$

\$18.9

$\$ 14.2$

$\$ 0.0$

$\$ 0.0$

斗 24.5

$\$ 19.8$

5.6

$\$ 0.8$

$\$ 0.0$ \$.

$\begin{array}{rr}450 & 0 \\ 0 & 0 \\ 0 & 0\end{array}$

\begin{tabular}{|c|c|c|}
\hline 8.5 & & 8.0 \\
\hline 20,561 & 5,001 & 0 \\
\hline 8 & 8 & 0 \\
\hline 2 & 0 & 8 \\
\hline 8. 522 & 0.580 & ERR \\
\hline 8,858 & 2,151 & 0 \\
\hline 8 & 0 & 0 \\
\hline$\theta$ & 0 & $\theta$ \\
\hline 215.8 & 215.8 & ERR \\
\hline
\end{tabular}


M LB. MOLES:YEAR

COMPONENT $\# 1$
COMPONENT $\# 2$
COMPONENT $\# 3$
COMPONENT $\# 4$
COMPONENT $\# 5$
COMPONENT $\# 5$
COMPONENT $\# 7$
COMPONENT $\# 8$
COMPONENT $\# 3$
COMPONENT $\# 19$
COMPONENT $\# 11$
COMPONENT $\# 12$
COMPONENT $\# 13$
COMPONENT $\# 14$
COMPONENT $\# 15$
COMPONENT $\# 15$
COMPONENT $\# 17$
COMPONENT $\# 13$
COMPONENT $\# 19$
COMPONENT $\# 20$
WATER
SOLUENT

SOLVENT

TOTAL (G)

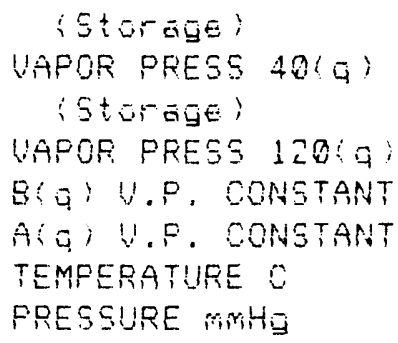

\begin{tabular}{|c|c|c|c|}
\hline \multicolumn{4}{|c|}{ DISTILLATION CALCULATIONS } \\
\hline 35 & 35 & 37 & 38 \\
\hline HBS \#1 & HES \#1 & BYPRODUCT & RAFF \#1 \\
\hline MAKE & TAILS & FEE:I & FEEO \\
\hline 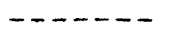 & $\cdots \cdots-$ & $\cdots-\cdots-$ & $-\cdots-\cdots$ \\
\hline 0 & $B$ & 0 & 0 \\
\hline 0 & 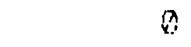 & 0 & 0 \\
\hline 0 & 0 & 250 & 250 \\
\hline 0 & 0 & 0 & 2 \\
\hline D & 6 & 385 & 424 \\
\hline 0 & 3 & 8 & 8 \\
\hline 12 & 0 & 17 & 17 \\
\hline 297 & 1 & 290 & 290 \\
\hline 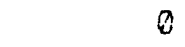 & 0 & 0 & 0 \\
\hline 78 & 8 & 78 & 78 \\
\hline 3 & 0 & 0 & a) \\
\hline 0 & 0 & 0 & 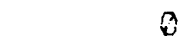 \\
\hline 0 & 0 & 8 & 0 \\
\hline$\theta$ & 0 & 0 & 0 \\
\hline 8 & $\theta$ & 0 & b) \\
\hline 0 & 8 & 0 & 0 \\
\hline 3 & 8 & $\theta$ & $\theta$ \\
\hline 0 & $\theta$ & $\infty$ & 8 \\
\hline 0 & $\theta$ & 0 & 0 \\
\hline 0 & 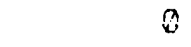 & $\theta$ & 3 \\
\hline 2 & 1 & 45,792 & $47: 779$ \\
\hline 0 & 15.843 & 8 & 0 \\
\hline$\cdots \cdots-$ & $--\cdots-\cdots$ & $-\cdots-\cdots$ & $\cdots-\cdots$ \\
\hline 333 & 15,046 & 47,723 & 48,838 \\
\hline 11 & 8 & 152 & $15 ?$ \\
\hline 27.7 & 8.1 & 55.8 & 55.8 \\
\hline $2 E J$ & $\frac{1}{2}$ &, \pm 831 & 3,925 \\
\hline 577.7 & 15.8 & 1557.6 & 1558.0 \\
\hline-4917.5 & -7747.8 & -5090.5 & -5091.2 \\
\hline 19.032 & 23.531 & 20.303 & 20.305 \\
\hline 123.4 & 드으. & 34.8 & 33.5 \\
\hline 753 & 913 & 758 & TEO \\
\hline
\end{tabular}

$10,3.8$ 10, 9.8
SOLUENT SOLUENT

$\begin{array}{rr}182.5 & 3915.4 \\ 19.7 & 939.4 \\ 2.117 & 2.117 \\ 2.858 & 2.858 \\ 3.255 & 8.598 \\ 5.927 & \\ 0.999 & .900 \\ 0.905 & \\ 0.801 & 1.900 \\ 1.200 & 2.117 \\ 2.954 & 1.006 \\ 10.9 & \\ 13.5 & \\ 10 & \end{array}$


M LE. MOLES YEAR

AETLIAL PLATES

PEESGURE WA HE R REUISED ?

TEMEERATLFE O TREUISED:

AUERAGE MELECULAR WEIGHT

GAS DENEITY - LDIOF

CEOSE SEOTIONAL AFEA - SE FT

EOLUMH HEIGHT - ET

COLUM DIAMETER

H. 1 :MFP;

H. : HEAT UAPORI?.-QUWLE:

Gr SHEAT CAPACITY - EtLILAF;

HEAT LOAD - MM BTWIHE

CONEENEF COOLINE WATER - EPM I

CALANRPIA GTEAM - MPFH ISO PSIE;

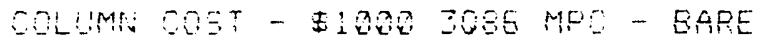

- ALL EAREON BTEEL

- C. W DOA E.5. TFAU

- ALL JOA STAINLESE STEEL

- ALL JRAL STAINLESS GTEEL

- HLL JL OTATNLESE GTEEL

OOMENGER OR CALANOFIA SURFAOE -

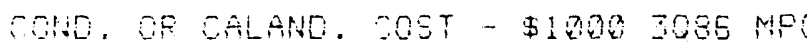

- CAREOR BTEE:

- TR4 ETAINLESS BTEEL

- IE STAINLSS STEEL.

MUVEL

\footnotetext{
BUETSTAL

GETGTAL.

GESTOAL

MIUYMU FEFLUX RATIO

ET EUETOTAL \#1

CA SUETETAL \#2

CA SUETOTAL \#Z

SA CHEC:

H. EUETOTHL \#1

H. SUETOTAL \#2

H. SIETGTAL \#

H. GUEO

MIN. FLATESEMOFMAL;

GO . OST- IS WOFMAL

COL DOST-SIS HORMAL

MANAEFLOR MORMAL:

○ O AREA HOFMAL

HEAT LOAES NOPWAL

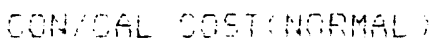

DISTILLATION CALCULATIONS

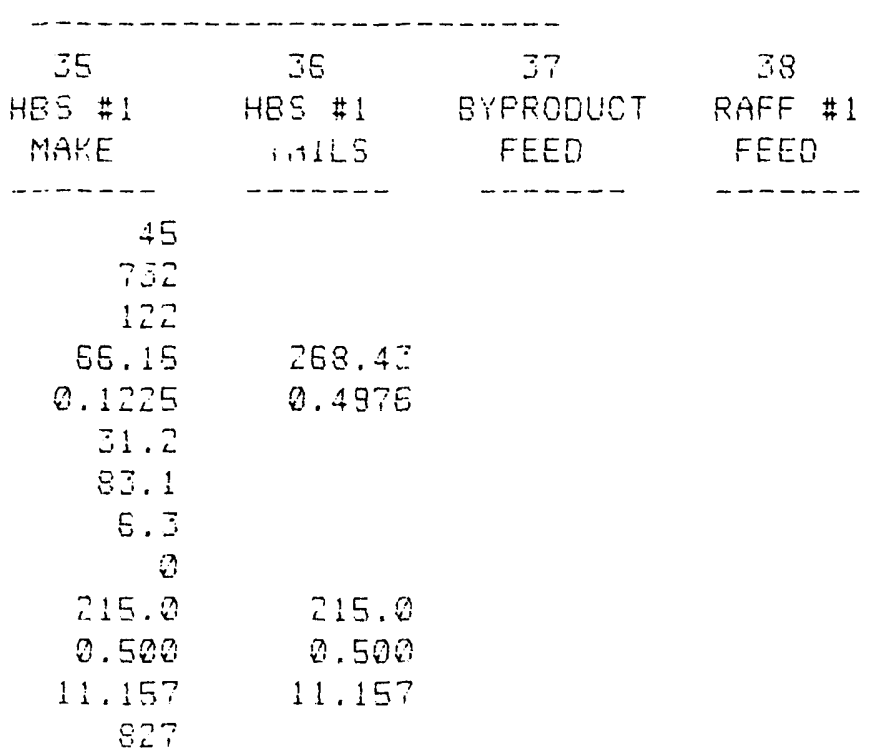

13.02

\$155.2

$\$ 181.7$

\$252.5

\$298.7

$\$ 54.3$

838

520

\$ES.5 \$2马.9

$\$ 51.1 \quad \$ 41.8$

$\$ 54.8 \quad \$ 44.8$

\$1.2 \$58.2

2.153,554

2., 15:, 502

2.153:502

18.9

455

12. 255

0. $50 x^{7}$

195

5,317

215.0$$
2.150 .502
$$

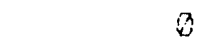

2.155 .502

6. 500

ด

925. 2015

215.0 
M LB. MOLES, YEAR

COMPONENT $\# 1$
COMPONENT $\# 2$
COMPONENT $\# 3$
COMPONENT $\# 4$
COMPONENT $\# 5$
COMPCNENT $\#$ TE
COMPONENT $\# 7$
COMPONENT $\# 8$
COMPONENT $\# 9$
COMPONENT $\# 10$
COMPONENT $\# 11$
COMPONENT $\# 12$
COMPONENT $\# 13$
COMPONENT $\# 14$
COMPONENT $\# 15$
COMPONENT $\# 15$
COMPONENT $\# 17$
COMFONENT $\# 13$
COMPONENT $\# 13$
COMPONENT $\# 20$
WATER
SOLVENT

TOTAL GG

(StOTEGE)

VAPOR PRESS $40(q)$

\{StUTEIES

VAPOR FRESS $120(q)$

E( U) U.P. CONGTANT

ALG: V.P. CONSTANT

TEMFERATURE $C$

PRESSURE $\mathrm{MMHG}$

ㄴ (COMPONENT \#)

K2 COOMPONENT \#)

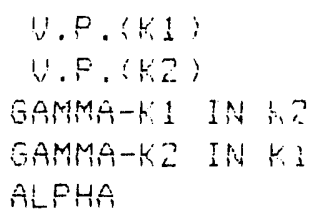

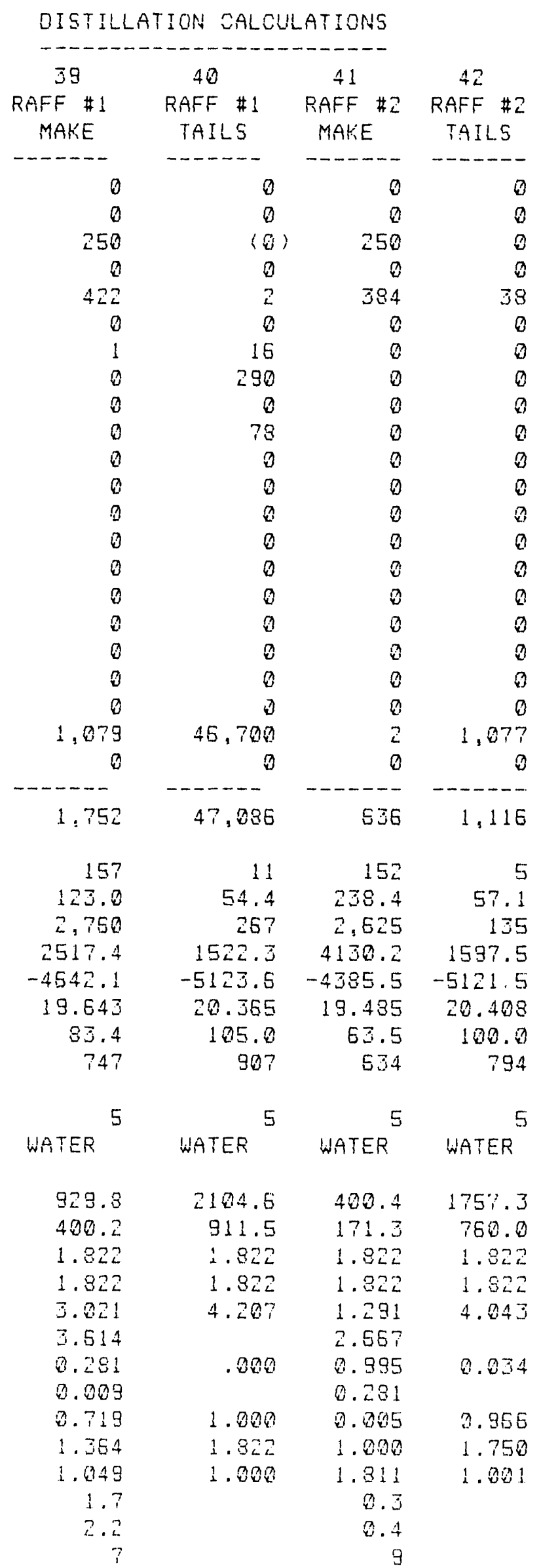


M LS. MOLESIYEAR

\section{ACTUAL PLATES}

PRESSURE MM HG (REUISED)

TEMPERATURE C 〈REUISED)

AUERAGE MOLECULAR WEIGHT

GAS DENSITY - LBICF

CROSG SECTIONAL AREA - SO FT

COLUMN HEIGHT - FT

COLUMN DIAMETER

$\therefore 1$ (MPPY)

HV (HEAT VAPORIZ, -BtulLb;

Cri (HEAT CAPACITY - BtuiLLiF)

HEAT LOAD - MM BtLIHT

CONDENSER COOLING WATER - GPM 41

CALANDRIA STEAM - MPFH I 150 PSIG;

COLUMN COST - \$100R 3QBE MPC - BARE EQUIPMENT

- ALL CARBON STEEL

- C.S WISRA S.S. TRAYS

- ALL 3O4 3TAINLESS STEEL

- ALL 3OAL STAINLESS STEEL

- ALL JIG STAINLESE STEEL

CONDENSER OR CALANDRIA SURFACE -

CONO. OF CALAHD. COST - \$1900 3085

- CAREON STEEL

- 304 STAINLESS STEEL

- 15 STAINLESE STEEL

MONEL

SUDTOTAL

SUETOTAL

SURTOTAL

MINIMUM REFLUX RATIO

ON SLETOTAL \#1

CI SUETOTAL \#2

CH SUBTOTAL \#3

Cr CHECK

HY SUETOTAL \#1

H. SUETOTAL \#2

H. SUETOTAL \#J

HV CHECY.

MIN. PLATESANOFMAL)

COL.COST-OIS NORMAL

COL.COST-SIS NORMAL

MIN.REFLUX NORMAL!

C. S. AREA:NORMAL)

HEAT LDADSNORMAL!

COWUCAL COST INORMAL)
DISTILLATION CALCULATIONG

\begin{tabular}{|c|c|c|c|}
\hline 39 & 48 & 41 & 42 \\
\hline RAFF \#1 & RAFF \#1 & RAFF \#Z & PAFF \#2 \\
\hline MALE & TAILS & MAKE & TAILS \\
\hline$--\cdots--$ & $\cdots \cdots$ & $-\cdots-\cdots$ & $-\cdots \cdots--$ \\
\hline 32 & & 40 & \\
\hline 779 & & 635 & \\
\hline 85 & & 54 & \\
\hline 30.51 & 18.58 & 50.72 & 13.98 \\
\hline 0.0555 & 0.0444 & 0.8959 & 0.0404 \\
\hline 19.2 & & 4.1 & \\
\hline$E 3.1$ & & 74.3 & \\
\hline 4.9 & & 2.3 & \\
\hline 19,442 & & 17.574 & \\
\hline 489.7 & 943.8 & 215.8 & 307.1 \\
\hline 0.682 & 0.985 & 0.501 & 0.953 \\
\hline 11.505 & 26.575 & 1.313 & 1. 391 \\
\hline 853 & & 97 & \\
\hline & 31.13 & & 1.52 \\
\hline
\end{tabular}

\$102. 4

$\$ 112.6$

$\$ 153.8$

$\$ 180.2$

$\$ 22 i, 1$

1,465

1,482

$\$ 60.5$

$\$ 56.5$

$\$ 97.9$

$\$ 107.7$

\$132.2

271

77

$\$ 54.8$

$\$ 75.7$

$\$ 82.2$

$\$ 55.3$

$\$ 77.4$

\$19.4

\$25. 8

क77.5

$\$ 32.9$

$\$ 55.9$

\$10.6

$\$ 14.9$

$\$ 15.9$

क20.7

19,409

19,406

13.405

Q. 3

1.7

354,105

841:352

841,362

17.805

541

12,146

1. 3.442

6. 582

7,312

341,362

๑. 935

275

5. 223

18,864

489.7
B15, 359

348.0
15, 120

0
-55

0. 501

E, $9: 1$

8

34

215.8
385
0
13.405
0.958
381
0
18.830
907.1 
M LB. MOLES Y YAAR

COMPONENT $\# 1$
COMPONENT $\# 2$
COMPONENT $\# 3$
COMPONENT $\# 4$
COMPONENT $\# 5$
COMPONENT $\# 5$
COMPONENT $\# 7$
COMPONENT $\# 8$
COMPONENT $\# 9$
COMPONENT $\# 10$
COMPONENT $\# 11$
COMPONENT $\# 12$
COMPONENT $\# 13$
COMPONENT $\# 14$
COMPONENT $\# 15$
COMPONENT $\# 16$
COMPONENT $\# 17$
COMPONENT $\# 18$
COMPONENT $\# 13$
COMPONENT $\# 20$
WATER
SOLVENT

TOTAL (

S Storage:

VAPOP PRESS 40(

(Storage)

VAPOR PRESS 120 (A)

EIQ) U.F. CONGTANT

ALG; U.P. CONSTANT

TEMPERATURE O

PRESSURE MMHG

K1 COOMPONENT \#)

YZ S COMPONENT \#

$$
\text { U.F. (K1) }
$$$$
\text { U.P.SKL; }
$$

GAMMA-KI IN $K 2$

GAMMA-KI IN KI

ALFHA

AUE COLUMA ALPHA

MOL FRACT. KI MAYKE OR TAILS)

MOL FRACT. K1 (FEED)

MOL FRACT. KL (MAKE OR TAILS)

ADJ. GAMMA-H IN IN

AOJ. GAMMA-KL IN K1

MINIMUM REFLUX RATIO (ADUUSTED)

ACTUAL REFLUX RATIO

MINIMUM PLATES
DISTILLATION CALCULATIONS

\begin{tabular}{|c|c|}
\hline 43 & 44 \\
\hline RAFF $\#$ & RAFF \#כ \\
\hline MAKE & TAILS \\
\hline$-\cdots \cdots$ & $\ldots$ \\
\hline 0 & $\theta$ \\
\hline 0 & . \\
\hline 249 & 1 \\
\hline 0 & D \\
\hline 1 & 383 \\
\hline 0 & 0 \\
\hline 0 & 0 \\
\hline 0 & 0 \\
\hline 0 & 0 \\
\hline 0 & a \\
\hline 0 & 0 \\
\hline 0 & 0 \\
\hline$D$ & 0 \\
\hline D & 0 \\
\hline 0 & 0 \\
\hline 0 & 0 \\
\hline 0 & $\theta$ \\
\hline$D$ & a \\
\hline a & 0 \\
\hline 0 & 8 \\
\hline 8 & 2 \\
\hline 0 & 0 \\
\hline$\ldots$ & $\ldots$ \\
\hline 250 & 387 \\
\hline 102 & 50 \\
\hline 407.3 & 129.1 \\
\hline 1,271 & 1,354 \\
\hline 5032.7 & 3509.1 \\
\hline-3882.2 & -5078.0 \\
\hline 18.414 & 21.08 .4 \\
\hline 85.0 & 105.0 \\
\hline 1,941 & 2,101 \\
\hline 3 & 3 \\
\hline 5 & 5 \\
\hline 1943.6 & 3.444 .3 \\
\hline 894.2 & 2104.5 \\
\hline 1.432 & 1.492 \\
\hline 1.432 & 1.492 \\
\hline 1.313 & 2.435 \\
\hline 1.874 & \\
\hline 0.397 & 0.003 \\
\hline 0.335 & \\
\hline 0.003 & 0.997 \\
\hline 1.000 & 1.483 \\
\hline 1.489 & 1.000 \\
\hline 0.7 & \\
\hline 0.3 & \\
\hline 19 & \\
\hline
\end{tabular}




\section{LB. MOLESIYEAR \\ ACTUAL PLATES}

PRESSURE MIM HO (REUISED)

TEMPERATURE C (REUISED)

AUERAGE MOLECULAR WEIGHT

GAS DENSITY - LBICF

CROSG SECTIONAL AREA - SO FT

COLUMN HEIGHT - FT

COLUMA DIAMETER

S 1 \&MPPY

HV (HEAT VAFORIZ, -EtUILE)

CI (HEAT CAPACITY - BtUILEIF)

HEAT LOAD - MM ELU/HR

CONDENGER COOLING WATER - GPM :

CALANOFIA STEAM - MPFH (150 PSIG)

COLUMA COST - \$2000 3085 MEC

- ALL CARBON STEEL

- C.S WIJR4 S.5. TRAYS

- ALL 304 STAINLESS STEEL

- ALL 3O4L STAINLESS STEEL

- ALL jIE STAINLESS STEEL

CONDENSER OR CALANDRIA SURFACE -

COND. OR CALAND. COST - \$1QOO $30 S$

-CAREON ZTEEL

- 304 STAINLESS STEEL

- JIE STAINLESS STEEL -MONEL.

\section{SUBTOTAL \\ SUIBTOTAL \\ SURTOTAL \\ MINIMUM REFLUX RATIO \\ CTI SUETOTAL \#1 \\ CN SUETOTAL \#2 \\ CT SUBTOTAL \#3 \\ Cr CHECL}

HV SUETOTAL \#I

HV SUBTOTAL \#2

HV SUBTOTAL \#3

HV CHECK

MIN. PLATES(NORMAL)

COL.COST-CIS NORMAL

COL.COST-S/S NORMAL

MIN.REFLUX? NORMAL)

C.S. AREA NORMAL)

HEAT LOADSNORMAL)

CONICAL COST:NORMAL)
DISTILLATION CALCULATIONS

\begin{tabular}{|c|c|}
\hline 43 & 44 \\
\hline RAFF \#3 & RAFF \#ت \\
\hline MARE & TAILS \\
\hline$\cdots-\cdots$ & $---\cdots$ \\
\hline 84 & \\
\hline $\begin{array}{r}1.766 \\
82\end{array}$ & \\
\hline 58.35 & 46.06 \\
\hline 0.2830 & 6.255 \\
\hline 1.5 & \\
\hline 148.8 & \\
\hline 1.4 & \\
\hline 14,457 & \\
\hline 215.0 & 215.5 \\
\hline 0.500 & 0.50 \\
\hline 0.335 & Q. 925 \\
\hline 52 & \\
\hline
\end{tabular}

$\$ 68.1$

$\$ 74.9$

$\$ 109.9$

$\$ 120.9$

$\$ 148.3$

$112 \quad 52$

MPC - BARE EOUIPMENT

\$12.2 \$3.3

$\$ 17.1 \$ 13.0$

$\$ 18.3 \$ 14.0$

$\$ 23.8 \$ 18.2$

$\begin{array}{rr} & 53 \\ & 35 \\ 0.7 & 35 \\ 7,243 & 3,877 \\ 0 & 0 \\ 0 & 35 \\ 0.500 & 0.501 \\ 3.114 & 3.817 \\ 0 & 0 \\ 0 & 54 \\ 215.0 & 215.5 \\ 58 & \\ 110 & \\ 2 & \\ 12 & 1\end{array}$


APPENDIX B. BASECASE STOICHIOMETRY WITH 15:1 RAFFINATE/CELLS RECYCLE NO RECOVERY OF RAFFINATE CHEMICALS 
MULTISTAGE EXTRACTIUE FERMENTATION OF EUTANOL NO RECOUERY OF RAFFINATE

BASECASE STOICHIOMETRY

SUMMARY

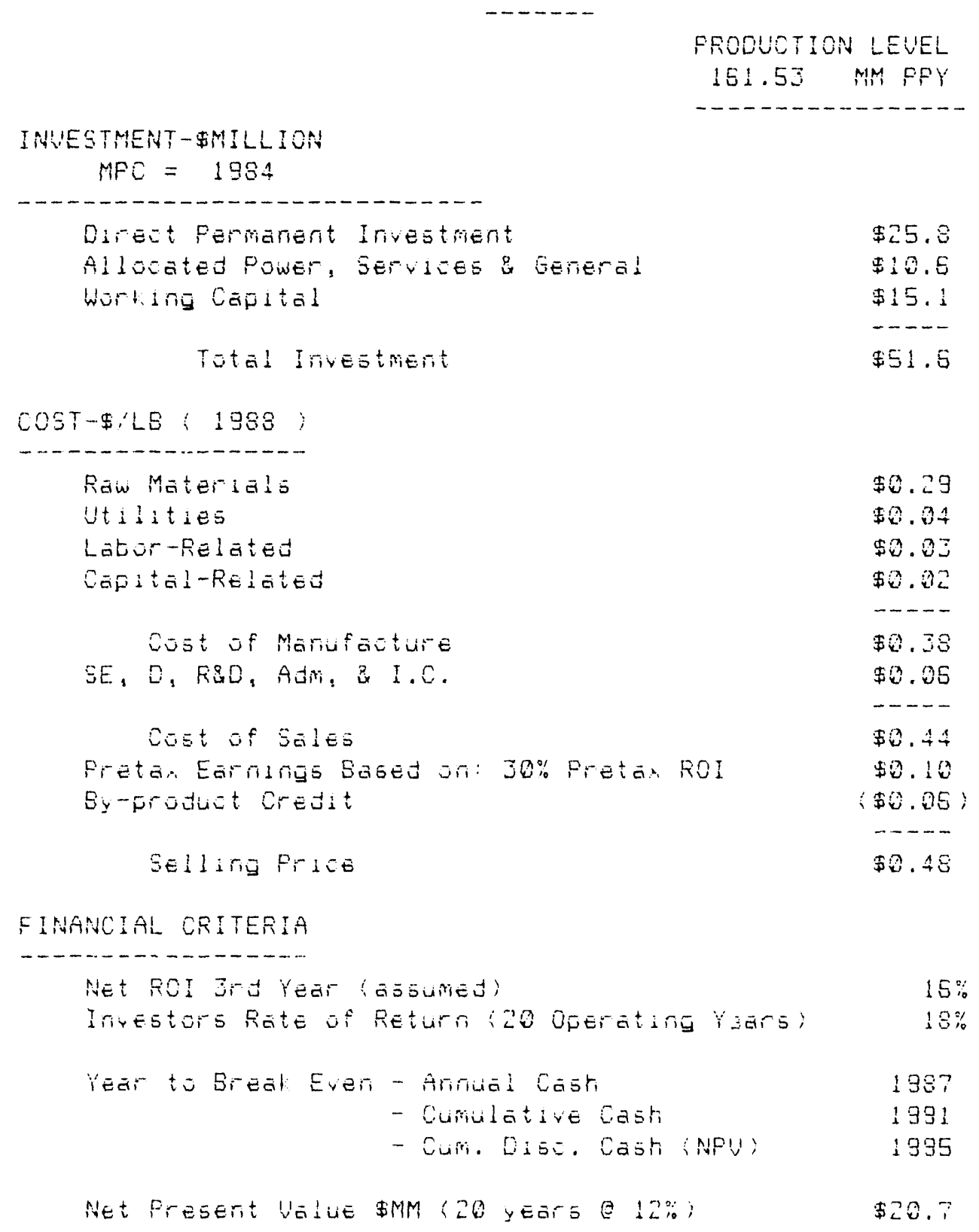




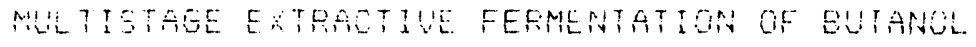

W PECOUEFY OF RAFFINATE

BASECASE STOHCHIOMETPY

INUESTMEN?

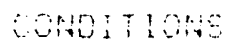

- . - ........

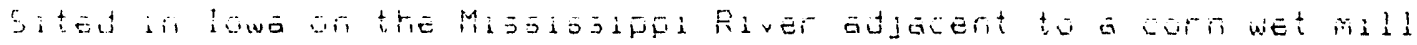

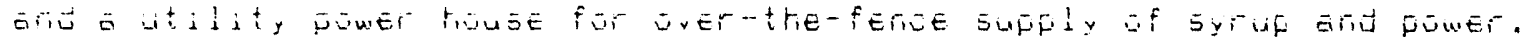

\begin{tabular}{|c|c|c|c|c|}
\hline & UNITS & & $\begin{array}{l}\text { TH1S } \\
\text { CASL } \\
\ldots . . .\end{array}$ & \\
\hline CAFACITY O OOGG HFS & $\mathrm{MMPY}$ & & 179.5 & \\
\hline 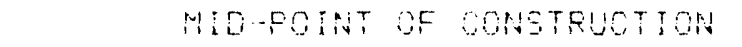 & YEAR & & 1984 & \\
\hline CONGTFUETION COEY MNEX & $198 x=19 x$ & & 120 & \\
\hline JHAESTMENT OOHT TNGENGY & : INGTALLED & * & $33:$ & \\
\hline 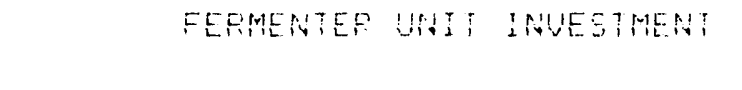 & $\begin{array}{l}\text { T:GR.GAL. - } \\
\text { \$:GR.GAL. - }\end{array}$ & $\begin{array}{l}00 \\
0,46\end{array}$ & $\begin{array}{l}+12 \cdot 92 \\
+16.94\end{array}$ & \\
\hline 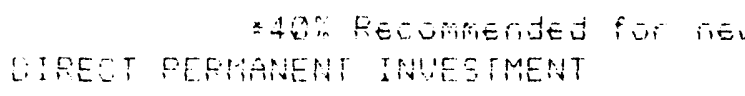 & FUすES5ES & & & \\
\hline 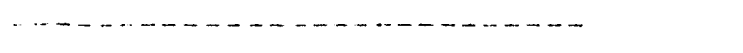 & SCALE & & TH & $\Xi C A S E$ \\
\hline & FAOTSR & & IMM & W ANH.LB. \\
\hline & $\cdots-\cdots$ & & $\cdots-\cdots$ & $---\cdots--$ \\
\hline 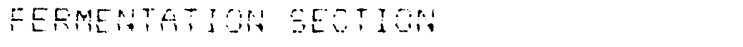 & & & & \\
\hline 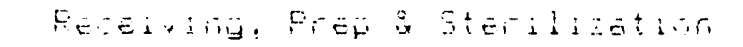 & $\Leftrightarrow .5 \hat{x}$ & & 4.88 & $\$ 0.822$ \\
\hline 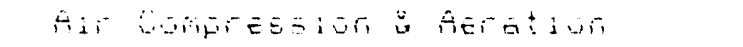 & A. Eis & & 8.03 & 0.800 \\
\hline Fermentatisin & $2.89 \cdots 1.80$ & & 7.46 & 0.042 \\
\hline E.; & & & 5.52 & Q \\
\hline 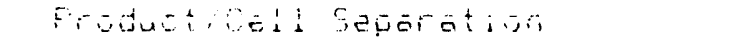 & 8.75 & & 0.37 & 0.085 \\
\hline & & & $\cdots-\cdots$ & $-\cdots-\cdots$ \\
\hline 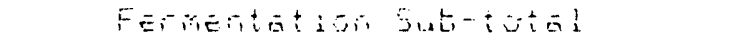 & & & $\$ 17.94$ & 90.100 \\
\hline QSOTYLLATOON SEOTIOH & STILLS & $H X \cdot 5$ & & \\
\hline BeE & $\pm(\hat{z}) .31$ & \$1. & $\$ 1,3$ & $\$ 0.907$ \\
\hline 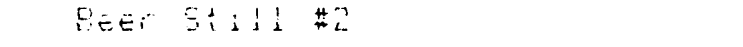 & 8.20 & D. 21 & 0.41 & 0.802 \\
\hline 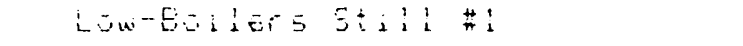 & 6. 23 & A. $1 \%$ & 0.40 & B. 102 \\
\hline 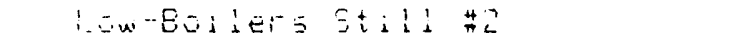 & A. 35 & A. 11 & 8.4. & 0.085 \\
\hline 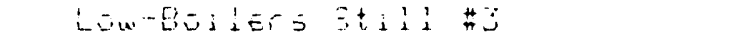 & A. .8 & A. $\theta 0$ & $0.0 \hat{i}$ & 0.306 \\
\hline 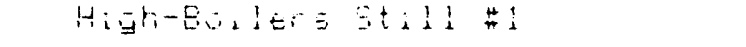 & 2.75 & 0.31 & $\therefore .87$ & 0.005 \\
\hline$r:-1+1-1+1$ & & & 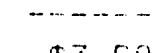 & 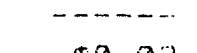 \\
\hline 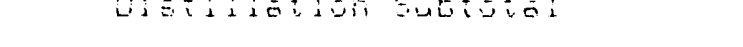 & & & $7 \div .58$ & 90.02 \\
\hline SRAGE SEOTION & & & & \\
\hline Stor age - Produst & & & $\$ 3.45$ & $\$ 2.019$ \\
\hline 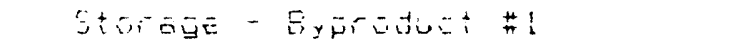 & & & 97.78 & 0.004 \\
\hline & & & $\cdots-\cdots-$ & $-\cdots--$ \\
\hline 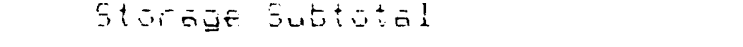 & & & $\$ 4.24$ & $\Phi\left[\begin{array}{l}\lambda \\
0\end{array}\right.$ \\
\hline TETAL OIFEOT PLANT & & & $\$ 25.35$ & $E 0.142$ \\
\hline
\end{tabular}


MULTISTAGE EXTRACTIUE FERMENTATION OF BUTANOL

NO RECOVERY OF RAFFINATE

BASECASE STOICHIOMETPY

INUESTMENT

ALLOCATED PERMANENT INVESTMENT

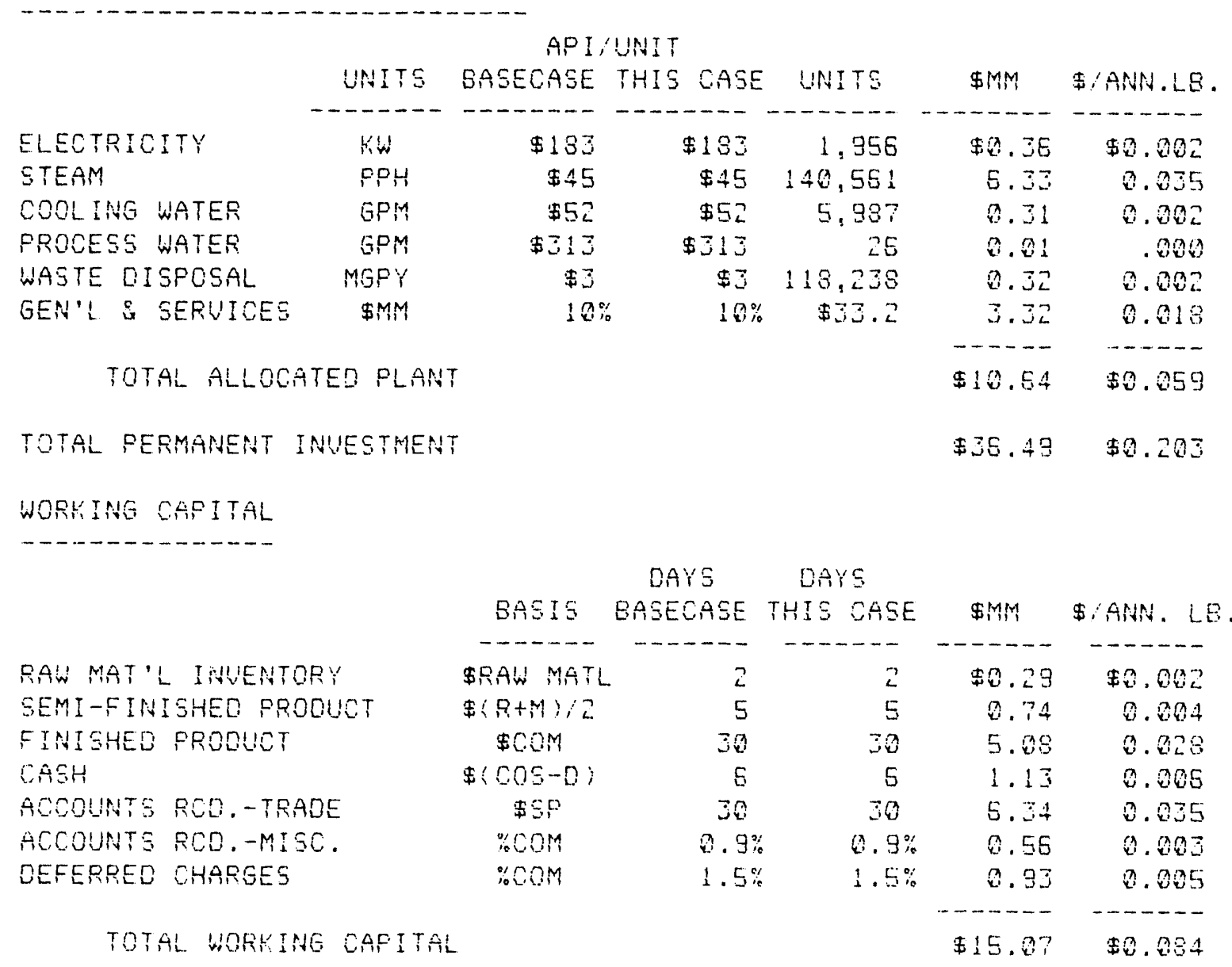

Note: $P=$ row materials: $M$ or COM = oot of manufacture;

COS = Esst of sales; SP = SElling price; D = Jefrediation.

$\$ 51.56 \quad+2.287$ 
MUL.TISTAGE EXTRACTIUE FERMENTATION OF BUTANOL NO RECOUERY OF RAFFINATE BASECASE GTOIOHIOMETRY

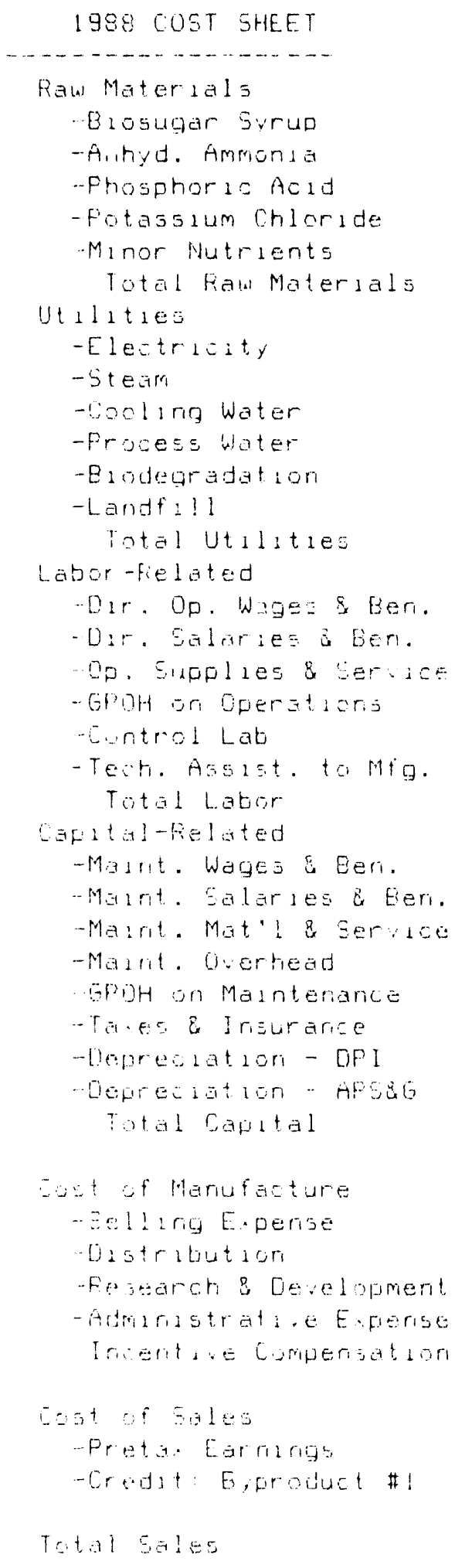

\begin{tabular}{|c|c|c|c|c|}
\hline RATE & IUNIT & $\begin{array}{l}\text { MILLLION } \\
\text { UINITS }\end{array}$ & \$MIL.LION & $\$ / 16$. \\
\hline$\ldots \ldots \ldots$ & $\cdots \cdots$ & $\cdots-\cdots-$ & $-\cdots-\cdots$ & $\cdots \cdots$ \\
\hline$\$ 0.065$ & ito. d.s. & 701.02 & $45.5 \%$ & 0.282 \\
\hline$\$ 0.046$ & itu. & 1.70 & 0.08 & .000 \\
\hline$\$ 0.155$ & ito. & 1.42 & 0.22 & 0.001 \\
\hline$\$ 0.053$ & ib. & 1.08 & 0.06 & .000 \\
\hline$\$ 0.451$ & / 1 t. & 0.49 & $0 . I I$ & 0.001 \\
\hline & & & $\$ 46.14$ & $\$ 0.286$ \\
\hline$\$ 0.040$ & AWH & 13.94 & 0.56 & 0.003 \\
\hline$\$ 2.20$ & M Ib. & 1.0013 & 2.20 & 0.011 \\
\hline$\$ 0.04$ & $M$ पail. & $\therefore .56$ & 0.10 & 0.001 \\
\hline 90.50 & 'M gal. & 0.01 & 0.01 & .000 \\
\hline$\$ 0.04$ & $\therefore$ Ib. d.s. & 100.74 & 4.03 & 0.025 \\
\hline$\$ 0.95$ & ith. d.5. & 0.00 & 0.00 & 0.000 \\
\hline & & & \$日. .11 & $\$ 0.043$ \\
\hline$\$ 25.40$ & /manthr. & 0.117 & 3.08 & 0.019 \\
\hline 18 & $\%$ DOW\&B & & 0.56 & 0.003 \\
\hline$E$ & $\% \quad 00 \omega \& B$ & & 0.19 & 0.001 \\
\hline 23 & $\%$ FGWSEG & & 0.34 & 0.005 \\
\hline \$19.วこ & man-hr. & 0.020 & 0.59 & 0.002 \\
\hline$\$ 22.06$ & $\operatorname{man} \cdots \mathrm{hr}$ & 0.003 & 0.0 .4 & .000 \\
\hline & & & $\$ 5.09$ & $\$ 0.032$ \\
\hline 1.7 & $\%$ UPPI & $\$ 25.0$ & 0.44 & 0.003 \\
\hline 25 & \% MWBB & & 0.11 & 0.001 \\
\hline 40 & $\%$ MWEB & & 0.18 & 0.001 \\
\hline 4 & $\%$ MWüB & & $0.0 \%$ & .000 \\
\hline $2 \ddot{3}$ & $\%$ MWS\&E & & 0.13 & 0.001 \\
\hline 0.3 & $\%$ DPI & $\$ .25 .8$ & 0.08 & .000 \\
\hline e & $\%$ DPI & $\$ 25.8$ & $\therefore .07$ & 0.013 \\
\hline$E$ & $\%$ APS\&E & $\$ 10 . E$ & 0.64 & 0.004 \\
\hline & & & $\$ 3.65$ & $\$ 0.023$ \\
\hline & & & $\ldots \ldots$ & $-\cdots \ldots \ldots$ \\
\hline & & & $\$ C 1.79$ & $\$ 0.383$ \\
\hline 3 & $\%$ Sales & $\$ 77.1$ & $2 . \pm 1$ & 0.014 \\
\hline$\$ 0.01$ & itt. & 161.5 & 1.65 & 0.010 \\
\hline 5 & $\%$ Bales & $\$ 7 \% .1$ & 3.47 & 0.021 \\
\hline ?. & $\because$ : ajes & $\$ 77.1$ & 1.54 & 0.010 \\
\hline$G$ & $\because$ FTE & $\$ 15.5$ & 0.93 & Q). $.00 E$ \\
\hline & & & $\ldots \ldots$ & $\ldots \ldots$ \\
\hline & & & $\$ 1.6 E$ & \$0. 4.44 \\
\hline 30.0 & $\because$ IIFF & $\$ 51.6$ & 15.47 & (1). $99 G_{j}$ \\
\hline$\$ 0.27$ & itt. & 57.0 & $(9.99)$ & $\left(O . O E_{2} ?\right)$ \\
\hline & & & $\ldots \ldots$ & $\cdots---$ \\
\hline & & & $\$ 77.14$ & $\$ 0.478$ \\
\hline
\end{tabular}


APPENDIX C. BASECASE STOICHIOMETRY

NO RAFFINATE/CELLS RECYCLE

WITH RECOVERY OF RAFFINATE CHEMICALS 
MULTISTAGE EXTRACTIUE FERMENTATION OF BUTANOL

WITH RECOUERY OF RAFFINATE

BASECASE STOICHIOMETPY

\section{SUMMARY}

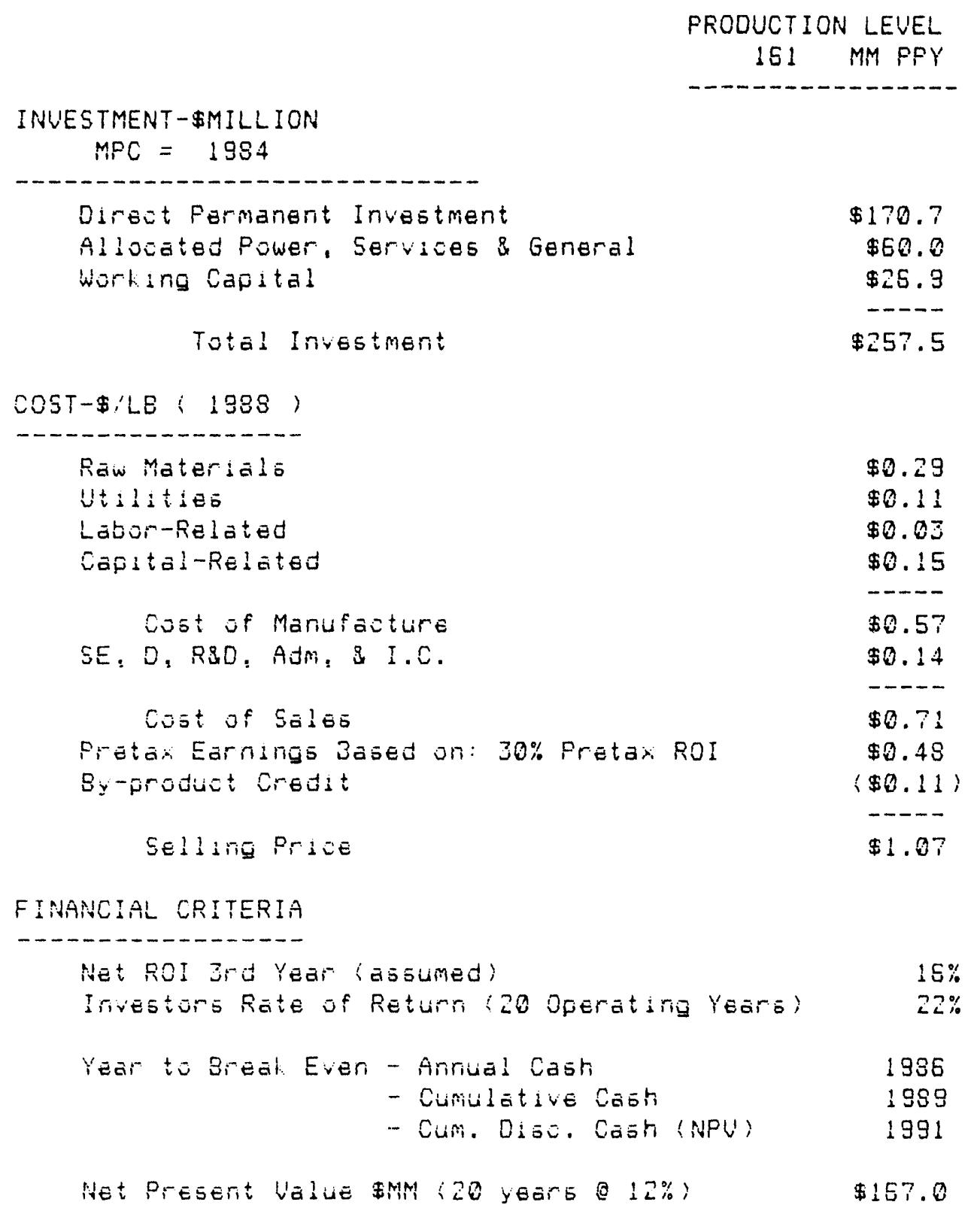


MULTISTAGE EXTRACTIUE FERMENTATION OF BUTANOL

WITH RECOVERY OF RAFFINATE.

BASECASE STOICHIOMETRY

INUESTMENT

CONOITIONE

Sited in lowa on the Misaissippi Rlver adjacent to a corn wet mill and a litility power house for voer-the-fence supply of syipo and power.

\begin{tabular}{|c|c|c|c|c|}
\hline & $\begin{array}{l}\text { UNITS } \\
\text { HN- }\end{array}$ & & $\begin{array}{l}\text { THIS } \\
\text { CASE } \\
-1 .-\end{array}$ & \\
\hline CAPACITY O 8000 HRS & MM PPY & & 173.4 & \\
\hline MID-POINT OF CONGTRUCTION & YEAR & & 1934 & \\
\hline CONGTRUETION COST INOEX & $1380=100$ & & 128 & \\
\hline INUESTMENT CONTINGENCY & $\%$ INSTALLE & & $30 \%$ & \\
\hline FERMENTER UNIT INUESTMENT & \$ /GR.GAL. - & OWTH & $\$ 10.94$ & \\
\hline $\begin{array}{l}\text { *40\% REOUMmERJEd for ne } \\
\text { DIRECT PERMANENT INUESTMENT }\end{array}$ & $\begin{array}{l}\text { \$GR.GAL. } \\
\text { PHOCES5E }\end{array}$ & $D D, n$ & $\$ 10.94$ & \\
\hline 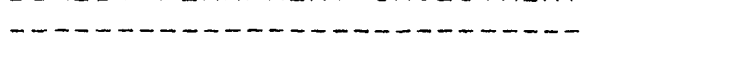 & SCALE & & $\mathrm{TH}$ & $\Xi$ CASE \\
\hline & FACTOR & & FMM & \$/ANHL: S. \\
\hline & $--\cdots$ & & $\cdots \cdots$ & 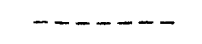 \\
\hline FERMENTPTION SECTION & & & & \\
\hline Receiving: Prep \& Sterilization & 0.50 & & $\$ 20.35$ & $\$ 0.113$ \\
\hline 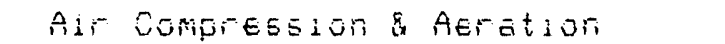 & 0.50 & & 0.00 & 0.000 \\
\hline Fermentation & $0.39-1.00$ & & 117.07 & 0.552 \\
\hline Extrotioti & & & 7.15 & 0.840 \\
\hline Product/Cell Separation & 0.75 & & 7.74 & 0.043 \\
\hline Ferrientation Sut-total & & & $\$ 152.32$ & $\$ 0.849$ \\
\hline DISTILLATION SECTION & STILLS & $4 \times 9$ & & \\
\hline BEET St:11 \#1 & $\$ 0.29$ & $\$ 1.37$ & $\$ 1.35$ & $\$ 0.009$ \\
\hline BeEt Still \#2 & 0.18 & 0.21 & 0.39 & 0.032 \\
\hline Low-Builers Stili \#1 & 0.11 & 0.09 & 0.20 & 0.001 \\
\hline Low-Doliers Still \#2 & 0.13 & 0.07 & 2.25 & 0.001 \\
\hline Low-Boilers Sti11 \# & 0.00 & 0.00 & 0.20 & 0.030 \\
\hline Higri-Eoilers Stidi \#1 & 0.82 & 0.30 & 1.12 & 0.005 \\
\hline Roffinate Still \#1 & 2.03 & 7.04 & 3.12 & 0.051 \\
\hline Raffinate Stil12 \#2 & 0.35 & 0.20 & 0.55 & 0.003 \\
\hline Raffiriate Stili \#J & 0.37 & 0.12 & 0.43 & 0.003 \\
\hline Ristillation Suttotal & & & $\$ 13.47$ & $\$ 2.03$ \\
\hline STORAGE SECTION & & & & \\
\hline Strirage - Dutariol & & & $\$ 3.45$ & $\$ 0.019$ \\
\hline Storage - Adeturie & & & $\$ 1.67$ & 0.005 \\
\hline Storage - Ethanol & & & $\$ 0.37$ & 0.002 \\
\hline & & & $-\cdots \cdots$ & $\cdots \cdots$ \\
\hline Stringe Sutototal & & & $\$ 4.89$ & $\$ 0.03$ \\
\hline TOTAL DIRECT FLANT & & & $\$ 170.59$ & $\$ 0.351$ \\
\hline
\end{tabular}




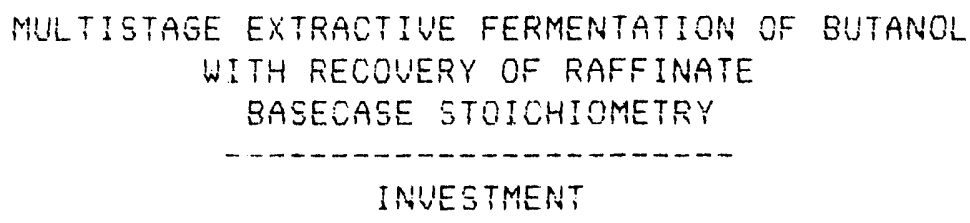


MU TISTAEE EXTEAETIUE FERMENTATION OF DUTABOH WITH REQOUERY OF RAFFINATE BASEOAE STOIOHIOMETRY

FPICES E COET FATTORS

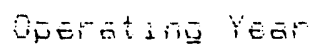

Raw Molerials

- Bujugar Sytug

- Arityd. Amonio

-Progetrota fojä

Potessiun Chít de

-Mirior Mutionts

\section{Utilities \\ - chistiditity \\ - Sten \\ - Coolitie dater \\ -Froege water

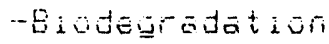 \\ -Lêtifil}

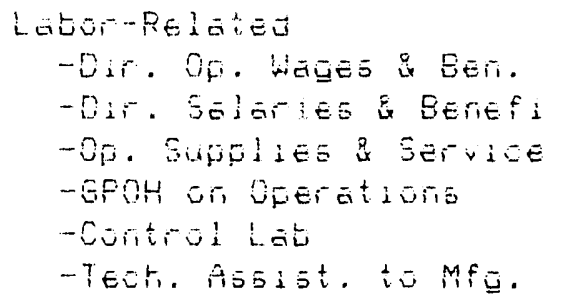

Capital-Reinted

-Marit. Wagé \& Beri.

-Mairt. jalariés Bún Ber.

-Marit. Mat'l s Servide

Mairit. Overriead

-GPOH or Mantenance

-Tábs i Irisurarise

- Depreaiation - DPI

-DEFTESIEtion - APS品

Cust of Marufãture

- Sellirg Ergente

- Distiritutivi

- Researri d Develuprierit

-Adrinistrative E,perise

-Iricentive Cumperisation

8ASEOASE
1989

\$2.055:15. A. .

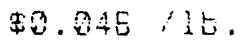

\$2.155:15.

$\$ 0.053 ; 15$.

$\$ 0.451: 1$ b.

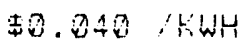

$\$ 工 .20 ; M 15$.

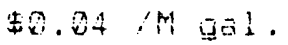

\$0.50, iM Gal.

$\$ 0.04$ １与. J.5.

\$a.

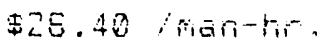

$18 \%$ DOWES

$5 \%$ OOWE

$23 \%$ OOWJ品

\$19.22, inantir.

\$2D. 05 imen-hit.

i. $7 \%$ DFI

$25 \%$ MWLB

$40 \%$ MUPB

$4 \%$ MWE

$23 \%$ MWSBD

Q $3 \%$ DPI

$8 \%$ EPI

6 i APSOE

$3 \%$ Sales

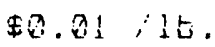

$4.5 \%$ Sales

$2 \%$ Solé

E \% PTE

ID $\%$ TIFR

\$. 27, i上.

$\$ 0.29 ; 1$.

$\$ 0.00 ; 15$.
IMELATION

FACTOP

1988

THIS CASE

-..........

1988
1.00 \$. क5 itt. 3.5.

1.90 की.045: is.

1.20 क0.155;15.

1.00 \$0.05\%:it.

1.00 \$o.451; it.

1.20

1.00

1. .09

1. 00

1.96

1.80

1.00

- -

$--$

$-\cdot$

1. 80

1. 20

$--$

-.

-

$--$

$-$

$-$

$--$

$\ldots$

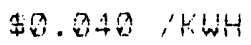

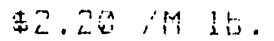

की.04 iM 可这.

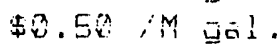

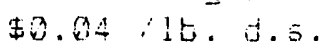

\$0.05 ilt. d.5.

\$25. 40 ifan-th.

$18 \%$ DOWLE

$6 \%$ DOW

$23 \%$ DOWSES

$\$ 19.22$ imarir.

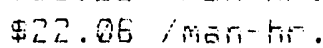

$1.7 \%$ DPI

$25 \%$ MWg

$40 \%$ MWDS

$4 \%$ MWL

23\% MWS品

จ. $\%$ D.

$8 \%$ OPI

5 i APS品

3 is Sales

\$0.01 its.

$5 \%$ Sales

2 \% Soles

E $\because$ PTE

$30 \%$ TIFR

1.80

1.00

1.00
$\$ 0.27$ 化。

$\$ 0.29 ; 15$.

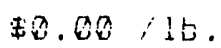


SALARIES \& WAGES
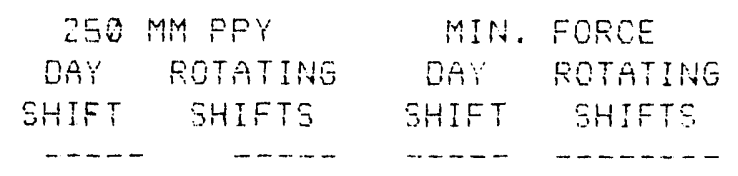

DIREST OFERATORE

SYRUP RECEIUING \& TRANGFER

CHEMICALS RECEIUING \& TRANCFER

INNOCULUM PREPARATION

MEOIUM FREFARATION

STERILIZATION

FERMENTATION \& EXTRACTION

- CONTROL ROOM

-PATPOL

-AIR COMPRESEION O AMMONIA FEEO

-TURNAROUNO

BEE다 FILTER \& CELL RECYCLE

OISTILLATION

TOTAL DAY \& 4.Z-EHIFT OFE

TOTAL OPERATORS

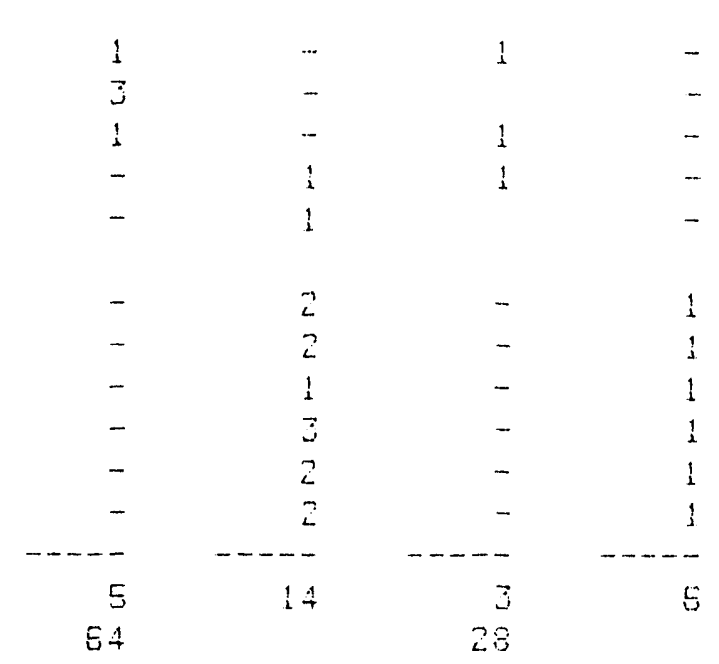

CONTROL LABORATORY

BIOLOGICAL ANALYSIS

CHEMICAL ANALYSIS

OTHER

$$
\begin{aligned}
& \text { TOTAL DAY \& 4.2-SHIFT TECHS } \\
& \text { W' SUPERVISION E } \\
& \text { TOTAL LAB FORCE }
\end{aligned}
$$
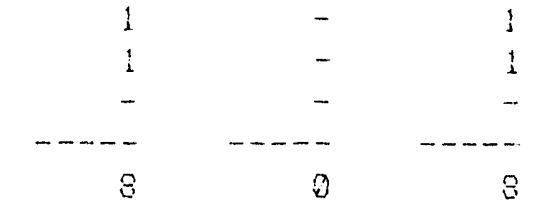

TECHNICAL ASEISTANCE TO MANUFACTURING

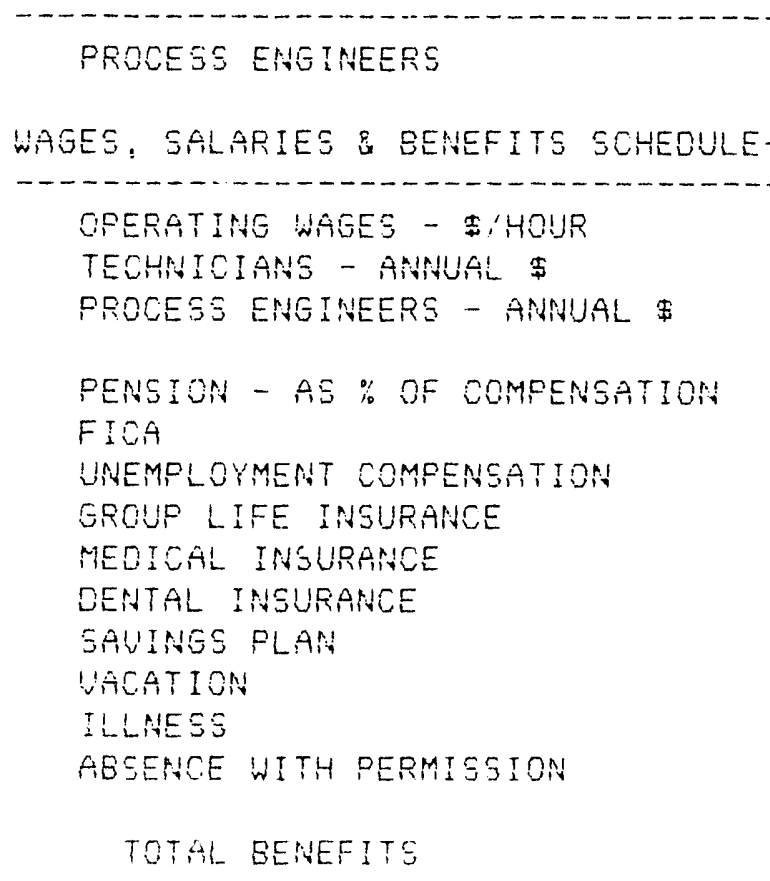

$$
\begin{aligned}
& 3.1 \% \\
& 5.8 \% \\
& 0.5 \% \\
& 0.7 \% \\
& 3.5 \% \\
& 0.8 \% \\
& 2.5 \% \\
& 7.4 \% \\
& 1.4 \% \\
& 0.2 \% \\
& -31.1 \%
\end{aligned}
$$




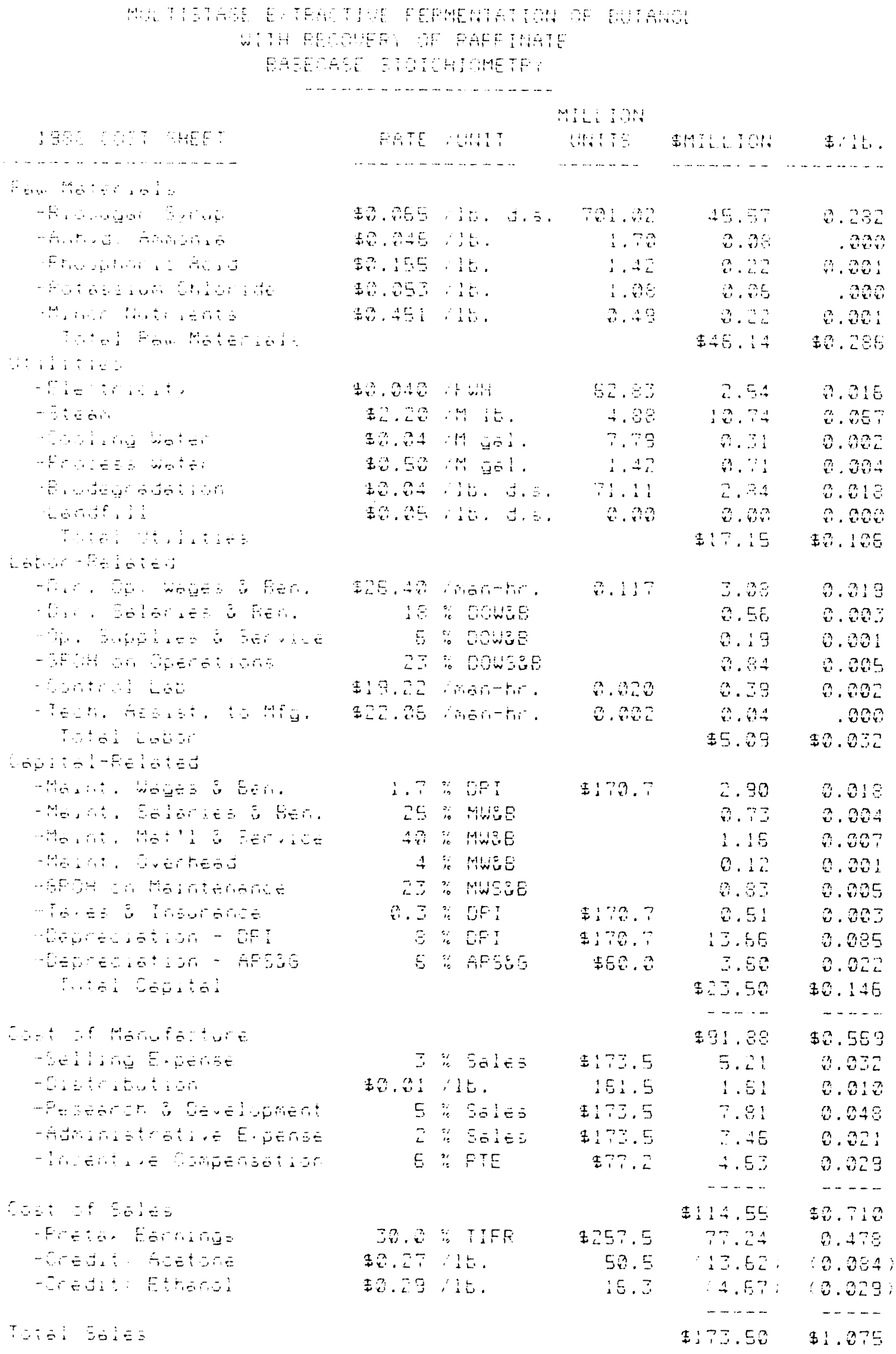


MULTISTAGE EXTRACTIUE FERMENTATION OF BUTANOL WITH RECOUERY OF RAFFINATE

BASECASE STOICHIOMETRY

CASH FLOW :MILLION DOLLARS, YEAR:

SEerianto:

1. Investrient oplit evenly over three construction years.

2. Flarit operates at $50 \%$ of full 50 ale the firet year.

j. " " $75 \%$ " " the jecort year.

4. " " $100 \%$ " " the thirtyear.

5. " $" 100 \% "$ " thereafter.

5. Five year deprediation rate; half-year corverition;

(20, 32, 19.2, 11.5, 11.5, 5.8\%). Tan: $34 \%$ federal, 3\% 5 tate.

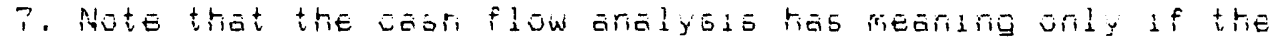
product oan be sold at the prive indicated on the cost sheet.

\begin{tabular}{|c|c|c|c|c|c|c|c|}
\hline \multirow[b]{2}{*}{ YEAR } & \multicolumn{2}{|c|}{ INUESTMENT } & \multirow{2}{*}{ DEP. } & \multirow{2}{*}{$\cos T^{\top}$ EX $D$} & \multirow{2}{*}{ SALES } & \multirow{2}{*}{ NET EARN } & \multirow{2}{*}{ ANA CASH } \\
\hline & $P I$ & $W C$ & & & & & \\
\hline$\cdots$ & $\ldots$ & $-\cdots$ & & & & & \\
\hline 1983 & $\$ 55.90$ & & & & & & $: \$ 55.90)$ \\
\hline 1984 & $\$ 55.30$ & & & & & & $(\$ 55.30)$ \\
\hline 1985 & $\$ 55.90$ & $\$ 25.85$ & & & & & $(\$ 33.75)$ \\
\hline 1985 & & & $\$ 34.14$ & $\$ 52.81$ & $\$ 35.90$ & $\$ 5.54$ & $\$ 12.92$ \\
\hline 1937 & & & $\$ 54.52$ & $\$ 7.32$ & $\$ 143.34$ & $\$ 9.83$ & $\$ E J .70$ \\
\hline 1988 & & & $\$ 32.77$ & $\$ 97.30$ & $\$ 191.79$ & $\$ 38.83$ & $\$ 7 ! .55$ \\
\hline 1983 & & & $\$ 19.53$ & $\$ 97.30$ & $\$ 191.73$ & $\$ 77.15$ & \$ES. 79 \\
\hline 1930 & & & $\$ 19.53$ & $\$ 97.30$ & $\$ 131.79$ & $\$ 47.15$ & \$ES. 79 \\
\hline 1891 & & & $\$ 9.90$ & $\$ 97.30$ & $\$ 191.79$ & $\$ 53.29$ & \$5j.19 \\
\hline 1992 & & & & $\$ 37.50$ & $\$ 191.79$ & $\$ 59.53$ & $\$ 53.53$ \\
\hline 1993 & & & & $\$ 97.30$ & $\$ 191.79$ & $\$ 59.5 .3$ & $\$ 59.53$ \\
\hline 1994 & & & & $\$ 97.30$ & $\$ 191.73$ & $\$ 59.53$ & $\$ 59.5 \overline{3}$ \\
\hline 1995 & & & & $\$ 97.30$ & $\$ 191.73$ & $\$ 59.53$ & $\$ 59.53$ \\
\hline 1995 & & & & $\$ 37.30$ & \$191.79 & $\$ 53.53$ & $\$ 59.53$ \\
\hline 1997 & & & & $\$ 97.30$ & $\$ 191.79$ & $\$ 59.53$ & $\$ 59.53$ \\
\hline 1398 & & & & $\$ 97.30$ & $\$ 191.73$ & $\$ 59.5 .5$ & $\$ 59.53$ \\
\hline 1989 & & & & $\$ 37.30$ & \$191.79 & $\$ 59.53$ & $\$ 59.53$ \\
\hline 2000 & & & & $\$ 97.30$ & $\$ 191.79$ & $\$ 59.53$ & $\$ 59.53$ \\
\hline 2001 & & & & $\$ 97.30$ & $\$ 191.79$ & $\$ 59.53$ & $\$ 53.53$ \\
\hline 2002 & & & & $\$ 97.30$ & \pm 131.73 & $\$ 53.53$ & $\$ 59.53$ \\
\hline 2003 & & & & $\$ 97.30$ & $\$ 191.79$ & $\$ 59.53$ & $\$ 53.53$ \\
\hline 2004 & & & & $\$ 97.30$ & $\$ 131.73$ & $\$ 53.53$ & $\$ 59.5 \%$ \\
\hline 2005 & & \$25.85; & & $\$ 97.30$ & $\$ 191.79$ & $\$ 59.53$ & $\$ 8 E .39$ \\
\hline
\end{tabular}

WET RETURN ON INUESTMENT-ZRD OPERATING YEAR = $15.5 \%$ 


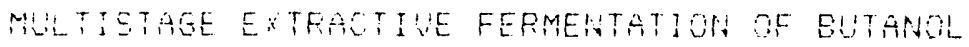
WTYH REOUUEG OF RAFFIHATE BASEOASE STOUTIOMETRY

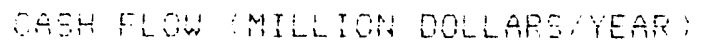

Sisitio

i. I

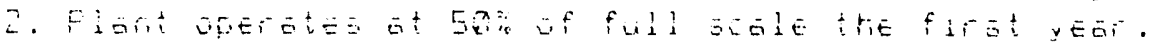

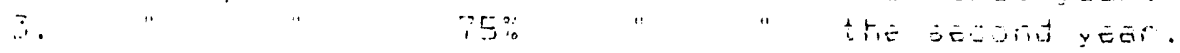

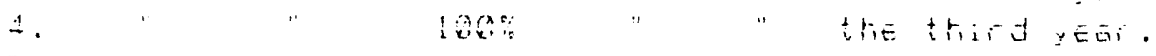

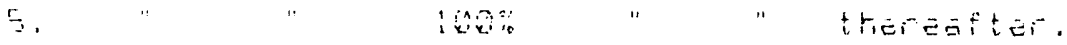

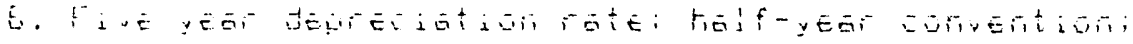

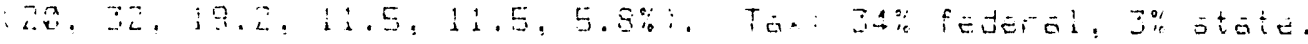

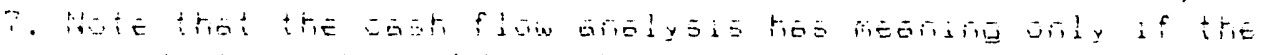

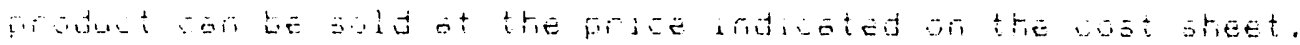

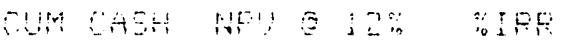

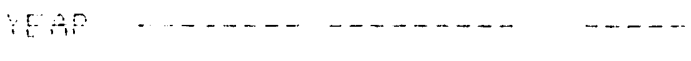

$1989 \quad+55.98 ;: 556.92:-0$

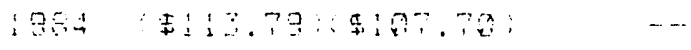

1985 :197.54:24:74.45:

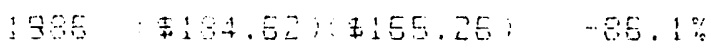

1997 : $120.92,+124.79, \quad-30.6 \%$

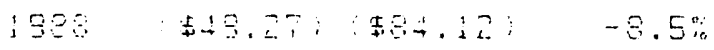

1999 \$17.52 :550.29; 2.3\%

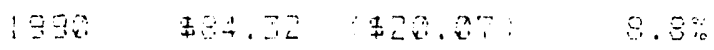

1991 \$147.51 \$5.5 $12.8 \%$

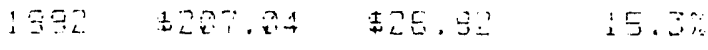

1992 +25E.57 +46.

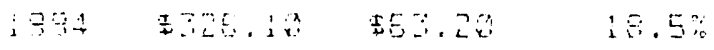

1990 \$25.65 $19.40 \quad 13.5 \%$

1995 +445.15 +93.12 $20.2 \%$

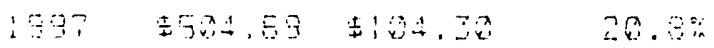

1998 \$5E-22 \$15.15 21.2\%

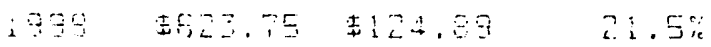

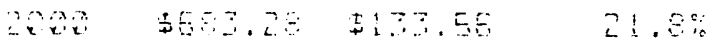

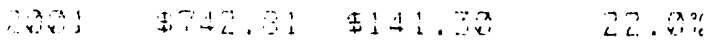

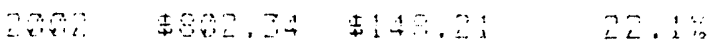

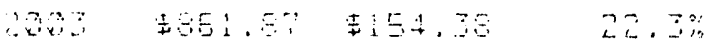

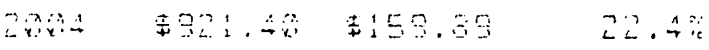

6...5 
MULTISTAGE EXTRACTIUE FERMENTATION OF EUTANOI

WITH RECOUERY OF FAFFINATE

BASEOASE STOICHIOMETRY

\begin{tabular}{|c|c|c|c|}
\hline \multicolumn{3}{|c|}{ BAEIC DATA } & \\
\hline ㄱ. 9 & rug & EE:1 & -MINDR NUTRIENTS \\
\hline 2.3 .5 & migi $\bar{y}$ & $5=113$ & -MESO4.7420 \\
\hline 8.01 & nig: & DE115 & -VISAMIN D: \\
\hline 1.25 & mg' & cells & $-F I$ \\
\hline 9.89 & mi & DE 115 & $-N 2612$ \\
\hline 0.72 & Nig & 리응 & -FEC13.5HaO \\
\hline 0.55 & irig: & EE115 & 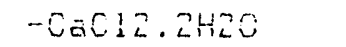 \\
\hline 3.54 & Nपू & 00115 & - HЗSOJ \\
\hline Q. 22 & MË: & EEIIS & - 25504.7420 \\
\hline 0.15 & mig & E2115 & -MnSO4. H2O \\
\hline 7.7 & $u \bar{g} ; \bar{y}$ & CE⿺1 & - C4504.5420 \\
\hline 5.4 & Uي & $E=115$ & - NaMoOA. $2 \mathrm{H}_{2} \mathrm{O}$ \\
\hline 4.3 & Lg, & VEll & -0.0012 .5420 \\
\hline
\end{tabular}

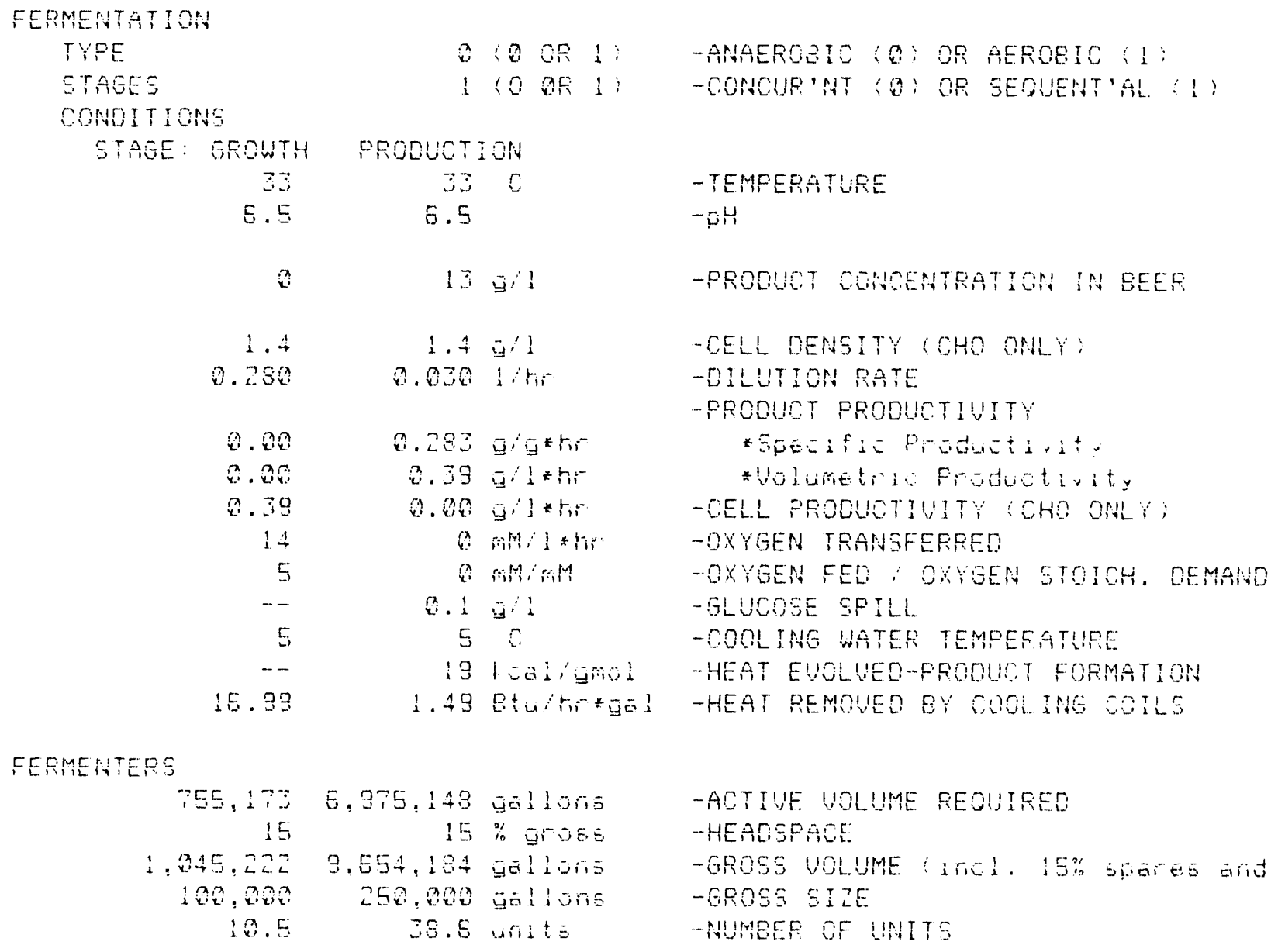

FROBUET GEFARATLOH

\begin{tabular}{|c|c|c|}
\hline & : 1 & SUO : EX FI!TEF \\
\hline $0.85 \%$ & 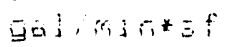 & -FILTER THROWGHPUT \\
\hline 56.846 & $50 \mathrm{ft}$ & -FILTEP SIFE \\
\hline
\end{tabular}

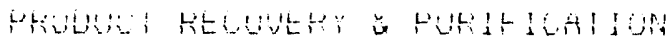


MULTTSTAGE EXTRACTIUE FERMENTATION OF BUTANOL

WITH REQOUERY OF RAFFINATE

BASECASE STOICHIOMETPY

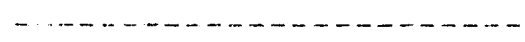

BASIC DATA

-..........

MATERIALS OF CONGTRULTION

FERMENTERS \& EXTRACTERS

STILL.

HEAT EXCHANGERS

STOFAGE TANLE

FOE WHTCH:

$\begin{array}{cc}\text { CHOICES } & \text { SELECTION } \\ 1,3 & 1 \\ 1,2.5,4.5 & 1 \\ 1,3,5.5 & 1 \\ 1.3 & 1\end{array}$

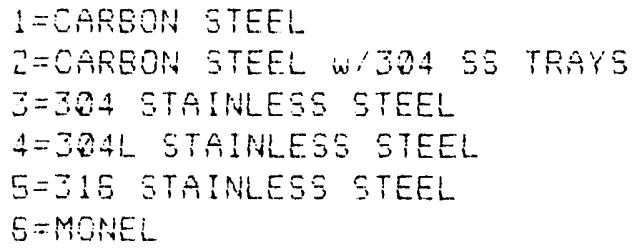

RETURA ON INUESTMENT

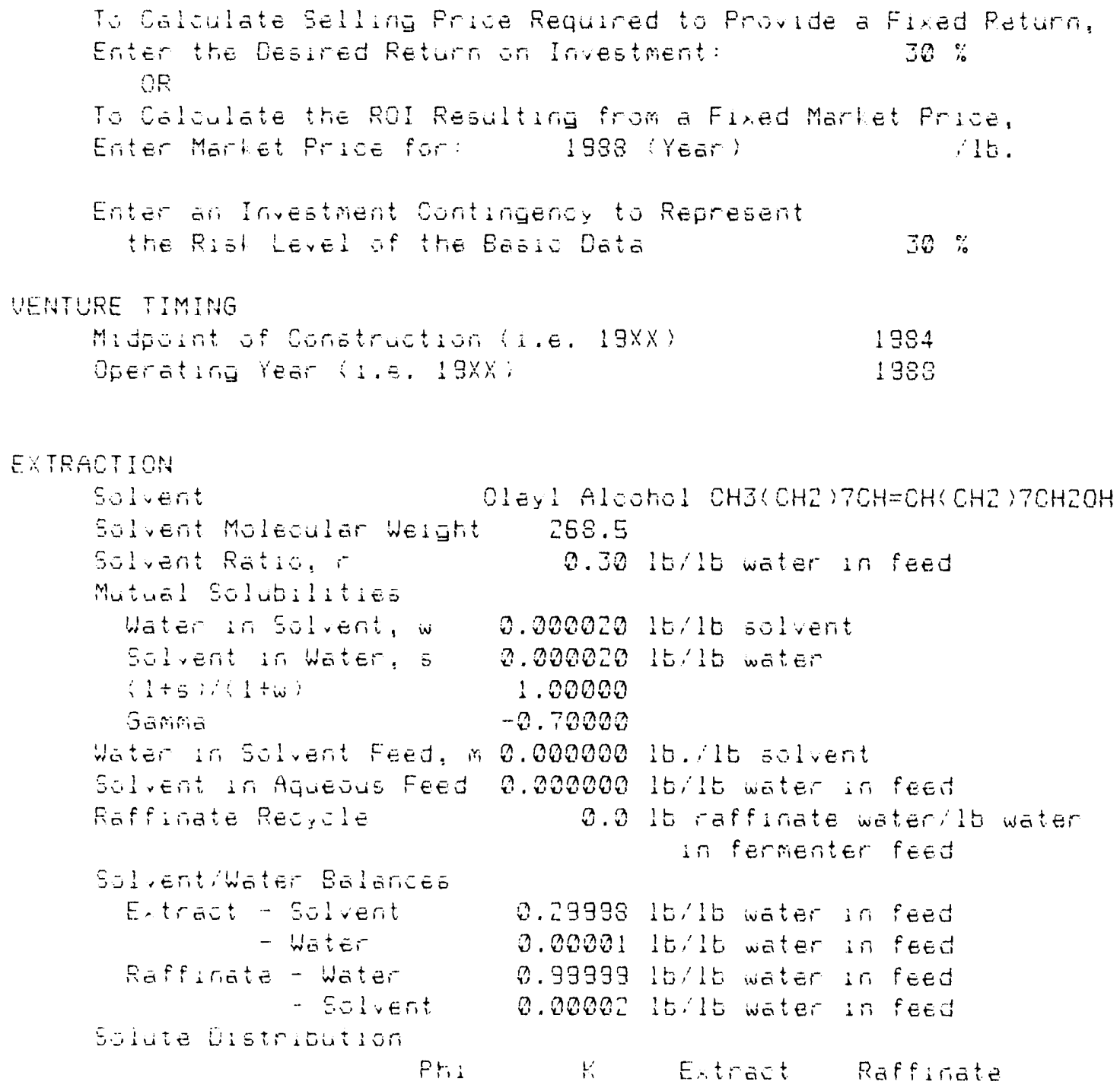


MULTISTAGE EXTRACTIUE FERMENTATION OF BUTANIOL WITH RECOUERY OF RAFFINATE BASECASE STOICHIOMETRY

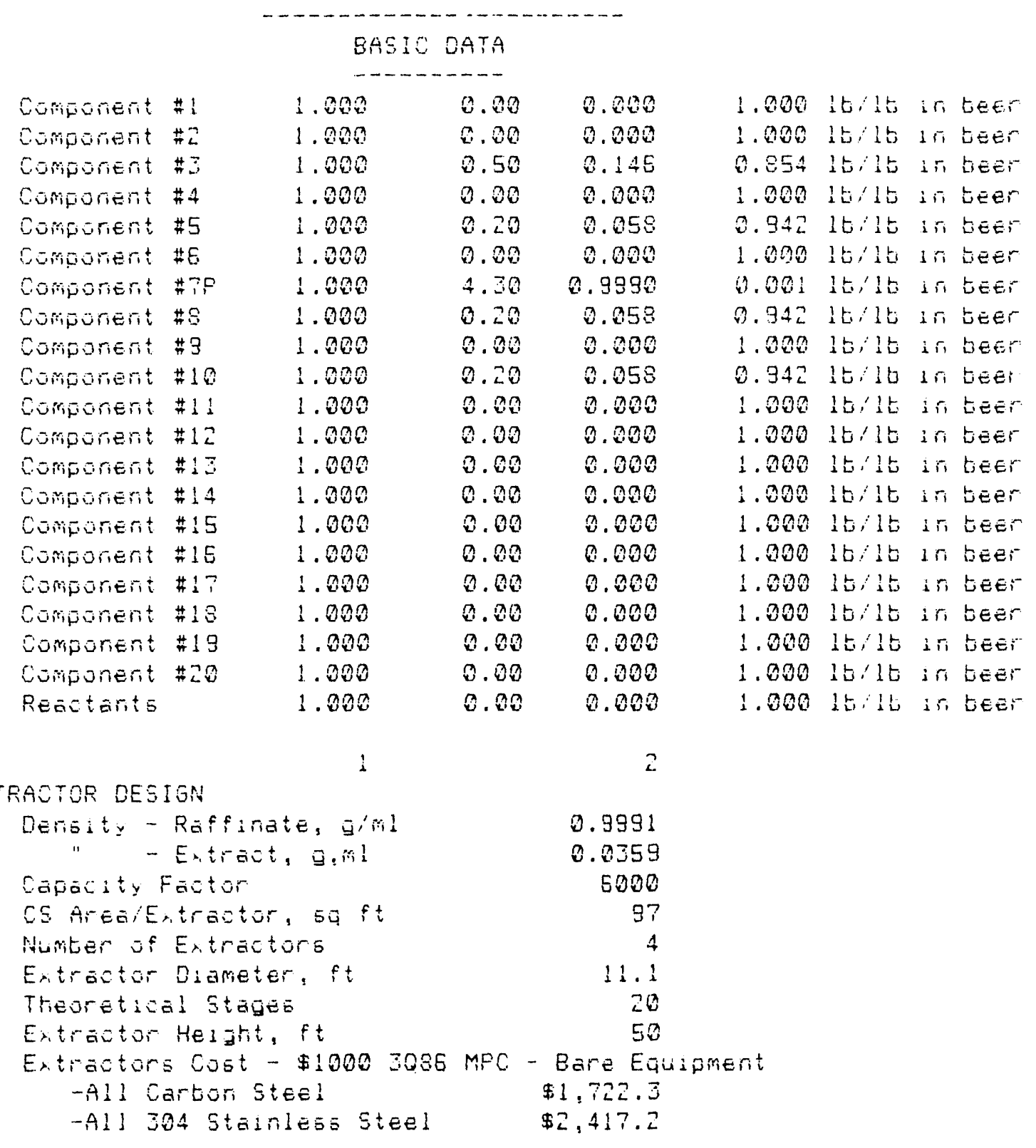




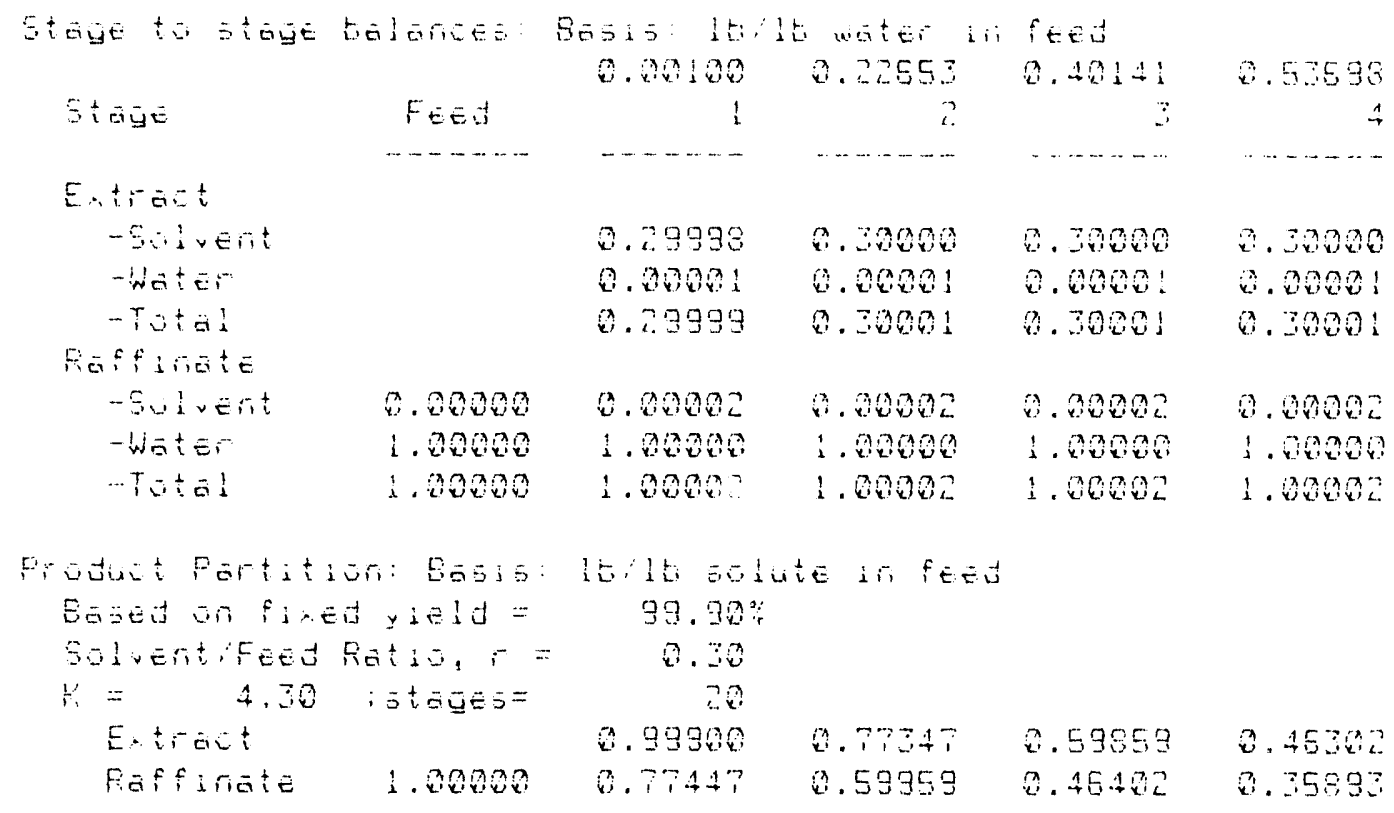




\begin{tabular}{|c|c|c|c|c|c|c|c|}
\hline A. $5420 \%$ & 6.7255 & 2.78650 & 0.83564 & 0.87553 & a 30301 & .32581 & 0.94349 \\
\hline 5 & 6 & $?$ & 8 & 9 & 10 & 11 & 12. \\
\hline$\therefore=3030$ & 0.38000 & 8.30000 & Q. 30000 & 0.30000 & 0. 30000 & 0.30030 & 0.30 \\
\hline 9.0201 & andol & ․․ำ1 & 0.03001 & 0.00001 & ๑. 00001 & 0.00801 & 0.00001 \\
\hline A Junal & ค. 30001 & 0.50301 & 0.30001 & 0.30001 & 0.30001 & 0.30001 & 0.30001 \\
\hline 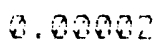 & A. $\operatorname{son} 20$ & Q. 00002 & 0.00902 & 0.00002 & 0.00002 & 0.0 & 0.0 \\
\hline 1.030000 & 1.00008 & 1.80000 & 1.00000 & 1.00000 & 1.000000 & 1.00000 & 1.00000 \\
\hline 1.86002 & 1.00202 & $1.90 \times 022$ & 1.00002 & 1.00002 & 1.00002 & 1.00002 & 1.00002 \\
\hline
\end{tabular}

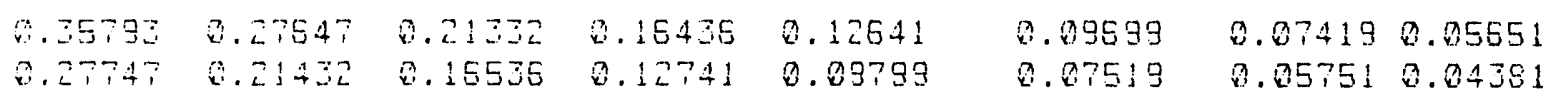




$\begin{array}{rrrrrrrr}0.95719 & 0.95782 & 0.97505 & 0.98244 & 0.98738 & 0.99122 & 0.99413 & 0.99550 \\ 13 & 14 & 15 & 15 & 17 & 13 & 19 & 20\end{array}$

a. 3030

0. 30000

$0.30000 \quad 0.30000$

(A. . 30060

Q. .30000

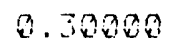

ค. 39090

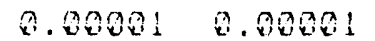

2.00001 0.000.1

2. 20031

0. 00001

3. $0000:$

0.00001

ค. 30001

3. 30001

0. 39001

0. 30301

0.30001

ค. 30091

b. 30001

$\begin{array}{llll}0.00002 & 0.00002 & 0.00002 & 0.00002 \\ 1.00000 & 1.00000 & 1.00000 & 1.00000 \\ 1.00802 & \text { i. } 00002 & 1.00002 & 1.00002\end{array}$

6. 90002

1. 00000

ด. 90932

ช. จดหลำ

0. 2000?

․ 80802

․ 00002

1. 00992

1. 08080

1. 930000

‥ 39999

1. 39002

1.00002

1. 0001

$\begin{array}{llllllll}0.04231 & 0.03218 & 0.02395 & 0.01755 & 0.01252 & 0.00878 & 0.00581 & 0.00350 \\ 0.03318 & 0.02435 & 0.01855 & 0.01352 & 0.00978 & 0.00681 & 0.00458 & 0.00271\end{array}$


Eutretit

........

R. 30303

2. 200020

A. 50000

A.0.2:3 


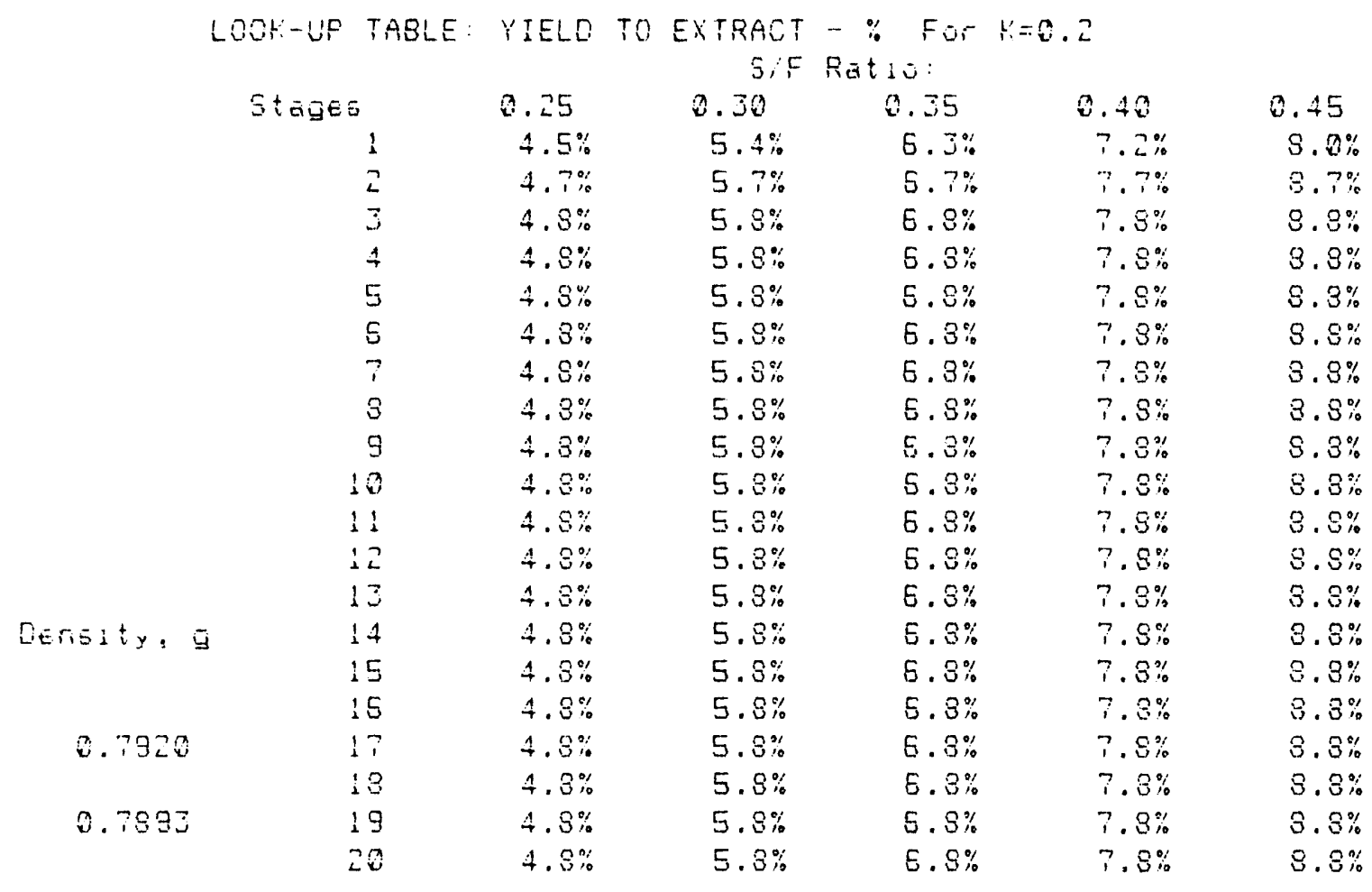




\begin{tabular}{|c|c|c|c|c|c|c|c|}
\hline 0.50 & 1.00 & 1.50 & 2.00 & 2.50 & 3.00 & 5.50 & 4.08 \\
\hline $8.9 \%$ & $16.8 \%$ & $22.0 \%$ & $28.3 \%$ & $33.1 \%$ & $37.4 \%$ & $41.1 \%$ & $44.3 \%$ \\
\hline $9.7 \%$ & $19.1 \%$ & $27.8 \%$ & $35.8 \%$ & $42.7 \%$ & $48.3 \%$ & $54.2 \%$ & $58.9 \%$ \\
\hline $9.8 \%$ & $19.5 \%$ & $27.0 \%$ & $38.3 \%$ & $45.6 \%$ & $53.9 \%$ & $60.4 \%$ & $55.1 \%$ \\
\hline $9.3 \%$ & $19.8 \%$ & $23.5 \%$ & $39.2 \%$ & $43.2 \%$ & $55.5 \%$ & $53.3 \%$ & $70.1 \%$ \\
\hline $9.8 \%$ & $1.3 .8 \%$ & $23.8 \%$ & $33.6 \%$ & $49.1 \%$ & $57.9 \%$ & $55.9 \%$ & $72.8 \%$ \\
\hline $3.8 \%$ & $19.8 \%$ & $29.8 \%$ & $39.7 \%$ & $43.5 \%$ & $58.8 \%$ & $E T .2 \%$ & $74.5 \%$ \\
\hline $9.8 \%$ & $13.8 \%$ & $23.9 \%$ & $39.8 \%$ & $49.7 \%$ & $59.2 \%$ & $58.1 \%$ & $75.8 \%$ \\
\hline $3.8 \%$ & $13.8 \%$ & $29.9 \%$ & $39.9 \%$ & $49.8 \%$ & $59.5 \%$ & $58.5 \%$ & $75.9 \%$ \\
\hline $9.8 \%$ & $13.8 \%$ & $29.3 \%$ & $39.9 \%$ & $49.8 \%$ & $59.5 \%$ & 69.1\% & $77.6 \%$ \\
\hline $9.8 \%$ & $13.8 \%$ & $29.9 \%$ & $39.3 \%$ & $43.8 \%$ & $53.7 \%$ & $59.3 \%$ & $78.0 \%$ \\
\hline $9.8 \%$ & $19.8 \%$ & $29.9 \%$ & $39.9 \%$ & $49.8 \%$ & $59.8 \%$ & $59.5 \%$ & $78.4 \%$ \\
\hline $3.8 \%$ & $19.8 \%$ & $23.3 \%$ & $33.9 \%$ & $49.8 \%$ & $53.3 \%$ & $53.5 \%$ & $78.7 \%$ \\
\hline $9.3 \%$ & $19.8 \%$ & $23.9 \%$ & $39.9 \%$ & $49.8 \%$ & $59.9 \%$ & $59.7 \%$ & $73.0 \%$ \\
\hline $9.8 \%$ & $19.8 \%$ & $23.3 \%$ & $39.9 \%$ & $43.8 \%$ & $59.9 \%$ & $59.8 \%$ & $73.2 \%$ \\
\hline $3.8 \%$ & $18.8 \%$ & $23.9 \%$ & $39.9 \%$ & $49.8 \%$ & $59.9 \%$ & E9.8\% & $79.3 \%$ \\
\hline $8.8 \%$ & $19.8 \%$ & $29.9 \%$ & $33.3 \%$ & $43.8 \%$ & $59.9 \%$ & $53.9 \%$ & $73.4 \%$ \\
\hline $9.8 \%$ & $13.8 \%$ & $23.9 \%$ & $39.9 \%$ & $49.8 \%$ & ㄷ‥ $9 \%$ & $59.9 \%$ & $79.5 \%$ \\
\hline $9.8 \%$ & $19.8 \%$ & $29.8 \%$ & j9.9\% & $43.8 \%$ & $59.9 \%$ & $53.9 \%$ & $73.5 \%$ \\
\hline $9.8 \%$ & $13.8 \%$ & $23.9 \%$ & $39.9 \%$ & $43.8 \%$ & 59. $9 \%$ & E马. $9 \%$ & Т. \\
\hline $9.8 \%$ & $13.8 \%$ & $29.9 \%$ & $39.9 \%$ & $43.8 \%$ & $59.9 \%$ & $59.9 \%$ & $73.8 \%$ \\
\hline
\end{tabular}




\begin{tabular}{|c|c|}
\hline 4.50 & 5.80 \\
\hline $47.3 \%$ & $43.9 \%$ \\
\hline $53.1 \%$ & $55.5 \%$ \\
\hline $70.8 \%$ & $74.9 \%$ \\
\hline $75.5 \%$ & $79.8 \%$ \\
\hline $75.6:$ & $83.3 \%$ \\
\hline $8 \pi .7 \%$ & $35.5 \%$ \\
\hline $82.4 \%$ & $87.4 \%$ \\
\hline $83.5 \%$ & $88.8 \%$ \\
\hline $84.6 \%$ & $33.9 \%$ \\
\hline $85.3 \%$ & $30.8 \%$ \\
\hline $85.8 \%$ & $31.5 \%$ \\
\hline $85.5 \%$ & $92.2 \%$ \\
\hline $87.0 \%$ & $92.8 \%$ \\
\hline $87.5 \%$ & $93.2 \%$ \\
\hline $87.7 \%$ & $33.7 \%$ \\
\hline $87.9 \%$ & $94.1 \%$ \\
\hline $83.2 \%$ & $94.4 \%$ \\
\hline $88.4 \%$ & $34.7 \%$ \\
\hline $8.5 \%$ & $94.9 \%$ \\
\hline $88.5 \%$ & $95.1 \%$ \\
\hline
\end{tabular}




\begin{tabular}{|c|c|c|c|c|c|c|c|}
\hline$O O K-U P$ & YIELD & $\begin{array}{l}\text { TO EXTRACT } \\
\text { SiF Ratio: }\end{array}$ & - \% For & $k=0.5$ & & & \\
\hline Stages & 0.25 & 0.30 & 0.35 & 0.40 & 0.45 & 0.50 & 1.00 \\
\hline 1 & $10.0 \%$ & $12.5 \%$ & $14.6 \%$ & $16.0 \%$ & $18.0 \% \%$ & $19.0 \%$ & $33.0 \%$ \\
\hline 2 & $11.0 \%$ & $14.0 \%$ & $16.0 \%$ & $13.0 \%$ & $21.0 \%$ & $24.0 \%$ & $42.0 \%$ \\
\hline $\bar{j}$ & $12.0 \%$ & $14.5 \%$ & $17.0 \%$ & $19.0 \%$ & $22.0 \%$ & $25.00 \%$ & $43.0 \%$ \\
\hline 4 & $12.0 \%$ & $14.6 \%$ & $17.1 \%$ & $20.0 \%$ & $23.0 \%$ & $25.0 \%$ & $48.0 \%$ \\
\hline 5 & $12.00 \%$ & $14.5 \%$ & $17.1 \%$ & $20.0 \%$ & $23.0 \%$ & $25.0 \%$ & $48.5 \%$ \\
\hline$E$ & $12.0 \%$ & $14.5 \%$ & $17.1 \%$ & $20.0 \%$ & $23.00 \%$ & $25.0 \%$ & $49.0 \%$ \\
\hline 7 & $12.0 \% \%$ & $14.5 \%$ & $17.1 \%$ & $20.0 \%$ & $23.0 \%$ & $25.0 \%$ & $49.5 \%$ \\
\hline 3 & $12.0 \%$ & $14.6 \%$ & $17.1 \%$ & $20.0 \%$ & $23.0 \%$ & $25.0 \%$ & $49.6 \%$ \\
\hline 9 & $12.0 \%$ & $14.6 \%$ & $17.1 \%$ & $20.00 \%$ & $23.0 \%$ & $25.0 \%$ & $49.7 \%$ \\
\hline 10 & $12.0 \%$ & $14.6 \%$ & $17.1 \%$ & $20.00 \%$ & $23.0 \%$ & $25.0 \%$ & $49.7 \%$ \\
\hline 11. & $12.8 \%$ & $14.5 \%$ & $17.1 \%$ & $20.0 \%$ & $23.00 \%$ & $25.0 \%$ & $49.7 \%$ \\
\hline 12 & $12.0 \%$ & $14.5 \%$ & $17.1 \%$ & $20.0 \%$ & $23.0 \%$ & $25.0 \%$ & $49.7 \%$ \\
\hline 15 & $12.0 \%$ & $14.5 \%$ & $17.1 \%$ & $20.0 \%$ & $23.0 \%$ & $25.0 \%$ & $43.7 \%$ \\
\hline 14 & $12.0 \%$ & $14.6 \%$ & $17.1 \%$ & $20.0 \%$ & $23.0 \%$ & $25.0 \%$ & $49.7 \%$ \\
\hline 15 & $12.0 \%$ & $14.5 \%$ & $17.1 \%$ & $20.0 \%$ & $23.0 \%$ & $25.0 \%$ & $49.7 \%$ \\
\hline 15 & $12.0 \%$ & $14.6 \%$ & $17.1 \%$ & $20.0 \%$ & $23.0 \%$ & $25.0 \%$ & $49.7 \%$ \\
\hline 17 & $12.0 \% \%$ & $14.5 \%$ & $17.1 \%$ & $20.0 \%$ & $23.0 \%$ & $25.0 \%$ & $49.7 \%$ \\
\hline 18 & $12.0 \%$ & $14.5 \%$ & $17.1 \%$ & $20.0 \%$ & $23.0 \%$ & $25.0 \%$ & $49.7 \%$ \\
\hline 19 & $12.0 \%$ & $14.6 \%$ & $17.1 \%$ & $20.0 \%$ & $23.06 \%$ & $25.0 \%$ & $48.7 \%$ \\
\hline 20 & $12.0 \%$ & $14.6 \%$ & $17.1 \%$ & $20.0 \%$ & $23.0 \%$ & $25.0 \%$ & 49.7 \\
\hline
\end{tabular}




\begin{tabular}{|c|c|c|c|c|c|c|c|}
\hline 1.50 & $=.80$ & 2.50 & 5.90 & 3.50 & 4.00 & 4.58 & 5.80 \\
\hline $42.0 \%$ & $49.0 \%$ & $55.3 \%$ & $59.5 \%$ & $53.8 \%$ & $5 E .0 \%$ & $59.0 \%$ & $71.0 \%$ \\
\hline $55.3 \%$ & $5 E .0 ;$ & $73.8 \%$ & $78.8 \%$ & $82.0 \%$ & $85.7 \%$ & $87.0 \%$ & $89.7 \%$ \\
\hline $55.0 \%$ & $74.9 \%$ & $82.0 \%$ & $87.0 \%$ & $31.8 \%$ & $93.8 \%$ & $94.8 \%$ & $95.0 \%$ \\
\hline $57.0 \%$ & $73.0 \%$ & $87.5 \%$ & $92.3 \%$ & $35.1 \%$ & $97.8 \%$ & $37.7 \%$ & $98.4 \%$ \\
\hline $69.0 \%$ & $33.0 \%$ & $31.0 \%$ & $55.0 \%$ & $97.0 \%$ & $88.3 \%$ & 马日. $1 \%$ & $99.3 \%$ \\
\hline $71.9 \%$ & $35.0 \%$ & $33.2 \%$ & $35.8 \%$ & $38.4 \%$ & $39.2 \%$ & $93.5 \%$ & $83.7 \%$ \\
\hline $71.5 \%$ & $87.0 \%$ & $9.4 .9 \%$ & $37.0 \%$ & $39.0 \%$ & $39.5 \%$ & $39.8 \%$ & $99.9 \%$ \\
\hline $72.5 \%$ & $88.0 \%$ & $95.0 \%$ & $98.0 \%$ & $39.5 \%$ & $99.8 \%$ & $73.9 \%$ & $100.0 \%$ \\
\hline $73.0 \%$ & $89.0 \%$ & $35.5 \%$ & $39.1 \%$ & $39.7 \%$ & 39. 9\% & $100.8 \%$ & $100.0 \%$ \\
\hline $73.5 \%$ & $90.0 \%$ & $97.0 \%$ & $39.5 \%$ & $99.8 \%$ & $100.0 \%$ & $180.8 \%$ & $100.0 \%$ \\
\hline $74.0 \%$ & $91.0 \%$ & $98.3 \%$ & $39.6 \%$ & $39.9 \%$ & $100.0 \%$ & $100.0 \%$ & $100.0 \%$ \\
\hline $74.2 \%$ & $32.0 \%$ & $98.5 \%$ & $39.7 \%$ & $99.9 \%$ & $100.0 \%$ & $100.0 \%$ & $100.0 \%$ \\
\hline $74.5 \%$ & $92.5 \%$ & $98.7 \%$ & $39.8 \%$ & $100 . \pi \%$ & $100.0 \%$ & $100.0 \%$ & $100.0 \%$ \\
\hline $74.5 \%$ & $93.0 \%$ & $93.0 \%$ & $83.3 \%$ & $10 \% .0 \%$ & $10 \%$ & $100.0 \%$ & $100.0 \%$ \\
\hline $74.5 \%$ & $33.5 \%$ & $33.2 \%$ & $39.9 \%$ & $100.0 \%$ & $100.0 \%$ & $10 \% .0 \%$ & $100.2 \%$ \\
\hline $74.6 \%$ & $34.0 \%$ & $39.4 \%$ & $93.9 \%$ & $100.0 \%$ & $100.0 \%$ & $100.0 \%$ & $100.0 \%$ \\
\hline $74.7 \%$ & $34.2 \%$ & $93.5 \%$ & $39.9 \%$ & $100.0 \%$ & $100.0 \%$ & $100.0 \%$ & $100.0 \%$ \\
\hline $74.7 \%$ & $34.5 \%$ & $33.6 \%$ & $99.9 \%$ & $100.0 \%$ & $100.0 \%$ & $100.0 \%$ & $100.0 \%$ \\
\hline $74.7 \%$ & $95.0 \%$ & $99.7 \%$ & $93.9 \%$ & $103.8 \%$ & $100.0 \%$ & $100.0 \%$ & $100.0 \%$ \\
\hline $74.8 \%$ & $35.0 \%$ & $39.8 \%$ & $99.3 \%$ & $100.0 \%$ & $100.0 \%$ & $100.0 \%$ & $100.0 \%$ \\
\hline
\end{tabular}




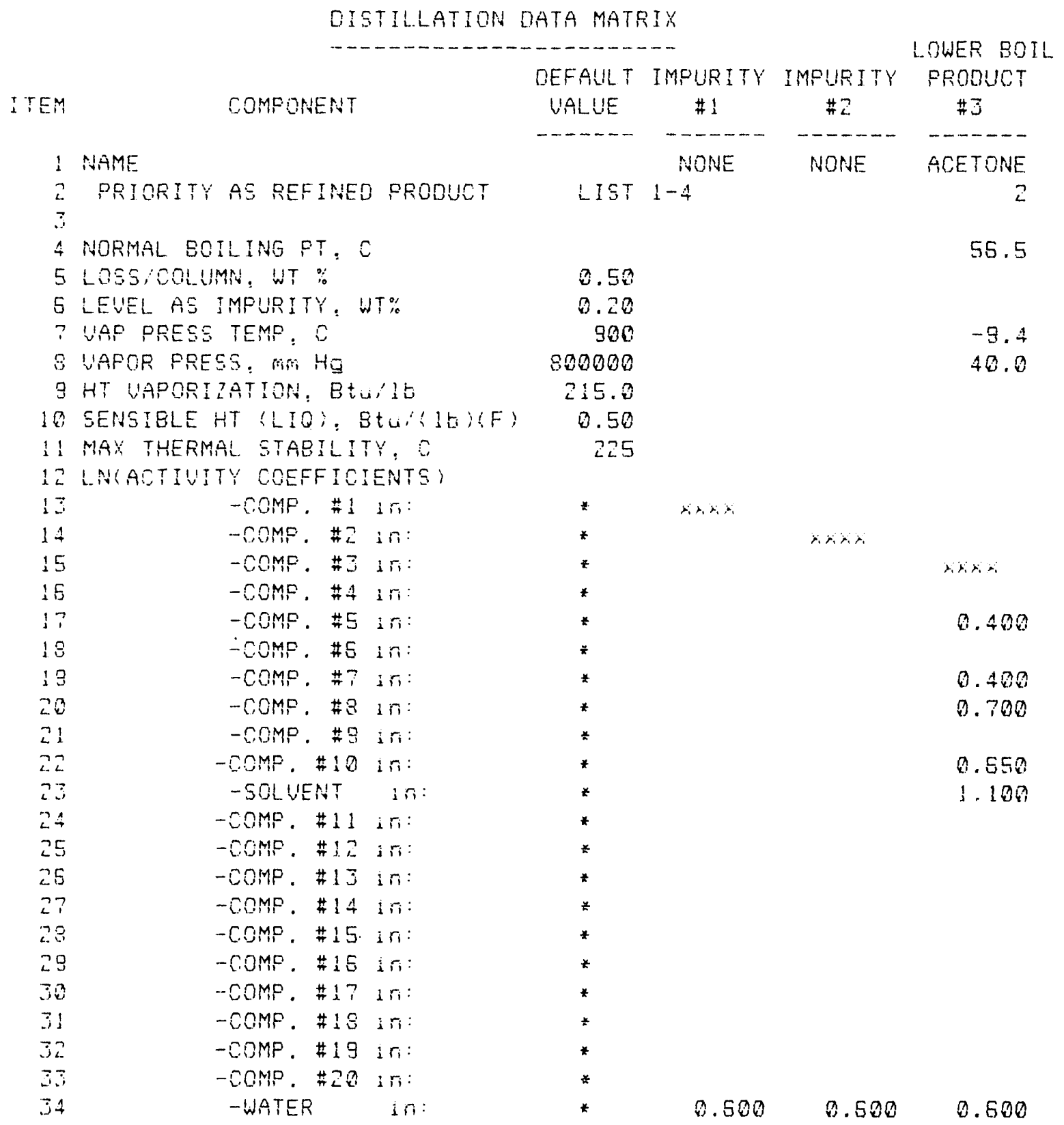

* The followirg values añ be used in lieu of actual activity coefficients; however: the uncertainty of the calculation is räised bigrificantly:

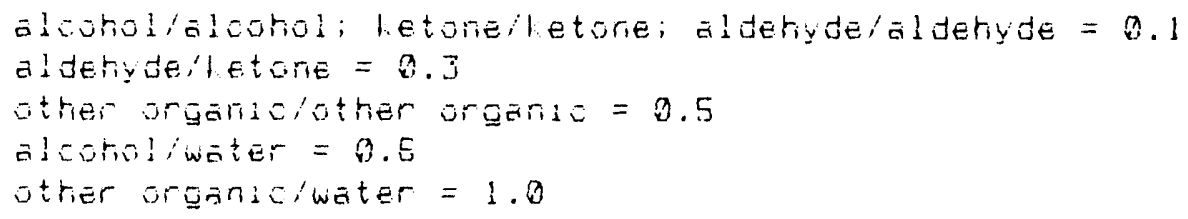

$\begin{array}{rrr}-2475.10 & -2476.10 & -3880.77 \\ 15.703 & 15.703 & 18.411 \\ 2422.2 & 2422.2 & 408.5 \\ 12121.9 & 12121.9 & 5096.7\end{array}$




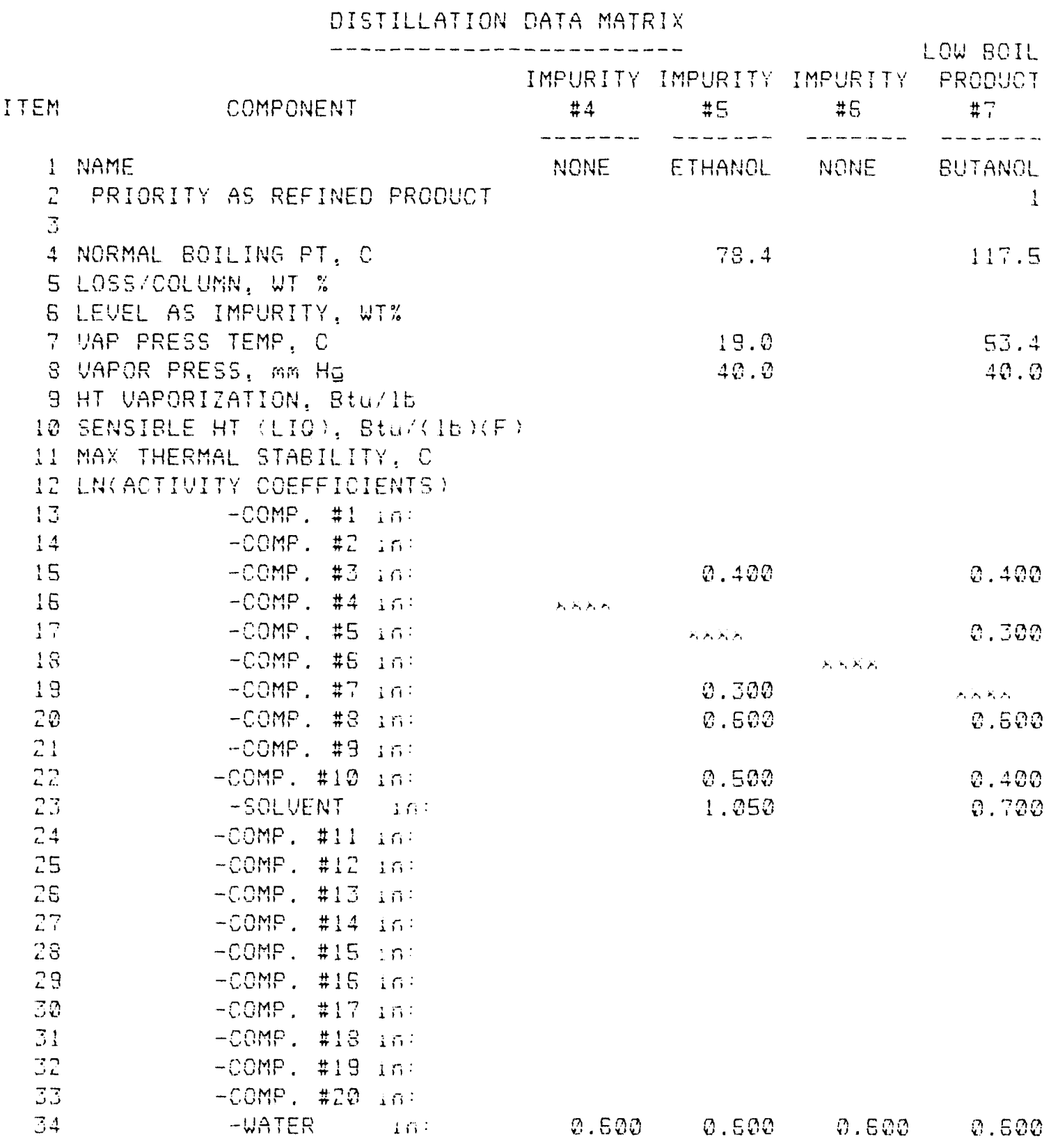

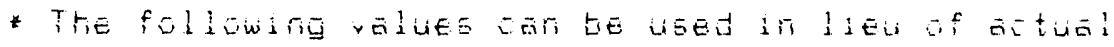
ätivity coefficienta; however, the bricertainty of

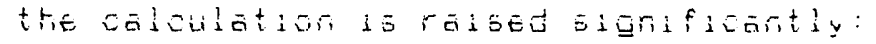

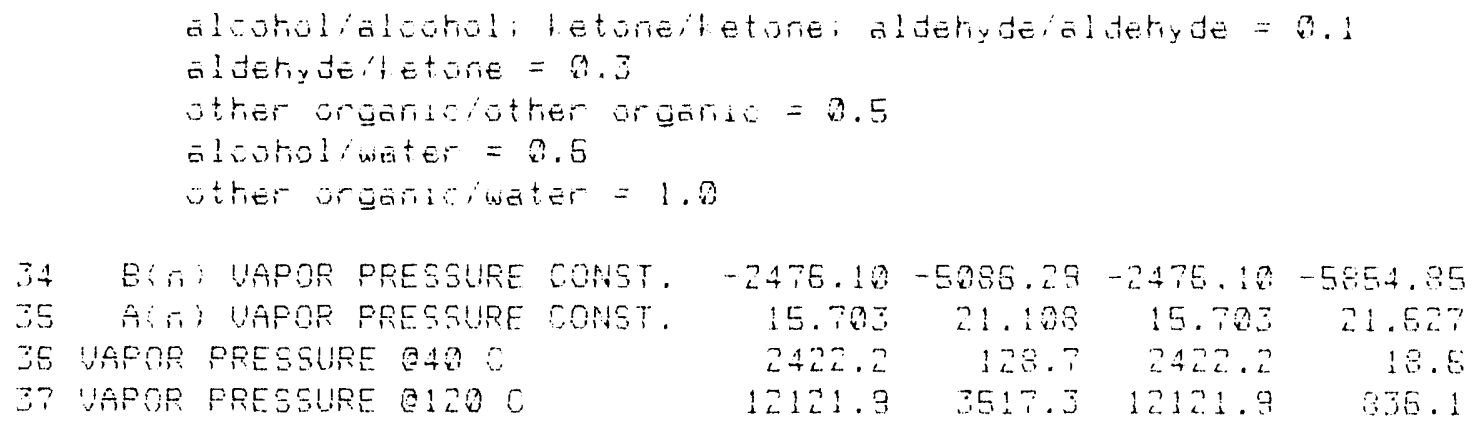




\section{DISTILLATION DATA MATRIX}

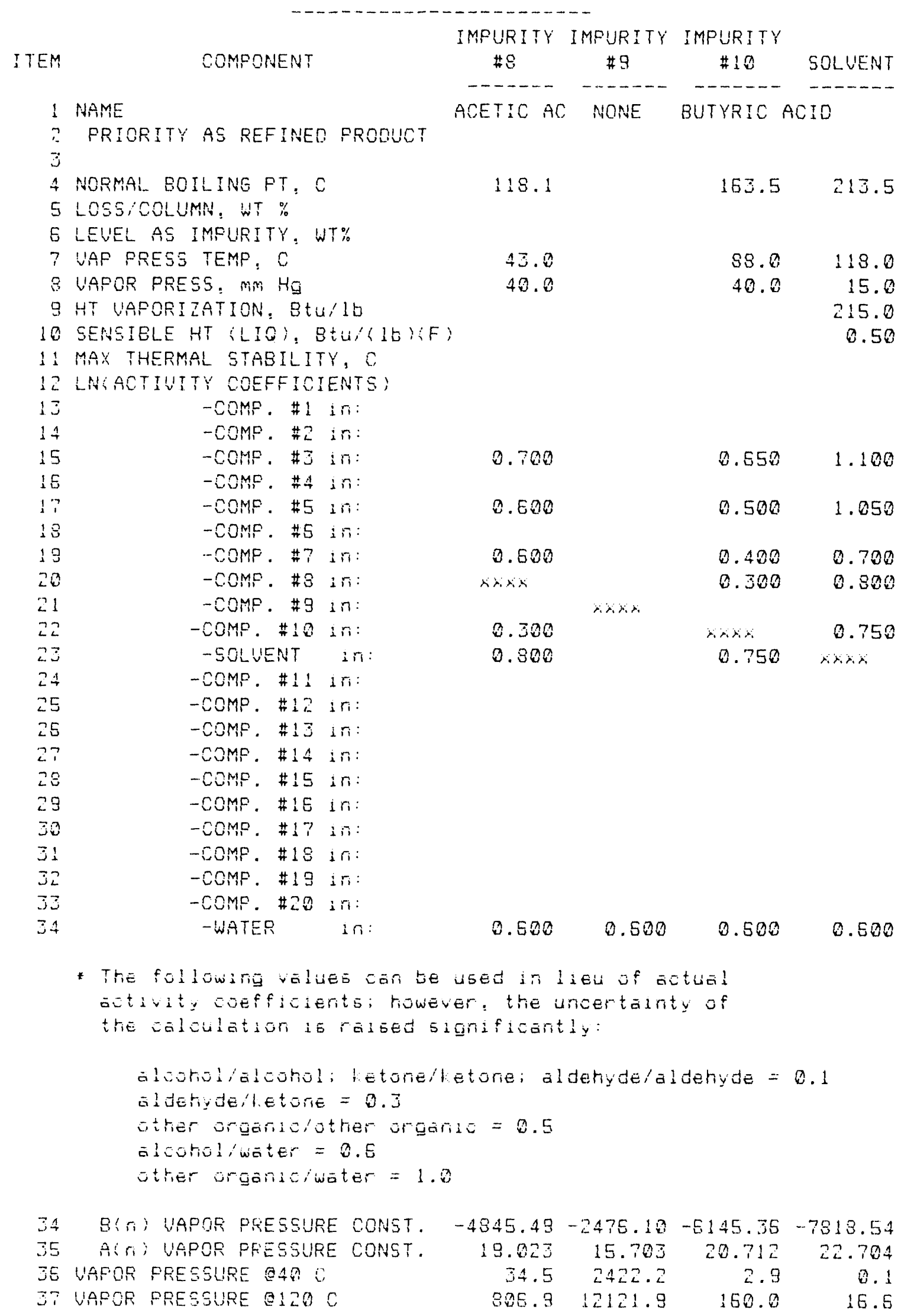




\begin{tabular}{|c|c|c|}
\hline ITEM & COMPONENT & WATER \\
\hline 1 & NAME & \\
\hline$\frac{2}{3}$ & FRIORITY AS REFINED PRODUCT & \\
\hline 4 & NORMAL BOILING PT:C & 100.0 \\
\hline 5 & LOSS,COLUMN, WT \% & \\
\hline 5 & LEVEL AS IMPURITY, WT\% & \\
\hline$?$ & WAP PRESS TEMP, 0 & 30.0 \\
\hline 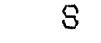 & VAFOR PRESS, mim Hg & 31.3 \\
\hline$g$ & HT WAPORIZATION, StUIL & 970.3 \\
\hline 10 & SENSIDLE HT (LIO;, BtU'(1t)(F) & 1.00 \\
\hline 11 & MAX THERMAL STABILITY, C & \\
\hline 12 & LNEACTIUITY COEFFICIENTS: & \\
\hline 13 & -COMP. \#1 in: & \\
\hline 14 & -COMP. \#2 in: & \\
\hline 15 & $-\operatorname{COMP} . \quad \# 3 i n_{i}:$ & 0.600 \\
\hline 15 & -COMP. \#4 in: & \\
\hline 17 & - COMP. \#5 in: & 0.500 \\
\hline 18 & - COMP. \#6 $1 \pi:$ & \\
\hline 13 & -COMP. \#7 in: & 8.500 \\
\hline 20 & -COMP. \#马 ir: & 0.500 \\
\hline 21 & - COMP. \#马 iri: & \\
\hline 22 & -COMP. \#10 in: & 0.500 \\
\hline 23 & -EOLVENT in: & \\
\hline$\therefore 4$ & -COMP. \#11 1n: & \\
\hline 25 & - COMP. \#12 in: & \\
\hline 25 & -COMP. \#13 in: & \\
\hline 27 & -COMP. \#14 i $r_{i}:$ & \\
\hline 28 & -COMP. \#15 in: & \\
\hline 23 & -COMP. \#1E in: & \\
\hline 30 & -COMP. \#17 in: & \\
\hline 31 & - COMP. \#18 $1 \mathrm{ri}_{\mathrm{i}}$ & \\
\hline 32 & -COMP. \#19 in: & \\
\hline 3 & - COMP. \#20 in: & \\
\hline 34 & -WATER & $x \times x \times$ \\
\hline & 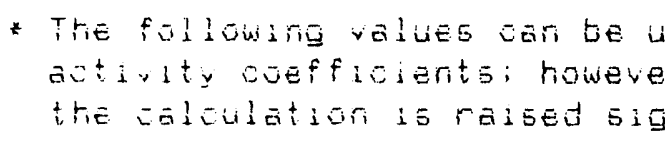 & $\begin{array}{l}\text { Weed in luel of actual } \\
\text { er, the uncertainty of } \\
\text { gnificantly: }\end{array}$ \\
\hline & 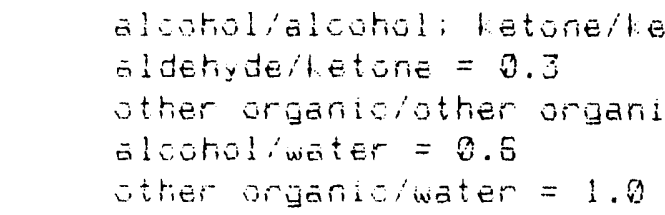 & $\begin{array}{l}\text { etorie; aldehideialdehyde }=0.1 \\
\text { is }=0.5\end{array}$ \\
\hline 3.4 & BSA: WAPOR PRESSURE CONST. & -5124.37 \\
\hline 35 & AGR: UAPOR PRESSURE CONST. & 20.372 \\
\hline 55 & UAPOR PRESGURE Q4DO & 54.5 \\
\hline 37 & UAFOR FRESSURE O1200 & 1529.1 \\
\hline
\end{tabular}


OFTEN USED PARAMETERS

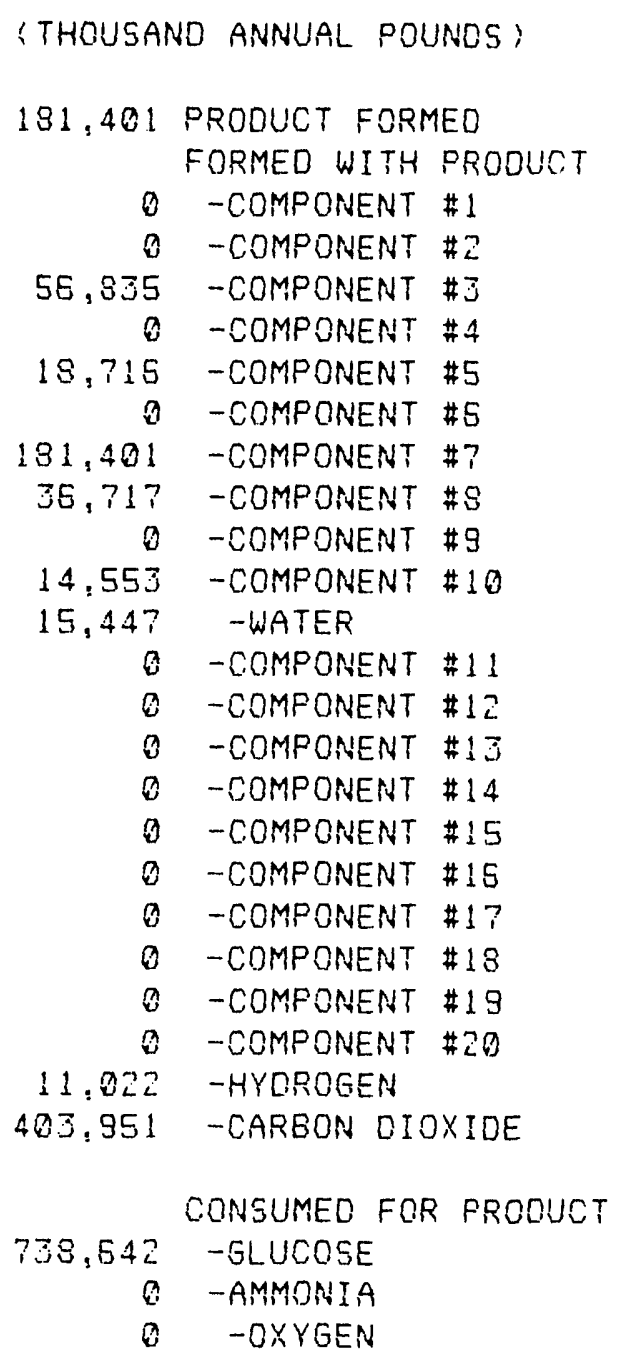

$$
\begin{aligned}
& 19,438 \text { CELLS PROOUCED - } 640 \\
& \text { 1.777 CELLS PROQUCED - NHZ } \\
& \text { FORMED WITH CELLS } \\
& 11,556 \text {-WATER-CHO } \\
& 1.00 \% \text {-WATER-NH2 } \\
& \text { 12.55E -WATER-TOTAL } \\
& \text { 23,493 - CARBON OIOXIDE-CHO } \\
& \text { CONSUMED FOR CELLS } \\
& \text { 33.875 - GLUCOSE-CHO } \\
& 1,888 \text {-AMMONIA-NHZ } \\
& \text { 20.722 -OXYGEN-CHO } \\
& 339-O X Y G E N-N H Z \\
& \text { 21,E11 -OXYGEN-TOTAL }
\end{aligned}
$$

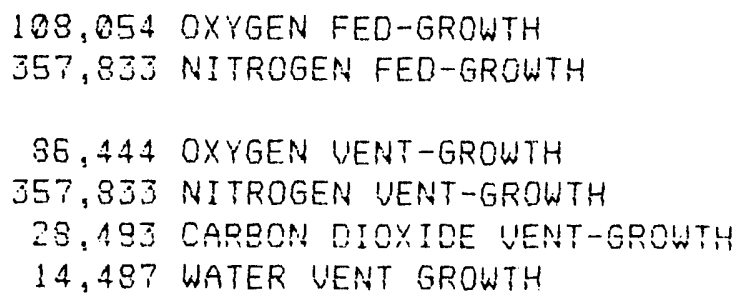




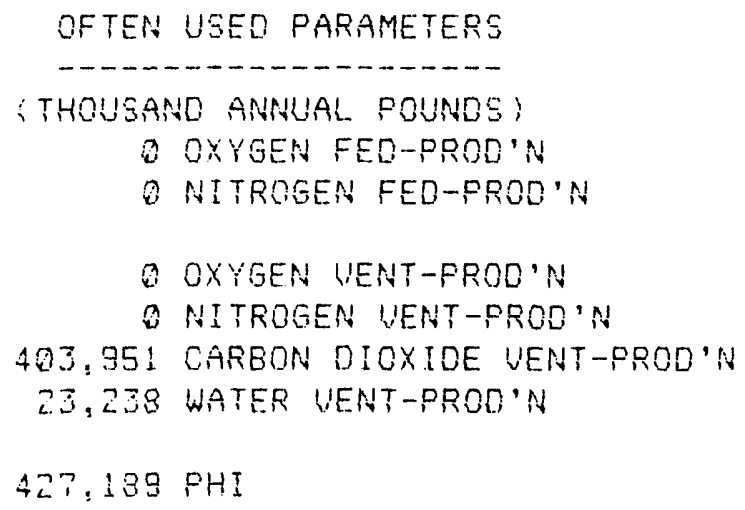




\section{MULTISTAGE EXTRACTIUE FERMENTATION OF EUTANOL WITH RECOUERY OF RAFFINATE BASECASE STOICHIOMETRY}

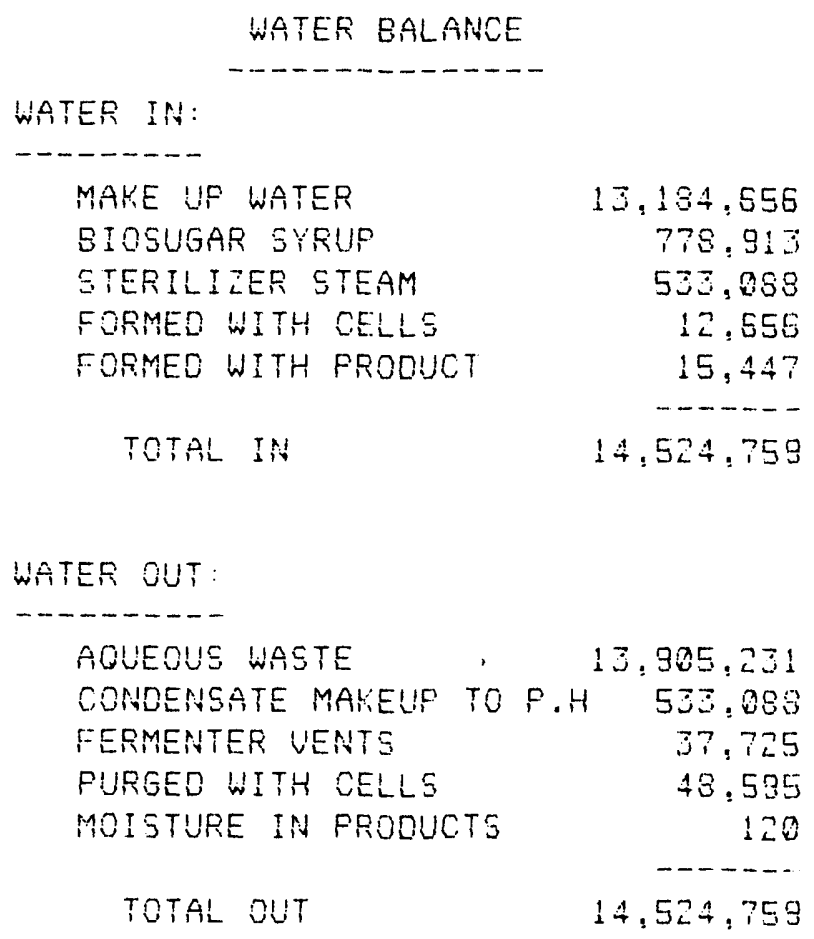




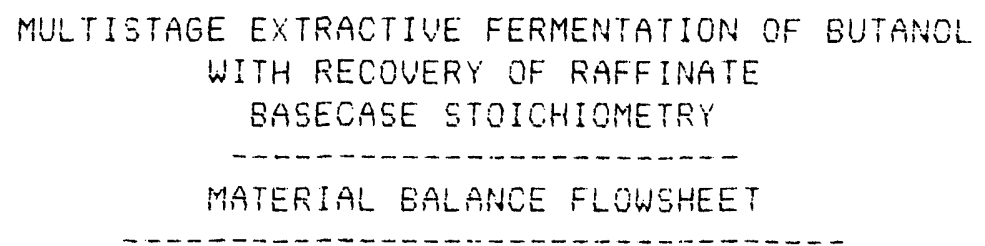

THOUGAND ANNUAL POUNDS S 330 DAYS) A CAPACITY

\begin{tabular}{|c|c|c|c|c|c|c|}
\hline & & $\begin{array}{c}1 \\
\text { CORH' } \\
\text { CYRUE }\end{array}$ & $\begin{array}{c}2 \\
\text { ANHYO } \\
\text { AMMONIA }\end{array}$ & $\begin{array}{l}3 \\
\text { NUTRIENTS }\end{array}$ & $\begin{array}{c}4 \\
\text { MIX } \\
\text { WATER }\end{array}$ & $\begin{array}{c}5 \\
\text { MIYED } \\
\text { MEOIUM }\end{array}$ \\
\hline$P$ & $\begin{array}{l}\text { STREAM } \\
\text { ST }\end{array}$ & SYRUF & AMMONIA & $\ldots \ldots$ & WATER & MEOIUM \\
\hline F & CELLS - CHO & 3 & 0 & 0 & 0 & 0 \\
\hline 0 & $-N+12$ & 0 & 0 & 0 & 0 & 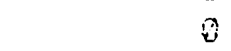 \\
\hline D & -MINERALS & 0 & 0 & 0 & 8 & D. \\
\hline y & -TOTAL & 0 & 0 & 0 & 3 & 3 \\
\hline$c$ & COMFONENT \#1 & 8 & 0 & 3 & 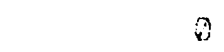 & 8 \\
\hline ? & COMPONENT \#Z & 0 & 0 & 8 & 8 & 3 \\
\hline$\# 2$ & COMPONENT \#3 & 0 & 8 & 8 & 8 & a \\
\hline & COMPONENT \#4 & 0 & 0 & 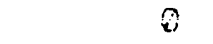 & 8 & 0 \\
\hline & COMPONENT \#5 & 2 & 0 & 0 & 0 & Q \\
\hline & COMPONENAT \#E & 8 & 0 & 0 & 0 & 8 \\
\hline \#1 & COMPONENT \#T & 8 & 0 & 0 & 0 & 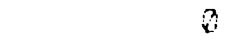 \\
\hline & COMFONENT \#B & 0 & 8 & 0 & 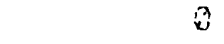 & 0 \\
\hline & COMPONENT \#9 & 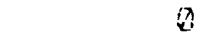 & 0 & 0 & 0 & 0 \\
\hline & COMPONENT $\# 10$ & 3 & 0 & 0 & 8 & 0 \\
\hline & COMPONENT \#11 & 8 & 0 & 0 & 0 & $\theta$ \\
\hline & COMPONENT \#12 & 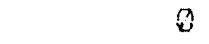 & 0 & $\theta$ & 6 & 0 \\
\hline & COMPONENT \#13 & 8 & 0 & 0 & 8 & 0 \\
\hline \# & COMFONENT \#14 & 0 & 0 & 0 & 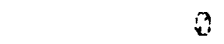 & 0 \\
\hline & COMPONENT \#15 & 8 & 0 & 8 & 8 & 0 \\
\hline & COMPONENT \#2E & 0 & 8 & 8 & 0 & 6 \\
\hline & COMPONENT \#17 & 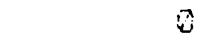 & 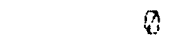 & 0 & 0 & 0 \\
\hline \# & COMOONENT \#13 & 0 & 8 & 8 & 8 & ? \\
\hline & COMPONENT \#13 & 0 & b) & 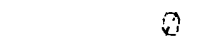 & 8 & 3 \\
\hline & COMPONENT \#ZO & 8 & 3 & 0 & $\theta$ & 0 \\
\hline & GLUCOSE & $778.31 \dot{0}$ & $\theta$ & 0 & 0 & 773,913 \\
\hline & AMMONIA & 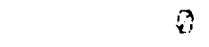 & 1.883 & 0 & 8 & \\
\hline & FHOSPUORIO ACID & 0 & 0 & 1,573 & 2 & 1,573 \\
\hline & FOTASSIUM OHLORIDE & 2 & 0 & 1.197 & 2 & 1.197 \\
\hline & MINOR RUTRIENTS & 0 & 8 & $5.4 \overline{3}$ & 0 & 543 \\
\hline & WATER & 778,913 & $\theta$ & 0 & $12,551,558$ & $13,430,481$ \\
\hline & SOLUENT & 0 & $\theta$ & $\theta$ & 0 & 8 \\
\hline & CAREON CIOKIOE & 0 & 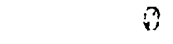 & 3 & 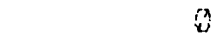 & 0 \\
\hline & OXYEEN & $\theta$ & 0 & 0 & 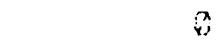 & $a$ \\
\hline & NITROGEN & 8 & 8 & 0 & $\hat{x}$ & 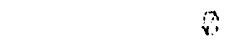 \\
\hline & HYOROGEN & 8 & $\theta$ & B & 8 & $\theta$ \\
\hline & 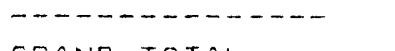 & $-\cdots---$ & $\cdots-\cdots$ & $\cdots \cdots-$ & $\cdots \cdots \cdots$ & $\cdots \cdots-\cdots$ \\
\hline & $\begin{array}{l}\text { GPANAE TOTAL } \\
\text { CHECL ON TOTAL }\end{array}$ & $1,557,825$ & 1,888 & 3,313 & $12,551,558$ & $14,212,707$ \\
\hline & TEMPERATURE, C & 28 & 20 & 20 & 20 & 20 \\
\hline & FRESGULE, PSIA & 14.7 & 14.7 & 14.7 & 20.8 & 25.0 \\
\hline & STATE & 506 in & LIOUID & SOLIOS & LIOUID & $50 \mathrm{n} \cdot \mathrm{n}$ \\
\hline
\end{tabular}


MULTISTAGE EXTRACTIUE FERMENTATION OF DUTANOL

WTTH RECOUERY OF RAFFINATE

DASEOASE STOICHIOMETPY

MATERIAL EALANCE FLOWEHEET

THOUSAND AHNUAL POUNDS 3 SO DAYS: O CAPACITY

\begin{tabular}{|c|c|c|c|c|c|c|}
\hline & & $\begin{array}{c}E \\
4 x^{6}=5 \\
\text { EFE }\end{array}$ & $\begin{array}{c}\text { ? } \\
\text { STEPILE } \\
\text { MEOIUM }\end{array}$ & $\begin{array}{c}8 \\
\text { HV TS } \\
\text { EFFLUENT }\end{array}$ & $\begin{array}{c}3 \\
\text { COOLEF } \\
\text { EFFLUENT }\end{array}$ & $\begin{array}{l}10 \\
\text { AIS } \\
\text { TO FERM }\end{array}$ \\
\hline 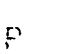 & SAERAT & 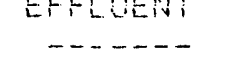 & MEDSUM & 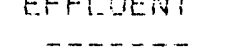 & EFHothin & 16tekn \\
\hline$P$ & CELES CHS & 8 & 8 & 6 & $\theta$ & $\theta$ \\
\hline a & $-N H 2$ & 8 & 0 & 8 & 0 & 0 \\
\hline$?$ & -MINERALS & 0 & 0 & 0 & 6 & 0 \\
\hline 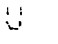 & -TGTAL & a) & $\theta$ & 8 & 0 & $\hat{\theta}$ \\
\hline$c$ & COMEONENT \#1 & 3 & 2 & 0 & 0 & 0 \\
\hline T & COMFONEHT \#2 & a & 8 & 0 & $\theta$ & $n$ \\
\hline$\# 2$ & COMPONENT \#Z & 8 & 0 & (4) & 0 & 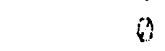 \\
\hline & COMPOHENA \#4 & a & $B$ & 0 & 0 & 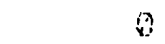 \\
\hline & GOMPONENT \#E & 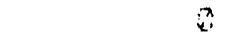 & 0 & $x^{3}$ & 0 & a \\
\hline & COMPONEENT \#S & a & 8 & 0 & $a$ & 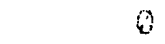 \\
\hline$\# 1$ & COMPONENT \#T & $\theta$ & 0 & 0 & $\theta$ & \\
\hline & COMPOHEAT \#S & a & $\hat{v}$ & 0 & 0 & \\
\hline & COMPONENT \#G & 0 & 0 & 0 & 0 & 0 \\
\hline & COMPONENT $\# 10$ & 0 & 8 & 0 & 8 & $\theta$ \\
\hline & COMPONENT $\# 11$ & 0 & 0 & i & 0 & 0 \\
\hline & OMOONEAT \#ZZ & 8 & 0 & a & 0 & 0 \\
\hline & COMFORENT & 0 & 0 & 0 & 8 & 0 \\
\hline$\#$ & COMPONER!T $\# 14$ & 0 & $\hat{0}$ & 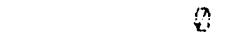 & a & 0 \\
\hline & OOMFONENT \#15 & 0 & 8 & 0 & 0 & 0 \\
\hline & SOMFOHEWT \#1E & 0 & 0 & 0 & 0 & 0 \\
\hline & COMEONENT \#17 & 0 & 0 & 3 & $\theta$ & 0 \\
\hline$\#$ & COMEONENT \#18 & ) & 3 & 0 & 0 & 0 \\
\hline & COMFONENT \#19 & 3 & 0 & 8 & 0 & 0 \\
\hline & COMPONENT $\# 20$ & 3 & 0 & $\theta$ & $\theta$ & $\theta$ \\
\hline & GLUCOSE & 778,913 & 778,913 & 779.913 & 778.913 & 8 \\
\hline & AMHONASA & 0 & 0 & 0 & 8 & 0 \\
\hline & PHOSPHORIC ACID & $: 573$ & 1,573 & 1.573 & 1,573 & - \\
\hline & POTASSTHM CHLOEIOE & 1,197 & 1.197 & 1,197 & 1,197 & \\
\hline & MTHOR MUTRIENTS & 543 & 543 & 543 & 543 & 0 \\
\hline & WATER & $13,430,481$ & $13,36 \dot{i}, 569$ & $13,983,559$ & $13,353,553$ & $\theta$ \\
\hline & SOLVENT & 0 & 0 & 0 & 0 & 0 \\
\hline & CAREON GIOKIDE & $\theta$ & 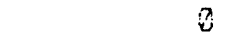 & 0 & 9 & 0 \\
\hline & OXYGEN & 8 & 8 & $\theta$ & $\theta$ & 183,054 \\
\hline & RITFOGEN & 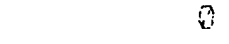 & ? & 0 & 0 & 357,833 \\
\hline & HEROGEN & $\dot{v}$ & 0 & 8 & $\theta$ & $\theta$ \\
\hline & $\ldots$ & $\ldots \ldots$ & $\cdots \cdots$ & $\ldots \ldots$ & $\ldots \ldots$ & $\ldots \ldots$ \\
\hline & $\begin{array}{l}\text { GPAHE TOTAL } \\
\text { CHECH ON TOTAL }\end{array}$ & $14,212,707$ & $14,745,795$ & $14,745,735$ & $14,745,735$ & 455,886 \\
\hline & TEMEERATURE: $\quad 0$ & 100 & 120 & 40 & 33 & 3 \\
\hline & PFESSUPE : PSIA & 25.8 & 25.0 & 25.0 & 25.0 & 60.0 \\
\hline & STATE & $50 L \cdot N$ & $\operatorname{soL} N$ & $50 L N$ & SOL. N & GAS \\
\hline
\end{tabular}


MULTISTAGE EXTRACTIUE FERMENTATION OF BUTANOL WITH RECQUERY OF RAFFINATE

BASECASE STOICHIOMETRY

MATERIAL BALANCE FLOWSHEET

THOUSAND ANNUAL POUNOS (ZJO DAYS: O CAPACITY

\begin{tabular}{|c|c|c|c|c|c|c|}
\hline & STREAM & $\begin{array}{c}11 \\
\text { COMBINED } \\
\text { FEED }\end{array}$ & $\begin{array}{c}12 \\
\text { BEER } \\
\# 1\end{array}$ & $\begin{array}{c}1 \Xi \\
B E E R \\
\# 2\end{array}$ & $\begin{array}{c}14 \\
\text { VENT } \\
\text { GROWTH }\end{array}$ & $\begin{array}{c}15 \\
\text { UENT } \\
\text { PROO'N }\end{array}$ \\
\hline$p$ & 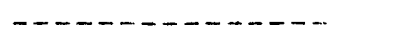 & $\ldots-\cdots$ & $\ldots \ldots$ & $\ldots \ldots$ & & \\
\hline R & CELLS - CHO & 0 & 19,433 & 13,433 & w & $y$ \\
\hline 0 & $-N H Z$ & 0 & 1,777 & $1,7,77$ & 2 & 0 \\
\hline $\mathrm{D}$ & -MINERALS & 8 & 543 & 543 & 0 & (2) \\
\hline $\mathrm{u}$ & -TOTAL & 0 & 21,753 & 21.758 & $\theta$ & 0 \\
\hline$C$ & COMFONENT \#1 & 0 & 0 & 0 & 0 & 8 \\
\hline T & COMPONENT \#Z & 0 & 9 & 0 & 0 & () \\
\hline$\# 2$ & COMPONENT \#Z & 0 & $\theta$ & $5 E, 335$ & 0 & v \\
\hline & COMPONENT \#A & 0 & 8 & 0 & 0 & () \\
\hline & COMPONENT \#5 & 0 & 8 & 18,715 & 0 & $y$ \\
\hline & COMPONENT \#E & 0 & 0 & 0 & $D$ & 3 \\
\hline$\# 1$ & COMPONENT \#7 & $D$ & 0 & 181,401 & 2 & 8 \\
\hline & COMPONENT \#B & 0 & 8 & 35.717 & 0 & $a$ \\
\hline & COMPONENT \#9 & 0 & 0 & 0 & 2 & 0 \\
\hline & COMPONENT \#10 & 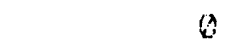 & 8 & 14,553 & 0 & 0 \\
\hline & COMPONENT \#11 & (2) & 0 & $\theta$ & a & 8 \\
\hline & COMPONENT \#12 & 3 & (6) & 8 & 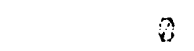 & 0 \\
\hline & COMPONENT \#13 & 3 & 0 & 6 & 8 & 4 \\
\hline \# & COMPONENT \#14 & 0 & 0 & 6 & 2 & \\
\hline & COMPONENT \#15 & 0 & 0 & 0 & 0 & \\
\hline & COMPONENT \#15 & 8 & 8 & : & 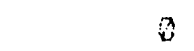 & \\
\hline & COMPONENT \#17 & 0 & 0 & 8 & $\theta$ & \\
\hline & COMEONENT \#13 & 0 & 0 & 0 & 0 & \\
\hline & COMPONENT \#19 & 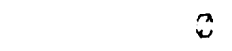 & 8 & 8 & 0 & \\
\hline & COMPONENT \#2Q & 0 & 8 & 0 & 0 & \\
\hline & GLUCOGE & 778.913 & 740.037 & 1,395 & 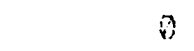 & \\
\hline & AMMONIA & 1,388 & 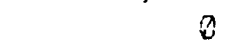 & 0 & $\theta$ & \\
\hline & PHOSFHORIC ACID & 1,573 & 1.573 & 1,573 & 8 & \\
\hline & POTASSIUM CHLORIDE & 1,197 & 1,197 & 1,197 & 8 & \\
\hline & MINOR NUTRIENTS & 543 & $\theta$ & 0 & $a$ & $\theta$ \\
\hline & WATER & $13,953,569$ & $13,951,738$ & $13,353,345$ & $1+, 487$ & 23,238 \\
\hline & SOLVENT & 0 & 0 & 0 & 0 & $\pi$ \\
\hline & CARBON DIOXIOE & 0 & 3 & $D$ & 28,493 & 403,351 \\
\hline & OXYGEN & 108.054 & 8 & 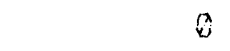 & 86,444 & $\theta$ \\
\hline & NITROGEN & 357.833 & 8 & 2 & 357,833 & 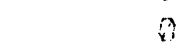 \\
\hline & HYDROGEN & 0 & 0 & 8 & 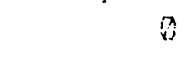 & 11.022 \\
\hline & 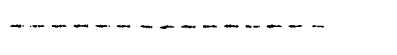 & $\cdots \cdots$ & $\cdots \cdots-$ & $\cdots \cdots$ & $\cdots \cdots$ & $\ldots \ldots$ \\
\hline & $\begin{array}{l}\text { GRANO TOTAL } \\
\text { CHECK ON TOTAL }\end{array}$ & 15.213 .571 & $14,726,503$ & $14,239,891$ & 487,257 & $458,21 \%$ \\
\hline & TEMPERATURE, O & 33 & 3.5 & $3 j$ & $3 j$ & 35 \\
\hline & PRESSURE, FSIA & $\cdots$ & 4.4 .7 & 44.7 & 14.7 & 14.7 \\
\hline & STATE & -- & SLUEFY & SLURR: & GAS & GAS \\
\hline
\end{tabular}


MULTISTAGE EXTRACTIUE FERMENTATION OF BUTANOL

WITH RECOUERY OF RAFFINATE

BPSECASE STOIOHIOMETRY

MATERTAL BALANUE FLOWSHEET

THOUSANO ANAUAL POUNOE I3JO DAYS: G CAPACITY

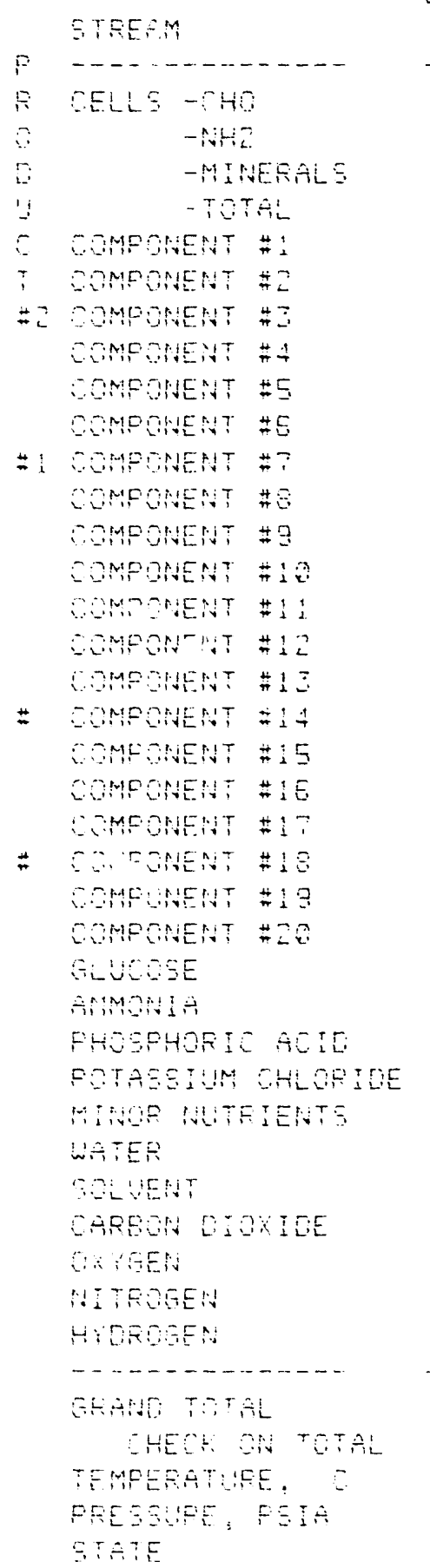


MULTISTAGE EXTRACTIUE FERMENTATION OF BUTANOL WITH RECOUERY OF RAFFINATE BASECASE STOICHIOMETPY

MATERIAL BALANCE FLOWSHEET

THOUSAND ANNLIAL POUNDS (330 DAYS) E CAPACITY

\begin{tabular}{|c|c|c|c|c|c|c|}
\hline & STREAM & $\begin{array}{l}\text { 21 } \\
\text { CELLS TO } \\
\text { DISPOSAL }\end{array}$ & $\begin{array}{c}22 \\
\text { FILTRATE } \\
\text { TO RAFF \#1 }\end{array}$ & EXTRACT & $\begin{array}{l}\text { 2A } \\
\text { BEER \#1 } \\
\text { FEED }\end{array}$ & $\begin{array}{l}25 \\
\text { BEER \#1 } \\
\text { MAKE }\end{array}$ \\
\hline$P$ & SIREAM & $\ldots$ & - & - - - - & $-\cdots-\ldots$ & - \\
\hline$R$ & CELLS - CHO & 19.433 & 0 & 0 & $D$ & 0 \\
\hline 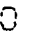 & $-N H 2$ & 1,777 & 0 & $\theta$ & (2) & 0 \\
\hline 0 & -MINERALS & 543 & 0 & 0 & 0 & 0 \\
\hline ' & -TOTAL & 21,758 & D & 0 & 0 & 0 \\
\hline c & COMPONENT \#1 & 0 & 0 & 0 & 0 & 0 \\
\hline$T$ & COMPONENT \#Z & 0 & 0 & 0 & 0 & 0 \\
\hline$\# 2$ & COMPONENT \#3 & 159 & 48,335 & 3,281 & 8,281 & 8,281 \\
\hline & COMPONENT \#4 & 0 & 0 & D & $\theta$ & 0 \\
\hline & COMPONENT \#5 & E1 & $17: 567$ & 1,088 & 1,038 & 1,083 \\
\hline & COMPONENT \#E & 0 & 0 & 0 & 0 & 0 \\
\hline$\# 1$ & COMFONENT \#? & 1 & 181 & 131,220 & 133,251 & 138,345 \\
\hline & COMPONENT \#B & 120 & 34,453 & 2,134 & 2,170 & 397 \\
\hline & COMPONENT \#3 & 8 & 0 & 0 & 0 & 0 \\
\hline & COMPONENT \#10 & 48 & 13,659 & 845 & 882 & 397 \\
\hline & COMPONENT \#11 & 0 & 0 & 0 & 0 & 6 \\
\hline & COMFONENT \#12 & 6 & 0 & 0 & 0 & 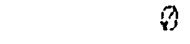 \\
\hline & COMPONENT \#Iذ & 0 & 0 & 0 & 0 & 0 \\
\hline$\#$ & COMPONENT \#14 & 9 & 0 & 0 & 0 & 0 \\
\hline & COMPONENT \#15 & 8 & 0 & 0 & 0 & 0 \\
\hline & COMPONENT \#1E & 0 & (i) & 0 & 0 & 0 \\
\hline & COMPONENT $\# 17$ & 0 & D & 0 & 0 & 0 \\
\hline \# & COMFONENT \#18 & 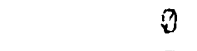 & $\theta$ & 0 & 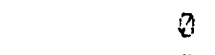 & 0 \\
\hline & COMEONENT $\# 13$ & 0 & 0 & 0 & 0 & 0 \\
\hline & COMPONENT \#ZO & 0 & 0 & 0 & 0 & 0 \\
\hline & GLUCOSE & 5 & 1,531 & (3) & 0 & 8 \\
\hline & AMMONIA & 0 & 0 & 0 & 0 & 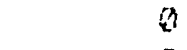 \\
\hline & PHOSFHORIC ACID & 5 & $1,5 E ?$ & 8 & 0 & 0 \\
\hline & POTASEIUM CHLORIDE & 4 & $1: 193$ & 0 & 0 & $n$ \\
\hline & MINOR NUTRIENTS & 0 & 0 & 0 & 0 & 0 \\
\hline & WATER & 48,595 & $1 \overline{3}, 905,263$ & 84 & 84 & 84 \\
\hline & SOLUENT & 1 & 278 & $4,135,905$ & $4,333,389$ & 198,345 \\
\hline & CARBON DIOXIDE & 0 & 0 & 0 & 0 & 8 \\
\hline & OXYGEN & 0 & 8 & 0 & 0 & 0 \\
\hline & NITROEEN & 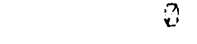 & 0 & 0 & 3 & 0 \\
\hline & HYOROGEN & 3 & D & 8 & 0 & 0 \\
\hline & $\cdots \cdots$ & $-\cdots--$ & $\cdots-\cdots$ & $\cdots-\cdots$ & $\cdots \cdots$ & $\cdots-\cdots$ \\
\hline & $\begin{array}{l}\text { GRAND TOTAL } \\
\text { OHECS ON TOTAL }\end{array}$ & 70.753 & $14,825,951$ & $4,379,555$ & $4,595,644$ & $405: 936$ \\
\hline & TEMFERATURE: $C$ & 3 & 33 & $\dot{j}$ & $\cdots-$ & 114 \\
\hline & PRESSURE F FIA & 14.7 & 24.7 & 11.7 & -- & 12.5 \\
\hline & STATE & SLUFFi & sol in & SOL in & $S O L \cdot A$ & $S O L \cdot N$ \\
\hline
\end{tabular}


MULTISTAEE EXTRACTIUE FERMENTATION OF EUTAROL WITH RECOULRY OF RAFFINATE

BASECASE STOICHIOMETRY

MATERIAL BALANCE FLOWSHEET

THOUSAND ANNUAL POUNDE :

\begin{tabular}{|c|c|c|c|c|c|c|}
\hline & STREAM & TAILS & MAKLE & TAILS & MAKE & TAILS \\
\hline$P$ & $\ldots$ & $\cdots \cdots$ & $\cdots \cdots$ & $\ldots \ldots$ & $\ldots \ldots$ & $\ldots \ldots$ \\
\hline$R$ & CELLS - CHC & 0 & 6 & 0 & 8 & 3 \\
\hline 0 & $-N_{4}+2$ & 0 & 0 & 0 & 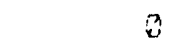 & 3 \\
\hline [ & -MINERALS & 8 & 8 & 0 & 0 & $a$ \\
\hline bid & -TOTAL & 8 & 8 & $\theta$ & 8 & 8 \\
\hline$C$ & COMPONENT \#! & 8 & R & 8 & 8 & 6 \\
\hline T & COMPLNENT \#Z & 2 & 8 & 6 & 0 & 6 \\
\hline$\# 2$ & COMPONENT \# & $\theta$ & 8.281 & 0 & 7.922 & 359 \\
\hline & COMPONENT \#4 & $\theta$ & 0 & 0 & 9 & 8 \\
\hline & COMPONENT \#5 & $\theta$ & 1,883 & 3 & 729 & 359 \\
\hline & COMFONENT \#E & 0 & 0 & 0 & 3 & 3 \\
\hline . & COMFONENT \#T & 306 & 180,314 & 13.031 & 902 & 179.412 \\
\hline & COMPONENAT \#B & 1.773 & JE 1 & 35 & 8 & JE! \\
\hline & COMPONENT \#9 & 6 & 0 & 0 & 0 & 0 \\
\hline & COMPONENT \#LB & 485 & 361 & is & 8 & 351 \\
\hline & COMFONENT \#11 & 6 & 8 & 0 & 8 & 0 \\
\hline & COMPONENT \#12 & 0 & 0 & 8 & 0 & 8 \\
\hline & COMPONENT \#ZZ & 0 & 8 & 0 & 0 & 0 \\
\hline \# & COMPONENT \#14 & $\theta$ & : & 8 & 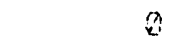 & 0 \\
\hline & COMPONENT \#15 & 3 & 0 & $\theta$ & 0 & 0 \\
\hline & COMPONENT \#15 & 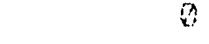 & 0 & 0 & $\theta$ & 8 \\
\hline & COMPONEHT \#17 & 8 & $\theta$ & D & 8 & 0 \\
\hline$\#$ & COMPONENT \#1B & 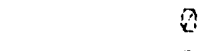 & 0 & 0 & 8 & 2 \\
\hline & COMPONENT \#19 & 8 & 0 & 0 & 0 & 0 \\
\hline & OOMFONENT \#2O & 8 & 3 & 0 & 0 & 0 \\
\hline & GLUCOSE & $\hat{z}$ & 0 & 8 & 0 & 0 \\
\hline & AMMONIA & 0 & 8 & 3 & 0 & (a) \\
\hline & PHOSPHOFIC ACIO & 8 & $\theta$ & 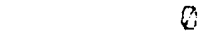 & 0 & 0 \\
\hline & POTASEIUM CHLORIDE & $\hat{i}$ & 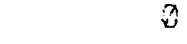 & $\theta$ & 0 & 0 \\
\hline & MINOR AUTRIENTS & 8 & 0 & 0 & $Q$ & 0 \\
\hline & WATEE & 0 & 34 & 3 & 8 & 34 \\
\hline & SOLUENT & 4.185 .544 & 351 & 197,985 & 8 & SEL \\
\hline & CAREOA EIOXIDE & 2 & 0 & 3 & 0 & 0 \\
\hline & OXYEEN & 8 & r & 8 & 0 & 8 \\
\hline & NITROGEN & 8 & 8 & 0 & $\theta$ & 8 \\
\hline & HYOROGER: & 3 & 8 & 0 & 8 & 8 \\
\hline & 西 & $-\cdots$ & $\cdots \cdots$ & 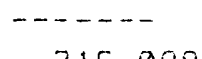 & . & \\
\hline & $\begin{array}{l}\text { GRANE TOTAL } \\
\text { OHECL ON TOTAL }\end{array}$ & 4.138 .708 & 130,948 & 216,098 & 9,552 & 181,295 \\
\hline & TEMPERATURE : & 220 & 170 & 220 & 127 & 175 \\
\hline & FRESOURE， PSIA & 14.3 & 39.2 & 90.2 & 39.1 & 100.5 \\
\hline & STATE & $50 \mathrm{~N}$ & $50 \mathrm{~L} n$ & $50 \mathrm{~L}$ ' & $50 \mathrm{~L} \cdot \mathrm{W}$ & $50 \mathrm{~L} \cdot \mathrm{H}$ \\
\hline
\end{tabular}


MULTIETAGE EXTRACTIUE FERMENTATION OF BUTANOL WITH RECOUERY OF RAFFINATE

BASECASE STOYCHIOMETRY

MATERIAL BALANCE FLOWEHEET

THOUGAND ANNUAL POUNDS 330 DAYS: E CAFACITY

\begin{tabular}{|c|c|c|c|c|c|c|}
\hline & STREAM & $\begin{array}{c}3 ! \\
\text { LBS \#2 } \\
\text { MAKE }\end{array}$ & $\begin{array}{c}32 \\
\text { LBS } \# 2 \\
\text { TAILS }\end{array}$ & $\begin{array}{c}53 \\
\text { LES \#3 } \\
\text { MAKE }\end{array}$ & $\begin{array}{c}34 \\
\text { LBS \#3 } \\
\text { TAILS }\end{array}$ & $\begin{array}{c}35 \\
\text { HPS \#1 } \\
\text { MAKE }\end{array}$ \\
\hline$P$ & 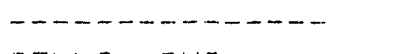 & $\cdots \cdots$ & $\cdots \cdots$ & $-\cdots-\cdots$ & $\cdots \cdots$ & $a$ \\
\hline$R$ & CELLS - CHO & 0 & 0 & 0 & 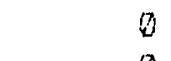 & $\theta$ \\
\hline 0 & $-\mathrm{NHHZ}$ & 0 & (⿻) & 0 & $\theta$ & 0 \\
\hline $\mathrm{D}$ & -MINERALS & 0 & 0 & 0 & 0 & $\theta$ \\
\hline ! & -TOTAL & 0 & 8 & 0 & 0 & 0 \\
\hline $\mathrm{C}$ & COMPONENT \#1 & 0 & 0 & 6 & 0 & $a$ \\
\hline$T$ & COMPONENT \#2 & 0 & 0 & 6 & 0 & $\pi$ \\
\hline$\# 2$ & COMPONENT \#Z & 7.882 & 43 & $\theta$ & $\theta$ & Q \\
\hline & COMPONENT \#4 & 0 & 8 & $\theta$ & $\theta$ & 0 \\
\hline & OOMPONENT \#5 & 16 & 713 & 0 & 0 & $\pi$ \\
\hline & COMPONENT \#S & 8 & 0 & 0 & 0 & 0 \\
\hline$\# 1$ & COMPONENT \#T & 8 & 902 & $\theta$ & 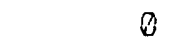 & $90 E$ \\
\hline & COMPONENT \#B & 8 & 0 & 0 & 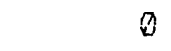 & 1,754 \\
\hline & COMPONENT \#B & 0 & 0 & 0 & $a$ & $\theta$ \\
\hline & COMPONENT \#10 & 0 & 0 & 0 & $\theta$ & 483 \\
\hline & COMPONENT \#11 & 2 & a & 0 & 0 & 0 \\
\hline & COMPONENT \#12 & 0 & 0 & 6 & $\theta$ & 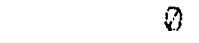 \\
\hline & COMFONENT \#13 & 0 & 0 & 0 & 0 & 0 \\
\hline \# & COMPONENT \#14 & 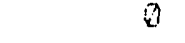 & 0 & 0 & 0 & 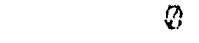 \\
\hline & COMFONENT \#15 & $\theta$ & 0 & 0 & 0 & ( \\
\hline & COMPONENT \#1E & 8 & 9 & 0 & $\theta$ & 0 \\
\hline & COMFONENT \#17 & 0 & 3 & 0 & 0 & 0 \\
\hline \# & COMPONENT \#18 & $\theta$ & 0 & 0 & 0 & 0 \\
\hline & COMFONENT \#13 & 8 & 0 & 0 & 0 & 0 \\
\hline & COMPONENT \#ZO & 0 & 0 & 0 & 0 & 0 \\
\hline & GLUCOSE & $\theta$ & 0 & $\pi$ & 6 & 0 \\
\hline & AMMONIA & 8 & 0 & 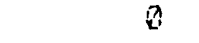 & 0 & 0 \\
\hline & FHOSFHORIC ACID & 0 & 0 & $\theta$ & 0 & 0 \\
\hline & FOTASEIUM CHLORIDE & 8 & 6 & 0 & 0 & 0 \\
\hline & MINOR NUTRIENTS & 0 & 8 & $\theta$ & a & 0 \\
\hline & WATEK & 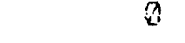 & 0 & 0 & 0 & 0 \\
\hline & SOLVENT & 8 & 0 & 0 & 0 & 1 \\
\hline & CARBON DIOXIDE & 0 & 0 & 0 & 0 & 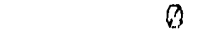 \\
\hline & OKYGEN & 0 & 0 & 0 & $Q$ & w \\
\hline & NITROGEN & 0 & 0 & 0 & 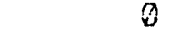 & $\psi$ \\
\hline & HYDROGEN & 8 & 0 & Q & 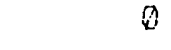 & 8 \\
\hline & $\begin{array}{l}\text { GRANO TOTAL } \\
\text { CHECK ON TOTAL }\end{array}$ & 7,838 & 554 & 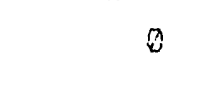 & 0 & 14 \\
\hline & TEMPERATURE, $\quad C$ & 37 & 120 & $\{273\}$ & 120 & 120 \\
\hline & PRESSURE, PSIA & 40.3 & $4 E .5$ & .0 & .0 & 14.1 \\
\hline & ETATE & SOL'N & SOL'N & SOL'N & SOL N & SOL'M \\
\hline
\end{tabular}


MULTISTAGE EXTPACTIUE FERMENTATIOH OF EUTAROL.

WITH FECOUEFY OF RAFFINATE

BASECASE STOICHIOMETFY

MATERIAL BALANCE FLOWBHEET

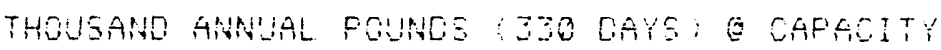

\begin{tabular}{|c|c|c|c|c|c|c|}
\hline & STREAM & $\begin{array}{l}\text { HES } \# 1 \\
\text { TAILS }\end{array}$ & $\begin{array}{c}\text { BHFROWUC } \\
\text { FEED }\end{array}$ & $\begin{array}{l}\text { PAFF \#1 } \\
\text { FEED }\end{array}$ & $\begin{array}{c}\text { FAFE } 1 \\
\text { MAYE }\end{array}$ & $\begin{array}{c}\text { PAFF \#1 } \\
\text { TAILS }\end{array}$ \\
\hline $\mathrm{F}$ & 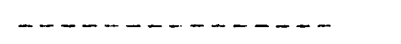 & $\ldots \ldots$ & $\ldots \ldots$ & $\ldots \ldots$ & $\ldots \ldots$ & $\ldots \ldots$ \\
\hline$R$ & CELLS - CHO & 0 & 8 & 8 & 8 & 3 \\
\hline 0 & $-N .14 Z$ & 0 & 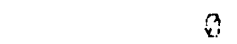 & 2 & 3 & 0 \\
\hline 0 & -MINERALS & 0 & 0 & 8 & 6 & 8 \\
\hline t: & -TOTAL & 3 & 0 & $a$ & 2 & $B$ \\
\hline c & GOMPONENT \#1 & 0 & 0 & 3 & 8 & 0 \\
\hline$T$ & COMPONENT $\# 2$ & 3 & 3 & 6 & 8 & 3 \\
\hline$\# 2$ & COMFONENT $\#$ & 0 & 48.424 & 48.424 & $4 E .424$ & 0 \\
\hline & OOMPOAENT \#4 & $\hat{\theta}$ & a & 3 & 3 & ? \\
\hline & COMPONENT \#5 & 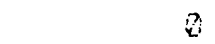 & 18,280 & 20.099 & 20.837 & $9:$ \\
\hline & COMPONENT \# & 2 & $D$ & $\hat{v}$ & 3 & $a$ \\
\hline$\# 1$ & COMFONENT \#7 & 0 & 1,032 & 1.235 & 40 & 1.045 \\
\hline & COMPONEHT \#S & 9 & 34,453 & $34: 4 E \Xi$ & 6 & 34,463 \\
\hline & COMPONENT $\# 9$ & 0 & 0 & 6 & 0 & 0 \\
\hline & COMPOHEAT \#10 & 2 & 15.659 & $1: 559$ & 0 & $1: 1,559$ \\
\hline & COMFONENT \#11 & 8 & $a$ & 3 & 0 & 0 \\
\hline & COMPONENT \#12 & 3 & 0 & 3 & : & 3 \\
\hline & COMPOHENT \#1Z & 0 & 8 & 0 & 8 & 3 \\
\hline$\#$ & COMPONENT \#14 & 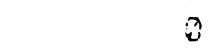 & 3 & 3 & 3 & ? \\
\hline & COMPONENT \#IS & 8 & 0 & 8 & 2 & 3 \\
\hline & COMPOHENT \#IS & 0 & 0 & 3 & 3 & 6 \\
\hline & SCMPONENT \#17 & $a$ & 0 & 0 & 0 & 0 \\
\hline \# & COMFONENT \#18 & 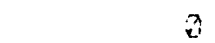 & c & 3 & (3) & $\lambda$ \\
\hline & COMFONENT \#19 & 0 & 0 & $a$ & 2 & 8 \\
\hline & COMPONENT $\# 2 Q$ & 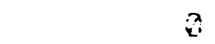 & D & 2 & 3 & 3 \\
\hline & GLUCOSE & 2 & $2: 591$ & $1: \div 31$ & 8 & 1,391 \\
\hline & AMMONIA & 8 & 0 & $\hat{x}$ & a & 0 \\
\hline & FHOSFHOEIC ACIO & 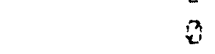 & $2.56 ?$ & 1.557 & 0 & 1.567 \\
\hline & POTASEIUM CHLORIDE & $a$ & $1,19 j$ & $1: 193$ & 8 & 1.193 \\
\hline & MINOR NUTEIENTS & 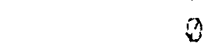 & 8 & 0 & 8 & 0 \\
\hline & WATER & $a$ & $1 \equiv, 305: 268$ & $13,325,233$ & 20,807 & $13,335,231$ \\
\hline & SOLUENT & $4: 135.543$ & 275 & 273 & 3 & 278 \\
\hline & GARSOH DIOXIDE & 0 & 6 & 0 & 6 & $\lambda$ \\
\hline & OX:GEN & 0 & 3 & 3 & 0 & 0 \\
\hline & MITEOGEN & 8 & 3 & 2 & 2 & 3 \\
\hline & HYDROGEN & 0 & 8 & $\theta$ & 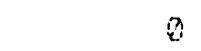 & 0 \\
\hline & $\ldots \ldots \ldots$ & $\cdots \cdots-$ & $\cdots \cdots-\cdots$ & $\ldots \ldots$ & $\ldots \ldots$ & $\ldots \ldots$ \\
\hline & $\begin{array}{l}\text { GRAHE TOTAL } \\
\text { OHEOK ON TOTAL }\end{array}$ & $4: 185: 554$ & $14,025,505$ & 14.047 .593 & 83.473 & $1 j, 958,320$ \\
\hline & TEMFEFATURE, & 220 & 3 & $3:$ & 74 & 105 \\
\hline & FRESSURE, FSIA & $1 E .1$ & $14 . ?$ & - & 14.4 & 27.6 \\
\hline & STATE & $30 L N$ & $50 L$ id & $50 \mathrm{~L} \%$ & $50 L$ 'H & SoL'i \\
\hline
\end{tabular}


MULTISTAGE EXTRACTIUE FERMENTATION OF BUTANOL

WITH RECOUERY OF RAFFINATE

BASECASE STOICHIOMEIPY

MATERIAL BALANCE FLOWGHEET

THOUSAND ANNUAL PQUNOS 33 OR DAYS G CAPACITY

\begin{tabular}{|c|c|c|c|c|c|c|c|}
\hline & STREAM & $\begin{array}{l}\text { RAFF \#2 } \\
\text { MAKE }\end{array}$ & $\begin{array}{l}\text { RAFF \#2 } \\
\text { TAILS }\end{array}$ & $\begin{array}{l}\text { RAFF \#S } \\
\text { MAKE }\end{array}$ & $\begin{array}{l}\text { RAFF \#3 } \\
\text { TAILS }\end{array}$ & $\begin{array}{l}\text { REFINED } \\
\text { BUTANOL }\end{array}$ & $\begin{array}{l}\text { REFINEO } \\
\text { ACETONE }\end{array}$ \\
\hline P & 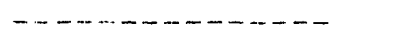 & $\cdots \cdots \cdots$ & $\ldots \ldots$ & 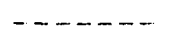 & $\ldots-\cdots$ & $\ldots \ldots$ & $\ldots \ldots$ \\
\hline 只 & CELLS -CHO & 0 & 0 & 8 & 8 & $\theta$ & 0 \\
\hline 0 & $-N+4 z$ & 0 & 0 & a) & 0 & 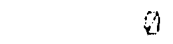 & 0 \\
\hline D & -MINERALS & 8 & 0 & 0 & 8 & 0 & 0 \\
\hline ! & -TOTAL & 0 & 0 & 0 & 0 & 0 & ? \\
\hline 0 & COMPONENT \#1 & 0 & (1) & n & $\theta$ & 0 & is \\
\hline T & COMPONENT \#Z & ? & 0 & 0 & 0 & 0 & $\theta$ \\
\hline$\# 2$ & COMPONENT \#3 & 43.424 & 0 & 48,182 & 242 & 359 & 55,055 \\
\hline & OOMPONENT \#4 & 0 & a & $a$ & $n$ & 6 & $\theta$ \\
\hline & COMPONENT \#5 & 18.189 & 1.819 & 35 & 19,832 & 359 & 112 \\
\hline & COMPONENT \#E & 0 & 8 & 0 & i & 0 & 0 \\
\hline$\# 1$ & COMFONENT \#? & 35 & 4 & 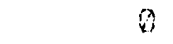 & 35 & 173,412 & 0 \\
\hline & COMPONENT \#G & 0 & 8 & a & $D$ & $3 E 1$ & Q \\
\hline & COMPONENT \#马 & 0 & i & 0 & 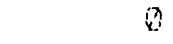 & 0 & $\theta$ \\
\hline & COMPONENT \#10 & 0 & 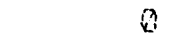 & 0 & 0 & 351 & 0 \\
\hline & COMPCINENT \#11 & 0 & 0 & 0 & 0 & $\theta$ & 0 \\
\hline & COMPONENT \#12 & 6 & 0 & 0 & 8 & 8 & 0 \\
\hline & COMPONENT \#13 & $\theta$ & (i) & 0 & 0 & 8 & 0 \\
\hline \# & COMEONENT \#14 & 8 & 8 & $\theta$ & 8 & 0 & $\theta$ \\
\hline & COMPONENT \#15 & 0 & 0 & 8 & 0 & a) & ? \\
\hline & COMPONENT \#IE & 0 & 0 & 3 & 8 & (i) & ()) \\
\hline & COMPONENT \#17 & 0 & 0 & 0 & 0 & 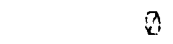 & 8 \\
\hline \# & COMPONENT \#18 & 0 & 0 & 0 & 0 & 8 & 0 \\
\hline & COMPONENT \#19 & 0 & 0 & 8 & $\theta$ & 3 & 8 \\
\hline & COMEOAENT \#2O & 8 & 0 & 0 & $\theta$ & $\theta$ & a) \\
\hline & GLUCosE & 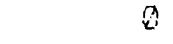 & 0 & 0 & 0 & 8 & $D$ \\
\hline & AMMONIA & 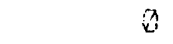 & 0 & (a) & 0 & 0 & 0 \\
\hline & PHOSPHORIC ACIO & 0 & 0 & 0 & i & (3) & a \\
\hline & FOTASSIUM CHLORIDE & 0 & 0 & $B$ & 0 & in & 0 \\
\hline & MINOR NUTRIENTS & 0 & 0 & 0 & 0 & $\theta$ & 8 \\
\hline & WATER & 36 & 19.971 & 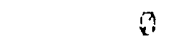 & 35 & 84 & 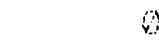 \\
\hline & SOLVENT & (x) & $\hat{i}$ & $\theta$ & 8 & 351 & 8 \\
\hline & CAREON DIOXIDE & 8 & 0 & $D$ & $\theta$ & 8 & 8 \\
\hline & OXYGEN & ix & 2 & 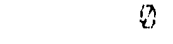 & 8 & 8 & 8 \\
\hline & NITROGEN & 8 & 0 & a & 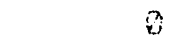 & 0 & a \\
\hline & HYDROGEN & 8 & $\theta$ & 0 & $\theta$ & 0 & D \\
\hline & $\begin{array}{l}\text { GRAND TOTAL } \\
\text { CHECK ON TOTAL }\end{array}$ & 56.585 & 21,794 & 48,273 & 18,407 & $\begin{array}{r}181: 295 \\
0\end{array}$ & $55,17 ?$ \\
\hline & TEMFERATURE， $\quad$ & 57 & 100 & 83 & 105 & 175 & 87 \\
\hline & PRESSURE, PSIA & 12.3 & 15.4 & 35.0 & 40.9 & 101 & 48 \\
\hline & STATE & $50 \mathrm{~L}$ in & $30 L$ in & $S O L N$ & $50 L N$ & 0 & $\theta$ \\
\hline
\end{tabular}


MUYZT TSAGE EXTFACTIUE FERMENTATION OF EUTANOL WTH REOOUEFY OF FAFFINATE

BASECASE STOTCHTOMETRY

MATERYAL BALANCE FLOWGHEET

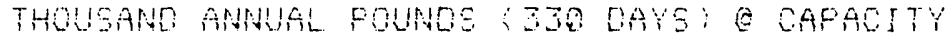

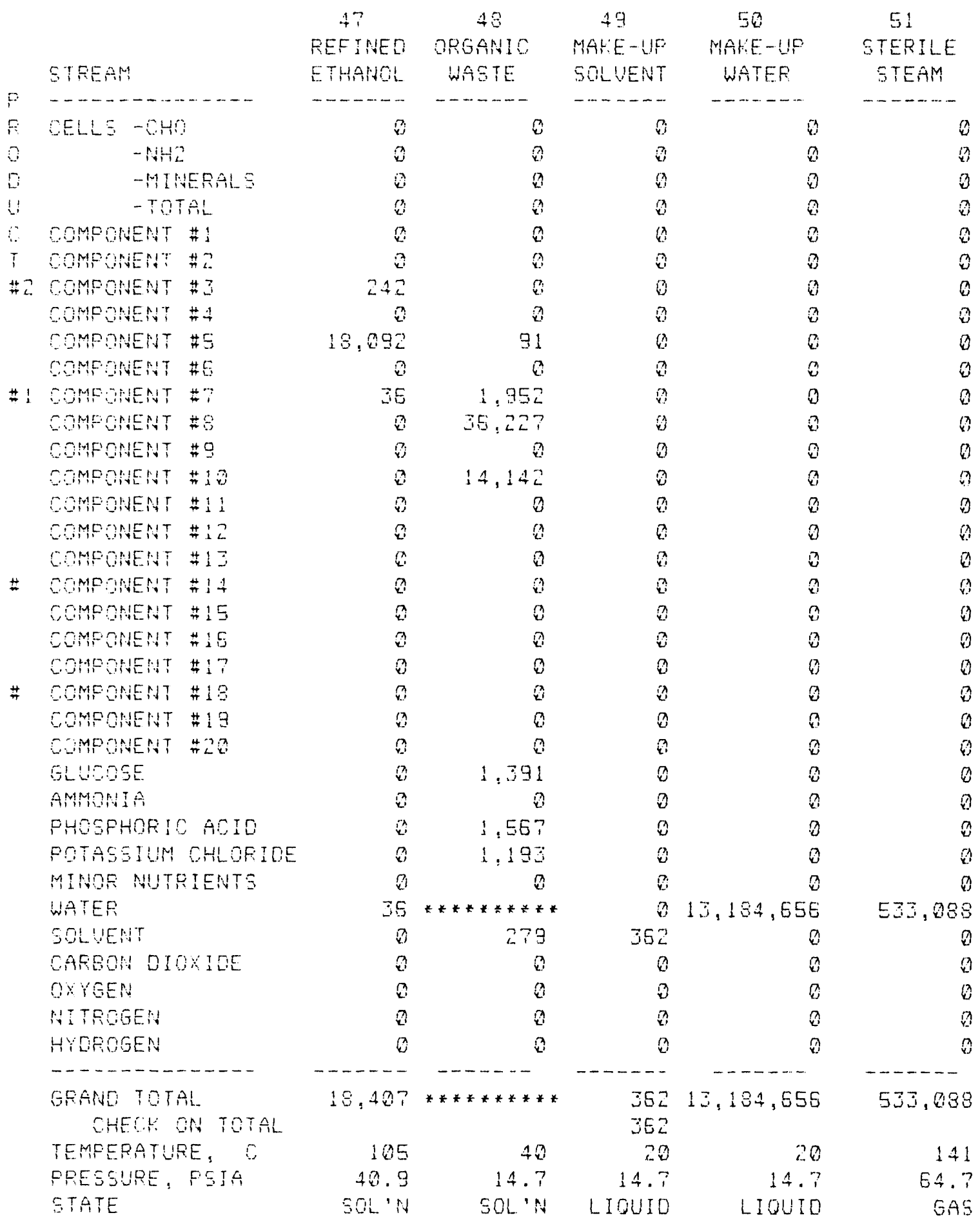


MULTISTAGE EKTRACTIUE FERMENTATION OF BUTANOL WITH RECOUERY OF RAFFINATE

BASECASE STOICHIOMETRY

MATERIAL BALANCE FLOWSHEET

THOUSAND ANNUAL POUNDS ISBO DAYS: O CAPACITY

\begin{tabular}{|c|c|c|c|c|c|c|}
\hline & & $\begin{array}{l}52 \\
\text { FESM'TR }\end{array}$ & $\begin{array}{c}53 \\
\text { DISTILL. }\end{array}$ & $\begin{array}{l}54 \\
\text { Cono. Mu }\end{array}$ & $\begin{array}{l}55 \\
\text { MELIUM }\end{array}$ & $\begin{array}{l}\text { SE } \\
\text { FERM. TR }\end{array}$ \\
\hline & STREAM & STEAM & STEAM & P'WR H'SE & COOL WTR & COOL WTR \\
\hline$P$ & 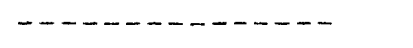 & $\ldots \ldots$ & $\cdots \cdots$ & $\ldots \ldots$ & $\ldots-\cdots$ & $\ldots \ldots$ \\
\hline$R$ & CELLE - CHO & 0 & 0 & 8 & 8 & 0 \\
\hline 0 & $-\mathrm{NH} \mathrm{H}_{2}$ & 6 & 0 & 0 & 0 & $a$ \\
\hline 0 & -MINERALS & 0 & 0 & 8 & 2 & 8 \\
\hline 4 & -TOTAL & $\theta$ & 0 & a & 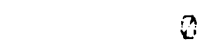 & 8 \\
\hline$c$ & COMPONENT \#1 & 0 & 0 & 0 & 0 & 0 \\
\hline$T$ & COMFONENT \#Z & 0 & 3 & 0 & 0 & 8 \\
\hline$\# 2$ & COMPONENT \#3 & 0 & 0 & 0 & 0 & 0 \\
\hline & COMPONENT \#4 & 8 & 0 & 0 & 0 & 0 \\
\hline & COMFONENT \#5 & $\theta$ & 8 & 0 & () & 0 \\
\hline & COMPONENT \#E & 0 & $B$ & 0 & 0 & 0 \\
\hline \#1 & COMPONENT \#? & a & ( & 8 & 0 & i \\
\hline & COMPONENT \#Q & 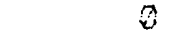 & 8 & 0 & 8 & 3 \\
\hline & COMFONENT \#G & 3 & 8 & 0 & 8 & i \\
\hline & COMPONENT \#10 & 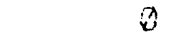 & 2 & 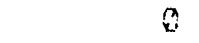 & Q & 8 \\
\hline & COMPONENT \#1: & 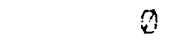 & $\theta$ & 8 & $\pi$ & a \\
\hline & COMPONENT \#12 & 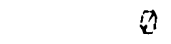 & 0 & 3 & 0 & 0 \\
\hline & COMFONENT \#13 & 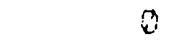 & D & 8 & 0 & $a$ \\
\hline \# & COMFONENT \#14 & 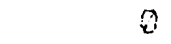 & 0 & 8 & 0 & $n$ \\
\hline & COMPONENT \#15 & 0 & 0 & 0 & 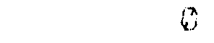 & D \\
\hline & COMPONENT \#IE & 0 & 0 & 0 & $\hat{\theta}$ & 0 \\
\hline & COMPONENT \#17 & 8 & $\hat{B}$ & 0 & 8 & 0 \\
\hline \# & COMPONENT \#18 & 0 & 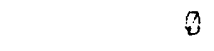 & $a$ & $\theta$ & 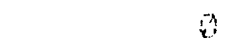 \\
\hline & COMPONENT \#19 & 8 & $\theta$ & 8 & $\theta$ & 0 \\
\hline & COMEONENT \#ZO & $\vec{v}$ & D & 3 & 3 & 6 \\
\hline & GLUCOSE & 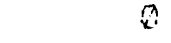 & 8 & 2 & 6 & $\hat{\theta}$ \\
\hline & AMMONIA & 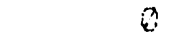 & 6 & 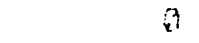 & $a$ & 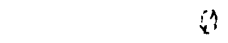 \\
\hline & PHOSFHORIC ACID & 8 & 8 & 8 & 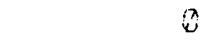 & 0 \\
\hline & POTASSIUM CHLORIDE & 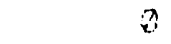 & 0 & 8 & $\hat{2}$ & 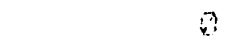 \\
\hline & MINOR NUTRIENTS & $\theta$ & 6 & $\theta$ & $\Leftrightarrow$ & 0 \\
\hline & WATER & 3 & $4,830,533$ & 533.088 & $5,881,571$ & $12,750,550$ \\
\hline & SOLUENT & 8 & 3 & c. & 6 & $a$ \\
\hline & CARBON DIOXIDE & 0 & 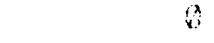 & ? & 0 & $\therefore$ \\
\hline & OXYGEN & $\theta$ & $\theta$ & 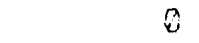 & 0 & 0 \\
\hline & NITROEEN & 0 & 0 & 3 & 8 & 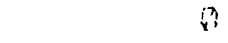 \\
\hline & HYOROGEN & $\theta$ & 3 & $B$ & \% & 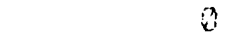 \\
\hline & $\ldots \ldots \ldots$ & $\cdots \cdots$ & $\ldots \ldots$ & $\cdots \cdots$ & $\cdots \cdots$ & $\ldots \ldots$ \\
\hline & $\begin{array}{l}\text { GEANO TOTAL } \\
\text { CHECK ON TOTAL }\end{array}$ & 0 & $4.990,533$ & 533.868 & $0.831,371$ & 12.750 .550 \\
\hline & TEMPERATURE, O & 141 & 186 & 110 & 5 & 5 \\
\hline & FRESGURE, PSIA & 54.7 & 154.7 & $2 \times .8$ & 14.7 & $14 . \%$ \\
\hline & STATE & GA5 & GAS & LIOUIC & Ligur & L. 10410 \\
\hline
\end{tabular}




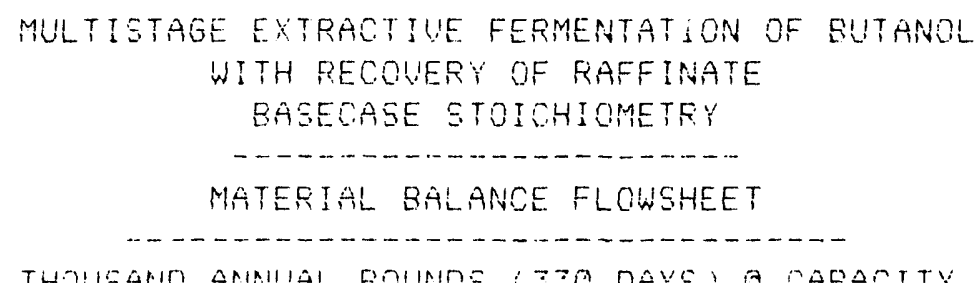

$5 ?$

DISTILL.

STREAM

COOL WTP

P

0

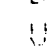


M LE. MOLESIYEAR

COMPONENT \#1
COMPONENT \#2
COMPONENT \#3
COMPONENT \#4
COMPONENT \#5
COMPONENT \#5
COMPONENT \#7
COMPONENT \#8
COMPONENT \#9
COMPONENT \#10
COMPONENT \#11
COMPONENT $\# 12$
COMPONENT \#13
COMPONENT \#14
COMPONENT \#15
COMPONENT $\# 15$
COMPONENT \#17
COMPONENT \#13
COMPONENT \#19
COMPONENT \#20
WATER
SOLVENT

$$
\text { TOTAL :G : }
$$$$
\text { (STSADE) }
$$$$
\text { VAPOR PRESS 40:G) }
$$$$
\text { (StUIJGE) }
$$$$
\text { UAPOR FRESS 12R (q) }
$$$$
\text { B!G) U.P. CONSTANT }
$$$$
\text { AG) U.F. CONSTANT }
$$$$
\text { TEMPERATURE } C
$$

PRESEURE NMHÜ

ㄴ (COMPONENT \#)

‥ (COMPONENT \#)

$$
\text { W.P.iks) }
$$$$
\text { U.F.(KI); }
$$

GAMMA-K1 IN K2

GAMMA-K2 IN

ALFHA

AUG COLUMN ALPHA

MOL FRACT. HI MMALE OR TAILE;

MOL FPACT. L1 (FEEC)

MOL FRACT. KE SMAKE OR TAILS:

ADS. GAMMA-KI IN KL

AOS. GAMMA-LZ IN LI:

MINIMUM REFLUX RATIO (AOJUSTED)

ADTUAL REFLUK RATIO

MINIMUM FLATES

\begin{tabular}{|c|c|c|c|}
\hline 23 & 24 & 25 & 26 \\
\hline & BEER \# 1 & BEER \#1 & BEER \# 1 \\
\hline EXTRACT & FEED & MAKE & TAILS \\
\hline$-\cdots---$ & $---\cdots--$ & $-\cdots---$ & $\cdots \cdots$ \\
\hline 0 & $\theta$ & $n$ & 0 \\
\hline 0 & 0 & 0 & 0 \\
\hline 143 & 143 & 143 & ? \\
\hline 0 & 0 & 0 & $\theta$ \\
\hline 24 & 24 & 24 & 8 \\
\hline 0 & 0 & 0 & 0 \\
\hline 2,445 & 2,688 & 2,575 & 12 \\
\hline 36 & 35 & 7 & 30 \\
\hline$\theta$ & 3 & 0 & 0 \\
\hline 10 & 10 & 5 & 5 \\
\hline 0 & 0 & 0 & $\theta$ \\
\hline 0 & 8 & 0 & 8 \\
\hline 0 & 0 & $\theta$ & 0 \\
\hline 0 & 0 & 0 & 0 \\
\hline 0 & 0 & 0 & 0 \\
\hline 0 & $\theta$ & 0 & 0 \\
\hline 0 & 0 & 0 & 0 \\
\hline$\theta$ & 0 & 0 & 6 \\
\hline 0 & 3 & 0 & $\theta$ \\
\hline 0 & 0 & 0 & 0 \\
\hline 5 & 5 & 5 & 0 \\
\hline 15,592 & 15,329 & 739 & 15,590 \\
\hline$-\cdots-\cdots$ & $-\cdots-\cdots$ & $\cdots-\cdots$ & $\cdots-\cdots$ \\
\hline 18,253 & 19,234 & 3,597 & 15,538 \\
\hline 108 & 112 & 111 & 1 \\
\hline 5.0 & 5.9 & 31.0 & 0.2 \\
\hline 2,384 & 3,088 & 3,053 & 35 \\
\hline 172.5 & 175.8 & 854.2 & 13.8 \\
\hline-5161.5 & -5200.3 & -5098.8 & -7119.7 \\
\hline 18.284 & 18.397 & 19.724 & 21.058 \\
\hline 33.0 & 35.8 & 108.7 & 220.0 \\
\hline 760 & 750 & 581 & 741 \\
\hline & & 7 & $?$ \\
\hline & & SOLUENT & SOLVENT \\
\hline & & 537.1 & 17164.0 \\
\hline & & 9.2 & 939.4 \\
\hline & & 2.014 & 2.014 \\
\hline & & 2.014 & 2.014 \\
\hline & & 39.260 & 36.755 \\
\hline & & 38.007 & \\
\hline & & 0.784 & 0.901 \\
\hline & & 0.141 & \\
\hline & & A. 216 & 9.999 \\
\hline & & 1. 853 & 2.012 \\
\hline & & 1.537 & 1.900 \\
\hline & & 0.0 & \\
\hline & & 0.0 & \\
\hline
\end{tabular}

DISTILLATION CALCULATIONS 
M LE. MOLES;YEAP

ACTUAL PLATES

PRESSURE Mir HG LREUISED:

TEMPERATURE C 'REUISED:

AUERAGE MOLECULAR WEIGHT

GAS DENEITY - LEYOF

CROSS SECTIONAL AREA - SO FT

COLUMN HEIGHT - FT

COLUMA DIAMETER

YI M MPPY)

HV : HEAT VAFORIZ, -BTUILE:

CS L HEAT CAFACITY - BEULLIF:

HEAT LOAD - MM BtUIH

CONDENSER COOLING WATER - GPM : 15 C UT:

CALAMURIA STEAM - MPPH I 150 PEIG:

COLUMN COET - 10005035 MPC - BARE EOUTPMENT

- ALL CARBON STEEL

- C.S w 304 S.S. TRAYS

- ALL 304 STAINLESS STEEL

- ALL 304L STAINLESS STEEL

- ALL JIE STAINLESS STEEL

CONDENSER OR CALANORIA GURFACE - SO FT

CONL. OR CALANE. COST - \$1000 3035 MPC - EARE EQUIFMENT

- CARBON STEEL

- JOA STAINLESS STEEL

- Z1S STATNLESS STEEL

-MONEL

EUETOTAL

SUETOTAL

SUETOTAL

MINIMUM REFLUX FATIO

CI SUETOTAL \#1

OR SUETOTAL \#2

Ci SURTOTAL \#3

Or OHECH

H. SUETOTAL

HV GUETOTAL \#L

H. BURTOTAL \#

H. CHECK

MIN. PLATESMONRMAL;

COL. COST-CIS NORMAL

OOL.COST-SIS NORMAL

MIN.REFLUX! NOFMAL:

O.E. AREASNORMAL;

HEAT LOADONORMAL:

OON!OAL COSTENORMAL:
DISTILLATION CALCULATIONS

\begin{tabular}{|c|c|c|c|}
\hline 23 & 24 & 25 & 25 \\
\hline & BEER $\# 1$ & SEER \#! & BEER \#! \\
\hline EXTRACT & FEED & MAK'E & TAILS \\
\hline$\cdots-\cdots$ & $-\cdots-\cdots$ & $\cdots \cdots---$ & $---\cdots-\cdots$ \\
\hline & & 10 & \\
\hline & & 599 & \\
\hline & & 114 & \\
\hline & & 113.14 & 257.38 \\
\hline & & 0.2045 & 0.4029 \\
\hline & & 25.3 & \\
\hline & & 30.7 & \\
\hline & & 5.8 & \\
\hline & & 198.345 & \\
\hline & & 215.2 & 215.0 \\
\hline & & 2.503 & 0.500 \\
\hline & & 12.150 & $183.58 E$ \\
\hline UT : & & 901 & \\
\hline & & & $12 E .7$ \\
\hline
\end{tabular}

ФE. .

$\$ 58.8$

$\$ 101.2$

\$111.3

\$13E. 5

1,005

$\$ 41.5$

$\$ 53.0$

$\$ 62.2$

$\$ 80.8$

5,03

$\$ 187.0$

\$251.7

$\$ 280.4$

$\$ 364.5$

$2,094,354$

2.. 92.772

$2,092,772$

(0). 4 )

$103,05 ?$

397

39. 256

0.500

44,558

171

42,725

215.2
453

1.129

$2,092,772$

0.500

135

485

899.892

215.8 
M LE. MOLES, YEAR

COMPONENT $\# 1$
COMPONENT $\# 2$
COMPONENT $\# 3$
COMPONENT $\# 1$
COMPONENT $\# 5$
COMFONENT $\#$ C
COMPONENT $\# 7$
COMPONENT $\# 3$
COMPONENT $\# 9$
COMPONENT $\# 10$
COMPONENT $\# 11$
COMPONENT $\# 12$
COMPONENT $\# 13$
COMPONENT $\# 14$
COMPONENT $\# 15$
COMPONENT $\# 15$
COMPONENT $\# 17$
COMPONENT $\# 13$
COMPONENT $\# 19$
COMPONENT $\# 20$
WATER
SOLVENT

TOTAL : G:

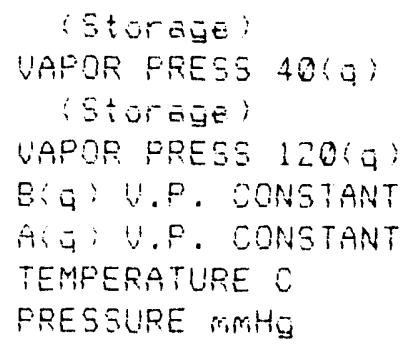

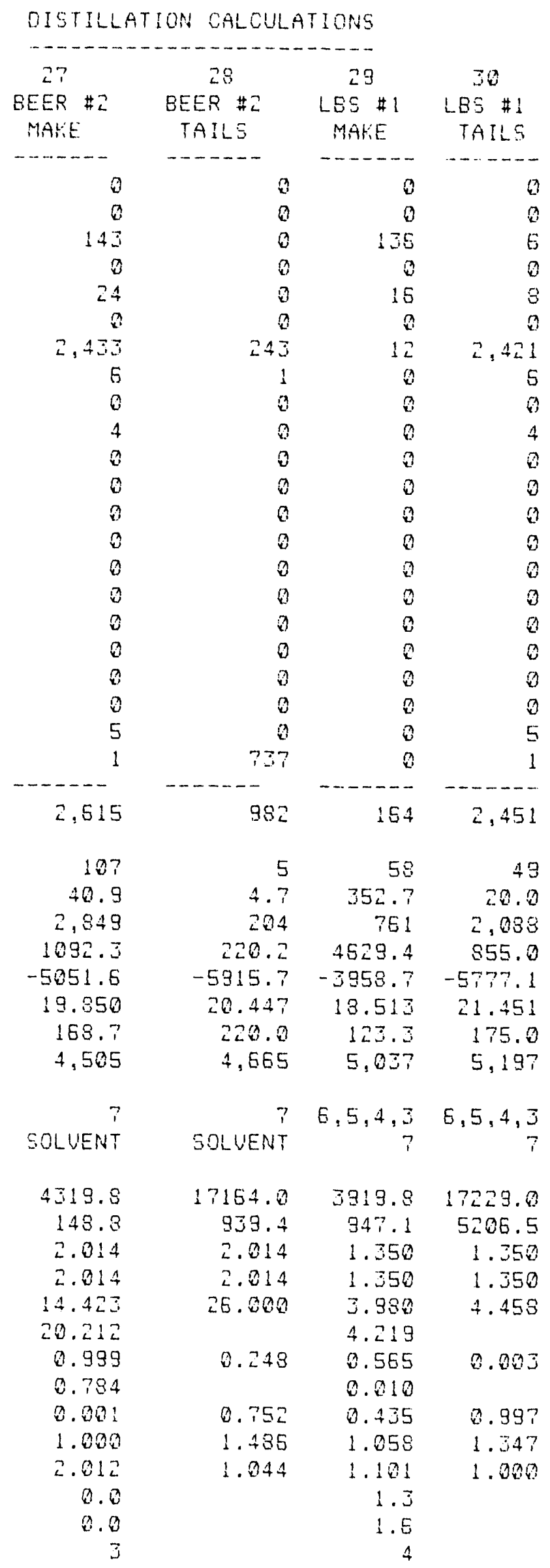


M LE. MOLES:YEAR

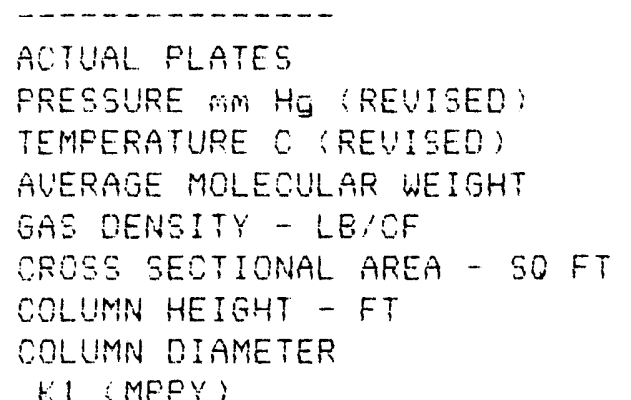

HV (HEAT VAFORIZ, - Etu/LE)

CI, SHEAT CAPACITY - BtwiLEIF;

HEAT LOAD - MM BtUIHF

CONOENGER COOLING WATER - GPM : 1

CALANIRIA STEAM - MPPH (150 PSIG:

COLUMN COST - 10003035 MPE - BARE EOUIPMENT

- ALL CARBON STEEL

- C.S WIJO4 S.S. TRAYS

- ALL 304 STAINLESS STEEL

- ALL 304L STAINLESS STEEL

- ALL. J15 STAINLESS STEEL

CONDENGER OR CALANDRIA SURFACE -

COND. OR CALAND. COST - \$1000 30SE MPO

-CARBON STEEL

-3O4 STAINLESS STEEL

- IIE STAIHLESS STEEL.

-MONEL

\begin{abstract}
SUETOTAL
SUSTOTAL

SUBTOTAL

CT SURTOTAL \#1

Cn SUBTOTAL \#2

CH SUBTOTAL \#3

CD CHECK

H. SUETOTAL \#1

$H_{v}$ SUBTOTAL \#2

Hv SURTOTAL \#3

H. CHECK
\end{abstract}

MINIMUM REFLUX RATIO

MIN. PLATESINORMAL)

COL.COST-CIS NORMAL

COL . COST-SIS NORMAL

MIN. REFLUX (NORMAL)

C. 3. AREA (NORMAL)

HEAT LOAO\&NORMAL :

CONICAL COST (NORMAL)

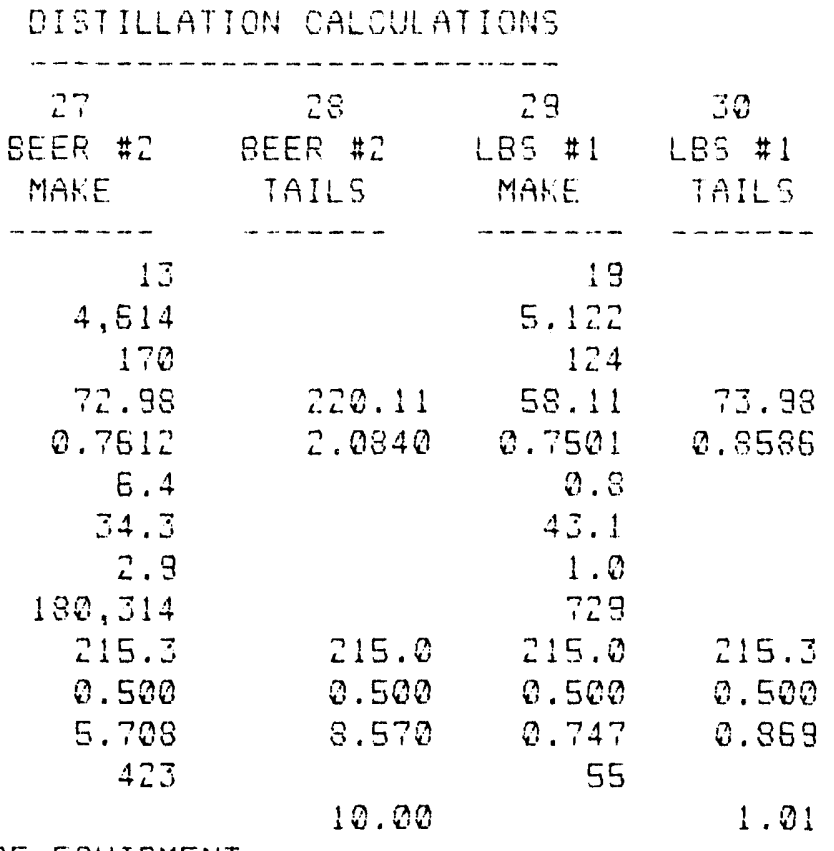

\$2.3.J

\$2 5.5

$\$ 39.4$

$\$ 4 \dot{j} .3$

$\$ 53.2$

55

43

\$9.5 \$9.1

\$13.J \$12.8

$\$ 14.3$ \$1 3.7

$\$ 18.5 \$ 17.8$

$\$ 35.8$

\$3.

$\$ 49.4$

103,044

98.992

93,932

90, $: 31$

254

264

(0.3)

94, 341

361

254

0.500

40,782

155

159

2. 15.3

9,915
36
93,932
0.500
$3,87 ?$
16
42,567
215.8

1. 3

4.775

30, 0E5

361

264

त. 500

2.. 054

0.500

38, 728

155

159

215.0

215.3

25

30

1

10

0. 87 
M LE. MOLES YEAR

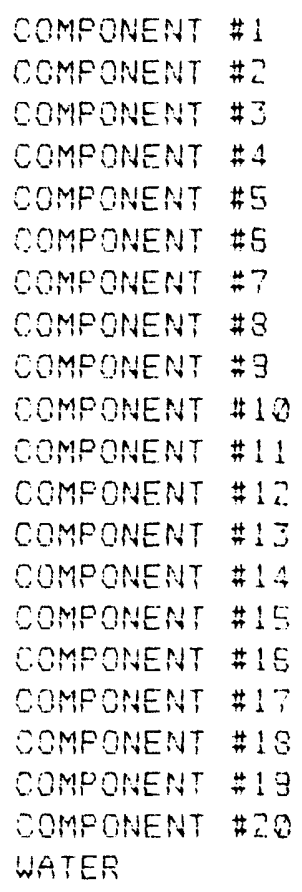

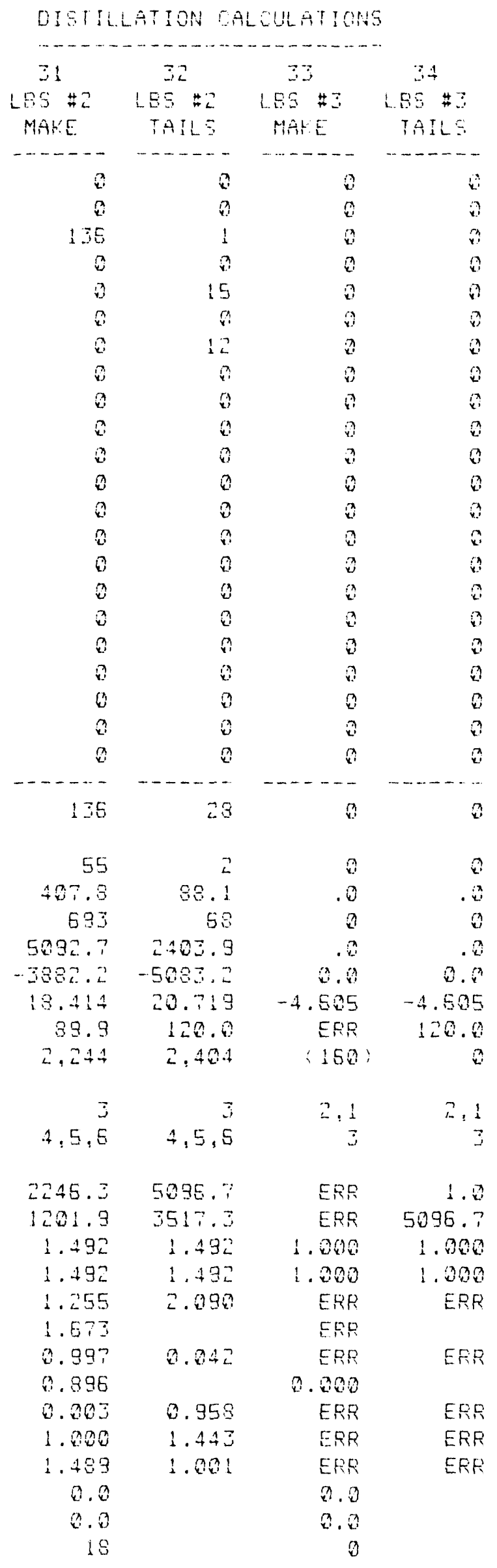


$\because$ LE, WHES, Y AS

ROTUAL PLATES

PRESGURE WU HE SFEUTEL

TEHPEFATLRE C AREUISED:

AUERAGE WOLECULAF WESGHT

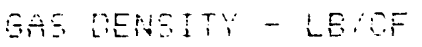

CROSO SEOTIOWAL AREA - SO FT

COLUMI HEIGLT - FT

ODUMHA OTAMETER

t.1 MNPPY

H. : HEAT GAFORIZ, -ETULL

CR : HEAT CAPAEITY - DELLLIE:

HEAT LOAD - MM DEU HH

CONELNEES EOOLING WATEF - GFM I

A ANOPIA STEAM - MPFH I 50 PSIG:

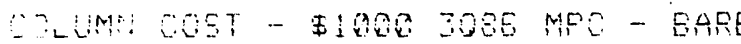

- ALL CAEEOH STEEL

- .5 wIRA S.S. TRAYS

- ALL OAT STATALESG GTEEL

- ALL JOAL GTAJHLESS BTEEL

- ALL j16 STAINLES STEEL

GONOENGER OP CALANDPYA SURFACE -

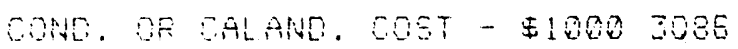

- CAREON STEEL

- ZRA GTATHLESE GTEEL

- ZIE STAINLESS STEEL

-MONEL

EUETOTAL
GUETOTAL
EUETOTAL

MININUM REFIUX FATIO

SA SUSTOTAL \#1

CA SUETOTAL \#2

CA SURTOTAL \#Z

CA BHECL

H. SUDTOTAL \#2

HV G!STOTAL \#2

HV SUSTOTAL \#

H. CHECH

MIN. FLATESBMGRMAL

BOL.COST-OIS MORMAL

COL . OOET-SIS NOFMAL

MIN.PEFLUKINORMAL :

E. S. AREACN RMAL!

HEAT LOAOLNOFMAL:

CONVCAL OOET:HORMAL:
D.STILATION CALCULATIONS

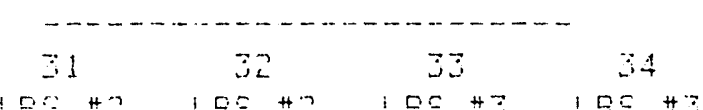

L83 \#2 LSE \#2 LOS \#S LOS \#

MALE TAILS MALE TAILS

80

‥ .855

87

58.05

(1) . 350

R. 4

134.5

0.7

7.882

$\because 15.8$

a. 50.

Q. 235

17

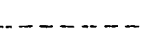

MAtic

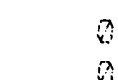

$\{2 \div 3$

$58.4 \%$ ERR ERR

Q. 3575 ERP ERP

T. D

(i)

ก. 9

?

215.8 ERP

(4.580 ERP

0.235

ล. 900

ล. . 27

$a$

E只只

B. 208

0.0

\&3.4

\$4 ᄂ. 2

\$5丁. 2

\$5口.5

$\$ 35.3$

29

MPO

\section{BARE EOUIFMENT}

$\$ 8.9$

\$11.5

$\$ 1 \hat{L} .1$

平15.?

\$क.

$\$ 0.6$

\$.

क日.

40.0

\$日.

0

0

$\$ 2.8$

$+10.0$

$\$ 0.8$

$\$ 0.0$

\$马,

$\$ 10.7$

古0.

$\$ 5.0$

$\$ 1.3 .9$

451

0

(0.8)

ㅍ. 949

(a)

3.0

8

0.5202

1, ES9

?

215.8

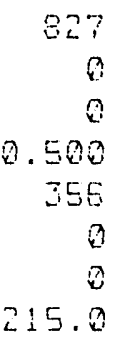

$\$ 0.0$

$\begin{array}{rr}0 & 0 \\ 0 & 0 \\ \text { ERR } & 0 \\ 0 & \text { ERR } \\ 0 & 0 \\ \text { ERR } & 0 \\ \text { ERR } & 0 \\ \text { ERR }\end{array}$


M LB. MOLES; YEAR

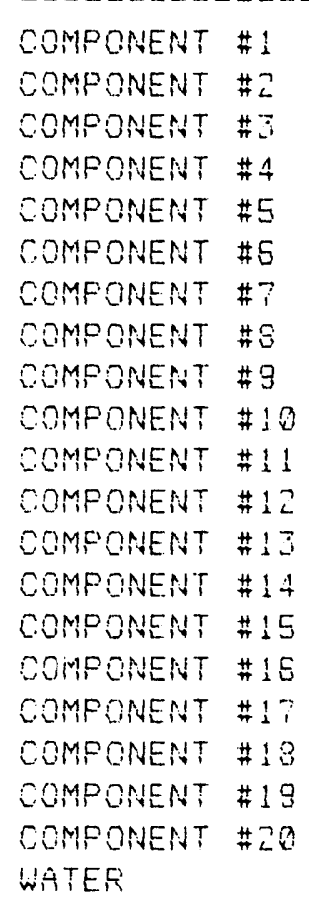

SOLUENT

TOTAL :

(St)TEEE)

UAPOR PRESS 406 ;

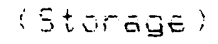

WAFOR PRESE 1206 G:

SUL) U.P. OONETANT

AIZ: U.P. CONSTANE

TEMPERATURE 0

PRESSURE W WHG

YI COMMPONENT \#:

H. (COMPONENT \#)

$$
\begin{aligned}
& \text { U.P. OL } \\
& \text { U.P. BZ }
\end{aligned}
$$

GAMM-KI IN KE

GAMMA-KZ IN 1

\section{ALPLH}

AUE EOLUMN ALPHA

MOL FRAOT. HI MALE OF TALL:

MOL FRACT. II IFEED:

MOL FFAET IZ MAHE OF TABS

ARI GAMMA-F! IN L

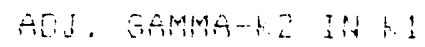

MINIMUM FEFLUK RARTO A ADIUGED

ACTUA! PEFLUK FATL

\begin{tabular}{|c|c|c|c|}
\hline \multicolumn{4}{|c|}{ DISTILLATION CALCULATIONS } \\
\hline 35 & 36 & 37 & 33 \\
\hline HES $\# 1$ & HES \#1 & EYPRODUCT & RAFF \#1 \\
\hline MALE & TAILS & FEED & FEED \\
\hline$-\cdots-\cdots$ & $-\cdots-\cdots-$ & $\ldots \ldots$ & $\cdots-\cdots$ \\
\hline 8 & 8 & 8 & 0 \\
\hline 8 & $\theta$ & 8 & 8 \\
\hline$?$ & $\mathrm{i}$ & 854 & $83 i$ \\
\hline 0 & $r$ & 0 & $a$ \\
\hline 8 & 3 & 397 & 458 \\
\hline 0 & 0 & 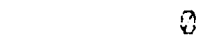 & 8 \\
\hline 12 & 8 & 15 & 15 \\
\hline 29 & 9 & 574 & 574 \\
\hline 3 & s. & 8 & 8 \\
\hline 5 & 8 & 155 & 155 \\
\hline 8 & 8 & ? & 8 \\
\hline 0 & $\hat{k i}$ & 8 & 8 \\
\hline 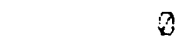 & $\theta$ & 0 & 9 \\
\hline 0 & 8 & 8 & 8 \\
\hline 8 & 8 & a & $\theta$ \\
\hline 0 & ? & $(2)$ & $\theta$ \\
\hline 3 & 0 & 8 & 8 \\
\hline$\theta$ & 8 & 8 & 0 \\
\hline 8 & 8 & i) & 3 \\
\hline 0 & 8 & 8 & 8 \\
\hline 8 & a & $7 \% 1.829$ & 772.957 \\
\hline 0 & 15.590 & 1 & 1 \\
\hline$\cdots \cdots$ & $-\cdots-\cdots$ & $\cdots \cdots$ & $\ldots-\ldots$ \\
\hline 47 & 15,531 & 779.804 & 774,952 \\
\hline 1 & 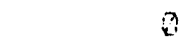 & 41 & 417 \\
\hline 26.7 & D. 1 & 55.2 & 55.8 \\
\hline 55 & B & 5,145 & 5,204 \\
\hline 739.2 & $1 E .5$ & 1533.2 & $15: 3.5$ \\
\hline-5105.8 & -7812.0 & -5117.4 & 5.117 .4 \\
\hline 19.537 & 22.588 & $20.35 E$ & 20.357 \\
\hline 121.5 & 220.0 & $\Xi \bar{B}$ & 33.9 \\
\hline 777 & 37 & 760 & 750 \\
\hline
\end{tabular}

MANUMUM FLATES $\begin{array}{rr}16,9,3 & 10,9.8 \\ \text { SOLVENT SOLVENT }\end{array}$

$153.9 \quad 3615.4$

17.9

2.117 2.11?

$2.858 \quad 2.858$

5.31 8.599

5. $35 x$

2. 999

. मxix

2. $6 x$

1. 203

ㄱ. 854

$9 \%$

$1 \times 9.8$

12
$.8 x$

1. Ax

…1?

1. An 
$M$ LE. MOLES YEAR

ACTHAL PLATES

FRESSURE WW HG (REUISED

TEMPERATURE C (REVISEO)

AVERAGE MOLECULAR WEIGHT

GAS RENBITY - LEICF

CROSE SEOTIONAL AREA - $30 \mathrm{FT}$

COLUNIN HEIELT ‥ FT

BOLUNA DTAMETER

I: MPFY:

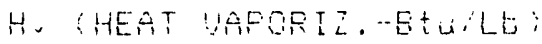

U HEAT CAPACITY - DELLLIF

HEAT LOAE - MM BAW HH

COWENGES COOLING WATEF - GPM 1

CHLANOFIA STEAM - MFPH 150 PSIG;

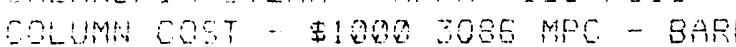

- Ail chebon zoes

- . . w. SA4 S.5. TFAY

ALL $3 \times 4$ STAINLESS STEEL

- ALL 3OAL STAINLESS STELL.

- AL DIE STAIHLES GTEEL

COMDENEEF OL GLANURIA SUEFAOE -

ConC. OS GLAND. COST - \$1000

- CAPEOR STELL

TRA BTATHLESS ETEE!

- ZIE STARMLES BTEEL

MONEL

SUETGTAL.

SETOTAL

GLETOTAL

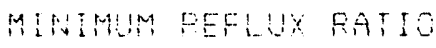

GI SUETOTAL \#1

CS SUETOTAL \#Z

OR GUETOTAL \#

a OHESh

H. ERTOTAL \#1

H. OIETOTAL \#2

H. GUETOTSI \#

H. CHEE

MIN, PLATESAMONAL

OL . COST-Y I AOPMAL

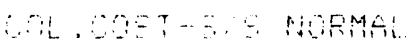

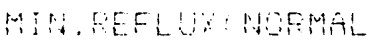

A S AFEA MOPMAL:

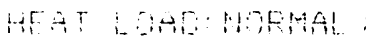

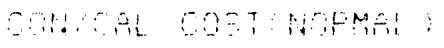

DISTILLATION CALCULATIONS

\begin{tabular}{|c|c|c|c|}
\hline 35 & 35 & $\ddot{3} 7$ & \\
\hline HES \#1 & HES \#1 & EYPRODUCT & RAFF \#1 \\
\hline MAKE & TAILS & FEED & FEED \\
\hline$\cdots--\cdots$ & $\ldots \ldots$ & $--\cdots-\cdots$ & $\cdots-\cdots$ \\
\hline 52 & & & \\
\hline 729 & & & \\
\hline 120 & & & \\
\hline 55.98 & 258.45 & & \\
\hline Q. 1245 & A.5:05 & & \\
\hline 23.7 & & & \\
\hline 32.9 & & & \\
\hline 6.0 & & & \\
\hline 0 & & & \\
\hline 215.0 & 215.6 & & \\
\hline 0.500 & 8.500 & & \\
\hline 10.355 & 10.355 & & \\
\hline$\because 57$ & & & \\
\hline & 12.98 & & \\
\hline
\end{tabular}

MEN

175.4

$\$ 19.4 .8$

$\$ 280.0$

$\$ 20.80$

$\$ 578.6$

805

575

C - BARE EOUIPMENT

$\$ 35.4$ \$28.5

$\$ 49.6 \quad \$ 39.9$

$\$ 53.2$ \$42.?

蛙3.1 155.5

\begin{tabular}{|c|c|}
\hline & $\begin{array}{l}2,992.777 \\
2.892,772 \\
2,892.772\end{array}$ \\
\hline $\begin{array}{r}87.2 \\
453\end{array}$ & \\
\hline 1.123 & \\
\hline 0 & $\therefore, B 92,77$ \\
\hline 9.570 & 8.50 \\
\hline 193 & \\
\hline 485 & \\
\hline 8 & $899 \cdot 39$ \\
\hline 215.9 & $=15$. \\
\hline
\end{tabular}


M LB. MOLESIYEAR

COMPONENT $\# 1$
COMPONENT $\# 2$
COMPONENT $\# 3$
COMPONENT $\# 4$
COMPONENT $\# 5$
COMPONENT $\# 5$
COMPONENT $\# 7$
COMPONENT $\# 8$
COMPONENT $\# 3$
COMPONENT $\# 10$
COMPONENT $\# 11$
COMPONENT $\# 12$
COMPONENT $\# 13$
COMPONENT $\# 14$
COMPONENT $\# 15$
COMPONENT $\# 16$
COMPONENT $\# 17$
COMPONENT $\# 13$
COMPONENT $\# 19$
COMPONENT $\# 20$
WATER
SOLVENT

TOTAL (q)

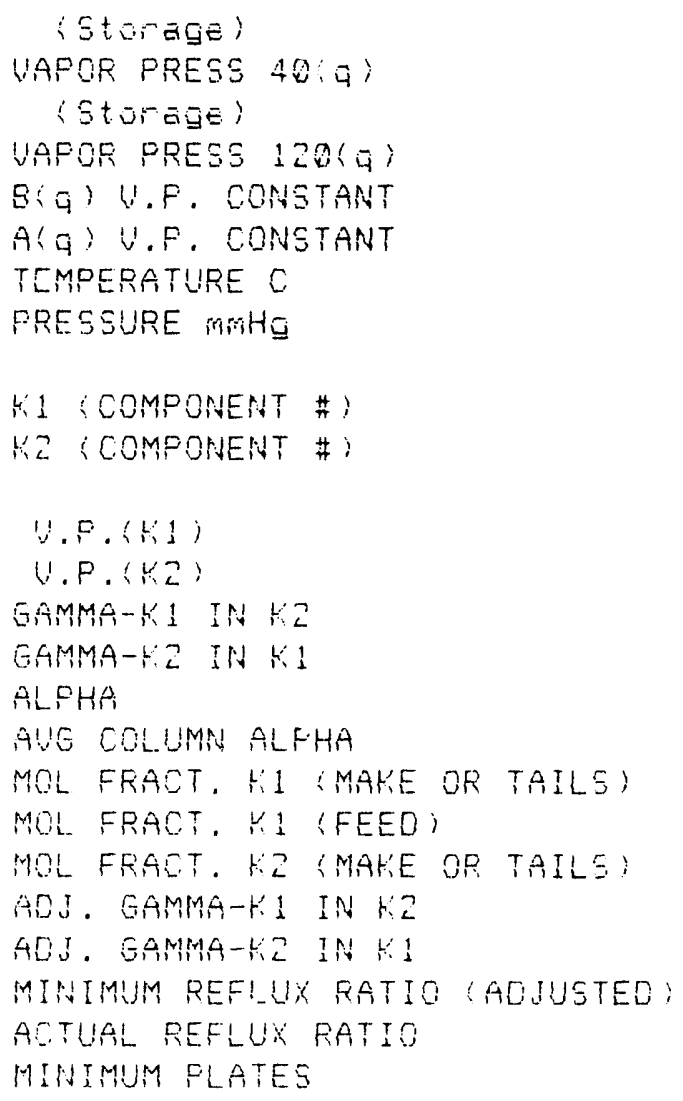

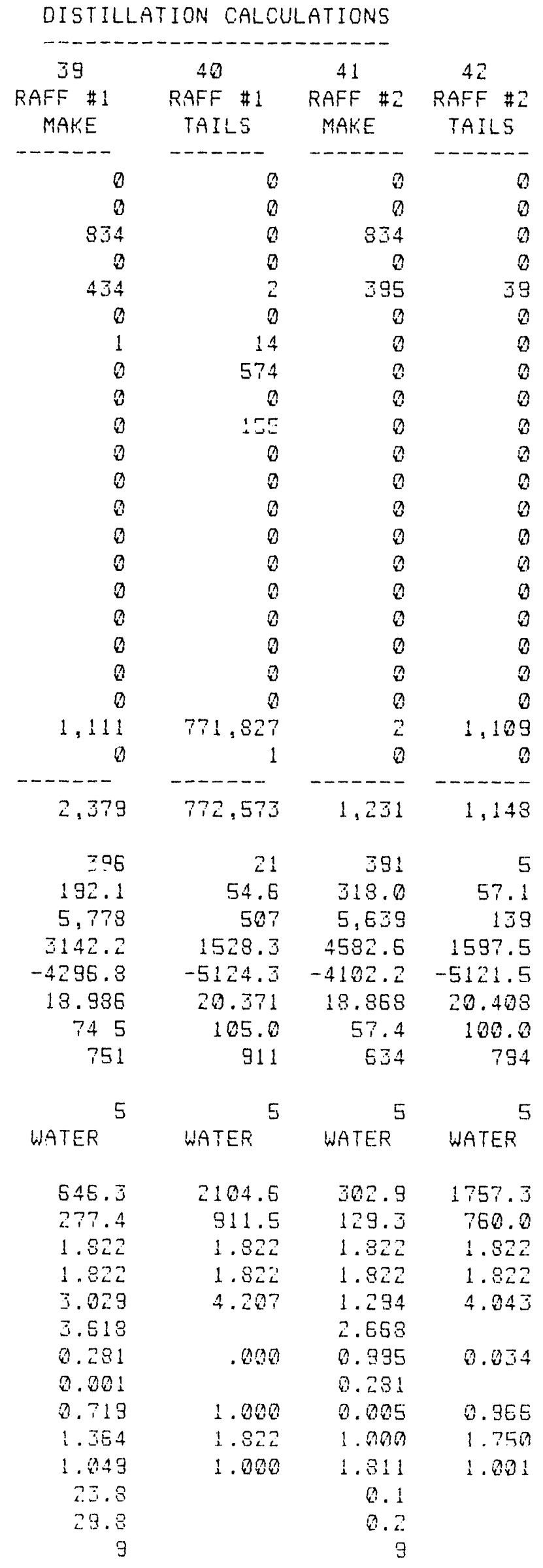


M LB. MOLES, YEAR

ACTUAL PLATES

FRESSURE MM HG (REUISED)

TEMPERATURE C (REUISEO)

AUERAGE MOLECULAR WEIGHT

GAS DENSITY - LEICF

CROSS SECTIONAL AREA - SO FT

COLUMN HEIGHT - FT

COLUMN DIAMETER

52 SMPPY)

HV (HEAT VAPOFIZ, -BtULL)

ON IHEAT CAPACITY - EtLILL,F)

HEAT LOAO - MM BELIHT

CONOENEER COOLING WATER - GPM 1

CALANDRIA STEAM - MPPH (15O PSIG)

COLUMN COST - \$10OO 3085 MPC - BARE EQUTPMENT

- ALL CAPBON STEEL

- C.S WOBA S.S. TRAYS

- AlL jo4 sTAINLESS gTEEL

- ALL 3Q4L STAINIEES STEEL

- all jie stainless steEl

OONDENGER OR CALANILRIA SURFACE -

COND. OE CALAND. COST - \$1900 3086

- CAREON F TEEL

- ZOA STAINLESE STEEL

- J1E STAINLESS STEEL

-MONEL

\section{SUBTOTAL \\ SURTOTAL \\ SUETOTAL}

MINIMLN REFLUY RATIO

CI SURTOTAL \#1

ON SUETOTAL \#2

CA BUSTOTAL \#3

G. CHECK

H. SUETOTAL \#1

H. SLETOTAL \#2

HV SUBTOTAL \#3

H. CUECL

MIN. PLATESINORMAL;

COL. COST-E:S NORMAL

COL . COST-SIS NOPMAL

MIN.REFLUXSWORMAL;

C. S. AREAONORMAL

HEAT LOADONORMAL:

OON CAL OOSTENORMAL:
DIGTILLATION CALCULATIONS

\begin{tabular}{|c|c|c|c|}
\hline 39 & 40 & 41 & 42 \\
\hline RAFF \#1 & RAFF \#1 & FAFF \#2 & RAFF \#2 \\
\hline MALE & TAILS & MALE & TALIS \\
\hline$-\cdots-\cdots$ & $-\cdots-\cdots$ & $-\cdots-\cdots$ & $\ldots-\cdots$ \\
\hline 42 & & 39 & \\
\hline 744 & & 5.36 & \\
\hline 74 & & 57 & \\
\hline 37.19 & 18.87 & 54.17 & 18.98 \\
\hline 0.0797 & 0.0435 & 0.1044 & 0.0404 \\
\hline 281.8 & & 7.1 & \\
\hline 77.6 & & 74.2 & \\
\hline 18.3 & & 3.0 & \\
\hline 20,007 & & 18.189 & \\
\hline 335.8 & 957.6 & 215.4 & 907.1 \\
\hline D. 513 & 6.998 & 0.500 & 0.358 \\
\hline 145.847 & 395.551 & 2.350 & 2.484 \\
\hline 18803 & & 174 & \\
\hline & AEZ & & \\
\hline
\end{tabular}

그. 2

$\$ 83.8$

\$1 โ2. 5

$\$ 154.8$

$\$ 155.5$

4949.7

22,892

22,031

594

138

MPC - EARE EQUIPMENT

$\begin{array}{rrrr}\$ 771.9 & \$ 737.6 & \$ 29.0 & \$ 13.3 \\ \$ 1.079 .3 & \$ 1.032 .5 & \$ 40.7 & \$ 13.5 \\ \$ 1.156 .4 & \$ 1.105 .4 & \$ 43.5 & \$ 20.0 \\ \$ 1.503 .4 & \$ 1.433 .3 & \$ 55.5 & \$ 25.3\end{array}$

13.973

19.971

19,971

0.1

23.8

$13,923,954$

$13,905,370$

$13,905,370$

34,235

$\theta$

24,061

$20,00713,305,370$

0.613

14,721

0. 393

245

(3) $\quad 10,545$

$13,41323,492,396$

385.8

85?.
35.325

911

36

6. 500

$14: 358$

19.97

0.958

392

0
-5

15
19.376

307.1 
M LD. MOLES/YEAR

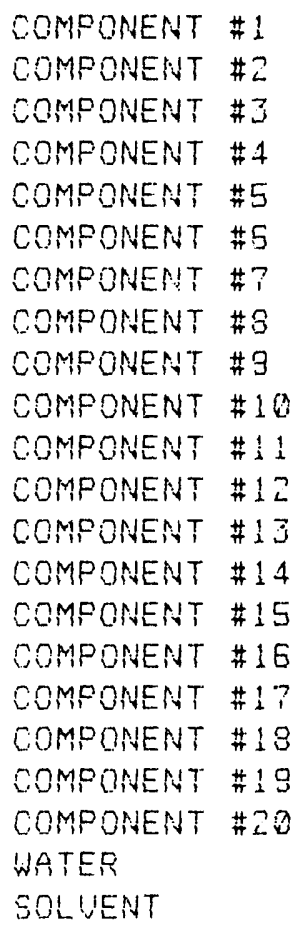

\section{DISTILLATION CALCULATIONS}

43

RAFF \#五 RAFF \#J

MAKE

TAILS

$\begin{array}{rr}0 & 0 \\ 0 & 0 \\ 30 & 4 \\ 0 & 0 \\ 2 & 333 \\ 0 & 0 \\ 0 & 0 \\ 0 & 0 \\ 0 & 0 \\ 0 & 0 \\ 0 & 0 \\ 0 & 0 \\ 0 & 0 \\ 0 & 0 \\ 0 & 0 \\ 0 & 0 \\ 0 & 0 \\ 0 & 0 \\ 0 & 0 \\ 0 & 0 \\ 0 & 2 \\ 0 & 0\end{array}$

332

393

$339 \quad 52$

$407.3 \quad 131.1$

$4,236 \quad 1,403$

$5092.7 \quad 3520.5$

$-3532.2-5059.1$

$18.414 \quad 21.039$

$35.2 \quad 105.0$

$1,352 \quad 2,12$

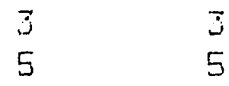

$$
\begin{array}{rr}
1354.5 & 3444.3 \\
1001.5 & 2104.6 \\
1.492 & 1.492 \\
1.492 & 1.492 \\
1.311 & 2.421 \\
1.365 & \\
0.997 & 0.011 \\
0.579 & \\
0.003 & 0.939 \\
1.000 & 1.479 \\
1.439 & 1.000 \\
0.0 & \\
0.0 & \\
17 &
\end{array}
$$




\begin{tabular}{|c|c|c|}
\hline & DISTIL & LATION CALCULA \\
\hline & 43 & 44 \\
\hline & RAFF $\# 3$ & RAFF \#3 \\
\hline MLE. MOLES Y YEAR & MAKE & TAILS \\
\hline 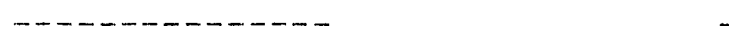 & $\ldots \ldots-$ & $\ldots \ldots$ \\
\hline ACTUAL PLATES & 75 & \\
\hline PRESSURE MM HE (REUISED) & 1,808 & \\
\hline TEMPERATURE O (REUISEDS) & 83 & \\
\hline AVERAGE MOLECULAR WEIEHT & 58.05 & 45.29 \\
\hline GAS DENEITY - LB/CF & 0.2353 & 0.2577 \\
\hline CROSS SECTIONAL AREA - SO FT & 2.6 & \\
\hline COLUMN HEIGHT - FT & 128.9 & \\
\hline COLUMN DIAMETER & 1.8 & \\
\hline K1 $(M P P Y)$ & 49,182 & \\
\hline HV (HEAT VAFORIZ. -BtUilLE\} & 215.0 & 215.5 \\
\hline CI KHEAT CAPACITY - BLUILLIF) & 0.500 & 0.501 \\
\hline HEAT LOAD - MM BtiLiLH & 1.442 & 1.551 \\
\hline CONDENSER COOLING WATER - GPM 11 & 187 & \\
\hline CALANDRIA STEAM - MPFH (150 PSIG) & & 1.81 \\
\hline COLUMN COST - $\$ 10003036 \mathrm{MPC}-$ BAF & ARE EQUIP & MENT \\
\hline - ALL CARBON STEEL & $\$ 79.5$ & \\
\hline - O.SW WO4 S.S. TRAYS & $\$ 97.4$ & \\
\hline - ALL 304 STAINLESS STEEL & $\$ 127.8$ & \\
\hline - ALL SQAL STAINLESS ETEEL & $\$ 140.5$ & \\
\hline - ALL J1G STAINLESS STEEL & $\$ 172.5$ & \\
\hline CONDENGER OR CALANORIA SURFACE - & 190 & 86 \\
\hline COND. OR CALANO. COST - $\$ 10003085$ & 35 MFC - & BARE EQUIPMENT \\
\hline -CARBON STEEL & $\$ 15.4$ & $\$ 11.0$ \\
\hline -304 STAINLESS STEEL & $\$ 21.5$ & $\$ 15.4$ \\
\hline - J1E STAINLESS SIEEL & $\$ 23.1$ & $\$ 16.5$ \\
\hline -MONEL & $\$ 30.0$ & $\$ 21.5$ \\
\hline SUETOTAL & & 55 \\
\hline SUETOTAL & & 35 \\
\hline SUBTOTAL & & JE \\
\hline MINIMUM REFLUX RATIO & $(0.5)$ & \\
\hline CN SUBTOTAL \#1 & 24,139 & 3,185 \\
\hline CN SUBTOTAL \#2 & 0 & 0 \\
\hline Cn SUBTOTAL \#3 & $\theta$ & 36 \\
\hline Cri CHECH & 0.500 & 0.501 \\
\hline HV SUETOTAL \#1 & 10,380 & 3,950 \\
\hline HV SUBTOTAL \#2 & 0 & 0 \\
\hline HV SUETOTAL \#J & $D$ & 35 \\
\hline HV CHECK & 215.0 & 215.5 \\
\hline MIA. PLATES!NORMAL) & & \\
\hline COL.COST-CIS NORMAL & 79 & \\
\hline COL.COST-SIS NORMAL & 123 & \\
\hline MIN.REFLUX（NORMAL; & & \\
\hline C.S. AREALNORMAL; & 3 & \\
\hline HEAT LOADQNORMAL: & & 1 \\
\hline CONICAL COST KNORMAL) & 15 & 11 \\
\hline
\end{tabular}


APPENDIX D. BASEC.ASE STOICHIOMETRY
NO RAFFINATE/CELLS RECYCLE
NO RECOVERY OF RAFFINATE CHEMICALS 
MULTISTAGE EXTRACTIUE FERMENTATION OF BUTANOL NO RECOUERY OF RAFFINATE

BASECASE STOICHIOMETRY

SUMMARY

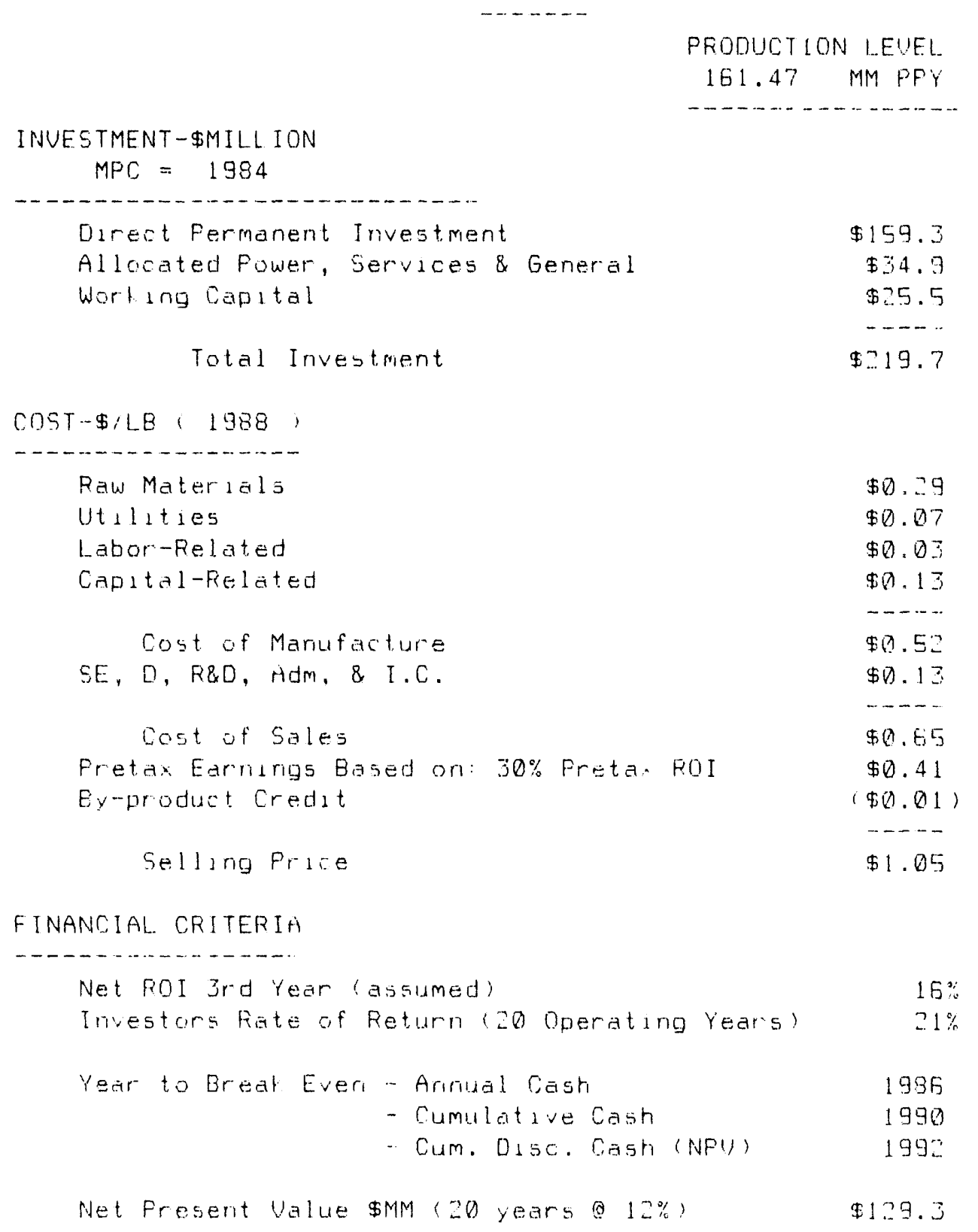


MII TISTAGE EXTPACTIUE FEFMENTATIUN OF BUTANOL

NO FECOUEFY OF RAFFINATE

BASECASE STOICHIOMETFY

INUESTMENT

ranII T IONS

Elted an Towe an the Mississippl River adjacent to a corn wet mill and a ut lity pouer house for over-the-ferice subply of syruf and pouer.

\begin{tabular}{|c|c|c|c|c|}
\hline & UNITS & & $\begin{array}{l}\text { THIS } \\
\text { CASF } \\
\end{array}$ & \\
\hline CAFAST: 9 GAMG HRS & MM PFY & & 179.4 & \\
\hline WIO-FIINT OF CONSTFIOTION & YFAF & & 1984 & \\
\hline CONSTREISTION COST INEEX & $1980=100$ & & 128 & \\
\hline IHWE IMENT CONT INGENCY & $\%$ INSTALLED & $\star$ & $30 \%$ & \\
\hline FEFMENTER UNIT INUEETMENT & S:GR.GAL. - - E & OWTH & $\$ 10.54$ & \\
\hline 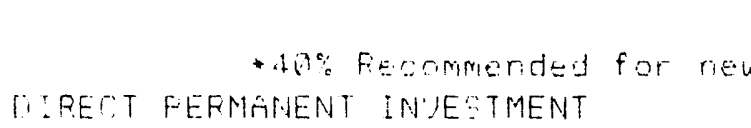 & $\begin{array}{l}\text { \$ GR.GAL. - P } \\
\text { W processes }\end{array}$ & 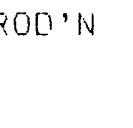 & $\$ 10.94$ & \\
\hline 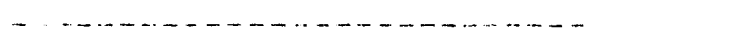 & SCALE & & THI & IS CASE \\
\hline & FACTOR & & कMM & \$:ANN.LB, \\
\hline & $\cdots-\cdots$ & & $\cdots \cdots$ & $-\cdots---$ \\
\hline FEFMENTATION SECTION & & & & \\
\hline Recelving, Prep \& Ster1lization & 0.60 & & $\$ 20.35$ & $\$ 0.113$ \\
\hline hip Compression \& Aeration & 0.60 & & 0.00 & 0.000 \\
\hline Fermentation? & $0.89-1.00$ & & 117.07 & 0.652 \\
\hline$E \cdot \operatorname{tr} a c \operatorname{lon}$ & & & 7.16 & 0.040 \\
\hline Froducticell separation & 0.75 & & 7.74 & 0.043 \\
\hline & & & $\cdots \cdots$ & $\cdots \cdots$ \\
\hline Firmentit don Sub-total & & & $\$ 152.32$ & $\$ 0.849$ \\
\hline OISTILLATION SECTION & STILLS & $H X \cdot S$ & & \\
\hline Eeer St111 \#1 & $\$ 0.29$ & $\$ 1.07$ & $\$ 1.36$ & $\$ 0.008$ \\
\hline 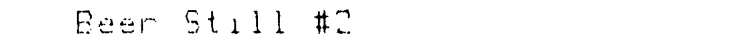 & 0.18 & 0.18 & 0.37 & 0.002 \\
\hline Low-Eg11Ers Sti11 \#1 & 0.11 & 0.09 & 0.20 & 0.001 \\
\hline Low-Eo11er.5 St:11 \#2 & 0.18 & 0.07 & 0.25 & 0.001 \\
\hline Low-Eotlers St111\#3 & 0.00 & 0.00 & 0.00 & 0.000 \\
\hline Hatr-Eo1 lersestil1 \#1 & 0.82 & 0.30 & 1.12 & 0.006 \\
\hline & & & $-\cdots \cdots$ & $---\cdots$ \\
\hline D stillation Subtotal & & & $\$ 3.29$ & $\$ 0.02$ \\
\hline STOFAGE EEUTION & & & & \\
\hline Storage - Product & & & $\$ 3.45$ & $\$ 0.019$ \\
\hline storage - Eyproduct \#1 & & & $\$ 0.21$ & 0.001 \\
\hline & & & $\cdots--\cdots$ & $-\cdots-\cdots$ \\
\hline Storage Sutitotal & & & $\$ 3.66$ & $\$ 0.02$ \\
\hline TOTAL DIRECT PLANT & & & $\$ 159.2 .7$ & $\$ 0.888$ \\
\hline
\end{tabular}


MULTISTAGE EXTRACTIUE FERMENTATION OF BUTANOL NO RECOUERY OF RAFFINATE

BASECASE STOICHIOMETRY

INUESTMENT

ALLOCATED PERMANENT INUESTMENT

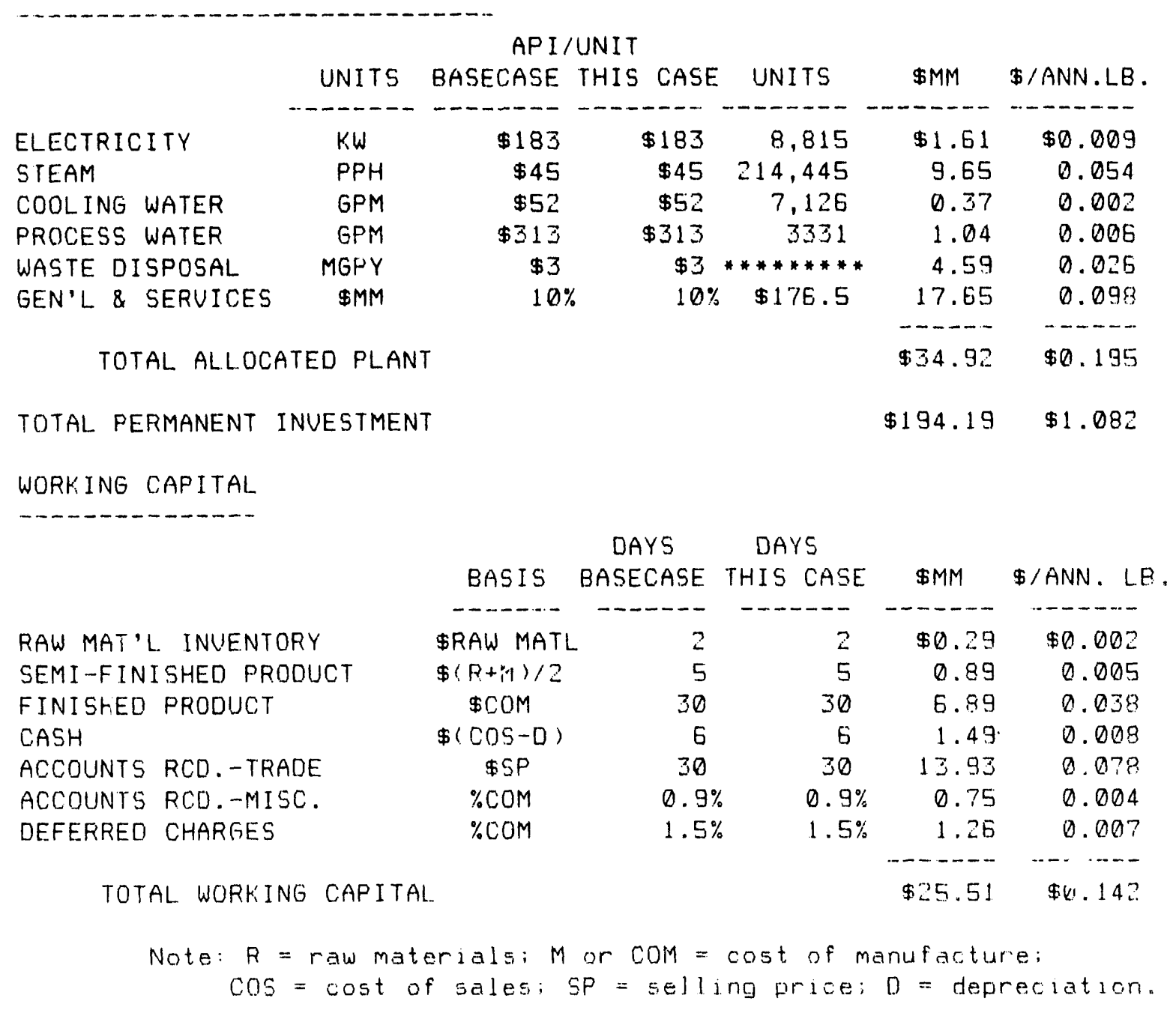

TOTAL INUESTMENT FOR RETURN

$\$ 219.69 \$ 1.225$ 
MII TISTAGE EXTRACTIUE FERMENTATION OF: BUTANOL NO RECOUERY OF RAFFINATE BASECASE $\triangle$ TOICHIOMETRY

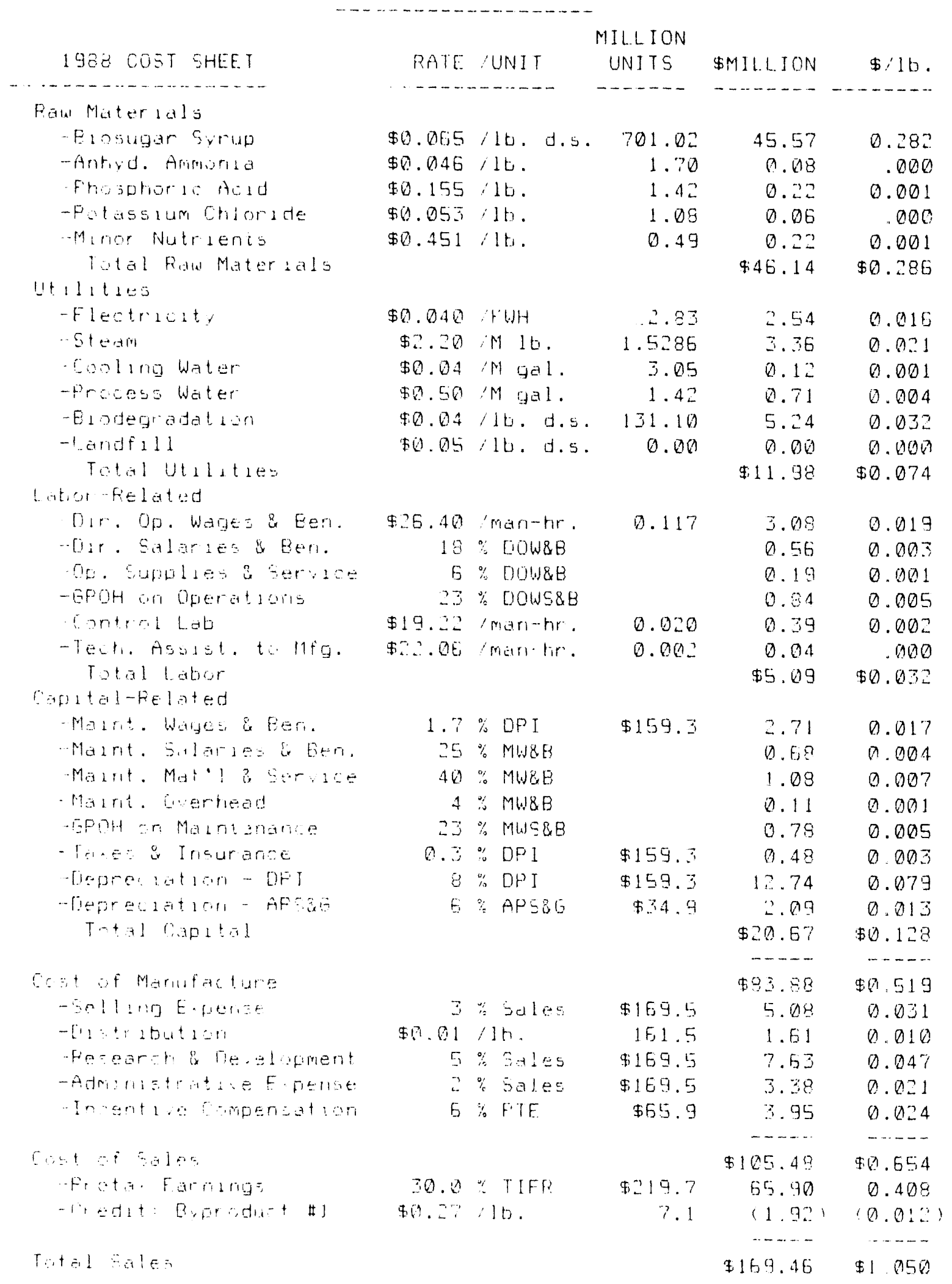


APPENDIX E. GOAL STOICHIOMETRY

WITH 27-1 RAFFINATE/CELLS RECYCLE 
MULTISTAGE EXTRACTIUE FERMENTATION OF BUTANOL WITH RECOUERY OF RAFFINATE

GOAL STOICHIOMETRY

SUMMARY

FRODUCTION LEVEL
162

COST-\$LB ( 1988 )

COST-\$/LB ( 1988$)$

Saw Materiala

$\$ 0.17$

Utilities

$\$ 0.02$

Labor-Related

$\$ 0.03$

Capital-Related

$\$ 0.02$

Cost of Manifacture

-...-

$\$ 0.24$

SE, D, R\&D, Adm, \& I.C.

$\$ 0.05$

Cost of Sales

Pretax Eaminga Based on: $30 \%$ Pretax ROI

$-1 .--$

$\$ 0.28$

By-product Cradit

$\$ 0.07$

Selling Price

(\$.00)

$-\cdots$

$\$ 0.35$

FINANCIAL CRITERIA

Net ROI Jid Year (assumed)

Irivestors Rate of Return (20 Operating Years)

Year te Break Even - Annual Cash

- Cumulative Cash

- Eum. Disc. Cash (NPU)

1991

1994

Net Present value \$MM (20 years Q $12 \%$ )

$\$ 15.0$ 
MULTISTAGE EXTRACTIUE FERMENTATION OF BUTANOL WITH RECOUERY OF RAFFINATE GOAL STOICHIOMETRY

INUESTMENT

CONDITIONG

Sited in lowa on the Mississipgi River adjacent to a corn wet mill arid a litility power house for over-the-ferice bupgly of syrug arid power.

\begin{tabular}{|c|c|c|c|c|}
\hline & $\begin{array}{l}\text { UNITS } \\
\text { - }\end{array}$ & & $\begin{array}{l}\text { THIS } \\
\text { CASE } \\
-\end{array}$ & \\
\hline CAPACITY O 8000 HRS & MiM pPY & & 180.3 & \\
\hline MIO-POINT OF CONSTRUCTION & YEAR & & 1384 & \\
\hline CONSTRUCTION COST INDEX & $1330=100$ & & 128 & \\
\hline INUESTMENT CONTINGENCY & $\%$ INSTALLEO & & $30 \%$ & \\
\hline FERMENTER UNIT INUESTMENT & \$IGR.GAL. -G & OWTS & $\$ 13.72$ & \\
\hline & $\$ / G R . G A L .-P$ & $0 D_{1} \mathrm{~N}$ & $\$ 10.94$ & \\
\hline $\begin{array}{l}\text { *48\% RECOMMENIEA for ne } \\
\text { DIRECT PERMANENT INUESTMENT }\end{array}$ & Wrocestes & & & \\
\hline - - & SCALE & & THI & IS CASE \\
\hline & FACTOR & & \$MM & \$/ANH.LB. \\
\hline & $----\cdots$ & & $\cdots \cdots-\cdots$ & $-\cdots--$ \\
\hline FERMENTATION SECTION & & & & \\
\hline Recelvirig, Prep \& Sterilization & 0.50 & & $\$ 2.81$ & $\$ 0.015$ \\
\hline Air Compression \& Aeration & 0.50 & & 0.00 & 0.000 \\
\hline Fempentation & $0.89-1.00$ & & 7. 43 & 0.041 \\
\hline Extastion & & & 4.24 & 0.024 \\
\hline Product/Cell Separation & 0.75 & & 0.55 & 0.004 \\
\hline Fermeritation Sub-totai & & & $\$ 15.18$ & $\$ 2.084$ \\
\hline DISTILLATION SECTION & STILLS & $H \times \cdot S$ & & \\
\hline Beer Still \#1 & $\$ 0.28$ & $\$ 0.85$ & $\$ 1.15$ & $\$ 0.005$ \\
\hline Beer Still \#2 & 0.18 & 0.20 & 0.38 & 0.002 \\
\hline Low-Builers Stili \#1 & 0.00 & 0.00 & 0.00 & 0.000 \\
\hline Luw-Boniers Sti11 \#2 & $2 \pi .00$ & 0.00 & 0.00 & 0.000 \\
\hline Low-Builers Still \#z & 0.00 & D. $D$ & 0.00 & 0.000 \\
\hline High-Eoilers Still \#1 & 0.00 & 0.00 & 0.00 & 0.000 \\
\hline 只ffirite Stild \#1 & 0.00 & 0.00 & 0.80 & 0.008 \\
\hline Raffinate Stil1 \#2 & 0.00 & 0.80 & 0.00 & 0.000 \\
\hline Räflinate Still \#产 & 0.80 & 0.00 & 0.00 & 0.000 \\
\hline Distillativen Suttotal & & & $\$ 1.50$ & $\$ 2.81$ \\
\hline ETORAGE SECTION & & & & \\
\hline Storage - Butanol & & & $\$ 3.44$ & $\$ 0.013$ \\
\hline Gtorage - ADetorie & & & $\$ 0.00$ & 0.020 \\
\hline Storage - Ethinol & & & $\$ .00$ & .020 \\
\hline & & & $\ldots-\cdots$ & $\cdots \cdots$ \\
\hline Storage Sutitotal & & & $\$ 3.44$ & $\$ 0.02$ \\
\hline TOTAL DIRECT PLANT & & & $\$ 20.12$ & $\$ 0.112$ \\
\hline
\end{tabular}


MULTISTAGE EXTRACTIVE FEEMENTATION DF BUTANOL WITH RECOUERY OF RAFFINATE GOAL STOICHIOMETRY

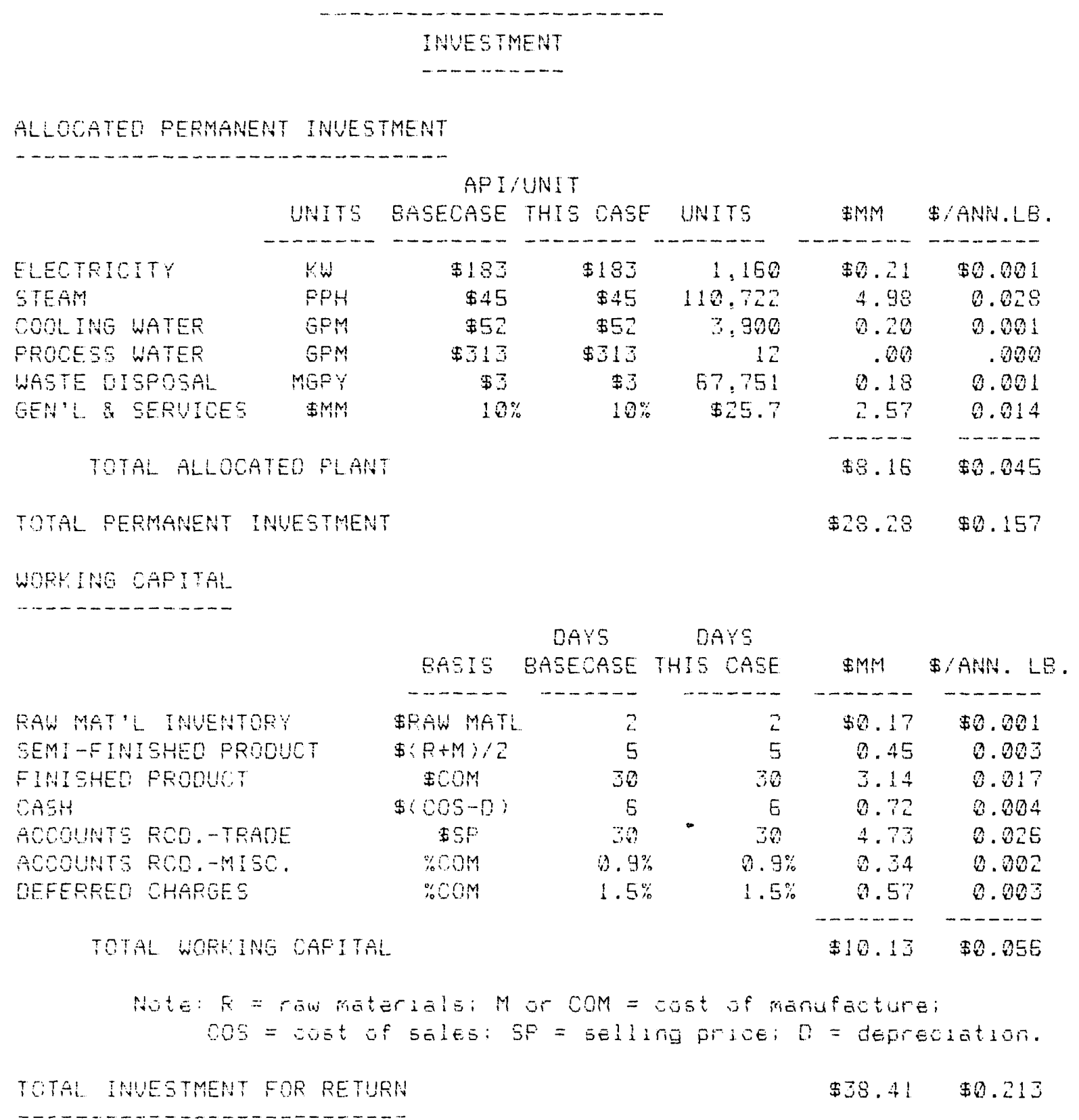


MULTISTAGE EXTRACTIUE FERMENTATION OF BUTANOL WITH REOOUERY OF RAFFINATE GOAL STOICHIOMETRY

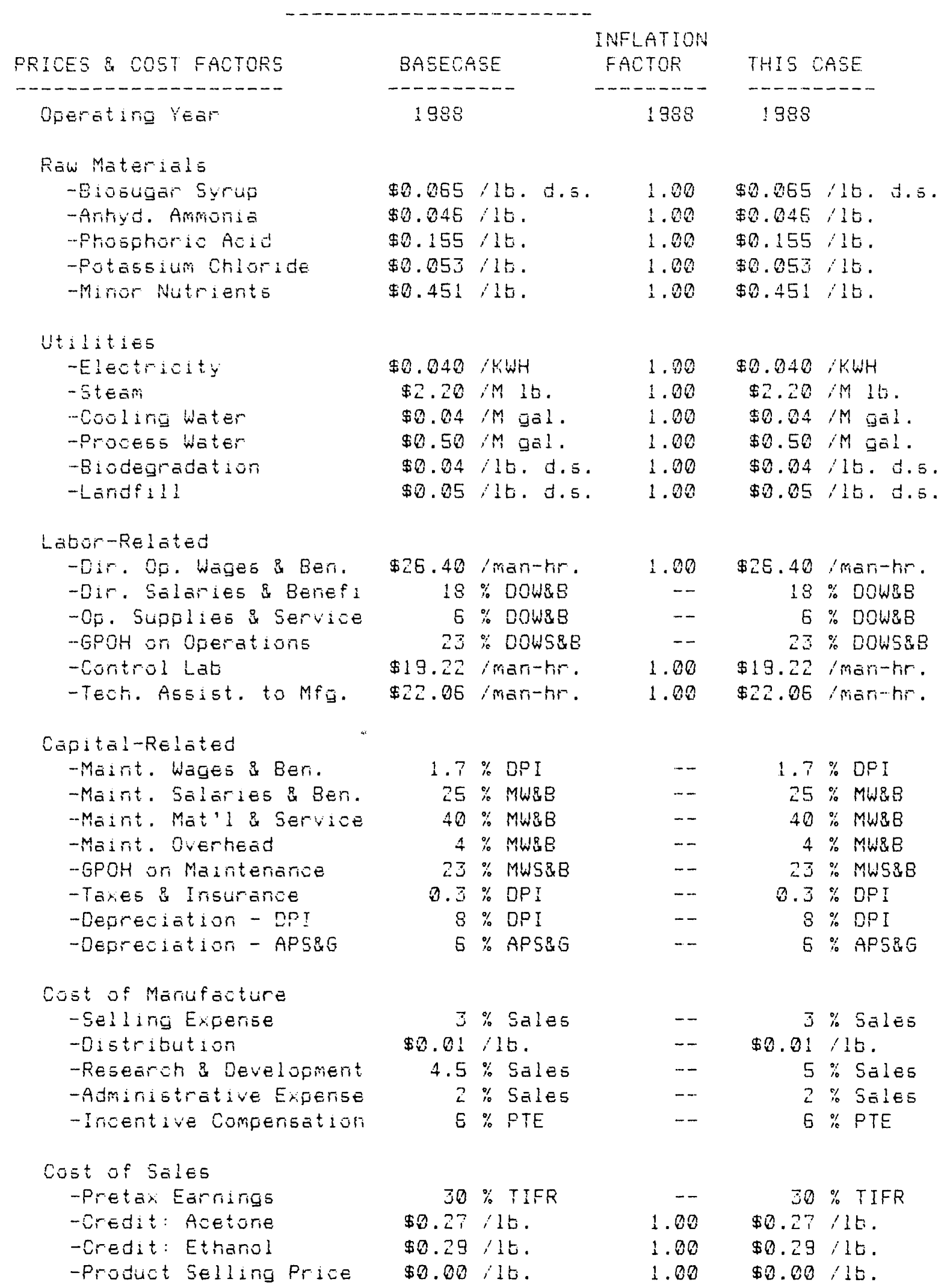


SALARIES OW WAES

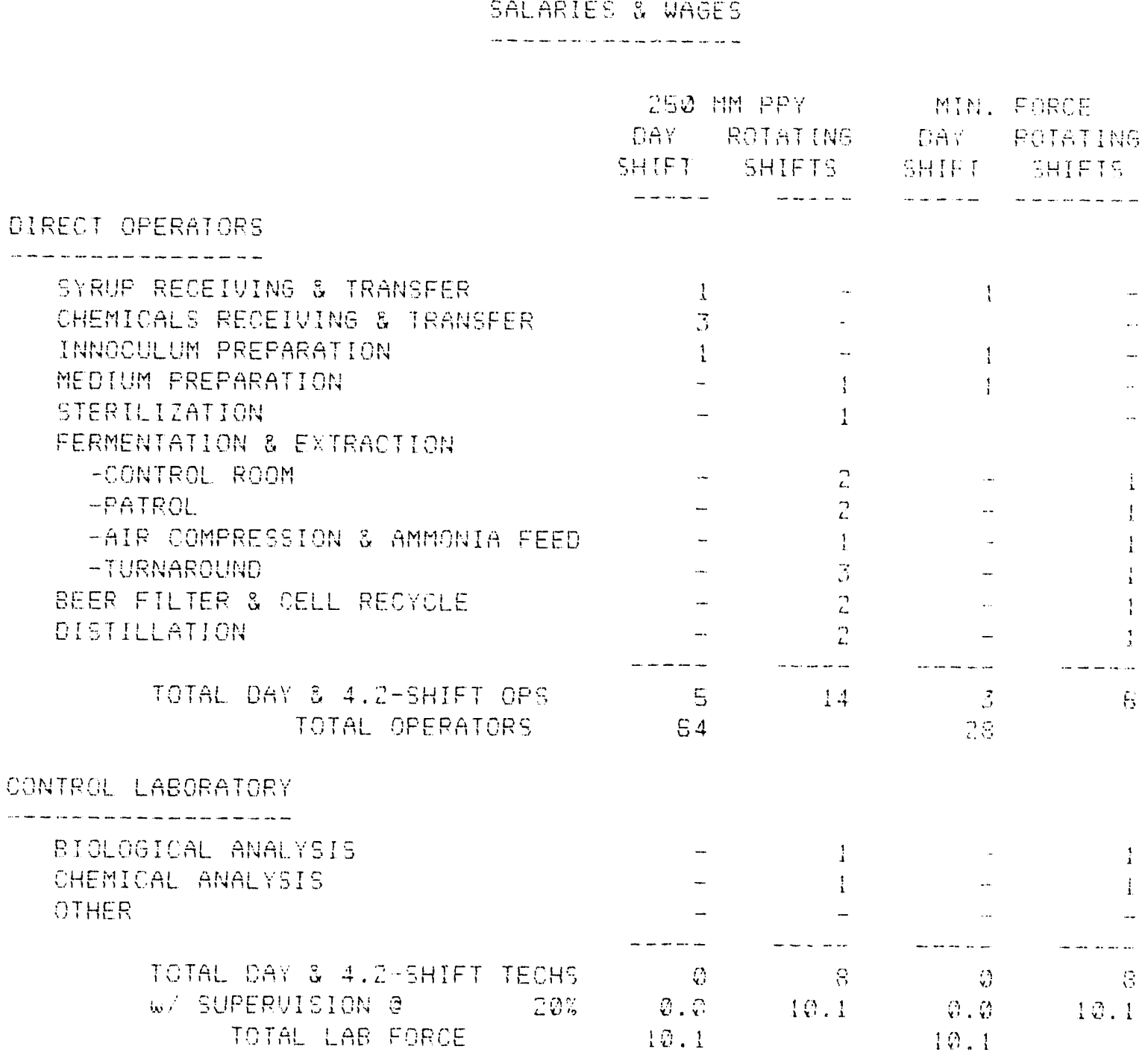

TECHNICAL AESTETANCE TO MANUFACTURING

FROOESE ENGINEERS

WAGES. SALARIES B EENEFITS OCHEDULE- 1998

\begin{tabular}{|c|c|}
\hline OFERATIHG WAEES - \$ HOUR & $\$ 2 \pi \cdot 14$ \\
\hline TECHALOAAMS - ANAULAL $\$$ & $\$ 3 x: 500$ \\
\hline PROCESS ENGENEERS - AMHUAL & \pm 5.000 \\
\hline PENETON - AS $\%$ OF COMPENSATION & 8. $1 \%$ \\
\hline FIOA & $5.8 \%$ \\
\hline WNEMELOYMENT COMPENSATIOA & $0.5 \%$ \\
\hline GROUP I. IFE INGUFANCE & $0.7 \%$ \\
\hline MECIICAL MUSURANOE & $3.5 \%$ \\
\hline OENTAL INGURANCE & $0.8 \%$ \\
\hline SAUTHES FLAN! & $2.5 \%$ \\
\hline VACATION & i. $4 \%$ \\
\hline ILLHESS & $1.4 \%$ \\
\hline ABSFNCE WITH PEFMISSION & A. $2 \%$ \\
\hline TOTHL DENEFI & $\therefore 1.1 \%$ \\
\hline
\end{tabular}


MLLTISTAGE EYTRACTIUE FERMENTATION OF EUTAROL WITH RECOUERY OF RAFFINATE GOAL STOJOHIOMETRY

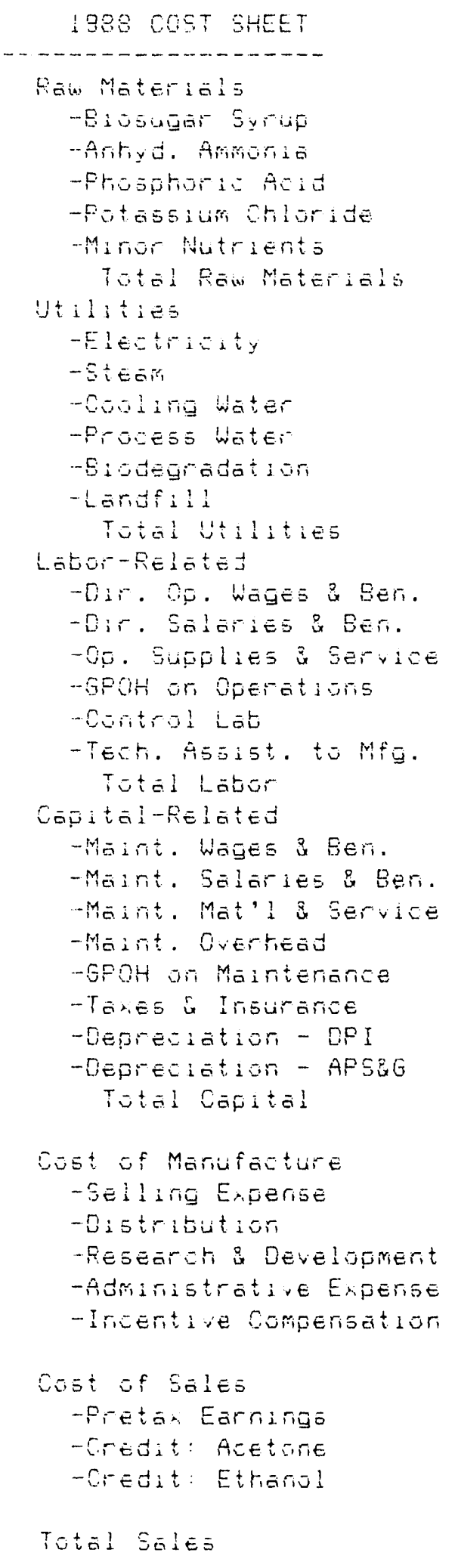

\begin{tabular}{|c|c|c|c|c|}
\hline RATE & IUNIT & $\begin{array}{l}\text { MILLION } \\
\text { UNTTS }\end{array}$ & \$MLLION & 专; \\
\hline$\cdots \cdots$ & $\ldots-\cdots$ & $-\cdots-\cdots$ & $\ldots \ldots \ldots-\cdots$ & $-\cdots-\cdots$ \\
\hline 事. & it. d.5. & 418.35 & 27.23 & 0.168 \\
\hline$\$ 3.046$ & 侣 & 1.01 & 0.85 & .000 \\
\hline$\$ 0.155$ & iti. & 0.84 & 0.13 & 0.001 \\
\hline$\$ 0.053$ & $\therefore$ 让. & 0.54 & 6.83 & .030 \\
\hline \multirow[t]{2}{*}{$\$ 0.45:$} & $i 1 t$ & 0.29 & 0.13 & 2.201 \\
\hline & & & $\$ 2.7 .58$ & $\$ 0.17 \%$ \\
\hline$\$ 0.940$ & ;KWH & 8.27 & 0.33 & 8.002 \\
\hline 生工. . & Ｍ IL. & 8.79 & 1.74 & 0.011 \\
\hline$\$ 0.64$ & M gal. & 1.57 & 8.67 & .000 \\
\hline$\Phi .50$ & M פ̄il. & D. 1 & .90 & .000 \\
\hline$\$ 0.84$ & 沾. d.5. & 14.54 & 0.59 & 0.004 \\
\hline \multirow{2}{*}{$\$ 0.05$} & $\therefore 15.4 .5$. & 0.003 & 0.00 & 0.000 \\
\hline & & & $\$ 2.73$ & $\$ 0.017$ \\
\hline$\$ 25.40$ & manthr. & 0.117 & 3.83 & 0.019 \\
\hline 18 & \% DOW & & 0.55 & 0.033 \\
\hline 5 & $\%$ DOWBE & & 0.19 & 0.001 \\
\hline 23 & \% DOWSŨ & & 0.84 & 0.005 \\
\hline$\$ 17.22$ & iman-hr. & 0.020 & 0.39 & 0.802 \\
\hline \multirow[t]{2}{*}{ \$2.. } & imaritrir. & 0.802 & 0.84 & .000 \\
\hline & & & 邫, 10 & $\$ 0.031$ \\
\hline 1.7 & $\% D P I$ & 需 20.1 & 0.34 & 0.802 \\
\hline 25 & $\because$ MWE & & 0.09 & 0.001 \\
\hline 40 & $\because$ Mwg & & 0.14 & 0.001 \\
\hline 4 & $\because M W E B$ & & 0.01 & .000 \\
\hline 23 & $\%$ MWS\&B & & 0.10 & 0.001 \\
\hline A. $\overline{5}$ & $\because D P I$ & $\$ 20.1$ & 8.05 & .000 \\
\hline 3 & $\because D P I$ & $\$ 20.1$ & 1.51 & 0.510 \\
\hline \multirow[t]{4}{*}{ E } & \% APSBG & $\$ 3.2$ & 0.43 & 0.003 \\
\hline & & & $\$ 2.84$ & $\$ 0.017$ \\
\hline & & & $\cdots-\cdots$ & $-\cdots--$ \\
\hline & & & $\$ 38.24$ & $\$ 0.235$ \\
\hline 3 & $\%$ Sales & $\$ 57.5$ & 1.73 & 0.011 \\
\hline$\$ 0.01$ & 结。 & 152.3 & 1.62 & 0.010 \\
\hline 5 & $\%$ Sales & $\$ 57.5$ & 2.59 & 0.016 \\
\hline 2 & $\%$ Sales & $\$ 57.5$ & 1.15 & 0.907 \\
\hline \multirow[t]{3}{*}{5} & \% PTE & $\$ 11.5$ & 0.59 & 0.004 \\
\hline & & & $-\cdots--$ & ----- \\
\hline & & & 生45.01 & $\$ 0.284$ \\
\hline 30.0 & $\%$ TIFR & $\$ 38.4$ & 11.51 & 0.071 \\
\hline 92.27 & , 15. & 6.8 & 0.00 & 0.060 \\
\hline \multirow[t]{3}{*}{$\$ 0.29$} & it & .0 & $(.00)$ & $\{.000\rangle$ \\
\hline & & & $-\cdots$ & $-\cdots$ \\
\hline & & & $\$ 57.52$ & $\$ 2.355$ \\
\hline
\end{tabular}


MULTISTAGE EXTRACTIVE FERMENTATION OF EUTANOL WITH RECOVERY OF RAFFINATE GOAL STORCHIOMETRY

BASH FLOW OMLLION DOLLARS, YEAR;

Ecentiotio:

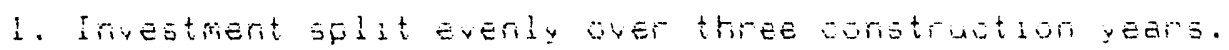

2. Plant operates at $50 \%$ of full soale the first year.

i. " " $75 \%$ " "the eecond year.

4 4. $100 \% "$ " "the thisd $"$ " "

5. " $" 100 \%$ " " thereafter.

E. Five year Jepreciatlor ratel half-year correritidit

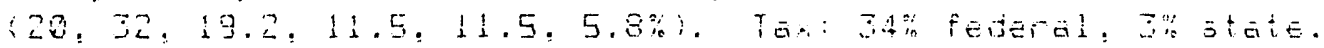

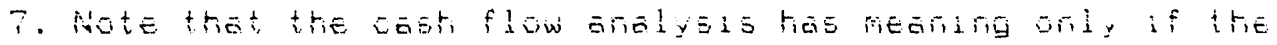

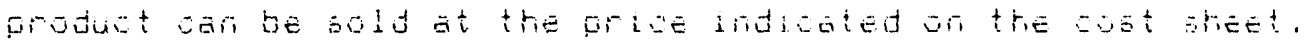

\begin{tabular}{|c|c|c|c|c|c|c|c|}
\hline & \multicolumn{2}{|c|}{ INUESTMENT } & DEP. & $\operatorname{cost} 5 x$ & SALES & NET EARA & ANA CASH \\
\hline YEAE & $\mathrm{FI}$ & we & $\cdots \cdots$ & $\cdots \cdots-\cdots$ & $\cdots-\cdots$ & $\cdots \cdots \cdots$ & $-\cdots \cdots \cdots$ \\
\hline$-\cdots$ & $-\cdots$ & $\cdots---$ & & & & & \\
\hline 1383 & \$E. 71 & & & & & & :\$E.T: \\
\hline 1984 & WE. : & & & & & & $\div 5.71$ \\
\hline 1985 & $\$ 5.71$ & $\$ 10.15$ & & & & & $: \pm 16.04:$ \\
\hline 1385 & & & 44.02 & $\$ 25.92$ & \pm 26.75 & +10.13 & +6.29 \\
\hline 1987 & & & 55.44 & $\$ 35.89$ & +45.14 & 50.51 & $\$ 5.95$ \\
\hline 1986 & & & $\$ 3.85$ & $\$ 43.91$ & $\$ 57.52$ & \$5.14 & tकी. \\
\hline 1989 & & & 筷?. & $\$ 43.91$ & 557.52 & 生: ! ! & 49.43 \\
\hline 1980 & & & 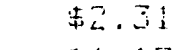 & $\$ 4.91$ & $\$ 5 ? .5 \%$ & \pm 7.11 & \pm 9.4 \\
\hline 1391 & & & 31.17 & 443.31 & $\$ 57.52$ & \pm 7.94 & +9.92 \\
\hline 1952 & & & & \pm 42.91 & 957.52 & $16.5 ;$ & $48.5 ;$ \\
\hline 1393 & & & & +43.91 & +57.52 & \pm 8.57 & 58.57 \\
\hline 199.4 & & & & $\$ 4: .9 !$ & $157.5 \%$ & +8.5? & $40.5 \%$ \\
\hline 1995 & & & & 445.91 & $\$ 57.52$ & 65.5 & $16.5 \%$ \\
\hline 1995 & & & & \pm 43.91 & 957.52 & $48.5 \%$ & $78.5 \%$ \\
\hline 1997 & & & & 443.91 & $\$ 57.52$ & $46.5 \%$ & $4.5 \%$ \\
\hline 1998 & & & & $+4 \overrightarrow{5} .91$ & 457.52 & +6.57 & $\$ 6.57$ \\
\hline 1999 & & & & $\$ 45.91$ & $\$ 5 \% .5$ & 40.5 & 19,57 \\
\hline 2006 & & & & \pm .43 .91 & \pm 57.52 & $+8,5$ & $\pm 5 ;$ \\
\hline 2001 & & & & $4+3.91$ & $55 \% .52$ & 96.57 & 48.57 \\
\hline $29 \times 2$ & & & & 索4 & $45 \% .52$ & 46,59 & $+8.5 \%$ \\
\hline 2023 & & & & 943.91 & 953.52 & +6.57 & 19.57 \\
\hline 2004 & & & & 943.31 & $\$ 57.52$ & $+9.5 \%$ & 5.5 \\
\hline 205 & & 10.13 & & 4.9 .91 & 757.52 & $46.5 \%$ & \pm 15.70 \\
\hline
\end{tabular}

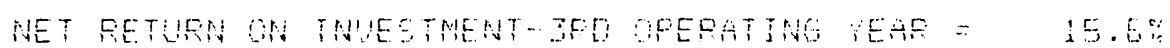


MULTISTAEE EXTRACTIUE FERMENTATION OF BUTANOL WITH RECOUERY OF RAFFINATE GOAL STOICHIOMETRY

CASH FLOW MNLLION DOLLARS,YEAR;

SLeñátio:

i. indestirerit afilt everily over three ooristruction years.

2. Plant operates at $59 \%$ of full soale the first year.

i. " " $"$ " " " " the second year.

4 4. " $" 100 \%$ " " "he third year.

5. " $100 \% "$ " " thereafter.

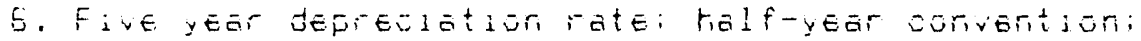

\{20, 32, 19.2, 11.5, 11.5, 5.3\%;. Ta. 34\% federal, 3\% state.

i. Note that the cast flow andalysis has mearing orily if the pioduct anr te sold at the price indloated on the cost sheet. CUM CAEH NFU O $12 \%$ \%RP

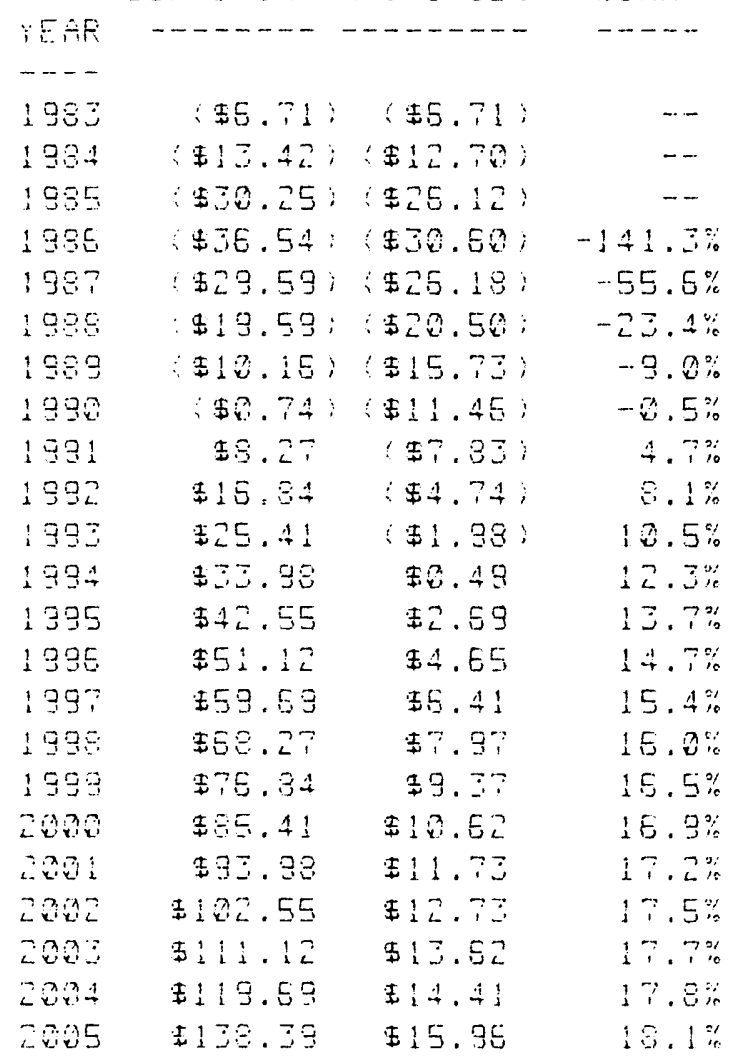


MULTISTAGE EXTRACTIUE FERMENTATION OF BUTANOL WITH RECOUERY OF RAFFINATE GOAL STOICHIOMETRY

EASIC DATA
$152.25 \mathrm{MM} \mathrm{PPY}$
$180.28 \mathrm{MM}$ PPY
$90 \%$
$153.25 \mathrm{MM} P P Y$
$181.40 \mathrm{MM} P P Y$
$95.0 \%$
$2.56 \mathrm{LI} / 1 \mathrm{~L}$
$74.12 \mathrm{MOL} W \mathrm{WT}$

PRODUCT STOICHIOMETRY
ANNUAL PROOUCTION LEUEL ANINUAL CAPACITY OPERATING UTILITY PROOUCT IN BEER PRODUCT IN BEER AT CAPACITY MOLAR YIELD-GLUC. TO PROO. IN EEER KE GLUCOSE DEMANDIPROD IEXOL. SPILL;

PFODUCT MOLECULAR WEIGHT

$$
\begin{aligned}
& \text { MOL. WT. MOLESIMOL PROQUCT COMPONENT } \\
& \text { 180.1E I. OOOOO ,MOL PROD. -GLUCOSE CONSUMED } \\
& \text { Z2. OO O.DADOQ IMOL PROD. -OXYGEN CONSUMED } \\
& \text { 17.0L O.OOAOQ MOL PROD. -AMMONIA CONSUMED } \\
& .000 .00000 \text { IMOL PROD. -COMPONENT \#1 FORMED } \\
& .00 \text { O.OONOO IMOL PROO. -COMPONENT \#2 FORNED } \\
& \text { 58.08 } 0.00000 \text { IMOL PROD. COMPONENT \#J FORMED (ACETONE) } \\
& \text {. RO O. DOQOO IMOL PROD. -COMPONENT \#4 FORMED } \\
& 45.07 \text { Q. DQOOO IMOL PROD. -COMPONENT \#5 FORMED (ETHANOL) } \\
& \text {.DO O.RODOR IMOL PROD. -COMPONENT \#G FORMED } \\
& \text { T. } 12 \text { 1.00OOQ MMOL PROD. -COMPONENT \#7 FORMED (BUTANOL) }
\end{aligned}
$$

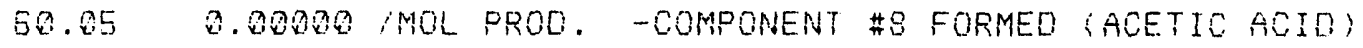

$$
\begin{aligned}
& \text {.OQ } 2.00000 \text { MMOL PROL. -COMPONENT \#9 FORMED }
\end{aligned}
$$

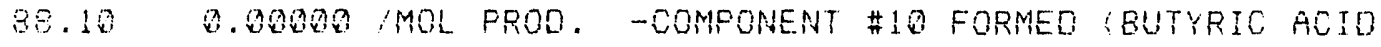

$$
\begin{aligned}
& \text { 18.02 1.00000 MMOL FROD. WATER FORMED }
\end{aligned}
$$

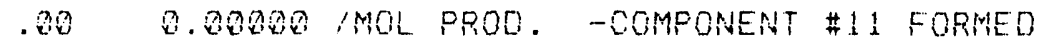

$$
\begin{aligned}
& \text {. DO O.QOOOO IMOL PROO. -COMPONENT \#12 FORMED }
\end{aligned}
$$

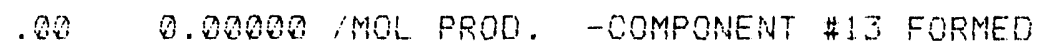

$$
\begin{aligned}
& \text {. DO O.0OOOO MNOL FROO. COMPONENT \#14 FORMEO } \\
& .30 \text { O.00000 MOL PROO. -COMPONENT \#15 FORMED } \\
& \text {. DO D. OOQOO IMOL PROO. COMPONENT \#LE FORMED }
\end{aligned}
$$

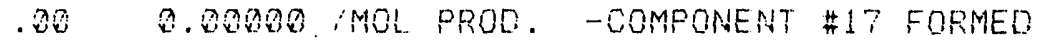

$$
\begin{aligned}
& \text {. OO O.OOOOO IMOL PROD. COOMPONENT \#18 FORMED }
\end{aligned}
$$

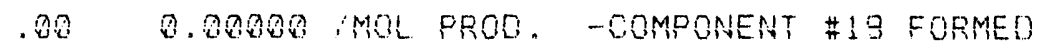

$$
\begin{aligned}
& .30 \text { O. OOQOO IMOL PROO -COMPONENT \#20 FORMED } \\
& \text { 44.01 2.0OOOO IMOL PROO. -CARBON DIOXIOE FORMEO } \\
& \text { ב.O. O.00000 IMOL FROD. HYOROGEN FORMED }
\end{aligned}
$$

NUTFIENTS IN FLRMENTER FEEG

$$
8 \%
$$

-N IN OELLS AS \%CHO

8. 9 rigig dells -H3POA

51.6 rgig alls -

23.9 MGIG CEL15 WMINOR MUTRIENTS 
MULTISTAGE EXTRACTIUE FERMENTATION OF BUTANOL WITH RECOUERY OF RAFFINATE

GOAL STOICHIOMETRY

\begin{tabular}{|c|c|c|c|}
\hline \multicolumn{4}{|c|}{ BASIC DIATA } \\
\hline 27.9 & mg'g & $\Delta \in 115$ & -MINOR NUTRIENTS \\
\hline 23.6 & mg'g & cells & $-\mathrm{MgSO} 4.7 \mathrm{HLO}$ \\
\hline 0.01 & $m g / g$ & ce115 & -VITAMIN BL \\
\hline 1.25 & $M g / g$ & cells & $-K I$ \\
\hline 0.39 & $r_{i g} / g$ & cells & $-N i C 12$ \\
\hline 0.72 & moig & cells & $-F \in C 13.5420$ \\
\hline 6.55 & $\mathrm{mg} / \mathrm{g}$ & sells & - \\
\hline 0.54 & mgig & cel15 & $-\mathrm{H} 3 \mathrm{BO} 3$ \\
\hline 0.22 & $\mathrm{mg}$ & $\operatorname{cel15}$ & $-2 n 504.7420$ \\
\hline 0.15 & $m g ' g$ & cel15 & $-M n S O 4 . H_{2} O$ \\
\hline 7.7 & $4 g / G$ & $\operatorname{cells}$ & -CuS04.5H2O \\
\hline 5.4 & ugig & cel15 & -KOMOOO4.2H2O \\
\hline 4.3 & Uigig & cells & -60012.6420 \\
\hline
\end{tabular}

\section{FERMENTATION}

TYPE

STAGES

CONDITIONS

STAGE : GROWTH

PRODUCTION
J3 C

33

E.5

0

22.7

0.230

0.00

0.00

5.35

221

5

$--$

5

--

2"IE.5E
$0<0$
$1 \leqslant 0$
$T 10 N$
30
5

$1 \bar{j} 91$

$21.25: 1$

Q. 451 1, hir

$0.283 g / g * 5.6$

$5.93 \mathrm{~g} / 1 * h \mathrm{hm}$

D. 00 gil $1 * 1 \%$

Q mMil $*$ tir

D mimim

$0.19 / 1$

50

19 hod Gmol

22.5* Btultir*gal
-ANAEROBIC (O) OR AEROBIC : $1:$

-CONCUR'NT $(O)$ OR SEQUENT'AL (1)

-TEMPERATURE

$-T H$

-PRODUCT CONCENTRATION IN BEER

-CEL! DENSITY (CHO ONLY;

-DILUTION RATE

-FRODUCT PROOUCTIUITY

* Specifio Productivity

* Volumetrie Productivity

-CELL PRODUCTIVITY (CHO ONLY)

-OXYGEN TRANSFERRED

- OXYGEN FED; OXYGEN STOICH. DEMANO

- GLUCOSE SPILL

- COOLING WATER TEMPERATURE

-HEAT EVOLUED-PRODUCT FORMATION

- TIEAT REMOUED BY COOLING COILS

FERMENTERS

$\begin{array}{rr}27.686 & 458,939 \text { gallons } \\ 15 & 15 \% \text { gros } \\ 33.320 & 535.279 \text { gallons } \\ 103.000 & 250.000 \text { gallons } \\ 0.4 & 2.5 \text { uritts }\end{array}$

-ACTIVE UOLUME REQUTRED

- HEADEPACE

- GROSE VOLUME \& inCl. 15\% 5pares and

-GROSS SI ZE

-NUMBER OF UNITS

PRODUCT SEPARATION

$4009 ; 1$

0.053 Ḡil, mintosf

2. 489 50 ft
-CELL CONC. (CHO) EX FILTEF

-FILTER THROUGHPUT

-FILTER SIZE 
MULTISTAGE EXTRACTIUE FERMENTATION OF BUTANOL

WITH RECOUERY OF RAFFINATE

GOAL STOICHIOMETRY

BASIC DATA

MATERIALS OF CONSTRUCTION

FERMENTERS \& EXTRACTERS

STILLS

HEAT EXCHANGERS

STOFAGE TANKS

FOR WHICH:

$\begin{array}{cc}\text { CHOICES } & \text { SELECTION } \\ 1,3 & 1 \\ 1,2,3,4,5 & 1 \\ 1,3,5,5 & 1 \\ 1,3 & 1\end{array}$

$$
\begin{aligned}
& 1=\text { CARBON STEEL } \\
& 2=\text { CARBON STEEL W'304 SS TRAYS } \\
& 3=304 \text { STAINLESS STEEL } \\
& 4=304 L \text { STAINLESS STEEL } \\
& 5=315 \text { STAINLESS STEEL } \\
& 5=\text { MONEL. }
\end{aligned}
$$

RETURN ON INUESTMENT To Calculate Salling Price Required to Frovide a Fixed Return,
Enter the Desired Return on Investinerit: $O R$

To Calculate the ROI Resulting from a Fixed Market Price, Eniter Market Price for: 1988 (Year) , lt.

Enter an Investment Contingency to Represent the R1sk Level of the Basic Data

$30 \%$

UENTURE TIMING

Midooint of Construction (i.e. 13xX) 1984

Qperating Vear (i.e. 19xx)

\section{EXTRACTIOM}

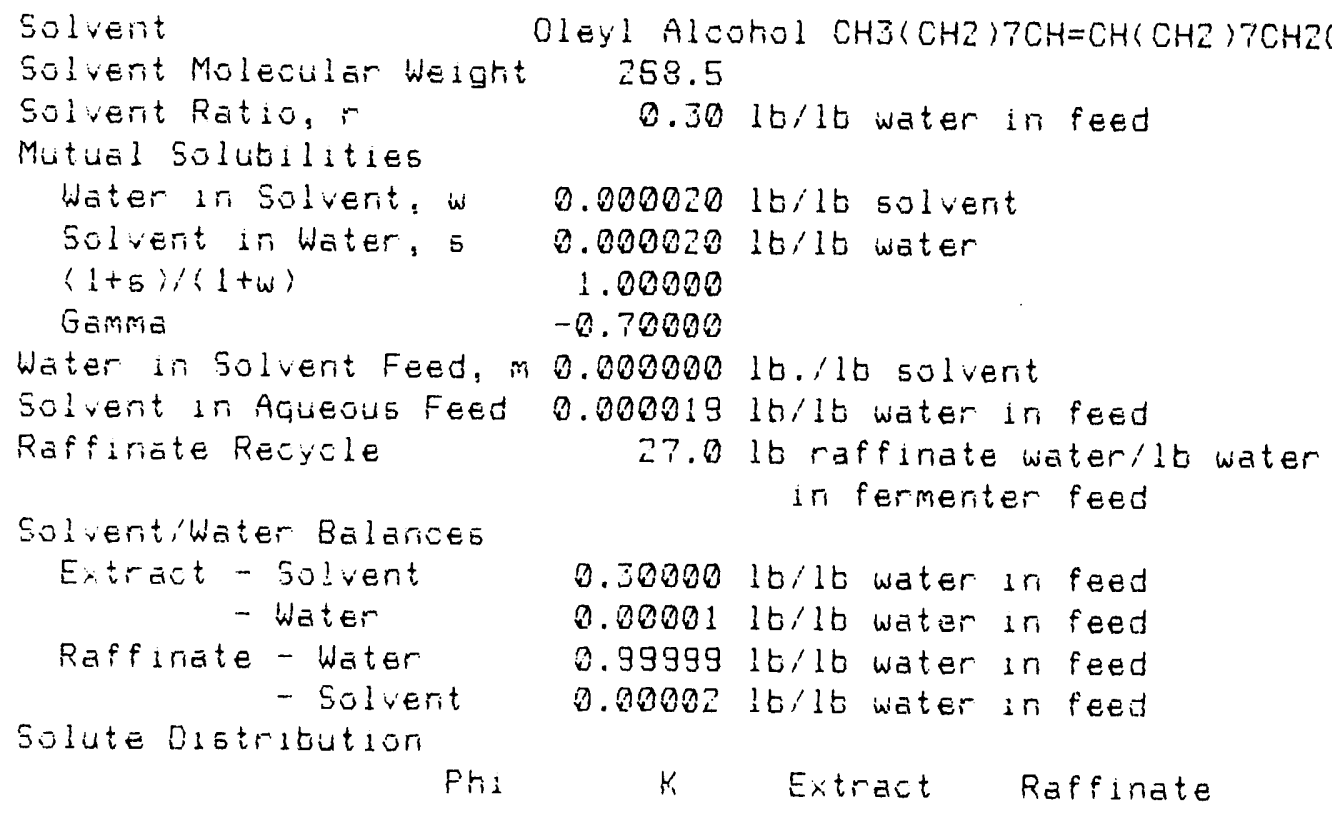


MULTISTAGE EXTRACTIUE FERMENTATION OF BUTANOL.

WITH RECOUERY OF RAFFINATE GOAL STOICHIOMETPY

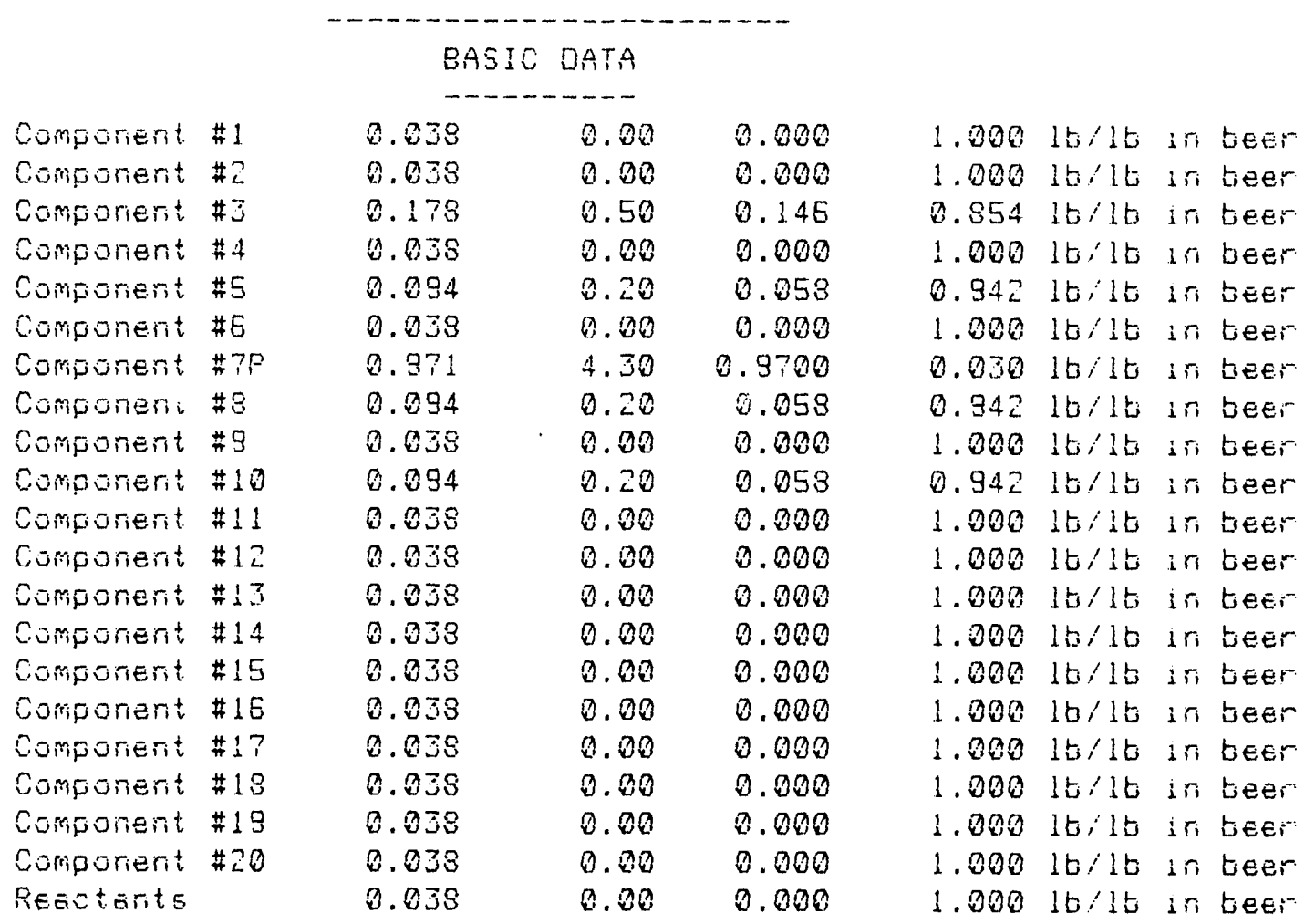

\section{EXTRACTOR DESIGN}

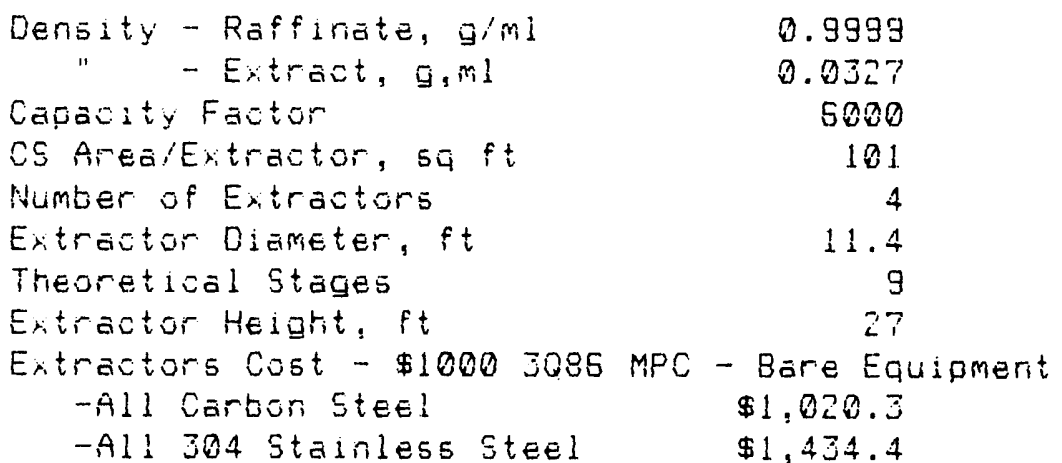




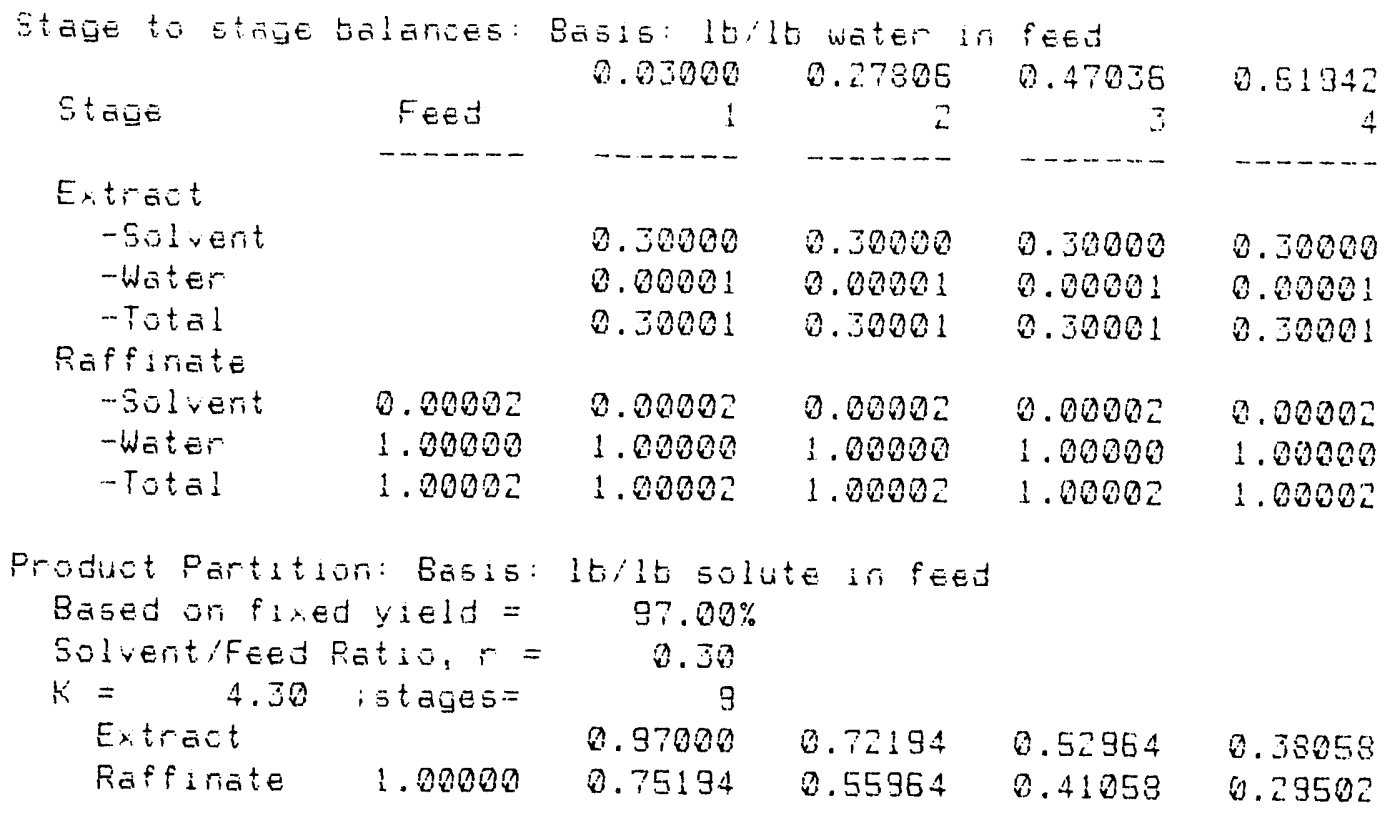




\begin{tabular}{|c|c|c|c|c|c|c|c|}
\hline $\begin{array}{r}5.7438 \\
5\end{array}$ & $\begin{array}{r}8.8245 E \\
E\end{array}$ & $\begin{array}{r}0.83402 \\
i\end{array}$ & $\begin{array}{r}0.94783 \\
8\end{array}$ & $\begin{array}{r}0.98956 \\
9\end{array}$ & $\begin{array}{r}1.02130 \\
10\end{array}$ & $\begin{array}{r}1.04698 \\
11\end{array}$ & $\begin{array}{r}1.05542 \\
12\end{array}$ \\
\hline D. 30200 & A. 30030 & 0.38000 & 3. 30900 & 0.30000 & 00000 & .30000 & 0.20000 \\
\hline$\therefore .8001$ & 0.30901 & 0. 80,$30 ;$ & 0.00001 & 0.00001 & 0. & 8.00001 & 0.00001 \\
\hline$\Delta .50001$ & $\therefore .30001$ & 0.50001 & 0.20001 & 0.30001 & 0.50001 & 0.30001 & 0.30001 \\
\hline ด. & 322 & 0.0 & . & & & & 6.0 \\
\hline 1.00030 & 1.90000 & $\therefore .00000$ & 1.20000 & 1.00000 & 1.00000 & 1.00000 & 1.00000 \\
\hline 1.00002 & 1.30002 & 1.00002 & 1.00002 & 1.00002 & 1.00002 & 1.00002 & 1.00002 \\
\hline
\end{tabular}

D.25502 ‥17544

0.20544

6. 13500

0.10530

0.8521?

$0.0521 ?$

2. 2.2844

0.01044

0.08818
$-0.02190$

$-0.01538$
$-0.04538$

$-0.03542$ 
$\begin{array}{rrrr}1.08149 & 1.09317 & 1.10222 & 1.10924\end{array}$

1.11890

$\begin{array}{rr}1.12217 & 1.12471 \\ 19 & 20 \\ -\ldots .0 & -\ldots . .0\end{array}$

0.30000

0.30000

0.30000

2. 30000

0.30000

0.30000

0. 30000

0.53000

0. 00001

0.00001

0.00001

0.00001

0.00001

0.00001

0. 00001

0.00001

0.30001

0.30001

0.30001

0. 30001

0.30001

0.30001

0.30001

$0.00002 \quad 0.00002 \quad 0.00002 \quad 0.00002$

$0.00002 \quad 0.00002 \quad 0.00002$

1. 00000

1.000001 .00000

0.00002

1.00000

1.00000

1. 000000

1.00000

1.00002

1.00002

1. 00002

0.93999

1. 00001

$-0.08149-0.09317-0.10222-0.10924$

$-0.11468-0.11890-0.12217-0.12471$

$-0.05317-0.07222-0.07924-0.03489$

$-0.03830-0.09217-0.09471-6.09567$ 
ELvert

.........

2. 30000

ด. . 28023

2. 30203

- A. 12EE? 


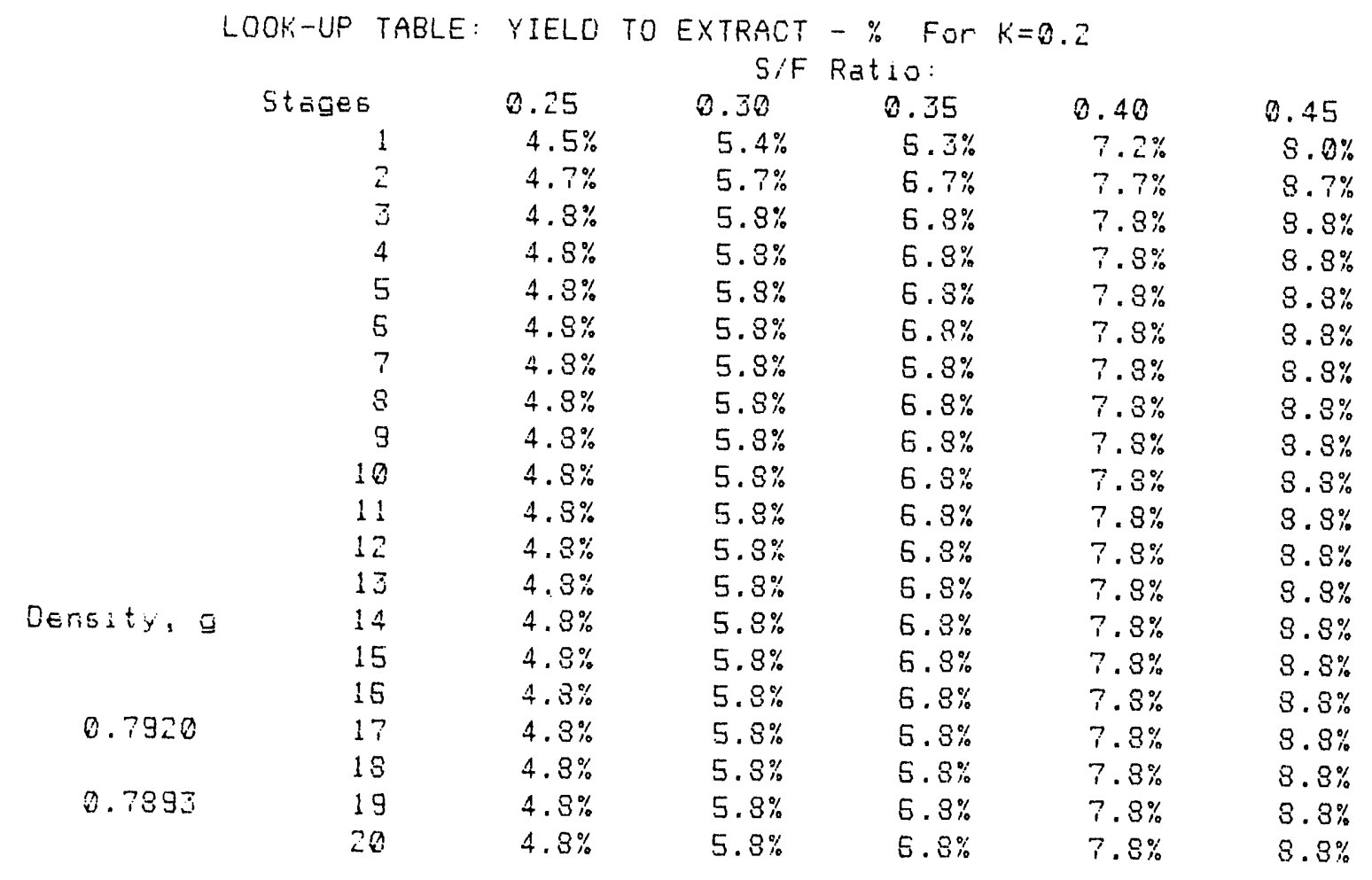




\begin{tabular}{|c|c|c|c|c|c|c|c|}
\hline 0.50 & 1.00 & 1.50 & 2.00 & 2.50 & 3.80 & 3.50 & 4.00 \\
\hline $8.5 \%$ & $15.0 \%$ & $22.00 \%$ & $28.3 \%$ & $33.1 \%$ & $37.4 \%$ & $41.1 \%$ & $44.3 \%$ \\
\hline $9.7 \%$ & $13.1 \%$ & $27.0 \%$ & $35.8 \%$ & $42.7 \%$ & $48.8 \%$ & $54.2 \%$ & $53.9 \%$ \\
\hline $9.3 \%$ & $19.5 \%$ & $27.00 \%$ & $38.3 \%$ & $4 E .6 \%$ & $53.9 \%$ & $50.4 \%$ & $56.1 \%$ \\
\hline $9.8 \%$ & $19.8 \%$ & $29.6 \%$ & $33.2 \%$ & $48.2 \%$ & $55.5 \%$ & $53.8 \%$ & $70.1 \%$ \\
\hline $9.8 \%$ & $19.8 \%$ & $23.8 \%$ & $39.5 \%$ & $49.1 \%$ & $57.9 \%$ & $55.9 \%$ & $72.8 \%$ \\
\hline $3.8 \%$ & $19.8 \%$ & $29.8 \%$ & $39.7 \%$ & $49.5 \%$ & $58.3 \%$ & ET. $2 \%$ & $74.5 \%$ \\
\hline $3.8 \%$ & $19.3 \%$ & $29.9 \%$ & $33.8 \%$ & $49.7 \%$ & $59.2 \%$ & $58.1 \%$ & $76.0 \%$ \\
\hline $3.8 \%$ & $19.8 \%$ & $29.9 \%$ & $39.9 \%$ & $49.8 \%$ & $59.5 \%$ & $58.5 \%$ & $76.6 \%$ \\
\hline $9.8 \%$ & $19.3 \%$ & $23.9 \%$ & $33.9 \%$ & $49.8 \%$ & $53.5 \%$ & $53.1 \%$ & $77.5 \%$ \\
\hline $9.8 \%$ & $19.8 \%$ & $23.3 \%$ & 亏马. 9\% & $49.5 \%$ & $59.7 \%$ & $69.3 \%$ & $78.0 \%$ \\
\hline $3.8 \%$ & $19.8 \%$ & $29.9 \%$ & $39.9 \%$ & $49.8 \%$ & $59.8 \%$ & E. $9.5 \%$ & $78.4 \%$ \\
\hline $3.8 \%$ & $18.8 \%$ & $23.3 \%$ & $39.3 \%$ & $49.8 \%$ & $59.9 \%$ & $59.6 \%$ & $78.7 \%$ \\
\hline $3.8 \%$ & $19.8 \%$ & $29.9 \%$ & $39.9 \%$ & $49.8 \%$ & $59.9 \%$ & $69.7 \%$ & $79.0 \%$ \\
\hline $3.8 \%$ & $19.8 \%$ & $29.9 \%$ & 39.8\% & $49.8 \%$ & $59.9 \%$ & $59.8 \%$ & $79.2 \%$ \\
\hline $3.9 \%$ & $13.8 \%$ & $29.9 \%$ & $39.9 \%$ & $49.8 \%$ & $59.3 \%$ & $69.8 \%$ & $79.3 \%$ \\
\hline $9.8 \%$ & $18.3 \%$ & $29.9 \%$ & $39.9 \%$ & $43.8 \%$ & $59.9 \%$ & $59.9 \%$ & $79.4 \%$ \\
\hline $3.8 \%$ & $19.8 \%$ & $29.9 \%$ & $33.9 \%$ & $49.3 \%$ & $59.9 \%$ & $69.9 \%$ & $79.5 \%$ \\
\hline $9.8 \%$ & $19.8 \%$ & $23.9 \%$ & $59.9 \%$ & $49.8 \%$ & $59.9 \%$ & E3. $9 \%$ & $79.5 \%$ \\
\hline $9.8 \%$ & $19.8 \%$ & $29.9 \%$ & $39.9 \%$ & $49.8 \%$ & $59.9 \%$ & $53.9 \%$ & $79.7 \%$ \\
\hline $9.8 \%$ & $13.8 \%$ & $29.9 \%$ & $39.9 \%$ & $49.8 \%$ & $59.9 \%$ & $59.9 \%$ & $79.8 \%$ \\
\hline
\end{tabular}




\begin{tabular}{|c|c|}
\hline 4.58 & 5.00 \\
\hline $47.3 \%$ & $49.9 \%$ \\
\hline $83.1 \%$ & EE. $6 \%$ \\
\hline $70.8 \%$ & $74.9 \%$ \\
\hline $.6 \%$ & $79.9 \%$ \\
\hline$\therefore 8.8 \%$ & $33.3 \%$ \\
\hline $80.7 \%$ & $85.6 \%$ \\
\hline $92.4 \%$ & $37.4 \%$ \\
\hline $83.5 \%$ & $88.8 \%$ \\
\hline $84.5 \%$ & $89.9 \%$ \\
\hline $85.3 \%$ & $90.8 \%$ \\
\hline $85.0 \%$ & $91.5 \%$ \\
\hline $85.5 \%$ & $32.2 \%$ \\
\hline $37.0 \%$ & $32.8 \%$ \\
\hline $37.3 \%$ & $33.3 \%$ \\
\hline $87.7 \%$ & $93.7 \%$ \\
\hline $87.3 \%$ & $94.1 \%$ \\
\hline $8.2 \%$ & $34.4 \%$ \\
\hline $5.4 \%$ & $34.7 \%$ \\
\hline $.5 \%$ & $34.3 \%$ \\
\hline $8.5 \%$ & $95.1 \%$ \\
\hline
\end{tabular}




\begin{tabular}{|c|c|c|c|c|c|c|c|}
\hline$F-U F$ & & $\begin{array}{l}0 \text { EXTRA } \\
\text { SIF Rat }\end{array}$ & & & & & \\
\hline Stajes & 0.25 & 0.30 & 0.35 & 0.40 & 0.45 & 0.50 & 1.00 \\
\hline 1 & $10.0 \%$ & $12.5 \%$ & $14.5 \%$ & $1 E .0 \%$ & $18.8 \%$ & $19.0 \%$ & $3 \Xi .0 \%$ \\
\hline 2 & $11.0 \%$ & $14.8 \%$ & $1 E .0 \%$ & $18.0 \%$ & $21.0 \%$ & $24.0 \%$ & $42.0 \% \%$ \\
\hline $\bar{j}$ & $12.0 \%$ & $14.5 \%$ & $17.8 \%$ & $19.0 \%$ & $22.0 \%$ & $25.0 \%$ & $43.0 \%$ \\
\hline 4 & $12.0 \% \%$ & $14.6 \%$ & $17.1 \%$ & $20.0 \%$ & $23.0 \%$ & $25.0 \%$ & $48.2 \%$ \\
\hline 5 & $12.0 \%$ & $14.5 \%$ & $17.1 \%$ & $20.0 \%$ & $23.0 \%$ & $25.0 \%$ & $48.5 \%$ \\
\hline 5 & $12.0 \%$ & $14.8 \%$ & $17.1 \%$ & $20.0 \%$ & $23.0 \%$ & $25.0 \%$ & $49.0 \%$ \\
\hline 7 & $12.0 \%$ & $14.6 \%$ & $17.1 \%$ & $20.0 \%$ & $23.0 \%$ & $25.03 \%$ & $43.5 \%$ \\
\hline 8 & $12.0 \%$ & $14.5 \%$ & $17.1 \%$ & $20.0 \%$ & $23.0 \%$ & $25.0 \%$ & $49.5 \%$ \\
\hline 9 & $12.0 \%$ & $14.6 \%$ & $17.1 \%$ & $20.0 \%$ & $23.0 \%$ & $25.8 \%$ & $43.7 \%$ \\
\hline 10 & $12.0 \%$ & $14.6 \%$ & $17.1 \%$ & $20.0 \%$ & $23.00 \%$ & $25.0 \%$ & $49.7 \%$ \\
\hline 11 & $12.0 \%$ & $14.5 \%$ & $17.1 \%$ & $20.0 \%$ & $23.0 \%$ & $25.0 \%$ & $49.7 \%$ \\
\hline 12 & $12.0 \%$ & $14.5 \%$ & $17.1 \%$ & $20.0 \%$ & $23.0 \%$ & $25.0 \%$ & $49.7 \%$ \\
\hline 13 & $12.0 \%$ & $14.5 \%$ & $17.1 \%$ & $20.0 \%$ & $23.0 \%$ & $25.0 \%$ & $49.7 \%$ \\
\hline 14 & $12.80 \%$ & $14.6 \%$ & $17.1 \%$ & $20.0 \%$ & $23.0 \%$ & $25.0 \%$ & $49.7 \%$ \\
\hline 15 & $12.0 \%$ & $14.6 \%$ & $1.7 .1 \%$ & $20.0 \%$ & $23.0 \%$ & $25.0 \%$ & $49.7 \%$ \\
\hline 16 & $12.0 \%$ & $14.6 \%$ & $17.1 \%$ & $20.0 \%$ & $23.0 \%$ & $25.0 \%$ & $49.7 \%$ \\
\hline 17 & $12.8 \%$ & $14.6 \%$ & $17.1 \%$ & $20.0 \%$ & $23.0 \%$ & $25.0 \%$ & $49.7 \%$ \\
\hline 13 & $12.0 \%$ & $14.6 \%$ & $17.1 \%$ & $20.0 \%$ & $23.0 \%$ & $25.6 \%$ & $43.7 \%$ \\
\hline 19 & $12.0 \%$ & $14.5 \%$ & $17.1 \%$ & $20.0 \%$ & $23.0 \%$ & $25.0 \%$ & $49.7 \%$ \\
\hline 20 & 1工. $0 \%$ & $14.5 \%$ & $17.1 \%$ & $20.0 \%$ & $23.0 \%$ & $25.0 \%$ & $49.7 \%$ \\
\hline
\end{tabular}




\begin{tabular}{|c|c|c|c|c|c|c|c|}
\hline $1.5 \hat{0}$ & 2.80 & 2.50 & 3.00 & 3.50 & 4.00 & 4.50 & 5.80 \\
\hline $42.0 \%$ & $49.0 \%$ & $55.3 \%$ & $59.5 \%$ & $53.0 \%$ & $55.0 \%$ & $53.0 \%$ & .8 \\
\hline $55.0 \%$ & EE. $2 \%$ & $73.0 \%$ & $78.0 \%$ & $82.6 \%$ & $85.8 \%$ & $8 \% .0 \%$ & 9.8 \\
\hline $55.0 \%$ & $74.9 \%$ & $82 . x \%$ & $87.0 \%$ & $31.6 \%$ & $93.0 \%$ & $94.0 \%$ & $.0 \%$ \\
\hline $57.0 \%$ & $73.8 \%$ & $87.5 \%$ & $92.3 \%$ & $85.1 \%$ & $37.0 \%$ & $97.7 \%$ & $: \%$ \\
\hline $59.9 \%$ & $83.0 \%$ & $91.0 \%$ & $95.0 \%$ & $97.0 \%$ & $98.3 \%$ & $99.1 \%$ & \\
\hline $71.0 \%$ & $85.0 \%$ & $33.2 \%$ & $35.0 \%$ & $98.4 \%$ & $99.2 \%$ & $39.5 \%$ & \\
\hline $71.5 \%$ & $87.0 \%$ & $34.9 \%$ & $37.0 \%$ & $39.0 \%$ & $99.6 \%$ & $93.8 \%$ & \\
\hline $72.5 \%$ & $80.2 \%$ & $35.0 \%$ & $38.0 \%$ & $93.5 \%$ & $39.8 \%$ & $99.9 \%$ & 100 \\
\hline $73.0 \%$ & $83.0 \%$ & $35.5 \%$ & $39.1 \%$ & $39.7 \%$ & $99.9 \%$ & $100.0 \%$ & 0.2 \\
\hline $73.5 \%$ & $92.0 \%$ & $37.0 \%$ & $33.5 \%$ & $93.3 \%$ & $100.0 \%$ & $100.0 \%$ & 80 \\
\hline $74.0 \%$ & $91.0 \%$ & $93.6 \%$ & $39.5 \%$ & $39.9 \%$ & $100.0 \%$ & $100.00 \%$ & 180. \\
\hline $74.2 \%$ & $32 . \%$ & $39.5 \%$ & $39.7 \%$ & $39.3 \%$ & $100.0 \%$ & $100.0 \%$ & 100.6 \\
\hline $74.5 \%$ & $92.5 \%$ & $93.7 \%$ & $39.8 \%$ & $100.0 \%$ & $100.0 \%$ & $100.0 \%$ & 100.8 \\
\hline $74.5 \%$ & $93.0 \%$ & $39.0 \%$ & $39.9 \%$ & $100.0 \%$ & $100.0 \%$ & $100.8 \%$ & 100.8 \\
\hline $74.5 \%$ & $93.5 \%$ & $39.2 \%$ & $39.9 \%$ & $100.0 \%$ & $100.0 \%$ & $100.0 \%$ & 100.6 \\
\hline $74.5 \%$ & $34.0 \%$ & $39.4 \%$ & $39.9 \%$ & $100.0 \%$ & $100.0 \%$ & $100.0 \%$ & 100.0 \\
\hline $7.4 .7 \%$ & $3.4 .2 \%$ & $99.5 \%$ & $39.9 \%$ & $100.0 \%$ & $100.0 \%$ & $100.0 \%$ & 100.8 \\
\hline $74.7 \%$ & $34.5 \%$ & $99.5 \%$ & $39.3 \%$ & $100.0 \%$ & $100.0 \%$ & $100.0 \%$ & 100.0 \\
\hline $74.7 \%$ & $35.0 \%$ & $99.7 \%$ & $99.3 \%$ & $100.0 \%$ & $100.0 \%$ & $100.0 \%$ & 100.0 \\
\hline $74.8 \%$ & $35.0 \%$ & $53.8 \%$ & $93.9 \%$ & $100.0 \%$ & $100.0 \%$ & $100.0 \%$ & 100.0 \\
\hline
\end{tabular}


EISTILLATION DATA MATRIX

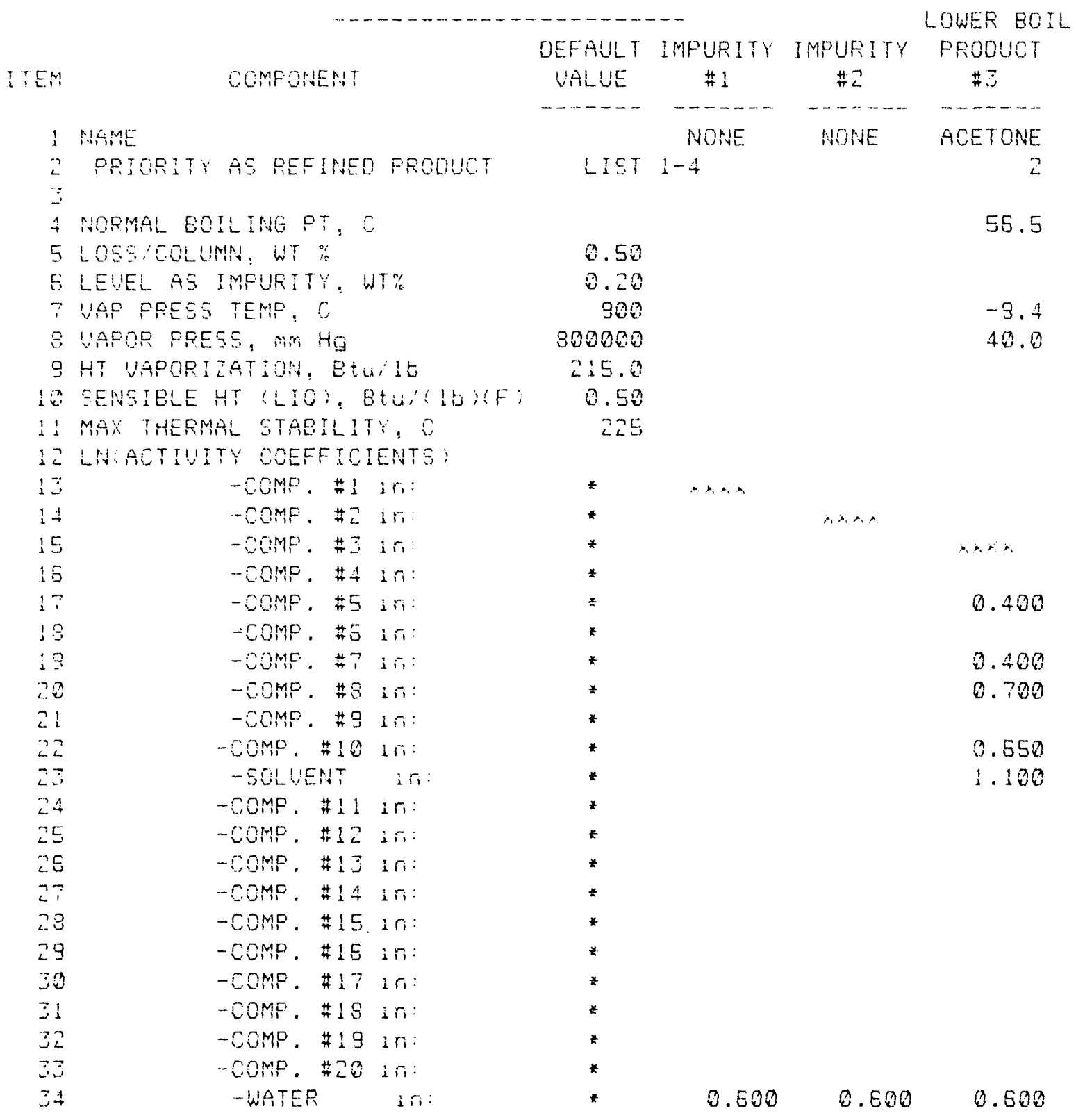

* The following values cari be used in lieu of actual activity coefficlents; however, the uncertairity of the caliulation is rábed 5 ignificantly:

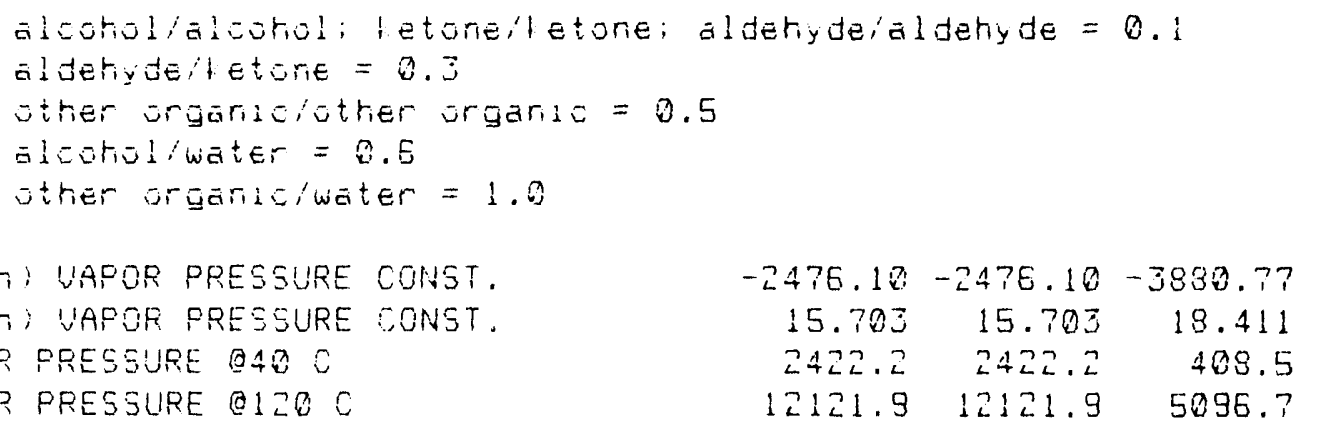




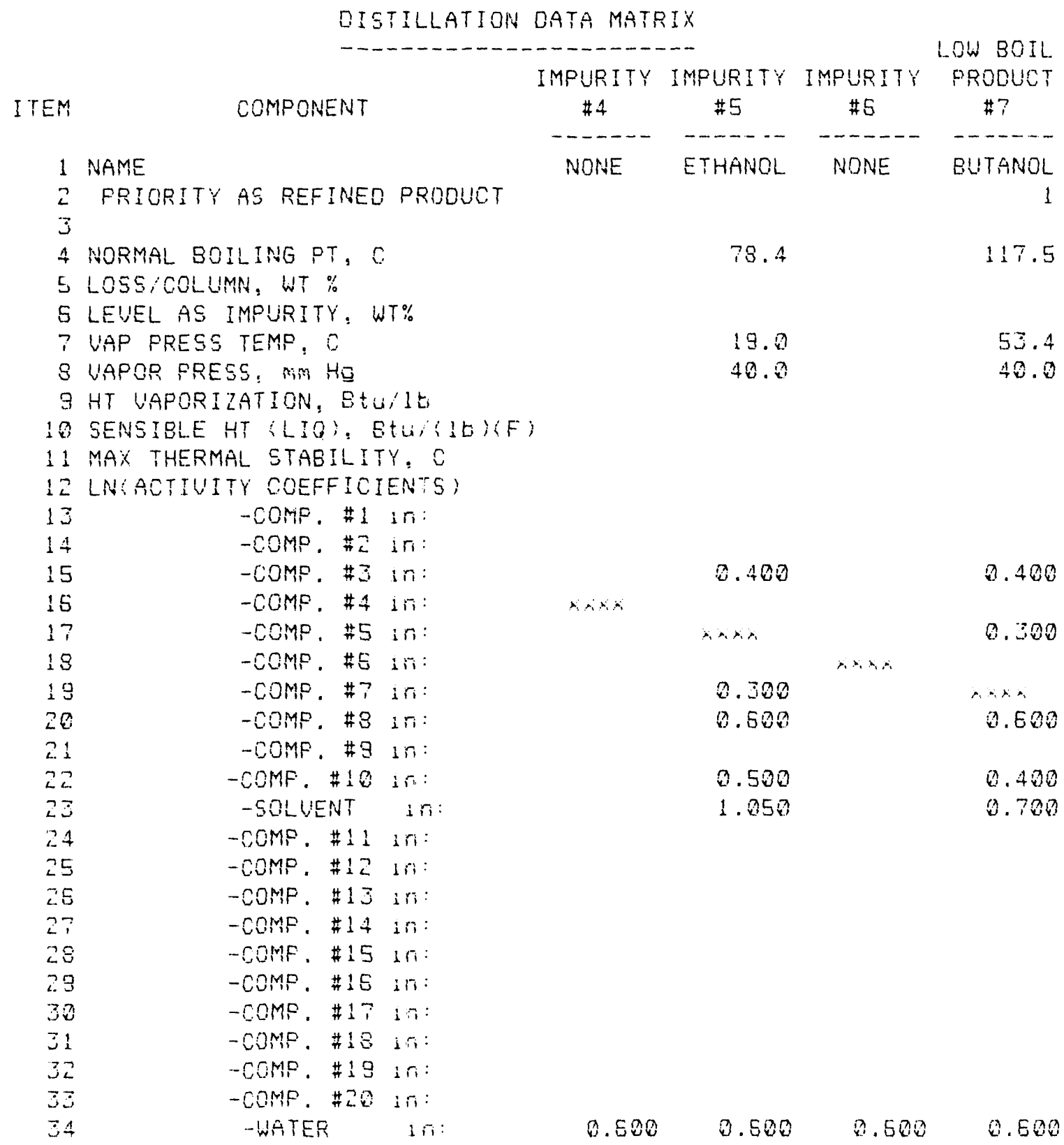

* The followirg values can be used lr lieu of gotual activity cuefficientz; however, the iricertainty of the caloulation is rabed aigrificantly:

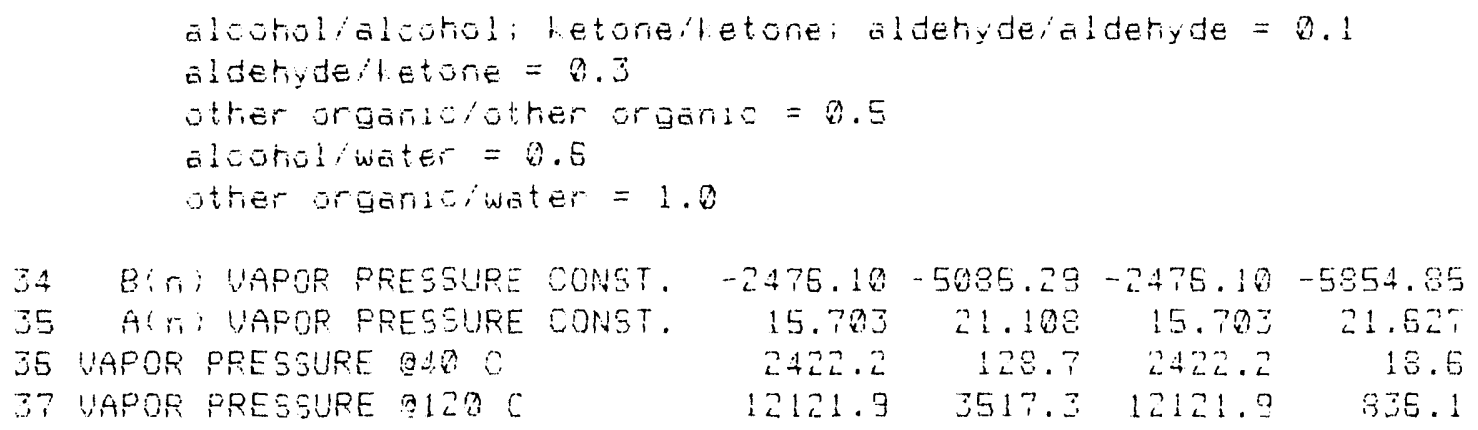




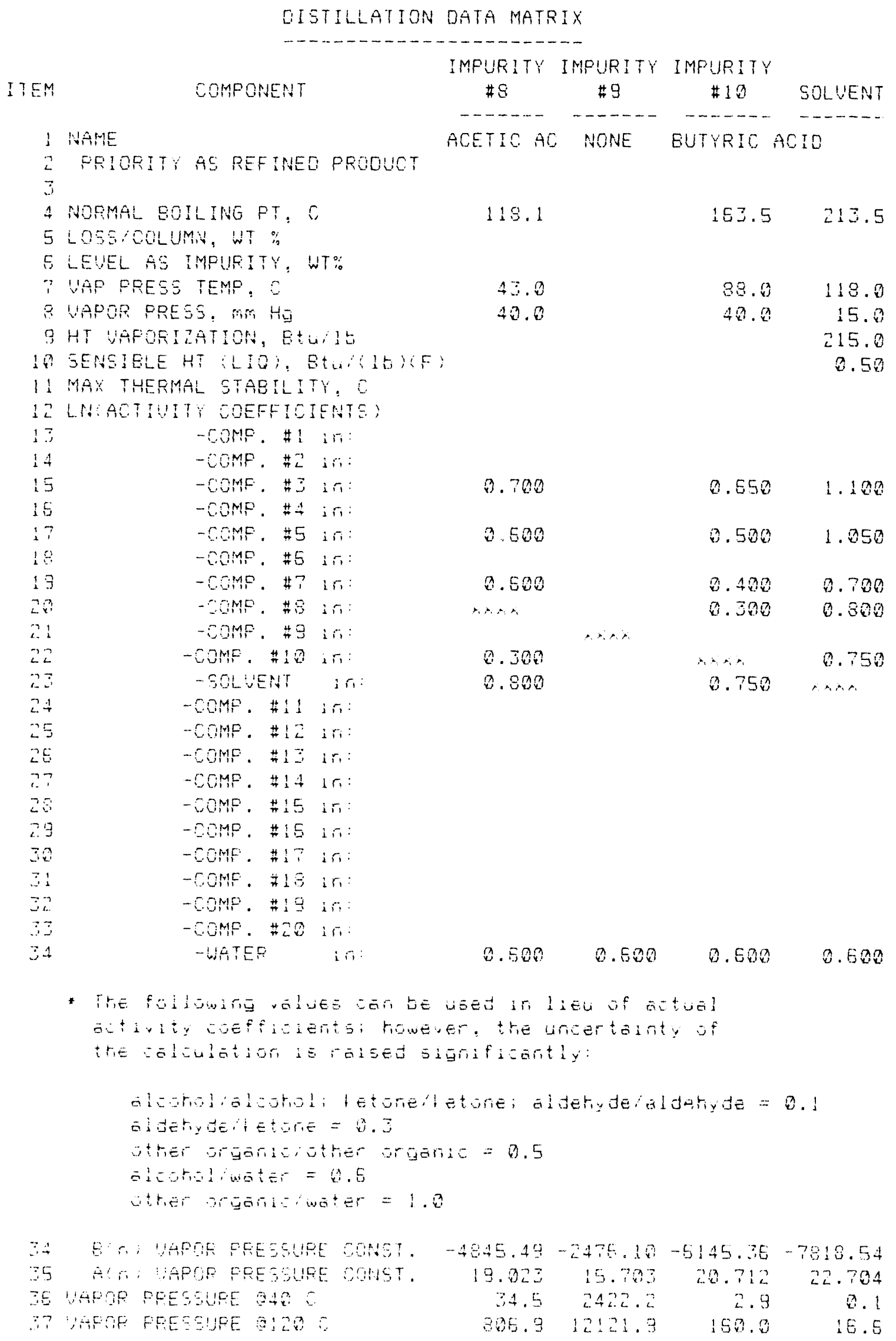




\section{DISTILLATION DATA MATRIX}

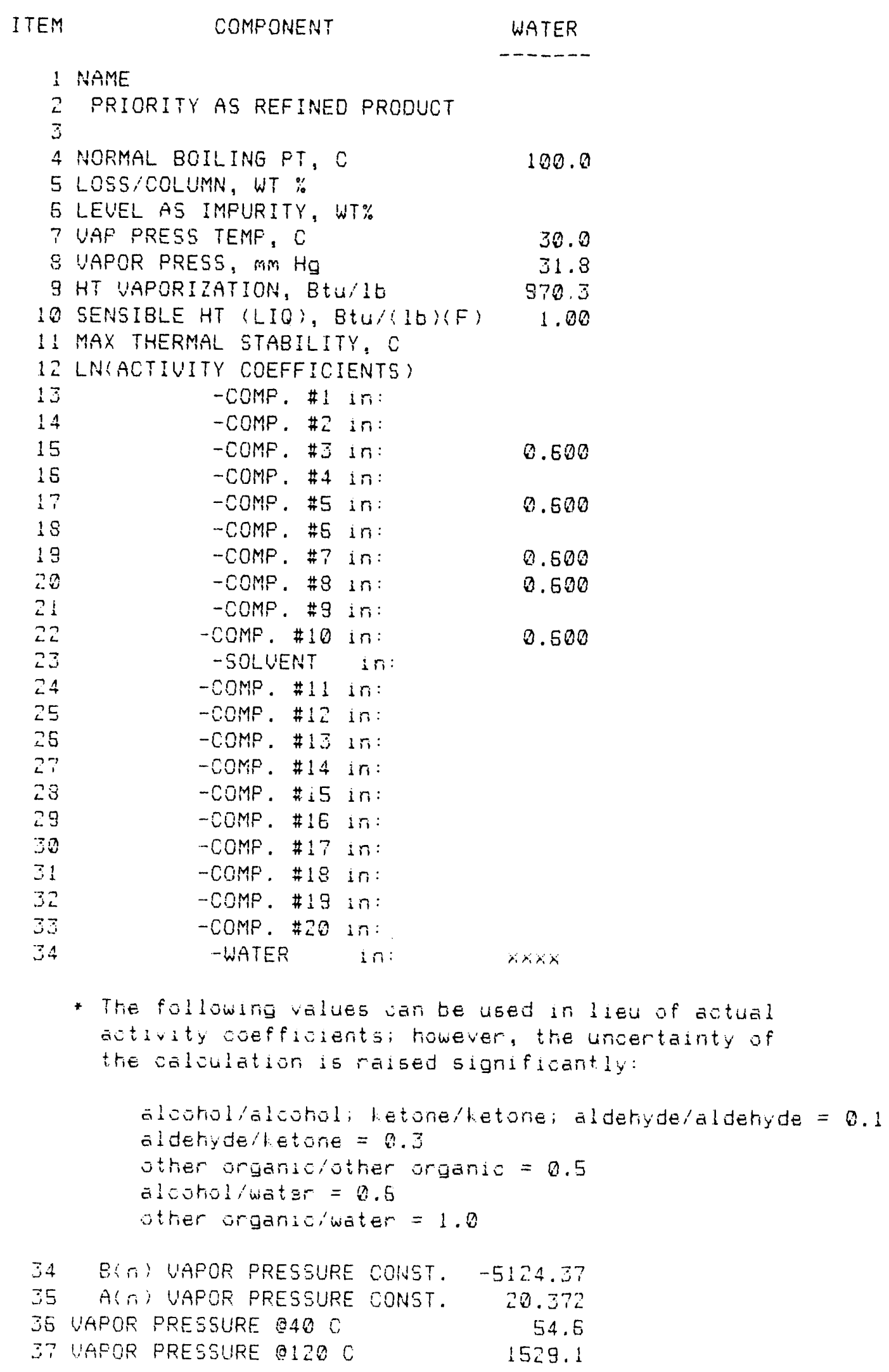


OFTEN USED PARAMETERS

(THOUSAND ANNUAL POUNDS:

\begin{tabular}{|c|c|c|}
\hline 01 & PRODUCT FORM & \\
\hline & FORMED WITH & PRODUCT \\
\hline 0 & -COMPONENT & $\# 1$ \\
\hline 6 & - COMPONENT & $\# 2$ \\
\hline$\theta$ & -COMFONENT & $\# 3$ \\
\hline 0 & -COMPONENT & $\# 4$ \\
\hline 8 & - COMPONENT & $\# 5$ \\
\hline 0 & - COMPONENT & $\# 5$ \\
\hline 181.401 & - COMPONENT & $\# 7$ \\
\hline 0 & - COMPONENT & $\# 8$ \\
\hline 0 & -COMPONENT & $\# 9$ \\
\hline 0 & -COMPONENT & $\# 10$ \\
\hline 44,092 & -WATER & \\
\hline (8) & - COMPONENT & $\# 11$ \\
\hline 0 & - COMPONENT & $\# 12$ \\
\hline 8 & - COMPONENT & $\# 13$ \\
\hline 0 & -COMPONENT & $\# 14$ \\
\hline 0 & - COMPONENT & $\# 15$ \\
\hline 0 & -COMPONENT & $\# 16$ \\
\hline D & - COMPONENT & $\# 17$ \\
\hline$\theta$ & -COMFONENT & $\# 18$ \\
\hline 8 & -COMPONENT & $\# 11=$ \\
\hline 0 & -COMFONENT & $\# 20$ \\
\hline$\theta$ & - HYDROGEN & \\
\hline 215,420 & -CARBON DIO & $X I D E$ \\
\hline & CONSUMED FOR & PRODU \\
\hline 440,914 & -GLUCOSE & \\
\hline 0 & -AMMONIA & \\
\hline 8 & -OXYGEN & \\
\hline
\end{tabular}




\section{OFTEN USED PARAMETERS}

- - - - - .

(THOUSAND ANNUAL POUNDS;

- OXYGEN FEO-PROO'N

- NITROGEN FED-PROD' i

8 OXYGEN UENT-PROD'N

- NITROGEN VENT-PROD'N

215,420 CARBON OIOXIDE VENT-PROD'N

7,767 WATER UIENT-PROD'N

223,187 PHI 
MULTISTAEE EXTRACTIUE FERMENTATION OF BUTANOL WITH RECOUERY OF RAFFINATE GOAL STOICHIOMETRY

WATER BALANCE

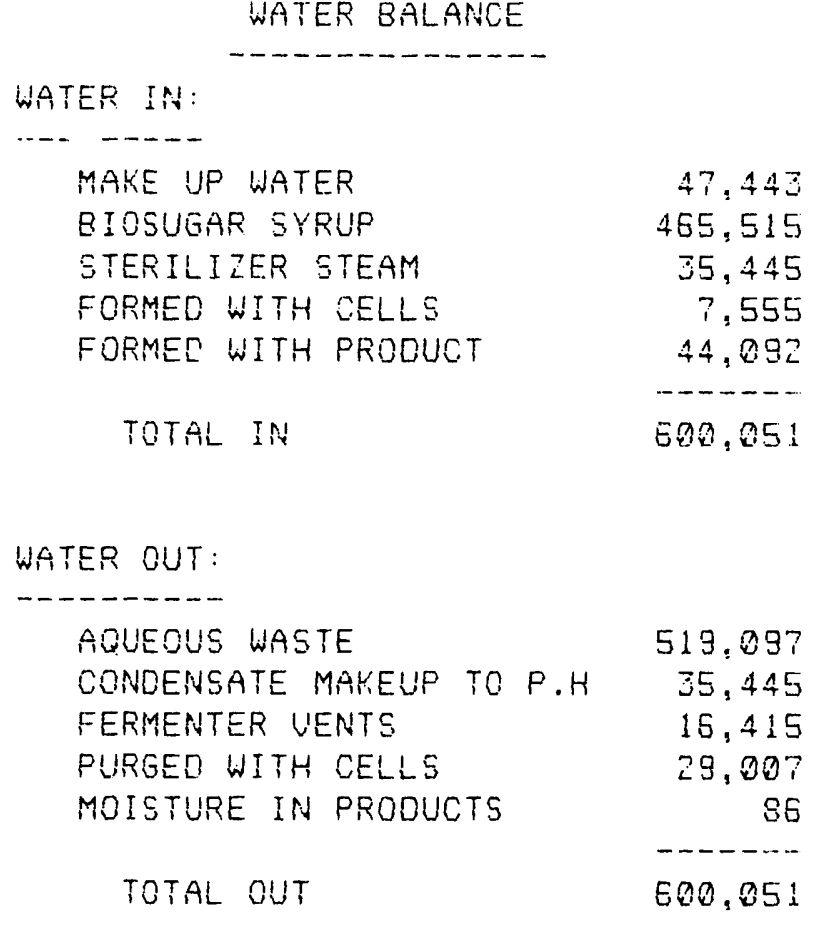


MULTISTAGE EXTRACTIUE FERMENTATION OF EUTANOL WITH RECOUERY OF RAFFINATE GOAL STOTOHIOHETRY

MATERIAL BALANOE FLOWGHEET

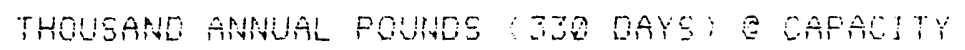

\begin{tabular}{|c|c|c|c|c|c|c|}
\hline & & $\begin{array}{c}1 \\
\cos \\
\cos \end{array}$ & $\begin{array}{c}2 \\
\text { AnHYYO } \\
\text { AMMONTA }\end{array}$ & $\begin{array}{c}3 \\
\text { MUTRIEHTS }\end{array}$ & $\begin{array}{ll}4 \\
\operatorname{Mix} \\
\text { WATE }\end{array}$ & $\begin{array}{c}5 \\
\text { MIXEO }\end{array}$ \\
\hline P & $\begin{array}{l}\text { STREAM } \\
\text { - }\end{array}$ & $\begin{array}{l}\text { GYRUP } \\
-\ldots\end{array}$ & $\begin{array}{l}\text { AMMONIA } \\
\text { AMAOH }\end{array}$ & $\ldots \ldots$ & WATER & MEUIOM \\
\hline i & CELLS - CHO & 0 & 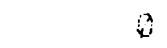 & $\therefore$ & 8 & 8 \\
\hline 9 & -5.42 & 0 & 3 & 0 & is & 3 \\
\hline D & MINESALS & 0 & 0 & 8 & 3 & $\theta$ \\
\hline 4 & -TOTAL & ? & 0 & a & $\hat{x}$ & a \\
\hline C & COMFOHENT $\# 1$ & 0 & 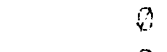 & 8 & 3 & 6 \\
\hline T & COMPONENT \#2 & $\therefore$ & 0 & $\hat{y}$ & 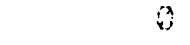 & 3 \\
\hline$\$ 2$ & COMPONENT \#Z & ( & 8 & 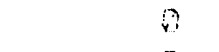 & 0 & a \\
\hline & OOMPONENT & B & 8 & 2 & 0 & $i$ \\
\hline & COMPOAENT \#Б & 3 & $\theta$ & 8 & 8 & 6 \\
\hline & OOHPONENE \#E & $a$ & 0 & 0 & 6 & 0 \\
\hline$\# 1$ & COMEONENT \#? & 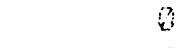 & 2 & 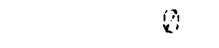 & 0 & 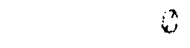 \\
\hline & OOMPONENT \#3 & 8 & 8 & 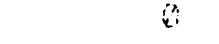 & 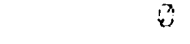 & $\Leftrightarrow$ \\
\hline & OOMPOHEM \# $\#$ & 8 & 8 & Q) & 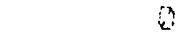 & 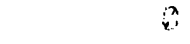 \\
\hline & COMOONEAT \#1O & 0 & 8 & ? & a & 8 \\
\hline & COMPONENT \#:1 & 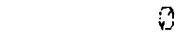 & 0 & i & 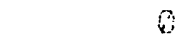 & ? \\
\hline & COMPONENT \#22 & $\theta$ & ? & 2 & 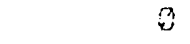 & $\because$ \\
\hline & COMEONENT \#15 & 0 & 8 & 0 & 0 & 0 \\
\hline$\#$ & COMPONELHT \#14 & 3 & 角 & 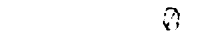 & i) & $\hat{3}$ \\
\hline & COMPONENT \#15 & $a$ & 8 & Q) & 3 & 8 \\
\hline & OOMPONEENT \# \#E & (8) & 0 & 8 & 0 & 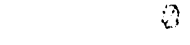 \\
\hline & COMFONENT \#1? & 3 & 2 & $\hat{\mathrm{i}}$ & 8 & 8 \\
\hline \# & COMPONENT \#18 & 8 & a & is & 6 & i) \\
\hline & COMFONENT \#19 & 0 & ?) & 8 & 0 & 8 \\
\hline & COMPONEA $\quad 420$ & 3 & $\hat{x}$ & $\hat{2}$ & $\theta$ & i) \\
\hline & FLUCOSE & 455.515 & 8 & $\theta$ & 8 & 455,515 \\
\hline & AMMOHEA & in & 1.127 & 6 & 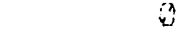 & a) \\
\hline & FHOSFHORIO ACIO & 0 & $\theta$ & 939 & D) & $3: 9$ \\
\hline & FOTAEEIUM CHLOPIDE & 8 & 8 & $\because 15$ & i & 715 \\
\hline & WINOR NUTRIENTS & 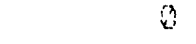 & ? & 327 & 8 & $\therefore 24$ \\
\hline & WATER & $455: 515$ & 8 & i) & 11,993 & $47 \div, 514$ \\
\hline & 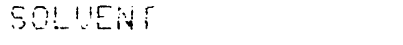 & 0 & 2 & 0 & is & 3 \\
\hline & CAREOA OIOKIDE & 8 & 8 & a & (9) & 0 \\
\hline & SVOGEN & 0 & 8 & $\hat{2}$ & 8 & 8 \\
\hline & AT TROGEN & 0 & 8 & 3 & $\hat{z}$ & $\hat{\imath}$ \\
\hline & HYSEOEEN & 8 & 0 & i) & $\hat{v}$ & ) \\
\hline & $\begin{array}{l}\text { GFANE TOTAL } \\
\text { CHEOK ON TOTAL }\end{array}$ & $931: 030$ & 1.127 & 1.979 & 11,358 & 945,025 \\
\hline & TEMPERATURE: C & 20 & 20 & 20 & 20 & 20 \\
\hline & PEEESURE， PSIA & 14.7 & 14.7 & 24.7 & 20.8 & 25.6 \\
\hline & STATE & 50 hit & 1.10410 & SOLIOS & LSOUID & $60 \mathrm{~L}$ \\
\hline
\end{tabular}


MUL TSTAGE EXTFAOTIUE FERMENTATION OF EUTANOL WITH FEOOUERY OF FAFFINATE GOAL BTOLHIOMETPY

MATERAA DALANCE FLOWGHEET

THOUSANE AHNUAL POUNOS 330 DAYS O CARACTTY

\begin{tabular}{|c|c|c|c|c|c|c|}
\hline & & $\begin{array}{c}5 \\
4 x^{5} 59 \\
505\end{array}$ & $\begin{array}{c}\bar{i} \\
\text { STERILE }\end{array}$ & $\begin{array}{c}8 \\
H K K^{2} \\
5 E 51\end{array}$ & $\begin{array}{c}9 \\
\text { COOLER }\end{array}$ & $\begin{array}{l}10 \\
A I R\end{array}$ \\
\hline 口 & STREAM & EFFLUENT & MEOIUM & EFFLUENT & EFFLUENT & TO FERM \\
\hline 5 & $C F:=-r 40$ & 0 & 0 & 0 & $a$ & a \\
\hline 5 & $-x+42$ & 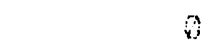 & 0 & 0 & 8 & $a$ \\
\hline $\mathrm{s}$ & MINERALS & 0 & 8 & 8 & 0 & 0 \\
\hline 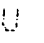 & -TSTAL & 0 & $a$ & 0 & 8 & 0 \\
\hline$\therefore$ & OOMEOMENT \#1 & 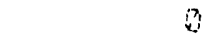 & ? & 0 & 8 & 0 \\
\hline T & COMPOHENT \#2 & $\theta$ & 0 & 8 & 0 & 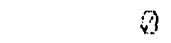 \\
\hline \# & CONEONENT \#Z & 8 & 0 & 0 & 8 & 0 \\
\hline & COMOONENT \#4 & $\Leftrightarrow$ & 0 & 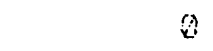 & 0 & 0 \\
\hline & OMMPONENT \#5 & 0 & 8 & $\theta$ & $\theta$ & $\theta$ \\
\hline & COMPOHENT \#E & $\theta$ & $B$ & D & 2 & 8 \\
\hline$\# 1$ & SOMFOHENT \#? & 8 & 8 & 8 & 8 & 0 \\
\hline & COMPONENT $\#$ & n & $n$ & 0 & 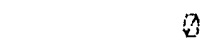 & 0 \\
\hline & COMPOHENT \#马 & in & 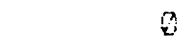 & 0 & 0 & 3 \\
\hline & COMPONENT \#10 & 0 & 0 & 0 & 2 & $\theta$ \\
\hline & COMFONENT \#:1 & 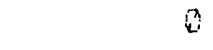 & B & $\theta$ & 0 & 0 \\
\hline & COMPOHENT \#12 & 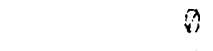 & 0 & $B$ & 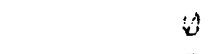 & 8 \\
\hline & COMFOHENT \#13 & in & 8 & 0 & 9 & 0 \\
\hline$\#$ & COMOONENA \#14 & $n$ & 0 & $n$ & 8 & $\theta$ \\
\hline & COMPONENT \#15 & 2 & 0 & $D$ & 0 & 0 \\
\hline & COMPONENT \#1E & 0 & $\theta$ & 0 & 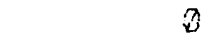 & 0 \\
\hline & OOMPONENT $\# 17$ & 8 & 0 & 0 & 8 & 0 \\
\hline \# & COMPONENT \#18 & $\therefore$ & 0 & 0 & 0 & 0 \\
\hline & OOMPONENT \#19 & 6 & 0 & (1) & 0 & 8 \\
\hline & COMPONENT $\# 20$ & 0 & 0 & a & 0 & 0 \\
\hline & GLUCOSE & 455.515 & 465,515 & 465.515 & 465,515 & D \\
\hline & AMMONIA & 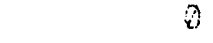 & 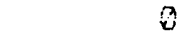 & 0 & 0 & 0 \\
\hline & PHOSPHORTO AOID & 333 & 939 & 939 & 933 & 8 \\
\hline & POTASSIUM OHLORIDE & 715 & 715 & 715 & 715 & 0 \\
\hline & MINOR NUTRIENTS & 324 & 324 & 324 & 324 & 0 \\
\hline & WATER & 477.514 & 512,953 & 512,359 & 512,353 & 0 \\
\hline & SOLVENT & 8 & 0 & 0 & 0 & 0 \\
\hline & CARBON DIOXIDE & $\theta$ & 0 & 0 & 0 & $\theta$ \\
\hline & OXYGEN & 0 & (3) & $\theta$ & 0 & 54,500 \\
\hline & NITROGEN, & 8 & $D$ & $a$ & 8 & 213.500 \\
\hline & HYOROGEN & 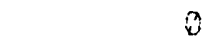 & 8 & 0 & 0 & 0 \\
\hline & - & $\ldots \ldots$ & $\ldots \ldots$ & $\ldots \ldots$ & $\ldots . . .-$ & $\ldots \ldots$ \\
\hline & $\begin{array}{l}\text { GPANE TOTAL } \\
\text { CHEOL ON TOTAL }\end{array}$ & 945.835 & 980.452 & 390,452 & 380,452 & 278,100 \\
\hline & TEMFERATURE， $C$ & 100 & 120 & 40 & 33 & 33 \\
\hline & FEESEURE， PSIA & 25.0 & 25.0 & 25.0 & 25.0 & 50.0 \\
\hline & STATE & SOL' & SOL N & $50 \mathrm{~L}$. & $S O L N N$ & GAS \\
\hline
\end{tabular}


MULTISTAGE EXTRACTIVE FERMENTATION OF DUTANOL WITH REQOUERY OF RAFFINATE GOAL STOICHIOMETRY

MATERIAL BALANCE FLOWSHEET

THOUSANO ARINUAL POUNDS (3ZO DAYE: G CAPACITY

\begin{tabular}{|c|c|c|c|c|c|c|}
\hline & STREAM & $\begin{array}{c}11 \\
\text { COMBINED } \\
\text { FEED }\end{array}$ & $\begin{array}{c}12 \\
\text { BEER } \\
\# 1\end{array}$ & $\begin{array}{c}15 \\
\text { BE도오 } \\
\text { \#2 }\end{array}$ & $\begin{array}{c}14 \\
\text { UENT } \\
\text { ORONTH }\end{array}$ & $\begin{array}{c}15 \\
\text { UENT } \\
\text { PROO }\end{array}$ \\
\hline p & - & $\ldots \ldots$ & ----- & $-\ldots \ldots \ldots$ & $\ldots \ldots$ & \\
\hline 只 & CELLS - CHO & $\theta$ & 11.503 & $304: 171$ & 8 & 8 \\
\hline 0 & $-N 4 k z$ & 0 & 1,051 & 27.810 & 0 & 0 \\
\hline D & -MINERALS & 0 & 324 & 8,500 & 0 & Q \\
\hline u & -TOTAL & 3 & 12,983 & 340,480 & 0 & 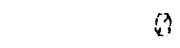 \\
\hline $\mathrm{C}$ & COMFONENT \#1 & 8 & 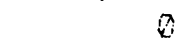 & 0 & 0 & $\theta$ \\
\hline$\tau^{\top}$ & COMEONENT \#2 & 0 & 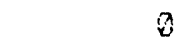 & 0 & 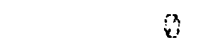 & $a$ \\
\hline$\# 2$ & COMFONENT \#3 & 0 & 0 & 0 & 6 & 0 \\
\hline & COMPONENT \#4 & 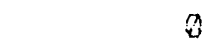 & 0 & a & 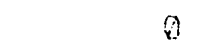 & $n$ \\
\hline & COMPONENT \#5 & 0 & 0 & 6 & 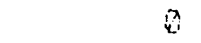 & 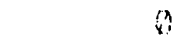 \\
\hline & COMPONENT \#E & 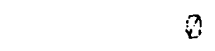 & 0 & 0 & 0 & 0 \\
\hline$\# 1$ & COMPONENT \#T & Q & 0 & $185: 731$ & 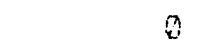 & 9 \\
\hline & COMPONENT \#S & 3 & $\theta$ & 0 & 0 & 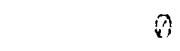 \\
\hline & COMPONENT \#B & 8 & $\theta$ & 8 & $\theta$ & $n$ \\
\hline & COMPONENT \#10 & 0 & 0 & 0 & 8 & 0 \\
\hline & COMFONENT \#11 & 0 & 8 & 8 & 0 & 0 \\
\hline & COMPONENT \#:Z & 0 & 0 & 0 & 0 & $Q$ \\
\hline & OOMPONENT \#13 & 0 & 0 & 0 & 0 & 0 \\
\hline \# & COMFONENAT \#14 & 0 & 0 & 3 & $\theta$ & a) \\
\hline & COMPONENT \#15 & 8 & 8 & 0 & 8 & 0 \\
\hline & COMPONENT \#1E & D & B & $\Leftrightarrow$ & 0 & 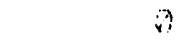 \\
\hline & COMPONENT \#1? & 8 & 0 & 0 & 0 & 8 \\
\hline \# & OOMOONENT \#18 & 0 & 0 & 0 & 0 & a \\
\hline & COMFONENT \#19 & 0 & Q & 0 & 0 & 0 \\
\hline & COMPONENT $\# 20$ & 0 & 0 & $a$ & 0 & 8 \\
\hline & GLUOOSE & 465,515 & 442.509 & 30,580 & a & 8 \\
\hline & AMMONIA & $i, 127$ & 8 & 0 & 8 & 8 \\
\hline & FHOSPHORIO ACIO & 939 & 939 & 24.607 & 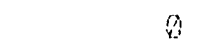 & 0 \\
\hline & POTAESIUM OHLORIOE & 715 & 715 & 18.737 & $a$ & 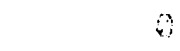 \\
\hline & MINOR HUTRIENTS & 32.4 & 0 & 0 & a & 8 \\
\hline & WATER & 512,959 & 511,856 & $14,358,560$ & 8,548 & 7.757 \\
\hline & SOLVENT & 0 & 8 & 270 & 0 & 0 \\
\hline & CAREOH DIOKIDE & $\mathrm{b}$ & $\theta$ & ? & $\because \% 000$ & 215.420 \\
\hline & OK:GEN & 64.500 & ? & 0 & 51.500 & 8 \\
\hline & WITROGEM & 213.500 & 6 & 0 & 213.584 & 9 \\
\hline & HYCFOGEN & 9 & $\theta$ & 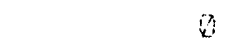 & 3 & 8 \\
\hline & (n) & $\cdots$ & $\cdots$ & $1 \cdots$ & (n) & 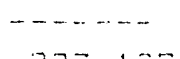 \\
\hline & $\begin{array}{l}\text { GOANE TOTAL } \\
\text { OHECL ON TOTAL }\end{array}$ & $1,253,679$ & $958: 315$ & 14.375 .022 & 230,855 & 273,197 \\
\hline & TEMPERATUPE：O & $j$ & 33 & $\because \because$ & 3 & 3 \\
\hline & FEESSURE, PSIA & $-\cdots$ & 44.7 & 44.7 & 14.7 & $14 . \because$ \\
\hline & STATE & - & SLURRY & SURR: & 645 & 6.95 \\
\hline
\end{tabular}


MULTISTAGE EXTRACTIVE FERMENTATION OF DUTANOL WITH RECOUEFY OF RAFFINATE GLAL STOICHIOMETRY

MATEFIAL BALANCE FLOWSHEET

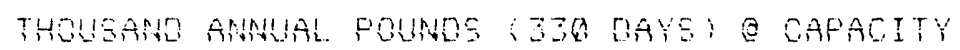

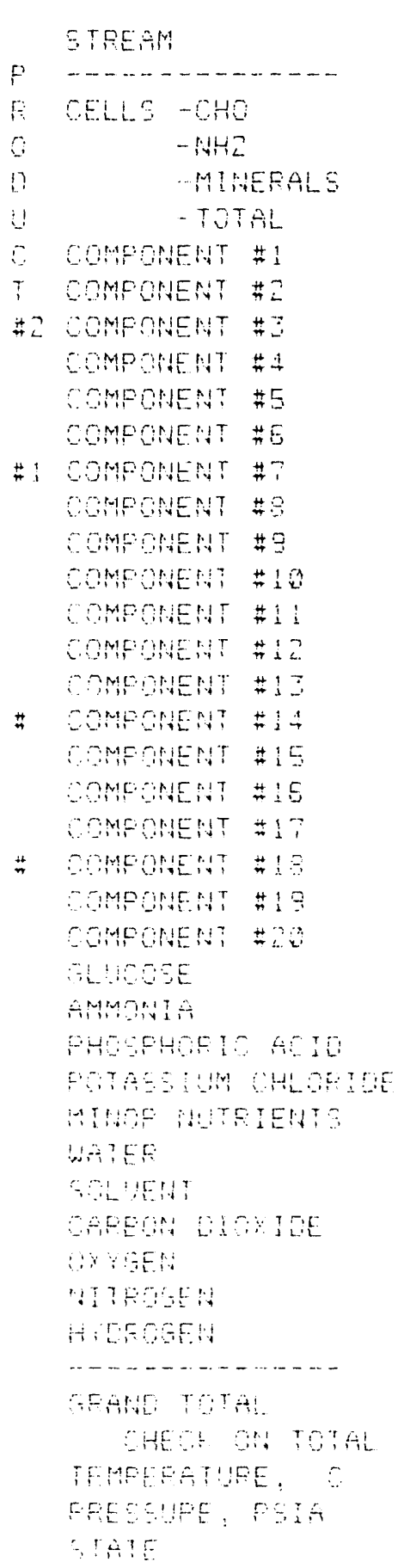

15

COMEIMED

o

0

COMPONEMT \#1

OOMEONENT \#2

GOMOOREN? \#?

OGMEGNENT \#?

Component \#

OANPOHEA

Gomponent

Compgrent \#12

CMCONENT

\#!

MPONENT \#1?

$\therefore$ LOSE

AnMngin

PHEFHETO AOIO

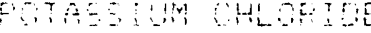

MTHEC UTEIEHT

G)

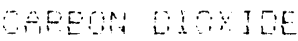

a)

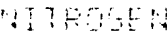

And TOT

Ge!

TPMEDATIPE.

$\therefore, 5$
VEN

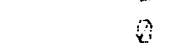

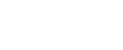

17
SOLUENT
FEEO

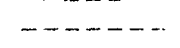

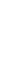

3

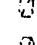

i)

in

8

$$
\text { a }
$$

$$
\text { ia }
$$$$
\text { (i) }
$$

8

0

8

a

8

\section{i)}

8

in

8

3

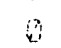

i

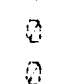

$a$

\section{2}

i

15. 415

232,428

5.580

21.603

?

512,043

-.........

$4,50,508$

3
$1+3$

26

i5

6,5

4.210 .558

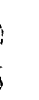

1. 10010
18

RAFF INATE

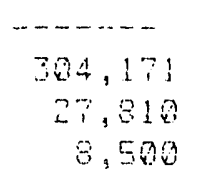

540,400

a

a

in

a

5,504

0

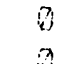

(2)

i

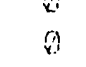

in

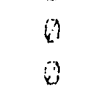

(3)

$$
\text { i) }
$$$$
\text { (n) }
$$$$
\begin{array}{r}
25 \\
25.598
\end{array}
$$$$
\begin{array}{r}
30.900 \\
0
\end{array}
$$$$
24.607
$$$$
19,75 \%
$$

19

RECYOLE
292.568
25,749
8,175

327,492

20
AOUEOUS

FURGE

11,003

11.503

1,861

324

12.983

0

2

(3)

0

(1)

214

$$
5.330
$$

39

8

is

0

$\theta$

$$
35.135
$$$$
0
$$$$
23.569
$$$$
18,022
$$$$
14,000,470
$$

614,360,47315,820,309

SLUEY

SLIPR! 
MULTISTAGE EXTRACTIUE FERMENTATION OF BUTANOL WITH RECOUEFY OF RAFFINATE GOAL STOICHIOMETRY

MATERIAL BALANCE FLOWSHEET

THOUSAND ANNUAL POUNDS 330 DAYS) E CAFACITY

\begin{tabular}{|c|c|c|c|c|c|c|}
\hline & & $\begin{array}{l}21 \\
\text { CELLS TO } \\
\text { DISPOSAL }\end{array}$ & $\begin{array}{c}22 \\
\text { FILTRATE } \\
\text { TO WASTE }\end{array}$ & $\begin{array}{c}23 \\
\text { EXTRACT }\end{array}$ & $\begin{array}{c}24 \\
\text { BEER \#1 } \\
\text { FEED }\end{array}$ & $\begin{array}{c}25 \\
\text { BEELR \#1 } \\
\text { MAKE }\end{array}$ \\
\hline$P$ & STREAM & - & $-\cdots-$ & -........ & $\ldots$ & $\ldots$ \\
\hline 只 & CELLS - CHO & 11,503 & 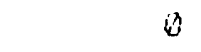 & 0 & 0 & 8 \\
\hline 0 & $-N_{H Z}$ & 1,061 & 0 & 0 & 0 & $\theta$ \\
\hline $\mathrm{D}$ & -MINERALS & 324 & 0 & $\theta$ & $\theta$ & 0 \\
\hline U & -TOTAL & 12,388 & 0 & 0 & 0 & 0 \\
\hline $\mathrm{C}$ & COMPONENT \#1 & 0 & 0 & 0 & 0 & 0 \\
\hline$T$ & COMPONENT \#Z & 0 & 0 & 0 & 0 & $\theta$ \\
\hline$\$ 2$ & COMPONENT \#Z & 0 & $\theta$ & 0 & 0 & 0 \\
\hline & COMPONENT \#4 & 0 & 0 & 0 & D & 0 \\
\hline & COMPONENT $\# 5$ & 0 & $\theta$ & 0 & (1) & 0 \\
\hline & COMPONENT \#5 & 0 & 0 & 0 & 0 & 0 \\
\hline$\# 1$ & COMPONENT \#? & 11 & 202 & 181,188 & 193,215 & 198,310 \\
\hline & COMPONENT \#B & 0 & 0 & 0 & 0 & 0 \\
\hline & COMFONENT $\# 9$ & 0 & $a$ & 0 & $\emptyset$ & 0 \\
\hline & COMPONENT \#10 & in & 0 & 0 & a & 0 \\
\hline & COMPONENT \#11 & 3 & 0 & $\theta$ & 0 & 3 \\
\hline & COMPONENT \#12 & 0 & 2 & 0 & 0 & 0 \\
\hline & COMFONENT \#13 & 0 & 0 & 0 & 0 & 0 \\
\hline \# & COMPONENT \#14 & 8 & 0 & 0 & 0 & 0 \\
\hline & COMPONENT \#15 & $\theta$ & 0 & 0 & 0 & 0 \\
\hline & COMPONENT \#1E & 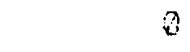 & 0 & 0 & D & $\theta$ \\
\hline & COMPONENT $\# 17$ & 0 & 0 & 0 & 0 & 0 \\
\hline$\#$ & COMPONENT \#18 & 0 & 8 & 0 & 0 & 0 \\
\hline & COMPOMENT \#19 & 0 & 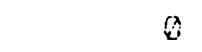 & 0 & 0 & 0 \\
\hline & COMPONENT \#2O & 8 & 0 & 6 & 0 & 0 \\
\hline & GLUCOSE & 74 & 1,322 & $\theta$ & $\theta$ & 0 \\
\hline & AMMONIA & 8 & 0 & 0 & 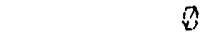 & 0 \\
\hline & PHOSFHORIO ACID & 50 & 889 & Q & $\theta$ & $\theta$ \\
\hline & POTASSTUM CHLORIDE & 38 & 577 & $\theta$ & $n$ & 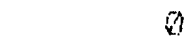 \\
\hline & MINOR NUTRIENTS & 0 & 0 & 8 & 0 & 0 \\
\hline & WPTER & 23,007 & 519,097 & 85 & 85 & 85 \\
\hline & SOLVENT & 1 & 10 & $4.310,557$ & $4,508,505$ & 198,310 \\
\hline & CAROON DIOXIOE & $\theta$ & $\theta$ & 0 & 0 & $a$ \\
\hline & OXYGEM & $\theta$ & 0 & 2 & 0 & 0 \\
\hline & NITROGEN & 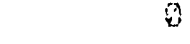 & 0 & 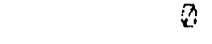 & $\theta$ & () \\
\hline & HYLROGEN & 0 & 0 & 0 & 0 & 0 \\
\hline & 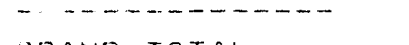 & - n & $\cdots$ & $\cdots$ & $\cdots \cdots-\infty$ & $\cdots \cdots$ \\
\hline & $\begin{array}{l}\text { GEANO TOTAL } \\
\text { CHECK ON TOTAL }\end{array}$ & 42,163 & 522,138 & $4,491,831$ & $4,707,808$ & 396,705 \\
\hline & TEMFERATURE, $Q$ & 33 & 33 & 33 & -- & 1.7 \\
\hline & PRESSURE, PSIA & 14.7 & 14.7 & 14.7 & $\cdots$ & 15.7 \\
\hline & OTATE & ELURRY & SOLN N & SOLN & $80 t n$ & SOL'N \\
\hline
\end{tabular}


MULTISTAGE EXTRACTIUE FERMENTATION OF DUTANOL WITH RECOUERY OF RAFFINATE GOAL STOICHIOMETRY

MATERIAL BALANCE FLOWSHEET

THOUSAND ANNUAL FOUNOS (3BR DAYS) O CAPACITY

\begin{tabular}{|c|c|c|c|c|c|c|}
\hline & & 25 & 2.7 & 28 & 29 & 30 \\
\hline & & BEER \#1 & BEER \#2 & BEER \#2 & $\operatorname{LBS} \# 1$ & L品 \#! \\
\hline & STREAM & TAILS & MAKE & TAILS & MAKE & TAILS \\
\hline$P$ & $\ldots-\cdots+\cdots-\cdots$ & $-\cdots--$ & $-\cdots--$ & $\ldots \ldots \ldots$ & $-\cdots \cdots$ & $\ldots \ldots$ \\
\hline$R$ & CELLS - CHO & 8 & 0 & 8 & 0 & 0 \\
\hline 0 & $-4+H_{2}$ & 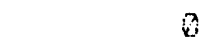 & 8 & 8 & 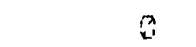 & 9 \\
\hline 0 & -MINERALS & $Q$ & 8 & 8 & 8 & 8 \\
\hline$!$ & -TOTAL & 8 & 0 & 0 & 8 & $B$ \\
\hline $\mathrm{C}$ & COMPONENT \#1 & 8 & $a$ & 8 & 8 & 0 \\
\hline$T$ & OOMPONENT \#Z & 0 & 0 & $\theta$ & 8 & 0 \\
\hline+2 & COMGONENT \#Z & $B$ & 0 & 8 & $\theta$ & $\theta$ \\
\hline & COMPONENT \#4 & $\theta$ & 0 & 9 & 9 & 0 \\
\hline & COMPONENT \#5 & 8 & 0 & 8 & 8 & 0 \\
\hline & COMPONENT \#S & 8 & 8 & 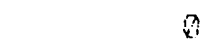 & a & $\theta$ \\
\hline$\# 1$ & COMPONENT \# & 906 & 180,232 & 18,028 & 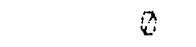 & 180,282 \\
\hline & COMPONENT \#B & R & 0 & 0 & $\theta$ & $\theta$ \\
\hline & COMPONENT \#G & 8 & 8 & 8 & 8 & 8 \\
\hline & COMPONENT \#1R & 9 & 8 & 3 & 0 & $a$ \\
\hline & COMPONENT \#11 & 0 & 8 & $\theta$ & 8 & 0 \\
\hline & OOMPONENT \#12 & 9 & $B$ & 0 & 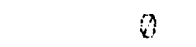 & $\theta$ \\
\hline & COMPONENT \#13 & 8 & 8 & 8 & i) & 0 \\
\hline$\#$ & COMPONENT \#14 & 8 & 7) & $\infty$ & ? & 0 \\
\hline & COMPONENT \#15 & 8 & 0 & 8 & 8 & $\theta$ \\
\hline & COMOONENT \#1E & g & $n$ & 8 & 9 & $\theta$ \\
\hline & GOMODNENT \#1? & 0 & 0 & 0 & 8 & 8 \\
\hline$\#$ & COMOONENT \#18 & $\theta$ & 8 & 8 & 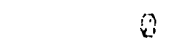 & 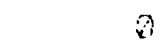 \\
\hline & CIMPONEMT \#19 & 8 & $\theta$ & 8 & 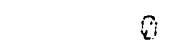 & 0 \\
\hline & COMPONENT \#2O & 8 & 0 & $\theta$ & 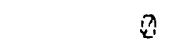 & $\theta$ \\
\hline & GLUCOSE & 8 & 8 & 8 & 8 & 0 \\
\hline & AMMONIA & 8 & $\theta$ & 3 & 8 & 0 \\
\hline & PHOSPLIORTO AOID & 8 & $a$ & 0 & 8 & n \\
\hline & POTAESIUM CHLOFIOE & Q & in & 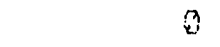 & $\theta$ & 3 \\
\hline & MINOF MUTRIENTS & 0 & 8 & 8 & 8 & 0 \\
\hline & WATER & 8 & 85 & 0 & 8 & 35 \\
\hline & GOLUENT & 4.310 .196 & 351 & 197.949 & 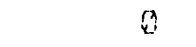 & 351 \\
\hline & CARBON OIOXIDE & $\theta$ & 3 & $\theta$ & 9 & 8 \\
\hline & GXYGEN & 0 & 8 & $\Leftrightarrow$ & $\theta$ & 8 \\
\hline & NITROGEN & 8 & 8 & 3 & 8 & 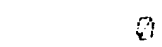 \\
\hline & HYOSOGEN & 8 & 0 & 0 & 0 & 0 \\
\hline & $\cdots \cdots-\cdots-\cdots-\cdots$ & $\cdots \cdots \cdots$ & $\cdots \cdots$ & $\cdots \cdots \cdots$ & $\cdots \cdots-\cdots$ & 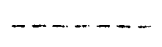 \\
\hline & $\begin{array}{l}\text { GRAND TOTAL } \\
\text { CHECL ON TOTAL }\end{array}$ & $4.311: 102$ & 180,728 & 215,977 & 0 & 180.728 \\
\hline & TEMEERATURE： C & 2.26 & 171 & 228 & $(273)$ & 175 \\
\hline & PRESBUPE, PSIA & 17.5 & 89.3 & 90.3 & 160.7 & 100.7 \\
\hline & BTATE & $5 O L N$ & SOLN & $501 . N$ & $60 L^{\prime} \mathrm{in}$ & SOL'N \\
\hline
\end{tabular}


MULISTAGE ETRACTIUE FERMENTATIOW OF BUIANOE

WITH REOQUERY OF RALFTHATF

GOAL GTOLBLIOMETRY

MATERIAL BALAACE FLOWSHEE I

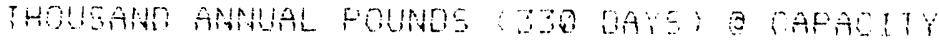

\begin{tabular}{|c|c|c|c|c|c|c|}
\hline & STREAM & WASE & TAILS & MSEE & $\begin{array}{l}230 \text { \#3 } \\
\text { TALS }\end{array}$ & $\begin{array}{l}\text { HES } \\
\text { MAKE }\end{array}$ \\
\hline$P$ & 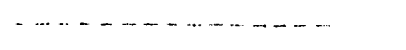 & $\ldots \ldots \ldots$ & $\cdots \cdots$ & $-\cdots$ & $\ldots \ldots$ & $\ldots \ldots$ \\
\hline 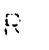 & CELLO -OHO & 0 & a & 8 & $x$ & 8 \\
\hline 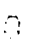 & -1428 & 3 & 8 & 2 & 3 & $a$ \\
\hline 0 & WINERALS & 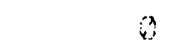 & in & 6 & $\pi$ & 8 \\
\hline !: & $-3 \% 13$ & 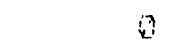 & $x$ & $a$ & 3 & ค \\
\hline 8 & GMNONENT \# & 3 & $\lambda$ & n & 8 & 6 \\
\hline$T$ & OMEOAENT & i) & 8 & ) & $\theta$ & 8 \\
\hline $4 ?$ & MANOMENT & 8 & 0 & $a$ & $\theta$ & 8 \\
\hline & OMPONE:IT $\# 4$ & a & i & 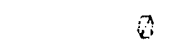 & 8 & 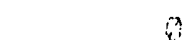 \\
\hline & GMEGEUT \#5: & a & 8 & 3 & $\pi$ & 3 \\
\hline & OMFOHENT \#E & 2 & 8 & 0 & $a$ & Q) \\
\hline 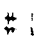 & OMFONEAT \#? & $\lambda$ & 0 & 8 & $n$ & $\theta$ \\
\hline & Ginernent $\#$ & ? & 8 & 8 & $\hat{x}$ & 8 \\
\hline & MOAOENT \# & $Q$ & 8 & 3 & 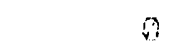 & i \\
\hline & Coméngnt \# & 2 & ?. & i & 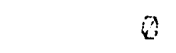 & a) \\
\hline & CONCONEAT \#II & $a$ & 0 & 9 & 9 & 8 \\
\hline & OHWOHE: & $a$ & 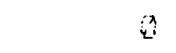 & a & 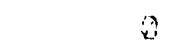 & 9 \\
\hline & MWronent \# & $\Leftrightarrow$ & 0 & $\theta$ & ? & 8 \\
\hline \# & OMPOHENT \#14 & 3 & 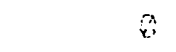 & 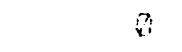 & 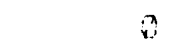 & 0 \\
\hline & OMFGHET \#15 & $a$ & 3 & $\theta$ & i) & 8 \\
\hline & OMFONE:H & i & 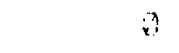 & 8 & $\therefore$ & 3 \\
\hline & GMPOHEAT $\# 1 ?$ & $\mathrm{a}$ & $\theta$ & $\therefore$ & i) & 8 \\
\hline it & OGMOUENT \#18 & $\ddot{x}$ & 8 & $x$ & ⿵ & 8 \\
\hline & OAPOHENT $\# 19$ & $\therefore$ & $n$ & 4 & 0 & $Q$ \\
\hline & GWFOHEH 427 & $\therefore$ & $\hat{\mathrm{k}}$ & i & $\hat{i}$ & 6 \\
\hline & $\because \because O E E$ & $\ddot{a}$ & 3 & a & $i$ & 8 \\
\hline & AMMlORIA & ? & ? & $\theta$ & $\hat{x}$ & 8 \\
\hline & FUAFHATE ATIO & $\therefore$ & 0 & i & $a$ & Q \\
\hline & POTAESIM OHUPUE & 6 & 3 & 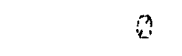 & 3 & n \\
\hline & MHOS HUTETENT & Q & 8 & 0 & 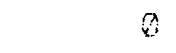 & 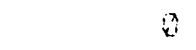 \\
\hline & WATE & ? & 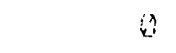 & 3 & 3 & $\theta$ \\
\hline & SEYUET & 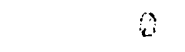 & $\hat{3}$ & 0 & 8 & i \\
\hline & GAOON LOUIDE & 2 & $?$ & a & 8 & 0 \\
\hline & SUYSOH & $\hat{3}$ & a & $\mathrm{a}$ & 0 & a \\
\hline & 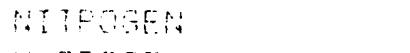 & 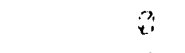 & 3 & ? & 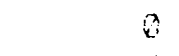 & a \\
\hline & H:C5OGE? & in & 0 & 9 & A & i \\
\hline & $-\ldots \ldots$ & - & $\cdots$ & $\cdots \cdots$ & $\cdots \cdots \cdots$ & $\cdots \cdots+\cdots$ \\
\hline & $\begin{array}{l}\text { GFAND COTAL } \\
\text { OHEOF OW TATAL }\end{array}$ & $Q$ & Q & 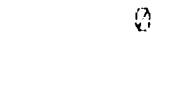 & 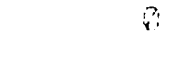 & is \\
\hline & THEEFATIRE, S & 2.75 & 120 & $423:$ & $12 x$ & 237 \\
\hline & PPESGLEE FOL & .3 & .9 & .6 & .3 & .0 \\
\hline & $\because A T E$ & 501.14 & $56 L^{\prime} n$ & $39 \mathrm{~L} N$ & $502 \cdot M$ & $501 \mathrm{~K}$ \\
\hline
\end{tabular}


MULTISTAEE EXTRACTIUE FERMENTATION OF EUTANOL WITH FECOVERY OF RAFFINATE GOAL STOICHIOMETRY

MATERIAL BALANCE FLOWSHELT

THOUSANO ANHUAL FOUNGE S IJR DAYS: G CAPAEIT'

\begin{tabular}{|c|c|c|c|}
\hline \multicolumn{4}{|c|}{ STREAM } \\
\hline Fi & CEUS & $-c 40$ & \\
\hline & & - & \\
\hline [ & & -MINE & ERALS \\
\hline !) & & - TOTA & \\
\hline 6 & Сомео & NENT & $\# !$ \\
\hline T & COMFO & NENAT & $\# 2$ \\
\hline 䩕 & Co IFO & NESET t & $\# \vec{Z}$ \\
\hline & COMPO & NENT & $\# 4$ \\
\hline & COMPO & NENT & $\# 5$ \\
\hline & COMPOI & NENT & $\# E$ \\
\hline$\# 1$ & COMFO & NENT & $\# ?$ \\
\hline & COMPO & NENTT & $\# 8$ \\
\hline & COMFOI & NENT & $\# 9$ \\
\hline & СOMPOH & NERIT \# & $\# 10$ \\
\hline & COMPO & NENT \# & $\# 11$ \\
\hline & COMPOH & NENT & $\# 12$ \\
\hline & COMPO & NENT & $\# 13$ \\
\hline 世 & COMPO & NEST \# & $\# 14$ \\
\hline & COMFO & NENT \# & $\# 15$ \\
\hline & COMPO & NENAT H & $\# 15$ \\
\hline & COMPO & NEYIT & $\# 17$ \\
\hline tt & COMPO & NENT & $\# 13$ \\
\hline & COMPO & NEAIT & $\# 19$ \\
\hline & COMPOI & NENT & $\# 20$ \\
\hline & GLUCO & & \\
\hline & AMMON & & \\
\hline
\end{tabular}

FHOSPHORIC ACIO POTASSIUM CHLORIOE MINOR NUTRIENTS WATER EOLUENT CAREON DIOKIDE OYYGEN MITROGEN HYOROGEN GRAND TOTAL CHECK ON TOTAL TEMPERATURE, C PRESSUEE, FSIA STATE

\begin{tabular}{|c|c|c|c|c|}
\hline $\begin{array}{c}3 E \\
\text { HES } 1 \\
\text { TAILS }\end{array}$ & $\begin{array}{l}\text { ST } \\
\text { BYFRODUCT } \\
\text { FEED }\end{array}$ & $\begin{array}{c}\text { SO } \\
\text { RAFF \# } \\
\text { FEED }\end{array}$ & $\begin{array}{c}33 \\
\text { FAFF \#1 } \\
\text { MAKE }\end{array}$ & $\begin{array}{l}40 \\
\text { CAEF \# } \\
\text { TALLS }\end{array}$ \\
\hline$\cdots$ & n & 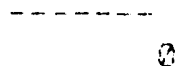 & 0 & $\cdots$ \\
\hline 0 & 0 & 0 & 0 & 0 \\
\hline 8 & 0 & (i) & a & 8 \\
\hline 0 & 0 & is & i) & $i^{2}$ \\
\hline 0 & 3 & 0 & 0 & 4 \\
\hline 3 & 준 & 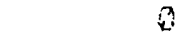 & a & 0 \\
\hline 0 & 0 & 8 & a & 8 \\
\hline 0 & $\theta$ & 0 & i) & $\theta$ \\
\hline$\theta$ & 0 & $\pi$ & B & 6 \\
\hline 0 & 8 & 0 & $\theta$ & 8 \\
\hline 0 & 0 & 0 & D & $\Leftrightarrow$ \\
\hline 0 & 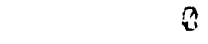 & i & a & 0 \\
\hline 2 & 0 & (2) & 6 & 0 \\
\hline 0 & 0 & 6 & 0 & 0 \\
\hline 0 & 8 & 8 & 8 & 0 \\
\hline 0 & 3 & 0 & 8 & 6 \\
\hline 8 & 0 & (3) & ह & 0 \\
\hline 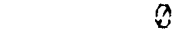 & $\theta$ & 0 & 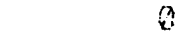 & 0 \\
\hline$\pi$ & 2 & 6 & 8 & 0 \\
\hline 0 & 3 & 0 & 0 & 6) \\
\hline 0 & 0 & a & 0 & 2 \\
\hline 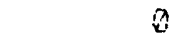 & 0 & 0 & D & $\theta$ \\
\hline 0 & 0 & (a) & 8 & $\theta$ \\
\hline iv & 6 & 0 & $D$ & $\theta$ \\
\hline$\theta$ & 0 & 8 & 0 & 8 \\
\hline 0 & 0 & 3 & 0 & 0 \\
\hline$\theta$ & 0 & 0 & 0 & in \\
\hline 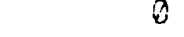 & 0 & 0 & 0 & 8 \\
\hline 0 & $\theta$ & 0 & 0 & 6 \\
\hline 0 & 0 & 0 & 6 & 0 \\
\hline 0 & 0 & 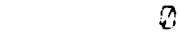 & $\theta$ & 0 \\
\hline 0 & 0 & 3 & 0 & 6 \\
\hline 0 & $\theta$ & 6 & 0 & a \\
\hline 0 & $\theta$ & 0 & 0 & 0 \\
\hline 0 & a & 0 & 0 & (3) \\
\hline- & 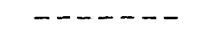 & $\cdots \cdots$ & $\cdots \cdots$ & --1 \\
\hline 0 & 0 & 6 & 2 & 6 \\
\hline 220 & 33 & 0 & 0 & $\hat{\varepsilon}$ \\
\hline .0 & 14.7 & - & 0.0 & 0.0 \\
\hline $5 O L \cdot N$ & SOL N & $S O L ' N$ & $50 \mathrm{~L} \cdot 1 !$ & SOL N \\
\hline
\end{tabular}




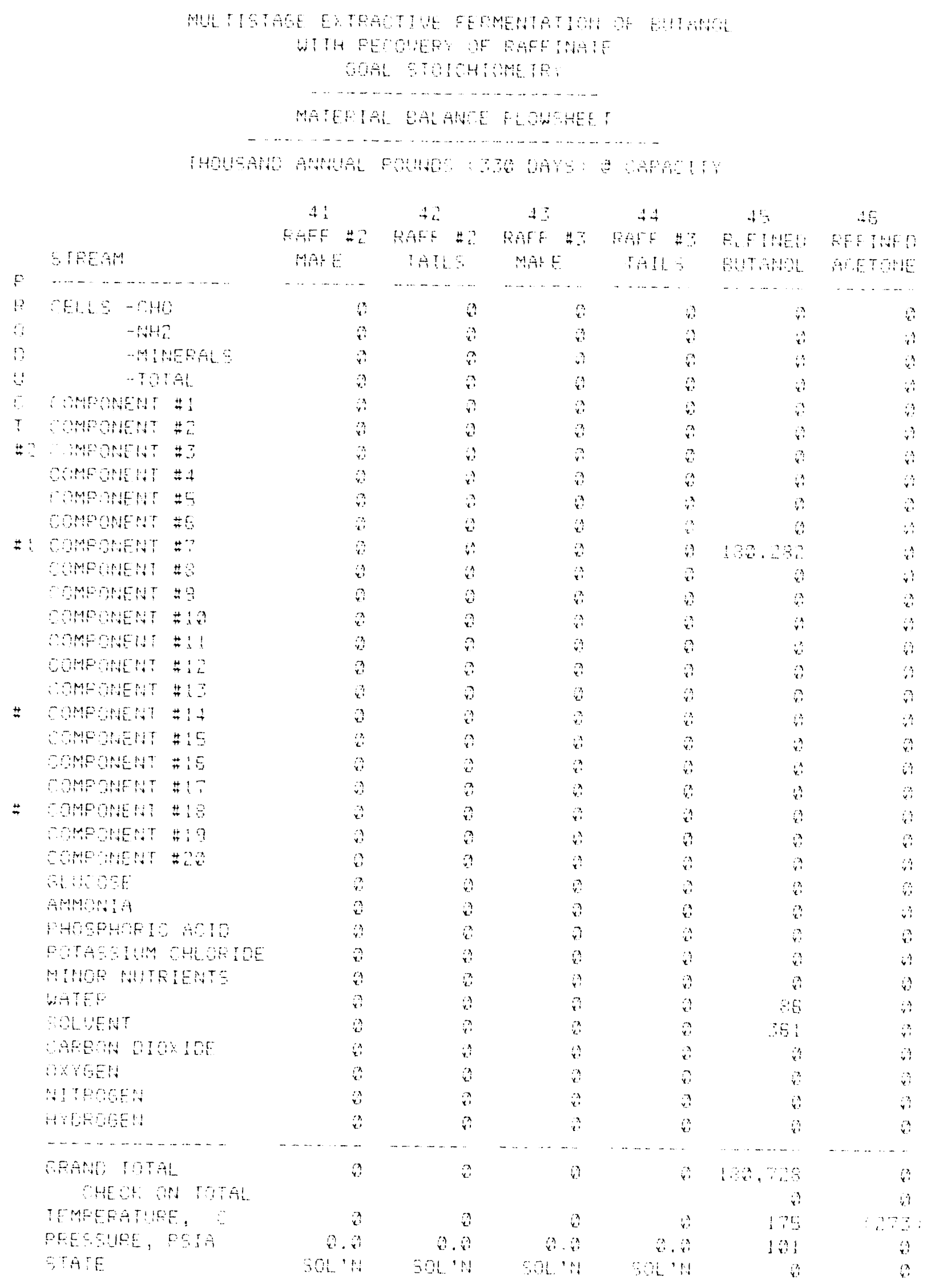




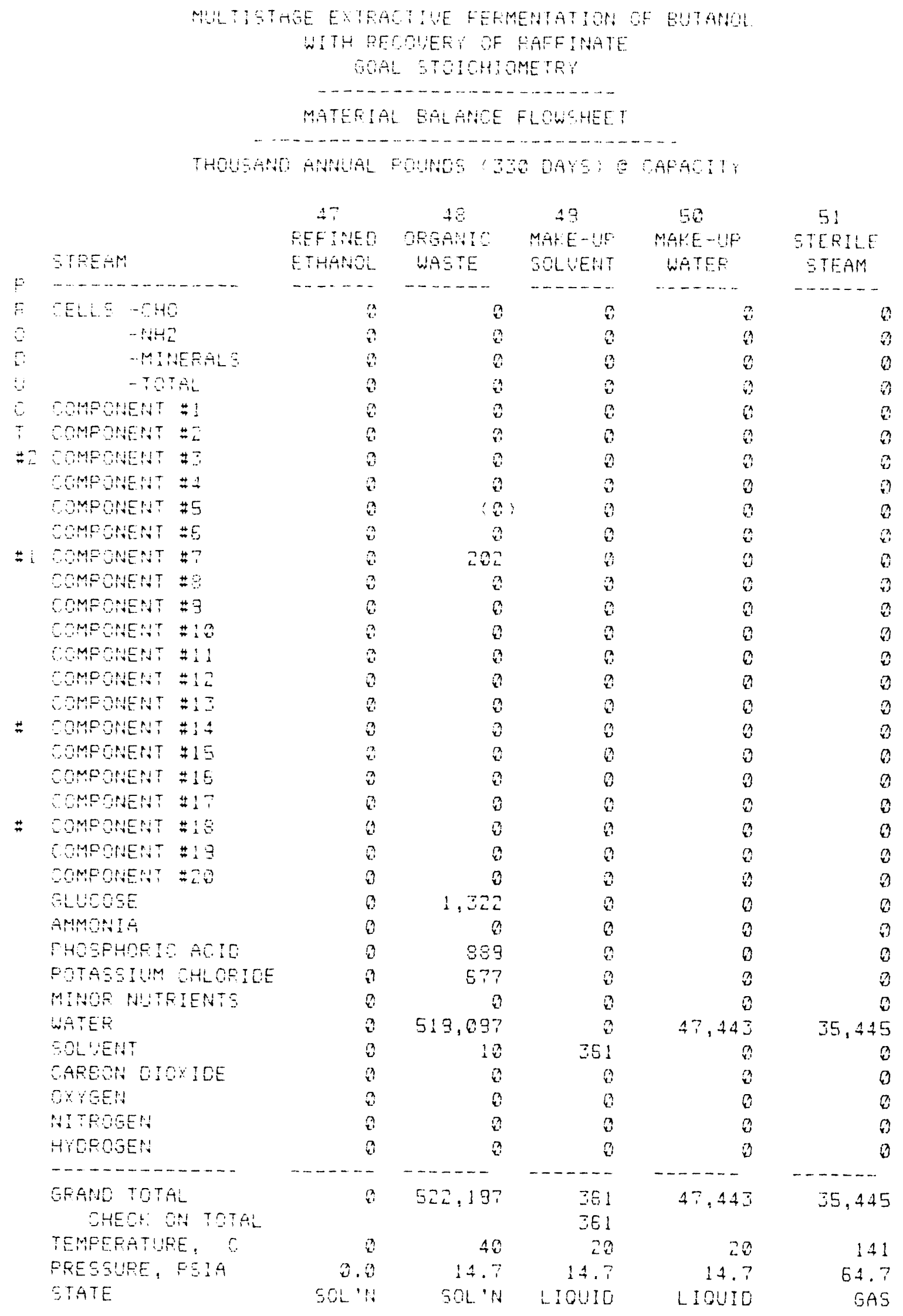


MULTISTAGE EXTRACTIUE FERMEMTATION OF EUTANGL WITH RECQUER: OF RAFFINATE

GOAL GTOIOHIOMETRY

MATERIAL BALANOE FLOWGHET

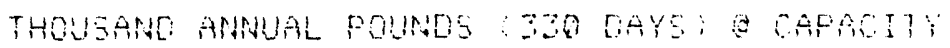

\begin{tabular}{|c|c|c|c|c|c|c|}
\hline & & 52 & 53 & 54 & & \\
\hline & & FEFN'T & OISTILL. & Contr. M! & MEDI!:M & EEPMP TP \\
\hline & SPREAM & STEAM & STEAM & P'WR HOE & OOOL WTF & SOTL WTE \\
\hline$P$ & - & $-\cdots \cdots$ & $\ldots \ldots$ & $\cdots \ldots$ & $-\cdots \cdots+$ & $\ldots \ldots-\cdots$ \\
\hline P & CELO -CHI & 8 & $\hat{i}$ & 6 & 3 & 8 \\
\hline 9 & $-N 4 Z$ & $\hat{2}$ & $a$ & $\hat{n}$ & ) & (3) \\
\hline$\Gamma$ & -MINESALS & 8 & $\vec{x}$ & 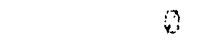 & $\therefore$ & 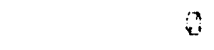 \\
\hline$y$ & -TOTAL & 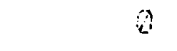 & 3 & 8 & $\therefore$ & $\therefore$ \\
\hline$\therefore$ & GOMEONENT $\#$ & 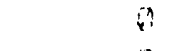 & 4 & 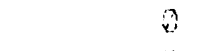 & is & 8 \\
\hline$T$ & EOMOGNENT $\# 2$ & $\therefore$ & $\theta$ & $\therefore$ & 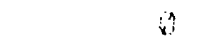 & a \\
\hline$\because 2$ & GOMFOWENT & 8 & A & $a$ & 8 & $\hat{i}$ \\
\hline & OOMFOHENT \#4 & 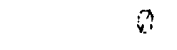 & 8 & 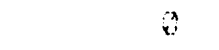 & () & 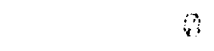 \\
\hline & COMPONENT \#S & $\theta$ & $a$ & $\hat{x}$ & 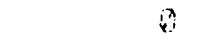 & 3 \\
\hline & BOMPONENT \#E & 8 & 3 & $\therefore$ & $\theta$ & 6 \\
\hline$\#$ & COMFONENT \#? & $\theta$ & $\therefore$ & 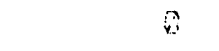 & 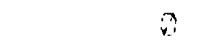 & 3 \\
\hline & COMEOHENT $\#$ & $\theta$ & $\hat{i}$ & 8 & $\therefore$ & $A$ \\
\hline & COMOONEAT $\# 3$ & 8 & 8 & 4 & ? & 3 \\
\hline & GMPONEWT \#20 & $\hat{x}$ & 8 & 2 & 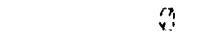 & $\hat{3}$ \\
\hline & MMPONEMT & 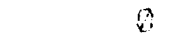 & is & $a$ & $\because$ & i \\
\hline & BNFOHENT \#12 & $\theta$ & $\hat{i}$ & $\therefore$ & $\therefore$ & 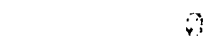 \\
\hline & GOMFONEN: $\$ 1 \vdots$ & ? & 0 & 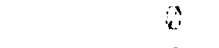 & $\therefore$ & 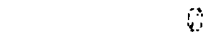 \\
\hline 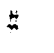 & GMongent $=14$ & 3 & 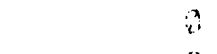 & i & $\therefore$ & $\rightarrow$ \\
\hline & COMPONENT $=15$ & i & 8 & $\therefore$ & 8 & 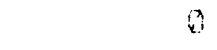 \\
\hline & E GMPOMEAT \#1E & 0 & i & $\therefore$ & $\hat{v}$ & $\hat{3}$ \\
\hline & TOMFONENA \#17 & $\mathrm{A}$ & i) & $\hat{2}$ & ? & 3 \\
\hline$\#$ & GMEONENT \#1B & 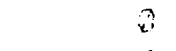 & $a$ & (1) & $\hat{A}$ & 3 \\
\hline & CMOMnEMT \#19 & 4 & 3. & 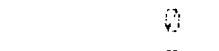 & $\therefore$ & 9 \\
\hline & OHPOHEM \#Z & $\hat{A}$ & $\therefore$ & $a$ & $\therefore$ & 3 \\
\hline & $\therefore 10.95$ & 0 & 3 & 0 & 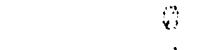 & 8 \\
\hline & AMtMrigt & $\theta$ & 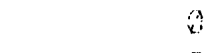 & ) & 4 & ; \\
\hline & PHSPHOFIS ACO & $\therefore$ & a & $\hat{i}$ & 3 & 3 \\
\hline & OTTAES OU OHOFSOE & 8 & 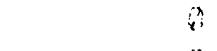 & i & $\therefore$ & $\therefore$ \\
\hline & 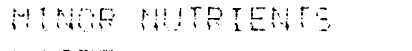 & 3 & $\theta$ & $\hat{3}$ & $\therefore$ & $\therefore$ \\
\hline & 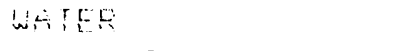 & $\therefore$ & $6+1,49$ & 35,45 & 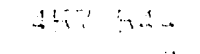 & $9.916, \%$ \\
\hline & $\because \because 18.1 T$ & $a$ & $a$ & $a$ & $\therefore$ & $\hat{x}$ \\
\hline & 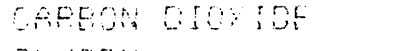 & 3 & is & $\therefore$ & $\therefore$ & $\therefore$ \\
\hline & $5 \times \mathrm{SOH}$ & $\therefore$ & 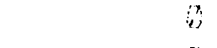 & $\therefore$ & $\therefore$ & 3 \\
\hline & ATSOSE & 9 & i) & $\therefore$ & $\therefore$ & $\because$ \\
\hline & HOPOGEA & O & $(i)$ & $\because$ & $\dot{4}$ & 3 \\
\hline & $\cdots$ & $\cdots \cdots$ & $-\cdots \cdots \cdots$ & $\cdots \cdots$ & $\ldots \cdots$ & $\cdots \ldots \ldots$ \\
\hline & 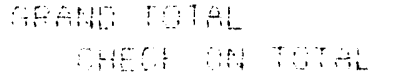 & $\because$ & $241,4: 5$ & $\therefore 5,46$ & $\therefore 47, \angle, A$ & 9.969 \\
\hline & 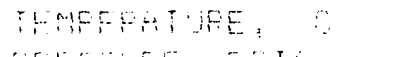 & 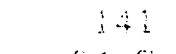 & 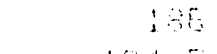 & $1:$ & $\ddots$ & \\
\hline & 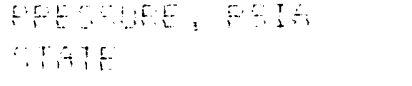 & $\begin{aligned} 62 \% \\
965\end{aligned}$ & $\begin{array}{r}164 \\
649\end{array}$ & 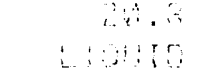 & 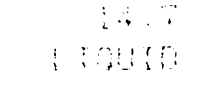 & $\begin{array}{r}14 \\
0 \quad 10\end{array}$ \\
\hline
\end{tabular}


MUL TISTAGE EXTFACTIUE FERMENTATION UE BUTANOL WITH PEOOVEFY OF DAFFINATE GOAL STOICHIOMETRY

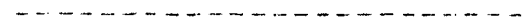

MATEFIAL EALANCE FLOWSHEET

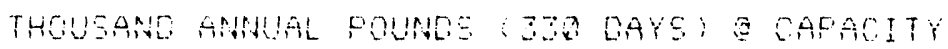

BTPEAM

57

UISIILL. 600 wip

F $\ldots \ldots$

OEL1 5 - -

- 142

DVIVESHLS

-TOTAL

CMmoremt $\#$

MOMERENT \#

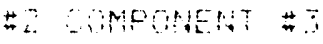

COMFPRENT \#4

COMfOMEMt

COMEORENT \#5

\# SOMPONERT \#?

COMFONEWT \#

Omporet.; \#

Somponent \#is

Gomponets \#:

COHPORENT \#12

COMPGRENT \#13

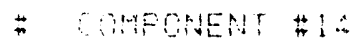

GOMEOMENT \#15

Componellt

CONDORERT \#?

* Componert

COMOOHER

OMPOine?t

GLDOUE

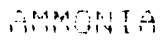

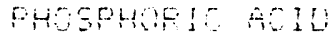

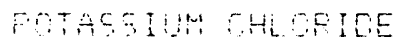

MIPG MUTSEL?

WATER

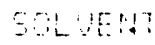

CAPEOH LIOY IOE

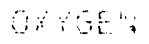

HTFOE:

HeFegen
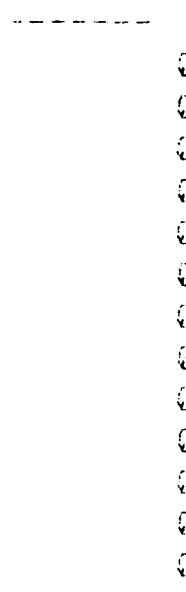

(2.....................

GESHA TATHL

CHES OHI TOTAL

TEMFEFATPE

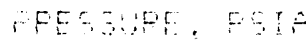

$\therefore+T_{1}$

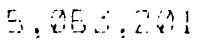

(1)

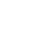

(a)

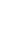


M LE. MOLES YEAR

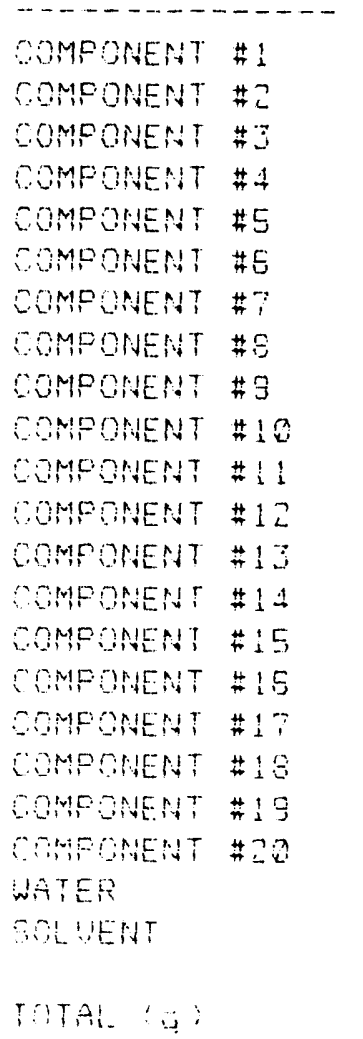

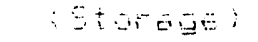

WOOR PEFS 404 .

$\therefore+\theta$ G

MPCHE PEESE 1204

En : U. COASAnt

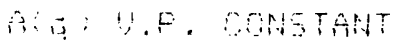

TEMEEFAT IFE

PRES IFE WHHE

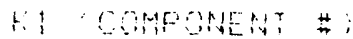

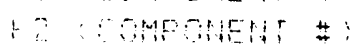

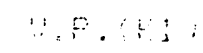

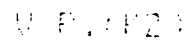

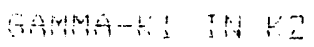

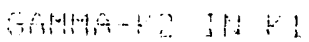

A. Plitis

AU POLOMH ALFHA

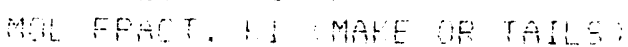

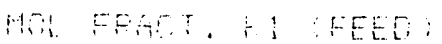

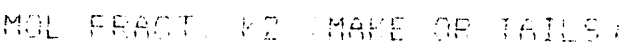

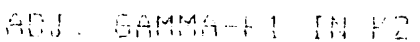

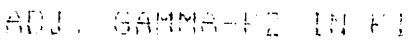

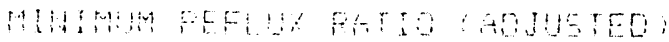

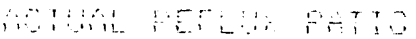

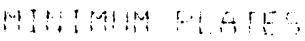

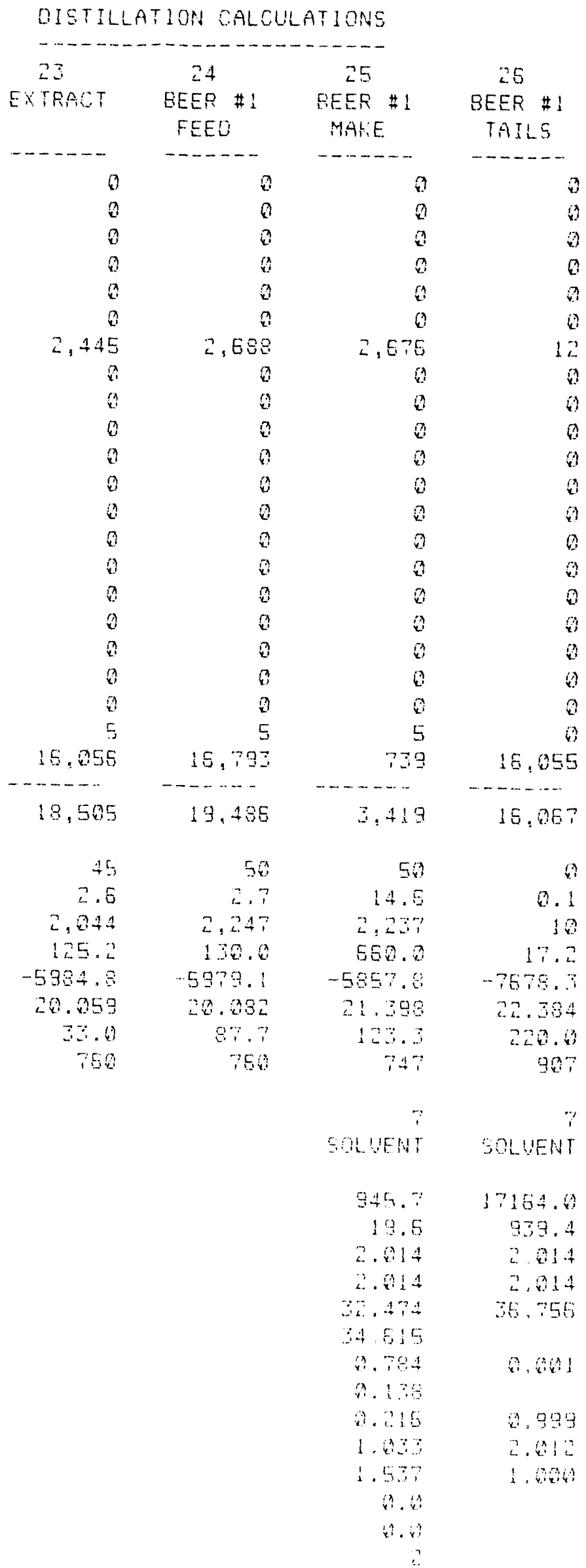




\begin{tabular}{|c|c|c|c|}
\hline DISTIL & TION CALO & ATIOAS & \\
\hline 23 & 24 & 25 & 25 \\
\hline EXTRACT & EEER \#1 & BEER \#1 & SEER \# 1 \\
\hline MLD. 1OLES:YEAD & FEED & MALE & TAILS \\
\hline$\ldots-\ldots-n-\ldots-n$ & $---\ldots$ & $\ldots \ldots-\ldots$ & $-\ldots-\ldots$ \\
\hline ACTUAL PLATES & & 11 & \\
\hline FRESSURE MI HE (REUISED) & & 864 & \\
\hline TEMFERATIIRE C (REUISED) & & 127 & \\
\hline AUERAGE NOLECULAR WEIGHT & & 115.03 & 258.32 \\
\hline GAS DENSITY - LBICF & & 0.2505 & $0.493 \%$ \\
\hline CROSS SECTIONAL AREA - SO FT & & 23.2 & \\
\hline COLLIMN HEIGHT - FT & & 31.1 & \\
\hline COLWHAN DIAMETER & & 5.4 & \\
\hline ㄴ MPPY) & & $138: 310$ & \\
\hline 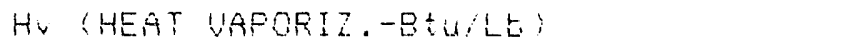 & & 2.15 .2 & 215.0 \\
\hline OR (MEAT OAPACITY - EAGLEG) & & 0.580 & 0.500 \\
\hline HEAT LOAD - MM EtUiHi & & 11.855 & 83.143 \\
\hline COMDENSER COOLING WATER - GPM $15 \mathrm{C}$ UT' & & 879 & \\
\hline CALANOEIA STEAM - MPPH ( 150 PSIG) & & & 37.02 \\
\hline COLUMN COST - \$1800 SOBS MPC - EARE EQU & ENT & & \\
\hline - ALL CARBON STEEL & & $\$ E D .1$ & \\
\hline - O.SW $3045.5 . T P A Y S$ & & \$5E.1 & \\
\hline - ALL 304 STAIHLESE STEEL & & $\$ 97.3$ & \\
\hline - ALL JOAL STAIMLESS GTEEL & & \pm 107.0 & \\
\hline - ALL IIS STAIMLESS ETEEL & & $\$ 131.4$ & \\
\hline CONDENSER OR CALANDFIA SURFACE - SO FT & & 847 & $4: 013$ \\
\hline COND. OR CALAND, COST - \$1000 J08E MFO & AFE EOU & & \\
\hline - CAPEOW STEEL & & $\$ 36.8$ & +145.1 \\
\hline -304 STAINLESS STEEL & & $\$ 51.5$ & 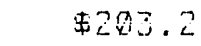 \\
\hline - J1E STAINLESE STEEL & & $\$ 55.1$ & $\$ 2.7 .7$ \\
\hline -MONEL & & $\$ 71.7$ & $\$ 285.0$ \\
\hline SUETSTAL & & & $2.155,551$ \\
\hline SUETOTAL & & & $2.155,898$ \\
\hline SUETOTAL & & & $2,155,938$ \\
\hline MINIMUM REFLUX RATIO & & $(6.4)$ & \\
\hline CH EUETOTAL \#1 & & 99,155 & 453 \\
\hline CA SUETOTAL \#2 & & 0 & \\
\hline ON SUBTOTAL \#3 & & 99,241 & $2,155,898$ \\
\hline SII CHECK & & 0.508 & 0.508 \\
\hline HW SUETOTAL \#1 & & $42, E 37$ & 195 \\
\hline H. SUETOTAL \#2 & & 0 & \\
\hline H. EUETTOTAL \#Z & & 42,720 & 926,592 \\
\hline H. CHECL & & 215.2. & 215.8 \\
\hline MING PLATESBNORMAL； & & & \\
\hline COL.COST-EIS MORMAL & & & \\
\hline OOL.OOST-SIS NORMAL & & & \\
\hline MIN.REFLUX NORMAL： & & & \\
\hline O. A. AFEAMORMAL： & & & \\
\hline HEAT LGADENOFHAL: & & & \\
\hline MHOAL COST NOPMA & & & \\
\hline
\end{tabular}


M LE. MOLES:YEAR

COMPONENT $\# 1$
COMFONENT $\# 2$
COMPONENT $\# 3$
COMPONENT $\# 4$
COMPONENT $\# 5$
COMPONENT $\#$ G
COMPONENT $\# 7$
COMPONENT $\# 3$
COMPONENT $\# 9$
COMPONENT $\# 10$
COMPONENT $\# 11$
COMPONENT $\# 22$
COMPONENT $\# 13$
COMPONENT $\# 14$
COMPONENT $\# 15$
COMPONENT $\# 16$
COMEONENT $\# 17$
COMPONENT $\# 18$
COMFONENT $\# 13$
COMPONENT $\# 26$
WATER
SOLUENT

TOTAL \&

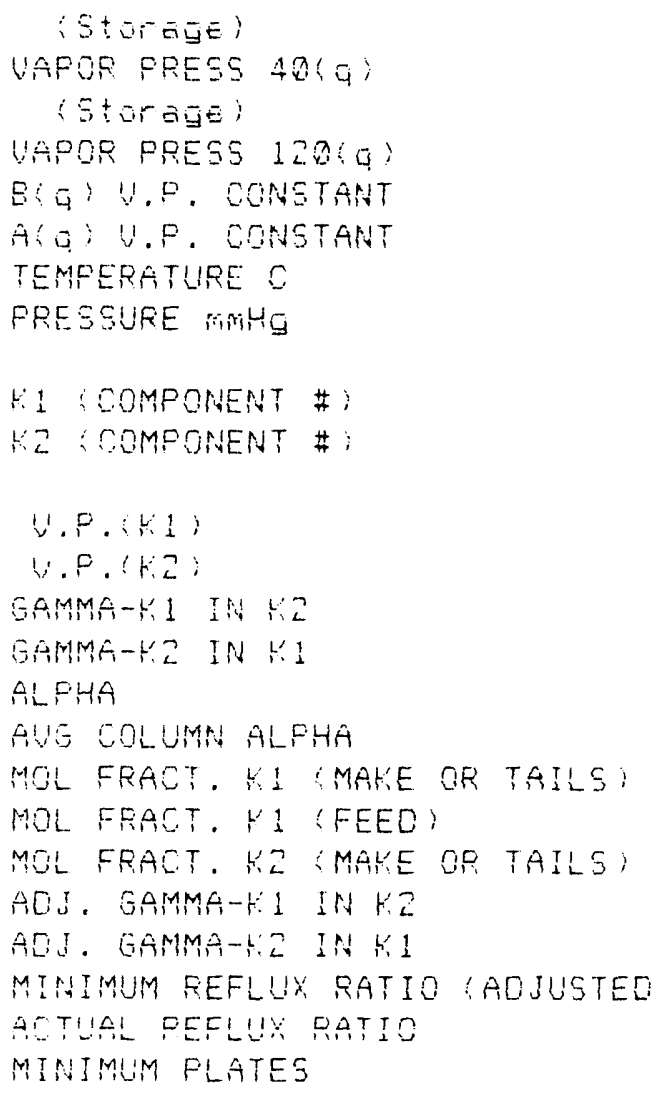

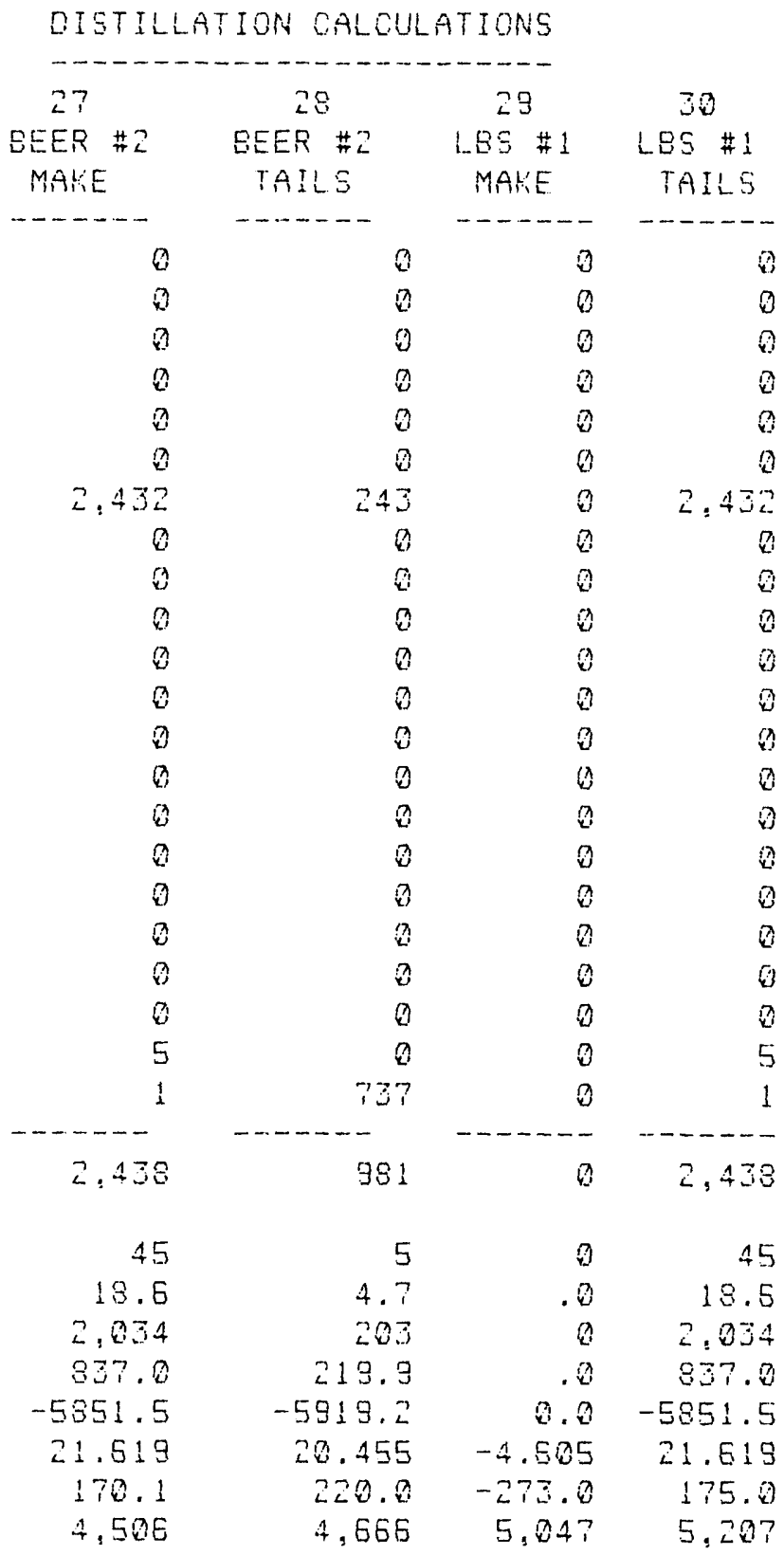

\begin{tabular}{|c|c|c|c|}
\hline$?$ & 7 & $5,5,4,3$ & $6,5,4,3$ \\
\hline OLUENT & SOLUENT & 7 & \\
\hline 4505.7 & 17164.0 & ERR & 17229.0 \\
\hline 157.5 & 333.4 & ERR & 5206.5 \\
\hline 2.014 & 2.014 & 1.350 & 1.350 \\
\hline 2.014 & 2.014 & 1.350 & 1.350 \\
\hline 14.221 & 25.000 & ERP & 4.457 \\
\hline 20.110 & & ERR & \\
\hline 0.393 & 0.248 & ERR & 0.000 \\
\hline 0.784 & & 0.000 & \\
\hline 2.801 & 0.752 & ERE & 1.000 \\
\hline 1.0 .90 & 1.436 & $E R F$ & 1.350 \\
\hline 2.012 & 1.044 & $E R P$ & 1.000 \\
\hline 0.0 & & 0.0 & \\
\hline$\hat{u} \cdot \hat{v}$ & & 6.6 & \\
\hline 3 & & 0 & \\
\hline
\end{tabular}


M LE. MOLES:YEAR

AOTUAL PLATES

PRESSURE MM HE (REUISED)

TEMPERATURE C (REUISED)

AUERAGE MOLECULAR WEIGHT

GAS DENSITY - I.DIOF

CROSS SECTIONAL AREA - SO FT

COLUMN HEIGHT - FT

COLUMN DIAMETEF

il (MPFY)

H. SHEAT UAPORIZ, -ETUILE:

CO QHEAT CAPACITY - BELILEIF)

HEAT LOAD - MM ETLIH

CONOENSEP COOLING WATEP - GPM

CALANORIA STEAM - MPFH ( 15 O PSIG)

COLUMN COST - \$1300 3035 MPC - BARE EOUIPMENT

- ALL CAREON STEEL

- C.S WIJOA 5.5. TFAYS

- ALL 304 STAINLESS STEEL

- ALL 3OHL STAINLESS STEEL

- hll jie stainless steEl

OONDENSER OR CALANDRIA SURFACE

COND. OR CALAND. COST - \$10OO JQBE

- CAREON STEEL

- 3R4 STAINLESS STEEL

- J1E STAINLESS STEEL -MONEL

\section{SUBTOTAL \\ SUBTOTAL \\ SUETOTAL}

MINIMUM REFLUX RATIO

CI, SUBTOTAL \#1

Cri SUBTOTAL \#2

OR SUBTOTAL \#3

Cri CHECK

H. SUBTOTAL \#!

H. SUBTOTAL \#2

HV SUBTOTAL \#

H. CHECK

MIN. PLATES NORMAL)

COL.COST-CIS NORMAL

COL.COST-EIS NORMAL

MIN.REFLUX (NORMAL;

C.S. AREACNORMAL ;

HEAT LOAD NORMAL :

CONICAL COSTINORMAL)
DIETILLATION CALCULATIONS

$$
27
$$

응

EEER \#2

MALE

13

4.515

171

74.12

6. 7709

5.0

34.4

2.8

180,282

215.4

3. 500

5.405

491

2B
BEEP $\# 2$
TAILS
TAL

23

LBS

MAKE

30

LSS \#1

ADL

TAL

a

5, 207

(27\%)

ERR 74.12

EFR 0.8613

S. 2

B. 6

0. 3

215.8

ERQ

215.4

0. 580

도모

7. 911

‥ 900

0.500

0. 800

9.23

0.00

\$3. 2

\$4 2.

$\$ 62.9$

\$69. 2

$\$ 84.9$

25E

MPC - BARE

$\$ 18.2$

$\$ 25.5$

\$27. 3

$\$ 35.5$

$\$ 2.0$

$\$ 0.9$

$\$ 0.0$

$\$ 0.0$

$\$ 0.0$

439

EOUIPMENT

$\$ 0.6$ \$Q.

$\$ 0.0$ \$ 10.0

$\$ 0.0 .00 .0$

$\$ 0.0 \quad \$ 0.0$

$\$ 33.8$

\$35.2

$\$ 47.8$

107,389
98,375
38,975

$(1.0)$

30,141

30
255

9,014

0.500

38,751

9,014
0
98,975
0.500
3,375
0
42,559
215.0

161

215.4

215.0

0.0

00

(1) 255

ERR 0.580

i) 33,751

161

ERR 2.15 .4 $\theta$

ERR

ERR ERR 
MLE. MOLESYCAR

OOMOONENT \#1 COMFONENT \#2 COMPONENT \#3 COMPONENT \#4 OOMOONENT \#S OOMPONENT \#G COMPONENT \#? COMPONENT \#B COMPONENT \#G COMPONENT \#10 COMPONENT \#11 COMPONENT \#2 COMPONENT \#13 COMPONENT \#14

COMOONENT \#15

COMFONENT \#15 COMPONENT \#17 COMFONENT \#18 COMPONENT \#13 COMFONENT \#2O

WAT도

SOLVENT

TOTAL :9:

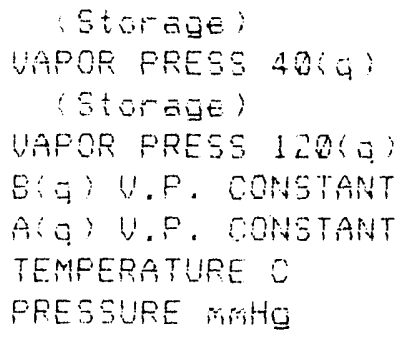

\begin{tabular}{|c|c|c|c|}
\hline 31 & 32 & 33 & 54 \\
\hline $189 \#$ & $\angle B S \# 2$ & LES & LGS $\#$ \\
\hline FALELE & TATLS & MAY'E & TAILS \\
\hline . & $\cdots \cdots$ & $-\cdots \cdots \cdots$ & $\ldots$ \\
\hline 0 & 0 & 0 & 0 \\
\hline 0 & 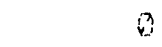 & 8 & $n$ \\
\hline 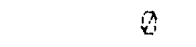 & 0 & 8 & 8 \\
\hline 0 & 8 & 8 & 3 \\
\hline 0 & 8 & 8 & Q \\
\hline 0 & 8 & 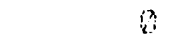 & 3 \\
\hline 8 & 8 & 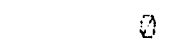 & 8 \\
\hline$\theta$ & 8 & 8 & 8 \\
\hline 0 & 8 & is & $\theta$ \\
\hline 0 & 8 & 8 & 9 \\
\hline 3 & $B$ & 8 & () \\
\hline$\theta$ & $a$ & 0 & 3 \\
\hline 3 & 8 & 0 & (i) \\
\hline 8 & 8 & 0 & (3) \\
\hline 8 & ? & 8 & के \\
\hline 0 & in & (3) & 0 \\
\hline is & 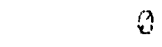 & $a$ & 9 \\
\hline 8 & $\pi$ & 0 & 0 \\
\hline$n$ & $\theta$ & 3 & i) \\
\hline 8 & $Q$ & $Q$ & 8 \\
\hline 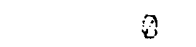 & 8 & 8 & 0 \\
\hline 0 & 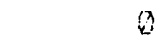 & 2 & 8 \\
\hline$\cdots \cdots$ & $\cdots \cdots$ & $\cdots \cdots$ & $\ldots \ldots$ \\
\hline 0 & 0 & 0 & $x$ \\
\hline 3 & 8 & 0 & 8 \\
\hline 8 & . ? & .8 & .2 \\
\hline 3 & 8 & 0 & 0 \\
\hline .8 & .8 & .3 & .2 \\
\hline 0.0 & is. 8 & Q. 0 & 0.6 \\
\hline-4.505 & -4.605 & -4.505 & -4.505 \\
\hline$E R R$ & 120.0 & ERR & 120.0 \\
\hline$(158)$ & $\theta$ & $(150)$ & 0 \\
\hline$\Xi$ & 3 & $2: 1$ & $2: 1$ \\
\hline $4,5,5$ & $4,5,5$ & 3 & 3 \\
\hline ERP & 5095.7 & ERP & 1.0 \\
\hline ERF & 2517.3 & EPR & 5096.7 \\
\hline 1.492 & 1.432 & 1. $20 \%$ & 1.800 \\
\hline$\therefore .492$ & 1.492 & 1..$\Delta B$ & 1. $\sin 2$ \\
\hline 동 & ERP & EPR & EF: \\
\hline ERF & & $58 R$ & \\
\hline ERR & ERP & ERP & $E R$ \\
\hline EAF & & ERF & \\
\hline$E R_{1}$ & ERP & ERP & ERF \\
\hline ERP & ERP & ERR & ERF \\
\hline ERR & $E R R$ & ERR & EPR \\
\hline 0.0 & & 0.8 & \\
\hline$\hat{\mathrm{u}} \cdot \hat{\mathrm{u}}$ & & . & \\
\hline$Q$ & & 0 & \\
\hline
\end{tabular}


$M$ LE. MOLESYEAR

AOTUAL PUATES

FPESOURE WF HG AREUTSED:

TEMEERATURE C :PEUISEO:

AUERAGE MOLECULAE WEIGHT

GAS DENGITY - LESOF

CFOSB SECTIONAL AREA - SO FT

COLUMN HEIGLT - FT

OOUMU DIAMETER

HI :MPQY

H, SHEAT UAPORIZ,-BEULE:

GH HEAT OAPAOITY - EtUILEF:

HEAT LOAD - MM EDLIHT

BONOENEEP COOLING WATER - GPM

OALANDFIA STEAM - MPPH 150 PSIG;

COLUMA COST - \$1000 3096 MPC - EAPE

- ALL CARBON STEEL

- OS W JQ4 E.S. TRAYS

- ALL JA4 BTAINLESE STEEL

- ALL JXAL STAINLESS GTEEL

- ÁlL jle stainless gteEl

CONDENGER OP CALANDRIA SURFACE

COHO. OR CALARD. COST - \$10OO

- CAREON STEEL.

- JRA GTAINLESS STEEL

- T1S STAINLESE ETEEL

-MONEL

$$
\begin{aligned}
& \text { SUBTOTAL } \\
& \text { SUBTOTAL } \\
& \text { SUBTOTAL }
\end{aligned}
$$

MINIMUM REFLUK RATIO

Cr SURTOTAL \#1

CH SUBTOTAL \#2

CH SUIBTOTAL \#

CO CHECL

HV SUETOTAL \#1

H. SUETOTAL \#2

HY SUETOTAL \#3

H. CHECK

MIN. PLATES (NORMAL)

OOL. COST-CIS NORMAL

COL . COST-S!S NORMAL

MIN.PEFLUX!NORMAL)

C.S. AREA (NORMAL)

\begin{tabular}{|c|c|c|c|}
\hline 31 & 33 & 33 & 34 \\
\hline LSS \#. & $1.85 \quad 42$ & $\angle E S \# 3$ & $185 \quad \# 3$ \\
\hline MAEE & TAILS & MALE & THL \\
\hline$\ldots-\cdots$ & $\cdots$ & $\cdots \cdots-\cdots$ & $\ldots \ldots$ \\
\hline 0 & & 0 & \\
\hline 0 & & 8 & \\
\hline$(273)$ & & $(273)$ & \\
\hline ERP & ERR & ERR & ERR \\
\hline ERR & ERP & ERS & $E R R$ \\
\hline 9.8 & & 0.0 & \\
\hline 0.0 & & Q. 0 & \\
\hline 8.8 & & 0.0 & \\
\hline 3 & & 3 & \\
\hline ERR & ERF & ERR & ERP \\
\hline ERP & ER⿱口龰 & ERP & ERP \\
\hline 0.000 & 9. 200 & 0.000 & 3.000 \\
\hline 0 & & 8 & \\
\hline $5 ;$ & 0.00 & & 0.80 \\
\hline
\end{tabular}

HEAT LOAOU NORMAL:

COMICAL COST:HORMAL;
$\$ 0.0$

$\$ 0.0$

$\$ 0.8$

$\$ 8.0$

$\$ 0.8$

a

$3086 \mathrm{MPE}$

$\$ 0.0$

$\$ 8.6$

$\$ 0.0$

$\$ 0.0$

DARE EQ

中O.

$\$ 8.0$

象. 8

本司.

0

Q

D. 0

0
0
ERR
0
0
0

ERF

0
0
0
ERR
0
0
0
ERR

$\$ 0.0$

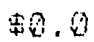

$\$ 2.0$

$\$ 0.0$

$\$ 8.8$

O

0

$\$ 2.0$

$\$ 0.8$

$\$ 0.0$

$\$ 0.6$

$\$ 0.0$

\$Q .0

$\$ 0.0$

$\$ 2$.

0

0.0

0

8

ERR

0

0

ERR
0
0
0
0
0
0
ER 
M LB. MOLES YEAR

\begin{tabular}{|c|c|}
\hline COMEONENT & $\# 1$ \\
\hline COMPONENT & $\# 2$ \\
\hline COMPONENT & $\# 3$ \\
\hline COMPONENT & $\# A$ \\
\hline COMPONENT & $\# 5$ \\
\hline COMPONENT & $\# E$ \\
\hline COMPONENT & $\# ?$ \\
\hline COMPONENT & $\# 8$ \\
\hline COMPONENT & $\# 3$ \\
\hline SOMPONENT & $\# 10$ \\
\hline COMPONENT & $\# 11$ \\
\hline COMFONENT & $\# 12$ \\
\hline COMPONENT & $\# 13$ \\
\hline COMFONENT & $\# 14$ \\
\hline COMPONENT & $\# 15$ \\
\hline COMPONENT & \#1E \\
\hline COMPONENT & $\# 17$ \\
\hline COMPONENT & $\# 18$ \\
\hline SOMPONENT & $\# 19$ \\
\hline $\begin{array}{l}\text { COMPONENT } \\
\text { WATES } \\
\text { SOL UENT }\end{array}$ & $\# 20$ \\
\hline
\end{tabular}

TOTAL $: \bar{a}$

(StDíñe)

UAPOF PRESS 40:

(Storrige)

VAFOP FRESS 120 :

B(g) U.P. TONSTANT

AGQ U.P. CONSTANT

TEMPERATURE $C$

PRESGURE MIHU

H S COMPONENT \#

F. S COMPONENT \#

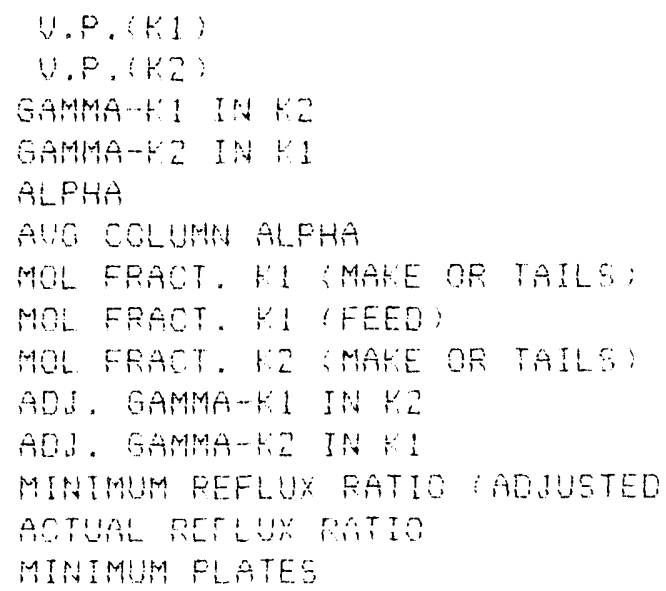

DISTILLATION CALCULATIONS

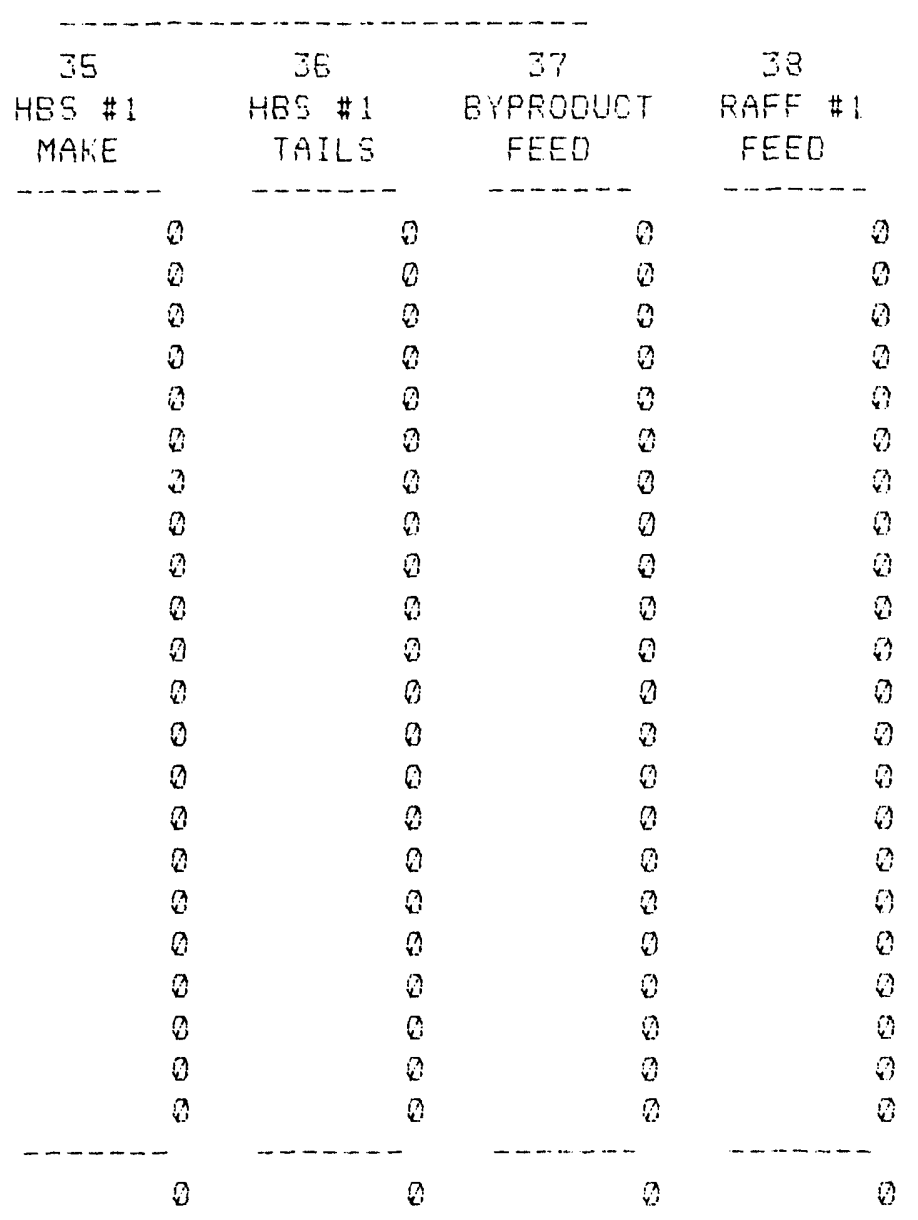


M LE, MOES YEAS
AOTUAL PLATES

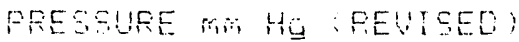

TEAFERATUPE O I PEUTEL:

AUERAGE MOLEOULAR WETEHT

GAS OLUSITY - LEAS

CROES GEOTOWAL AFEA - 90 FT

COLUMA HEIGHT - F?

COL UMA OAMETEP

Himp?

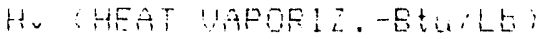

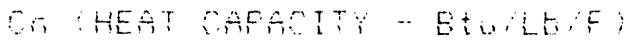

HEAT LGAT M MA Ba H

CONDENEE OOOLTHE WATER - GEY

CALAIOFIA STEAM -. MPFH 150 PSIG:

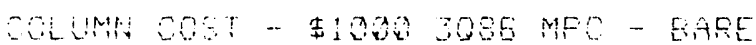

- ALL CAFEON STEEL

- 5.5 w. 3.4 5.S. TPAYS

- AL YHA STAINLESS STEEL

- ALL TOH STALULSG STEEL

AL 315 GTAINLESG STEEL

OONDENGER OP OALANDETA BUPFACE

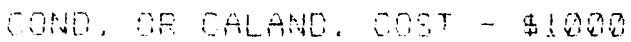

- AESOH STEEL

TOA GTATHLES STEEL

- IDE STAYHESO STEEL

-MONHEL.

E!ETOTAL

SIETOTAL

EVEOOTHL

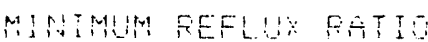

Ci SUETOTHL \#1

Cr, SETOTAL \#2

O- GUPTOTA \#

G. OHES

H. OULTOTAL \#1

H. SESTOTAL \#2

H. S!g?

H. CHE H

HAU, PLATEE NOFMAL

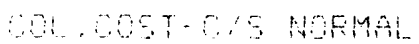

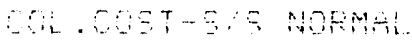

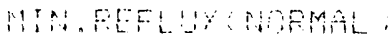

$\therefore, \sigma$, ARE $A$ HOWAL

HEFT lOAD HOPHAL:

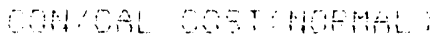

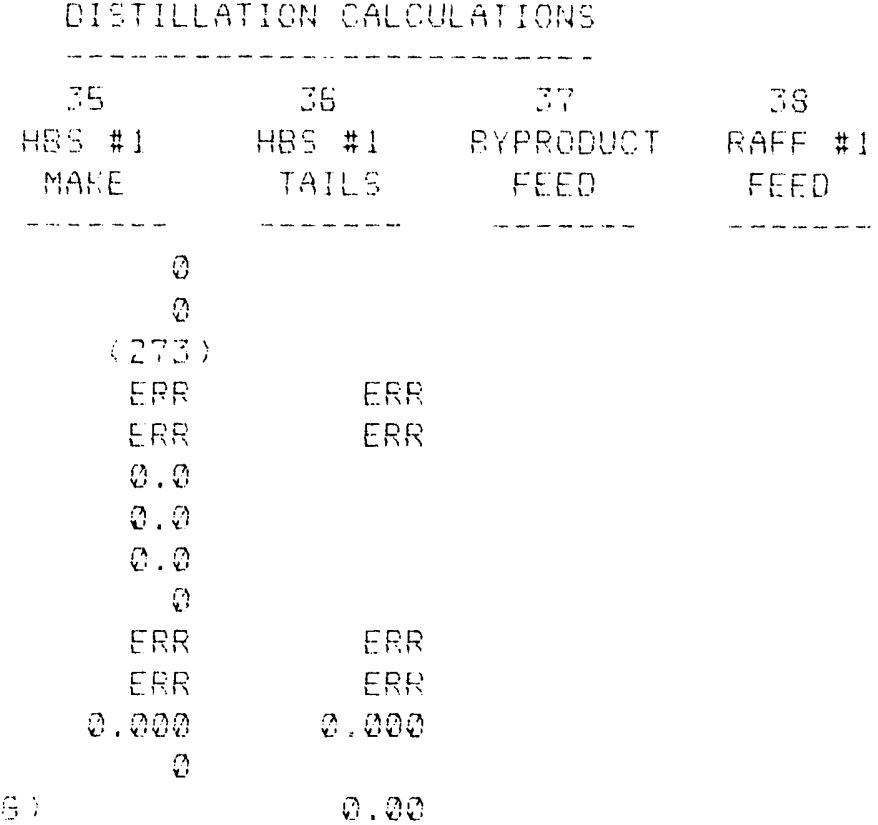

争, 8

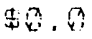

\$日.

क日.

की.

B

BARE COUIPMENT

\$क. 0 की

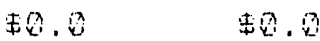

क日.

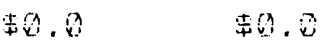

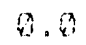

i)

3

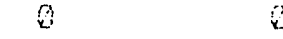

ERP FRP

( ) $D$

in

ERR ERP 
M LE. MOLES, YEAR

COMPONENT \#1 COMPONENT \#Z COMPONENT \#3 COMPONENT \#A COMPONENT \#5 COMPONENT \#S COMPONENT \#? COMPONENT \#B COMPONENT \#G COMPONENT \#19 COMPONENT \#11 COMPONENT \#12. COMPONENT \#13 COMPONENT \#14 COMPONENT \#15 COMPONENT \#1E COMPONENT \#1? COMPCNENT \#13 COMPONENT \#19 COMPONENT \#ZO WATER

SOLUENT

TOTAL (G)

$$
\text { (Storage) }
$$

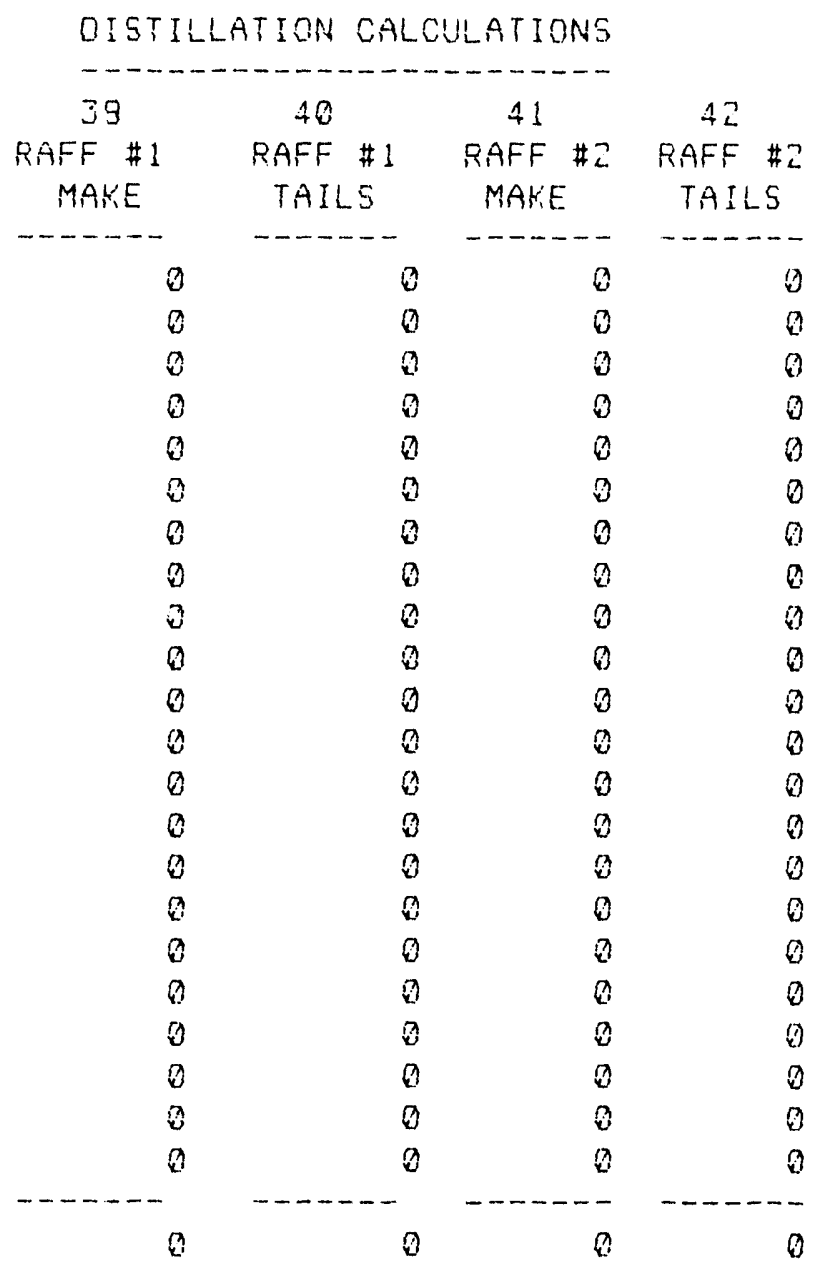




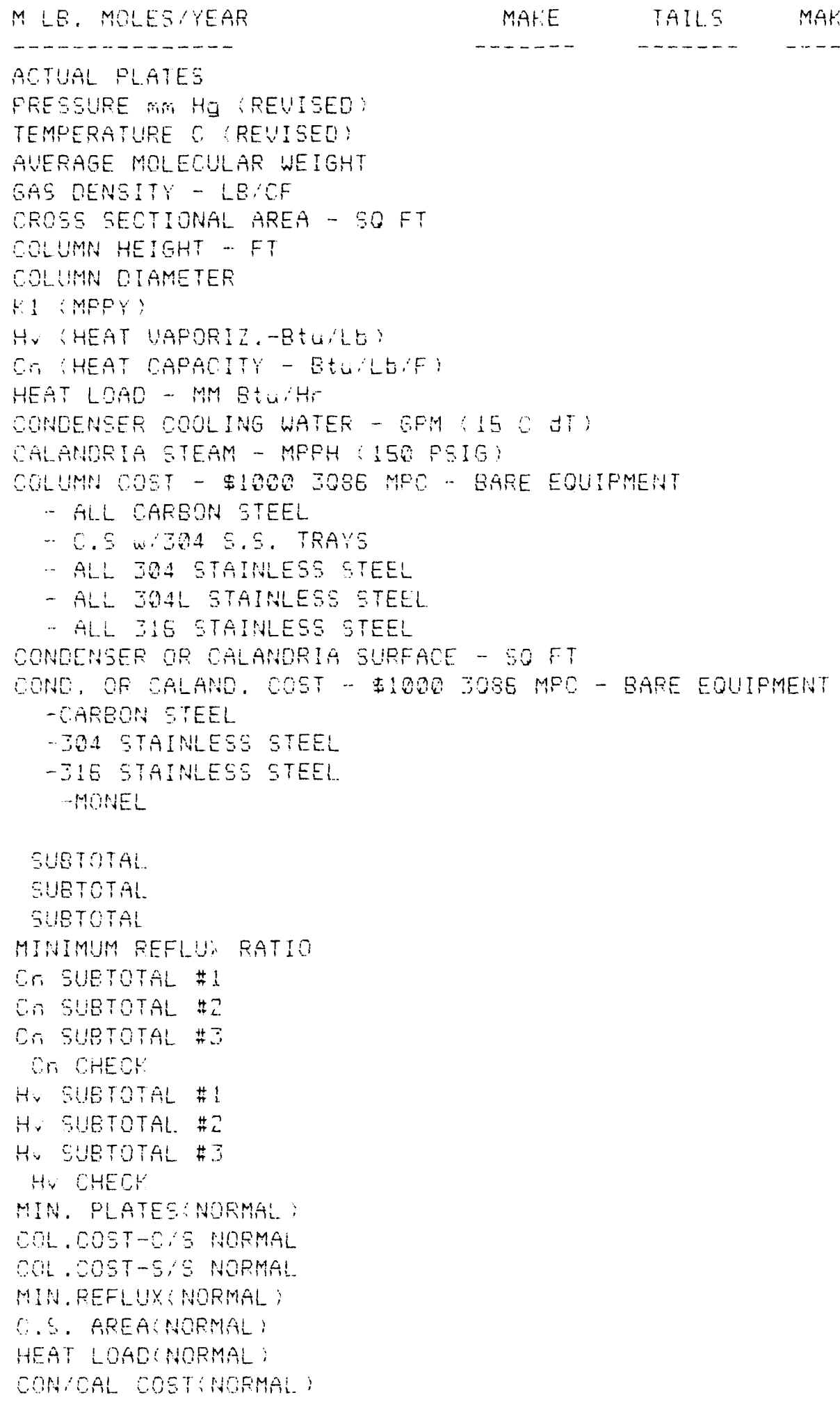


$\because$ LE. MOLES YEAR

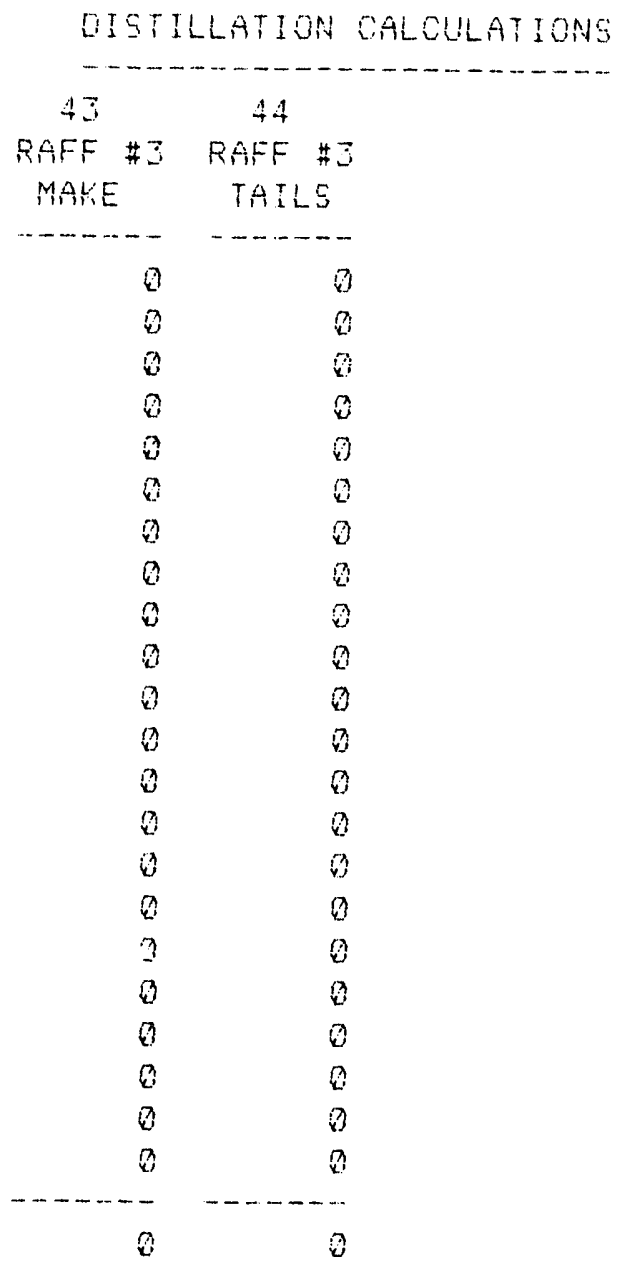

TOTAL :G :

COMPONENT \#!

COMFONENT \#2

COMPONENT $\#$ J

COMPONENT \#4

COMPONENT \#S

COMPONENT \#E

COMPONENT \#T

COMPONENT \#B

COMPONENT $\#$

COMPONENT \#IO

COMOONENT \#11

GOMPONENT \#12

COMPONENT \#1S

GOMPGNENT \#14

COMPONENT \#15

COMFONENT \#15

OOMPONENT \#17

OIMPONENT \#18

COMPONENT \#13

COMFONENT \#ZA

WATER

GOLUENT

(Stor age;

UAPOR FRESS 40:

(Storage)

WAFOR PFESE 120 O

BU U.P. CONSTANT

AS F UF OONSTANT

TEMFEFATURE $C$

PRESGURE MUHE

H1 C COMPONENT \#;

L C COMPONENT \#;

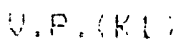

U.P. :

GAMMA-LI IN LIZ

GAMMA-HZ IN

ALPLA

AUG COLUMN ALPHA

MOL FRACT. KL MAKE OR TAILS:

MOL FRAET. HL (FEED)

MOL FPACT. KL IMAKE OR TAILS;

ADO. GAMMA-K1 IN

ADJ. GAMMA-KL IN KI

MININUM REFLUX RATIO (AOUUSTEQ)

AETUAL FEFLUX FATIO

MINIMUM PLATES 

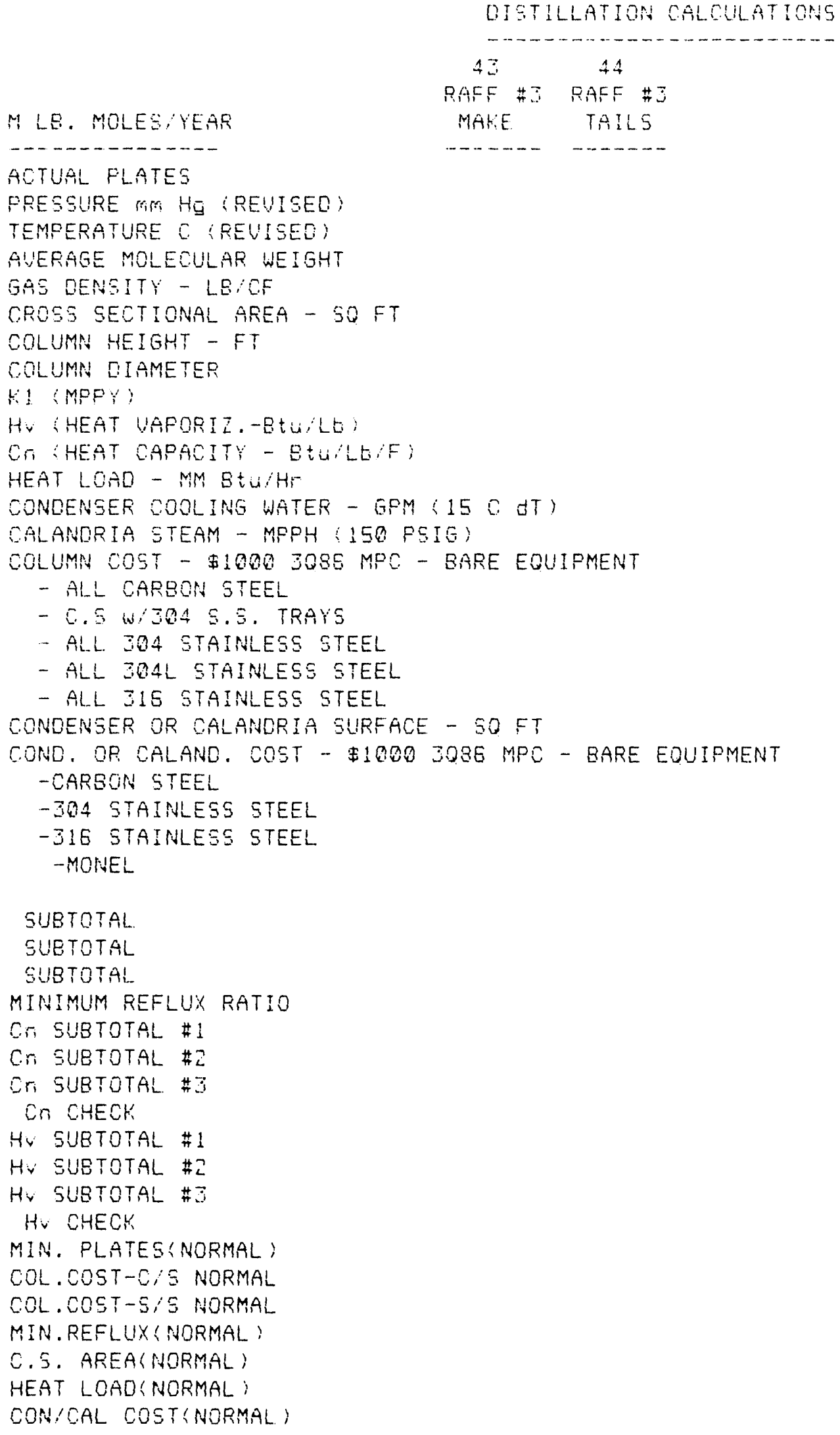
APPENDIX F. IN SITU EXTRACTION NO RECOVERY OF RAFFINATE RECYCLE 
SINOLE-STAGE INEITU LXTFACTIUE FERMEHTATION OF EUTANOL

NO RECOUERY OF RAFFINATE CHEMICALE

BASECASE STOICHIOMETRY

INUESTHENT

CONLITIONS

Slted an luwa on the $M_{1}$ isjsslppl River adjacent to a corn wet mill and a utility power house for over-the-fence supply of syrup and power.

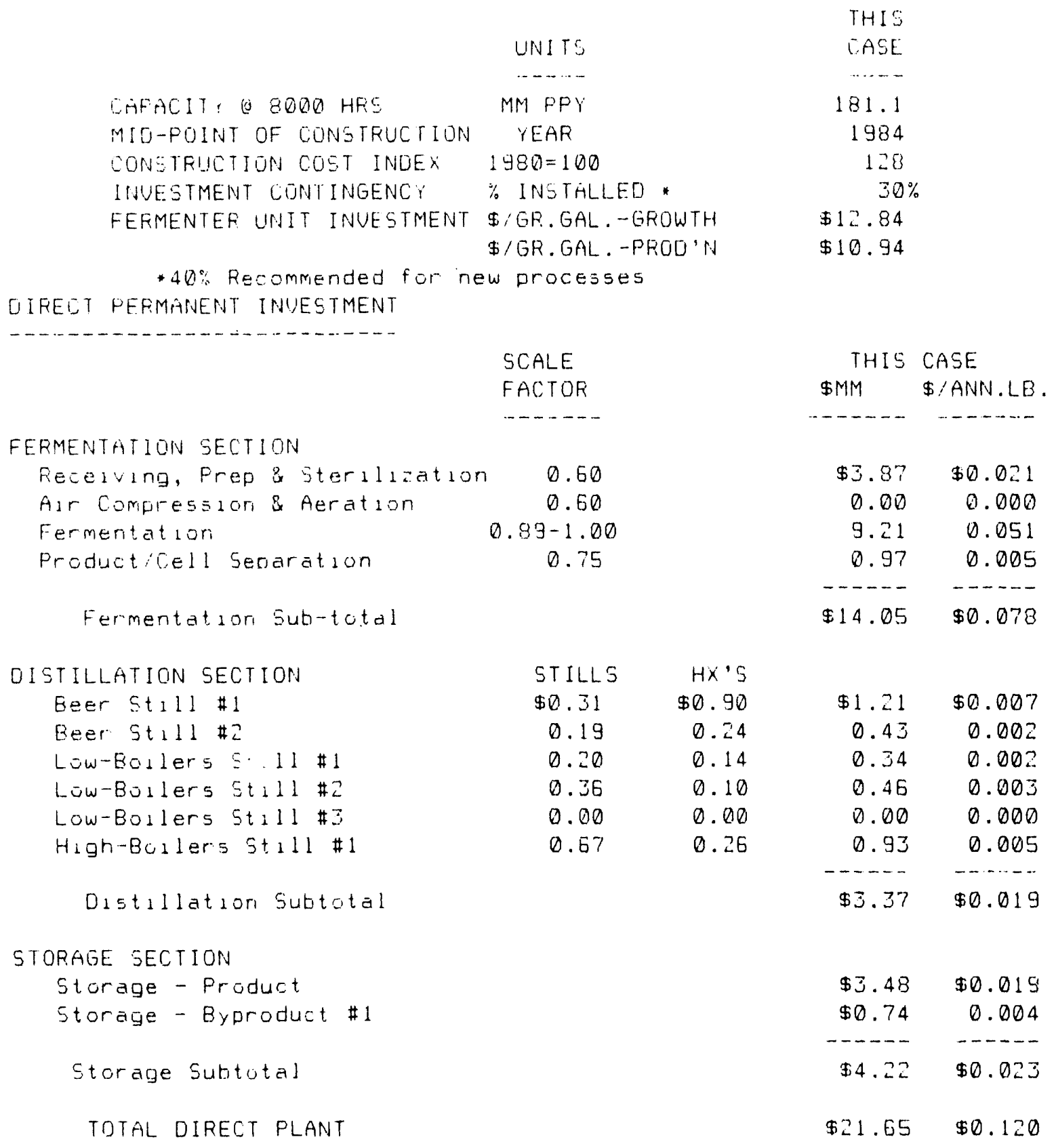




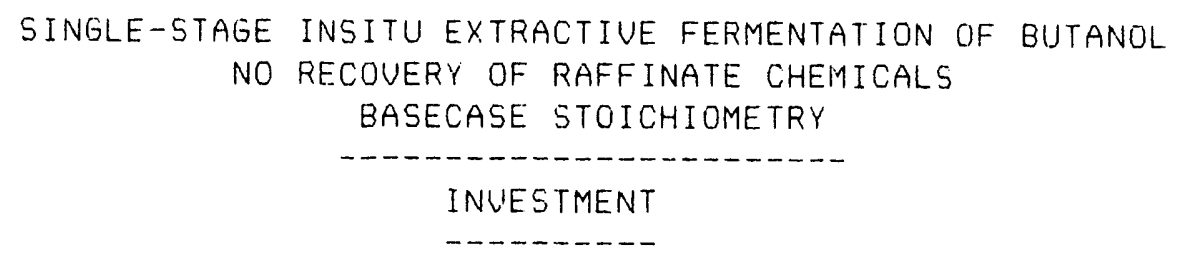

ALLOCATED PERMANENT INUESTMENT

\begin{tabular}{|c|c|c|c|c|c|c|}
\hline & & API $/$ & UNIT & & & \\
\hline & UNITS & BASECASE & THIS CASE & UNITS & $\$ M M$ & $\$ / A N N$. LE. \\
\hline ELECTRICITY & fiw & $\$ 183$ & $\$ 18=$ & 2997 & 5078 & \pm 0 ר \\
\hline STEAM & $\mathrm{PPH}$ & $\$ 45$ & $\$ 45$ & 124.940 & $\begin{array}{r}90.38 \\
5.5 ?\end{array}$ & $\$ 0.002$ \\
\hline COOLING WATER & GPM & $\$ 52$ & $\$ 52$ & 6,119 & 0.32 & 0.002 \\
\hline FROCESS WATER & GPM & $\$ 313$ & $\$ 313$ & 28 & 0.01 & .000 \\
\hline WASTE DISPOSAL & MGFY & $\$ 3$ & $\$ 3$ & 128,606 & 0.35 & 0.002 \\
\hline GEN'L \& SERUICES & \$MM & $10 \%$ & $10 \%$ & $\$ 28.3$ & 2.83 & 0.016 \\
\hline TOTAL ALLOO & TED PL & ANT & & & $\$ 9.51$ & $\$ 0.053$ \\
\hline TOTAL PERMANENT & NUES & & & & $\$ 31.16$ & $\$ 0.172$ \\
\hline
\end{tabular}

WORKING CAPITAL

\begin{tabular}{|c|c|c|c|c|c|}
\hline & BASIS & $\begin{array}{l}\text { DAYS } \\
\text { BASECASF }\end{array}$ & $\begin{array}{l}\text { DAYS } \\
\text { THIS CASF }\end{array}$ & $\$ M M$ & DiANN. LB. \\
\hline & $\ldots-\ldots$ & $\ldots$ & -........ & $-\cdots$ & n \\
\hline RAW MAT'L INUENTORY & SRAW MATL & 2 & 2 & $\$ 0.31$ & $\$ 0.002$ \\
\hline SEMI-F INISHED PRODUCT & $\$(R+M) / 2$ & 5 & 5 & 0.79 & 0.004 \\
\hline FINISHED PROOUCT & $\$ C O M$ & 30 & 30 & 5.37 & 0.036 \\
\hline CASH & $\$(\cos -0)$ & E & 6 & 1.20 & 0.007 \\
\hline ACCOUNTS RCD. - TRADE & $\$ \subseteq P$ & 30 & 30 & 6.58 & 0.036 \\
\hline ACCOUNTS RCD.-MISC. & $\% \mathrm{COM}$ & $0.9 \%$ & $0.9 \%$ & 0.59 & 0.003 \\
\hline DEFERREU CHARGES & $\% \mathrm{COM}$ & $1.5 \%$ & $1.5 \%$ & 0.98 & 0.005 \\
\hline TOTAL. WORFING CAF & TAL & & & $\$ 15.82$ & $\$ 0.087$ \\
\hline
\end{tabular}

TOTAL INUESTMENT FOR RETURN

$\$ 45.98 \quad \$ 0.259$ 
SINGLE - STAGE INSITU EXTRACTIUE FERMENTATION OF EUTANOL.

NO RECOUEFY OF RAFFLNATE CHEMICALS

\section{BASECASE STOICHIOMETRY}

FFIEES \& COSI FACTOFS

Coerating Year

Raw Mèterlals

- Elosugar Syrup

- Aritiya. Ammorila

- Phosphor lo Acia

- Fotassiur Erlorlde

-Minor Nutrient:

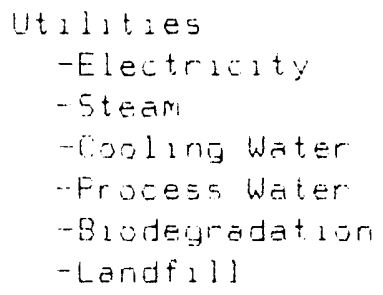

BASECASE

$\$ 0.065 / 1$ b. d.s. $\$ 0.046 / 10$. $\$ 0.155 / 10$. $\$ 0.05 \% / 16$. $\$ 0.451 / 1 b$.

INFLATION FACTOF

$\$ 0.040 / \mathrm{KWH}$
$\$ . .20 / M 16$.
$\$ 0.04 / M 991$.
$\$ 0.50 / M 9 a 1$.
$\$ 0.04 /$ it.
$\$ 0.05 / 16 . d .5$.

1.00

1.00

1.00

1.00

1.00

1.00

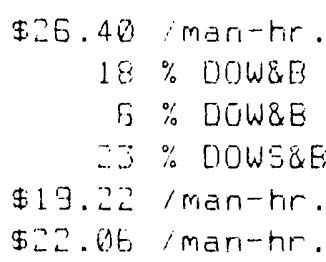

$\therefore .00$

--

$-$

$-$

1.00

1.00
THIS CASE

1988

$$
\begin{aligned}
& 1.7 \% \text { DPI } \\
& 25 \% \text { MWQE } \\
& 40 \% \text { MWEE } \\
& 4 \% \text { MWSE } \\
& 23 \% \text { MLSÜ } \\
& 0.3 \div \text { OPI } \\
& 8 \% \text { DPI } \\
& 6 \% \text { APSBE }
\end{aligned}
$$

$3 \%$ Sales

\begin{tabular}{|c|c|c|}
\hline$\ldots$ & $1.7 \%$ & OPPI \\
\hline- & $25 \%$ & MW\&B \\
\hline- & $40 \%$ & MWEE \\
\hline- & $4 \%$ & HWQE \\
\hline - & $23 \%$ & MWS\&B \\
\hline- & $0.3 \%$ & DFI \\
\hline- & $3 \%$ & OPI \\
\hline$\ldots$ & $E \%$ & APS\&G \\
\hline
\end{tabular}

50.01 ; 1 t.

$4.5 \%$ sales

$2 \%$ Sales

6 \% PTE

$20 \%$ TIFF

\$0. $2 ?$; it.

\$0. $00 / 15$.
$--$

$\ldots$

-.

$-$

$\cdots$

$-$

1.30

1.00

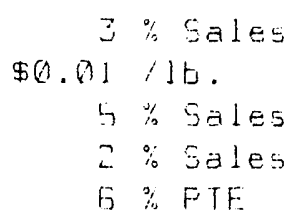

$30 \%$ TIFF

$\$(2.2 \%: 16$.

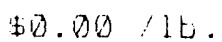

$\$ 0.040$ iFWH $\$ 2.20 / M 16$. $\$ 0.04 / M$ gal. $\$ 0.50$ iM Gal. $\$ 0.05$ ilt. U.5. $\$ 0.04 / 16.0 .5$.

$\$ 0.065 / 16 . d .5$. $\$ 0.046 / 1 b$. $\$ 0.155 / 1 b$. $\$ 0.053 / 16$. $\$ 0.451 / 1$ t

$\$ 26.40$ imär-hr. $18 \%$ DOWEB

$6 \%$ COOW\&B $23 \%$ DOWSEB

$\$ 19.22 / m a n-h i r$. \$2. OE /man-hr. 
SALARIES \& WAGES

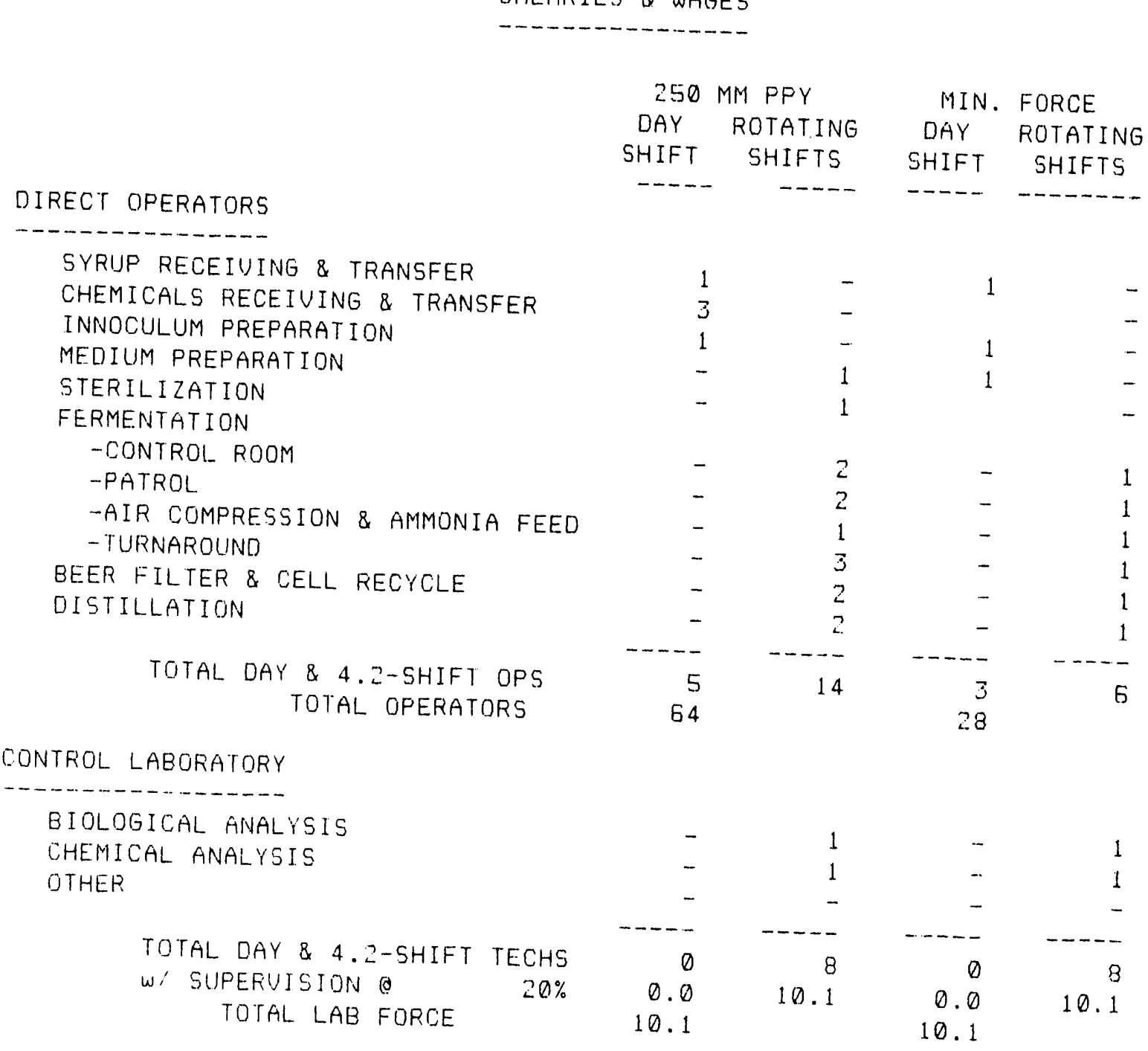

TECHNICAL ASSISTANCE TO MANUFACTURING

PROCESS ENGINEERS

WAGES, SALARIES \& BENEFITS SCHEDULE-

OPEFATING WAGES - \$/HOUR

TECHNICIANS - ANNUUAL \$

PROCESS ENGINEERS - ANINUAL \$

PENSION - AS\% OF COMPENSATION

FICA

UNEMPLOYMENT COMPENSATION

GROUP LIFE INSURANCEE

MEOICAL INSURANCE

DENTAL INOURANCE

SAUINGS PLAN

VACATION

ILLNEES

ABSENCE WITH PERMISSION

TOTAL EENEFITS
$\$ 20.14$

$\$ 30,500$

$\$ 35,000$

$8.1 \%$

$5.8 \%$

$0.6 \%$

$0.7 \%$

J. $6 \%$

$0.0 \%$

$2.5 \%$

$7.4 \%$

$1.4 \%$

D. $2 \%$

$31.1 \%$ 
SINGLE-STAGE INSITU EXTRACTIUE FERMENTATION OF BUTANOL. NO FECOVERY OF RAFFINATE CHEMICAL.S BASECASE STOICHIOMETFY

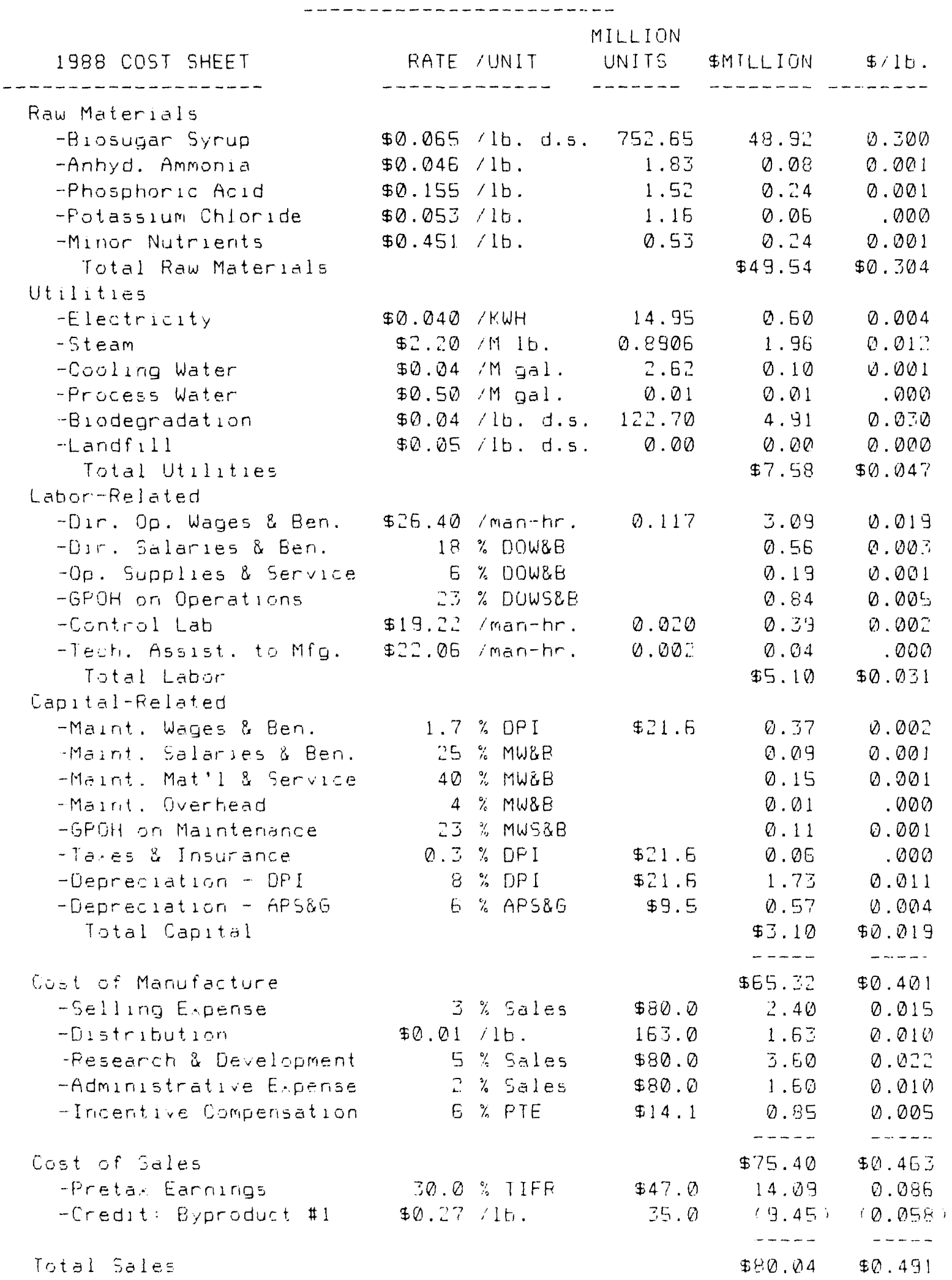


SINGLE-STAGE INEITU EXTRACI IUE FERMENTATION OF BIITANOL NO RECOUERY OF RAFFINATE CHEMICALS

\author{
BASECASE STOICHIOMETRY
}

CASH FLOW (MILIION DOLLARS/YEAR)

Seeriar $10:$

1. Irvestment split evenly over three coristruction years.

$\therefore$ Flarit operates at $50 \%$ of full $5 r a l e$ the first yaar.

3 . " $75 \%$ " " " $"$ "

4. " $100 \%$ " " " the third year.

5. " " $100 \%$ " " thereafter.

6. Five yeai defreclation rate; half-year conventson;

$(20,32,13.2,11.5,11.5,5.3 \%)$. Tas: $34 \%$ federal, $3 \%$ state.

\begin{tabular}{|c|c|c|c|c|c|c|c|}
\hline & \multicolumn{2}{|c|}{ INUESTMENT } & DEP & COST EX O & SALES & NET EARN & ANN CASH \\
\hline YEAR & PI & WC & - & $\ldots-\cdots \cdots$ & $-\cdots-\ldots$ & $\ldots \ldots \ldots$ & $\ldots-\ldots \ldots$ \\
\hline$\ldots-\cdots$ & $\ldots \ldots$ & $-\cdots-$ & & & & & \\
\hline 1933 & $\$ 7.23$ & & & & & & $(\$ 7.22)$ \\
\hline 1904 & $\$ 7.27$ & & & & & & $(\$ 7.22)$ \\
\hline 1983 & $\$ 7.22$ & $\$ 15.82$ & & & & & $(\$ 23.03)$ \\
\hline 1986 & & & $\$ 4.35$ & $\$ 39.63$ & $\$ 44.75$ & $\$ 0.50$ & $(\$ 10.99)$ \\
\hline 1987 & & & $\$ 6.93$ & $\$ 5 ? .77$ & $\$ 67.12$ & $\$ 1.53$ & $\$ 8.45$ \\
\hline 1969 & & & $\$ 4.16$ & $\$ 73.10$ & $\$ 89.49$ & $\$ 7.71$ & $\$ 11.87$ \\
\hline 1989 & & & \$2.49 & $\$ 73.10$ & T89. 49 & $\$ 8.76$ & $\$ 11.25$ \\
\hline 1990 & & & 42.49 & $\$ 73.10$ & $\$ 89.49$ & $\$ 8.76$ & $\$ 11.25$ \\
\hline 1991 & & & $\$ 1.26$ & $\$ 73.10$ & \$89.49 & $\$ 9.54$ & $\$ 10.73$ \\
\hline 1992 & & & & $\$ 73.10$ & $\$ 89.49$ & $\$ 10.33$ & $\$ 10.33$ \\
\hline 1993 & & & & $\$ 73.10$ & $\$ 99.49$ & $\$ 10.33$ & $\$ 10.33$ \\
\hline 1994 & & & & $\$ 73.10$ & $\$ 89.49$ & $\$ 10.33$ & $\$ 10.33$ \\
\hline 1395 & & & & $\$ 73.10$ & $\$ 89.49$ & 由10.33 & $\$ 10.33$ \\
\hline 1996 & & & & $\$ 73.10$ & $\$ 89.49$ & $\$ 10.33$ & $\$ 10.33$ \\
\hline 1997 & & & & $\$ 73.10$ & $\$ 89.49$ & $\$ 10.33$ & $\$ 10.33$ \\
\hline 1998 & & & & $\$ 73.10$ & $\$ 89.49$ & $\$ 10.33$ & $\$ 10.33$ \\
\hline 1999 & & & & $\$ 75.10$ & $\$ 89.49$ & $\$ 10.33$ & $\$ 10.33$ \\
\hline$\therefore 000$ & & & & $\$ 73 \ldots 0$ & $\$ 89.49$ & $\$ 10.33$ & $\$ 10.33$ \\
\hline 2001 & & & & $\$ 73.10$ & $\$ 89.49$ & $\$ 10.33$ & 910.33 \\
\hline 2002 & & & & $\$ 73.10$ & $\$ 89.49$ & $\$ 10.33$ & $\$ 10.33$ \\
\hline 2003 & & & & $\$ 73.10$ & $\$ 89.49$ & $\$ 10.33$ & $\$ 10.33$ \\
\hline 2004 & & & & $\$ 73.10$ & $\$ 89.49$ & $\$ 10.33$ & $\$ 10.33$ \\
\hline 2005 & & $(\$ 15.82)$ & & $\$ 73.10$ & $\$ 89.49$ & $\$ 10.33$ & $\$ 26.15$ \\
\hline
\end{tabular}

NET PETIRN ON INUESTMENT-3RO DPERATING YEAR $=15.6 \%$ 
SINGLE-STAGE INSITU EXTRACTIUE FERMENTATION OF BUTANOL.

NO RECOUERY OF RAFFINATE CHEMICALS

BASECASE STOICHIOMETRY

CASH FLOW (MILLION DOLLARS/YEAR)

Scenario:

1. Investment split evenly over three construction years.

2. Plant operates at $50 \%$ of full scale the first vear.

3. " $75 \%$ " " " " "

4. " $100 \%$ " " "he thirdyear.

5. " " $100 \%$ " " thereafter.

6. Five year depreciation rate; half-year convention; $(20,32,19.2,11.5,11.5,5.8 \%)$. Tax: 34\% federal, 3\% state.

CUM CASH NPV @ $12 \% \%$ IRR

$\begin{array}{lccc}\text { YEAR } & \ldots & & \\ \cdots & & & \\ 1983 & (\$ 7.22) & (\$ 7.22) & - \\ 1994 & (\$ 14.13) & (\$ 13.66) & - \\ 1985 & (\$ 37.46) & (\$ 32.02) & - \\ 1986 & (\$ 48.45) & (\$ 39.84) & -151.8 \% \\ 1987 & (\$ 40.00) & (\$ 34.47) & -60.8 \% \\ 1988 & (\$ 28.13) & (\$ 27.74) & -27.5 \% \\ 1989 & (\$ 16.88) & (\$ 2 . .04) & -12.2 \% \\ 1990 & (\$ 5.63) & (\$ 16.95) & -3.2 \% \\ 1991 & \$ 5.16 & (\$ 12.59) & 2.4 \% \\ 1992 & \$ 15.49 & (\$ 8.86) & 6.1 \% \\ 1993 & \$ 25.82 & (\$ 5.54) & 8.7 \% \\ 1994 & \$ 36.15 & (\$ 2.57) & 10.6 \% \\ 1995 & \$ 46.48 & \$ 0.08 & 12.0 \% \\ 1996 & \$ 56.81 & \$ 2.45 & 13.1 \% \\ 1997 & \$ 67.14 & \$ 4.57 & 14.0 \% \\ 1998 & \$ 77.47 & \$ 6.45 & 14.6 \% \\ 1999 & \$ 87.80 & \$ 8.14 & 15.2 \% \\ 2000 & \$ 98.13 & \$ 9.64 & 15.6 \% \\ 2001 & \$ 108.46 & \$ 10.99 & 15.9 \% \\ 2002 & \$ 118.79 & \$ 12.19 & 16.2 \% \\ 2003 & \$ 129.12 & \$ 13.26 & 16.4 \% \\ 2004 & \$ 139.45 & \$ 14.21 & 16.6 \% \\ 2005 & \$ 165.59 & \$ 16.37 & 17.0 \%\end{array}$


EINGLE-STAGE INZJII EXTFÁTIUE FEFMENTATION OF RUTANOL

HO RECOUERY DF RAFFINATE CHEMISALS

EASECASE STOICHIOINETFY

\begin{tabular}{|c|c|c|c|}
\hline \multicolumn{4}{|c|}{ EHSIC IIATA } \\
\hline$=7.9$ & $m g i g$ & $c \in 11 s$ & -MINOF NUTFIENTS \\
\hline 23.6 & $m g: g$ & $\Leftrightarrow \Leftrightarrow 11 \mathrm{~s}$ & $-M g 5 n 4.7 H_{2} 0$ \\
\hline 0.01 & $\operatorname{mig}$ & cells & - UITHMIN EJ \\
\hline 1.25 & $\operatorname{mg}: \bar{s}$ & $c e 11 s$ & $-k^{\prime} I$ \\
\hline 0.89 & Mğ & cells & $\cdots N 1 \subseteq 12$ \\
\hline 0.7 .9 & $\mathrm{mo} / \mathrm{g}$ & 00115 & -FeCil $\mathrm{S} .6 H_{2}: 0$ \\
\hline 0.55 & mg:g & cells & $-\mathrm{CaC} 12 . \mathrm{TH} H \mathrm{O}$ \\
\hline 0.54 & $m g i$ & $c \in 115$ & $-H 38153$ \\
\hline (1. . . & $m g / g$ & $\operatorname{cel} 1 \mathrm{~s}$ & $-Z_{n}=04.7 H_{2}^{-} O$ \\
\hline 0.15 & $\operatorname{mg}: g$ & cells & $-M n 504.420$ \\
\hline 7.? & $\operatorname{Lig}: \bar{y}$ & $\therefore=115$ & $-E \cup=04.5 \mathrm{H}^{2} \mathrm{O}$ \\
\hline 5.4 & यद: & cel1s & -NaMOO4. $2 \mathrm{H} 2 \mathrm{O}$ \\
\hline 4.3 & ug & $c \in 11 s$ & -EOCII. EHבO \\
\hline
\end{tabular}

FERMENTATION

TYPE:

Q (O) DF 1 ) ANAEROBIL O) DIF AEROBIC :1)

STAGES

CIONOITIONS

STAGE: GFOWTH PFODUTTION

$\begin{array}{rrr}5 & 3 & \\ 6.5 & 6.5 & \end{array}$

- TEMPERATURE

\begin{tabular}{|c|c|c|}
\hline$\emptyset$ & $=08.6$ & $9 i 1 *$ \\
\hline$\theta$ & 13.0 & \\
\hline ㅍ. & $\therefore 2.4$ & $9 i 1$ \\
\hline 0.28 & 0.050 & $1 / \mathrm{hr}$ \\
\hline 0.00 & 6.53 & $g / 1 * h r$ \\
\hline 5.20 & 0.00 & $g / l * h r$ \\
\hline $21 E$ & 0 & $m M i l * h r$ \\
\hline 5 & 0 & MMIMM \\
\hline
\end{tabular}

-PRODUCT CONCENTAATION IN BEFR

- Before E.traction

* After e.traction

-CELL DENSITY (CHO ONLY)

-DILUTION F.ATE

-PRODUCT PRODUCTIUTTY

-CELL PRDOUCTIUITY :EHO ONLY;

- OXYGEN TRANGFERRED

-OXYGEN FED / OXYGEN STOICH. DEMAND

- 0.1 gil

$5 \quad 5 \mathrm{c}$

-GLUCOSE SPILL.

$-\cdot$

19 lcal/gmol

-COOLING WATER TEMPERATURE

- HEAT EUOL VED-PROUUCT FORMATION

270

I4 Etu/hr*gal

-HEAT REMOUED BY COOLING COILS

FERMENTEFS

$\begin{array}{rr}50,574 & 465,5239 \text { alloris } \\ 15 & 15 \% \text { gross } \\ 69.999 & 759,655 \text { ga11ons } \\ 100.000 & 250,000 \text { gallons } \\ 0 . ? & 3.0 \text { units }\end{array}$

FPOUUT SEPAFATION

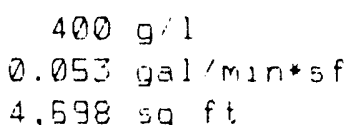

PFOOUCT FECQUERY \& PURIFICATION

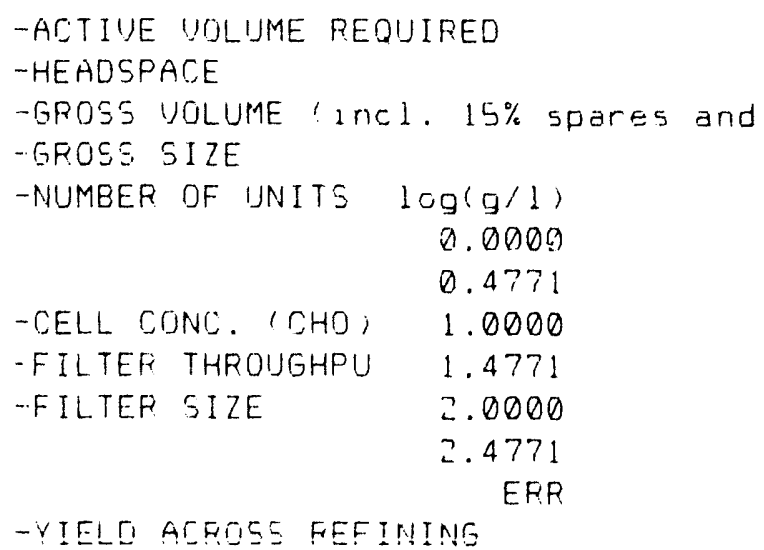


SINGLE-STAGE INSITU EXTRACTIVE FERMENTATION UF EUTANUL

NO RECOUERY OF RAFFINATE CHEMISALS

BASECASE STOICHIOMETRY

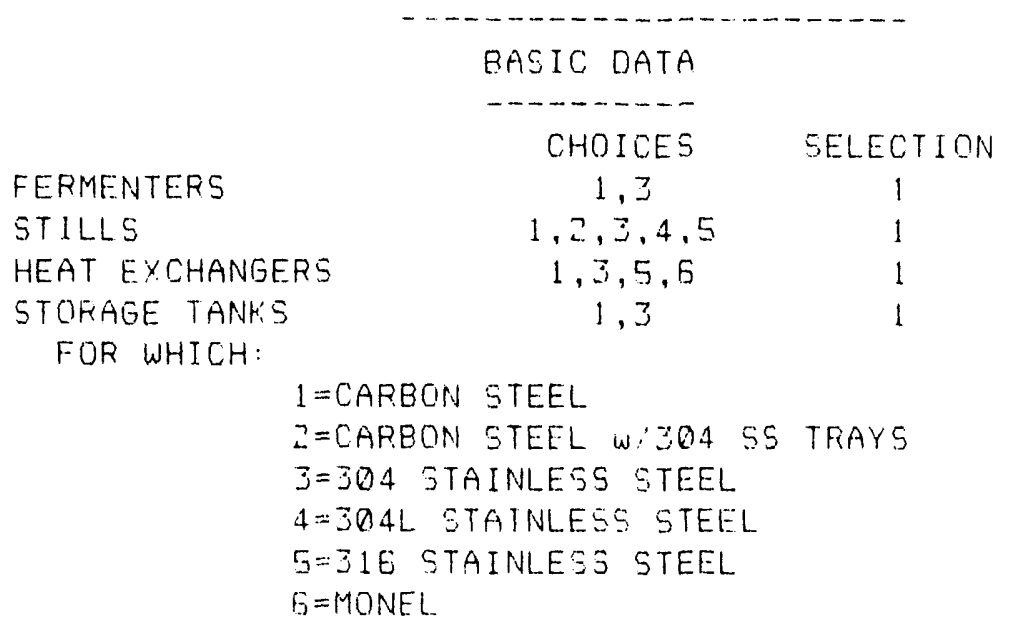

RETURN ON INUESTMENT

To Calculate Selling Price Required to Provide a Fised Retirn, Enter the Desired Fieturn on Investment: $30 \%$ OP

To Calculate the ROI Resultirg from a Flaed Marlet Priae. Enter the Martet Price for 1985 : ilt.

Enter an lrivestment Contingency to Represent.

thie fist Level of the Bajle Data $30 \%$

\section{VENTURE TIMING}

Midpoint of Construction (1.e. 19xX) 1984

Dperatirig Year (1.e. 19xx) 190

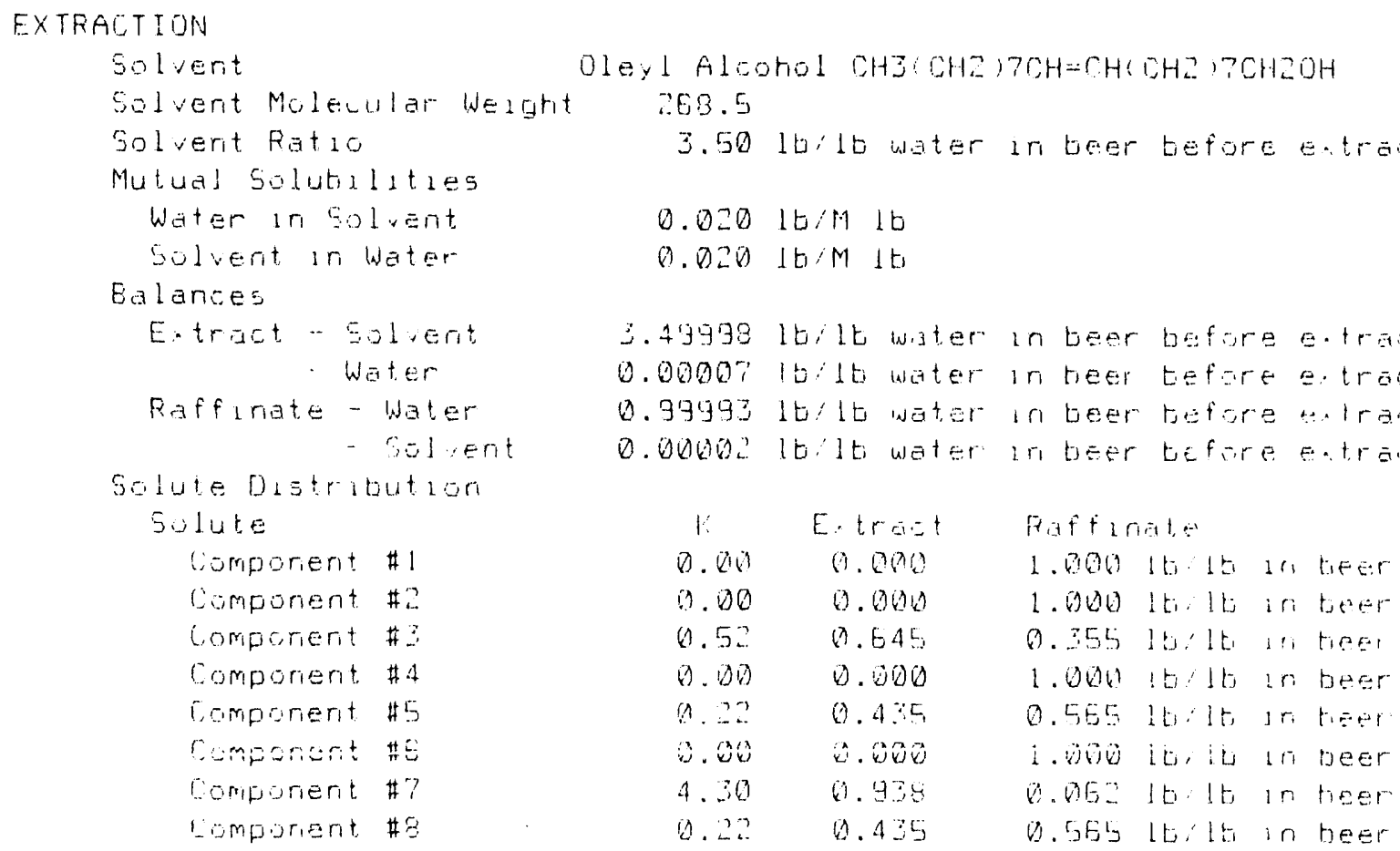




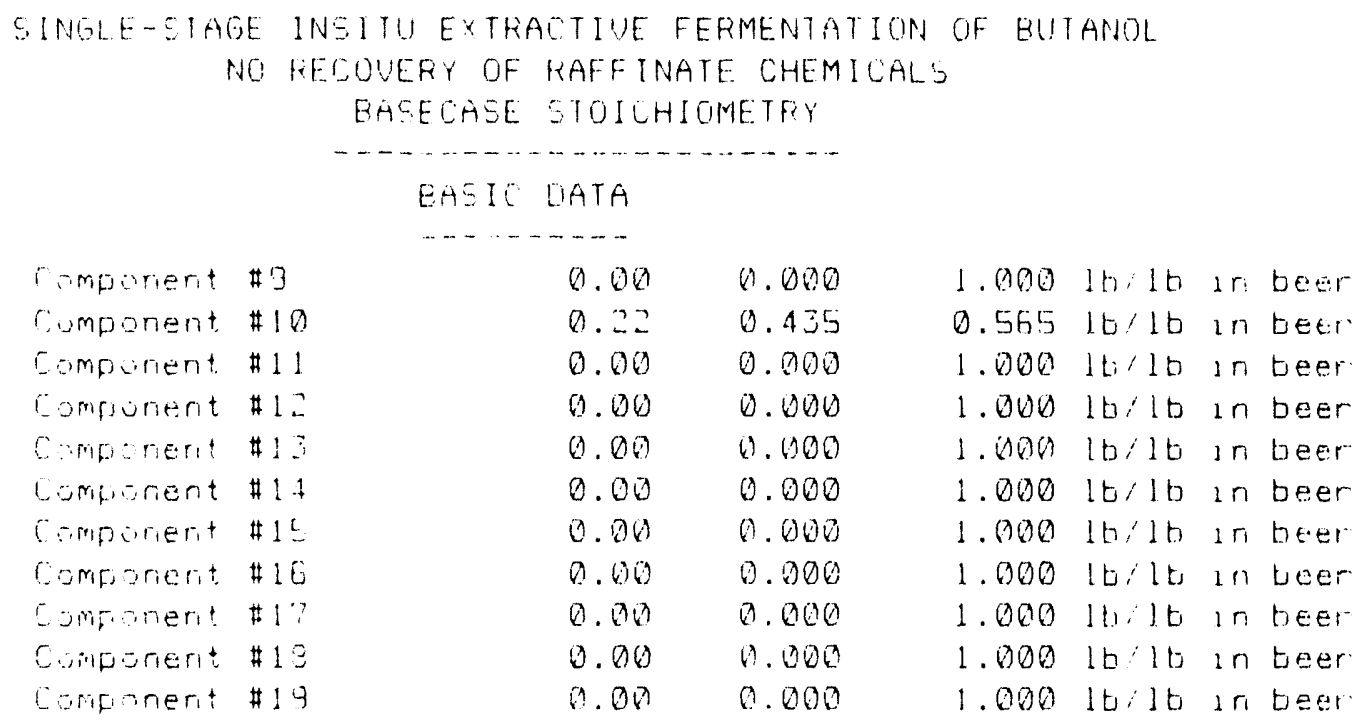




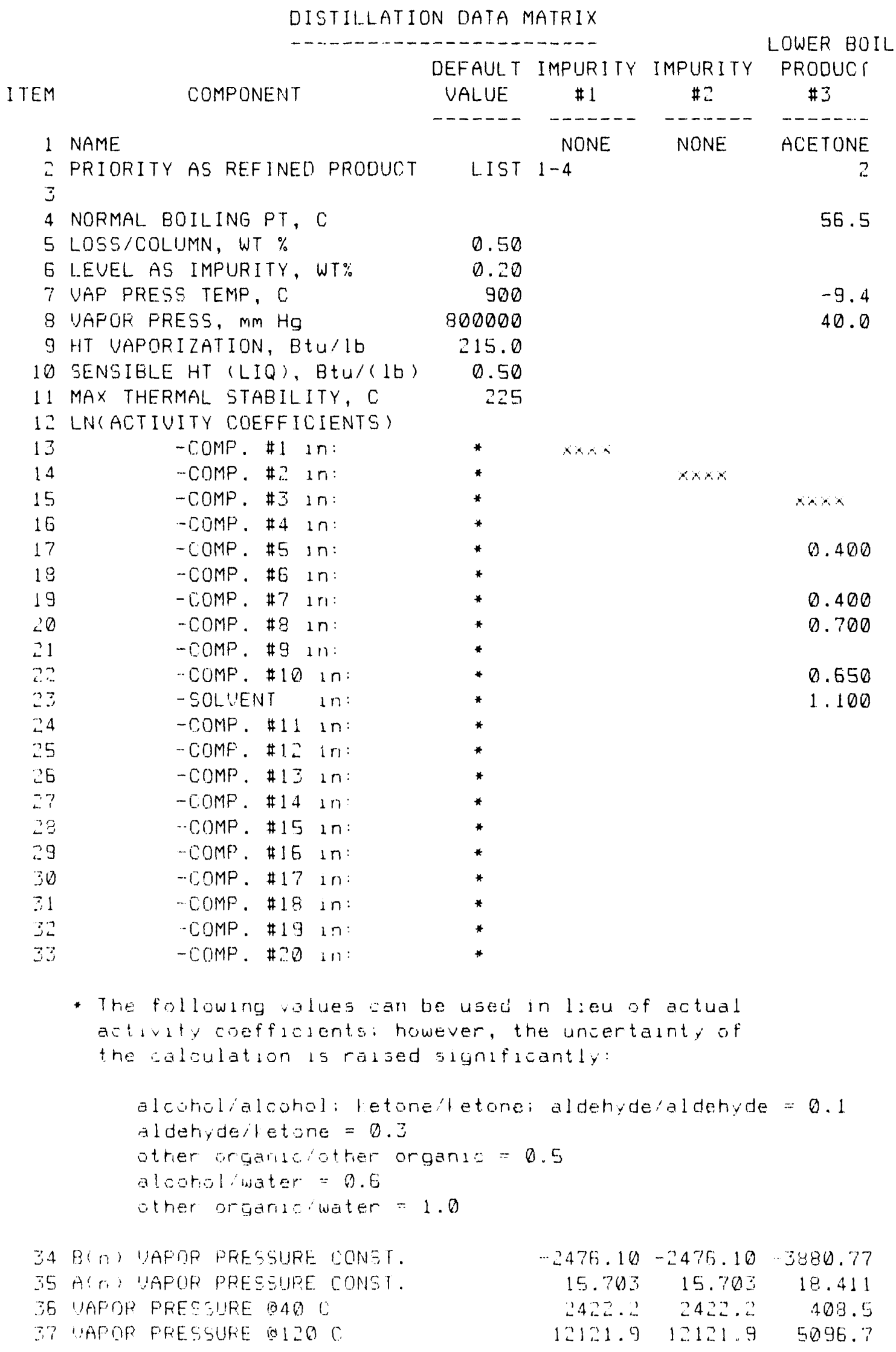




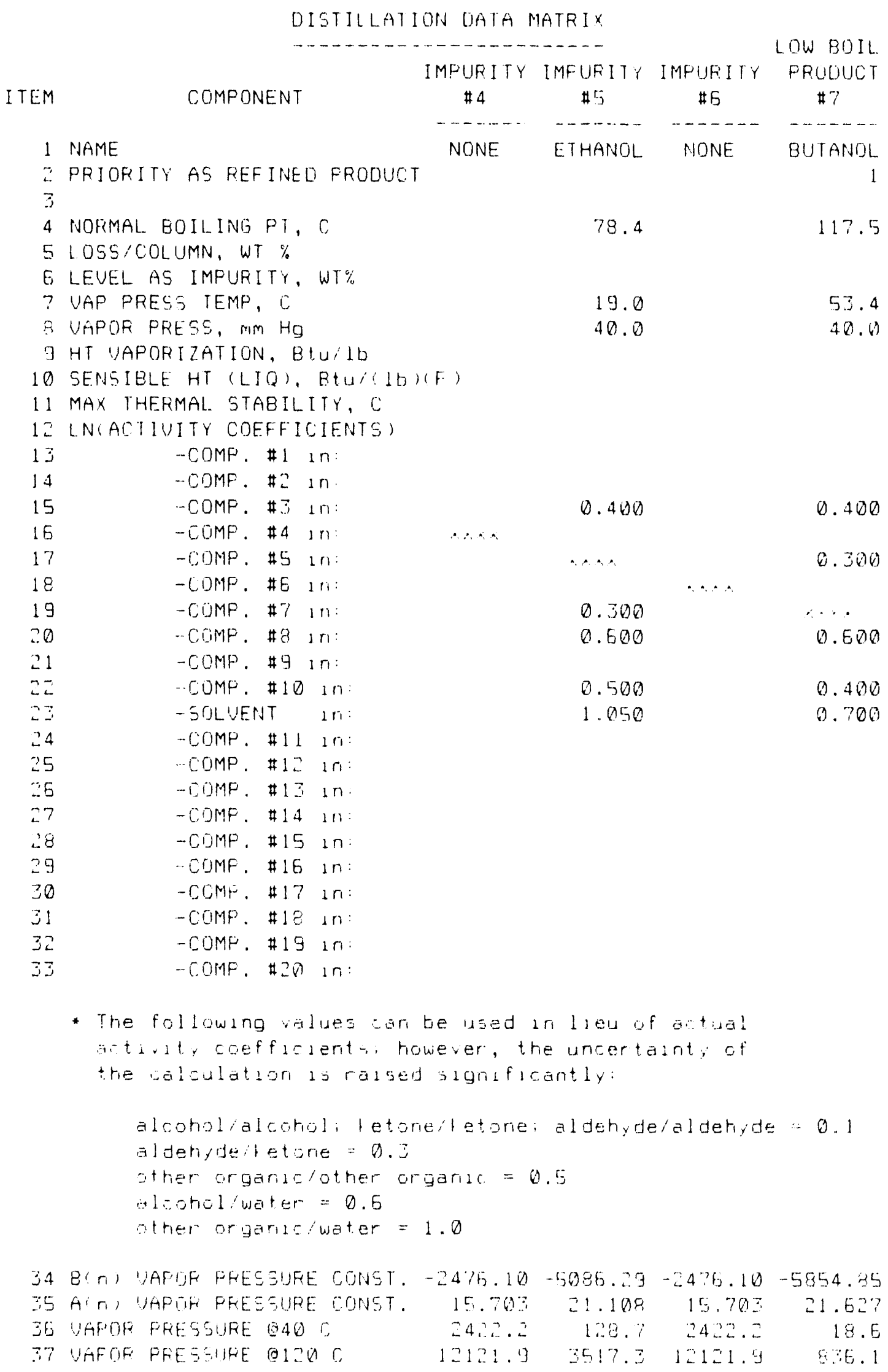




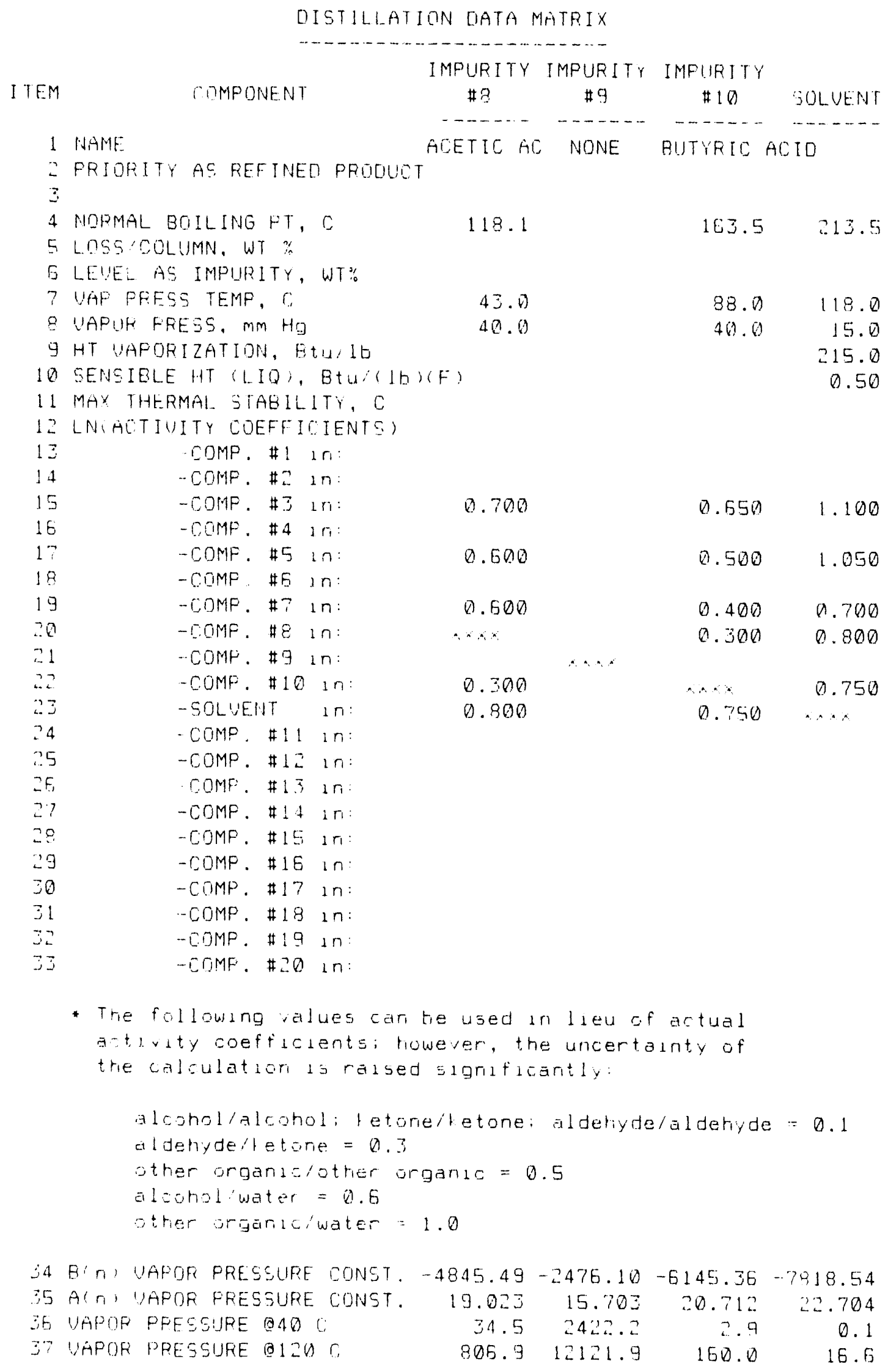



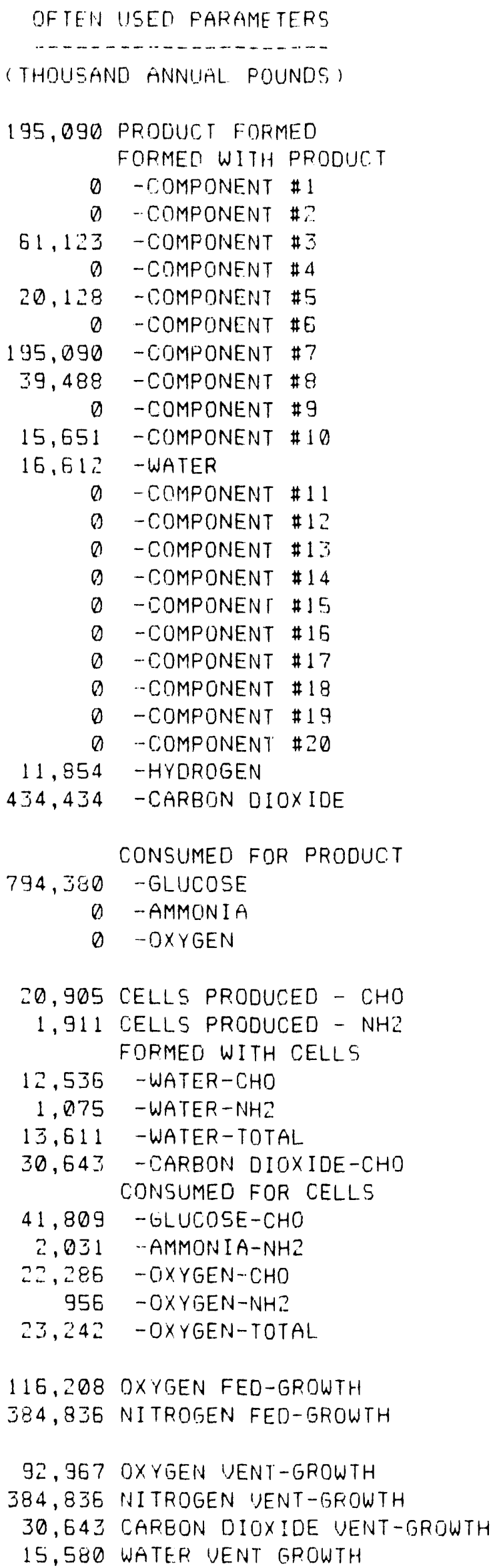


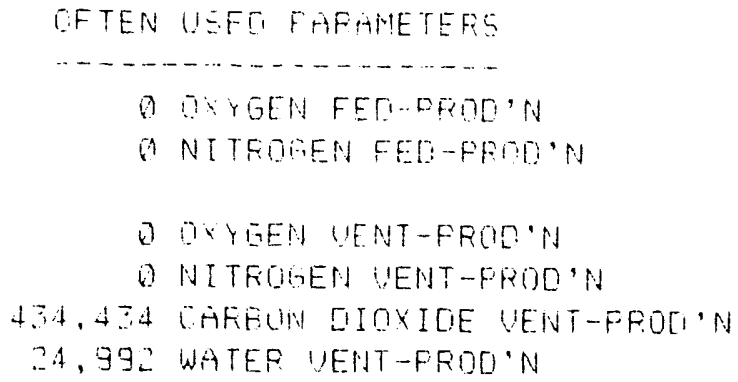




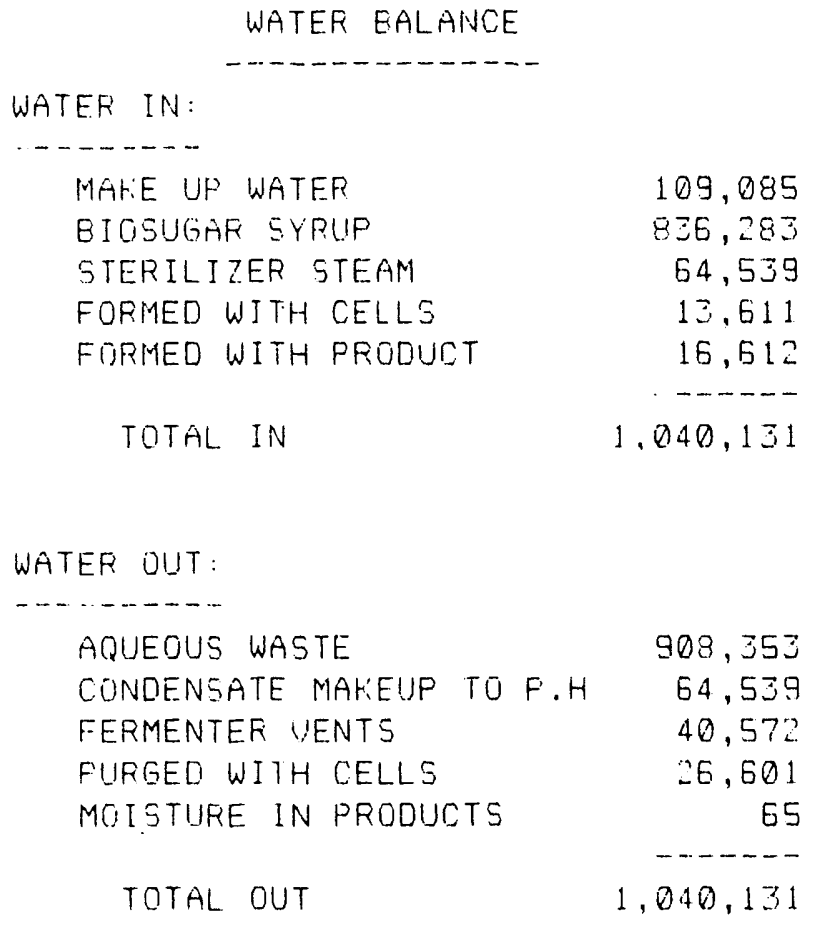


SINGLE-STAGE INSITU EXTRACTIUE FERMENTATION OF BUTANOL

NO RECOUERY OF FAFFINATE CHEMICALS

BASECASE STOICHIOHETRY

MATERIAL BALANCE FLOWSHEET

THDUSAND ANNUAL. POUNDS (JZO DAIS) E CAPACITY

\begin{tabular}{|c|c|c|c|c|c|}
\hline STREAMM & $\begin{array}{c}1 \\
\text { CORN } \\
\text { SYRUP }\end{array}$ & $\begin{array}{c}2 \\
\text { ANHYI } \\
\text { AMMONTA }\end{array}$ & $\begin{array}{c}3 \\
\text { NUTRIENTS }\end{array}$ & $\begin{array}{c}4 \\
\text { MIX } \\
\text { WATEF }\end{array}$ & $\begin{array}{c}5 \\
\text { MIXEO } \\
\text { MEOIUA }\end{array}$ \\
\hline ST KEEMM & - Sim & (........... & $\ldots \ldots$ & WHIR.K & ME: \\
\hline CELLS - CHO & 0 & 0 & $\theta$ & Q & 0 \\
\hline$-N H 2$ & $\emptyset$ & D) & $\theta$ & a & 0 \\
\hline -MINERALS & 0 & a & 0 & $\emptyset$ & D \\
\hline -TOTAL & 0 & 0 & 0 & $\theta$ & i) \\
\hline COMPONENT \#1 & 0 & 0 & 0 & 0 & 0 \\
\hline COMPONENT \#Z & $\theta$ & 0 & 0 & (i) & a \\
\hline COMPONENT \#. & 0 & $\theta$ & 0 & $\emptyset$ & 0 \\
\hline COMFONENT \#4 & 0) & 0 & 0 & n & 0 \\
\hline COMPONENT \#5 & 0 & 0 & $\theta$ & 0 & 0 \\
\hline COMPONENT \#- & $\theta$ & 0 & $n$ & 8 & i \\
\hline COMPONENT \#7 & (i) & 0 & 0 & $e$ & 0 \\
\hline COMPONENT \#Q & 0 & $\theta$ & 0 & 8 & 0 \\
\hline COMPONENT \#G & i) & $\theta$ & 0 & (1) & 0 \\
\hline COMFONENT \#10 & 0 & $\emptyset$ & $\theta$ & 0 & (1) \\
\hline COMFONENT \#11 & 0 & $\theta$ & $\theta$ & 8 & $n$ \\
\hline COMPUNENT \#12 & 0 & 0 & $\theta$ & n) & 9 \\
\hline COMPONENT \#13 & 0 & 0 & 0 & 0 & 0 \\
\hline COMPONENT \#14 & 9 & $\theta$ & 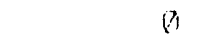 & 0 & $n$ \\
\hline COMFONENT $\# 15$ & $\theta$ & 0 & 0 & $D$ & a \\
\hline COMPONENT \#16 & 0 & 0 & (1) & (a) & (1) \\
\hline COOMFONENT $\# 17$ & 0 & 0 & 0 & $\theta$ & $\theta$ \\
\hline COMPONENT \#18 & $\theta$ & 0 & 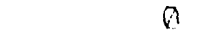 & a & a \\
\hline CODMPONENT \#19 & 0 & D) & 0 & 0 & 0 \\
\hline COMPONENT $\#: O$ & D & $\theta$ & (3) & 0 & $\pi$ \\
\hline GLUEDSE & 535,233 & 0 & D & (1) & 936.29. \\
\hline AMMONIÁ & 0 & 2,031 & $\emptyset$ & 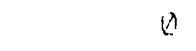 & $\theta$ \\
\hline PHOSPHORLO ALIO & $\emptyset$ & 0 & 1,691 & () & 1.671 \\
\hline FOTAESIUM CHIOORIDE & $a$ & a & 1.288 & (1) & 1.284 \\
\hline MINOR NHTFIENTS & 0 & 0 & 594 & 0 & 50.4 \\
\hline WATEF & 836.283 & 0 & 0 & 44.5 .17 & 380,820 \\
\hline SOLVENT & $\theta$ & $\lambda$ & $\theta$ & 0 & 0 \\
\hline EAFEON DIOXIOEE & () & $\nabla$ & $n$ & $n$ & a \\
\hline DXYGEN & 0 & $\theta$ & 0 & Q) & 0 \\
\hline NITFRGEN & $(\pi$ & $\theta$ & 0 & (1) & 8 \\
\hline HYDFOGEN & $\theta$ & (1) & a & ? & h \\
\hline$\ldots$ & $\ldots \ldots$ & $\ldots \ldots$ & $\ldots \ldots$ & (n) & $\cdots \cdots$ \\
\hline $\begin{array}{l}\text { GPAND TOTAL. } \\
\text { CHECH ON TOTAL }\end{array}$ & $i, 572,567$ & $\therefore 021$ & $3,5 F_{3} 3$ & $44.54^{-}$ & $1,720.675$ \\
\hline TFMPEPATUFE, & 20 & 20 & 20 & $\therefore 0$ & $\therefore 0$ \\
\hline PFESSUFE, PSIA & 14.7 & 14.7 & 14.7 & $\therefore 0$ & 25. \\
\hline STATE & $50 \mathrm{~L}$ H & Lioula & GOLIOE & 1.1010 & \\
\hline
\end{tabular}


SINGLE-STAGE INSITU EXTRACTIUE FERMENTATION OF BUTANOI.

NO RECOUERY OFF RAFFINATE CHEMICALS

BASECASE STOICHIOMETRY

ITTERIAL BALANCE FLOWSHEET

THOUSAND ANNUAL POUNOS (J3O DAYS) (O CAPACITY

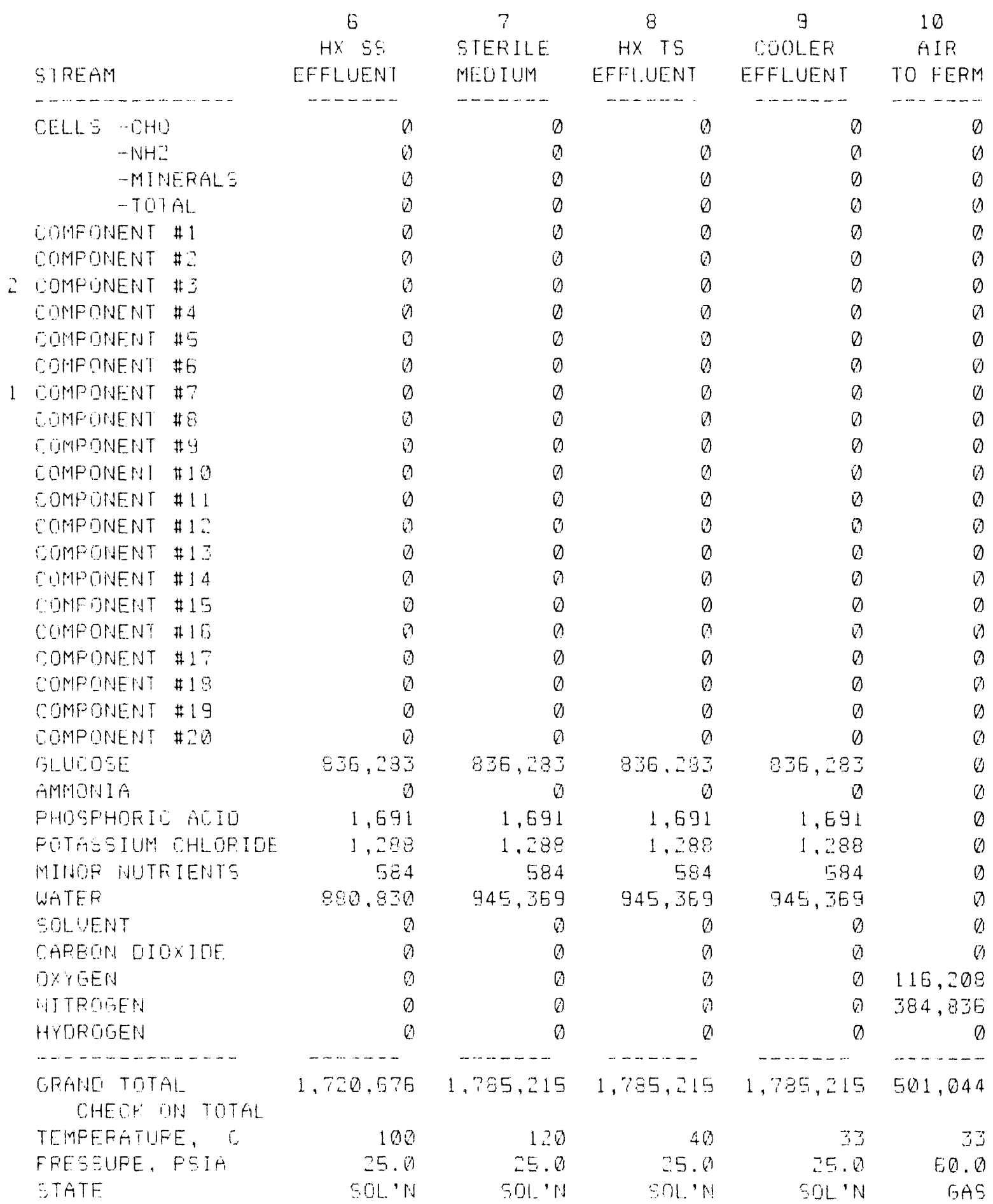


SINGLE-STAGE INSITU EXTRACTIVE FERMENTATION OF BUTANOL NO RECDUERY OF RAFFINATE CHEMICALS

BASECASE STOICHIOMETRY

MATERIAL. BALANCE FLOWSHEET

THOUSAND ANNUAL POUNDS ( 330 DAYS) CAPACITY

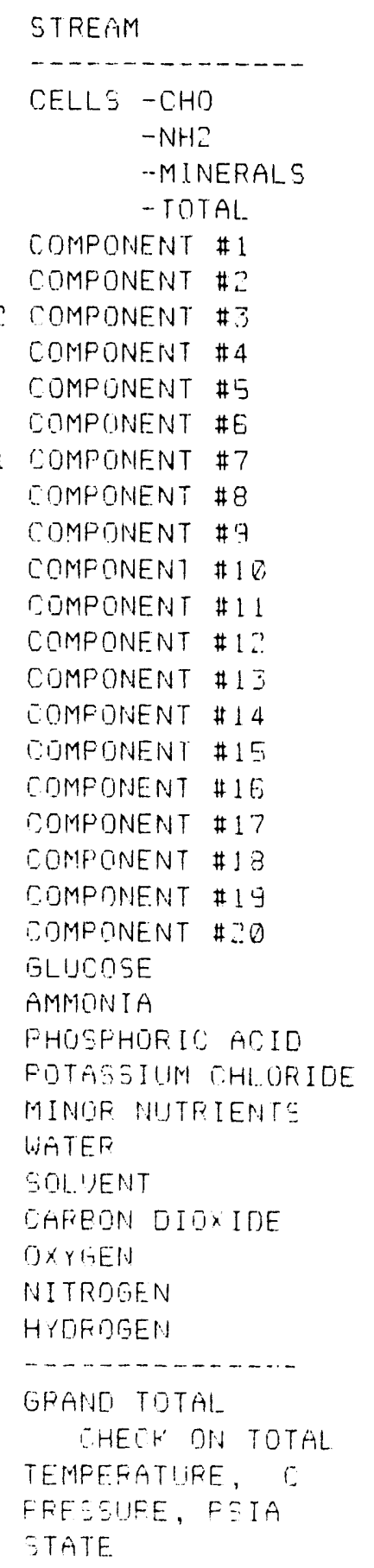

11

COMBINED

FEED

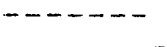

0

$8.36,283$

2,031

$1, E 91$

1. 288

584

945,369

1

115,209

394,830

……

.., 288,290

33

..
12

BEER

$\# 1$

20,905

1,911

584

23,400

$\emptyset$

0

$\emptyset$

$\emptyset$

0

0

$\theta$

0

0

$\theta$

0

0

0

0

0

0

$\theta$

$\theta$

0

794,474

1,691

1. 288

0

943,399

0

0

0

0

-........

$1,764,252$

33

44.7

SLUFAY
13

BEER

$\# 2$

20.905

1,911

584

23,400

$\theta$

61,123

20,128

195,090

39488

0

15,651

0

0

0

0

0

$\theta$

$\theta$

0

94$$
1.691
$$

1,298

$\theta$

935.020

$\theta$

0

0

.......

$1,292,973$

32

44.7

SLIJFFr

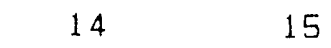

VENT VENT

GROWTH PROO'N

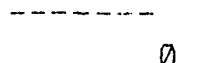

$\cdots \cdots$

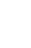

)

$\emptyset$

$\theta$

$\theta$

0

0

$\theta$

0

0

0

0

0

0

0

0

$\theta$

$\theta$

ก

$\theta$

$\theta$

$\theta$

$\theta$

$n$

0

0

0

0$$
15,580 \quad 24,992
$$

30,543

92, 967

$384,83 E$

0

524,025

11,854

471,230

33

14.7

GAS 
SINGLE-STAGE INSITU EXTRACTIVE FERMENTATION OF EUTANOL

NO RECOUERY DF RAFFINATE CHEMICALS

BASECASE STOICHIOMETFY

MATERIAL BALANCE FLOWSHEET

THOUSANO ANNUAL PQUNOS ( 330 DAYS) (C CAPACITY

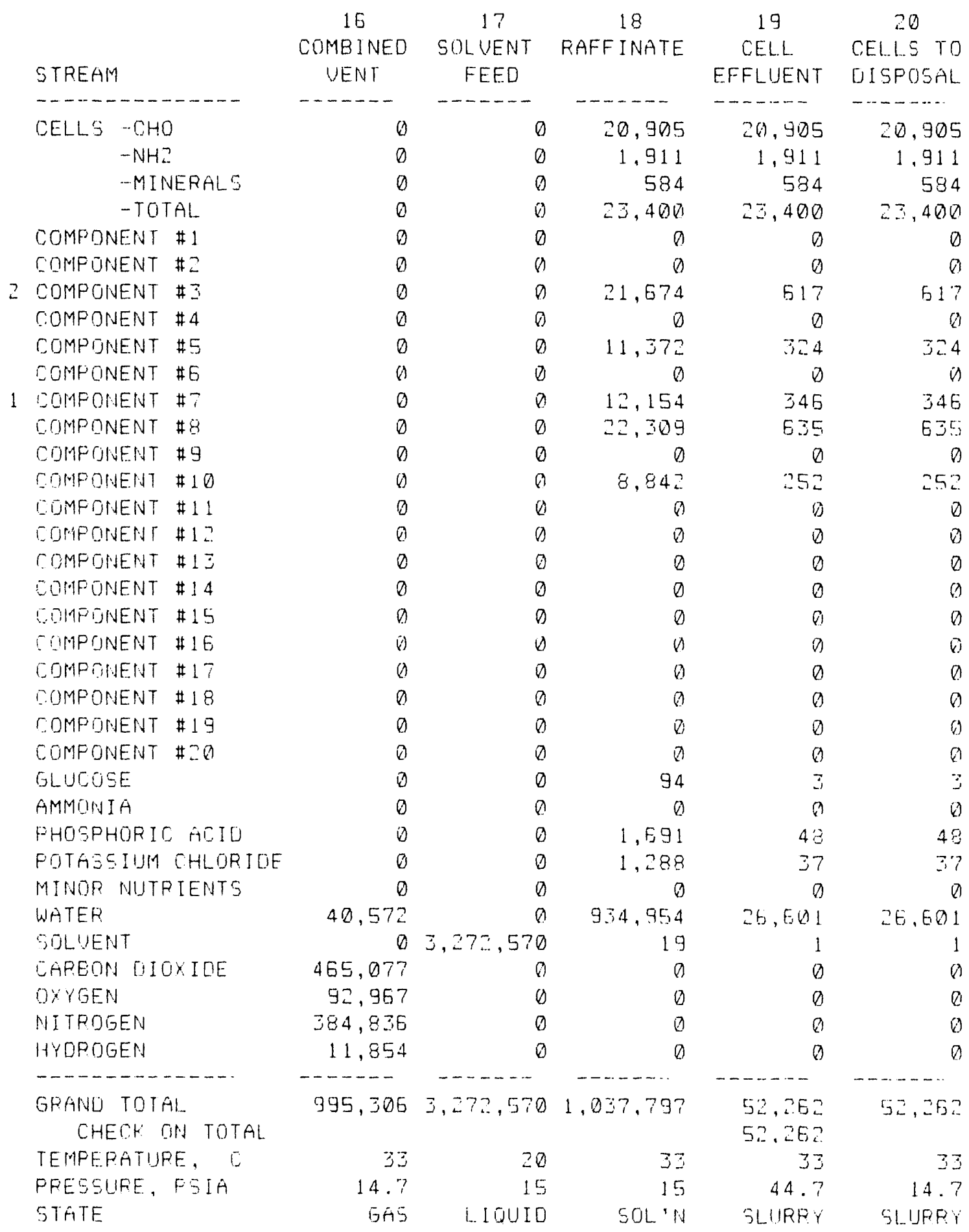


SINGLE-STAGE INSITU EXTRACTIUE FFRMENTATION OF EUTANOL

NO RECOUEFY OF FAFFINATE CHEMICALS

EMSECASE STOICHIOHETFY

MATERIAL BAL.ANCE FLOWSHEET

THOUSAND ANNUAL POUNDS 330 DAYS: E CAPACITY

\begin{tabular}{|c|c|c|c|c|c|}
\hline & 21 & 22 & 23 & 24 & 2.5 \\
\hline & FILTRATE & & BEER \# 1 & BEER \#1 & BEER \#1 \\
\hline STREAM & TO WASTE & EXTRACT & FEED & MAKE & TAILS \\
\hline$\ldots$ & $\ldots \ldots$ & 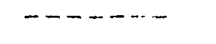 & $\ldots-\cdots$ & $\cdots \cdots$ & \\
\hline CELLS - CHO & $\theta$ & 0 & $\theta$ & 0 & $\emptyset$ \\
\hline$-N+22$ & $\emptyset$ & (1) & $\emptyset$ & $\emptyset$ & Q \\
\hline -MLNEFALS & $\theta$ & 8 & $\emptyset$ & $\theta$ & $\theta$ \\
\hline -TOTAL & $\theta$ & 0 & 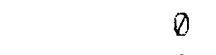 & 0 & 0 \\
\hline COMPONENT \#! & $\emptyset$ & 0 & $\theta$ & 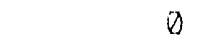 & 0 \\
\hline COMPONENT \#Z & $\theta$ & 0 & 0 & $\theta$ & $\emptyset$ \\
\hline COMPONENT \#Z & 21,057 & 33,449 & 39,449 & 39.449 & $\theta$ \\
\hline COMPUNENT \# 4 & 0 & 0 & $\emptyset$ & 0 & $n$ \\
\hline COMPONNENT \#5 & 11,048 & $8,75 ?$ & 8,757 & 8,757 & $\emptyset$ \\
\hline COMFONENT \#G & 0 & 0 & $\emptyset$ & 0 & $\emptyset$ \\
\hline COMPONENT \#? & 11,909 & 182.936 & 201,138 & 200,223 & 915 \\
\hline COMFONENT \#Q & $\therefore 1,674$ & 17,179 & 17.215 & 17,128 & 16,815 \\
\hline COMPONENT \# & $\theta$ & $Q$ & $\emptyset$ & $\theta$ & 0 \\
\hline COIMPONENT \#10 & 8.591 & 6.809 & 6,845 & 5,811 & $E, 445$ \\
\hline COMFONENT \#11 & $\emptyset$ & $\theta$ & 0 & $\theta$ & $\theta$ \\
\hline EOMPONENT \#1? & $\theta$ & $\theta$ & $\emptyset$ & $\emptyset$ & $\theta$ \\
\hline EOMFONENT \#IJ & $\theta$ & $\emptyset$ & $\emptyset$ & 0 & 0 \\
\hline COMFUNENT \#14 & $\theta$ & 0 & $\emptyset$ & $\emptyset$ & $\emptyset$ \\
\hline COMFONENT \#15 & 0 & $\theta$ & $\theta$ & 0 & $\theta$ \\
\hline COMPONENT \#15 & $\theta$ & $\emptyset$ & 0 & $n$ & 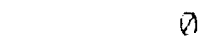 \\
\hline COMFONENT \#17 & D & 0 & 0 & $\emptyset$ & 0 \\
\hline COMPONENT \#18 & $\emptyset$ & $\theta$ & 0 & $\emptyset$ & Q) \\
\hline EOMPONENT \#19 & $\theta$ & $\emptyset$ & $\emptyset$ & 0 & $\theta$ \\
\hline COMPUNENT $\#=0$ & $\theta$ & 0 & $\emptyset$ & 0 & 0 \\
\hline $\begin{array}{l}\text { GLUCOSE } \\
\text { AMMUNIA }\end{array}$ & 91 & $\theta$ & $\theta$ & $\theta$ & $\theta$ \\
\hline \multirow{2}{*}{$\begin{array}{l}\text { AMMONIA } \\
\text { PHOSPHOFIC ACIO }\end{array}$} & $\emptyset$ & 0 & $\theta$ & 0 & $\emptyset$ \\
\hline & 1,643 & 0 & 0 & $\emptyset$ & 0 \\
\hline POTASSIUM CHLORIDE & 1,251 & 0 & $\theta$ & 0 & Q) \\
\hline MINOR NUTRTENTS & $\theta$ & $\theta$ & $\emptyset$ & 0 & (1) \\
\hline WATER & 908,353 & 65 & 65 & 65 & $\emptyset$ \\
\hline SOLUENT & 18 & $3.272,551$ & $3,472,410$ & 200.223 & $3,272,187$ \\
\hline CAREON DIOXIDE & 0 & 0 & $\emptyset$ & $\theta$ & $\emptyset$ \\
\hline OXYGEN & $\emptyset$ & $\theta$ & $\emptyset$ & $\emptyset$ & $\theta$ \\
\hline NITROGEN & $n$ & 0 & $\emptyset$ & $\theta$ & $\theta$ \\
\hline HYOROGEN & $\theta$ & 0 & $\emptyset$ & 8 & 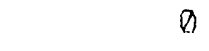 \\
\hline$\ldots$ & $\cdots \cdots-\cdots$ & $\cdots \cdots-\cdots$ & $\cdots \cdots$ & ----- & $\cdots-\cdots$ \\
\hline $\begin{array}{l}\text { GRAND TOTAL } \\
\text { CHECK ON TOTAL }\end{array}$ & 985,535 & $3,527,746$ & $3,745,879$ & 472,658 & $3,295,361$ \\
\hline TFMPEFATUFE, C & 33 & 33 & -- & 94 & 220 \\
\hline FRESSURE, FISIA & 14.7 & 14.7 & $\cdots$ & $\begin{aligned} 12.1 \\
001\end{aligned}$ & $\begin{aligned} 12.9 \\
601 . N\end{aligned}$ \\
\hline STATE & SOL'N & SOL.N & SOL'N & SOL'N & SOL'N \\
\hline
\end{tabular}




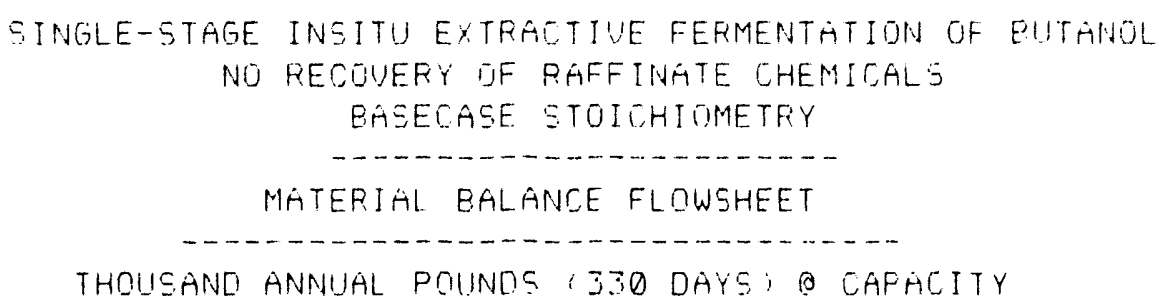

THOUSAND ANNUAL POUNOS 330 DAYS: O CAFACITY

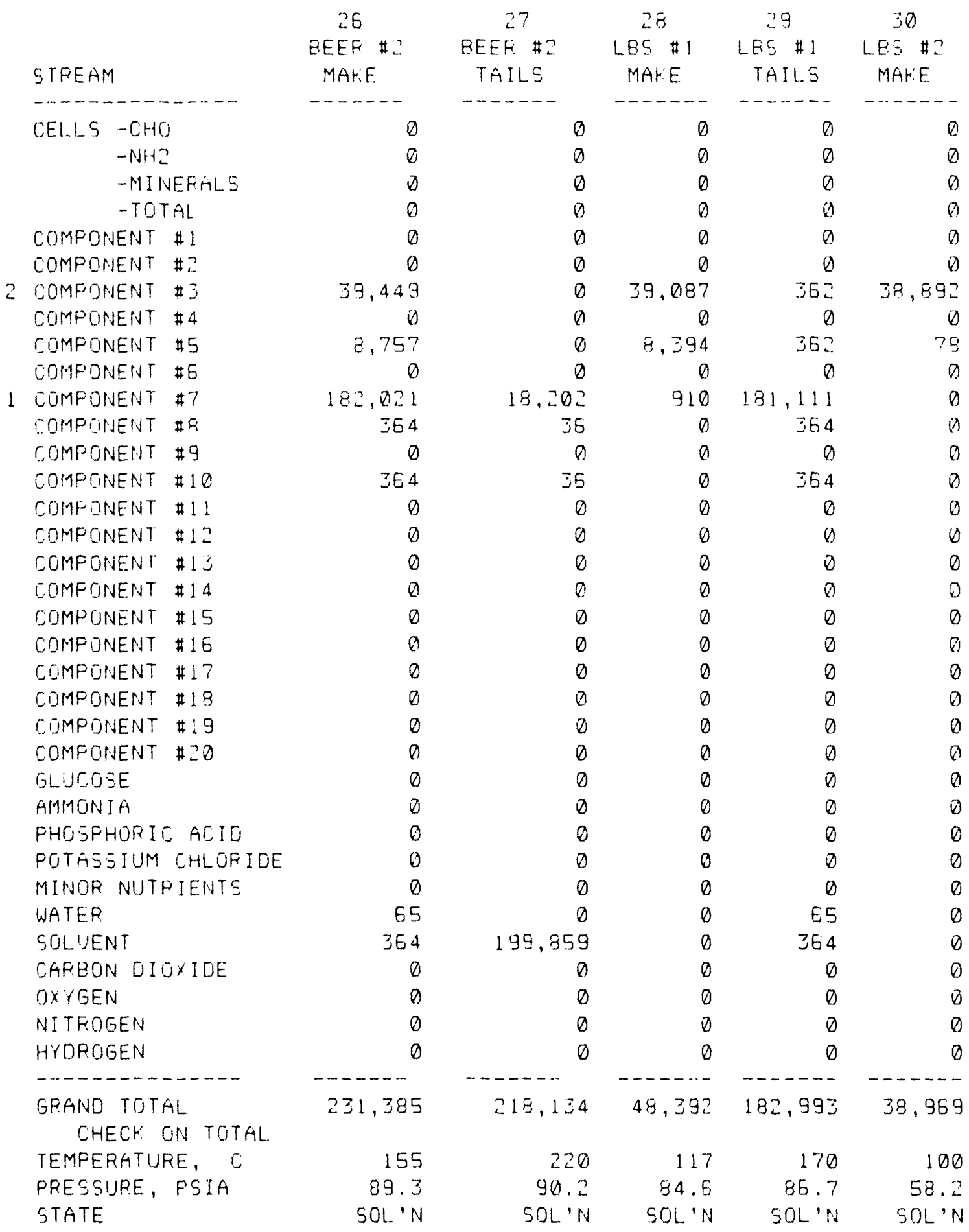




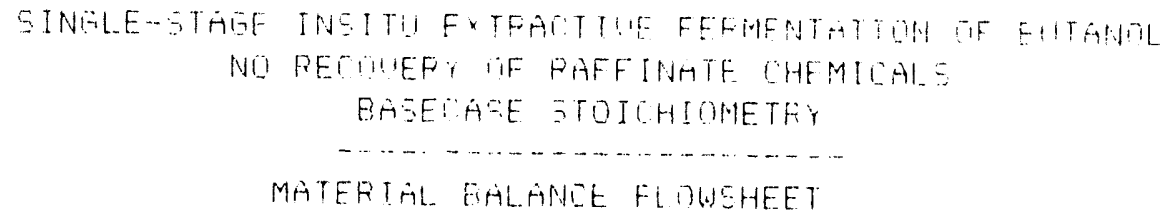

\begin{tabular}{|c|c|c|c|c|c|}
\hline STREAM & TAILS & 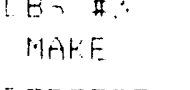 & $\begin{array}{l}\text { T.ESE } \\
\text { TAILE }\end{array}$ & $\begin{array}{l}\text { HES \#1 } \\
\text { MḦFE }\end{array}$ & $\begin{array}{l}\text { HE: } \\
\text { TAILS }\end{array}$ \\
\hline ELLO $-\mathrm{L} H \mathrm{HO}$ & 0 & & & & \\
\hline 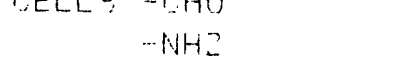 & 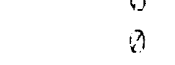 & b) & bi & 3 & \\
\hline MINEFIALS & n & i) & n & i & \\
\hline -TOTAL & 0 & 0 & $n$ & s & \\
\hline COMFINANAT \# 1 & D & $\theta$ & $\theta$ & (1) & \\
\hline COMPONENT \#? & ⿵ & 0 & $n$ & ? & \\
\hline COMFONENT \#Z & 195 & n & $\theta$ & (1) & \\
\hline COMPONENT \#4 & 0 & 0 & (1) & (1) & \\
\hline OOMPONEN $\# S$ & 9,217 & $\theta$ & 0 & 0 & \\
\hline CONHENENT \#6 & i) & $\emptyset$ & $n$ & 0 & \\
\hline COMFONENT \#? & 910 & (3) & 0 & 915 & \\
\hline CONFONENT HO & 0 & (1) & (7) & $16,-21$ & $\therefore$ \\
\hline COMPGNENT \#9 & $\theta$ & 0 & i) & 0 & \\
\hline COMPONENT \#LA & $\theta$ & 0 & $n$ & $6.41:$ & \\
\hline COMPONENT \#11 & $\theta$ & D & i) & 0 & \\
\hline CONPONENT \#3? & 0 & i) & () & 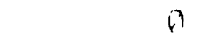 & \\
\hline IOMFONENT $\# 13$ & 0 & () & (3) & $a$ & \\
\hline ZMFONENT \#14 & 0 & 3 & 0 & 0 & \\
\hline COMFONENT \#15 & (]) & (2) & (1) & 0 & \\
\hline CIMFONENT \#IE & (1) & (1) & 0 & $n$ & \\
\hline OIMPONENT \#1? & 0 & $\theta$ & $n$ & (1) & \\
\hline COMFONENT \#13 & ? & $\emptyset$ & $n$ & (i) & \\
\hline COMPONENT \#19 & 6 & 1] & 0 & i & \\
\hline UMFONENT \#2O & $n$ & 0 & Q & $n$ & \\
\hline Gucose & $\theta$ & 0 & 0 & $\theta$ & \\
\hline AMMONIA & $\theta$ & $\theta$ & 0 & $\theta$ & \\
\hline FHOSFHOFIC ALE & $\theta$ & n & a & 8 & \\
\hline FOTHESIUM CHLORIOE & 0 & $b$ & 0 & i & \\
\hline MINGR RUIFIENTS & 0 & 6 & ? & Q & \\
\hline МATEF & 0 & n) & 0 & Q & \\
\hline DLUENT & $n$ & D & 0 & 13 & $3, \therefore 7.174$ \\
\hline CAREON UITIIDE & 0 & 0 & $\theta$ & $D$ & \\
\hline CXYGEN & $\theta$ & (7) & 0 & 0 & 0 \\
\hline MITFOGEN & b) & l & $\theta$ & (1) & \\
\hline $\begin{array}{l}\text { HOROESH } \\
\text { HOW }\end{array}$ & a & 0 & 0 & $\theta$ & b \\
\hline 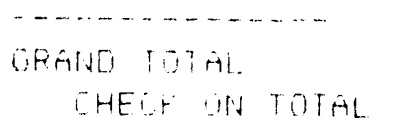 & 9,422 & 0 & i & 24.071 & $\therefore, 272,290$ \\
\hline IEMPERATURE, $\square$ & $1: 0$ & $1273:$ & 120 & 123 & 228 \\
\hline PFESEURE, FSIA & $5 E .3$ & .0 & .0 & 14.3 & $17 .:$ \\
\hline STATE. & $60 \mathrm{~L}$ & $50 L \cdot N$ & $50 L \cdot N$ & $\operatorname{sol}, N$ & SOL' \\
\hline
\end{tabular}




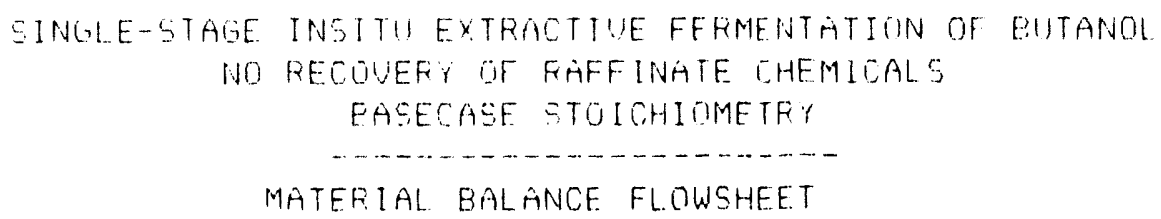

THOUSAND ANNUAL POUNOS 300 TAYS, $\bar{O}$ CAFACITY

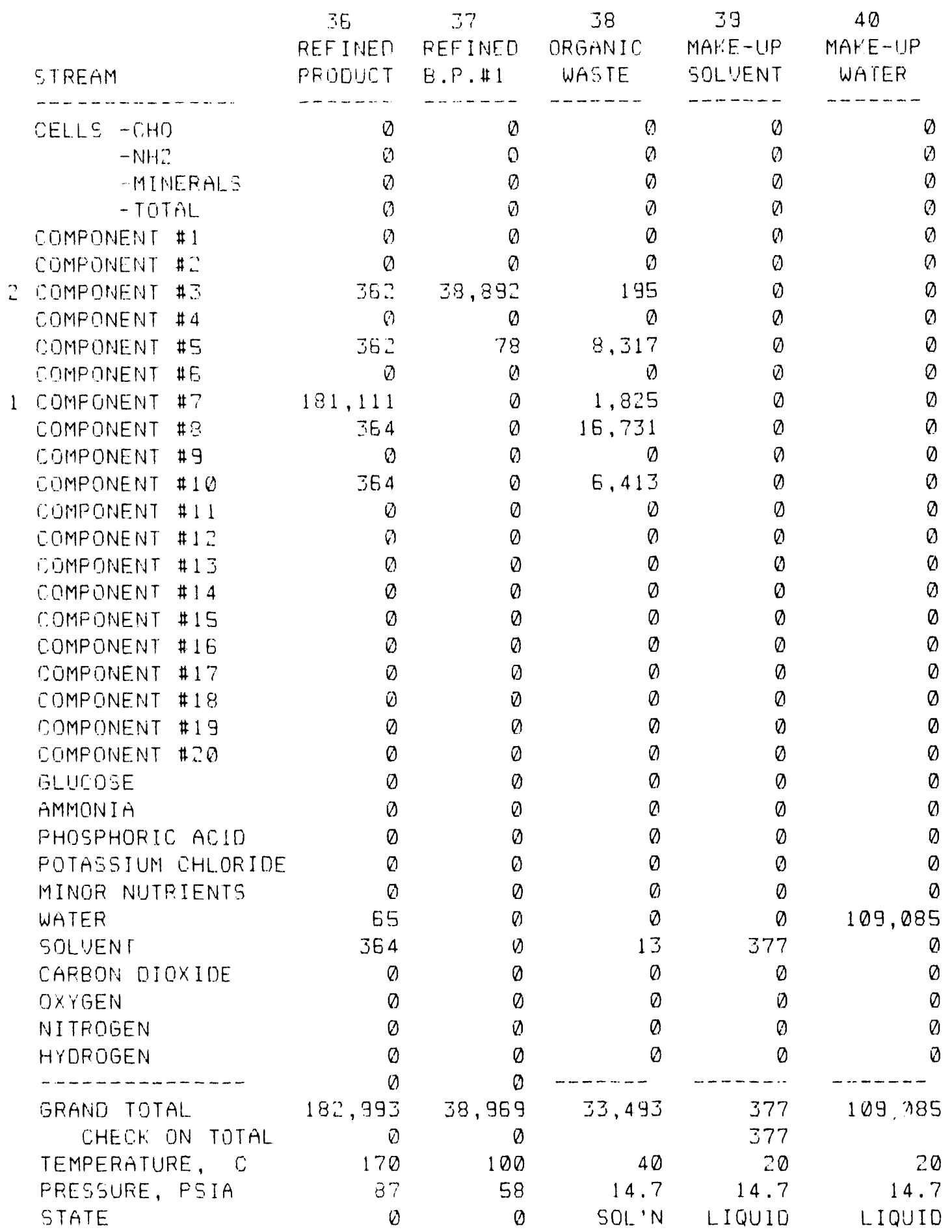


SINGLE-STAGE INSITU EXTRACTIUE FERMENTATIUN OFF EUTANOL

NO RECOUEPY DF FAFFINATE CHEMICALS

BASECASE STOICHIOMETRI

MATERIAL BALANIE FLOWSHEET

THOUSAND ANNUAL POUNGS 330 DAYS 9 CARACITH

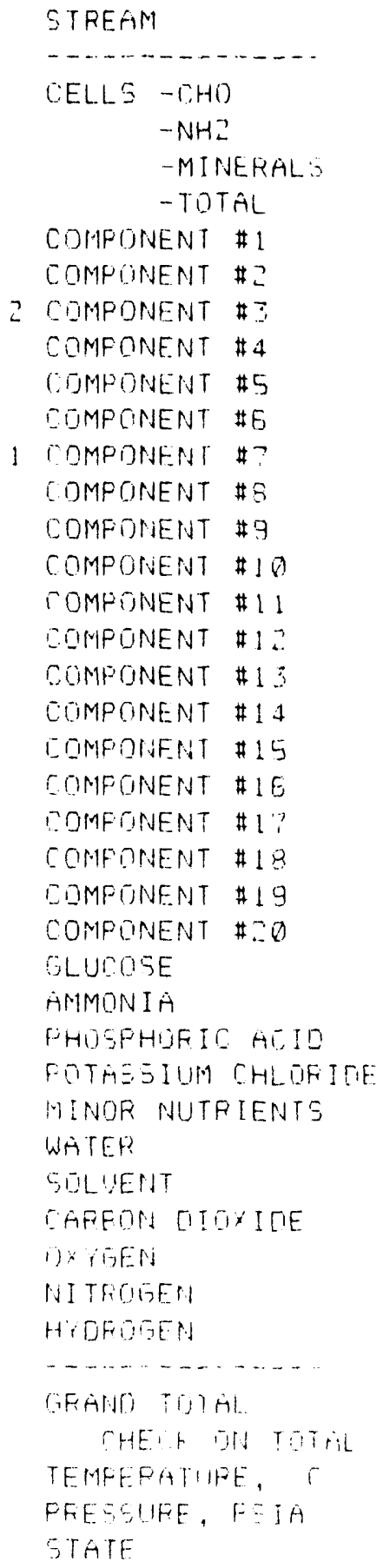

\begin{tabular}{|c|c|c|c|c|}
\hline 41 & 42 & $4 \%$ & 44 & 45 \\
\hline STEFTLE & FFRM' TF & DIST ILL. & GuPN, MU & ME.O!!M \\
\hline STEAM & STEAM & STEAM & F'WF H'CF & COOL WTE \\
\hline$\cdots \cdots$ & $\cdots$ & $\cdots$ & $\ldots \ldots$ & $\cdots \cdots$ \\
\hline$\theta$ & 0 & 0 & 1 & 0 \\
\hline$\theta$ & (1) & $n$ & (7) & 0 \\
\hline$\theta$ & 0 & 0 & $\theta$ & 0 \\
\hline 0 & 6 & $\theta$ & Q & 0 \\
\hline b & 0 & (1) & $\theta$ & O \\
\hline 0 & (1) & a & 6 & (1) \\
\hline 6 & $\theta$ & 0 & 0 & 0 \\
\hline 0 & n & 0 & 0 & 6 \\
\hline 0 & $\theta$ & 0 & 0 & 0 \\
\hline$\theta$ & 0 & 0 & 6 & () \\
\hline (1) & 0 & 0 & (1) & 0 \\
\hline$\theta$ & 0 & a & 0 & in \\
\hline (i) & 0 & 0 & 0 & $\theta$ \\
\hline 0 & 0 & $n$ & n & 0 \\
\hline$B$ & 0 & a & 0 & $D$ \\
\hline D & $\theta$ & (b) & A & 6 \\
\hline 0 & 0 & $a$ & 0 & 0 \\
\hline 0 & 0 & (3) & $b$ & $n$ \\
\hline n & 0 & 0 & v) & D \\
\hline 0 & D & $n$ & $\theta$ & 8 \\
\hline i) & (1) & $\theta$ & 6 & 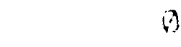 \\
\hline a & $a$ & 0 & 0 & () \\
\hline i) & D) & 0 & 9 & 0 \\
\hline 0 & 0 & 0 & () & $\theta$ \\
\hline 0 & $\theta$ & D & Q & 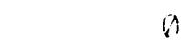 \\
\hline (1) & 0 & 0 & i & $\theta$ \\
\hline (3) & b & $\theta$ & 0 & ( ) \\
\hline a & $D$ & 0 & 6 & $n$ \\
\hline 0 & 3 & 0 & 0 & 0 \\
\hline 64,539 & in & 924,954 & 64.539 & $=35,100$ \\
\hline 0 & 0 & $\theta$ & i) & D \\
\hline$n$ & b) & 8 & 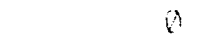 & 6 \\
\hline D) & B) & B & 3 & a \\
\hline (i) & 0 & $n$ & 6 & C \\
\hline 3 & D) & 8 & a & 0 \\
\hline$\cdots$ & $\cdots-\ldots-\cdots-$ & $\ldots \ldots \ldots \ldots$ & $\ldots \ldots$ & $\ldots \ldots$ \\
\hline $54,5: 3$ & 0 & $924.9 \div 4$ & $6,4,539$ & $=\therefore, 100$ \\
\hline$|4|$ & 141 & 136 & $1: 0$ & 4 \\
\hline $54 . \because$ & 64.7 & 164. & $\therefore 0$. & 14 \\
\hline$O A$ & $58 \%$ & $6 r 9$ & $110,1] 1)$ & $110 ! 110$ \\
\hline
\end{tabular}




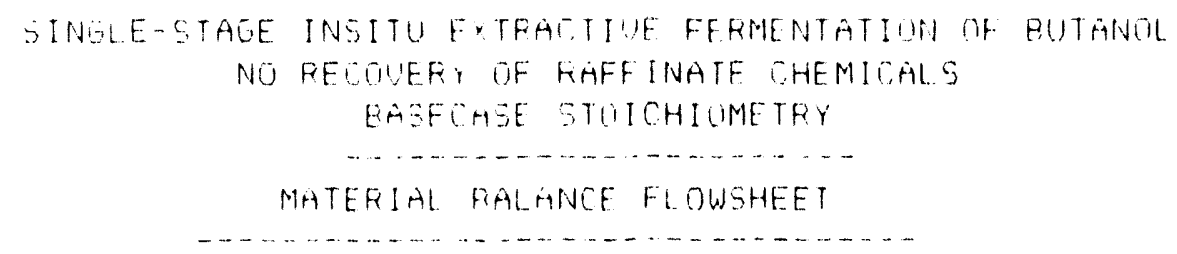

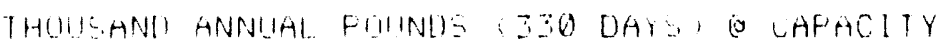

\begin{tabular}{|c|c|c|}
\hline & 46 & 47 \\
\hline & FERM. TF & $0118 T 11 \mathrm{~L}$. \\
\hline STFFriM & COOLL WIR & lOODL WTP \\
\hline 西 & $\ldots \ldots$ & $\ldots$ \\
\hline CtLLE $=-1 H 11$ & 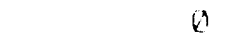 & 0 \\
\hline 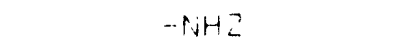 & $\emptyset$ & () \\
\hline MINEFALS & 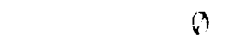 & $\theta$ \\
\hline$-r O T H L$ & 0 & 0 \\
\hline GOMEONENT $\#$ ! & 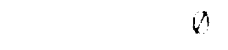 & $n$ \\
\hline COMFONENT H: & () & 0 \\
\hline ? TINPONENT $\# Z$ & $a$ & $\theta$ \\
\hline COMFONENT \#4 & 0 & 0 \\
\hline CIMPONENA $\$ 5$ & (1) & $\theta$ \\
\hline COMPONENT \#E & h & 0 \\
\hline COMHONENT \#? & 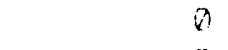 & $\theta$ \\
\hline COMFUNAN! $\#$ S & (1) & $\emptyset$ \\
\hline DOMMENWEIT $\#$ O & $n$ & $\theta$ \\
\hline COMFENANT \#:O & ? & 0 \\
\hline SIMPOHENI \#11 & ()) & $a$ \\
\hline 1. IMFUNENIT \#1 Z & 0 & $\theta$ \\
\hline OAFANENA \#13 & (4) & 0 \\
\hline EOMPONENT \# I & () & 0 \\
\hline COMFONENT \#15 & a & 0 \\
\hline COMPONENT \#1E & ) & $\theta$ \\
\hline OOMFONFIT H1? & () & 0 \\
\hline GOMPONENT HL & 8 & 0 \\
\hline COMF THENT \#19 & i) & 0 \\
\hline COMFONENT \#ZA & $\theta$ & 0 \\
\hline SLUODSE & 0 & 0 \\
\hline AMMONIA & $n$ & $\theta$ \\
\hline PHOSPHOKE RELO & 0 & 0 \\
\hline FOTAS IUM CHLOROTE & $\theta$ & $\theta$ \\
\hline MINTF HUTPIENT: & b) & $\theta$ \\
\hline WFIEF. & $\therefore .0 .49 .059$ & $9,739,159$ \\
\hline GOLIENT & (i) & 0 \\
\hline CAFEDIN DICIXIDF & D & (1) \\
\hline DXrGEN & 0 & $\theta$ \\
\hline NITEOEEN & (1) & 0 \\
\hline HIDFOLEN & D & $n$ \\
\hline$\ldots$ & $\cdots \cdots$ & 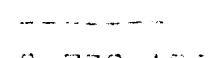 \\
\hline $\begin{array}{l}\text { GFAND TOIAL } \\
\text { CHESH ON TOTAL }\end{array}$ & $1 \therefore, 543,059$ & $9,739,158$ \\
\hline TEMFERATULE, C & 5 & 0 \\
\hline FFEE OUAFE, FSIH & 1.7 .7 & 14.7 \\
\hline STATE & 110010 & LIOUIO \\
\hline
\end{tabular}


$M$ LE. MOLES/ YEAR

M LE. MOLES/YEAR

COMPONENT \#1 COMPDNENT \#? COMPONENT \#3 COMPONENT \#4 COOMPONENT \#S COMPONENT \#G COMPONENT \#? COMPONENT \#Q CUMPONENT \#9 COMPONENT \#10 COMPONENT \#11 COMPONENT \#12 COMPUNENT \#13 CIJMPIJNENT \#14 COMPONENT \#15 COMPONENT \#16 GGMPONENT \#17 COMPONENT \#18 COMMPONENT \#19 COMPONENT \#LO WATER. SOLUEN!

TOTAL. 19 :

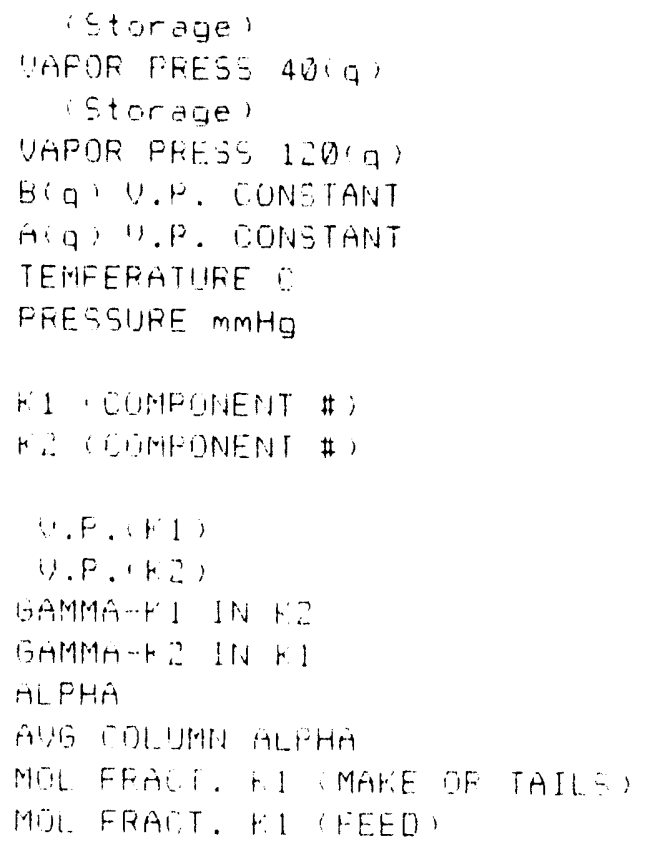

\begin{tabular}{|c|c|c|c|}
\hline \multicolumn{4}{|c|}{ DISTILLATION CALCULATIONS } \\
\hline 22 & 23 & 24 & 25 \\
\hline & BEER \#1 & BEER \#1 & BEER \#1 \\
\hline EXTRACT & FEED & MAKE & TAILS \\
\hline$---\cdots \cdots-$ & $\cdots-\cdots$ & $\cdots \cdots$ & $-\cdots--\cdots-$ \\
\hline \multicolumn{4}{|c|}{ DISTILLATION CALCULATIONS } \\
\hline \multicolumn{4}{|c|}{ 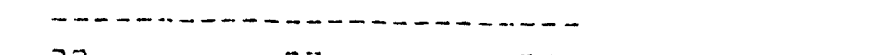 } \\
\hline$\Xi 2$ & 23 & 24 & 25 \\
\hline & BEER \#1 & BEER \#1 & BEER \#1 \\
\hline EXTRACT & FEED & MAKE & TAILS \\
\hline$--\cdots \cdots$ & $-\cdots-\cdots$ & $\cdots-\cdots$ & $\cdots-\cdots$ \\
\hline 0 & $\theta$ & 0 & 0 \\
\hline 0 & 0 & $\theta$ & 0 \\
\hline 079 & 679 & 679 & 0 \\
\hline$\theta$ & $\theta$ & $\emptyset$ & $\theta$ \\
\hline 190 & 190 & 190 & $\theta$ \\
\hline 0 & 0 & $\theta$ & 0 \\
\hline$\therefore 468$ & 2.714 & 2,701 & 12 \\
\hline 286 & 287 & 285 & 280 \\
\hline$\emptyset$ & $\theta$ & 0 & $u$ \\
\hline 77 & $7 \overline{5}$ & 77 & 7.3 \\
\hline 0 & $\theta$ & 0 & (1) \\
\hline () & $\theta$ & $\theta$ & $\theta$ \\
\hline$\emptyset$ & $\theta$ & 0 & 0 \\
\hline 0 & $\theta$ & $\theta$ & $(1)$ \\
\hline$\emptyset$ & $\theta$ & 0 & $\theta$ \\
\hline 0 & $\theta$ & 0 & $\theta$ \\
\hline$\emptyset$ & 0 & 0 & $\theta$ \\
\hline 0 & $\theta$ & $\theta$ & 0 \\
\hline$\theta$ & $\theta$ & $\theta$ & 0 \\
\hline 0 & 0 & (1) & 0 \\
\hline 4 & 4 & 4 & 0 \\
\hline 12,190 & 12,934 & 7.46 & 12,188 \\
\hline$\cdots \cdots-\cdots$ & $-\cdots-\cdots$ & $---\cdots$ & $\cdots-\cdots-\cdots$ \\
\hline 15,894 & 16.385 & 4,683 & 12,554 \\
\hline 358 & 362 & $36 ?$ & 10 \\
\hline 23.6 & 21.5 & 77.4 & 0.9 \\
\hline$E, 4: 37$ & 6,643 & 6,631 & 243 \\
\hline 417.7 & 406.1 & 1418.8 & 35.9 \\
\hline-4485.6 & -4515.5 & -4473.2 & -5657.5 \\
\hline 17.449 & 17.497 & 18.640 & 17.976 \\
\hline 33.0 & $E E .9$ & 87.3 & 220.010 \\
\hline 760 & 760 & 505 & 655 \\
\hline & & 7 & 7 \\
\hline & & SOL_YENT & SOLUENT \\
\hline & & 216.4 & 17164.0 \\
\hline & & 2.7 & 439.4 \\
\hline & & 2.014 & $\therefore 011$ \\
\hline & & 2.014 & $\therefore .014$ \\
\hline & & 53.255 & 35.747 \\
\hline & & 44.979 & \\
\hline & & $\begin{array}{l}0.784 \\
0.17 \%\end{array}$ & $0.0(1) 1$ \\
\hline
\end{tabular}




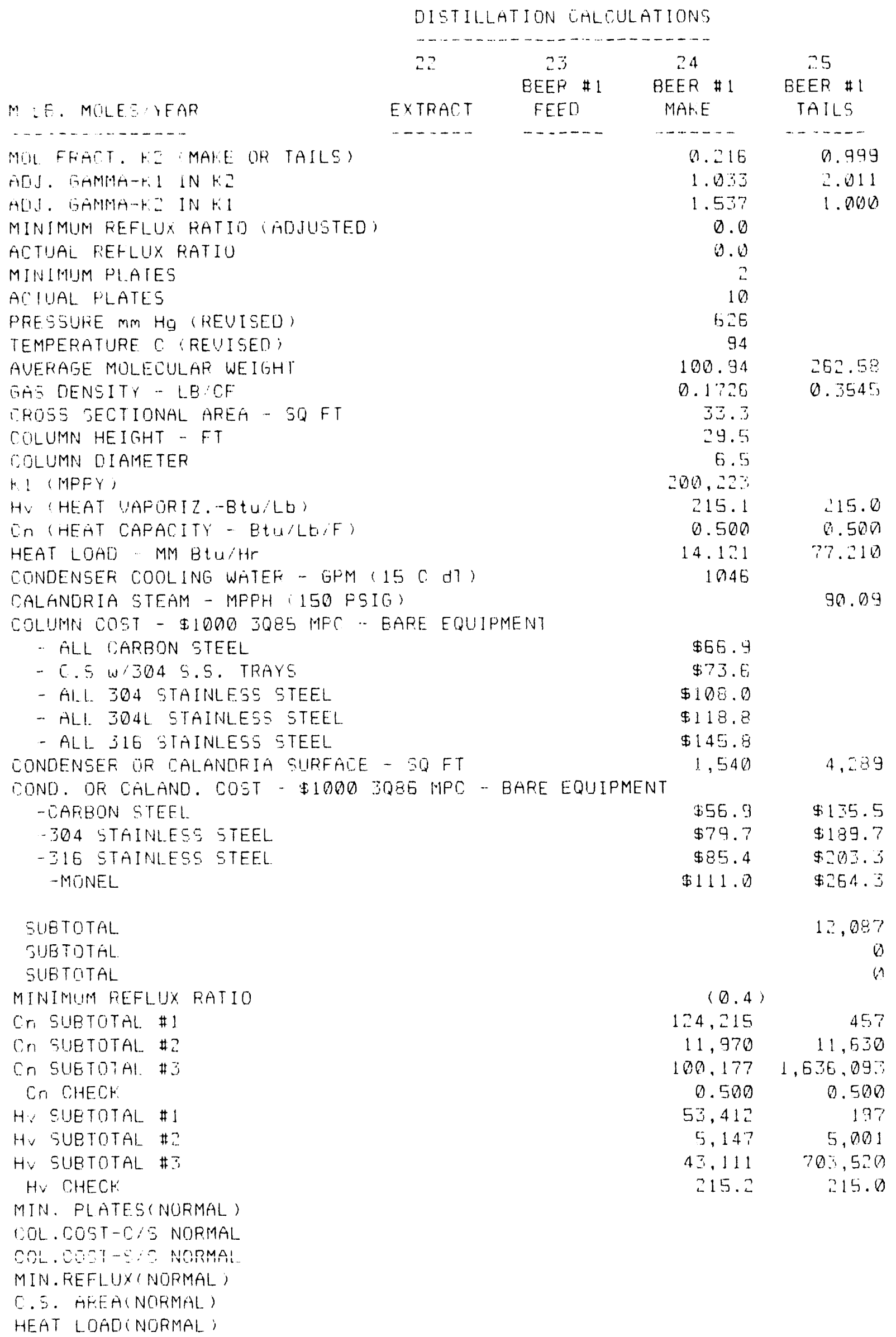


M LB. RIILES YYEAR

CON, LAl. CUST(NORMAL)
DISTILLATION CALCULATIONS
22

\begin{tabular}{cc}
23 & 24 \\
BEER \#1 & BEER \# \\
FEED & MAKE \\
\hline
\end{tabular}

25

BEER \# 1

EXTRACT

-......

-..... 
MLB. MOLES/YEAR

M LE. MOLESIYEAR

COMPONENT $\# 1$
COMPONENT $\# 2$
COMPONENT $\# 3$
COMPONENT $\# 4$
COMPONENT $\# 5$
COMPONENT $\# 6$
COMFONENT $\# 7$
COMPONENT $\# 8$
COMPONENT $\# 9$
COMPONENT $\# 10$
COMPONENT $\# 11$
COMPONENT $\# 12$
COMPONENT $\# 13$
COMPONENT $\# 14$
COMFONENT $\# 15$
COMPONENT $\# 16$
COMPONENT $\# 17$
COMPONENT $\# 18$
COMPONENT $\# 19$
COMPONENT $\# 20$
WATER
SOLUENT

TOTAL (a)

(Storage)

UAPOR PRESS $40(q)$

(Storage)

VAPOR FRESE $120(q)$

BIQ' U.P. CONSTANT

AIG) U.P. CONSTANT

TEMPERATURE $C$

PRESSURE MMHG

H. (COMF'JNENT \#)

HE COMPONENT \#)

$$
\text { U.F.(F.1) }
$$$$
\text { U.P.IHE; }
$$

GAMMA-KI IN HIZ

GAMMA-HI I IN KI

ALPHA

AUG COLUMN ALPHA

MOL FRACT. HI MAKE OR TAILS!

MOL FRACT. HI (FEED)
OISTILLATION CALCULATIONS

$\begin{array}{cccc}\text { LE } & 27 & 28 & 29 \\ \text { BEEF \#2 } & \text { BEEF \#2 } & \text { LBS \#1 } & \text { LBS \#1 } \\ \text { MATE } & \text { TAILS } & \text { MAKE } & \text { TAILS }\end{array}$

DISTILIATION CALCULATIONS

\begin{tabular}{|c|c|c|c|}
\hline 26 & 27 & 28 & 29 \\
\hline BEER \#2 & BEER \#2 & LES \#1 & LES \#1 \\
\hline MAK'E & TAILS & MALIE & TAILS \\
\hline$\ldots-\cdots$ & $\ldots \ldots-\cdots$ & $--\cdots-\cdots$ & $-\cdots \cdots$ \\
\hline 0 & $\theta$ & $\theta$ & 0 \\
\hline 0 & 0 & 0 & 0 \\
\hline 579 & $\theta$ & 673 & E \\
\hline 0 & 0 & 0 & $\theta$ \\
\hline 190 & 0 & 182 & 8 \\
\hline 0 & 0 & 0 & 0 \\
\hline 2,456 & 240 & 12 & $\because, 443$ \\
\hline 6 & 1 & $\theta$ & 6 \\
\hline$\theta$ & 0 & 0 & 0 \\
\hline 4 & 0 & $\theta$ & 4 \\
\hline$\theta$ & 3 & 0 & 0 \\
\hline 0 & 0 & 0 & $\theta$ \\
\hline$\theta$ & 0 & 0 & 0 \\
\hline 0 & 0 & $\theta$ & 0 \\
\hline 0 & 0 & 0 & D \\
\hline$\theta$ & 0 & 0 & 0 \\
\hline$\theta$ & 0 & 0 & 0 \\
\hline 0 & 0 & $\theta$ & 0 \\
\hline 0 & $\theta$ & 0 & $\theta$ \\
\hline$\theta$ & $\theta$ & 0 & 0 \\
\hline 4 & 0 & 0 & 4 \\
\hline 1 & 744 & $\emptyset$ & 1 \\
\hline$-\cdots---$ & $---\cdots$ & ------ & $---\cdots--$ \\
\hline 3,340 & 991 & 868 & 2,473 \\
\hline 348 & 5 & 299 & 49 \\
\hline 104.1 & 4.7 & 344.2 & 19.9 \\
\hline$E, 189$ & 206 & 4,081 & 2,108 \\
\hline 1852.9 & 220.2 & 4704.6 & 852.4 \\
\hline-4427.0 & -5915.7 & -4021.0 & -5779.9 \\
\hline 18.789 & 20.447 & 18.688 & $? 1.455$ \\
\hline 153.7 & 220.0 & $11 E .8$ & 170.0 \\
\hline 4,505 & 4,665 & 4,323 & 4,483 \\
\hline
\end{tabular}

SOLUENT

$76,5,4,3$

$6,5,4,5$

SOI.VENT

7

2707.3

79.8

2.014

2.014

16.870

21.435

0. 999

0.784

$\begin{array}{rrr}17164.0 & 3160.4 & 15156.9 \\ 939.4 & 739.2 & 4492.5 \\ 2.014 & 1.350 & 1.350 \\ 2.014 & 1.350 & 1.350 \\ 26.000 & 3.290 & 4.545 \\ & 3.918 & \\ 0.249 & 0.937 & 0.003 \\ & 0.072 & \end{array}$


M L.P. MOLES/YEAR

MOL FRACT. K. (MAKE OR TAILS)

ADJ. GAMMIAT-KI IN K2

ADJ. GAMMA-KZ IN KI

MINIMUM REFLUX RATIO (ADJUSTED

ACTUAL REFLUX RATIO

MINIMUM PLATES

ACTUAL PLATES

PRESSURE $\mathrm{MM} \mathrm{HG} \mathrm{(REUICED)}$

TEMPERATURE C (REVISED)

AUERAGE MOLECULAR WEIGHT

GAS DENSITY - LB/CF

CROSS SECTIONAL AREA - SQ FT

COLUMN HEIGHT - FT

COLUMN DIAMETER

KI (MPPY)

HV (HEAT VAPORIZ.-BtULL)

CN (HEAT CAPACITY - BtUILD/F,

HEAT LOAD - MM BtuiHr

CONDENSER CUOLING WATEF - GPM

CALANDRIA STEAM - MPDH (150 PSIG)

COLUMN COST - \$1000 3085 MPC - BARE ALL CAREON STEEL

- C.S W/304 S.S. TRAYS

- ALL 304 STAINLESS 5TEEL

- ALL 304L STAINLESS STEEL

AILL $31 B$ STAINLESS STEEL

CONDENSER OF CALANDRIA SURFACE

- CARBON STEEL

- 304 STAINLESS STEEL

31O STAINLESS STEEL

-MONEL
COND. OR CALAND. COST - \$1000 3086

DISTILLATTON GALCULATIONS

\begin{tabular}{|c|c|c|c|}
\hline 26 & 27 & 28 & $\therefore 9$ \\
\hline BEER \#2 & BEER \#2 & L.ES \#1 & LeS \#1 \\
\hline MAKE & TAILS & MATE. & TAILS \\
\hline - & - & 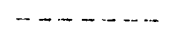 & $\cdots$ \\
\hline 0.001 & 0.752 & 0.063 & 0.997 \\
\hline 1.000 & 1.480 & 1.001 & 1.347 \\
\hline 2.012 & 1.044 & 1.301 & 1.000 \\
\hline 0.0 & & 0.5 & \\
\hline 0.0 & & 0.7 & \\
\hline 3 & & E & \\
\hline 1.3 & & 28 & \\
\hline 4,615 & & 4,372 & \\
\hline 155 & & 117 & \\
\hline 69.27 & 220.11 & 55.78 & 74.00 \\
\hline 0.7479 & 2.0840 & 0.6254 & 0.7493 \\
\hline 7.8 & & 3.0 & \\
\hline 34.0 & & 56.7 & \\
\hline 3.2 & & 2.0 & \\
\hline 182,021 & & 8,394 & \\
\hline 215.2 & 215.0 & 215.0 & 215.3 \\
\hline 0.500 & 0.500 & 0.500 & 0.500 \\
\hline 5.916 & 10.361 & 2.425 & 2.776 \\
\hline 513 & & 180 & \\
\hline & 12.09 & & 3.24 \\
\hline
\end{tabular}

$\$ 42.9$

$\$ 47.1$

$\$ 70.2$

$\$ 77.2$

$\$ 94.8$

193

154

385

MPC - BAFE EQUIFMENT

\$2. 3

$\$ 31.3$

$\$ 33.5$

$\$ 43.5$

$(0.8)$

115,113

364

247

0.500

49,499

$15 \%$

14.2

215.2

HV CHECH:

MIN. PLATESINORMAL)

COL. COST-C.S MURMAL

COL. COST SIS NGRMÁ.

MIN.REFLUX (NORMAL)

C.S. AREA ( NORMAL)

HEAT LOAO (NORMAL)
$\$ ? 3.5$

$\$ 39.9$

$\$ 42.7$

$\$ 55.5$

$\$ 15.5$

\$2.1.?

$\$ 23.3$

$\$ 30.2$

9,139

0

$\$ 14.0$

$\$ 19.6$

$\$ 21.0$

$\$ 27.2$

$90,95 ?$

33

33

0.5

$\begin{array}{rrr}9.161 & 24.196 & 30.918 \\ 36 & 0 & 354 \\ 99.929 & 0 & 247 \\ 0.500 & 0.500 & 0.500 \\ 3.91 .2 & 10.404 & 39.095 \\ 16 & 0 & 157 \\ 42.970 & 0 & 142 \\ 215.0 & 215.0 & 215.3\end{array}$

43

$? \theta$ 
M I. . MOLE:YEAR

CON: LHL OOST(NORMAL)

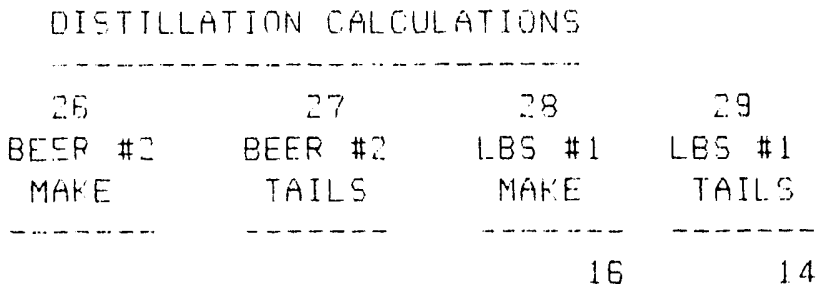


M L.B. MOL.ES/YEAR

$M$ LE. MOLES/YEAR

\begin{tabular}{|c|c|}
\hline COMPONENT & $\# 1$ \\
\hline COMPONENT & $\# 2$ \\
\hline COMPONENT & $\# 3$ \\
\hline COMPONENT & $\# 4$ \\
\hline COMPONENT & $\# 5$ \\
\hline COMPONENT & $\# 6$ \\
\hline COMPONENT & $\# 7$ \\
\hline COMPONENT & $\# 8$ \\
\hline COMPONENT & $\# 9$ \\
\hline COMPONENT & $\# 10$ \\
\hline COMPONENT & \#11 \\
\hline COMPONENT & $\# 12$ \\
\hline COMPONENT & $\# 13$ \\
\hline COMPONEN ${ }^{-}$ & $\# 14$ \\
\hline COMPONENT & $\# 15$ \\
\hline COMPONENT & $\# 16$ \\
\hline COMPONENT & $\# 17$ \\
\hline COMPONENT & $\# 18$ \\
\hline COMPONENT & $\# 19$ \\
\hline $\begin{array}{l}\text { COMPONENT } \\
\text { WATER } \\
\text { COI UFNT }\end{array}$ & $\# 2 \theta$ \\
\hline
\end{tabular}

TOTAL (q)

(Storage)

UAPOR PRESS 40 (q)

(storage)

UAPOR PRESS 120 (q)

$B(q)$ U.P. CONETANT

A (q) U.P. CONSTANT

TEMPERATUFE. C

PRESSURE MMHG

HI (COMPONENT \#)

$H 2$ (COMPINENT \#)

U.P. (k)

U.F.KZ)

GAMMA-KI IN K2

GAMMA-KL IN KL

กiLfrín

AUG COLLMP ILPHA

MOL FRACT. HI MAHE DR TAILS;

MOL FRACT. HL (FECD)
DISTILLATION CALCULATIONS

$\begin{array}{cccc}30 & 31 & 32 & 33 \\ \text { LBS } \# 2 & \text { LBS \#2 } & \text { LBS \#3 } & \text { LBS \#3 } \\ \text { MAKE } & \text { TAILS } & \text { MAKE } & \text { TAILS } \\ \text { DISTILLATION TALCULATIONS }\end{array}$

\begin{tabular}{|c|c|c|c|}
\hline 30 & 31 & 32 & 33 \\
\hline LBS \#2 & LBS \#2 & LBS \#3 & LBS \#3 \\
\hline MAKIE & TAILS & MAK'E. & TAILS \\
\hline$-\cdots-\cdots$ & $-\cdots-\cdots$ & $\cdots \cdots-\cdots$ & $\cdots-\cdots$ \\
\hline 0 & $\emptyset$ & $\emptyset$ & 0 \\
\hline$\theta$ & $\theta$ & $\emptyset$ & D \\
\hline 670 & 3 & $\emptyset$ & 0 \\
\hline$\theta$ & 0 & $\theta$ & $\theta$ \\
\hline 2 & 181 & $\emptyset$ & 0 \\
\hline$\theta$ & 0 & b & $\theta$ \\
\hline$\theta$ & 12 & 0 & $n$ \\
\hline 0 & $D$ & $\theta$ & $\theta$ \\
\hline$\emptyset$ & 0 & Q & $\emptyset$ \\
\hline$\theta$ & 0 & 0 & 8 \\
\hline 0 & $\emptyset$ & $\theta$ & $\alpha$ \\
\hline 0 & $\theta$ & $\theta$ & $\theta$ \\
\hline 0 & 0 & 0 & (1) \\
\hline$\theta$ & $\theta$ & 0 & $\theta$ \\
\hline 0 & (7) & $v$ & $\emptyset$ \\
\hline 0 & Q & $\theta$ & 0 \\
\hline$\theta$ & $\emptyset$ & 0 & 0 \\
\hline$\theta$ & 0 & $\theta$ & (1) \\
\hline 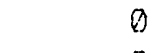 & 0 & $\theta$ & $\theta$ \\
\hline$\emptyset$ & $D$ & 0 & 0 \\
\hline$\emptyset$ & $\theta$ & (1) & $\theta$ \\
\hline$\emptyset$ & $\theta$ & $\theta$ & $\theta$ \\
\hline$c-$ & $--\cdots+$ & - & 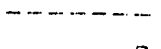 \\
\hline 671 & 196 & $\theta$ & Q \\
\hline
\end{tabular}
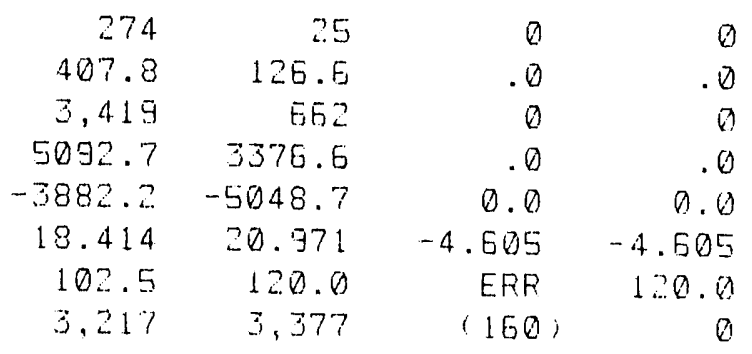

$4,5,6$

3.19 .7

1926.5

1.492

1.492

1. 12.5

1. .2.6

0. $99 \%$

0.787

$$
4,5,5
$$

5096.7

3517.3

1.492

1.4. 42

$\therefore 130$

0.019
3
3

ERE $\quad 1.0$

ERP 5096.7

$1.000 \quad 1.000$

1.0001 .000

EFFE EFF
ERR ERR

0.000 


\begin{tabular}{|c|c|c|c|c|}
\hline & $\begin{array}{l}\mathrm{CI} \\
-\cdots \mathrm{TI}\end{array}$ & LATION & LCULATION & \\
\hline & 30 & 31 & 32 & 33 \\
\hline & LBS \#2 & LES \#2. & LBS \#J & LES \#ذ \\
\hline M LB. MULES: MEAH & MAT.E. & TAILS & MATE. & TAILS \\
\hline$\ldots \ldots \ldots \ldots$ & $\ldots \ldots$ & $-\cdots-\cdots$ & $\ldots \ldots-\cdots$ & $-\cdots-\cdots$ \\
\hline MOL FFACT, FE MHFE OF TAILE: & 0.003 & $0.98 ?$ & ERR & ERR \\
\hline ADJ. GrimMA-FI IN KI & 1.000 & 1.470 & ERF & ERP \\
\hline ALJ. GAMMA-F IN KL & 1.489 & 1. $\operatorname{lng}$ & EPR & ERR \\
\hline WINIMUM FEFLUX FATIO :ADJUSTED & 0.0 & & ERP & \\
\hline ACTUAL FEFL FATID & 0.0 & & ERR & \\
\hline MINIMUM PLATES & 20 & & 0 & \\
\hline ACTUAL FLATES & 92 & & 3 & \\
\hline PRESGIRE MM HE AFEUISEO: & 3.008 & & Q & \\
\hline TEMHEFATIIRE C :REUISEO; & 100 & & $(273)$ & \\
\hline AUEFGGE MOLESULAF WE IGHT & 58.05 & 49.83 & ERP & EFR \\
\hline GAS LEMITY $\cdots$ LESCF & $0.4 E 8 ?$ & 0.4129 & ERR & ERP \\
\hline CROE = SECTYOHAL APEA - CQFT & 1.7 & & 0.0 & \\
\hline OOL UNM HE IGHT - FT & $15 \therefore .3$ & & 0.0 & \\
\hline ODLUMI OIAMETEF & 1.5 & & 0.0 & \\
\hline F. : MFF, ; & $\therefore 8,692$ & & 0 & \\
\hline H. HEAT WAFOFIZ. -EtulLb: & 215.0 & $\therefore 15.0$ & ERF & EFE \\
\hline ¿n HEAT OHFACTTY - EtULEIF: & 0.500 & 0.500 & ERR & ERI? \\
\hline HEAT LMAD - MM Eti Hr & 1.154 & 1.167 & 0.000 & 0.000 \\
\hline CONDENEEP COOLINE WATER GPM & $8 E$ & & 0 & \\
\hline CALANUFIA STEAM - MFFH : $15 D$ FEI & IE ; & $1 . \pm 6$ & & $Q .0 Q$ \\
\hline 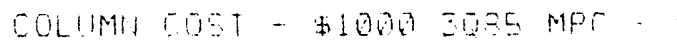 & EAAFE EQU & IPMENT & & \\
\hline - ALL TAFEON ETEEL & 韦PE.己 & & $\$ 0.0$ & \\
\hline-5.5 a $304=.5$. TFirs & $\$ 83.8$ & & $\$(\lambda .0)$ & \\
\hline .. MLLL . JO4 ETHIHLESE STEEL & $\$ 123.5$ & & $\$ 0.0$ & \\
\hline - MLL BUHL GTAINEEE- STFEL & $\$ 134.8$ & & \$0. & \\
\hline - ÍLL ZIS ETÁINLESS ETEE!. & $\$ 165.4$ & & $\$ 0.0$ & \\
\hline EONLENEFF OF GHLANDPIA SUFFACE & 115 & 65 & Q & \\
\hline ONLE DK ALANL GOET - $\$ 10003$ & $3096 \quad M P C$ & - BÄFE ED & IFMENT & \\
\hline -AFEON ETEEL. & $\$ 12.3$ & \pm 10.0 & कQ & $\$ 0.0$ \\
\hline JOA ZTHEESE ETEEI & $\$ 1 \% .3$ & $\$ 14.0$ & $\$ 0.0$ & $\$ 0.0$ \\
\hline ZIE STAINLESS GTEFL & $\$ 18.5$ & $\$ 15.9$ & $\$ 0 . Q$ & $\$ 0.0$ \\
\hline -MDNEL & $\$ 24 \cdot 1$ & $\$ 19.5$ & $\$ 0.0$ & $\$ Q .0$ \\
\hline$=1 E T O A L$ & & 455 & & \\
\hline G!ETOTHL & & 0 & & \\
\hline$=$ EETSTHL. & & 0 & & \\
\hline MIHIMUA PEFLUX FATIO & $(\theta . E)$ & & ERF & \\
\hline$\therefore r$ EUETGTAL \#1 & 19,455 & 4,711 & $\theta$ & 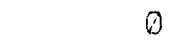 \\
\hline AO SUETOTHL H. & $\theta$ & 0 & $\hat{v}$ & \\
\hline EO EUETATHI \# & $\theta$ & $\theta$ & 0 & 0 \\
\hline Er EHELH & 0.500 & 0.500 & ERP & ERF \\
\hline H. VIPTTtAL $\# 1$ & 9,579 & $\because 0 \Omega E$ & $\theta$ & \\
\hline H: IJETOTAL \#? & 0 & $\theta$ & $\theta$ & 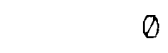 \\
\hline H. =UETETAL \#Z & 0 & i) & $\emptyset$ & \\
\hline H. SHE H & $\because 15.0$ & $=15.0$ & EFF & ERF \\
\hline MIM. Fl RTE :UPMAL) & & & & \\
\hline 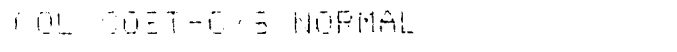 & & & & \\
\hline 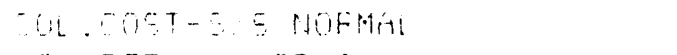 & & & & \\
\hline MUA. FEFIUX MOFMAL & & & & \\
\hline$\therefore \bar{A}$ APEA ROFMAL: & & & & \\
\hline 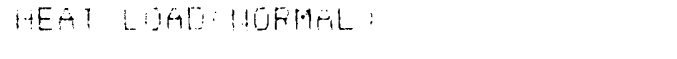 & & & & \\
\hline
\end{tabular}


M LB. MOLESIYEAR

CON/CAL COST(NORMAL)

\begin{tabular}{cccc}
\multicolumn{4}{c}{ DISTILLATION } \\
\hline 30 & 31 & 32 & 33 \\
LBS \#2 & LBS \#2 & LBS \#3 & LBS \#3 \\
MAKE & TAILS & MAKE & TAILS
\end{tabular}


M LB. MOLESIYEAR

M LE. MOLES Y YEAF

COMPONENT \#1
COMPONENT \#2
COMPONENT $\# 3$
COMPONENT $\# 4$
COMPONENT $\# 5$
COMPONENT $\# 5$
COMPONENT $\# 7$
COMPONENT $\# 8$
COMPONENT $\# 9$
COMPONENT $\# 10$
COMPONENT $\# 11$
COMPONENT \#12
COMPONENT $\# 13$
COMPONENT $\# 14$
COMPONENT $\# 15$
COMPONENT $\# 16$
COMPONENT $\# 17$
COMPONENT \#18
COMPONENT \#19
COMPONENT $\# 20$
WATER
SOLUENT
TOTAL (

(Storage)

UAPOR PFESS 4 (Q) (q)

(Storage)

UAFOR PRESS 120 (a)

B(a) U.P. CONSTANT

A!q) U.P. CONSTANT

TEMPERATURE C

PRESSURE MMHG

H1 (COMPONENT \#)

H. (COMPONENT \#)

U.P. (H)

U.P. (KI)

GAMMA-HI IN KL

GHPIVIA-RI IN $\because 1$

ALPHA

AUG COLUMN ALPHA

MOL FFIGT. HI (MAKE ORE TAILS;

MOL FRACT. HI (FEEC)
DISTILLATION CALCULATIONS

\begin{tabular}{cc}
34 & 35 \\
HBS \#1 & HBS \#1 \\
MAKE & TAILS \\
DISTILLATION LALCULATIONS \\
34 & 35 \\
MAS \#1 & HBS \#1 \\
\hline
\end{tabular}

$\begin{array}{rr}10 & 0 \\ 27.7 & 0.1 \\ 247 & 1 \\ 678.4 & 16.7 \\ -4919.6 & -7768.0 \\ 19.038 & 22.581 \\ 123.6 & 220.0 \\ 760 & 920 \\ 10.9 .8 & 10.9,8 \\ 501.15 N 1 & 50 L V E N \\ 184.5 & 3815.4 \\ 19.9 & 939.4 \\ 2.117 & 2.117 \\ 2.858 & 2.859 \\ 3.247 & 8.598 \\ 5.923 & \\ 0.999 & .000 \\ 0.00 E & \end{array}$


M L.E. MOLES/YEAR

MUL FRACT. KO

MULL FRAOT. HZ (MAKE OR TAILS)

ADJ. GAMMA-KI IN K2

ADJ. GAMMA-KZ IN KI

MINIMUM REFLUX FATIO ¿ADJUSTED

ACTUAL REFLUX RATIO

MINIMUM PLATES

ACTUAL. PLATES

PRESSURE MM HQ (REUISED)

TEMPERATURE C (REUISED)

AUERAGE MOLECULAR WEIGHT

GAS DENSITY - LB/CF

CROSS SECIIONAL AREA - SQ FT

COLUMN HEIGHT - FT

COLUMN DIAMETER

H.1 IMPPY)

Hv (HEAT UAPORIZ.-Btu/Lb)

CN (HEAT CAFACITY - Btu/Lb/F)

HEAT LOAD - MM BtU/Hr

CONDFISEF COOLING WATER - GPM

CALFNURIA STERM - MPPH ( 150 PSIG)

COLUMN COST - $\$ 10003 Q 85 \mathrm{MPC}$ - BARE

- ALL CARBON STEEL

- C.S w/304 5.5. TRAYS

- ALL 304 STAINLESS STEEL

- ALL 304L STAINLESS STEEL

- ALL. 316 STAINLESS STEEL

CONDENSER OR CALANDRIA SURFACE

COND. OP. CALAND. COST - \$1000 3086

-CARBON STEEL.

-3Ө4 STAINLESS STEEL

- 31E STAINLESS STEEL.

-MDINEL

SUBTOTAL.

SUBTOTAL

SUETOTAL

MINIMUM REFLUX RATIO

Cn SUIETOTAL. \#1

Cn SUBTOTAL \#2

Cn SUETOTAL \#3

CN CHECH

HV SUBTOTAL \#1

HV SUBTOTAL \#2

HV SUETOTAL \#.3

HV CHECK.

MIN. PLATES (NOKMAL)

COL.COST-C/S NORMAL

COL. COST-SIS NORMAL.

MIN.REFLUX (NORMAL)

C.5. AREA(NORMAL)

HEAT LOAD(NORMAL)
DISTILLATION CALCULATIONS
34

HES \#

MAKE

-...-.

0.001

1.000

2.854

8.7

10.9

10

45

741

123

66.17

0.1239

23.8

82.5

5.5

215.0

0.500

8.576

636
35

HBS \#1

TAILS

$\cdots \cdots \cdots$

1.000

2. 117

1. 390

268.42

0.5014

215.0

0.500

8.576
10.01

$\$ 144.5$

$\$ 158.9$

$\$ 229.9$

$\$ 252.9$

$\$ 310.3$

642

MPC - BARE EQUIPMENT

$\$ 30.5 \quad \$ 25.3$

$\$ 42.8 \$ 35.5$

$\$ 45.8 \$ 38.0$

$\$ 59.6 \quad \$ 49.4$

65
6
6

$\begin{array}{rr}8.7 & \\ 457 & 0 \\ 11.572 & 58 \\ 6 & 1,636.087 \\ 0.500 & 0.550 \\ 1.97 & 0 \\ 4.976 & 25 \\ 3 & 703.517 \\ 2.5 .0 & 290.0\end{array}$


M LB. MOLES/YEAR

DISTILLATION GALLCULATIONS

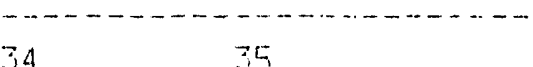

$34 \quad 35$

HBS \#1 HES \#1

MAKE TAILS

CON!CAL COSTINORMAL) 
APPENDIX G. EXTERNAL EXTRACTOR MODE NUMBER OF EXTRACTOR STAGES REQUIRED FOR VARIOUS SOLVENT/FEED RATINS AND YIELD TO EXTRACT 


Solvent
Water
Ratio

0.11
0.15
0.20
0.30
0.40
0.50
1.00
1.50
2.00
2.50
3.00
3.50

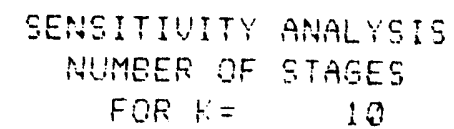

70.00\% Solute Yald as is of solute in feedi

$\begin{array}{rrrrrrrr}70.00 \% & 90.00 \% & 90.00 \% & 95.00 \% & 98.00 \% & 99.00 \% & 99.90 \% & 99.99 \% \\ 3 & 3 & 5 & 9 & 17 & 20 & 20 & 220 \\ 2 & 3 & 4 & 5 & 8 & 9 & 15 & 20 \\ 2 & 2 & 3 & 4 & 5 & 6 & 10 & 13 \\ 1 & 2 & 2 & 3 & 4 & 4 & 5 & 9 \\ 1 & 2 & 2 & 2 & 3 & 4 & 5 & 7 \\ 1 & 1 & 2 & 2 & 3 & 3 & 5 & 5 \\ 1 & 1 & 1 & 2 & 2 & 2 & 3 & 4 \\ 1 & 1 & 1 & 2 & 2 & 2 & 5 & 4 \\ 1 & 1 & 1 & 1 & 2 & 2 & 3 & 4 \\ 1 & 1 & 1 & 1 & 2 & 2 & 3 & 3 \\ 1 & 1 & 1 & 1 & 2 & 2 & 7 & 3 \\ 1 & 1 & 1 & 1 & 2 & 2 & 2 & 3\end{array}$

Solvert:
water
Ratio

\begin{tabular}{|c|c|c|c|c|c|c|c|}
\hline & & SE & $\begin{array}{l}\text { ASITIUITY } \\
\text { UUMBER OF } \\
\text { FOR K= }\end{array}$ & $\begin{array}{c}\text { ANAL YS } \\
\text { STAGES } \\
5\end{array}$ & & & \\
\hline & Solut & E Ýield & as \% of & solute & in feed? & & \\
\hline $70.80 \%$ & $30.08 \%$ & $90.00 \%$ & $95.00 \%$ & $98.00 \%$ & $93.00 \%$ & $93.90 \%$ & $99.99 \%$ \\
\hline 3 & $I$ & $E$ & 10 & 19 & $32 \pi$ & $>20$ & $\geq 20$ \\
\hline 2 & 3 & 5 & 8 & 13 & 16 & $\geq 20$ & $>20$ \\
\hline 2 & 2 & 3 & 4 & s & 7 & $=11$ & 15 \\
\hline 1 & 2 & 3 & 3 & 4 & 5 & 3 & 10 \\
\hline 1 & 2 & 2 & $\Xi$ & 4 & 4 & 5 & 号 \\
\hline 1 & 1 & 2 & 2 & 3 & 3 & 4 & 5 \\
\hline 1 & 1 & 1 & 2 & 2 & 3 & 4 & 5 \\
\hline 1 & 1 & 1 & 2 & 2 & 2 & 3 & 4 \\
\hline 1 & 1 & 1 & 2 & 2 & 2 & $\Xi$ & 4 \\
\hline 1 & 1 & 1 & 2 & 2 & 2 & $\Xi$ & 4 \\
\hline 1 & 1 & 1 & 1 & 2 & $z$ & 3 & 4 \\
\hline 1 & 1 & 1 & 1 & 2 & 2 & 3 & 3 \\
\hline
\end{tabular}

Solvent,
Water
Ratio

SENSITIUITY ANALYSIS

NUMMEE OF STAGES

FOR $R=4.3$ Solute Yield he \% of solute in feed:

\begin{tabular}{|c|c|c|c|c|c|c|c|c|}
\hline 0.24 & 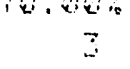 & $\begin{array}{c}50.60 \\
5\end{array}$ & $\begin{array}{c}56 \\
5\end{array}$ & $\begin{array}{c}95.00 \% \\
11\end{array}$ & $\begin{array}{c}93.00 \% \\
220\end{array}$ & $\begin{array}{c}39.0 \times 10 \\
>20\end{array}$ & $\begin{array}{c}39.90 \% \\
>20\end{array}$ & $\begin{array}{c}39.99 \% \\
>20\end{array}$ \\
\hline 0.30 & 2 & $\exists$ & 5 & 7 & 10 & 13 & $\geq 20$ & $>20$ \\
\hline 0.40 & 2 & 2 & $j$ & 5 & E & 7 & 12 & 15 \\
\hline 0.50 & 2 & 2 & 3 & 4 & 5 & $E$ & 9 & 12 \\
\hline 1.80 & 1 & 1 & 2 & 2 & 3 & J & 5 & 7 \\
\hline 1. 50 & 1 & 1 & 2 & 2 & 3 & 3 & 4 & 5 \\
\hline 2.00 & 1 & 1 & 2 & 2 & $?$ & $\Xi$ & 4 & 5 \\
\hline 2.50 & 1 & 1 & 1 & 2 & 2 & 2 & 5 & 4 \\
\hline 3.00 & 1 & 1 & 1 & ?. & $?$ & 2 & $\Xi$ & 4 \\
\hline 3.50 & 1 & 1 & 1 & 2 & L & 2 & $\Xi$ & $A$ \\
\hline 4.00 & 1 & 1 & 1 & 2 & 2 & 2 & 3 & 4 \\
\hline 4.50 & 1 & 1 & 1 & 1 & 2 & $I$ & 3 & 4 \\
\hline
\end{tabular}




$$
\begin{aligned}
& \text { Solvent } \\
& \text { water } \\
& \text { Ratio } \\
& \\
& 0.53 \\
& 1.00 \\
& 1.50 \\
& 2.00 \\
& 2.50 \\
& 3.00 \\
& 3.50 \\
& 4.00 \\
& 4.50 \\
& 5.00 \\
& 5.50 \\
& 6.00
\end{aligned}
$$

Solvent/ Water Rat 10

1.05

1.50

2.00

2.50

3.00

3.50

4.00

4.50

5.00

5.50

6.00

5. 50

Solvent/ Water

Rat 10

1.40

\begin{tabular}{|c|c|c|c|c|c|c|c|}
\hline \multicolumn{8}{|c|}{ as } \\
\hline $70.00 \%$ & $80.00 \%$ & $90.00 \%$ & $95.00 \%$ & $98.00 \%$ & $99.00 \%$ & 99.902 & $99.99 \%$ \\
\hline 3 & 4 & 7 & 13 & 20 & $>20$ & $>20$ & $>20$ \\
\hline 2 & 2 & 3 & 4 & 5 & 6 & 9 & 13 \\
\hline 1 & 2 & 2 & 3 & 4 & 4 & 6 & 9 \\
\hline 1 & 1 & 2 & 2 & 3 & 4 & 5 & 7 \\
\hline 1 & 1 & 2 & 2 & 3 & 3 & 5 & 6 \\
\hline 1 & 1 & 2 & 2 & 3 & 3 & 4 & 6 \\
\hline 1 & 1 & 2 & 2 & 2 & 3 & 4 & 5 \\
\hline 1 & 1 & 2 & 2 & 2 & 3 & 4 & 5 \\
\hline 1 & 1 & 1 & 2 & 2 & 3 & 4 & 5 \\
\hline 1 & 1 & 1 & 2 & 2 & 2 & 3 & 4 \\
\hline 1 & 1 & 1 & 2 & 2 & 2 & 3 & 4 \\
\hline 1 & 1 & 1 & 2 & 2 & 2 & 3 & 4 \\
\hline
\end{tabular}

1.50

2.00

2.50

3.00

3.50

4. .00

4.50

5.00

5.50

6.00

6.50
SENSITIUITY ANALYSIS

NUMBER OF STAGES

FOR $K=\quad 2$

$\begin{array}{cccccccc}70.00 \% & 80.00 \% & 90.00 \% & 95.00 \% & 98.00 \% & 99.00 \% & 99.90 \% & 99.99 \% \\ 3 & 4 & 8 & 14 & 20 & >20 & >20 & .>20 \\ 2 & 3 & 4 & 5 & 8 & 9 & 15 & 20 \\ 2 & 2 & 3 & 4 & 5 & 6 & 9 & 13 \\ 1 & 2 & 3 & 3 & 4 & 5 & 7 & 10 \\ 1 & 2 & 2 & 3 & 4 & 4 & 6 & 9 \\ 1 & 2 & 2 & 3 & 3 & 4 & 6 & 8 \\ 1 & 1 & 2 & 2 & 3 & 4 & 5 & 7 \\ 1 & 1 & 2 & 2 & 3 & 3 & 5 & 6 \\ 1 & 1 & 2 & 2 & 3 & 3 & 5 & 6 \\ 1 & 1 & 2 & 2 & 3 & 3 & 4 & 6 \\ 1 & 1 & 2 & 2 & 3 & 3 & 4 & 6 \\ 1 & 1 & 2 & 2 & 2 & 3 & 4 & 5\end{array}$

SENSITIUITY ANALYSIS

NUMBER OF STAGES

FOR $K=0.75$
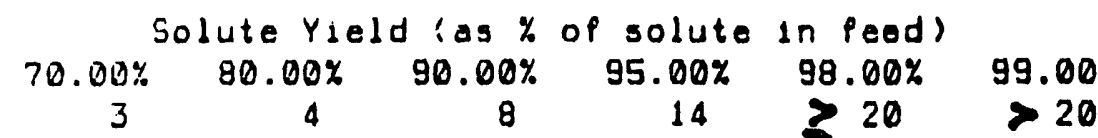

$>20$

8
6

10

16

$>20$

$\begin{array}{rr}902 & 99.99 x \\ 20 & >20 \\ 9 & 13 \\ 6 & 9 \\ 5 & 7 \\ 5 & 6 \\ 4 & 6 \\ 4 & 5 \\ 4 & 5 \\ 4 & 5 \\ 3 & 4 \\ 3 & 4 \\ 3 & 4\end{array}$

34

2

3

5

$$
8
$$

6

15

10

2

3

3

3

3

3

2 


Solvent'
Water
Ratio
2.10
2.50
3.00
3.50
4.00
4.50
5.00
5.50
5.00
5.50
7.00
7.50

Solvent/ Water Rat 10

4.20

4.50

5.00

5.50

6.00

6.50

7.00

7.50

8.00

8.50

9.00

9.50
SENSITIUITY ANALYSIS

NUMBER OF STAGES

FOR $K=0.5$

$\begin{array}{ccccccccc}7 & \text { Solute Yield (as } \% \text { of solute in feed) } & & & \\ 70.00 \% & 80.00 \% & 90.00 \% & 95.00 \% & 98.00 \% & 99.00 \% & 99.90 \% & 99.99 \% \\ 3 & 5 & 9 & 15 & \geq 20 & 720 & -20 & >20 \\ 2 & 3 & 5 & 8 & 11 & 14 & 20 & >20 \\ 2 & 3 & 4 & 5 & 8 & 9 & 15 & 20 \\ 2 & 2 & 3 & 4 & 6 & 7 & 11 & 15 \\ 2 & 2 & 3 & 4 & 5 & 6 & 9 & 13 \\ 2 & 2 & 3 & 3 & 5 & 5 & 8 & 11 \\ 1 & 2 & 3 & 3 & 4 & 5 & 7 & 10 \\ 1 & 2 & 2 & 3 & 4 & 5 & 7 & 9 \\ 1 & 2 & 2 & 3 & 4 & 4 & 6 & 8 \\ 1 & 2 & 2 & 3 & 3 & 4 & 6 & 8 \\ 1 & 2 & 2 & 3 & 3 & 4 & 6 & 8 \\ 1 & 2 & 2 & 3 & 3 & 4 & 5 & 7\end{array}$

SENSITIUITY ANALYSIS

NUMBER OF STAGES

FOR $K=0.25$

$\begin{array}{cccccccc}\text { Solute Yleld (as \% of solute in foed) } & & & \\ 70.00 \% & 80.00 \% & 90.00 \% & 95.00 \% & 98.00 \% & 99.00 \% & 99.90 \% & 99.99 \% \\ 2 & 5 & 9 & 16 & 220 & >20 & >20 & >20 \\ 2 & 4 & 6 & 10 & 16 & 220 & >20 & 720 \\ 2 & 3 & 5 & 7 & 11 & 14 & 220 & >20 \\ 2 & 3 & 4 & 6 & 9 & 11 & 18 & 720 \\ 2 & 3 & 4 & 5 & 7 & 9 & 15 & 220 \\ 2 & 2 & 4 & 5 & 7 & 8 & 13 & 17 \\ 2 & 2 & 3 & 4 & 6 & 7 & 11 & 15 \\ 2 & 2 & 3 & 4 & 6 & 7 & 10 & 14 \\ 2 & 2 & 3 & 4 & 5 & 6 & 9 & 13 \\ 2 & 2 & 3 & 4 & 5 & 6 & 9 & 12 \\ 2 & 2 & 3 & 3 & 5 & 5 & 8 & 11 \\ 1 & 2 & 3 & 3 & 4 & 5 & 8 & 10\end{array}$




\section{INTERNAL DISTRIBUTION}

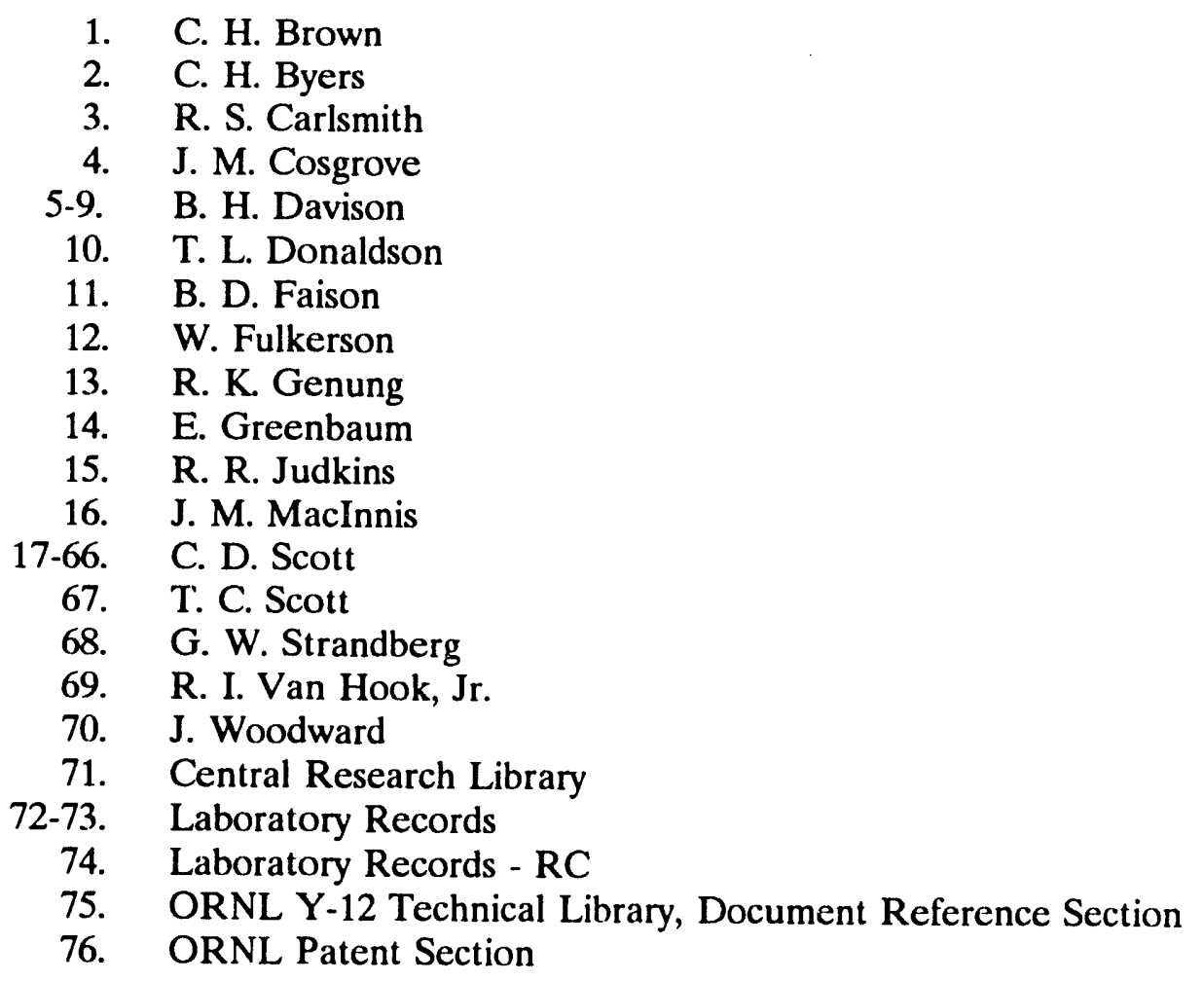

\section{EXTERNAL DISTRIBUTION}

77-101. R. M. Busche, Bio En-Gene-Er Associates, Inc., 533 Rothbury Rd., Wilmington, DE 19803-2439

102. P. Dugan, Idaho National Engineering Laboratory, P. O. Box 1625, Idaho Falls, ID 83415

103-127. M. F. Erker, National Corn Growers Association, 1000 Executive Parkway, Ste. 105, St. Louis, MO 63141

128-137. M. E. Gunn, Jr., Office of Industrial Technologies, U.S. Department of Energy, Forrestal Building, 1000 Independence Avenue, SW, Washington, DC 20585

138. E. E. Hoffman, U.S. Department of Energy, DOE Field Office, Oak Ridge, P. O. Box 2001, Oak Ridge, TN 37831-8600

139. L. Johnson, Idaho National Engineering Laboratory, P. O. Box 1625, Idaho Falls, ID 83415 
140. L. Keay, U.S. Department of Energy - Idaho Operations Office, 1 Energy Drive, Idaho Falls, ID 93402-1220

141. J. Kintzle, National Corn Growers Association, 1000 Executive Parkway, Ste. 105, St. Louis, MO 63141

142. Office of Assistant Manager, U.S. Department of Energy, DOE Field Office, Oak Ridge, P. O. Box 2001, Oak Ridge, TN 37831-8501

143. Lee R. Lynd, Dartmouth College, Hanover, New Hampshire

144. O. P. Manley, U.S. Department of Energy, GTN/ER-15, Washington, DC 20545

145-154. G. Petersen, Jet Propulsion Laboratory, M/S 89-2, 1800 Oak Grove Drive, Pasadena, CA 91109

155. W. M. Polansky, U.S. Department of Energy, GTN/ER-16, Washington, DC 20545

156. P. H. Salmon-Cox, Office of Industrial Technologies, U.S. Department of Energy, Forrestal Building, 1000 Independence Avenue, SW, Washington, DC 20585

157. W. W. Schertz, Argonne National Laboratory, 9700 S. Cass Avenue, ES/362, Argonne, IL 60439-4816

158. D. E. Wilcy, Office of Industrial Technologies, U.S. Department of Energy, Forrestal Building, 1000 Independence Avenue, SW, Washington, DC 20585

159. J. Wise, Battelle-Pacific Northwest Laboratory, P. O. Box 999, MS K6-66, Richland, WA 99352

160. C. E. Wyman, Solar Energy Research Institute, 1617 Cole Boulevard, Golden, Colorado 80401

161-170. Office of Scientific and Technical Information, P. O. Box 62, Oak Ridge, TN 37831 

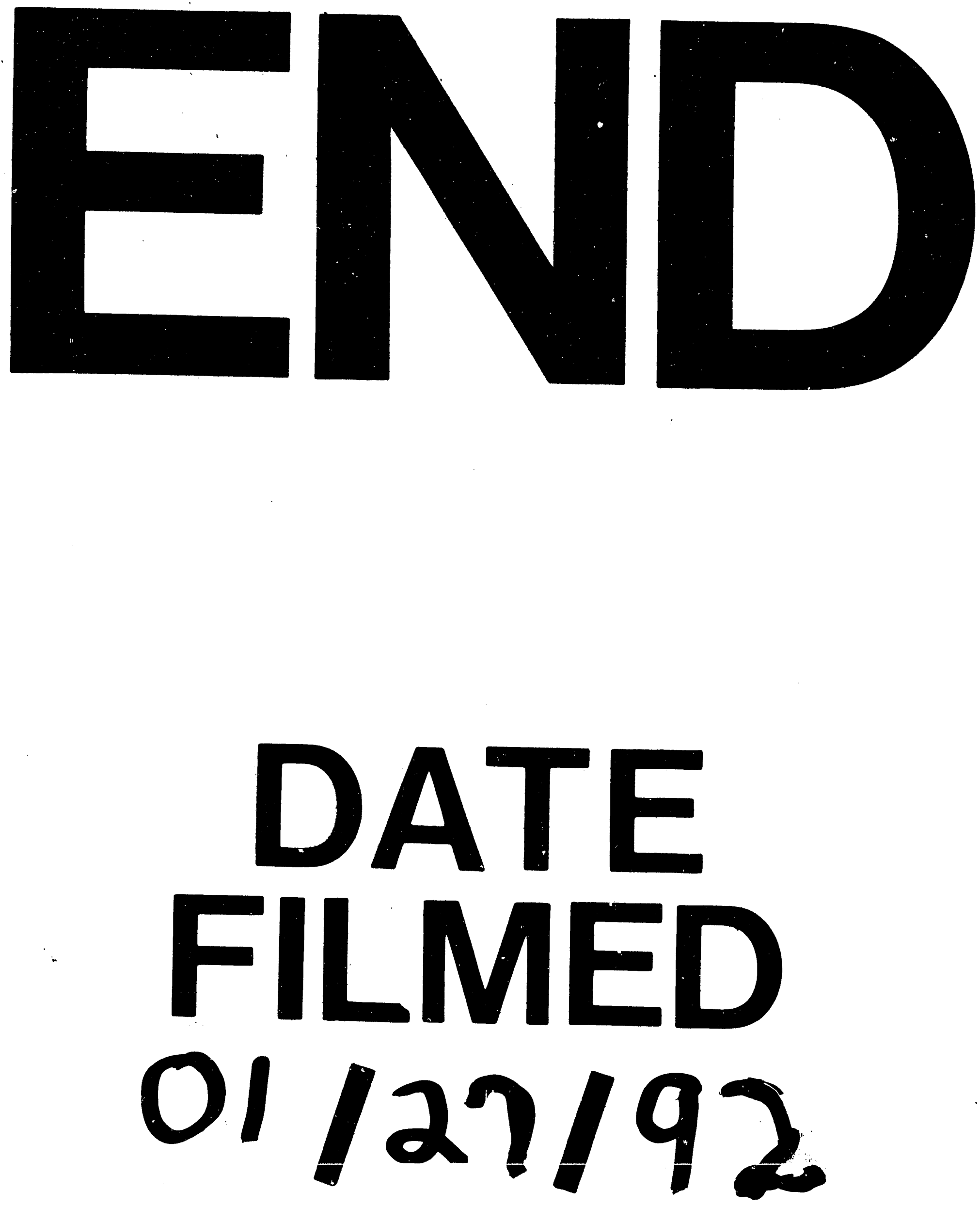
